\title{
ÍNDICE DE NÃO-DETECÇÃO DE ERROS GROSSEIROS NO PROCESSO DE ESTIMAÇÃO DE ESTADO EM SISTEMAS ELÉTRICOS DE POTÊNCIA
}

Tese apresentada à Escola de Engenharia de São Carlos da Universidade de São Paulo, como parte dos requisitos para obtenção do título de Doutor em Ciências, Programa de Engenharia Elétrica.

Área de Concentração: Sistemas Elétricos de Potência

Orientador: Prof. Dr. João Bosco Augusto London Júnior

Co-orientador: Prof. Dr. Luís Fernando Costa Alberto

São Carlos

2011 
AUTORIZO A REPRODUÇÃO E DIVULGAÇÃO TOTAL OU PARCIAL DESTE TRABALHO, POR QUALQUER MEIO CONVENCIONAL OU ELETRÔNICO, PARA FINS DE ESTUDO E PESQUISA, DESDE QUE CITADA A FONTE.

Ficha catalográfica preparada pela Seção de Tratamento da Informação do Serviço de Biblioteca - EESC/USP

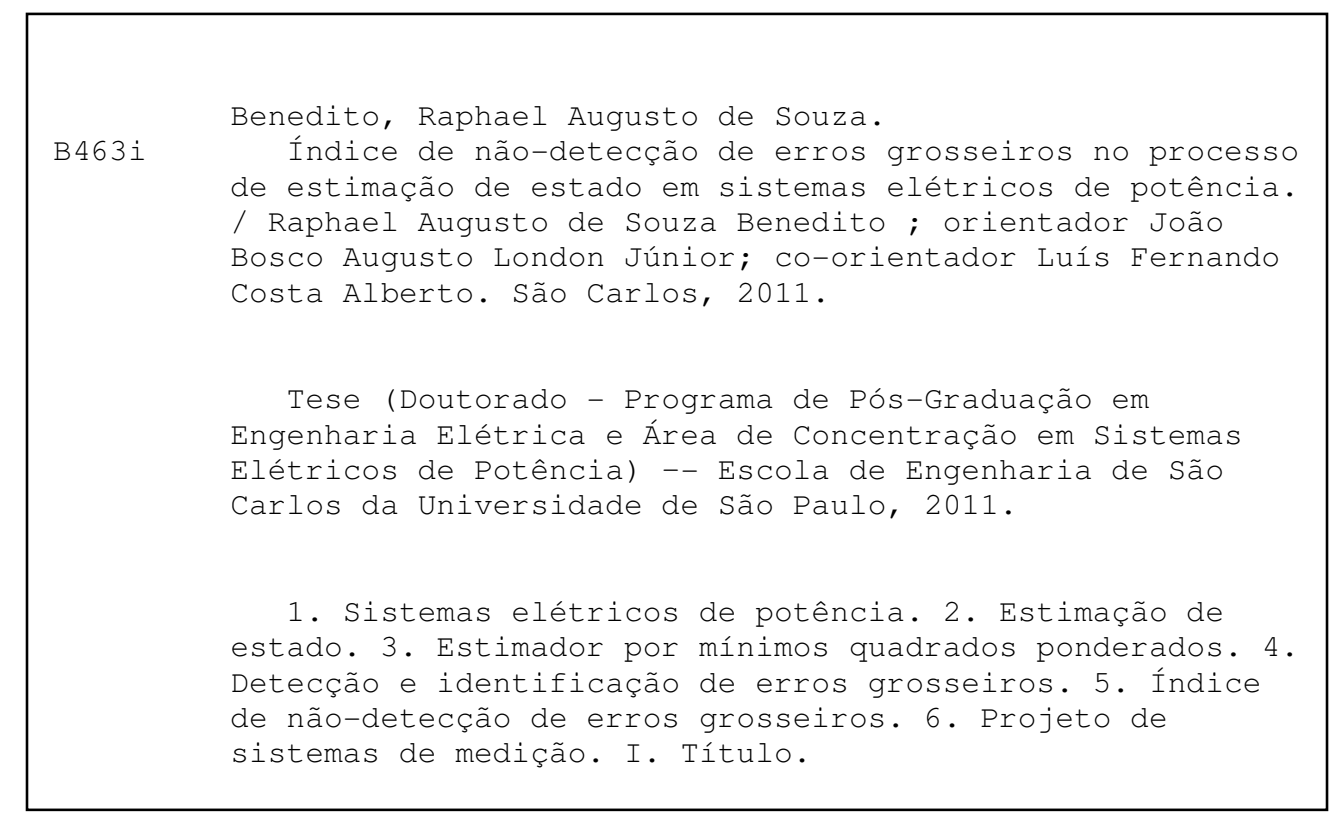


FOLHA DE JULGAMENTO

Candidato(a): Engenheiro RAPHAEL AUGUSTO DE SOUZA BENEDITO.

Tese defendida e julgada em 03/06/2011 perante a Comissão Julgadora:

hasoscantont. AI ROUADO

Prof. Dr. JOAO BOSCO AUGUSTO LONDON JUNIOR - (Orientador)

(Escola de Engenharia de São Carlos/USP)

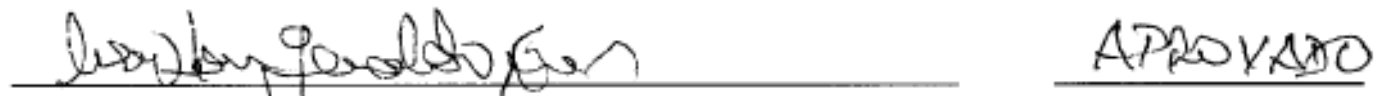

Prof. Titular NEWTION GERALDO BRETAS

(Escola de Engenharia de São Carlos/USP)

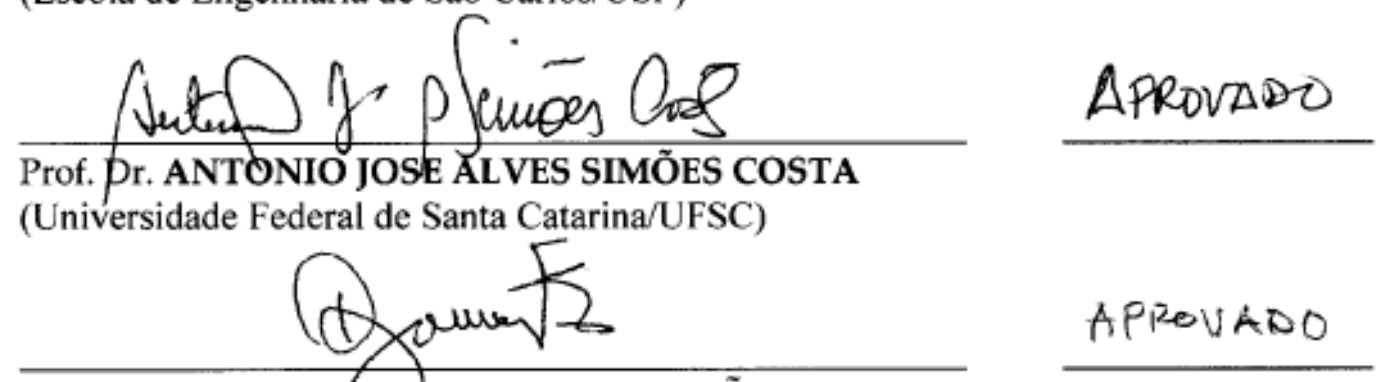

Prof. Titular DJALM(A MOSQUEIRA FALCÃO

(Universidade Federal do Rio de Janeiro/UFRJ)

Ande lenbrä pio harts

APROVADO

Prof. Dr. ANDRÉ CHRISTÓVÃO PIO MARTINS

(Universidade Estadual Paulista "Júlio de Mesquita Filho"/UNESP/campus de Bauru)

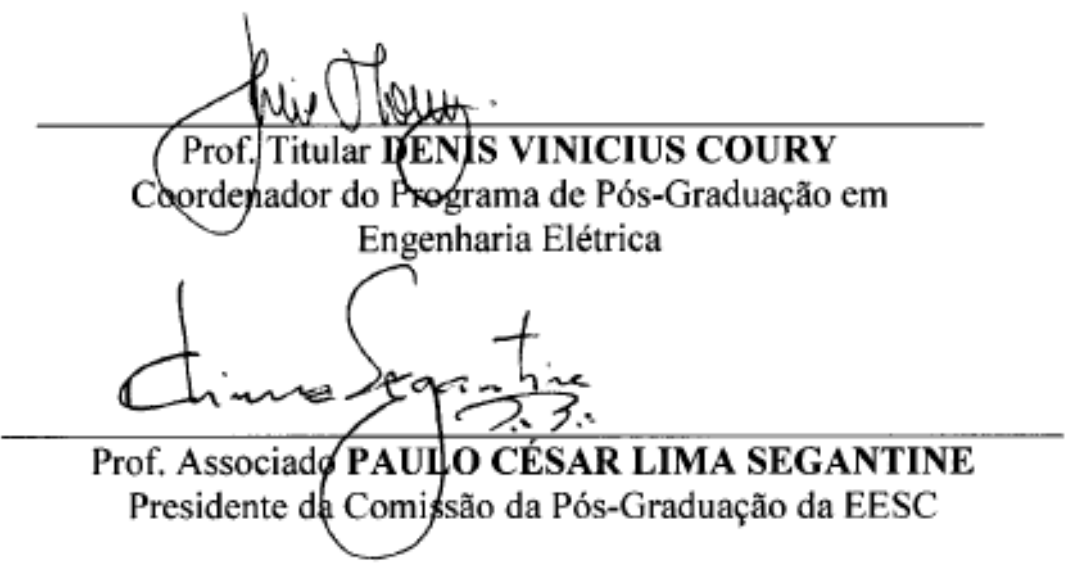



Dedico este trabalho à minha mãe Maria, à tia Ivani, ao tio José Lopes e ao meu irmão Jaime. 



\section{Agradecimentos}

Ao Prof. Dr. João Bosco Augusto London Jr., que nos anos de convivência, muito me ensinou, contribuindo para meu crescimento científico e intelectual.

Ao Prof. Dr. Luís Fernando Costa Alberto, pela co-orientação e atenção durante este trabalho.

Ao Prof. Dr. Newton G. Bretas, pela oportunidade de realização de meu curso de mestrado e doutorado, e também, pelos anos de convivência, discussões e aprendizagem.

À Magnífica Universidade de São Paulo (USP), por todo o suporte necessário para realização deste trabalho.

À Coordenação de Aperfeiçoamento de Pessoal de Nível Superior (CAPES), pela concessão da bolsa de doutorado.

Aos amigos: Saulo, Robson, Nanni, Castoldi, Lizandra, Carolina, Madeleine, Antônio Carlos (Maranhão), Fabíolo, Carlisson Jr., Tubota, Elmer, Edson, Augusto, Rafael, Gabriel, Danilo, Fernando Fabrizzi, Daniel, Moussa, Guilherme, Camila, Thais, Tatiane, Josaphat, Roberto, Igor, Pedro, Alexandre Ciabatari, Renato Ubukata e Wilson, por toda amizade.

Aos funcionários do Departamento de Engenharia Elétrica: Marisa, Jussara, Verinha, Rosane e Odair, que, de um modo ou de outro, me auxiliaram ao longo desses anos.

A todos, meus sinceros agradecimentos.

Raphael Augusto de Souza Benedito São Carlos, Abril de 2011. 

"Se eu vi mais longe, foi por estar de pé sobre ombros de gigantes"

Isaac Newton 



\section{Resumo}

BENEDITO, R. A. de S. Índice de Não-Detecção de Erros Grosseiros no Processo de Estimação de Estado em Sistemas Elétricos de Potência. 2011. Tese (Doutorado) - Escola de Engenharia de São Carlos, Universidade de São Paulo, São Carlos, 2011.

A partir de uma análise geométrica, do estimador de estado por mínimos quadrados ponderados, propõe-se, neste trabalho, um índice para classificação das medidas de acordo com as suas características de não refletirem grande parcela de seus erros nos resíduos do processo de estimação de estado, por mínimos quadrados ponderados. O índice proposto foi denominado Índice de Não-Detecção de Erros, ou apenas UI (Undetectability Index). As medidas com maiores UI são aquelas cujos erros grosseiros (EGs) são mais difíceis de serem detectados através de métodos que fazem uso da análise dos resíduos. Isto porque os erros dessas medidas são, de certa forma, "mascarados", isto é, não são refletidos nos resíduos das mesmas. Nesse sentido, a medida crítica é o caso limite de medidas cujos erros são mascarados, isto é, possui $U I$ infinito e seu resíduo é igual a zero, independente de a mesma ter ou não EG. Para obtenção dos índices $U I$ das medidas, desenvolveu-se um algoritmo simples e de fácil implementação. Tomando por base o índice $U I$, propõe-se, também, uma metodologia para processamento de EGs e dois algoritmos para projeto ou fortalecimento de sistemas de medição. Esses algoritmos possibilitam a obtenção de sistemas de medição confiáveis (observáveis e isentos de medidas críticas e de conjuntos críticas de medidas), de baixo custo e formados por medidas com índices $U I$ menores que um valor pré-estabelecido. Para validar o índice $U I$ e as suas aplicações propostas neste trabalho, realizaram-se diversas simulações computacionais nos sistemas de 14 e 30 barras do IEEE, tendo sido satisfatórios todos os resultados obtidos.

Palavras-chave: Sistemas Elétricos de Potência, Estimação de Estado, Estimador por Mínimos Quadrados Ponderados, Detecção e Identificação de Erros Grosseiros, Índice de Não-Detecção de Erros Grosseiros, Projeto de Sistemas de Medição. 



\section{Abstract}

BENEDITO, R. A. de S. Undetectability Index of Gross Errors in Power Systems

State Estimation. 2011. Thesis - Escola de Engenharia de São Carlos, Universidade de São Paulo, São Carlos, 2011.

The present thesis proposes an index, called Undetectability Index (UI), to classify the measurements according to their characteristics of not reflecting their errors into the residuals of the weighted least squares state estimation process from a geometric analysis of this estimator. Gross errors in measurements with higher UIs are very difficult to be detected by methods based on the residual analysis, as the errors in those measurements are "masked", i.e., they are not reflected in the residuals. In this sense, critical measurements are the limit case of measurements that mask errors, that is, they have infinite $U I$ and their residuals are always zero independently of their having or not gross errors. Based on the UI a methodology for gross error processing and two algorithms for metering system planning are also proposed in this thesis. These algorithms enable the obtaining of reliable measurement systems (observable and free from critical measurements and critical sets of measurements) with low investment and containing only measurements with UIs lower than a pre-established value. Several simulation results (with IEEE 14-bus and 30-bus systems) have validated the UI and its application.

Keywords: Electric Power Systems, Real-Time Operation, Least Square State Estimation, Gross Errors Detection and Identification, Undetectability Index, Metering System Planning. 



\section{Lista de Figuras}

Figura 1.1: Diagrama de blocos das funções principais do sistema de análise de redes 2

Figura 2.1: Modelo $\pi$ generalizado de um ramo do sistema elétrico $\quad 8$

Figura 2.2: Modelo de estimação generalizada 26

Figura 2.3: Estrutura básica de uma PMU (EHRENSPERGER, 2004) 27

Figura 3.1: Classificação de erros grosseiros em medidas 31

Figura 3.2: Função de densidade de probabilidade $(f)$ para uma distribuição qui-quadrado com

8 graus de liberdade $\left(\chi_{8}^{2}\right): \lambda=\chi_{8,(1-0,05)}^{2}=15,5$, ou seja, $\quad 40$

Probabilidade $\left(\chi_{8}^{2}>15,5\right)=0,05$.

Figura 3.3: Diagrama do sistema de 3 barras 46

Figura 3.4: Espaço de fator do sistema de 3 barras apresentado na fig.3.3: $l_{1}$ e $l_{2}$ são, respectivamente, a primeira e segunda coluna da matriz $\tilde{H}^{t}$

Figura 3.5: Sistema de 3 barras e 5 medidas utilizados no exemplo 1

Figura 4.1: Interpretação geométrica do operador $K$ atuando sobre o vetor $\Delta \mathrm{z}$

Figura 4.2: Interpretação geométrica considerando dois erros distintos em z1 62

Figura 4.3: Sistema de duas barras e duas linhas $\quad 63$

Figura 4.4: Exemplo de erro grosseiro em medida com alto UI 63

Figura 4.5: Exemplo de erro grosseiro em medida com baixo UI 64

Figura 4.6: Sistema de 14 barras IEEE

Figura 4.7: Índice UI para as medidas ativas. Sistema de 14 barras - medidas sem ruído 69

Figura 4.8: Índice UI para as medidas reativas. Sistema de 14 barras - medidas sem ruído 69

Figura 4.9: Gráfico de tendência para índice UI X Mínimo para detecção de erros em medidas 71

Figura 5.1: Gráfico de tendência para índice UI X Mínimo para detecção de EGs em medidas - valores médios para 100 amostras de medidas com ruído

Figura 5.2: Sistema de 30 barras IEEE 93

Figura 5.3: Índice UI para as medidas ativas. Sistema de 30 barras - medidas sem ruído 98

Figura 5.4: Índice UI para as medidas reativas. Sistema de 30 barras - medidas sem ruído 98

Figura 5.5: Gráfico de tendência para índice UI X Mínimo para detecção de erros em medidas. Sistema de 30 barras - medidas sem ruído

Figura 5.6: Gráfico de tendência para índice UI X Mínimo para detecção de EGs em medidas- valores médios para 100 amostras de medidas com ruído

Figura 6.1: Sistema de duas barras e duas linhas 
Figura 7.1: Variação do índice $U I$ das medidas FA:10-11 e FR:10-11com o aumento da redundância local

Figura 7.2: Variação do índice $U I$ das medidas IA:5 e IR:5 com o aumento da redundância

Figura 7.3: Índice $U I$ das medidas ativas após a instalação de todas as medidas de potência e tensão

Figura 7.4: Índice UI das medidas reativas após a instalação de todas as medidas de potência e tensão

Figura 7.5: Índice $U I$ das medidas ativas após a retirada de medidas com $U I$ elevado

Figura 7.6: Índice $U I$ das medidas reativas após a retirada de medidas com $U I$ elevado

Figura 7.7: Fluxograma do algoritmo 1 para o projeto de sistemas de medição

Figura 7.8: Índice $U I$ das medidas ativas do sistema de medição obtido com o Algoritmo 1 Sistema de 14 barras IEEE

Figura 7.9: Índice $U I$ das medidas reativas do sistema de medição obtido com o Algoritmo 1 Sistema de 14 barras IEEE

Figura 7.10: Índice $U I$ das medidas ativas do sistema de medição obtido com o Algoritmo 1 Sistema de 30 barras IEEE

Figura 7.11: Índice $U I$ das medidas reativas do sistema de medição obtido com o Algoritmo 1 - Sistema de 30 barras IEEE

Figura 7.12: Fluxograma do algoritmo 2 para o projeto de sistemas de medição

Figura 7.13: Índice $U I$ das medidas ativas do sistema de medição obtido com o Algoritmo 2 Sistema de 14 barras IEEE

Figura 7.14: Índice UI das medidas reativas do sistema de medição obtido com o Algoritmo 2 - Sistema de 30 barras IEEE

Figura 7.15: Índice $U I$ das medidas ativas do sistema de medição obtido com o Algoritmo 2 Sistema de 30 barras IEEE

Figura 7.16: Índice $U I$ das medidas reativas do sistema de medição obtido com o Algoritmo 2

- Sistema de 30 barras IEEE

Figura C.1: Fluxograma do algoritmo para Identificação do Nível de Detecção

Figura D.1: Exemplo de crossover

Figura D.2: Ótimo local, global e espaço de busca

Figura D.3: Sistema de 14 barras IEEE e seu cromossomo 


\section{Lista de Tabelas}

Tabela 3.1: Valores de interesse para o teste da matriz "chapéu" e para o teste da estatística de projeção, para o exemplo 1

Tabela 3.2: Valores de interesse para o teste da matriz "chapéu" e para o teste da estatística de projeção, para o exemplo 2

Tabela 4.1: Valores das medidas sem ruído e respectivos desvios padrões para o sistema de 14 barras

Tabela 4.2: Variáveis de estado estimadas para o sistema de 14 barras

Tabela 4.3: Índice $J(\mathrm{x})$, valores estimados das medidas, índice $U I$ e resíduos normalizados $r^{N}$

Tabela 4.4: Identificação do nível de detecção de erro

Tabela 4.5: Índices $U I$ e valores de interesse para o teste da matriz "chapéu" e para o teste da estatística de projeção, para o sistema de medição 1

Tabela 5.1: Resultado numérico da adição de EG de $10 \sigma$ na medida IA:1

Tabela 5.2: Resultado numérico da adição de EG de $10 \sigma$ na medida IA:5

Tabela 5.3: Resultados numéricos da adição de erro grosseiro de $10 \sigma$ para cada medida (em uma medida de cada vez) considerando o sistema de medição da tabela 4.1

Tabela 5.4: Resultados numéricos da adição de EG de $20 \sigma$ para cada medida (em uma medida de cada vez) considerando o plano de medição da tabela 4.1

Tabela 5.5: Resultados do índice $U I$, resíduo normalizado e índice J(x), para 100 amostras de medidas com ruído e sem EG

Tabela 5.6: Identificação do nível de detecção de EGs para 100 amostras de medidas com ruído

Tabela 5.7: Resultados do índice UI, resíduo normalizado e índice J(x) para 100 amostras de medidas com ruído e com EG de 10 sigmas em cada medida

Tabela 5.8: Resultados do índice UI com alterações nos valores das medidas - sistema 14 barras

Tabela 5.9: Valores de $F E$ para o sistema de 30 barras IEEE

Tabela 5.10: Valores das medidas sem ruído e respectivos desvios padrões para o sistema de 30 barras

Tabela 5.11: Valor do índice $J(\mathrm{x})$, valores estimados das medidas, índice $U I$ e resíduos normalizados $r^{N}$ - sistema de 30 barras IEEE

Tabela 5.12: Resultados numéricos da simulação do sistema de medição da tabela 5.10 sistema de 30 barras IEEE

Tabela 5.13: Resultados numéricos da adição de EG de $10 \sigma$ para cada medida (uma medida de 
cada vez) considerando o sistema de medição da tabela 5.10

Tabela 5.14: Resultados numéricos da adição de EG de $20 \sigma$ para cada medida (uma medida de 103 cada vez) considerando o sistema de medição da tabela 5.10

Tabela 5.15: Resultados do índice UI, resíduo normalizado e índice J(x) para 100 amostras de medidas com ruído e sem EG

Tabela 5.16: Identificação do nível de detecção de erro para 100 amostras de medidas com ruído

Tabela 5.17: Resultados do índice UI, resíduo normalizado e índice J(x) para 100 amostras de medidas com ruído e com EG de 10 sigmas em cada medida

Tabela 5.18: Resultados do índice UI com alterações nos valores das medidas - sistema de 30 barras

Tabela 6.1: Resultados numéricos da adição de EG de $10 \sigma$ na medida IA:1

Tabela 6.2: Resultados numéricos da adição de EG de $10 \sigma$ na medida IA:5

Tabela 6.3: Resultados numéricos da adição de EG de $10 \sigma$ para cada medida (uma de cada vez) considerando o sistema de medição 1

Tabela 6.4: Resultados numéricos da adição de EG de 20 б para cada medida (uma de cada vez) considerando o sistema de medição 1

Tabela 6.5: Resultados numéricos da adição de EG de 9 бi à medida z1

Tabela 6.6: Resultados numéricos da adição de EG de 9 бi à medida z2

Tabela 6.7: Resultados numéricos da adição de EG de $10 \sigma$ à medida IA:4

Tabela 6.8: Resultados numéricos da adição de EG de $10 \sigma$ na medida FA:3-4

Tabela 6.9: Valores médios e desvios padrões do índice $U I$, resíduo normalizado e erro composto em desvio padrão para 100 amostras de medidas com ruído e com EG simples de 10 sigmas em cada medida

Tabela 6.10: Dados do número de casos em que falharam a detecção ou identificação de erros para 100 amostras de medidas com ruído e adição de erro grosseiro de 10 sigmas em cada medida

Tabela 6.11: Divisão das medidas considerando o índice $U I$ e a possibilidade de falhas para detecção e identificação de erro grosseiro simples de 10 sigmas - sistema de 14 barras do IEEE

Tabela 6.12: Valores médios e desvios padrões do índice $U I$, resíduo normalizado e erro composto em desvio padrão para 100 amostras de medidas com ruído e com EG de 10 sigmas em cada medida - sistema de 30 barras do IEEE

Tabela 6.13: Dados do número de casos em que falharam a detecção ou identificação de erros para 100 amostras de medidas com ruído e adição de EG de 10 sigmas em cada medida

Tabela 6.14: Divisão das medidas considerando o índice $U I$ e a possibilidade de falhas para detecção e identificação de EG simples de 10 sigmas - sistema de 30 barras IEEE

Tabela 7.1: Variação do índice $U I$ das medidas FA:10-11 e FR:10-11 e, também, das novas 
medidas adicionadas

Tabela 7.2: Variação do índice $U I$ das medidas IA:5 e IR:5 com a adição de novas medidas

Tabela 7.3: Medidas com índice UI maior que 1,1 L considerando todas as medidas instaladas

Tabela 7.4: Medidas com índice $U I$ maior que $1,1 \mathrm{~L}$

Tabela 7.5: Medidas com índice $U I$ maior que $1,1 \mathrm{~L}$

Tabela 7.6: Medidas com índice UI maior que 1,65 considerando todas as medidas instaladas

Tabela 7.7: Medidas com índice UI maior que 1,65

Tabela 7.8: Medidas do plano de medição resultante e respectivos valores de $U I$ (sistema de 14 barras)

Tabela 7.9: Medidas com índice $U I$ maior que 1,1 considerando todas as medidas instaladas

Tabela 7.10: Medidas com índice UI maior que 1,1

Tabela 711: Medidas com índice $U I$ maior que 1,1

Tabela 7.12: Medidas com índice $U I$ maior que 1,65 considerando todas as medidas instaladas

Tabela 7.13: Medidas com índice UI maior que 1,65

160

Tabela 7.14: Medidas com índice UI maior que 1,65

Tabela 7.15: Medidas com índice UI maior que 1,65

Tabela 7.16: Medidas com índice $U I$ maior que 2,20 considerando todas as medidas instaladas

Tabela 7.17: Sistema de medição confiável para sistema de 14 barras do IEEE

Tabela 7.18: Medidas com UI maior que 2,20 (caso base)

Tabela 7.19: Medidas com UI maior que 2,20 (adição 1)

Tabela 7.20: Medidas com UI maior que 2,20 (adição 2)

Tabela 7.21: Medidas com UI maior que 2,20 (adição 3)

Tabela 7.22: Medidas com UI maior que 2,20 (adição 4)

Tabela 7.23: Medidas com UI maior que 2,20 (adição 5)

Tabela 7.24: Medidas com UI maior que 2,20 (adição 6)

Tabela 7.25: Medidas com UI maior que 2,20 (adição 7)

Tabela 7.26: Variação do índice $U I$ de medidas (cujo valor inicial está acima de 2,20) com a adição de novas medidas

Tabela 7.27: Medidas do sistema de medição obtido após a Etapa 2 e respectivos valores de $U I$ (sistema de 30 barras)

Tabela 7.28: Medidas com UI maior que 2,20 (caso base)

Tabela 7.29: Medidas com UI maior que 2,20 (adição 1)

Tabela 7.30: Medidas com UI maior que 2,20 (adição 2)

Tabela 7.31: Medidas com UI maior que 2,20 (adição 3)

Tabela 7.32: Medidas com UI maior que 2,20 (adição 4)

Tabela 7.33: Medidas com UI maior que 2,20 (adição 5)

Tabela 7.34: Variação do índice $U I$ de medidas (cujo valor inicial está acima de 2,20) com a adição de novas medidas

Tabela B.1: Valores de todas as medidas e respectivos desvios padrões para o sistema de 14 
Tabela B2: Valores de todas as medidas e respectivos desvios padrões para o sistema de 30 barras

Tabela B.3: Medidas do sistema de medição obtido pelo Algoritmo 1 e respectivos valores de $U I$ (sistema de 30 barras)

Tabela B.4: Medidas do sistema de medição obtido pelo Algoritmo 2 e respectivos valores de $U I$ (sistema de 14 barras)

Tabela B.5: Medidas do sistema de medição obtido pelo Algoritmo 2 e respectivos valores de $U I$ (sistema de 30 barras) 


\section{SUMÁRIO}

\section{Capítulo 1}

1. INTRODUÇÃO 1

1.1 Objetivos do Trabalho 5

1.2 Estrutura do Trabalho 6

\section{Capítulo 2}

2. ESTIMAÇÃO DE ESTADO EM SISTEMAS ELÉTRICOS DE POTÊNCIA

2.1 Modelagem de Linhas e Transformadores: Equações de Fluxo de Carga

2.2 Método da Equação Normal 10

2.3 Estimador de Estado Desacoplado Rápido

2.4 Estimador de Estado Linearizado 15

2.5 Aspectos sobre a Equação Normal e Condicionamento Numérico 17

2.6 Modelagem de Injeções Nulas

2.7 Método da Matriz Aumentada e Tableau Esparso

2.8 Método baseado em Transformação Ortogonal

2.9 Estimador Generalizado 25

2.10 Medição Sincronizada de Fasores

\section{Capítulo 3}

3. DETECÇÃO E IDENTIFICAÇÃO DE ERROS GROSSEIROS 29

3.1 Introdução

3.2 Propriedades dos Resíduos das Medidas

3.3 Detecção e Identificação de Erros Grosseiros 


\section{Capítulo 4}

4. ÍNDICE DE NÃO-DETECÇÃO DE ERROS GROSSEIROS 55

4.1 Interpretação Geométrica do Estimador WLS 56

4.2 Decomposição do Erro de Medida $\quad 59$

4.3 Proposição do Índice de Não-Detecção de Erros $\quad 60$

$\begin{array}{ll}4.4 \text { Conclusões do Capítulo } & 74\end{array}$

\section{Capítulo 5}

5. TESTES REALIZADOS

5.1 Simulações no Sistema de 14 Barras do IEEE 77

5.2 Simulações no Sistema de 30 Barras do IEEE 92

5.3 Conclusões do Capítulo 115

\section{Capítulo 6}

6. APLICAÇÃO DO ÍNDICE DE NÃO-DETECÇÃO DE ERROS PARA DETECÇÃO E IDENTIFICAÇÃO DE ERROS GROSSEIROS

6.1 Utilização do Índice UI para Detecção e Identificação de Erros Grosseiros

6.2 Conclusões do Capítulo

\section{Capítulo 7}

7. UTILIZAÇÃO DO ÍNDICE DE NÃO-DETECÇÃO DE ERROS PARA PROJETO OU FORTALECIMENTO DE SISTEMAS DE MEDIÇÃO

7.1 Influência da Redundância de Medidas sobre o Índice de NãoDetecção de Erros

7.2 Algoritmo para Projeto ou Fortalecimento de Sistemas de Medição:

Algoritmo 1

7.3 Algoritmo para Projeto ou Fortalecimento de Sistemas de Medição:

Algoritmo 2

7.4 Conclusões do Capítulo 


\section{Capítulo 8}

8. CONCLUSÕES

$\begin{array}{ll}8.1 \text { Conclusões } & 179\end{array}$

$\begin{array}{ll}8.2 \text { Perspectivas Futuras } & 181\end{array}$

Referências Bibliográficas

Anexo A Demonstrações Matemáticas não Apresentadas no Capítulo 4

Anexo B Tabelas com Dados e Resultados dos Sistemas Testes

Anexo C Fluxograma do Algoritmo para Identificação do Nível de Detecção

Anexo D Projeto de Sistemas de Medição Confiáveis Através de Algoritmos Evolutivos e Matriz $H_{\Delta}{ }^{\mathrm{t}}$

Anexo E Publicações do Autor Durante o Período de Doutoramento 



\section{Capítulo 1}

\section{Introdução}

A operação de sistemas elétricos de potência, em tempo-real, tem como principal objetivo manter o sistema elétrico operando, sem sobrecargas de equipamento, e atendendo a todos os consumidores em qualquer condição de operação. Para alcançar tal objetivo, é necessário que os níveis de tensão, freqüência, fluxos nas interligações, carregamento de linhas e equipamentos, sejam mantidos dentro de faixas, ou limites de segurança previamente estabelecidos.

Conseqüentemente, a operação dos sistemas elétricos exige que uma grande quantidade de informações esteja disponível, para os operadores dos sistemas. Essas informações devem permitir a determinação do estado operativo atual do sistema, isto é, se o sistema está ou não operando adequadamente e, caso não o esteja, devem indicar o que deve ser feito para corrigir tal operação inadequada.

O monitoramento, a análise e a coordenação da operação dos sistemas elétricos são de responsabilidade dos Sistemas de Gerenciamento de Energia (EMS, do inglês Energy Management Systems), que constituem os modernos centros de supervisão e controle das companhias do setor (DY LIACCO, 2002).

Os modernos centros de controle dispõem basicamente de dois sistemas: o sistema supervisório de controle e aquisição de dados (SCADA, do inglês Supervisory Control and Data Acquisition System), responsável pelo monitoramento da rede elétrica; e o sistema de análise de redes, que aglutina várias funções ou programas matemáticos distintos, que auxiliam na tomada de decisão da operação. 
Dentre as funções principais do sistema de análise de redes, enquadram-se: préfiltragem de informações, configurador de redes, estimador de estado, algoritmo de previsão de carga, fluxo de potência, análise de segurança, fluxo de potência ótimo e outros. A figura 1.1, a seguir, ilustra um diagrama de blocos contendo as funções principais do sistema de análise de redes, bem como o relacionamento entre elas (WU, 1988; BALU et al., 1992):

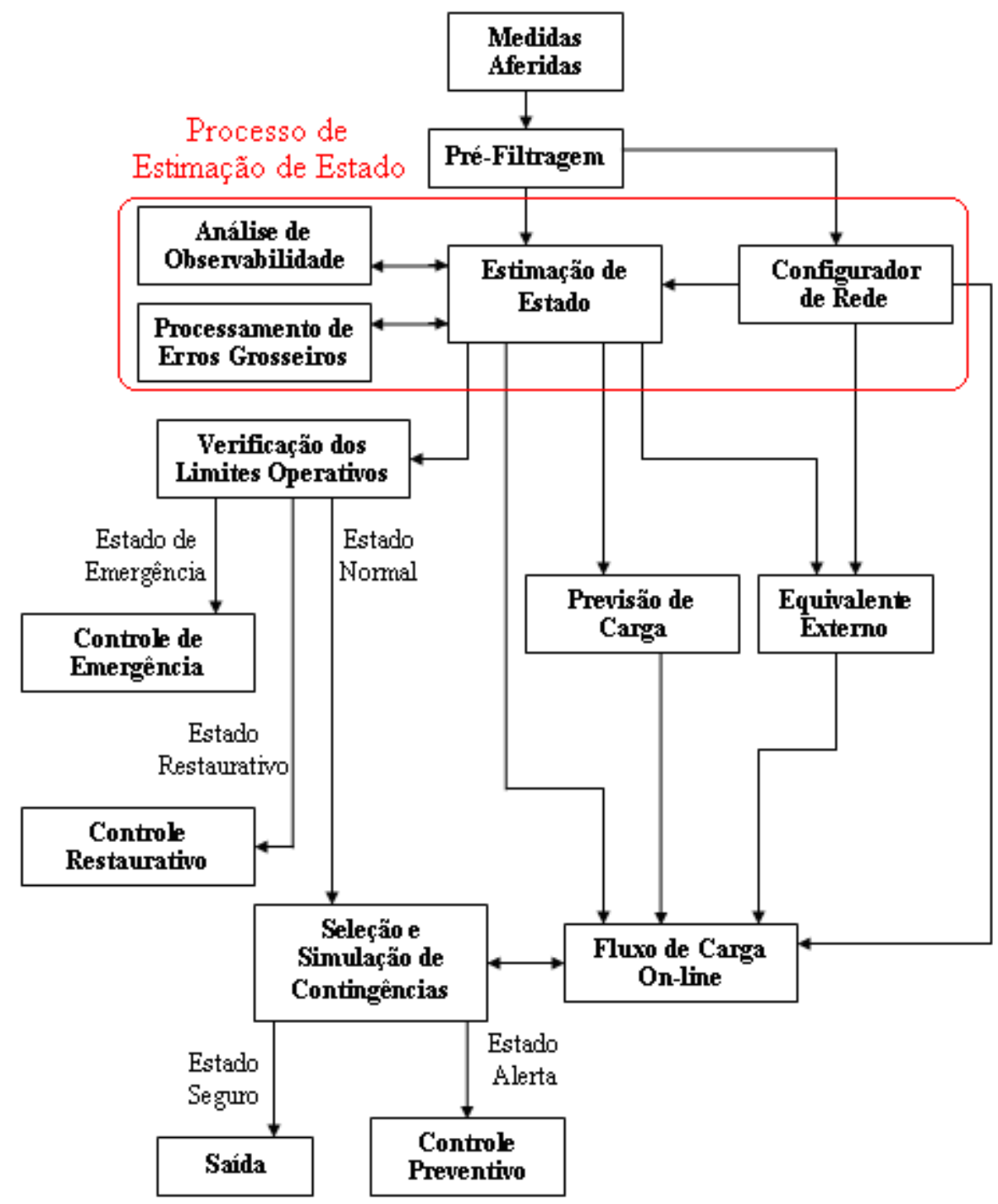

Figura 1.1: Diagrama de blocos das funções principais do sistema de análise de redes

O aprofundamento teórico sobre essas funções não será apresentado no presente texto, o qual se limitará ao processo de estimação de estado. Entretanto, uma vasta revisão bibliográfica sobre as mesmas e suas evoluções, ao longo de mais de três décadas, pode ser obtida em trabalhos de Dy Liacco (1974-1991-2002), Wu (1988), Wu et al. (2005) e Balu et al.(1992). 
Como pode ser visto em destaque através da figura 1.1, o processo de estimação de estado (em vermelho) exerce uma função essencial e importantíssima ao monitoramento e análise de um sistema elétrico, já que as demais funções necessitam de informações oriundas desse processo.

O estimador de estado processa informações analógicas redundantes e contaminadas por ruído, visando à melhor estimativa para as tensões complexas nas barras pertencentes ao sistema supervisionado. Tradicionalmente, o processo de estimação de estado é divido em 4 funções, como ilustrado na figura 1.1, sendo:

- Configurador de rede: responsável pela obtenção, em tempo-real, da topologia e a correspondente configuração de medidores, no modelo barra linha, que corresponde ao diagrama unifilar da rede. Para isto, o configurador processa medidas digitais, transmitidas pelo sistema SCADA, que consistem em informações lógicas sobre os estados de disjuntores/chaves;

- Análise de observabilidade: verifica se as informações contidas nas telemedidas são suficientes para o cálculo das tensões complexas, em todas as barras do sistema. Em caso negativo, o algoritmo deve indicar ou as ilhas observáveis do sistema interno, ou as pseudo-medidas (dados históricos ou de previsão de carga) necessárias à restauração da observabilidade;

- Estimação de estado propriamente dita: fazendo uso da modelagem matemática do sistema elétrico, através de equações algébricas não-lineares, realiza-se o cálculo das variáveis de estado da porção observável do sistema;

- Processamento de erros grosseiros: esta função é responsável pela detecção e identificação de medidas com grau de imprecisão muito maior que o suposto pelo modelo de medição, ou seja, de medidas portadoras de erros grosseiros (EGs). Caso o algoritmo permita detectar e identificar alguma medida com EG, esta é removida (ou o seu efeito) e as variáveis de estado são estimadas novamente.

A habilidade em detectar e identificar EGs é um dos atributos mais importantes do processo de estimação de estado em sistemas elétricos. Alguns EGs são óbvios e podem ser identificados a priori e eliminados do processo de estimação, através de uma simples verificação dos dados de entrada (medidas). Tais erros podem ser: valores absurdos de tensão elétrica eficaz; valores muito além dos esperados para medidas de potência e/ou corrente elétrica, etc (ABUR e EXPÓSITO, 2004). Entretanto, nem todos os tipos de EGs são facilmente detectáveis ou identificáveis dessa forma, exigindo o emprego de outras metodologias, que serão estudadas no decorrer deste trabalho. 
O estimador de estado por Mínimos Quadrados Ponderados (WLS, Weight Least Squares) funciona bem quando os ruídos nas medidas são Gaussianos, mas falha na ocorrência de um ou mais EGs (ABUR e EXPÓSITO, 2004). Para superar essa limitação, desenvolveram-se métodos para detecção e identificação de EGs, dentre os quais os mais utilizados baseiam-se na análise dos resíduos das medidas. Isto porque os resíduos fornecem informações sobre eventuais violações das suposições relativas ao modelo de medição.

O estimador WLS, associado com técnicas para processamento de EGs a partir da análise dos resíduos das medidas, apresenta desempenho satisfatório na ocorrência de EG simples, ou quando ocorrem EGs múltiplos não-interativos. Todavia, pode falhar ou até mesmo falham, em algumas situações:

i) EGs associados a medidas com baixa redundância (medidas críticas ou pertencentes a conjuntos críticos de medidas);

ii) EGs múltiplos interativos;

iii) EGs em medidas que possuam a característica de altamente influentes, ou seja, de atraírem a convergência do processo de estimação de estado, chamadas tradicionalmente medidas ponto de alavancamento (MONTICELLI, 2000).

Nota: Definições e conceitos pertinentes à redundância de medidas, aos tipos de EGs em medidas, assim como, à análise de resíduos das medidas serão detalhados no decorrer deste trabalho, mais precisamente no capítulo 3.

Em Pires et al. (1999), fazendo uso do método para identificação de pontos de alavancamento proposto em Mili et al. (1996), desenvolveu-se um estimador WLS que altera a ponderação das medidas, de forma iterativa, com objetivo de minimizar o efeito das medidas pontos de alavancamento suspeitas de estarem com EGs.

Como alternativa ao método WLS e à análise de seus resíduos, outros estimadores de estado considerados mais robustos estatisticamente foram aplicados em sistemas elétricos de potência, como o método do Mínimo Valor Absoluto Ponderado (WLAV, Weighted Least Absolute Value) e o método da Mínima Mediana do Resíduo Ponderado ao Quadrado (WLMS, Weighted Least Median of Squares).

O método WLAV, aplicado em sistemas elétricos de potência (IRVING et al., 1978; KOTIUGA e VIDYASAGAR, 1982; ABUR e CELIK, 1991; FALCÃO e ARIAS, 1994) mostrou-se mais robusto que o WLS na presença de EGs simples e 
múltiplos. Contudo, este método falha na ocorrência de erros em medidas ponto de alavancamento (discussão da referência FALCÃO e ARIAS, 1994).

Mili et al. (1991) propuseram a utilização do método WLMS em sistemas elétricos de potência. Este estimador foi o primeiro estimador de estado robusto estatisticamente capaz de identificar EGs em medidas classificadas como ponto de alavancamento. Entretanto, tal estimador requer uma busca combinatorial, que inviabiliza sua aplicação em tempo real (FALCÃO e ARIAS, 1994; MONTICELLI, 2000).

Dada a inviabilidade prática do estimador WLMS e, tendo em vista que o estimador WLS, associado à análise dos resíduos das medidas, é o mais utilizado na prática, pretende-se investigar com este trabalho um modo simples de verificar quais medidas apresentam problemas para detecção e identificação de possíveis EGs, com a utilização daquele estimador, e com isso, investigar possíveis maneiras de melhorar não somente o processamento de EGs (detecção/identificação de EGs), mas também o processo de estimação de estado como um todo.

A seguir, são delineados os objetivos principais e a estrutura deste trabalho.

\subsection{Objetivos do Trabalho}

A partir de uma análise geométrica, do estimador WLS, propõe-se, neste trabalho, um índice para classificação das medidas de acordo com as suas características de não refletirem grande parcela de seus erros nos resíduos do processo de estimação de estado, por mínimos quadrados ponderados. O índice proposto foi denominado Índice de Não-Detecção de Erros, ou, simplesmente, UI (Undetectability Index), como apresentado em Bretas et al. (2009).

As medidas com maiores UI são aquelas cujos EGs são mais difíceis de serem detectados através de métodos que fazem uso da análise dos resíduos do estimador WLS. Isto porque os erros dessas medidas são, de certa forma, "mascarados", isto é, não são refletidos nos resíduos das mesmas. Nesse sentido, a medida crítica é o caso limite de medidas cujos erros são mascarados, isto é, possui $U I$ infinito e seu resíduo é igual a zero, independente de a mesma ter ou não EG.

Para obtenção dos índices $U I$ das medidas, desenvolveu-se um algoritmo simples e de fácil implementação, que possibilita qualificar todas as medidas disponíveis, para 
estimação de estado, de acordo com as suas características de "mascararem" os seus erros.

Com base no índice $U I$ propõe-se, também, uma metodologia para processamento de EGs e dois algoritmos para projeto ou fortalecimento de sistemas de medição. Esses algoritmos possibilitam a obtenção de sistemas de medição confiáveis (observáveis e isentos de medidas críticas e de conjuntos críticas de medidas), de baixo custo e formados por medidas com índices $U I$ menores que um valor pré-estabelecido.

Para validar o índice UI e as suas aplicações propostas neste trabalho, realizaram-se diversas simulações computacionais nos sistemas de 14 e 30 barras do IEEE, tendo sido satisfatórios todos os resultados obtidos.

\subsection{Estrutura do Trabalho}

Com o intuito de delinear o processo de estimação de estado e cumprir os objetivos supracitados, este trabalho está estruturado na seguinte forma:

- O capítulo 2 apresenta uma revisão dos principais métodos e técnicas aplicadas à estimação de estado em sistemas elétricos de potência;

- No capítulo 3 é descrito o problema de detecção e identificação de EGs apresentando as suas principais causas, os tipos de erros e os métodos de análise mais empregados;

- No capítulo 4 é proposto o índice de não-detecção de erros (UI) através da interpretação geométrica do estimador WLS;

- No capítulo 5 são apresentados os resultados dos testes utilizados para validar os aspectos teóricos do capítulo 4;

- O capítulo 6 apresenta uma aplicação do índice de não-detecção de erros para prover uma melhora substancial no processamento de erros grosseiros;

- No capítulo 7 são propostos dois algoritmos para projeto ou fortalecimento de sistemas de medição, baseados no índice $U I$;

- Por fim, o capítulo 8 apresenta as conclusões mais relevantes do trabalho, e as perspectivas futuras da pesquisa em questão.

As referências bibliográficas e os anexos são apresentados após as conclusões. 


\section{Capítulo 2}

\section{Estimação de Estado em Sistemas Elétricos de Potência}

Os estados operativos de um sistema elétrico em regime estacionário dependem diretamente das suas variáveis de estado, isto é, das tensões complexas nas barras do sistema. Portanto, a obtenção de tais grandezas é de vital importância para a segurança e confiabilidade da operação, em tempo-real, dos sistemas elétricos de potência.

As variáveis de estado de um sistema são obtidas a partir do processamento e/ou “filtragem" de medidas analógicas que as relacionam. Assim, para execução de tal tarefa, os centros de controle dispõem de um conjunto de programas desenvolvíveis em computador, denominado Estimador de Estado.

Logo, pode-se dizer que a estimação de estado em sistemas elétricos de potência consiste na obtenção das variáveis de estado de um determinado sistema, através de um conjunto redundante de medidas com ruído. Atualmente, as medidas podem ser classificadas como convencionais ou como fasoriais sincronizadas. As medidas convencionais são aquelas oriundas do sistema supervisório SCADA, sendo este constituído usualmente por medidas de: fluxo e injeção de potência ativa e reativa; magnitude de tensão nas barras e magnitude de corrente nas linhas.

Por outro lado, as medidas fasoriais sincronizadas, tais como as tensões e correntes fasoriais, são aferidas pelos chamados PMUs (Phasor Measurements Units) e sincronizadas através do sistema de geoposicionamento por satélite (GPS).

A partir dos primeiros trabalhos publicados por Schweppe, no final da década de 1960 e início de 1970 (SCHWEPPE, WILDES, RON, 1968; SCHWEPPE e WILDES, 1970; SCHWEPPE e RON, 1970; e SCHWEPPE, 1970), os quais delinearam vários 
conceitos e a natureza geral do problema, a estimação de estado em sistemas elétricos tornou-se alvo de inúmeras pesquisas. Em trabalhos como os de Bose et al. (1987), Coutto Filho et al. (1990), Wu (1990), Monticelli (1999) e Abur e Expósito (2004) apresenta-se-nos uma vasta bibliografia referente aos estudos em estimação de estado, a partir de 1968.

O estimador de estado pode ser dinâmico ou estático. No caso do estimador dinâmico $^{2}$, as variações das grandezas, de interesse em relação à variável 'tempo', são consideradas na modelagem da rede. Por outro lado, no estimador estático, o modelo de rede utilizado é estático, ou seja, uma fotografia do sistema num instante de tempo fixo. Dessa forma, o comportamento matemático do sistema elétrico é traduzido utilizando-se apenas equações algébricas não-lineares, sem o emprego de equações diferenciais. Ademais, o mesmo pode ser considerado como uma generalização do problema clássico de fluxo de carga (HANDSCHIN et al., 1975).

Na seção 2.1, a seguir, apresentar-se-ão as equações de fluxo de carga, por serem utilizadas na formulação do processo de estimação de estado em sistemas elétricos.

\subsection{Modelagem de Linhas e Transformadores: Equações de Fluxo de Carga}

Neste trabalho adotou-se, como representação de um ramo do sistema elétrico, uma generalização do modelo equivalente de linhas de transmissão, trafos em fase e trafos defasadores, a partir do modelo apresentado em Monticelli (1983). A figura 2.1, a seguir, ilustra esse modelo:

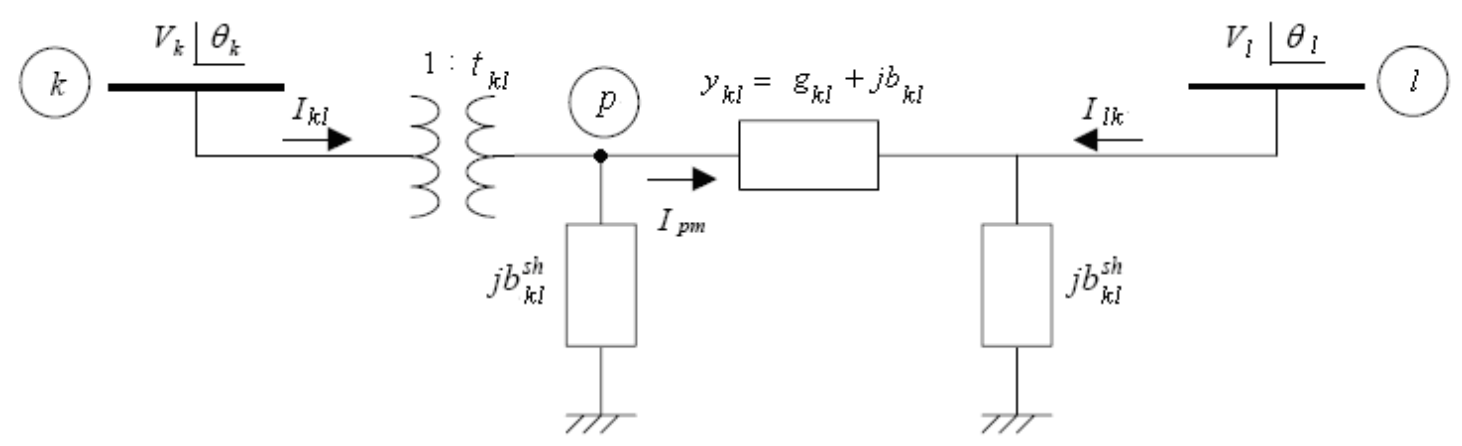

Figura 2.1: Modelo $\pi$ generalizado de um ramo do sistema elétrico.

Sendo:

- $g_{k l}$ a condutância série do componente;

\footnotetext{
${ }^{2}$ Uma vasta revisão bibliográfica sobre estimadores dinâmicos pode ser encontrada em Shivakumar e Jain (2008) e Coutto-Filho e Souza (2009).
} 
- $b_{k l}$ a susceptância série do componente;

- $b_{k l}^{s h}$ a susceptância shunt para linhas de transmissão;

- $t_{k l}=a_{k l} \cdot e^{j \varphi}$ a relação de transformação para transformadores;

- $V_{k}$ e $V_{l}$ as magnitudes de tensão elétrica das barras $k$ e $l$, respectivamente;

- $\theta_{k}$ e $\theta_{l}$ os ângulos de fase da tensão elétrica das barras $k$ e $l$, respectivamente.

A partir da aplicação das leis de Kirchhoff, sobre o modelo $\pi$ generalizado representado na figura 2.1, obtêm-se as seguintes expressões para os fluxos de potência ativa $\left(P_{k l}\right)$ e reativa $\left(Q_{k l}\right)$ no ramo que conecta as barras $k$ e $l$ (MONTICELLI, 1983):

Da barra $k$ para barra $l$ :

$$
\begin{aligned}
P_{k l} & =a_{k l}^{2} \cdot V_{k}^{2} \cdot g_{k l}-a_{k l} \cdot V_{k} \cdot V_{l} \cdot g_{k l} \cdot \cos \left(\theta_{k l}+\varphi\right)+ \\
& -a_{k l} \cdot V_{k} \cdot V_{l} \cdot b_{k l} \cdot \operatorname{sen}\left(\theta_{k l}+\varphi\right) \\
Q_{k l} & =-a_{k l}^{2} \cdot V_{k}^{2} \cdot\left(b_{k l}+b_{k l}^{s h}\right)+a_{k l} \cdot V_{k} \cdot V_{l} \cdot b_{k l} \cdot \cos \left(\theta_{k l}+\varphi\right)+ \\
& -a_{k l} \cdot V_{k} \cdot V_{l} \cdot g_{k l} \cdot \operatorname{sen}\left(\theta_{k l}+\varphi\right)
\end{aligned}
$$

Da barra $l$ para barra $k$ :

$$
\begin{aligned}
P_{l k} & =V_{l}^{2} \cdot g_{k l}-a_{k l} \cdot V_{k} \cdot V_{l} \cdot g_{k l} \cdot \cos \left(\theta_{l k}-\varphi\right)+ \\
& -a_{k l} \cdot V_{k} \cdot V_{l} \cdot b_{k l} \cdot \operatorname{sen}\left(\theta_{l k}-\varphi\right) \\
Q_{l k} & =-V_{l}^{2} \cdot\left(b_{k l}+b_{k l}^{s h}\right)+a_{k l} \cdot V_{k} \cdot V_{l} \cdot b_{k l} \cdot \cos \left(\theta_{l k}-\varphi\right)+ \\
& -a_{k l} \cdot V_{k} \cdot V_{l} \cdot g_{k l} \cdot \operatorname{sen}\left(\theta_{l k}-\varphi\right)
\end{aligned}
$$

No equacionamento da injeção de potência complexa, em qualquer barra de um sistema elétrico, deve considerar-se a existência de possíveis elementos shunts conectados a ela. Levando-se isto em conta, as expressões para as injeções de potência ativa e reativa, na barra genérica $k$ (fig. 2.1), podem ser escritas como:

$$
\begin{aligned}
& P_{k}=\sum_{l \in \Omega k} P_{k l} \\
& Q_{k}=-V_{k}^{2} \cdot b_{k}^{s h}+\sum_{l \in \Omega k} Q_{k l},
\end{aligned}
$$

onde $b_{k}^{s h}$ é a susceptância shunt de um reator/capacitor, conectado à barra $k ; \Omega k$ representando o conjunto de barras adjacentes à barra $k$. Os detalhes do equacionamento completo das expressões de fluxo de potência podem ser encontrados em Monticelli $(1983,1999)$. 
Nas próximas seções, serão abordados os principais métodos de solução do problema de estimação de estado estática, em sistemas elétricos de potência.

\subsection{Método da Equação Normal}

A estimação de estado consiste no cálculo de variáveis de estado desconhecidas, através de um conjunto de medidas não exatas. Logo, a estimação obtida para as variáveis de estado desconhecidas também não será exata.

Assim, o problema da estimação consiste em encontrar uma forma de atingir-se a melhor estimativa, e para isto, dos muitos critérios estatísticos existentes, o que vem sendo mais utilizado em sistemas elétricos é o dos mínimos quadrados ponderados (WLS, Weighted Least Squares).

Neste estudo, considera-se (por hipótese) um modelo determinístico para representar o sistema, isto é, admitir-se-á a não existência de erros nos parâmetros do modelo. Tal representação é ilustrada pelo diagrama a seguir:

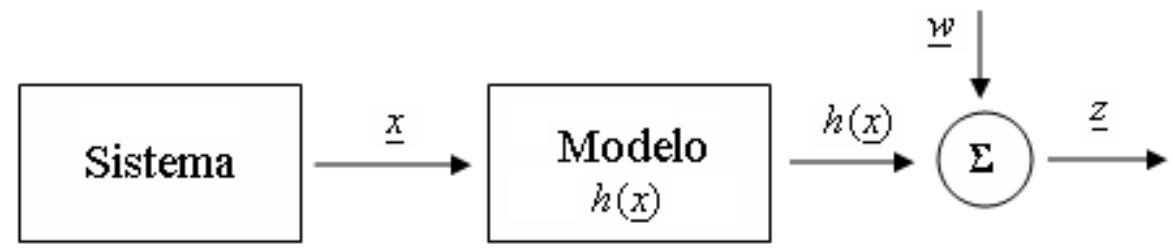

Com relação a esse diagrama, as equações não-lineares para estimação de estado em sistemas elétricos são representadas como:

$$
\underline{z}=h(\underline{x})+\underline{w},
$$

sendo:

$\underline{z}:$ vetor de medidas $(m \times 1)$;

$h($.$) : vetor de funções não-lineares, que relaciona as medidas com as variáveis$ de estado $(m \times 1)$;

$\underline{x}$ : vetor de variáveis de estado $(n \times 1)$;

$\underline{w}$ : vetor de erros das medidas $(m \times 1)$;

$m$ : número de medidas;

$n$ : número de variáveis de estado a serem estimadas. 
Os erros das medidas (termos de $\underline{w}$ ) são considerados como variáveis aleatórias independentes, com distribuição Gaussiana de média zero (SCHWEPPE e HANDSCHIN, 1974). Chamando de $R$ a matriz de covariância do vetor de erros das medidas, com dimensão $m \times m$, tem-se:

$$
R=\left[\begin{array}{cccc}
\sigma_{1}^{2} & & & \\
& \sigma_{2}^{2} & & \\
& & \ddots & \\
& & & \sigma_{m}^{2}
\end{array}\right],
$$

onde, $\sigma_{i}^{2}$ é a variância do erro da medida $i$. Assim, $\underline{w}_{i} \sim N\left(0, R_{i i}\right)$ para todo " $i$ ".

Através da aplicação da teoria de mínimos quadrados ponderados, a melhor estimativa do vetor de variáveis de estado $\underline{x}$, designada por $\underline{\hat{x}}$, pode ser obtida determinando-se o valor de $\underline{x}$ que torne mínimo o índice $J(\underline{x})$ (SCHWEPPE $\mathrm{e}$ HANDSCHIN, 1974), dado por:

$$
J(\underline{x})=\frac{1}{2} \cdot \underline{w}^{t} \cdot R^{-1} \cdot \underline{w},
$$

$\mathrm{ou}$

$$
J(\underline{x})=\frac{1}{2} \cdot[\underline{z}-h(\underline{x})]^{t} \cdot R^{-1} \cdot[\underline{z}-h(\underline{x})]
$$

sendo $R^{-1}$ o inverso da matriz de covariância do vetor de erros das medidas, aqui utilizado como a matriz de ponderação das medidas.

O índice $J(x)$ torna-se mínimo quando:

$$
\frac{\partial J(\underline{x})}{\partial \underline{x}}=0
$$

$\mathrm{ou}$

$$
H^{t}(\underline{\hat{x}}) \cdot R^{-1} \cdot[\underline{z}-h(\underline{\hat{x}})]=0,
$$

sendo $H(\underline{\hat{x}})$ a matriz de primeiras derivadas das funções não lineares do vetor $h(\underline{x})$, conhecida como matriz Jacobiana, calculada no ponto representado pelo vetor de variáveis de estado estimado $\underline{\hat{x}}$, e representada por:

$$
H(\underline{\hat{x}})=\left.\frac{\partial h(\underline{x})}{\partial \underline{x}}\right|_{x=\hat{x}} .
$$

\section{Linearização do Modelo de Medição}


Em razão de o índice $J(\underline{x})$ ser representado por uma função quadrática, expressa em termos de um vetor de equações não-lineares, a solução de $\underline{\hat{x}}$ exige a aplicação de um método iterativo, que resolva uma equação linear a cada iteração $v$, de modo a calcular a estimativa corrente do vetor de variáveis de estado, através de sucessivas correções (SCHWEPPE e HANDSCHIN, 1974), dadas por:

$$
\underline{x}^{v+1}=\underline{x}^{v}+\Delta \underline{x}^{v} \text {. }
$$

No entanto, para determinar a correção $\Delta \underline{x}^{v}$, efetua-se a linearização das equações de $h(\underline{x})$ em torno do ponto $\underline{x}^{v}$, representadas pela expressão

$$
h\left(\underline{x}^{v+1}\right) \cong h\left(\underline{x}^{v}\right)+H\left(\underline{x}^{v}\right) \cdot \Delta \underline{x}^{v} .
$$

Reescrevendo a equação (2.4), em relação às aproximações realizadas em $h(\underline{x})$, obtêm-se o modelo de medição que se converteu em linear:

$$
\underline{z}=h\left(\underline{x}^{v}\right)+H\left(\underline{x}^{v}\right) \cdot \Delta \underline{x}^{v}+\underline{w},
$$

ou ainda

$$
\Delta \underline{z}\left(\underline{x}^{v}\right)=\underline{z}-h\left(\underline{x}^{v}\right)=H\left(\underline{x}^{v}\right) \cdot \Delta \underline{x}^{v}+\underline{w},
$$

sendo $\Delta \underline{z}\left(\underline{x}^{v}\right)$ definido como vetor dos resíduos de medição.

A partir do modelo de medição linear, a função objetivo $J(\Delta \underline{x})$ torna-se:

$$
J(\Delta \underline{x})=\frac{1}{2} \cdot\left[\Delta \underline{z}\left(\underline{x}^{v}\right)-H\left(\underline{x}^{v}\right) \cdot \Delta \underline{x}^{v}\right]^{t} \cdot R^{-1} \cdot\left[\Delta \underline{z}\left(\underline{x}^{v}\right)-H\left(\underline{x}^{v}\right) \cdot \Delta \underline{x}^{v}\right],
$$

cujo mínimo é calculado a partir de

$$
\frac{\partial J(\Delta \underline{x})}{\partial \Delta \underline{x}}=H\left(\underline{x}^{v}\right)^{t} \cdot R^{-1} \cdot\left[\Delta \underline{z}\left(\underline{x}^{v}\right)-H\left(\underline{x}^{v}\right) \cdot \Delta \underline{x}^{v}\right]=0 .
$$

Logo, a solução pode ser processada pela seguinte expressão:

$$
\Delta \underline{x}^{v}=\left[H\left(\underline{x}^{v}\right)^{t} \cdot R^{-1} \cdot H\left(\underline{x}^{v}\right)\right]^{-1} \cdot H\left(\underline{x}^{v}\right)^{t} \cdot R^{-1} \cdot \Delta \underline{z}\left(\underline{x}^{v}\right),
$$

que é denominada Equação Normal, onde:

$$
H\left(\underline{x}^{v}\right)^{t} \cdot R^{-1} \cdot H\left(\underline{x}^{v}\right)=G\left(\underline{x}^{v}\right)
$$

representa a matriz ganho $(G)$, ou matriz de informação.

O processo iterativo começa a partir de uma estimativa inicial $\underline{x}^{0}$, e, a cada iteração $v$, as correções nas variáveis de estado $\Delta \underline{x}$ são obtidas através da equação (2.17). A atualização do vetor de variáveis de estado é obtida através da relação iterativa representada na equação (2.11), até que algum critério de parada seja satisfeito, por exemplo: 


$$
\begin{gathered}
\max \left|\Delta \underline{x}^{v}\right| \leq \mathcal{E}, \quad \text { ou } \\
J\left(\underline{x}^{v}\right)-J\left(\underline{x}^{v-1}\right) \leq \varepsilon
\end{gathered}
$$

no qual $\varepsilon$ denota uma tolerância previamente estabelecida. Assim, esses critérios indicarão que o processo iterativo é encerrado quando a magnitude dos ajustes nas variáveis de estado for desprezível, ou quando a variação do índice $J(x)$ também for desprezível.

O algoritmo do processo de estimação de estado por mínimos quadrados ponderados é apresentado a seguir:

\section{Algoritmo}

Passo 1: Fazer $v=0$ e escolher uma solução inicial $\underline{x}^{v}=\underline{x}^{0}$;

Passo 2: Calcular as matrizes $H\left(\underline{x}^{v}\right)$ e $G\left(\underline{x}^{v}\right)$ no ponto $\underline{x}=\underline{x}^{v}$;

Passo 3: Obter a correção nas variáveis de estado através da equação normal e atualizar as variáveis:

$$
\begin{gathered}
\Delta \underline{x}^{v}=G\left(\underline{x}^{v}\right)^{-1} \cdot H\left(\underline{x}^{v}\right)^{t} \cdot R^{-1} \cdot \Delta \underline{z}\left(\underline{x}^{v}\right) \\
\underline{x}^{v+1}=\underline{x}^{v}+\Delta \underline{x}^{v}
\end{gathered}
$$

Passo 4: Testar a convergência: se $\max \left|\Delta \underline{x}^{v}\right| \leq \varepsilon$, ou $J\left(\underline{x}^{v}\right)-J\left(\underline{x}^{\nu-1}\right) \leq \varepsilon$, o processo convergiu. Caso contrário, faça $v=v+1$ e volte ao passo 2 .

\subsection{Estimador de Estado Desacoplado Rápido}

O maior esforço computacional envolvendo o algoritmo do estimador WLS apresentado na seção anterior está associado ao cálculo da matriz $G$ e, posteriormente, à sua fatoração a cada iteração. Assim como no problema de fluxo de carga, uma alternativa encontrada para reduzir os cálculos envolvidos no processo de estimação de estado é manter constante, mas aproximada, a matriz Jacobiana que relaciona as equações das medidas com as variáveis de estado.

Para tanto, com base no princípio de que as potências ativas são influenciadas predominantemente pelos ângulos de fase de tensão e de que as potências reativas são influenciadas predominantemente pelas magnitudes de tensão, várias versões de estimadores de estado desacoplados e desacoplados rápidos foram propostas na literatura (HORISBERGER et al., 1976; ASCHMONEIT et al., 1976; HORTON e MASIELLO, 1977; GARCIA et al., 1979; ALLEMONG et al., 1982; MONTICELLI e 
GARCIA, 1990). Dentre estas versões, destaca-se a desenvolvida por Monticelli e Garcia (1990), em razão de chegar a resultados satisfatórios, mesmo em sistemas com relação $x / r$ baixa. Dada esta vantagem, o mesmo é apresentado a seguir.

\section{Formulação do Estimador Desacoplado Rápido}

Considere a seguinte formulação desacoplada, para o sistema de equações nãolineares, que representam o problema de estimação de estado em sistemas elétricos:

$$
\begin{aligned}
& h_{p}(\underline{x}) \cong \underline{z}_{p}, \\
& h_{q}(\underline{x}) \cong \underline{z}_{q},
\end{aligned}
$$

sendo:

- $p$ sub-índice que representa o conjunto ativo, formado por medidas de injeção e fluxo de potência ativa;

- $q$ sub-índice que representa o conjunto reativo, formado por medidas de injeção e fluxo de potência reativa, além das medidas de magnitude de tensão elétrica;

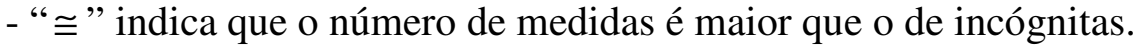

O vetor de variáveis de estado $\underline{x}$ é decomposto da seguinte forma:

$$
\underline{x}=\left[\begin{array}{l}
\underline{\theta} \\
\underline{V}
\end{array}\right],
$$

onde $\underline{V}$ é o vetor de magnitudes de tensão elétrica $(n b \times 1)$ e $\underline{\theta}$ é o vetor de ângulos de fase de tensão elétrica $((n b-1) \times 1)$, sendo $n b$ o número de barras do sistema.

A partir da divisão do conjunto de medidas e da decomposição do vetor de variáveis de estado, pode-se decompor a matriz Jacobiana da seguinte forma:

$$
H(x)=\frac{\partial h(x)}{\partial x}=\left[\begin{array}{ll}
\frac{\partial h_{p}(\underline{x})}{\partial \theta} & \frac{\partial h_{p}(\underline{x})}{\partial V} \\
\frac{\partial h_{q}(\underline{x})}{\partial \theta} & \frac{\partial h_{q}(\underline{x})}{\partial V}
\end{array}\right] \stackrel{\Delta}{=}\left[\begin{array}{cc}
H_{p \theta} & H_{p V} \\
H_{q \theta} & H_{q V}
\end{array}\right] .
$$

A solução ótima do vetor $\underline{x}$, através do estimador WLS desacoplado rápido (MONTICELLI e GARCIA, 1990), é obtida através de um procedimento iterativo, cujas correções $\Delta \underline{\theta}$ e $\Delta \underline{V}$ são calculadas separadamente. Nessa abordagem os efeitos das sub-matrizes $H_{p V}$ e $H_{q \theta}$ não são totalmente desprezados, mas são levados em conta através de uma aproximação na sub-matriz $H_{q V}$. Veja o algoritmo a seguir. 


\section{Algoritmo Desacoplado Rápido (versão “primal” ou $B X$ )}

As sub-matrizes $H_{p \theta}$ e $H_{q V}$ são consideradas fixas ao longo das iterações, sendo as mesmas calculadas com $\underline{\theta}=\underline{0}^{\circ}, \underline{V}=\underline{1} p u$ (flat start) e desprezando as resistências série na formação da sub-matriz $H_{q V}$. A partir dessas considerações, a solução ótima por mínimos quadrados ponderados pode ser obtida através de dois passos (MONTICELLI e GARCIA, 1990):

i) Cálculo da iteração ativa $P \theta$ :

$$
\begin{gathered}
\Delta \underline{\theta}^{v}=\left(H_{p \theta}^{t} \cdot R_{p}^{-1} \cdot H_{p \theta}\right)^{-1} \cdot H_{p \theta}^{t} \cdot R_{p}^{-1} \cdot\left[\underline{z}_{p}-h_{p}\left(\underline{\theta}^{v}, \underline{V}^{v}\right)\right], \\
\underline{\theta}^{v+1}=\underline{\theta}^{v}+\Delta \underline{\theta}^{v} .
\end{gathered}
$$

ii) Cálculo da iteração reativa $Q V$ :

$$
\begin{gathered}
\Delta \underline{V}^{v}=\left(H_{q V}^{t} \cdot R_{q}^{-1} \cdot H_{q V}\right)^{-1} \cdot H_{q V}^{t} \cdot R_{q}^{-1} \cdot\left[\underline{z}_{q}-h_{q}\left(\underline{\theta}^{v+1}, \underline{V}^{v}\right)\right], \\
\underline{V}^{k+1}=\underline{V}^{k}+\Delta \underline{V}^{k} .
\end{gathered}
$$

O processo iterativo encerra-se quando as magnitudes das correções $\Delta \theta$ e $\Delta V$ forem menores que os critérios de parada adotados, por exemplo, quando

$$
\begin{gathered}
\max \left|\Delta \underline{\theta}^{v}\right| \leq \varepsilon_{p \theta}, \mathrm{e} \\
\max \left|\Delta \underline{V}^{v}\right| \leq \varepsilon_{q V},
\end{gathered}
$$

no qual $\varepsilon_{p \theta}$ e $\varepsilon_{q V}$ denotam, respectivamente, a tolerância de parada do modelo ativo e do modelo reativo. Ademais, quando uma das tolerâncias é alcançada, fixa-se o respectivo vetor de estado e processa-se apenas a meia-iteração, na qual a tolerância não foi obtida. Conseqüentemente, o processo torna-se ainda mais rápido.

\subsection{Estimador de Estado Linearizado}

O estimador de estado linearizado, conquanto pouco interessante em aplicações práticas, tem importância considerável nos estudos dos métodos e técnicas referentes à estimação de estado. Isto porque, as hipóteses simplificadoras em que o mesmo se

\footnotetext{
${ }^{3}$ Em Monticelli e Garcia (1990) e Monticelli (1999), o algoritmo desacoplado rápido é apresentado tanto em versão "primal”, quanto em versão "dual” ou $X B$, o qual não será abordado neste trabalho.
} 
baseia transformam o problema de estimação em uma forma não-iterativa, simplificando-o conseqüentemente.

O estimador linearizado baseia-se nas equações de fluxo de carga linear, desprezando-se as perdas e considerando todas as magnitudes de tensão iguais a 1 p.u. Em função destas aproximações, calcula-se o fluxo de potência ativa entre duas barras genéricas $k$ e $l$, bem como a injeção de potência ativa na barra $k$, respectivamente, pelas seguintes expressões (MONTICELLI, 1999):

$$
\begin{gathered}
P_{k l}=\frac{\theta_{k}-\theta_{l}}{x_{k l}} \mathrm{e} \\
P_{k}=\sum_{l \in \Omega k}\left(\frac{\theta_{k}-\theta_{l}}{x_{k l}}\right),
\end{gathered}
$$

sendo: $\theta_{k}$ e $\theta_{l}$ os ângulos de tensão nas barras $k$ e $l$, respectivamente; $x_{k l}$ a reatância da linha de transmissão, que liga as barras $k$ e $l$; e $\Omega_{k}$ o conjunto das barras vizinhas à barra $k$.

A equação do fluxo de potência ativa, escrita dessa forma, permite analogias com a lei de Ohm, para um circuito de corrente contínua (CC). Em razão disto, o fluxo de potência linear é conhecido como fluxo de carga $\mathrm{CC}$, donde o estimador de estado linearizado também ser conhecido como estimador CC.

\section{Formulação do Estimador de Estado Linear}

$\mathrm{Na}$ formulação linear, o modelo de medição apresentado na seção 2.2 torna-se (MONTICELLI, 1999):

$$
\underline{z}_{p}=H_{p \theta} \cdot \underline{\theta}_{v}+\underline{w}_{p},
$$

sendo:

$\underline{\theta}_{v}$ : vetor de variáveis de estado $\left(n_{p} \times 1\right)$, que na formulação linear corresponde aos ângulos de tensão nas barras do sistema;

$\underline{z}_{p}$ : vetor de medidas de potência ativa $\left(m_{p} \times 1\right)$;

$H_{p \theta}$ : matriz Jacobiana ativa $\left(m_{p} \times n_{p}\right)$;

$\underline{w}_{p}:$ vetor de erros das medidas de potência ativa $\left(m_{p} \times 1\right) ;$

$m_{p}$ : número de medidas de potência ativa;

$n_{p}$ : número de variáveis de estado a serem estimados. 
A estimação do vetor de estado $\underline{\theta}_{v}$, designada por $\underline{\hat{\theta}}$, obtida através do método de mínimos quadrados ponderados é o valor de $\underline{\theta}$ que torna mínimo o índice $J(\underline{\theta})$, dado por:

$$
J(\underline{\theta})=\underline{w}_{p}^{t} \cdot R_{p}^{-1} \cdot \underline{w}_{p}=\left[\underline{z}_{p}-H_{p \theta} \cdot \underline{\theta}_{v}\right]^{t} \cdot R_{p}^{-1} \cdot\left[\underline{z}_{p}-H_{p \theta} \cdot \underline{\theta}_{v}\right] .
$$

Assim, a solução ótima pode ser calculada por:

$$
\underline{\hat{\theta}}=\left[H_{p \theta}^{t} \cdot R_{p}^{-1} \cdot H_{p \theta}\right]^{-1} \cdot H_{p \theta}^{t} \cdot R_{p}^{-1} \cdot \underline{z}_{p} .
$$

\subsection{Aspectos sobre a Equação Normal e Condicionamento Numérico}

\section{Aspectos sobre a Equação Normal}

No sistema linear expresso pela equação (2.17), a matriz $R^{-1}$ é diagonal. Devido a isto, o produto matricial $H\left(\underline{x}^{v}\right)^{t} \cdot R^{-1} \cdot H\left(\underline{x}^{v}\right)$ [matriz $G\left(\underline{x}^{v}\right)$ ] é aproximadamente duas vezes mais denso que a matriz $H\left(\underline{x}^{v}\right)$. Por outro lado, a matriz $H\left(\underline{x}^{v}\right)$ é esparsa e

portanto a matriz $G\left(\underline{x}^{v}\right)$ possui também um número reduzido de elementos não-nulos, o que possibilita o uso de técnicas de compactação e esparsidade.

Ademais, a matriz $G\left(\underline{x}^{v}\right)$ é simétrica em estrutura e valor, e definida positiva para sistemas observáveis, facilitando a sua fatoração e, por conseguinte, a solução do sistema linear representado pela equação normal. Dentre os métodos de fatoração tradicionalmente utilizados em estimação de estado, temos: Método de Eliminação de Gauss; fatoração $L U$ ou $L D U$; fatoração de Cholesky, decomposição $Q R$, entre outros.

\section{Condicionamento Numérico}

A precisão da solução da equação normal não dependerá apenas do método de fatoração empregado, mas também do condicionamento numérico da matriz Ganho (EBRAHIMIAN e BALDICK, 2001).

O número de condição de uma matriz não singular $A(K(A))$ pode ser definido como em Quarteroni et al. (2000) ou Ebrahimian e Baldick (2001):

$$
K(A)=\|A\| \cdot\left\|A^{-1}\right\|,
$$

onde II.II representa qualquer norma de matriz. 
Um aumento no número de condição produz uma grande sensibilidade da solução do sistema linear, em relação às mudanças de dados. Além disso, um número de condição de alto valor indica que a matriz em análise está mal condicionada.

Para o caso de uma matriz $A$ singular, $K(A)$ tende ao infinito. Assim, pode-se dizer que quanto maior o número de condição de uma matriz, mais próxima ela estará da singularidade.

Em Quarteroni et al. (2000) é provado que o número de condição de uma matriz, calculado através da norma-2, reduz-se a:

$$
K_{2}(A)=\|A\|_{2} \cdot\left\|A^{-1}\right\|_{2}=\frac{\sigma_{\max }(A)}{\sigma_{\min }(A)},
$$

onde $\sigma_{\max }$ e $\sigma_{\min }$ representam, respectivamente, o valor singular máximo e mínimo de $A$. Para o caso em que uma matriz $B\left(B=A^{t} A\right)$ é simétrica e definida positiva, tem-se:

$$
K_{2}(B)=\frac{\lambda_{\text {max }}(B)}{\lambda_{\text {min }}(B)}=\frac{\lambda_{\text {max }}\left(A^{t} A\right)}{\lambda_{\text {min }}\left(A^{t} A\right)}=\frac{\sigma_{\text {max }}^{2}(A)}{\sigma_{\text {min }}^{2}(A)}=\left(K_{2}(A)\right)^{2},
$$

sendo: $\lambda_{\max }$ o maior autovalor e $\lambda_{\min }$ o menor autovalor de $B$.

A partir desses resultados e considerando que $R^{-1}$ seja igual à matriz identidade, o número de condição da matriz ganho, da equação normal, pode ser calculado como:

$$
K_{2}(G)=\frac{\lambda_{\max }(G)}{\lambda_{\min }(G)} \cong\left(K_{2}(H)\right)^{2}
$$

Desse modo, pode-se concluir que o método de equação normal é intrinsecamente mal condicionado, já que o produto $H^{t} H$, existente no cálculo da matriz $G$, faz com que $G$ seja "pior" condicionada que $H$, numa ordem de grandeza ao quadrado (GOLUB et al., 1996).

$\mathrm{Na}$ literatura sobre a matéria, várias pesquisas apontam algumas situações em que se agrava o problema de condicionamento numérico da matriz Ganho (HOLTEN et al., 1988; EBRAHIMIAN e BALDICK, 2001; ABUR e EXPÓSITO, 2004). Como situações agravantes, podemos citar:

- disparidade entre as ponderações das medidas (elementos de $R^{-1}$ );

- medidas de injeção em barras conectadas a várias linhas;

- grande número de medidas de injeção;

- existência de linhas curtas e longas. 
Nas seções subseqüentes deste capítulo, serão apresentadas formulações derivadas do método WLS tradicional, que visam ao objetivo de diminuir o número de cálculos processados e de informações armazenadas em computador, ou buscam uma melhoria substancial na precisão numérica da solução.

\subsection{Modelagem de Injeções Nulas}

Comumente, em um sistema elétrico, existem barras cujas injeções de potência ativa e reativa são nulas. Tais barras são denominadas barras de transferência ou de passagem, sendo que as informações a elas associadas (injeções de potência nulas) são chamadas de medidas virtuais.

As medidas virtuais merecem tratamento especial no processo de estimação de estado, já que fornecem valores precisos de medidas de injeção. Tradicionalmente, este tipo de medida é modelado de duas formas (ABUR e EXPÓSITO, 2004):

i) Como medidas de injeção de potência de alta precisão, atribuindo-se-lhes ponderações elevadas. Apesar de tratar-se de um procedimento simples, a presença de ponderações diferentes, num mesmo conjunto de medidas, pode contribuir com um aumento no número de condição da matriz Jacobiana e prejudicar numericamente a convergência do estimador;

ii) Outra forma de tratar as injeções nulas é representá-las como restrições de igualdade no problema de mínimos quadrados ponderados, com o intuito de diminuir os problemas numéricos. Assim, o problema de estimação torna-se:

$$
\begin{gathered}
\text { minimizar } J(\underline{x})=\frac{1}{2}\left[[\underline{z}-h(\underline{x})]^{t} \cdot R^{-1} \cdot[\underline{z}-h(\underline{x})]\right] \\
\text { sujeito a } c(\underline{x})=0
\end{gathered}
$$

onde $c(\underline{x})=0$ representa o vetor de equações não-lineares de injeções de potência nulas e $R^{-1}$ a matriz de ponderação das demais medidas.

Conseqüentemente, a solução do problema, representado pela equação (2.39), é formulado através da função Lagrangeana:

$$
£(\underline{x}, \underline{\lambda})=\frac{1}{2} \cdot\left[[\underline{z}-h(\underline{x})]^{t} \cdot R^{-1} \cdot[\underline{z}-h(\underline{x})]\right]+\underline{\lambda}^{t} \cdot c(\underline{x}),
$$


na qual, $\underline{\lambda}$ é o vetor dos multiplicadores de Lagrange, relacionados às restrições de igualdade, cuja dimensão é igual ao número de injeções nulas.

Aplicando à equação (2.40) as condições ótimas de $1^{\text {a }}$ ordem, obtém-se o seguinte conjunto de equações:

$$
\begin{gathered}
\frac{\partial £(\underline{x}, \underline{\lambda})}{\partial \underline{x}}=0 \Rightarrow-H^{t}(\underline{x}) \cdot R^{-1} \cdot[z-h(\underline{x})]+C^{t}(\underline{x}) \cdot \underline{\lambda}=0 \\
\frac{\partial £(\underline{x}, \underline{\lambda})}{\partial \underline{\lambda}}=0 \Rightarrow c(\underline{x})=0
\end{gathered}
$$

onde, $C(\underline{x})=\frac{\partial c(\underline{x})}{\partial \underline{x}}$ é a matriz de primeiras derivadas das funções não-lineares do vetor $c(\underline{x})$, com relação ao vetor de variáveis de estado $\underline{x}$.

Como o conjunto de equações (2.41) engloba funções não-lineares, o mesmo exige a solução iterativa de um sistema linear, com a seguinte forma:

$$
\begin{gathered}
-H^{t}\left(\underline{x}^{k}\right) \cdot R^{-1} \cdot\left[\underline{z}-h\left(\underline{x}^{k}\right)-H\left(\underline{x}^{k}\right) \cdot \Delta \underline{x}^{k}\right]+C^{t}(\underline{x}) \cdot \underline{\lambda}^{k}=0 \\
c\left(\underline{x}^{k}\right)+C\left(\underline{x}^{k}\right) \cdot \Delta \underline{x}=0
\end{gathered}
$$

que ainda pode ser representado matricialmente por:

$$
\left[\begin{array}{cc}
H^{t}\left(\underline{x}^{k}\right) \cdot R^{-1} \cdot H\left(\underline{x}^{k}\right) & C^{t}\left(\underline{x}^{k}\right) \\
C\left(\underline{x}^{k}\right) & 0
\end{array}\right] \cdot\left[\begin{array}{c}
\Delta \underline{x}^{k} \\
\underline{\lambda}^{k}
\end{array}\right]=\left[\begin{array}{c}
H^{t}\left(\underline{x}^{k}\right) \cdot R^{-1} \cdot\left[\underline{z}-h\left(\underline{x}^{k}\right)\right] \\
-c\left(\underline{x}^{k}\right)
\end{array}\right] .
$$

Um inconveniente dessa abordagem é o fato de a matriz de coeficientes ser indefinida, mesmo para sistemas observáveis, de modo diferente ao caso da matriz ganho para sistemas observáveis. Para contornar esse problema, deve-se realizar a permutação de linhas durante a decomposição/fatoração do sistema de equações representado pela equação (2.43). Em Gjelsvik et al. (1985), esse procedimento é modificado através do "pivotamento" 2 × 2, que torna a solução numérica mais estável quando comparada à fatoração tradicional.

Pode-se ainda melhorar o condicionamento numérico da matriz de coeficientes, através de um simples escalonamento da função Lagrangeana, dada pela equação (2.40), da seguinte forma (ABUR e EXPÓSITO, 2004):

$$
£(\underline{x}, \underline{\lambda})=\alpha \cdot \frac{1}{2} \cdot\left[[\underline{z}-H(\underline{x})]^{t} \cdot R^{-1} \cdot[\underline{z}-H(\underline{x})]\right]+\underline{\lambda}_{s}^{t} \cdot c(\underline{x}),
$$

sendo $\lambda_{s}=\alpha \cdot \lambda$ e $\alpha$ um escalar, geralmente escolhido como 


$$
\alpha=\frac{1}{\max R_{i i}^{-1}} \text { ou } \alpha=\frac{m}{\sum_{i=1}^{m} R_{i i}^{-1}}
$$

Conseqüentemente, o sistema de equações a ser resolvido iterativamente passa a ser

$$
\left[\begin{array}{cc}
\alpha \cdot H^{t}\left(\underline{x}^{k}\right) \cdot R^{-1} \cdot H\left(\underline{x}^{k}\right) & C^{t}\left(\underline{x}^{k}\right) \\
C\left(\underline{x}^{k}\right) & 0
\end{array}\right] \cdot\left[\begin{array}{c}
\Delta \underline{x}^{k} \\
\underline{\lambda}_{s}^{k}
\end{array}\right]=\left[\begin{array}{c}
\alpha \cdot H^{t}\left(\underline{x}^{k}\right) \cdot R^{-1} \cdot\left[\underline{z}-h\left(\underline{x}^{k}\right)\right] \\
-c\left(\underline{x}^{k}\right)
\end{array}\right] .
$$

Apesar de a modelagem de injeções nulas, como restrições de igualdade, prover uma melhora substancial no condicionamento numérico da matriz de coeficientes, isto não elimina o problema de mau condicionamento (por exemplo, devido a linhas de baixa reatância e medidas com ponderações discrepantes) que afeta consideravelmente a estabilidade numérica.

Com o intuito de contornar essas limitações, surgiram alguns métodos numericamente superiores. Uma revisão sobre esses métodos pode ser encontrada em Holten et al. (1988), Wu (1989), Monticelli (2000) e Abur e Expósito (2004).

Dentre esses métodos, merecem destaque os seguintes: o método da Matriz Aumentada ou de Hachtel (GJELSVIK et al., 1985), o método Tableau Esparso (NUCERA e GILLES, 1991) e as técnicas baseadas em transformações ortogonais (SIMÕES-COSTA e QUINTANA, 1981a-1981b).

$\mathrm{Na}$ próxima seção, será apresentada a formulação do método da Matriz Aumentada e do Tableau Esparso, devendo o método baseado em transformações ortogonais ser apresentado na seção subseqüente.

\subsection{Método da Matriz Aumentada e Tableau Esparso}

\section{Formulação do Método da Matriz Aumentada de Hachtel}

Neste método, o vetor de resíduo $\underline{r}$ é considerado uma incógnita e sua equação associada é tratada como uma restrição de igualdade, na formulação do problema de estimação de estado, assim como realizado para as injeções nulas. A partir dessas considerações, o problema de otimização torna-se:

$$
\text { minimizar } J(\underline{x})=\frac{1}{2} \cdot \underline{r}^{t} \cdot R^{-1} \cdot \underline{r}
$$




$$
\text { sujeito a }\left\{\begin{array}{l}
c(\underline{x})=0 \\
\underline{r}-\underline{z}+h(\underline{x})=0
\end{array}\right.
$$

sendo $c(\underline{x})=0$ o vetor de equações não-lineares, relacionadas às medidas virtuais (injeções de potência nulas) e $R^{-1}$ a matriz de ponderação das demais medidas, também denominadas como regulares (ABUR e EXPÓSITO, 2004).

Conseqüentemente, forma-se a função Lagrangeana, apresentada a seguir:

$$
£(\underline{x}, \underline{r}, \underline{\lambda}, \underline{\mu})=\frac{1}{2} \cdot\left[\underline{r}^{t} \cdot R^{-1} \cdot \underline{r}\right]-\underline{\lambda}^{t} \cdot c(\underline{x})-\underline{\mu}^{t} \cdot[\underline{r}-\underline{z}+h(\underline{x})],
$$

sendo: $\underline{\lambda}$ o vetor dos multiplicadores de Lagrange relacionados às medidas virtuais; $\mathrm{e}$ $\underline{\mu}$ o vetor dos multiplicadores de Lagrange relacionados às restriçõos de resíduo.

A partir da aplicação das condições de otimalidade de primeira ordem, obtém-se o seguinte conjunto de equações (ABUR e EXPÓSITO, 2004):

$$
\begin{gathered}
\frac{\partial £(\underline{x}, \underline{r}, \underline{\lambda}, \underline{\mu})}{\partial \underline{x}}=0 \Rightarrow C^{t}(\underline{x}) \cdot \underline{\lambda}+H^{t}(\underline{x}) \cdot \underline{\mu}=0 ; \\
\frac{\partial £(\underline{x}, \underline{r}, \underline{\lambda}, \underline{\mu})}{\partial \underline{r}}=0 \Rightarrow R^{-1} \cdot \underline{r}-\underline{\mu}=0 ; \\
\frac{\partial £(\underline{x}, \underline{r}, \underline{\lambda}, \underline{\mu})}{\partial \underline{\lambda}}=0 \Rightarrow c(\underline{x})=0 ; \\
\frac{\partial £(\underline{x}, \underline{r}, \underline{\lambda}, \underline{\mu})}{\partial \underline{\mu}}=0 \Rightarrow \underline{r}-\underline{z}+h(\underline{x}) .
\end{gathered}
$$

Analisando-se a segunda equação da expressão (2.48), verifica-se que $\underline{r}=R \cdot \underline{\mu}$. Isso permite que a variável $\underline{r}$ seja eliminada do equacionamento. Conseqüentemente, a solução iterativa do conjunto de equações acima, representado pela expressão (2.48), pode ser obtida pela seguinte equação matricial:

$$
\left[\begin{array}{ccc}
R & H\left(\underline{x}^{k}\right) & 0 \\
H^{t}\left(\underline{x}^{k}\right) & 0 & C^{t}\left(\underline{x}^{k}\right) \\
0 & C\left(\underline{x}^{k}\right) & 0
\end{array}\right] \cdot\left[\begin{array}{c}
\underline{\mu}^{k} \\
\Delta \underline{x}^{k} \\
\underline{\lambda}^{k}
\end{array}\right]=\left[\begin{array}{c}
{\left[\underline{z}-h\left(\underline{x}^{k}\right)\right]} \\
0 \\
-c\left(\underline{x}^{k}\right)
\end{array}\right] .
$$

Assim como a matriz dos coeficientes do sistema da equação (2.43), a matriz de coeficientes da equação (2.49) (matriz de Hachtel) também é indefinida. Logo, a aplicação de pivotamento 2 × 2 também é recomendada para tornar a solução numérica mais estável (GJELSVIK et al., 1985). Outro ponto a ser destacado resulta de o número de condição da matriz de coeficientes ser menor para esta modelagem, se comparada 
àquela apresentada na eq. (2.43). Além disso, o número de condição da matriz ainda pode ser reduzido, através de um simples escalonamento na função Lagrangeana, assim como realizado na seção 2.6.

\section{Formulação do Método Tableau Esparso}

O método Tableau Esparso pode ser visto como um caso particular do método da matriz aumentada. Isto porque, nessa abordagem, o conjunto de medidas é dividido em dois: um conjunto formado pelas medidas de fluxo de potência e magnitudes de tensão (conjunto $F$ ); e outro conjunto formado pelas medidas de injeção de potência (conjunto I). Desse modo, o sistema de equações Tableau torna-se (ABUR e EXPÓSITO, 2004):

$$
\left[\begin{array}{cccc}
R_{F} & 0 & H_{F} & 0 \\
0 & R_{I} & H_{I} & 0 \\
H_{F}^{t} & H_{I}^{t} & 0 & C^{t} \\
0 & 0 & C & 0
\end{array}\right] \cdot\left[\begin{array}{c}
\underline{\mu}_{F}^{k} \\
\underline{\mu}_{I}^{k} \\
\Delta \underline{x}^{k} \\
\underline{\lambda}^{k}
\end{array}\right]=\left[\begin{array}{c}
{\left[\underline{z}_{F}-h_{F}\left(\underline{x}^{k}\right)\right]} \\
{\left[\underline{z}_{I}-h_{I}\left(\underline{x}^{k}\right)\right]} \\
0 \\
-c\left(\underline{x}^{k}\right)
\end{array}\right]
$$

Essa ordenação diminui a ocorrência dos chamados fill-in's, ou seja, os elementos não nulos que surgem durante a fatoração da matriz dos coeficientes. Isso influi diretamente na precisão da solução do sistema de equações, já que traz como benefício a redução de possíveis erros numéricos oriundos da fatoração.

\subsection{Método baseado em Transformação Ortogonal}

Primeiramente, considere o modelo transformado da equação normal (2.17), cuja representação é dada por:

$$
\left(\tilde{H}^{t} \cdot \tilde{H}\right) \cdot \Delta \underline{x}=\tilde{H}^{t} \cdot \Delta \underline{\tilde{z}},
$$

onde, $\tilde{H}=R^{-1 / 2} H$ e $\Delta \underline{\tilde{z}}=R^{-1 / 2}(\underline{z}-h(\underline{x}))$ representam, respectivamente, a matriz Jacobiana ponderada e o vetor $\Delta \underline{z}$ ponderado.

Através do método conhecido como $Q R$ de transformação ortogonal (SIMÕESCOSTA e QUINTANA, 1981a-b), a matriz Jacobiana ponderada $\tilde{H}$ pode ser decomposta em:

$$
\tilde{H}=Q \cdot R_{t},
$$


sendo: $Q$ uma matriz ortogonal $\left(Q \cdot Q^{t}=I\right.$ ou $\left.Q^{t}=Q^{-1}\right) m$ x $m$; e $R_{t}$ uma matriz trapezoidal superior $m \times n$.

Ordenando-se adequadamente $Q$ e $R_{t}$, a decomposição dada por (2.52) pode ser escrita como a seguir:

$$
\tilde{H}=Q \cdot R_{t}=\left[\begin{array}{ll}
Q_{n} & Q_{0}
\end{array}\right] \cdot\left[\begin{array}{l}
U \\
0
\end{array}\right]=Q_{n} \cdot U,
$$

na qual $Q_{n}$ é uma sub-matriz $m \times n$, dada pelas primeiras $n$ colunas de $Q ; U$ é uma matriz triangular superior $n \times n$.

Substituindo a expressão (2.52) em (2.51), tem-se o seguinte desenvolvimento:

$$
\begin{aligned}
& \left(Q \cdot R_{t}\right)^{t} \cdot\left(Q \cdot R_{t}\right) \cdot \Delta \underline{x}=\left(Q \cdot R_{t}\right)^{t} \cdot \Delta \underline{\tilde{z}} \\
& R_{t}^{t} \cdot Q^{t} \cdot Q \cdot R_{t} \cdot \Delta \underline{x}=R_{t}^{t} \cdot Q^{t} \cdot \Delta \underline{\tilde{z}} \\
& R_{t}^{t} \cdot R_{t} \cdot \Delta \underline{x}=R_{t}^{t} \cdot Q^{t} \cdot \Delta \underline{\tilde{z}}
\end{aligned} .
$$

Agora, utilizando a partição dada em (2.53), tem-se que:

$$
\begin{aligned}
& U^{t} \cdot U \cdot \Delta \underline{x}=U^{-t} \cdot Q_{n}^{t} \cdot \Delta \underline{\tilde{z}} \\
& U \cdot \Delta \underline{x}=Q_{n}^{t} \cdot \Delta \underline{\tilde{z}}
\end{aligned} .
$$

Em Simões-Costa e Quintana (1981a), a função $J(x)$ representada na norma-2, foi tomada como ponto de partida para formulação do problema. Assim, a expressão (2.55) é obtida através do chamado método de Golub, que é baseado em sucessivas transformações ortogonais de Householder, sobre o sistema de equações. Já em SimõesCosta e Quintana (1981b), a aplicação do método de Rotação de Givens é investigada e comparada à anterior. Como resultado, verifica-se que o método baseado em rotação de Givens apresenta o mesmo comportamento numérico robusto e exige menos tempo de processamento que o outro. Desse modo, esse método torna-se mais atrativo para aplicações práticas e em tempo-real.

\section{Método Híbrido}

Em Monticelli et al. (1985), foi proposto um método híbrido, que combina a robustez numérica da transformação ortogonal com a estrutura esparsa do método da equação normal. Nessa abordagem, preserva-se o lado direito da equação normal, e altera-se apenas o esquerdo. Desse modo, a equação (2.51) é reescrita como: 


$$
\begin{aligned}
& \left(\tilde{H}^{t} \cdot \tilde{H}\right) \cdot \Delta \underline{x}=\tilde{H}^{t} \cdot \Delta \underline{\tilde{z}} \\
& R_{t}^{t} \cdot Q^{t} \cdot Q \cdot R_{t} \cdot \Delta \underline{x}=\tilde{H}^{t} \cdot \Delta \underline{\tilde{z}}, \\
& R_{t}^{t} \cdot R_{t} \cdot \Delta \underline{x}=\tilde{H}^{t} \cdot \Delta \underline{\tilde{z}}
\end{aligned}
$$

como $R_{t}^{t} \cdot R_{t}=U^{t} \cdot U$, a equação do método híbrido fica

$$
U^{t} \cdot U \cdot \Delta \underline{x}=\tilde{H}^{t} \cdot \Delta \underline{\tilde{z}} .
$$

Desde que a matriz ganho $\left(\tilde{H}^{t} \cdot \tilde{H}\right)$ seja simétrica e definida positiva, a matriz $U$, resultante da fatoração ortogonal de $\tilde{H}$, será a mesma obtida com a fatoração Cholesky da matriz ganho (MONTICELLI et al., 1985).

\subsection{Estimador Generalizado}

A modelagem de elementos de impedância nula, proposta em Monticelli e Garcia (1991) e Monticelli (1993a - 1993b), iniciou uma nova fase na estimação de estado em sistemas elétricos. Inovou, no sentido de modelar componentes que não eram tradicionalmente considerados, como disjuntores e chaves, possibilitando a sua estimação. Além desses dispositivos, parâmetros como impedância de linhas de transmissão e transformadores, elementos shunt, podem também ser estimados. A esse modelo mais geral deram-lhe a denominação de estimação de estado generalizada (ALSAÇ et al., 1998).

No modelo tradicional de estimação, o configurador fornece a topologia da rede, no modelo barra linha, com associação das medidas aos componentes, e o estimador considera tais informações como exatas. Assim, o tratamento da topologia da rede e o processamento das medidas analógicas são realizados de forma independente. Por outro lado, como o modelo generalizado representa um único conjunto, o mesmo possibilita a identificação de possíveis erros, com o tratamento simultâneo de medidas, dados de topologia da rede e parâmetros.

O modelo de estimação generalizada é ilustrado na figura 2.2 (ASADA, 2004). 


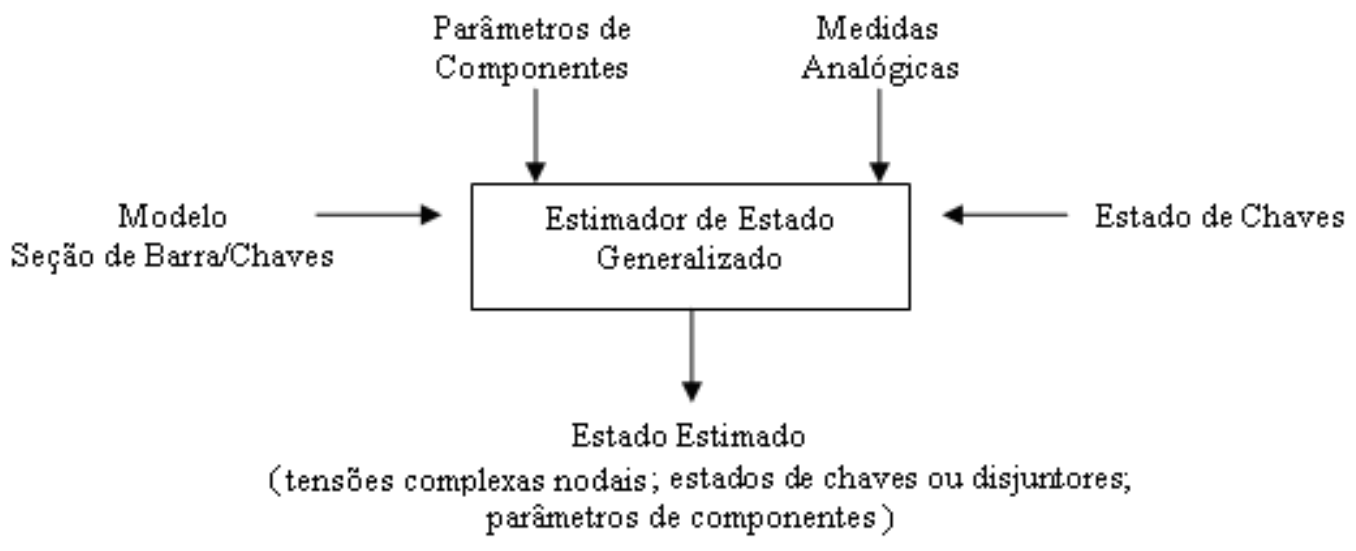

Figura 2.2: Modelo de estimação generalizada.

No modelo generalizado, o próprio fluxo de potência, circulante em dispositivos de impedância nula ou desconhecida, é representado como variável de estado. Desta forma, novas pseudo-medidas são também introduzidas no modelo, tais como: diferença de magnitude e ângulo de fase de tensão em dispositivos seccionadores; fluxo nulo de potência ativa e reativa em disjuntores abertos; diferença na magnitude de corrente para impedâncias desconhecidas; e diferença na admitância shunt para modelos $\pi$ equivalentes. Com isso, permite-se-nos uma melhor discriminação entre EGs em medidas analógicas, erros topológicos e erros de parâmetros.

Uma revisão completa dos aspectos principais relacionados à estimação de estado generalizada é apresentada em Monticelli (1999).

\subsection{Medição Sincronizada de Fasores}

Além do método matemático aplicado, o sucesso do estimador de estado dependerá também da qualidade das informações que lhe são fornecidas. Isto porque, erros nessas informações poderão levar o processo de estimação a valores estimados muito distantes dos valores verdadeiros, ou, até mesmo, à não convergência.

Dentre as causas de EGs, nas medidas fornecidas ao estimador de estado, destacam-se os erros nos canais de comunicação das telemedidas e também a falta de sincronismo dos dados obtidos pelo sistema SCADA, devido à ausência de uma fonte de sincronização das medições aferidas sobre o sistema elétrico.

Entretanto, nas duas últimas décadas, vários trabalhos de pesquisa têm abordado a possibilidade da obtenção de um sistema de medição sincronizada de Fasores (SMSF) (THORP et al., 1985; PHADKE et al., 1986; PHADKE, 1993), sendo que, atualmente, 
a implantação de equipamentos responsáveis por essas aferições, as Unidades de Medição Fasorial (PMUs, do inglês Phasor Measurement Units), já é uma realidade que se nos apresenta como uma forte tendência de utilização.

A PMU é um instrumento de medição desenvolvido em meados da década de 80, que realiza a amostragem sincronizada das tensões e correntes analógicas trifásicas. A base do tempo utilizado para a sincronização é dada pelo sinal de relógio do Sistema de Geoposicionamento por Satélite - GPS. Uma ilustração da estrutura básica de uma PMU é mostrada na figura 2.3 a seguir.

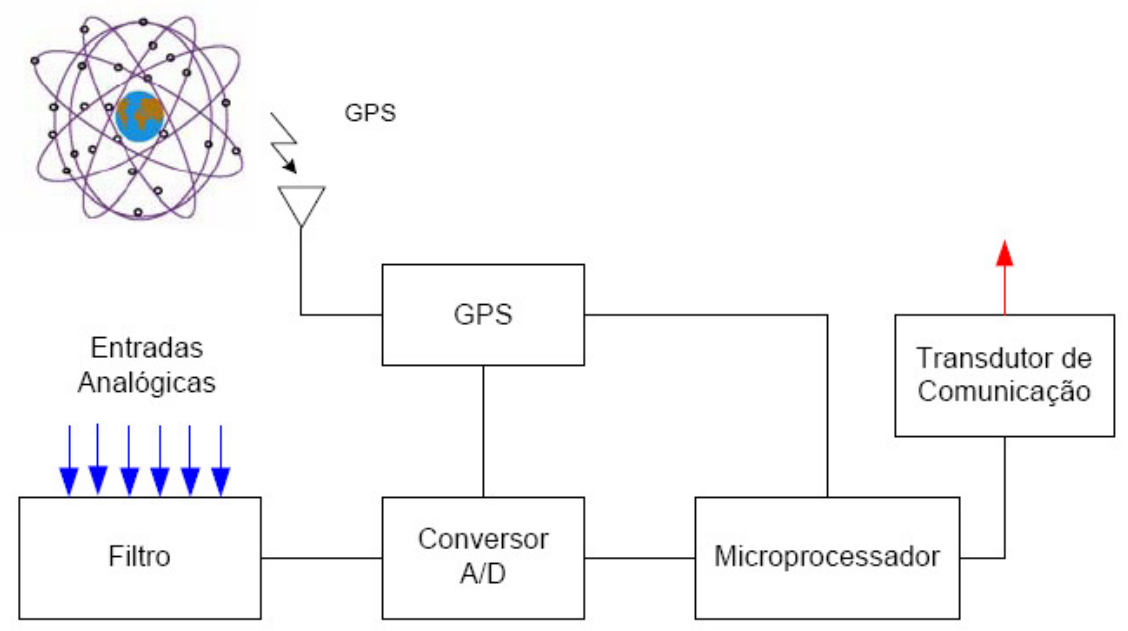

Figura 2.3: Estrutura básica de uma PMU (EHRENSPERGER, 2004)

Visando à melhoria do processo de estimação de estado em sistemas elétricos, ultimamente se nos apresentam diversas propostas, para a inclusão das medidas fasoriais.

Em trabalhos como os de THORP et al.(1985), PHADKE et al. (1986), ZIVANOVIC; CAIRNS (1996) e ZHOU et al. (2006), demonstra-se que, quando as medidas fasoriais são adicionadas às medidas convencionais, no processo de estimação de estado, a sua precisão aumenta. Além disto, desde que todas as tensões de barra do sistema sejam monitoradas por PMUs, o problema de estimação pode resolver-se utilizando somente medidas fasoriais (PHADKE, 2002; CEASE e FELDHAUS, 1999). Neste caso, tal problema torna-se linear, já que as medidas relacionarão diretamente as variáveis de estado, ao contrário do que acontece com as medidas convencionais de potência, as quais relacionam medidas e variáveis, através de funções não lineares.

Entretanto, a maioria dos sistemas elétricos ainda não é observável, considerando apenas as PMUs. Além disto, resultados de alguns estudos indicam que a utilização em conjunto, de medidas convencionais (obtidas pelo sistema SCADA) com 
medidas fasoriais, torna mais eficiente o processo de filtragem de erros, que o uso apenas de medidas fasoriais (ZIVANOVIC e CAIRNS, 1996), por serem as variáveis de estado estimadas mais sensíveis aos erros nas medidas fasoriais, que aos erros nas medidas de potência.

Importa destacar que uma das principais dificuldades dos estimadores de estado, que fazem uso de medidores convencionais e de PMUs, é o tratamento dado à informação angular, isto é, à determinação do ângulo de referência a ser utilizado.

O desenvolvimento de metodologias, para o tratamento de questões relacionadas à observabilidade e redundância das medidas, é outro ponto de muita relevância nesse contexto, o qual vem sendo alvo de várias pesquisas (ZHOU et al., 2008; LONDON Jr. et al., 2009). 


\section{Capítulo 3}

\section{Detecção e Identificação de Erros Grosseiros}

Neste capítulo, apresenta-se-nos uma descrição do problema de detecção e identificação de erros grosseiros (EGs), no processo de estimação de estado em sistemas elétricos de potência. Para tanto, as seções deste capítulo estão dispostas na seguinte ordem:

- Na seção 3.1, uma breve introdução ao problema de detecção e identificação de EGs, bem como as principais causas, tipos e métodos de análise de EGs são apresentados.

- Na seção 3.2, as propriedades dos resíduos das medidas são estudadas.

- Na seção 3.3, são descritas as metodologias principais para detecção e identificação de EGs através da análise dos resíduos das medidas.

- Na seção 3.4, é abordado o conceito de medidas ponto de alavancamento e seus efeitos nos estimadores de Mínimo Quadrado Ponderado, Mínimo Valor Absoluto Ponderado e Mínima Mediana do Resíduo Ponderado ao Quadrado. Além disso, três métodos para identificação de medidas ponto de alavancamento são apresentados no final da seção.

\subsection{Introdução}

Como as informações referentes ao processo de estimação de estado apresentam naturezas distintas, o mesmo está sujeito aos seguintes tipos de erros: EGs (presentes em medidas analógicas); erros topológicos (devido a informações erradas, quanto aos 
estados de chaves e/ou disjuntores) e erros de parâmetros ${ }^{4}$ (causados por informações erradas de parâmetros, dentre os quais: susceptância e condutância série de linhas de transmissão; susceptância shunt de linhas de transmissão; tap de transformadores, etc).

A habilidade de detectar e identificar EGs em medidas é um dos atributos mais importantes do processo de estimação de estado em sistemas elétricos.

Outros tipos de erros a serem destacados são os erros aleatórios, que existem devido à precisão finita dos medidores e dos canais de comunicação. Entretanto, esperase que tais erros sejam filtrados pelo processo de estimação, desde que exista redundância suficiente de medidas para tal. A natureza desta "filtragem" dependerá do método específico de estimação empregado (ABUR e EXPÓSITO, 2004).

Alguns EGs são óbvios e podem ser identificados a priori e eliminados do processo de estimação, através de uma simples verificação dos dados de entrada (medidas). Como exemplos de EGs têm-se: valores absurdos de tensão elétrica eficaz; valores muito além dos esperados para medidas de potência e/ou corrente elétrica, etc (ABUR e EXPÓSITO, 2004). Entretanto, nem todos os tipos de EGs são facilmente detectáveis ou identificáveis dessa forma, requerendo o emprego de métodos que realizem a análise de resíduos, após a estimação de estado propriamente dita.

Os métodos de detecção/identificação de EGs, que se baseiam na análise de resíduos das medidas, associados ao estimador WLS, são os mais utilizados em estimação de estado em sistemas elétricos. Isto porque os resíduos indicam eventuais violações das suposições relativas ao modelo de medição. Dada esta importância, a seção 3.2 apresentará as propriedades dos resíduos das medidas.

A figura 3.1, a seguir, mostra como tradicionalmente os EGs em medidas podem ser classificados (MONTICELLI, WU e YEN, 1986):

\footnotetext{
${ }^{4}$ Uma vasta revisão bibliográfica sobre erros de parâmetros no processo de Estimação de Estado em Sistemas Elétricos de Potência pode ser vista no trabalho de Castillo-Albertini (2010).
} 


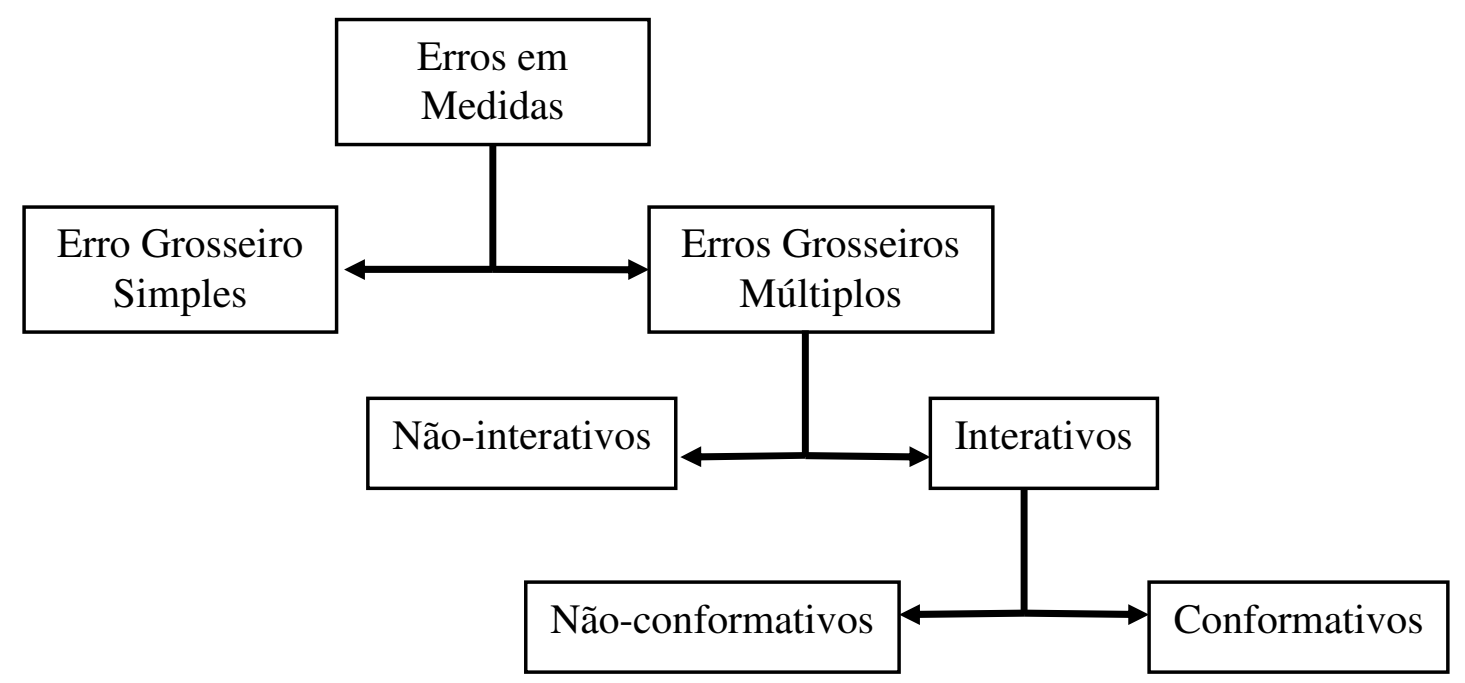

Figura 3.1: Classificação de erros grosseiros em medidas

Breves definições:

a) Erro Grosseiro Simples: ocorre quando apenas uma medida contém EG.

b) Erros Grosseiros Múltiplos: ocorrem quando mais de uma medida contém EG. Tais erros podem ainda ser classificados como interativos ou não-interativos.

b.1) Erros Não-Interativos: ocorrem quando os resíduos das medidas com EGs apresentam fraca interação com os demais resíduos, ou seja, quando os resíduos são fracamente correlacionados. Nessa situação, o EG em uma medida não afeta ou afeta pouco o resíduo das demais medidas disponíveis.

b.2) Erros Interativos: ocorrem quando as medidas com EGs apresentam resíduos fortemente correlacionados com os resíduos de outras medidas, geralmente na sua vizinhança. Assim, o erro em uma medida influência muito o resíduo das medidas que lhe são correlacionadas. Os EGs interativos podem ser classificados como conformativos ou não-conformativos.

b.2.1) Não-Conformativos: são EGs interativos, cujo efeito não é "mascarado" nos resíduos das medidas portadoras de EGs. Desta forma, as medidas com EGs irão apresentar resíduos normalizados elevados.

b.2.2) Conformativos: são EGs interativos cujo efeito é "mascarado" nos resíduos das medidas portadoras de EGs, fazendo com que as mesmas atuem "conforme" as medidas sem EG. Desta forma, as medidas com EGs irão apresentar resíduos normalizados pequenos, e medidas "sadias" (sem EGs) podem ser identificadas como portadores de EGs. 
O estimador WLS funciona bem quando os erros em medidas são Gaussianos, mas não apresenta estimativas boas na ocorrência de um ou mais EGs (ABUR e EXPÓSITO, 2004).

Como citado na introdução deste trabalho, os métodos para detecção/identificação de EGs, que se baseiam na análise de resíduos associados ao método WLS, são os mais utilizados na prática e apresentam desempenho satisfatório na ocorrência de EG simples, ou quando ocorrem EGs múltiplos não-interativos. Todavia, os métodos baseados na análise de resíduos podem falhar ou até mesmo falham, em algumas situações:

i) erros grosseiros associados a medidas com baixa redundância (vide definições logo a seguir);

ii) erros grosseiros múltiplos interativos;

iii) erros grosseiros em medidas que possuam a característica de serem altamente influentes, isto é, de atraírem a convergência do processo de estimação de estado, chamadas tradicionalmente medidas ponto de alavancamento (MONTICELLI, 2000) (vide seção 3.4).

Devido ao fato de a redundância das medidas afetar a capacidade de se detectar e identificar possíveis EGs, antes do aprofundamento nas propriedades e análise de resíduos em medidas, serão apresentadas as definições de medidas críticas, conjuntos críticos de medidas, conjuntos $p$-críticos e nível de redundância de medidas.

\section{Medida Crítica}

Uma medida é considerada crítica, quando a supressão dessa medida, de um sistema observável, torna-o não-observável. Além disso, é impossível detectar EG em tais medidas, já que apresentam resíduo nulo no estimador WLS (CLEMENTS et al., 1981).

As medidas não críticas são chamadas redundantes e somente tais medidas podem ter resíduos não nulos no estimador WLS (CLEMENTS et al., 1981). Entretanto, as medidas redundantes podem ou não pertencer a conjuntos críticos de medidas (veja o conceito a seguir). 


\section{Conjunto Crítico de Medidas}

Conjunto crítico de medidas, também conhecido na literatura como "minimally dependent sets of measurements", ou "bad data groups", pode ser definido segundo Ayres e Haley (1986) de duas formas:

Definição numérica: os conjuntos críticos de medidas são aqueles correspondentes às submatrizes, da matriz covariância dos resíduos (equação 3.14, a ser apresentada), com posto igual a 1;

Definição topológica: conjunto crítico de medidas é o conjunto de medidas formado por medidas redundantes (que não são críticas), em que a supressão de uma medida qualquer, a ele pertencente, torna as demais medidas deste conjunto críticas.

A identificação dos conjuntos críticos é importante para um desempenho confiável do estimador de estado (SIMÕES-COSTA et al., 1990). Isto porque, além de esses conjuntos críticos representarem um risco à observabilidade de um sistema elétrico, a identificação de EGs em medidas pertencentes a um conjunto crítico é impossível, pois seus resíduos normalizados (vide seção 3.3) apresentam valores iguais em módulo (MILI et al., 1984).

\section{Conjunto p-crítico}

Em London Jr. et al. (2004), definiu-se conjunto p-crítico de medidas como conjunto de " $p$ " medidas, associadas a um sistema elétrico observável, no qual a perda de todas essas " $p$ " medidas torna o sistema não observável. Além disso, a remoção de qualquer conjunto de $k$ medidas, pertencentes a um conjunto p-crítico, com $k<p$, não causa a perda da observabilidade do sistema. Através dessa definição, pode-se verificar que:

- Para $\mathrm{p}=1$, o conjunto $\mathrm{p}$-crítico é a medida crítica;

- Para $p=2$, par crítico de medidas;

- Para $p=3$, trio crítico de medidas; e assim por diante.

\section{Nível de Redundância de uma Medida}

Uma medida tem nível de redundância (NR) igual a ( $p$-1) se o conjunto $p$-crítico, com menor número de medidas a que ela pertencer, possuir " $p$ " medidas. Por exemplo, uma medida crítica apresenta $\mathrm{NR}=0$; já uma medida não crítica, que aparece em pelo menos um par crítico de medidas, apresenta $\mathrm{NR}=1$. 


\subsection{Propriedades dos Resíduos das Medidas}

O modelo linearizado do estimador de estado será utilizado nesta seção, para delinear as propriedades dos resíduos das medidas e também para o tratamento de EGs, já que se trata de um modelo mais simples que o modelo não-linear.

Por simplicidade de notação, as matrizes e vetores do modelo linear ativo (apresentados na seção 2.4) aparecerão, a partir desta seção, sem os sub-índices $p$ e $\theta$, e, além disso, o vetor de estado do modelo ativo será $\underline{x}=\underline{\theta}$.

Dadas as considerações acima e reescrevendo o modelo linear de medição como:

$$
\underline{z}=H \cdot \underline{x}+\underline{w},
$$

obtêm-se a solução ótima por mínimos quadrados ponderados através de (equação 2.34 do capítulo 2):

$$
\begin{aligned}
& \underline{\hat{x}}=\left(H^{t} \cdot R^{-1} \cdot H\right)^{-1} \cdot H^{t} \cdot R^{-1} \cdot \underline{z}, \\
& \underline{\hat{x}}=G^{-1} \cdot H^{t} \cdot R^{-1} \cdot \underline{z}
\end{aligned}
$$

sendo que os vetores e as matrizes acima conservam os significados anteriormente descritos.

Considerando o vetor de medidas estimadas como $\underline{\hat{z}}=H \cdot \underline{\hat{x}}$, e substituindo o valor de $\underline{\hat{x}}$, temos que:

$$
\begin{aligned}
& \underline{\hat{z}}=H \cdot\left(H^{t} \cdot R^{-1} \cdot H\right)^{-1} H^{t} \cdot R^{-1} \cdot \underline{z}, \\
& \underline{\hat{z}}=K \cdot \underline{z}
\end{aligned}
$$

onde $K=H \cdot\left(H^{t} \cdot R^{-1} \cdot H\right)^{-1} H^{t} \cdot R^{-1}$ é a chamada matriz "chapéu" ou de projeção. Esta matriz será simétrica apenas quando todas as variâncias dos erros de medida forem iguais, ou seja, $R=c I$ (“c" é um escalar e $I$ é a matriz identidade).

A seguir, apresentam-se-nos algumas propriedades importantes da matriz $K$, bem como suas respectivas provas:

Propriedade P.1: $K$ é uma matriz idempotente, ou seja, $K \cdot K=K$.

Prova P.1: De forma direta:

$$
\begin{aligned}
& K \cdot K=\left[H \cdot\left(H^{t} \cdot R^{-1} \cdot H\right)^{-1} H^{t} \cdot R^{-1}\right] \cdot\left[H \cdot\left(H^{t} \cdot R^{-1} \cdot H\right)^{-1} H^{t} \cdot R^{-1}\right] \\
& K \cdot K=H \cdot(G)^{-1} \cdot G \cdot(G)^{-1} H^{t} \cdot R^{-1} \\
& K \cdot K=H \cdot G^{-1} H^{t} \cdot R^{-1}=K
\end{aligned}
$$


Propriedade P.2: $K \cdot H=H$.

Prova P.2: De forma direta:

$$
\begin{aligned}
& K \cdot H=\left[H \cdot\left(H^{t} \cdot R^{-1} \cdot H\right)^{-1} H^{t} \cdot R^{-1}\right] \cdot H \\
& K \cdot H=H \cdot G^{-1} \cdot G=H
\end{aligned} .
$$

Propriedade P.3: $(I-K) \cdot H=0$.

Prova P.3: De forma direta:

$$
\begin{aligned}
& (I-K) \cdot H=H-K \cdot H, \quad \text { como } K \cdot H=H \\
& (I-K) \cdot H=H-H=0
\end{aligned}
$$

Propriedade P.4: $K^{t} \cdot R^{-1} \cdot K=K^{t} \cdot R^{-1}=R^{-1} \cdot K$

Prova P.4: $\quad$ Sabendo-se que $\quad K=H \cdot\left(H^{t} \cdot R^{-1} \cdot H\right)^{-1} H^{t} \cdot R^{-1}, \quad R^{-1}=\left(R^{-1}\right)^{t} \quad$ e $G^{-1}=\left(G^{-1}\right)^{t}$, temos que:

$$
\begin{aligned}
& K^{t} \cdot R^{-1}=\left[\left(R^{-1}\right)^{t} \cdot H \cdot\left(G^{-1}\right)^{t} \cdot H^{t}\right] \cdot R^{-1}, \\
& K^{t} \cdot R^{-1}=R^{-1} \cdot K
\end{aligned}
$$

a partir desse resultado, e lembrando que $K$ é uma matriz idempotente, obtém-se facilmente a seguinte expressão:

$$
\begin{aligned}
& K^{t} \cdot R^{-1} \cdot K=\left(R^{-1} \cdot K\right) \cdot K \\
& K^{t} \cdot R^{-1} \cdot K=R^{-1} \cdot K=K^{t} \cdot R^{-1},
\end{aligned}
$$

e com isso provamos a propriedade P.4.

Propriedade P.5: $K \cdot R \cdot K^{t}=K \cdot R=R \cdot K^{t}=H \cdot G^{-1} \cdot H^{t}$

Prova P.5: Como $K=H \cdot\left(H^{t} \cdot R^{-1} \cdot H\right)^{-1} H^{t} \cdot R^{-1}, R=R^{t}$ e $G^{-1}=\left(G^{-1}\right)^{t}$, temos que:

$$
\begin{aligned}
& K \cdot R=\left(H \cdot G^{-1} \cdot H^{t} \cdot R^{-1}\right) \cdot R=H \cdot G^{-1} \cdot H^{t} \\
& R \cdot K^{t}=R \cdot\left[\left(R^{-1}\right)^{t} \cdot H \cdot\left(G^{-1}\right)^{t} \cdot H^{t}\right]=H \cdot G^{-1} \cdot H^{t},
\end{aligned}
$$

a partir desse resultado, obtém-se a seguinte expressão:

$$
\begin{aligned}
& K \cdot R \cdot K^{t}=K \cdot(K \cdot R), \\
& K \cdot R \cdot K^{t}=K \cdot R
\end{aligned}
$$

provando a propriedade P.5.

A redundância das medidas também pode ser investigada através dos elementos da matriz $K$. Isto porque, valores da diagonal principal próximos a 1 , ou muito maiores 
que os demais elementos da mesma linha, dão indício a uma baixa redundância da medida, referente àquela diagonal. Em tais situações, o valor estimado para a medida em questão será fortemente influenciado pela própria medida. Uma medida crítica “i” é o exemplo de um caso extremo no qual $K_{\mathrm{ii}}=1$ e $K_{\mathrm{ij}}=0$, para todo $\mathrm{j} \neq \mathrm{i}$.

Através da propriedade P.2, verifica-se que a aplicação de $K$ sobre $H$ implica na própria matriz $H$. Por outro lado, através da propriedade P.3, constata-se que a aplicação de $(I-K)$ sobre $H$ implica numa matriz nula.

Nota 3.1: No capítulo 4, mais detalhes sobre as matrizes $K$ e $(I-K)$ serão apresentados, sobretudo com o intuito de elucidar a interpretação geométrica do estimador WLS.

O vetor de resíduos de medida $(\underline{r})$ pode ser expresso como a diferença entre os valores das medidas e seus respectivos valores estimados, sendo o mesmo dado por:

$$
\underline{r}=\underline{z}-\underline{\hat{z}} .
$$

Substituindo a expressão (3.3) em (3.11), segue-se o seguinte desenvolvimento:

$$
\underline{r}=\underline{z}-K \cdot \underline{z}=(I-K) \cdot \underline{z},
$$

como $\underline{z}=H \cdot \underline{x}+\underline{w}$, segue que

$$
\begin{aligned}
& \underline{r}=(I-K) \cdot(H \cdot \underline{x}+\underline{w}) \\
& \underline{r}=(I-K) \cdot H \cdot \underline{x}+(I-K) \cdot \underline{w}
\end{aligned} .
$$

Através da propriedade P3, verifica-se que $(I-K) \cdot H \cdot \underline{x}=0$. Dessa forma, a expressão (3.11) pode ser expressa por:

$$
\begin{aligned}
\underline{r} & =(I-K) \cdot \underline{w}, \\
\underline{r} & =S \cdot \underline{w}
\end{aligned}
$$

onde $S$ é chamada matriz de sensibilidade do resíduo estimado, cuja dimensão é $m \times m$. Assim como a matriz $K$, a matriz $S$ será simétrica quando todas as variâncias dos erros de medidas forem iguais.

A matriz $S$ representa a influência, ou sensibilidade, nos resíduos das medidas, em relação aos erros das medidas (MONTICELLI, 2000). Tomando-se como exemplo uma medida crítica "i", tem-se que $K_{\mathrm{ii}}=1 \mathrm{e}$, por conseguinte, $S_{\mathrm{ii}}=\left(1-K_{\mathrm{ii}}\right)=0$ e $S_{\mathrm{ij}}=0$, para todo $\mathrm{j} \neq \mathrm{i}$. Assim, através da equação (3.14), o valor do respectivo resíduo $r_{\mathrm{i}}$ também será zero, independentemente do valor do erro $w_{\mathrm{i}}$.

A partir das matrizes $K$ e $S$, e também do vetor de resíduos $\underline{r}$, outras propriedades podem ser observadas facilmente: 
Propriedade P.6: $S$ é uma matriz idempotente, ou seja, $S \cdot S=S$.

Prova P.6: De forma direta:

$$
\begin{aligned}
& S \cdot S=(I-K) \cdot(I-K) \\
& S \cdot S=I-K-K+(K \cdot K), \text { como } K \cdot K=K \\
& S \cdot S=I-K=S
\end{aligned}
$$

Propriedade P.7: $S^{t} \cdot R^{-1} \cdot S=S^{t} \cdot R^{-1}=R^{-1} \cdot S$.

Prova P.7: Como $S=(I-K)$ e $K^{t} \cdot R^{-1}=R^{-1} \cdot K$, segue-se que

$$
\begin{aligned}
& S^{t} \cdot R^{-1}=(I-K)^{t} \cdot R^{-1}=R^{-1}-K^{t} \cdot R^{-1} \\
& S^{t} \cdot R^{-1}=R^{-1}-R^{-1} \cdot K=R^{-1} \cdot(I-K)=R^{-1} \cdot S
\end{aligned}
$$

a partir desse resultado, mostra-se que

$$
\begin{aligned}
& S^{t} \cdot R^{-1} \cdot S=\left(R^{-1} \cdot S\right) \cdot S \\
& S^{t} \cdot R^{-1} \cdot S=R^{-1} \cdot S
\end{aligned}
$$

Propriedade P.8: $S \cdot R \cdot S^{t}=S \cdot R=R \cdot S^{t}$.

Prova P.8: Sabendo-se que $S=(I-K), R=R^{t}$ e $K \cdot R=R \cdot K^{t}$, temos que:

$$
\begin{aligned}
& S \cdot R=(I-K) \cdot R=R-K \cdot R \\
& S \cdot R=R-R \cdot K^{t}=R \cdot(I-K)^{t}=R \cdot S^{t},
\end{aligned}
$$

e com isso, prova-se que

$$
\begin{aligned}
& S \cdot R \cdot S^{t}=S \cdot(S \cdot R) \\
& S \cdot R \cdot S^{t}=S \cdot R
\end{aligned} .
$$

Propriedade P.9: $K \cdot \underline{r}=0$.

Prova P.9: De forma direta:

$$
\begin{aligned}
& K \cdot \underline{r}=K \cdot(\underline{z}-\underline{\hat{z}})=K \cdot(I-K) \cdot \underline{z} \\
& K \cdot \underline{r}=(K-K \cdot K) \cdot \underline{z}=(K-K) \cdot \underline{z} \\
& K \cdot \underline{r}=0
\end{aligned}
$$

Propriedade P.10: $S \cdot \underline{r}=\underline{r}$.

Prova P.10: De forma direta:

$$
\begin{gathered}
S \cdot \underline{r}=S \cdot(S \cdot \underline{z}), \text { como } S \cdot S=S \\
S \cdot \underline{r}=S \cdot \underline{z}=\underline{r} .
\end{gathered}
$$


Antes de aprofundar nas técnicas específicas de detecção e identificação de EGs, através da análise dos resíduos de medidas, será apresentada, na próxima subseção, a distribuição de probabilidade dos resíduos de medidas.

\subsubsection{Distribuição de Probabilidade dos Resíduos de Medidas}

Considerando que os erros de medidas $\left(\underline{w_{\mathrm{i}}}\right)$ sejam variáveis aleatórias independentes de distribuição normal, com média zero e variância $R_{\mathrm{ii}}$, a média do vetor de resíduo de medida $E(\underline{r})$ torna-se:

$$
E(\underline{r})=E(S \cdot \underline{w})=S \cdot E(\underline{w})=0 .
$$

Por outro lado, a matriz de covariância do vetor resíduo de medidas $(\Omega)$ é calculada do seguinte modo:

$$
\Omega=E\left(\underline{r} \cdot \underline{r}^{t}\right)=E\left(S \cdot \underline{w} \cdot \underline{w}^{t} \cdot S^{t}\right)=S \cdot E\left(\underline{w} \cdot \underline{w}^{t}\right) \cdot S^{t},
$$

como $E\left(\underline{w} \cdot \underline{w}^{t}\right)$ corresponde à matriz de covariâncias dos erros de medidas, representada por $R$, então:

$$
\Omega=S \cdot R \cdot S^{t} .
$$

A expressão (3.24) pode ser simplificada, através da propriedade P.8, na seguinte expressão:

$$
\Omega=S \cdot R \cdot S^{t}=S \cdot R=R \cdot S^{t} .
$$

Outra expressão para $\Omega$ é a seguinte:

$$
\begin{aligned}
& \Omega=S \cdot R=(I-K) \cdot R=R-H G^{-1} H^{t} R^{-1} \cdot R \\
& \Omega=R-H G^{-1} H^{t}=R-R_{\hat{Z}}
\end{aligned} .
$$

onde $H G^{-1} H^{t}=R_{\hat{Z}}$ representa a matriz de covariância das medidas estimadas (MONTICELLI, 1999). Para uma medida "i" crítica, a variância da medida estimada será igual a variância da própria medida e, conseqüentemente, a variância do resíduo da medida $\left(\Omega_{\mathrm{ii}}\right)$ será zero.

A partir dos resultados da média do resíduo e de sua respectiva covariância, pode-se afirmar que o vetor resíduo apresenta distribuição normal multivariada, com média dada pelo vetor nulo e covariância representada pela matriz $\Omega$, ou seja:

$$
\underline{r} \sim N(\underline{0}, \Omega)=N(\underline{0}, S \cdot R) .
$$




\subsubsection{Distribuição de Probabilidade do Índice $J(x)$}

Primeiramente, considere o seguinte teorema sobre distribuição de formas quadráticas (Graybill, 1976):

Teorema 3.1: Seja $\underline{X}$ um vetor aleatório p-dimensional, de distribuição normal pvariada, com média $\underline{u}$ e matriz de covariância $\Sigma$ positiva definida, isto é, $\underline{X} \sim N_{p}(\underline{u}, \Sigma)$. Assim, uma variável $Y=\underline{X}^{t} \cdot A \cdot \underline{X}$ apresentará distribuição Qui-quadrado com $g l$ graus de liberdade $\left(\underline{X}^{t} \cdot A \cdot \underline{X} \sim \chi_{g l}^{2}\right)$, se “ $A \cdot \Sigma$ ” for idempotente, sendo $g l=\operatorname{posto}(\mathrm{A})$.

Escrevendo o índice $J(\underline{\hat{x}})$ como uma forma quadrática em termos de $\underline{w}$, tem-se que (HANDSCHIN et al., 1975):

$$
\begin{aligned}
& J(\underline{\hat{x}})=\underline{r}^{t} \cdot R^{-1} \cdot \underline{r} \\
& J(\underline{\hat{x}})=(S \cdot \underline{w})^{t} \cdot R^{-1} \cdot(S \cdot \underline{w}) . \\
& J(\underline{\hat{x}})=\underline{w}^{t} \cdot\left(S^{t} \cdot R^{-1} \cdot S\right) \cdot \underline{w}
\end{aligned}
$$

Através da propriedade P.7, o termo $S^{t} \cdot R^{-1} \cdot S=A$ pode ser simplificado pela expressão

$$
S^{t} \cdot R^{-1} \cdot S=R^{-1} \cdot S
$$

Pelo Teorema 3.1 e considerando $\underline{w} \sim N(\underline{0}, R)$, o índice $J(\underline{\hat{x}})$ terá distribuição Qui-quadrado se “ $\left(R^{-1} \cdot S\right) \cdot R$ ” for idempotente. Sendo assim, segue-se a verificação:

$$
\begin{aligned}
& \left(R^{-1} \cdot S \cdot R\right) \cdot\left(R^{-1} \cdot S \cdot R\right)=R^{-1} \cdot S \cdot S \cdot R \\
& \left(R^{-1} \cdot S \cdot R\right) \cdot\left(R^{-1} \cdot S \cdot R\right)=R^{-1} \cdot S \cdot R
\end{aligned},
$$

portanto, a matriz “ $\left(R^{-1} \cdot S\right) \cdot R$ ” é idempotente e o índice $J(\underline{\hat{x}})$ tem distribuição Quiquadrado. $\mathrm{O}$ grau de liberdade para distribuição de $J(\underline{\hat{x}})$ é igual à diferença entre o número de medidas e o número de estados a serem estimados $(m-n)$, que corresponde ao posto da matriz $\left(R^{-1} \cdot S\right)($ HANDSCHIN et al., 1975). Conclui-se então, que:

$$
J(\underline{\hat{x}}) \sim \chi_{m-n}^{2} .
$$




\subsection{Detecção e Identificação de Erros Grosseiros}

Tradicionalmente, as técnicas de detecção/identificação de EGs em medidas são (MONTICELLI, 1999): teste do índice $J(\underline{\hat{x}})$; teste dos resíduos normalizados; técnica de identificação por teste de hipótese (HTI).

\subsubsection{Teste do Índice $J(\underline{\hat{x}})$}

O primeiro método de detecção de EGs, proposto na literatura (SCHEWEPP, 1970), foi baseado no teste da distribuição qui-quadrado $\left(\chi^{2}\right)$. Assim, a detecção de EGs, através do índice $J(\underline{\hat{x}})$ (equação 3.28), obtido a partir dos resíduos de medidas calculados para $\underline{\hat{x}}$, pode ser realizada por intermédio de um teste de hipótese.

Primeiramente, considera-se uma hipótese nula Ho e uma hipótese alternativa H1, sendo estas representadas como (MONTICELLI, 1999): Ho: $E\{J(\underline{\hat{x}})\}=m-n$; e H1: $E\{J(\underline{\hat{x}})\}>m-n$. A partir dessas hipóteses, o seguinte teste pode ser realizado:

- Caso $J(\underline{\hat{x}})>\lambda$, rejeita-se a hipótese Ho;

- Caso $J(\underline{\hat{x}}) \leq \lambda$, aceita-se a hipótese Ho;

sendo $\lambda$ uma constante previamente determinada, fixando certa probabilidade $\rho$ de se tomar a decisão errada e supondo uma distribuição $\chi^{2}$, com $(m-n)$ graus de liberdade, para o índice $J(\underline{\hat{x}})$, isto é,

$$
\lambda=\chi_{m-n, 1-\rho}^{2} .
$$

Veja a figura 3.2 a seguir (MONTICELLI, 1999):

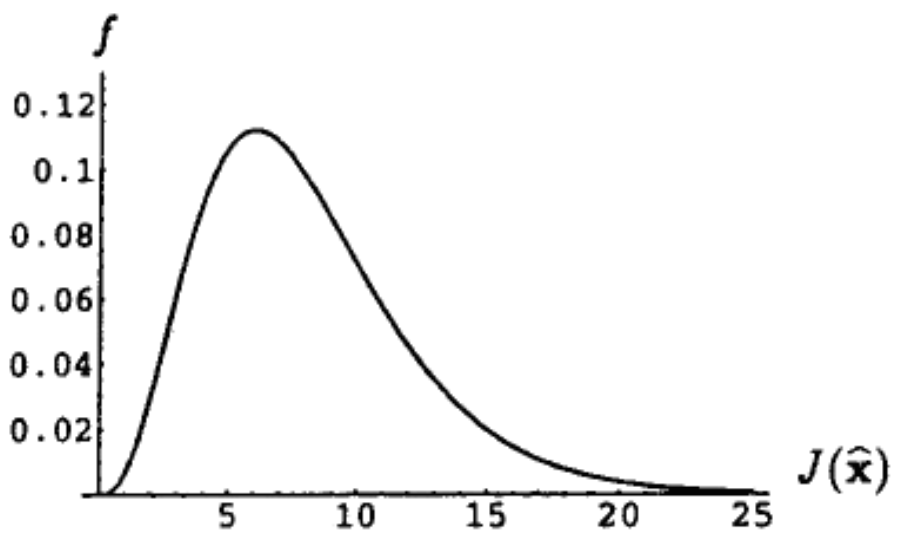

Figura 3.2: Função de densidade de probabilidade $(f)$ para uma distribuição qui-quadrado com 8 graus de liberdade $\left(\chi_{8}^{2}\right): \lambda=\chi_{8,(1-0,05)}^{2}=15,5$, ou seja, Probabilidade $\left(\chi_{8}^{2}>15,5\right)=0,05$. 
Conseqüentemente, o seguinte teste pode ser realizado para detecção de erros:

- Se $J(\underline{\hat{x}})>\lambda$, suspeita-se da existência de EG;

- Se $J(\underline{\hat{x}}) \leq \lambda$, não se suspeita da existência de EG.

Caso não se suspeite da existência de EG, consideram-se confiáveis os resultados obtidos pelo estimador de estado. Caso contrário, suspeita-se que exista algum EG em medida, ou, até mesmo, que o modelo assumido esteja inapropriado.

No caso em que as medidas portadoras de EGs sejam críticas, o método do índice $J(\underline{\hat{x}})$ não permite detectar EG, já que os resíduos das medidas críticas são nulos e, desse modo, não contribuem no valor do índice $J(\underline{\hat{x}})$. Além disso, esse método não possibilita realizar a identificação das medidas portadoras de EGs e, ainda, pode falhar em sistemas de grande porte, ou em sistemas cujo nível de ruído em medidas seja elevado (MONTICELLI e GARCIA, 1983).

\subsubsection{Teste dos Resíduos Normalizados}

Uma alternativa ao teste do índice $J(\underline{\hat{x}})$ é o método de detecção através do vetor de resíduos normalizados $\left(\underline{r}^{N}\right)$. Esse vetor é calculado a partir dos resíduos de medidas e da matriz de covariância de resíduos, pela seguinte relação:

$$
\underline{r}_{i}^{N}=\frac{\underline{r}_{i}}{\sqrt{\Omega_{i i}}} .
$$

Admitindo a hipótese de que os erros das medidas $\left(\underline{w}_{\mathrm{i}}\right)$ sejam variáveis aleatórias independentes, com distribuição normal de média zero e variância conhecida $R_{\mathrm{ii}}$, prova-se, em Monticelli (1999), que os elementos do vetor de resíduos normalizados apresentam distribuição normal padrão, ou seja:

$$
\underline{r}_{i}^{N} \sim N(0,1) .
$$

Desse modo, a existência de EGs pode ser verificada pelo seguinte teste:

- Se algum $\left|\underline{r}_{i}^{N}\right|>\beta$, com $i=1, \ldots, m$, suspeita-se que exista $\mathrm{EG}$

- Se todos $\left|\underline{r}_{i}^{N}\right| \leq \beta \operatorname{com} i=1, \ldots, m$, aceita-se a hipótese de que não exista EG.

O limiar estatístico $\beta$ é determinado admitindo-se certa probabilidade $\alpha$ de se tomar a decisão errada, para uma distribuição normal padrão, isto é $\beta=N(0,1), \alpha$. Usualmente assume-se $\beta=3$ (ABUR e EXPÓSITO, 2004). 
Depois de detectar a presença de EGs no conjunto de medidas em análise, tornase imprescindível a identificação das medidas portadoras de EG. Além de ser mais preciso que o teste do índice $J(\underline{\hat{x}})$, para detecção de EGs, o teste do resíduo normalizado possibilita ainda a identificação das medidas portadoras de EGs (ABUR e EXPÓSITO, 2004).

Considerando a hipótese de existência de uma única medida portadora de EG, e todas as demais medidas sem ruído algum, em trabalhos como o de Monticelli e Garcia (1983), bem como o de Abur e Expósito (2004), demonstra-se que, para um sistema de medição isento de medidas críticas e de conjuntos críticos de medidas, a medida portadora de EG apresentará o maior resíduo normalizado $\left(\underline{r}_{M A X}^{N}\right)$. Assim, tanto a detecção, quanto a identificação da medida portadora de EG, permite-nos verificar de uma só vez, ou seja, através do seguinte teste:

$$
\underline{r}_{M A X}^{N}>\beta \text { (limiar). }
$$

Na presença de EG simples, o método em pauta não permite identificar EG em medidas críticas, nem em medidas pertencentes aos conjuntos críticos de medidas. Isto porque, como já mencionado, as medidas críticas apresentam resíduo nulo (CLEMENTS et al., 1981) e as medidas de conjuntos críticos apresentam resíduos normalizados iguais em módulo (MILI et al., 1984).

Em caso de EGs múltiplos não interativos, esse teste permitirá identificar os EGs (de forma seqüencial), se os elementos fora da diagonal principal de $S$ forem baixos $\left(S_{i k} \approx 0\right)$, ou seja, quando tiverem baixa interação. Entretanto, se os resíduos das medidas portadoras de EGs tiverem forte interação, o teste do maior resíduo normalizado pode falhar, sendo os EGs conformativos ou não (ABUR e EXPÓSITO, 2004).

No trabalho de Clements e Davis (1986), uma interpretação geométrica para o resíduo normalizado foi apresentada, com o intuito de elucidar tanto a detecção quanto a identificação de erros grosseiros, através do teste dos resíduos normalizados. Além disso, são apresentados alguns exemplos para ilustrar a detecção e identificação de erros simples e múltiplos em medidas críticas, pares críticos e trios críticos. 


\subsubsection{Teste $\hat{b}$}

Em Monticelli e Garcia (1983), o chamado teste- $\hat{b}$ é utilizado em conjunto com o teste dos resíduos normalizados, com o intuito de melhorar a identificação de possíveis EGs.

O teste- $\hat{b}$ baseia-se numa estimativa do valor do EG, na medida considerada suspeita. Tal estimativa é obtida reescrevendo a equação (3.14), na forma mostrada a seguir (MONTICELLI e GARCIA, 1983):

$$
\underline{r}_{i}=S_{i i} \cdot \underline{w}_{i},
$$

sendo a medida " $i$ " considerada suspeita e não crítica.

A partir da equação (3.36), o erro $\underline{w}_{\mathrm{i}}$ pode ser escrito da forma seguinte:

$$
\begin{aligned}
& \underline{w}_{i}=\frac{\underline{r}_{i}}{S_{i i}}=\frac{R_{i i} \cdot \underline{r}_{i}}{\Omega_{i i}}=\sigma_{i} \cdot \frac{\sigma_{i} \cdot \underline{r}_{i}}{\Omega_{i i}}, \\
& \underline{w}_{i}=\sigma_{i} \cdot \underline{b}_{i}
\end{aligned}
$$

sendo

$$
\underline{\hat{b}}_{i}=\frac{\sigma_{i} \cdot \underline{r}_{i}}{\Omega_{i i}}=\frac{\sigma_{i} \cdot \underline{r}_{i}}{\sqrt{\Omega_{i i}} \cdot \sqrt{\Omega_{i i}}}=\frac{\sigma_{i} \cdot \underline{r}_{i}^{N}}{\sqrt{\Omega_{i i}}} .
$$

Com isso, verifica-se a existência de EG na medida "i" de maior resíduo normalizado, através do seguinte teste:

- Se $\left|\hat{b}_{i}\right|>c$, a medida apresenta EG;

- Se $\left|\hat{b}_{i}\right| \leq c$, a medida não apresenta EG.

Em Monticelli e Garcia (1983), o valor utilizado e sugerido para o limiar $c$ foi: $c=4$. Importa salientar que o teste- $\hat{b}$ apresenta as mesmas limitações do teste do resíduo normalizado, em relação a medidas críticas, conjuntos críticos e medidas ponto de alavancamento.

\subsubsection{Método de Identificação por Teste de Hipótese (HTI)}

Outra forma para identificar EGs foi proposta em Mili et al. (1984), baseando-se numa análise estatística concernente aos valores dos erros estimados em medidas, ao invés da análise dos resíduos normalizados. 
Os valores estimados dos erros em medidas podem ser calculados a partir da equação (3.39)

$$
\underline{\hat{w}}=S^{-1} \cdot \underline{r},
$$

onde $\underline{\hat{w}}$ é o vetor de erros estimados. Não nos permite, entanto, através dessa equação, estimar os erros de todas as medidas, já que o posto da matriz $S$ é menor que o número de medidas $m$, ou, com mais precisão: $\operatorname{posto}(S)=m-n$. Destarte, no máximo $(m-n)$ erros em medidas podem ser calculados, através de uma forma reduzida da matriz $S$.

A eficiência do método dependerá do conjunto reduzido escolhido, também denominado como conjunto suspeito. Conseqüentemente, esse conjunto deve ser escolhido de modo a incluir todos os possíveis erros, utilizando como critério os maiores resíduos normalizados.

Depois da formação do conjunto suspeito e do cálculo dos erros desse conjunto $\left(\underline{\hat{w}}^{s}\right)$, deve-se obter a distribuição de probabilidade para o erro estimado e, após isso, identificar os EGs, através de um teste de hipótese similar àquele apresentado para detecção de erros via índice $J(\underline{\hat{x}})$. Dessa forma, mais de uma medida com EG pode ser identificada de uma só vez, na ocorrência de EGs múltiplos.

Deve-se destacar que a escolha do conjunto suspeito através dos maiores resíduos normalizados pode ser comprometida, já que existe a possibilidade de uma ou mais medidas portadoras de EGs apresentarem baixos valores de resíduos normalizados (ABUR e EXPÓSITO, 2004) e, por conseguinte, são obtidas estimativas ruins para os valores dos erros.

\subsubsection{Tratamento de Medidas com Erros Grosseiros}

Após a identificação da medida portadora de EG, algum tratamento especial deve ser ministrado a essa medida, com o intuito de eliminar o seu efeito.

Tradicionalmente, o efeito da medida portadora de EG pode ser suprimido de duas formas (MONTICELLI e GARCIA, 1983):

i) retirada da medida com EG do conjunto de medidas e proceder-se à reestimação; 
ii) recuperação do valor da medida com EG, através do valor de erro estimado, ou $\hat{b}_{i}$, e re-estimação. A recuperação pode ser calcula a partir da equação (3.40) a seguir:

$$
\underline{z}_{i}^{\text {novo }}=\underline{z}_{i}^{\text {antigo }}-\sigma_{i} \cdot \underline{\hat{b}}_{i} .
$$

sendo:

$\underline{z}_{i}^{\text {novo }}$ o valor novo, ou valor recuperado, da medida com EG;

$\underline{z}_{i}^{\text {antigo }}$ o valor da medida utilizado na estimação de estado e que contém EG;

$\sigma_{i}$ o valor do desvio padrão da medida;

$\hat{b}_{i}$ definido anteriormente e calculado pela equação (3.38).

\subsection{Medidas Ponto de Alavancamento}

A designação ponto de alavancamento (leverage point) foi inicialmente empregada em análise de estatística robusta de regressão (HUBER, 1964; ROUSSEEUW e LEROY, 1987) e introduzida no problema de estimação de estado em sistemas elétricos por Mili et al. (1991).

Em Mili et al. (1991), os autores definem as medidas ponto de alavancamento como sendo aquelas que possuem posições discrepantes (“outliers"), em relação às demais medidas projetadas no espaço de fator, definido a seguir.

No processo de estimação de estado em sistemas elétricos de potência, espaço de fator é definido como sendo o espaço de dimensão $n$, gerado pelas linhas $\underline{l}_{i}(i=1, \ldots, m)$, da matriz Jacobiana ponderada $\tilde{H}\left(\tilde{H}=R^{-1 / 2} \cdot H\right)$. Desta forma, cada uma das linhas $\underline{l}_{i}^{t}$ representa um ponto no espaço de fator. Assim, a medida ponto de alavancamento corresponde a um ponto $\underline{l}_{-i}^{t}$, afastado dos demais no espaço de fator.

As seguintes situações tendem a gerar medidas ponto de alavancamento, em estimação de estado de sistemas elétricos (MILI et al., 1991):

- medidas de fluxo e de injeção de potência, adjacentes às linhas de transmissão que sejam relativamente curtas, quando comparadas com as demais linhas de transmissão do sistema; 
- medidas de injeção de potência, adjacentes às barras que apresentam elevado número de linhas incidentes.

\subsubsection{Exemplo de Medidas Ponto de Alavancamento}

Para caracterizar medidas ponto de alavancamento, em sistemas elétricos, utilizar-se-á o sistema de 3 barras ilustrado na figura 3.3, apresentado em Mili et al. (1991). Considera-se, inicialmente, que a reatância de todas as linhas daquele sistema seja igual a 1 p.u.

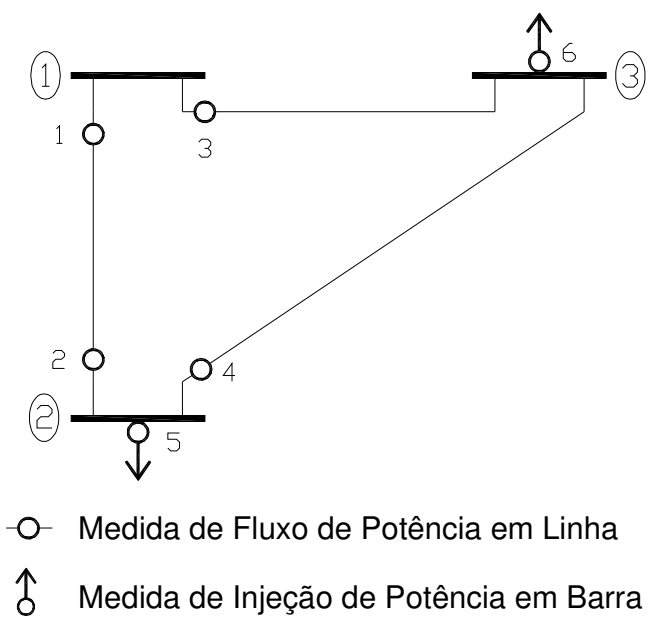

Figura 3.3: Diagrama do sistema de 3 barras.

Para simplificar a análise, considera-se o estimador WLS linear. Tomando como referência a barra $3\left(\theta_{3}=0^{\circ}\right)$ e considerando $R^{-1}=I$, sendo $I$ a matriz identidade de dimensão $(m \times m)$, temos que:

$$
\tilde{H}^{t}=\left[\begin{array}{cccccc}
1 & -1 & 1 & 0 & -1 & -1 \\
-1 & 1 & 0 & 1 & 2 & -1
\end{array}\right] .
$$

Pela figura 3.4(a), pode-se verificar que o sistema não apresenta medidas ponto de alavancamento, pois nenhum dos pontos se encontra afastado dos demais. Agora, se diminuirmos a reatância da linha 1-3 $\left(x_{13}\right)$, fazendo com que $1 / x_{13}$ seja igual a 5 p.u., os pontos 3 e 6 serão movidos em direção oposta, como mostra a figura 3.4(b). Estas medidas tornam-se medidas ponto de alavancamento, pois as mesmas correspondem, respectivamente, às colunas $[50]^{\mathrm{t}} \mathrm{e}[-5-1]^{\mathrm{t}}$ da nova matriz $\tilde{H}^{t}$. 
Por outro lado, se apenas a reatância da linha 2-3 for diminuída, então os pontos 4 e 5 serão movidos na direção positiva do eixo $l_{2}$, enquanto o ponto 6 mover-se-á na direção negativa, como mostra a figura 3.4(c).

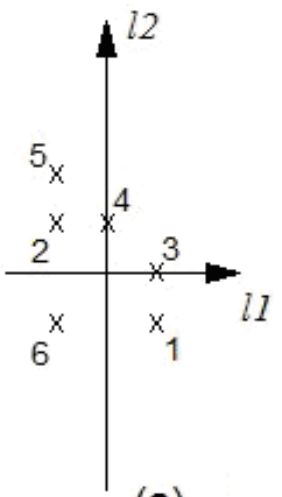

(a)

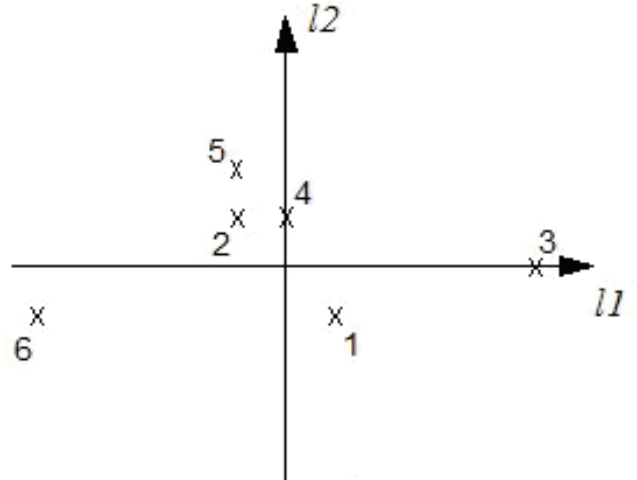

(b)

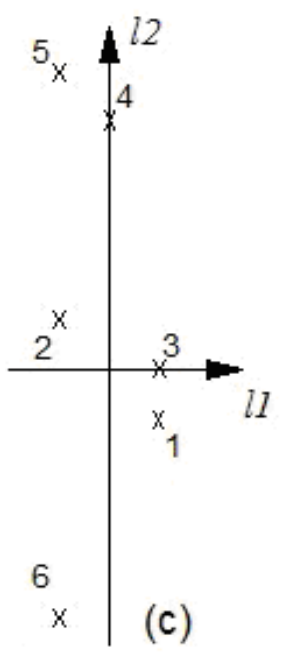

(c)

Figura 3.4: Espaço de fator do sistema de 3 barras, apresentado na figura 3.3: $l_{1}$ e $l_{2}$ são, respectivamente, a primeira e segunda linha da matriz $\tilde{H}^{t}$.

Pode-se observar, nesse exemplo, que medidas associadas às linhas com baixa reatância geram pontos afastados dos demais, no espaço de fator gerado pelas linhas da matriz $\tilde{H}^{t}$. Assim, tais medidas terão grande influência no processo de estimação de estado, sendo comumente denominadas como medidas ponto de alavancamento.

\subsubsection{Efeito de Pontos de Alavancamento nos Estimadores WLS, WLAV e WLMS}

Como mencionado na introdução deste capítulo, na presença de medidas com EGs simples, o estimador WLS falha, o que não acontece com o WLAV, por ser este insensível a medidas com EGs simples. O estimador WLS, associado à análise dos resíduos normalizados, apresenta um bom desempenho na ocorrência de EGs simples, mesmo quando ocorrem em uma única medida ponto de alavancamento (ABUR e EXPÓSITO, 2004).

Quando os EGs estão associados às medidas caracterizadas como ponto de alavancamento, o estimador WLAV é drasticamente afetado, produzindo estimativas incorretas.

Na presença de mais de uma medida ponto de alavancamente com EG, o teste do resíduo normalizado para o estimador WLS também falha. 
Finalmente, vale destacar que o estimador de estado WLMS é insensível às medidas com EGs, mesmo quando tais erros estejam associados às medidas classificadas como pontos de alavancamento.

\subsubsection{Identificação de Medidas Ponto de Alavancamento}

As medidas ponto de alavancamento podem ou não trazer prejuízo ao processo de estimação de estado; dessa forma, a identificação exata das mesmas é essencial para se ter idéia de quais medidas terão mais influência (negativa ou positiva) na resposta do estimador.

Os métodos usuais para identificação de medidas ponto de alavancamento, em estimação de estado em sistemas elétricos, são (ABUR e EXPÓSITO, 2004): método da matriz "chapéu", ou de projeção $K$; método da distância de Mahalanobis; e método da estatística de projeção.

\section{Método da Matriz "Chapéu” $K$}

Um método bastante difundido, para identificar medidas ponto de alavancamento, baseia-se na análise dos elementos da diagonal principal da matriz $K$, dada por:

$$
K=\tilde{H} \cdot\left(\tilde{H}^{t} \cdot \tilde{H}\right)^{-1} \cdot \tilde{H}^{t}
$$

Considerando que o valor esperado do $i$-ésimo elemento da diagonal de $K$ seja dado por:

$$
E\left[K_{i i}\right]=\bar{k}=\frac{1}{m} \sum_{i=1}^{m} K_{i i}=\frac{n}{m},
$$

se algum dos $K_{\mathrm{ii}}$ diferirem de modo significativo de $\bar{k}$, a correspondente medida $\underline{z}_{\mathrm{i}}$ é considerada como suspeita de ser um ponto de alavancamento, ou ainda, se a seguinte condição for satisfeita (ÇELIK e ABUR, 1992):

$$
K_{i i} \geq 2 \frac{n}{m} \text {. }
$$

A demonstração da equação (3.43) pode ser obtida da seguinte forma (ABUR e EXPÓSITO, 2004):

$$
\begin{aligned}
& \operatorname{tr}[K]=\operatorname{tr}\left[\tilde{H} \cdot\left(\tilde{H}^{t} \cdot \tilde{H}\right)^{-1} \cdot \tilde{H}^{t}\right] \\
& \operatorname{tr}[K]=\operatorname{tr}\left[\left(\tilde{H}^{t} \cdot \tilde{H}\right)^{-1} \cdot \tilde{H}^{t} \cdot \tilde{H}\right]=\operatorname{tr}\left[I_{n}\right]=n
\end{aligned}
$$




$$
\bar{k}=\frac{\operatorname{tr}[K]}{m}=\frac{1}{m} \sum_{i=1}^{m} K_{i i}=\frac{n}{m},
$$

sendo que $\operatorname{tr}[K]$ representa o traço da matriz $K$.

Este método de identificação de medidas ponto de alavancamento mostra-se eficaz para a ocorrência de um único ponto de alavancamento. No entanto, tal método falha na identificação de múltiplos pontos de alavancamento (MILI et al., 1991). O mesmo verificou-se em Çelik e Abur (1992).

Geometricamente, tal afirmação é justificada pelo fato de que $K_{\text {ii }}$ nos fornece a distância de um ponto $\left(l_{\mathrm{i}}, \underline{z}_{\mathrm{i}}\right)$ até o centróide $C$ do conjunto de medidas. Como $C$ é um ponto, cujas coordenadas resultam do cálculo da média aritmética das colunas da matriz $\tilde{H}$, o mesmo será atraído pelas medidas afastadas, mascarando os múltiplos pontos de alavancamento.

\section{Método da Distância de Mahalanobis}

Primeiramente se considera uma distribuição normal multivariada, para as linhas $\tilde{H}_{i}$ da matriz Jacobiana ponderada. A partir dessa consideração, o valor esperado da média $\bar{h}$ e da covariância $\bar{C}$ da amostra são calculados, respectivamente, por (ABUR e EXPÓSITO, 2004):

$$
\begin{gathered}
\bar{h}=\frac{1}{m} \sum_{i=1}^{m} \tilde{H}_{i}, \\
\bar{C}=\frac{1}{m-1} \sum_{i=1}^{m}\left(\tilde{H}_{i}-\bar{h}\right) \cdot\left(\tilde{H}_{i}-\bar{h}\right)^{t} .
\end{gathered}
$$

Uma forma de verificar-se a distância ou afastamento, entre $\tilde{H}_{i}$ e o resto da “nuvem" de medidas $\left(\tilde{H}_{j}\right)$, é através da Distância de Mahalanobis $(M D)$. O quadrado da distância de Mahalanobis para uma amostra é definido como:

$$
M D_{i}^{2}=\left(\tilde{H}_{i}-\bar{h}\right)^{t} \cdot C^{-1} \cdot\left(\tilde{H}_{i}-\bar{h}\right),
$$

que geometricamente pode interpretar-se como um elipsóide centrado em $\bar{h}$.

Se os vetores $\tilde{H}_{i}$ forem gaussianos, $M D_{i}^{2}$ terá aproximadamente uma distribuição qui-quadrado, com $n$ graus de liberdade $\left(\chi_{n}^{2}\right)$. Assim, a distância de Mahalanobis identificará os afastamentos $n$-dimensionais, quando $M D_{i}^{2}$ assumir valores 
maiores que o valor limite $\chi_{n,(1-\alpha)}^{2}$. Em outras palavras, uma medida $i$ será um ponto de alavancamento se:

$$
M D_{i}^{2}>\chi_{n,(1-\alpha)}^{2}
$$

sendo $\alpha$ a probabilidade de falso alarme, geralmente adotada como $\alpha=0,025$.

Este método, entretanto, não é robusto na presença de múltiplos pontos de alavancamento. Isto porque, pontos de alavancamento múltiplos podem ser mascarados, devido ao aumento nos valores da covariância $\bar{C}$, que leva a um aumento do elipsóide centrado em $\bar{h}$.

\section{Método da Estatística de Projeção}

Outro método para identificação de medidas ponto de alavancamento, aplicado à estimação de estado em sistemas elétricos, foi o método da Estatística de Projeção $\left(P S_{i}\right)$ (MILI et al., 1996). A estatística de projeção de um ponto $l_{i}$ indica o quão distante este ponto está dos demais pontos no espaço de fator, na pior dimensão unidimensional. Trata-se de um método mais robusto, em relação aos apresentados anteriormente, para identificação de múltiplos pontos de alavancamento. As projeções estatísticas podem ser calculadas por (ABUR e EXPÓSITO, 2004):

$$
P S_{i}=\max _{H_{k}} \frac{\left|\tilde{H}_{i}^{t} \cdot \tilde{H}_{k}\right|}{\beta}, \text { para } k=1,2, \ldots, \mathrm{m}
$$

sendo: $\quad \beta=1,1926 \cdot$ lomed $_{i}\left\{\right.$ lomed $\left._{j \neq i}\left\{\left|\tilde{H}_{i}^{t} \cdot \tilde{H}_{k}+\tilde{H}_{j}^{t} \cdot \tilde{H}_{k}\right|\right\}\right\}, \quad$ com $\quad 1 \leq i, j, k \leq m$; lomed $_{i}\{\underline{y}\}$ é a mediana baixa ("low median") de $m$ números em $\underline{y}=\left\{y_{1}, y_{2}, \ldots, y_{\mathrm{m}}\right\}$.

Admitindo-se uma distribuição qui-quadrada para $P S_{i}$ (MILI et al., 1996), uma medida $i$ é considerada como um ponto de alavancamento se a seguinte expressão for satisfeita:

$$
P S_{i}>\chi_{L i,(1-\alpha)}^{2}
$$

onde, o grau de liberdade $L i$ corresponde ao número de elementos não nulos na linha $i$ de $\tilde{H}$.

\section{Exemplo 1: Sistema de 3 barras com uma medida ponto de alavancamento}

Considere o sistema de 3 barras ilustrado na figura 3.5 (a seguir), cujas susceptâncias série de linhas são iguais a 1 p.u., exceto para a linha 1-3, que apresenta susceptância igual a 10 p.u. 


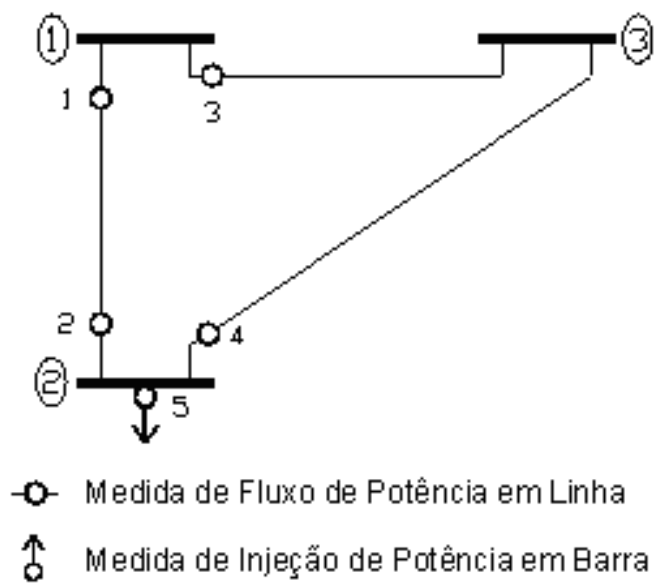

Figura 3.5: Sistema de 3 barras e 5 medidas utilizados no exemplo 1

Adotando-se a barra 3 como referência angular e $R^{-1}=I$, tem-se a seguinte matriz Jacobiana ponderada para este sistema:

$$
\tilde{H}^{t}=\left[\begin{array}{ccccc}
1 & -1 & 10 & 0 & -1 \\
-1 & 1 & 0 & 1 & 2
\end{array}\right] .
$$

Através da implementação em computador do método da matriz "chapéu" e do método da estatística de projeção, têm-se a seguir (tabela 3.1) os valores da diagonal principal da matriz de projeção $\left(K_{i i}\right)$, os valores da estatística de projeção $\left(P S_{i}\right)$, os graus de liberdade $(L i)$, e os valores da distribuição qui-quadrada para $L i$ graus de liberdade e probabilidade de falso alarme de $0,025\left(\chi_{L i, 0,975}^{2}\right)$.

Tabela 3.1: Valores de interesse para o teste da matriz "chapéu” e para o teste da estatística de projeção, para o exemplo 1

\begin{tabular}{c|c|c|c|c|c}
\hline \multirow{2}{*}{ Medida } & \multicolumn{2}{|c|}{ Teste da matriz $K$} & \multicolumn{3}{c}{ Teste da estatística de projeção } \\
\cline { 2 - 6 } & $K_{i i}$ & $2 n / m$ & $P S_{i}$ & $L i$ & $\chi_{L i, 0,975}^{2}$ \\
\hline F:1-2 & 0,1447 & 0,8 & 1,6770 & 2 & 7,38 \\
\hline F:2-1 & 0,1447 & 0,8 & 1,6770 & 2 & 7,38 \\
\hline F:1-3 & 0,9929 & 0,8 & 8,3850 & 1 & 5,02 \\
\hline F:2-3 & 0,1461 & 0,8 & 0,8385 & 1 & 5,02 \\
\hline I:2 & 0,5716 & 0,8 & 2,5155 & 2 & 7,38 \\
\hline
\end{tabular}

Diante dos valores da tabela 3.1, constata-se tanto pelo método da matriz $K$, quanto pelo método da estatística de projeção, que a medida F:1-3 é uma medida ponto de alavancamento. Isto porque, para essa medida F:1-3: 


$$
\begin{aligned}
& K_{i i}=0,9929>0,8=2 \cdot n / m, \mathrm{e} \\
& P S_{i}=8,3850>5,02=\chi_{L i, 0,975}^{2} .
\end{aligned}
$$

\section{Exemplo 2: Sistema de 3 barras com duas medidas ponto de alavancamento}

Considere o mesmo sistema de 3 barras ilustrado na figura 3.5, porém com a inclusão da medida de injeção de potência ativa na barra 3 (I:3).

Adotando-se, também, a barra 3 como referência angular e $R^{-1}=I$, tem-se a seguinte matriz Jacobiana ponderada para este sistema:

$$
\tilde{H}^{t}=\left[\begin{array}{cccccc}
1 & -1 & 10 & 0 & -1 & -10 \\
-1 & 1 & 0 & 1 & 2 & -1
\end{array}\right] .
$$

Considerando esta nova matriz Jacobiana ponderada e os métodos da matriz "chapéu" e da estatística de projeção, obtêm-se os valores tabelados a seguir (tabela $3.2)$.

Tabela 3.2: Valores de interesse para o teste da matriz "chapéu" e para o teste da estatística de projeção, para o exemplo 2

\begin{tabular}{c|c|c|c|c|c}
\hline \multirow{2}{*}{ Medida } & \multicolumn{2}{|c|}{ Teste da matriz $K$} & \multicolumn{3}{c}{ Teste da estatística de projeção } \\
\cline { 2 - 6 } & $K_{i i}$ & $2 n / m$ & $P S_{i}$ & $L i$ & $\chi_{L i, 0,975}^{2}$ \\
\hline F:1-2 & 0,1404 & 0,6667 & 1,6770 & 2 & 7,38 \\
\hline F:2-1 & 0,1404 & 0,6667 & 1,6770 & 2 & 7,38 \\
\hline F:1-3 & 0,5038 & 0,6667 & 8,3850 & 1 & 5,02 \\
\hline F:2-3 & 0,1278 & 0,6667 & 0,8385 & 1 & 5,02 \\
\hline I:2 & 0,5315 & 0,6667 & 2,5155 & 2 & 7,38 \\
\hline I:3 & 0,5560 & 0,6667 & 7,5465 & 2 & 7,38 \\
\hline
\end{tabular}

Através do teste da matriz "chapéu", nenhuma medida é suspeita de ser ponto de alavancamento. Isto porque, todos os $K_{i i}$ são menores que o valor limiar 0,6667. Entretanto, constata-se, pelo método da estatística de projeção, que as medidas F:1-3 e I:3 são medidas ponto de alavancamento, já que seus respectivos valores de $P S_{i}$ são maiores que seus limiares $\chi_{L i, 0,975}^{2}$. 
Os resultados do exemplo 2 mostram que o método da estatística de projeção é mais robusto que o método da matriz "chapéu", para identificar múltiplas medidas ponto de alavancamento. 
Capítulo 3 


\section{Capítulo 4}

\section{4 Índice de Não-Detecção de Erros Grosseiros}

A partir de uma análise geométrica do processo de estimação de estado através do método dos mínimos quadrados ponderados, propõe-se, neste capítulo, o Índice de Não-Detecção de Erros (INDE) ou, como foi apresentado em Bretas et al. (2009), UI (Undetectability Index).

As medidas com maiores $U I$ são aquelas cujos erros são mais difíceis de serem detectados, via teste dos resíduos normalizados e do índice $J(x)$. Isto porque uma grande parcela dos erros dessas medidas não aparece em seus resíduos, isto é, os erros nas mesmas são, de certa forma, "mascarados". Assim, se a medida $z_{1}$ possui UI maior que a medida $z_{2}$, o estimador WLS associado ao teste do resíduo normalizado será bem mais sensível aos erros na medida $z_{2}$, independentemente de ambas possuírem ou não a mesma redundância.

Face ao exposto, através do índice UI é possível qualificar as medidas de acordo com as suas características de "mascararem" os seus erros. Através da decomposição do espaço vetorial das medidas demonstrar-se-á, na seção 4.3 deste capítulo, que o índice $U I$ de uma determinada medida indica a proximidade da mesma para imagem da matriz Jacobiana do estimador WLS.

Nesse contexto, a medida crítica é o caso limite de medidas cujos erros são mascarados, isto é, ela pertence à imagem da matriz Jacobiana, possui índice UI infinito e seu resíduo é igual a zero, independente de a mesma ter ou não EG.

Além da proposição do índice $U I$, neste capítulo será apresentado um algoritmo para cálculo do $U I$ de cada uma das medidas disponíveis em um sistema elétrico, com 
exemplos ilustrativos. Para cumprir esses objetivos, as seções deste capítulo constam das seguintes partes:

- Na seção 4.1, apresenta-se-nos a interpretação geométrica do estimador WLS;

- Na seção 4.2, o erro de cada medida é decomposto em duas partes: uma detectável, e outra não-detectável;

- Na seção 4.3, encontra-se a proposição do índice UI e exemplos ilustrativos. Além disso, apresentar-se-á um exemplo numérico, comparando o índice UI com os métodos de identificação de medidas ponto de alavancamento, apresentados no capítulo 3 ;

- Por fim, na seção 4.4, acham-se as conclusões principais deste capítulo.

\subsection{Interpretação Geométrica do Estimador WLS}

Como visto em capítulos anteriores, as $m$ medidas realizadas e as $n$ variáveis de estado de um sistema elétrico estão relacionadas através do seguinte modelo de medição

$$
\underline{z}=h(\underline{x})+\underline{w},
$$

sendo que os vetores acima conservam os significados anteriormente descritos.

A partir do modelo representado pela expressão (4.1), tem-se que $\underline{z} \in R^{m}$, $\underline{x} \in R^{n}$ e $h: R^{n} \rightarrow R^{m}$ é uma função não-linear e continuamente diferenciável. Como o número de medidas $m$ é, em geral, maior que o número de variáveis de estado $n$, há mais equações que variáveis de estado a serem estimadas. Desta forma, a estimativa do vetor de variáveis de estado $\underline{x}$, pelo método WLS, é obtida através da minimização do índice (ou função) $J(\underline{x})$, dado por:

$$
J(\underline{x})=(\underline{z}-h(\underline{x}))^{t} \cdot R^{-1} \cdot(\underline{z}-h(\underline{x})),
$$

onde $R^{-1}$ é uma matriz simétrica e definida positiva (conforme apresentado no Capítulo 2, $R$ é a matriz de covariância das medidas).

Geometricamente, a função $J(\underline{x})$ é uma norma no espaço vetorial das medidas $R^{m}$, que é induzida pelo produto interno $\langle u, v\rangle=u^{t} \cdot R^{-1} \cdot v$, ou seja:

$$
\begin{aligned}
& J(\underline{x})=\|\underline{z}-h(\underline{x})\|_{R^{-1}}^{2}=<\underline{z}-h(\underline{x}), \underline{z}-h(\underline{x})>. \\
& J(\underline{x})=(\underline{z}-h(\underline{x}))^{t} \cdot R^{-1} \cdot(\underline{z}-h(\underline{x}))
\end{aligned}
$$


Conforme apresentado nos capítulos 2 e 3 , sendo $\underline{\hat{x}}$ o vetor de variáveis de estado estimadas, isto é, a solução do problema de minimização acima, o vetor de medidas estimadas $\underline{\hat{z}}$ e o vetor dos resíduos são dados respectivamente pelas seguintes equações:

$$
\begin{aligned}
& \underline{\hat{z}}=h(\underline{\hat{x}}), \\
& \underline{r}=\underline{z}-\underline{\hat{z}} .
\end{aligned}
$$

A linearização da equação (4.1), num certo ponto $\underline{x}^{*}$, resulta em:

$$
\Delta \underline{z}=H \cdot \Delta \underline{x}+\underline{w},
$$

sendo $H=\frac{\partial h}{\partial x}$ a matriz Jacobiana calculada em $\underline{x}^{*}, \Delta \underline{z}=\underline{z}-h\left(\underline{x}^{*}\right)$ o vetor de correção e $\Delta \underline{x}=\underline{x}-\underline{x}^{*}$

Se o sistema representado pela equação (4.6) é observável ( posto $(H)=n$ ), então o espaço vetorial das medidas $R^{m}$ pode ser decomposto numa soma direta de dois subespaços vetoriais, isto é,

$$
R^{m}=\mathfrak{R}(H) \oplus \mathfrak{R}(H)^{\perp},
$$

onde: $\Re(H)$ representa o subespaço vetorial de dimensão $n$ em $R^{m}$, denominado imagem de $H$; e $\mathfrak{R}(H)^{\perp}$ representa o complemento ortogonal de $\mathfrak{R}(H)$, isto é, se $\underline{u} \in \Re(H)$ e $\underline{v} \in \Re(H)^{\perp}$, então $<\underline{u}, \underline{v}>=\underline{u}^{t} \cdot R^{-1} \cdot \underline{v}=0$.

A equação (4.6), calculada em $\underline{x}$, que torna mínimo o índice $J(\underline{x})$, pode ser interpretada como uma projeção do vetor de correção $\Delta \underline{z}$ em $\mathfrak{R}(H)$. Assim, considere $P$ como um operador linear, que projeta o vetor $\Delta \underline{z}$ em $\mathfrak{R}(H)$, ou seja:

$$
\Delta \underline{\hat{z}}=P \cdot \Delta \underline{z}=H \cdot \Delta \underline{\hat{x}} .
$$

Desta forma, o vetor de resíduo $\underline{r}$, para o modelo linearizado, é dado por:

$$
\underline{r}=\Delta \underline{z}-\Delta \underline{\hat{z}}=\Delta \underline{z}-H \cdot \Delta \underline{\hat{x}} \text {. }
$$

A partir desses dois vetores, o operador $P$, que torna mínima a norma $J(\underline{x})$, é aquele que projeta $\Delta \underline{z}$ ortogonalmente em $\mathfrak{R}(H)$, em termos do produto interno $<\underline{u}, \underline{v}>=\underline{u}^{t} \cdot R^{-1} \cdot \underline{v}$, isto é, o vetor $\Delta \underline{\hat{z}}$ é ortogonal ao vetor $\underline{r}$. Mais precisamente:

$$
\begin{aligned}
& <\Delta \underline{\hat{z}}, \underline{r}>=\Delta \underline{\hat{z}}^{t} \cdot R^{-1} \cdot \underline{r}=0 \\
& <\Delta \underline{\hat{z}}, \underline{r}>=(H \cdot \Delta \underline{\hat{x}})^{t} \cdot R^{-1} \cdot(\Delta \underline{z}-H \cdot \Delta \underline{\hat{x}})=0
\end{aligned} .
$$

Resolvendo-se a equação (4.10) para $\Delta \underline{\hat{x}}$, obtém-se 


$$
\Delta \underline{\hat{x}}=\left(H^{t} \cdot R^{-1} \cdot H\right)^{-1} \cdot H^{t} \cdot R^{-1} \cdot \Delta \underline{z} .
$$

Como $\Delta \underline{\hat{z}}=P \cdot \Delta \underline{z}=H \cdot \Delta \underline{\hat{x}}$, a matriz projeção $P$ é:

$$
P=H \cdot\left(H^{t} \cdot R^{-1} \cdot H\right)^{-1} \cdot H^{t} \cdot R^{-1} .
$$

Conforme apresentado no capítulo 3, na literatura concernente à estimação de estado em sistemas elétricos, essa matriz é usualmente chamada de matriz "chapéu", representada pela letra $K$. Ante o exposto, a matriz de projeção $P$ será representada pela letra $K$. Ademais, todas as propriedades da matriz $K$, apresentadas no capítulo 3, são preservadas.

Caso a matriz de ponderação $R^{-1}$ seja dada por $R^{-1}=c \cdot I$, sendo $c$ um número real positivo e $I$ a matriz identidade de ordem $m$ x $m$, então $K=K^{t}$ e $K$ será uma projeção ortogonal.

A figura 4.1 a seguir ilustra o operador $K$ atuando sobre o vetor $\Delta \underline{z}$.

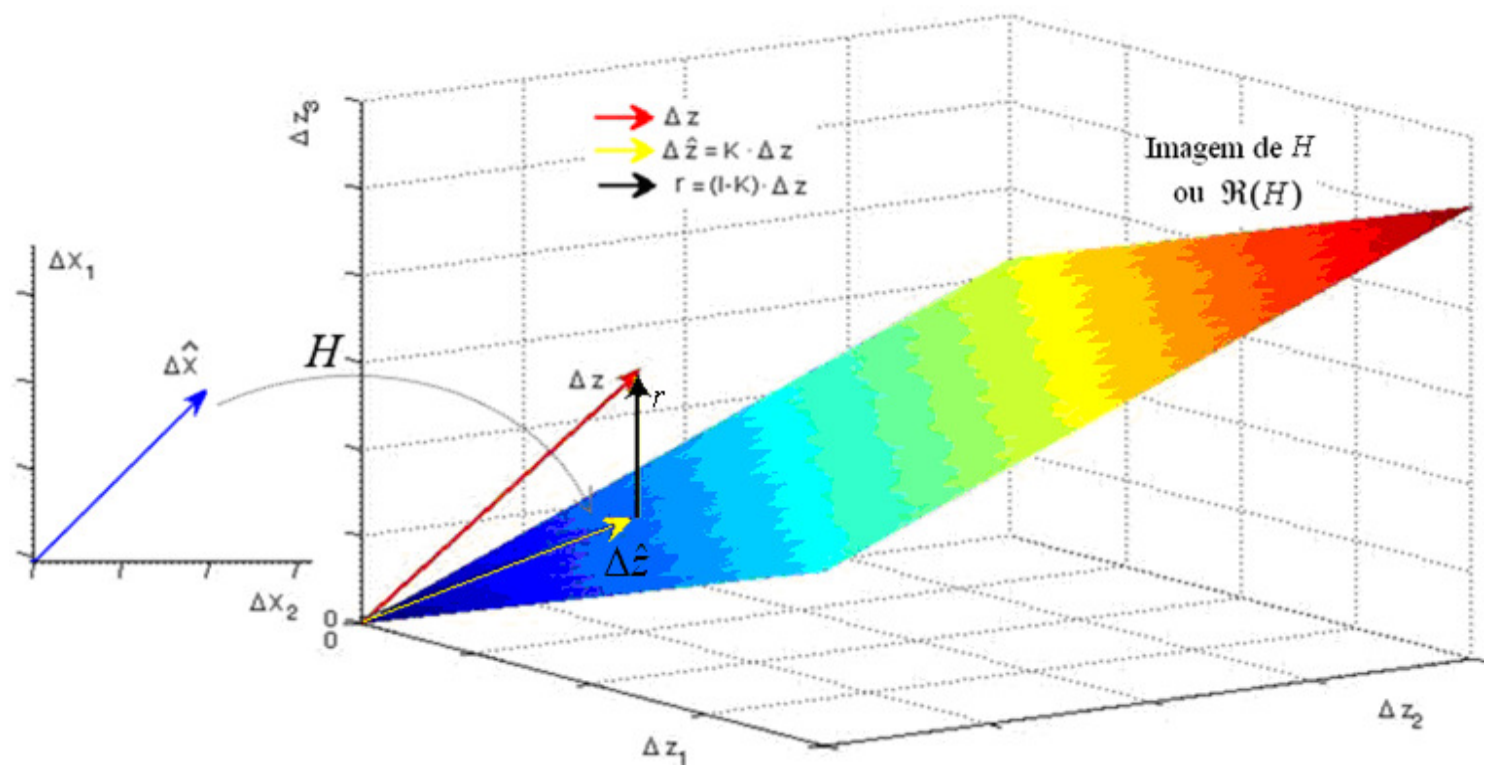

Figura 4.1: Interpretação geométrica do operador $K$, atuando sobre o vetor $\Delta \mathrm{z}$, para $m=3$ e $n=2$.

O vetor resíduo pode ser calculado, em termos da matriz de projeção $K$, através da seguinte expressão:

$$
\begin{aligned}
& \underline{r}=\Delta \underline{z}-\Delta \underline{\hat{z}}=\Delta \underline{z}-K \cdot \Delta \underline{z} \\
& \underline{r}=(I-K) \cdot \Delta \underline{z}=S \cdot \Delta \underline{z}
\end{aligned} .
$$

onde a matriz $(I-K)$, ou matriz de sensibilidade dos resíduos $S$, é um operador que projeta $\Delta \underline{z}$ em $\mathfrak{R}(H)^{\perp}$.

Como conclusões importantes desta seção, temos: 
i) A matriz de projeção $K$ projeta $\Delta \underline{z} \operatorname{em~} \Re(H)(\Delta \underline{\hat{z}}=K \cdot \Delta \underline{z})$;

ii) A matriz de sensibilidade $S$ projeta $\Delta \underline{z}$ em $\mathfrak{R}(H)^{\perp}(\underline{r}=S \cdot \Delta \underline{z})$.

\subsection{Decomposição do Erro de Medida}

Nesta seção, a decomposição do espaço vetorial das medidas, em uma soma direta de $\mathfrak{R}(H)$ e $\mathfrak{R}(H)^{\perp}$, será utilizada para decompor o vetor de erro das medidas $\underline{w}$ em duas componentes: uma componente será definida como detectável e outra como não-detectável. Para isso, seja $\underline{x}_{\text {verd }}$ o vetor de variáveis de estado verdadeiras, que na prática é desconhecido; então $\underline{z}_{\text {verd }}=h\left(\underline{x}_{\text {verd }}\right)$ será o correspondente vetor de medidas verdadeiras, isto é, sem erros. Considerando o modelo linearizado, no qual $\Delta \underline{x}_{v e r d}=\underline{x}_{v e r d}-\underline{\hat{x}}$ e $\Delta \underline{z}_{v e r d}=H \cdot \Delta \underline{x}_{v e r d}$, e assumindo que a matriz Jacobiana $H$ disponível esteja próxima daquela obtida com as medidas sem erros, então $\underline{z}_{\text {verd }}$ está próximo à $\mathfrak{R}(H)$ e $\Delta \underline{z}_{v e r d} \approx \underline{z}_{\text {verd }}-\underline{\hat{z}}$.

O vetor de erro das medidas é dado por:

$$
\underline{w}=\underline{z}^{-} \underline{z}_{v e r d} \approx \Delta \underline{z}-\Delta \underline{z}_{v e r d},
$$

e pode ser decomposto da forma seguinte:

$$
\underline{w}=K \cdot \underline{w}+(I-K) \cdot \underline{w},
$$

sendo $\underline{w}_{U}$ a componente não-detectável e $\underline{w}_{D}$ a componente detectável de $\underline{w}$, temos:

$$
\begin{gathered}
\underline{w}_{U}=K \cdot \underline{w}, \\
\underline{w}_{D}=(I-K) \cdot \underline{w} .
\end{gathered}
$$

A partir das equações acima, verificar-se que $\underline{w}_{U} \in \mathfrak{R}(H)$ e $\underline{w}_{D} \in \mathfrak{R}(H)^{\perp}$. Como conseqüência:

$$
\|\underline{w}\|_{R^{-1}}^{2}=\left\|\underline{w}_{U}\right\|_{R^{-1}}^{2}+\left\|\underline{w}_{D}\right\|_{R^{-1}}^{2} .
$$

Proposição 1: O vetor de resíduo $\underline{r}$, do estimador WLS, não é afetado pela componente não-detectável $\underline{w}_{U}$ do vetor de erro das medidas.

Prova: Reescrevendo a equação (4.14), em termos das componentes de $\underline{w}$, temos:

$$
\Delta \underline{z} \approx \Delta \underline{z}_{v e r d}+\underline{w} \approx \Delta \underline{z}_{v e r d}+\underline{w}_{U}+\underline{w}_{D},
$$

e como conseqüência: 


$$
\begin{aligned}
& \underline{r}=(I-K) \cdot \Delta \underline{z} \approx(I-K) \cdot\left(\Delta \underline{z}_{\text {verd }}+\underline{w}_{U}+\underline{w}_{D}\right) \\
& \underline{r} \approx(I-K) \cdot \Delta \underline{z}_{\text {verd }}+(I-K) \cdot \underline{w}_{U}+(I-K) \cdot \underline{w}_{D}
\end{aligned} .
$$

Desde que $\quad \Delta \underline{z}_{v e r d} \in \mathfrak{R}(H) \quad$ e $\quad \underline{w}_{U} \in \mathfrak{R}(H)$, então $\quad(I-K) \cdot \Delta \underline{z}_{v e r d}=0 \quad$ e $(I-K) \cdot \underline{w}_{U}=0$. Com isso, a equação (4.20) pode ser reescrita seguindo o desenvolvimento a seguir:

$$
\underline{r}=(I-K) \cdot \Delta \underline{z} \approx(I-K) \cdot \underline{w}_{D},
$$

como $\underline{w}_{D}=(I-K) \cdot \underline{w}$ e lembrando que $(I-K)$ é uma matriz idempotente, temos que

$$
\begin{aligned}
& \underline{r} \approx(I-K) \cdot \underline{w}_{D}=(I-K) \cdot(I-K) \cdot \underline{w} . \\
& \underline{r} \approx(I-K) \cdot \underline{w}=\underline{w}_{D}
\end{aligned}
$$

A proposição acima nos indica que a dificuldade, ou mesmo a impossibilidade de detectar erros em algumas medidas ocorre em razão de a componente não-detectável do erro dessa medida ser mais significante que a componente detectável. Como consequiência, os resíduos dessas medidas serão pequenos, mesmo quando o valor do erro for elevado, isto é, mesmo quando a medida for portadora de EG. Eis a razão de o teste dos resíduos normalizados falhar em determinadas situações.

Face ao exposto, valores baixos de resíduos normalizados não implicam necessariamente em valores baixos de erros em medidas, tendo em vista a existência das componentes não-detectáveis. Ou seja, quando os valores dos resíduos normalizados não forem maiores que o limiar de detecção, pode-se afirmar apenas, com certo grau de confiança, que somente os valores das componentes detectáveis dos erros em todas as medidas sejam pequenos. Por isso, torna-se essencial a verificação da componente nãodetectável dos erros de medidas, para uma análise mais confiável das estimativas obtidas. Para tanto, propõe-se, na próxima seção, o índice de não-detecção de erros $U I$.

\subsection{Proposição do Índice de Não-Detecção de Erros}

Nesta seção, tomando por base as componentes detectáveis e não-detectáveis do vetor de erro das medidas, propõe-se o índice $U I$, bem como um algoritmo para o cálculo do $U I$ de cada uma das medidas a serem utilizadas no processo de estimação de estado.

Nesse escopo, suponha a existência de um erro simples na $i$-ésima medida, isto é, 


$$
\underline{w}^{i}=b \cdot \underline{\delta}^{i},
$$

sendo o vetor $\underline{\delta}^{i}=[0, \ldots, 0,1,0, \ldots, 0]^{t}$ e $b$ um escalar qualquer representando a magnitude do erro. Através das equações (4.16) e (4.17), as componentes não-detectável e detectável são calculadas:

$$
\begin{gathered}
\underline{w}_{U}^{i}=K \cdot \underline{w}^{i}, \\
\underline{w}_{D}^{i}=(I-K) \cdot \underline{w}^{i} .
\end{gathered}
$$

A partir do que foi desenvolvido até aqui, definimos o $U I$ para a $i$-ésima medida como sendo:

$$
U I_{i}=\frac{\left\|\underline{w}_{U}^{i}\right\|_{R^{-1}}}{\left\|\underline{w}_{D}^{i}\right\|_{R^{-1}}}=\frac{\sqrt{\left(\underline{w}_{U}^{i}\right)^{t} \cdot R^{-1} \cdot\left(\underline{w}_{U}^{i}\right)}}{\sqrt{\left(\underline{w}_{D}^{i}\right)^{t} \cdot R^{-1} \cdot\left(\underline{w}_{D}^{i}\right)}} .
$$

Através do desenvolvimento a seguir, a equação acima pode ser expressa em função apenas das matrizes $K$ e $(I-K)$ :

$$
\begin{aligned}
& U I_{i}=\frac{\sqrt{\left(K \cdot \underline{w}^{i}\right)^{t} \cdot R^{-1} \cdot\left(K \cdot \underline{w}^{i}\right)}}{\sqrt{\left((I-K) \cdot \underline{w}^{i}\right)^{t} \cdot R^{-1} \cdot\left((I-K) \cdot \underline{w}^{i}\right)}} \\
& U I_{i}=\frac{\sqrt{\left(K \cdot b \cdot \underline{\delta}^{i}\right)^{t} \cdot R^{-1} \cdot\left(K \cdot b \cdot \underline{\delta}^{i}\right)}}{\sqrt{\left((I-K) \cdot b \cdot \underline{\delta}^{i}\right)^{t} \cdot R^{-1} \cdot\left((I-K) \cdot b \cdot \underline{\delta}^{i}\right)}} . \\
& U I_{i}=\frac{b \cdot \sqrt{\left(K \cdot \underline{\delta}^{i}\right)^{t} \cdot R^{-1} \cdot\left(K \cdot \underline{\delta}^{i}\right)}}{b \cdot \sqrt{\left((I-K) \cdot \underline{\delta}^{i}\right)^{t} \cdot R^{-1} \cdot\left((I-K) \cdot \underline{\delta}^{i}\right)}}
\end{aligned}
$$

No Anexo A, demonstra-se que o numerador e o denominador da equação (4.27) resultam, respectivamente, em:

$$
\begin{gathered}
\left\|\underline{w}_{U}^{i}\right\|_{R^{-1}}=b \cdot \sigma_{i}^{-1} \cdot \sqrt{K_{i i}}, \\
\left\|\underline{w}_{D}^{i}\right\|_{R^{-1}}=b \cdot \sigma_{i}^{-1} \cdot \sqrt{\left(1-K_{i i}\right)},
\end{gathered}
$$

Logo, podemos escrever o índice $U I$, para uma medida $i$, da forma seguinte:

$$
U I_{i}=\frac{\left\|\underline{w}_{U}^{i}\right\|_{R^{-1}}}{\left\|\underline{w}_{D}^{i}\right\|_{R^{-1}}}=\frac{\sqrt{\left(K_{i i}\right)}}{\sqrt{\left(1-K_{i i}\right)}},
$$

sendo $K_{i i}$ o elemento da diagonal principal da matriz de projeção $K$.

Como pode ser visto através da equação acima, o UI não depende da magnitude do erro $b$, mas somente da matriz de projeção $K$ e da matriz de sensibilidade do resíduo 
$S(S=I-K)$. Isto se deve ao fato de a componente não-detectável do erro estar relacionada diretamente com a matriz de projeção $K$, ou, com a imagem de $H$, enquanto que a componente detectável está relacionada com a matriz $S$, ou, com o complemento da imagem de $H$.

Um valor grande de $U I$, para uma determinada medida, indica que uma grande parcela do seu erro será "mascarada" no processo de estimação de estado. Desta forma, esse erro vai contribuir pouco (ou quase nada) para o valor do resíduo daquela medida. Em particular, se $\underline{w}^{i} \in \mathfrak{R}(H)$, então $\underline{w}_{D}^{i}=0$ e o $U I$ tende ao infinito $\left(U I_{i} \rightarrow \infty\right)$. Esta situação particular mostra que o UI, para uma medida crítica, é infinito, isto é, não tem componente detectável.

Através de uma análise geométrica, é possível verificar que a informação transmitida pelo $U I$ de uma dada medida é, de certa forma, a proximidade de um ponto definido por um vetor unitário, pertencente ao eixo de uma dada medida, até a imagem da matriz Jacobiana do estimador WLS, conforme apresentado na figura 4.2 para um sistema com duas medidas $(m=2)$ e uma variável de estado a ser estimada $(n=1)$.

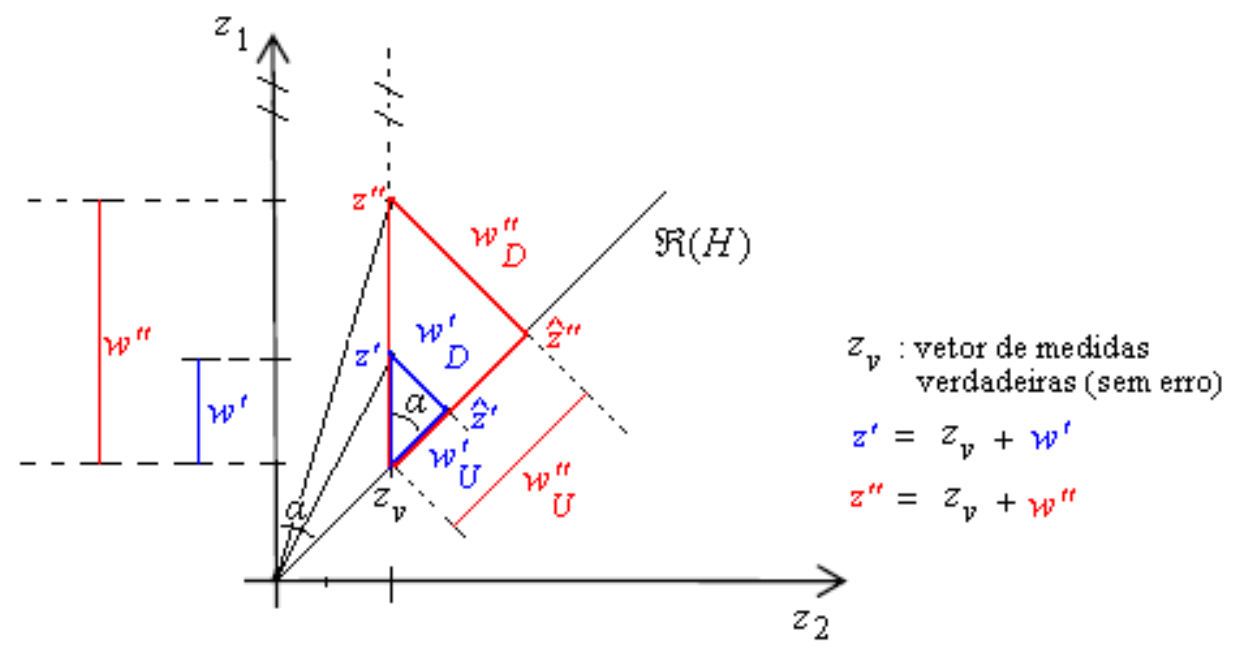

Figura 4.2: Interpretação geométrica considerando dois erros distintos em z1

$\mathrm{Na}$ figura 4.2 foram considerados dois vetores de erro, o primeiro com magnitude $w^{\prime}$ (azul) e o segundo com magnitude $w^{\prime}$ '(vermelho). Observe que o UI da medida $z_{1}$ vai ser o mesmo, independentemente da magnitude do erro, cujas componentes $w_{u}$ e $w_{d}$ crescem de forma proporcional com essa magnitude. Considerando a equação (4.26), podemos concluir ainda que o UI de uma determinada medida é igual à cotangente do ângulo formado pelo eixo da medida e pela imagem da matriz Jacobiana. 
Essa visão geométrica das componentes do vetor de erros possibilita uma compreensão mais ampla e simples do problema de detecção/identificação de EGs, do que o conceito de medidas ponto de alavancamento.

\subsubsection{Exemplo de Medidas com Alto e Baixo Valor de Índice de Não-Detecção}

Para evidenciar melhor as diferenças entre medidas com alto e baixo UI, consideremos o sistema de duas barras apresentado na figura 4.3, contendo duas medidas e uma variável a ser estimada (estimador WLS linear).

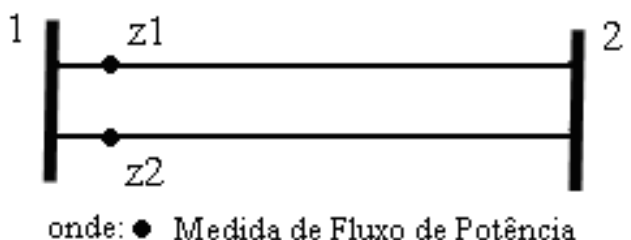

Figura 4.3: Sistema de duas barras e duas linhas

Vamos considerar que os parâmetros e a ponderação das duas medidas de fluxo, apresentadas na figura 4.3 , sejam tais que tornem a medida $\mathrm{z}_{1}$ uma medida com alto $U I$ e a medida $z_{2}$ uma medida com baixo UI. Destarte, se a medida $z_{1}$ for portadora de EG, esse erro vai ser de difícil detecção, pois o resíduo de tal medida será baixo (veja figura 4.4).

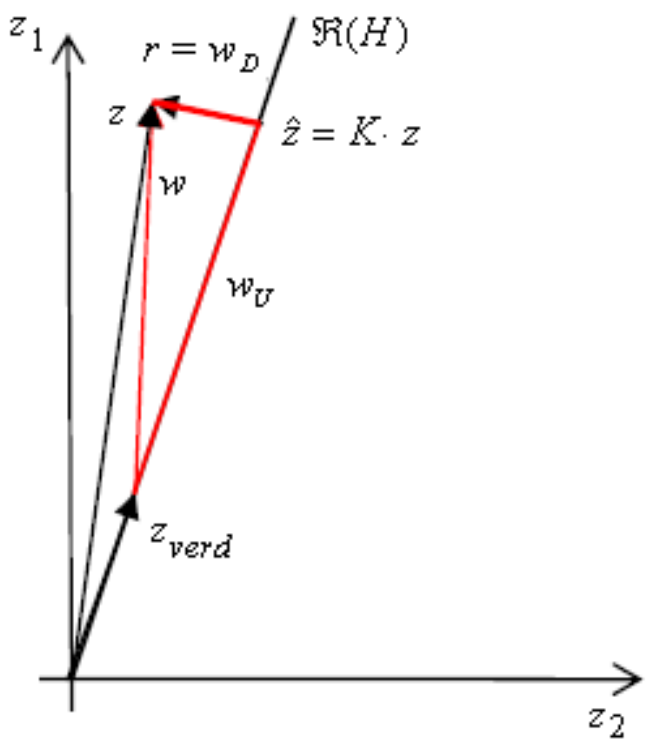

Figura 4.4: Exemplo de erro grosseiro em medida com alto $U I$ 
Isto ocorre porque a componente detectável do erro dessa medida é muito baixa, ou, em outras palavras, a medida z1 apresenta um UI muito alto. Por outro lado, se a medida $z_{2}$ for portadora de EG, tendo em vista que a componente detectável do erro dessa medida é superior à componente não-detectável, pois a medida z2 apresenta baixo UI, a mesma vai apresentar um resíduo elevado, tornando mais fácil a detecção de EG (figura 4.5).

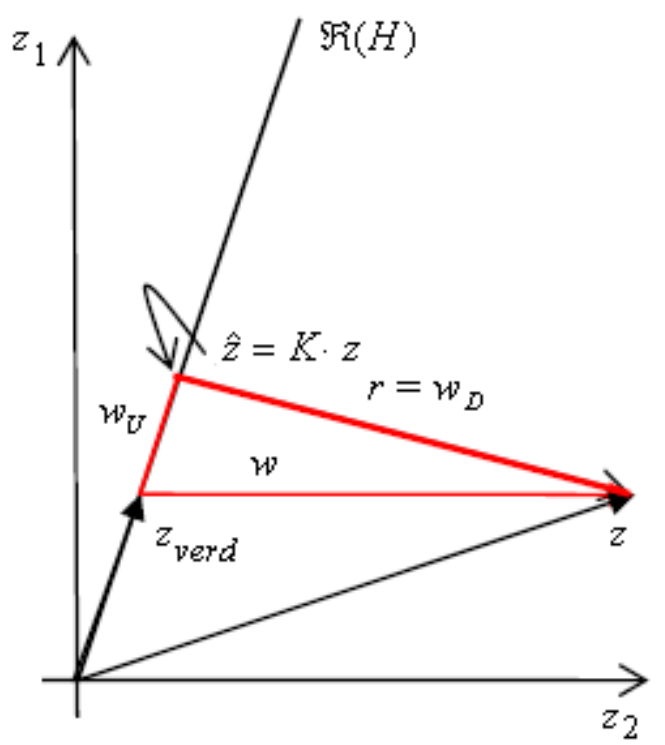

Figura 4.5: Exemplo de erro grosseiro em medida com baixo $U I$

A seguir, apresenta-se-nos um algoritmo para cálculo do UI, para cada uma das medidas que serão processadas pelo estimador de estado.

\subsubsection{Algoritmo para Cálculo do Índice $U I$}

Utilizando-se o estimador WLS convencional, faça:

Passo 1: Determine o valor estimado das variáveis de estado $(\underline{\hat{x}})$ através da solução iterativa da equação (2.17) (equação normal);

Passo 2: Calcule as matrizes $K$ e $(I-K)$ para $\underline{\hat{x}}$, ou apenas os elementos da diagonal principal dessas matrizes;

Passo 3: Para cada medida $i$, calcule o índice $U I$, diretamente pela equação (4.30), isto é

$$
U I_{i}=\frac{\left\|\underline{w}_{U}^{i}\right\|_{R^{-1}}}{\left\|\underline{w}_{D}^{i}\right\|_{R^{-1}}}=\frac{\sqrt{\left(K_{i i}\right)}}{\sqrt{\left(1-K_{i i}\right)}}, \operatorname{para} i=1, \ldots, m .
$$




\subsubsection{Simulações Utilizando o Sistema de 14 barras do IEEE}

Nesta seção, apresentaremos algumas simulações computacionais, mostrandonos que quanto maior o índice $U I$ de uma medida, pior será a sua capacidade em detectar possíveis erros, através do teste dos resíduos normalizados e do índice $J(x)$.

A figura 4.6 ilustra a topologia do sistema de 14 barras do IEEE, utilizado nas simulações que serão relatadas a seguir.

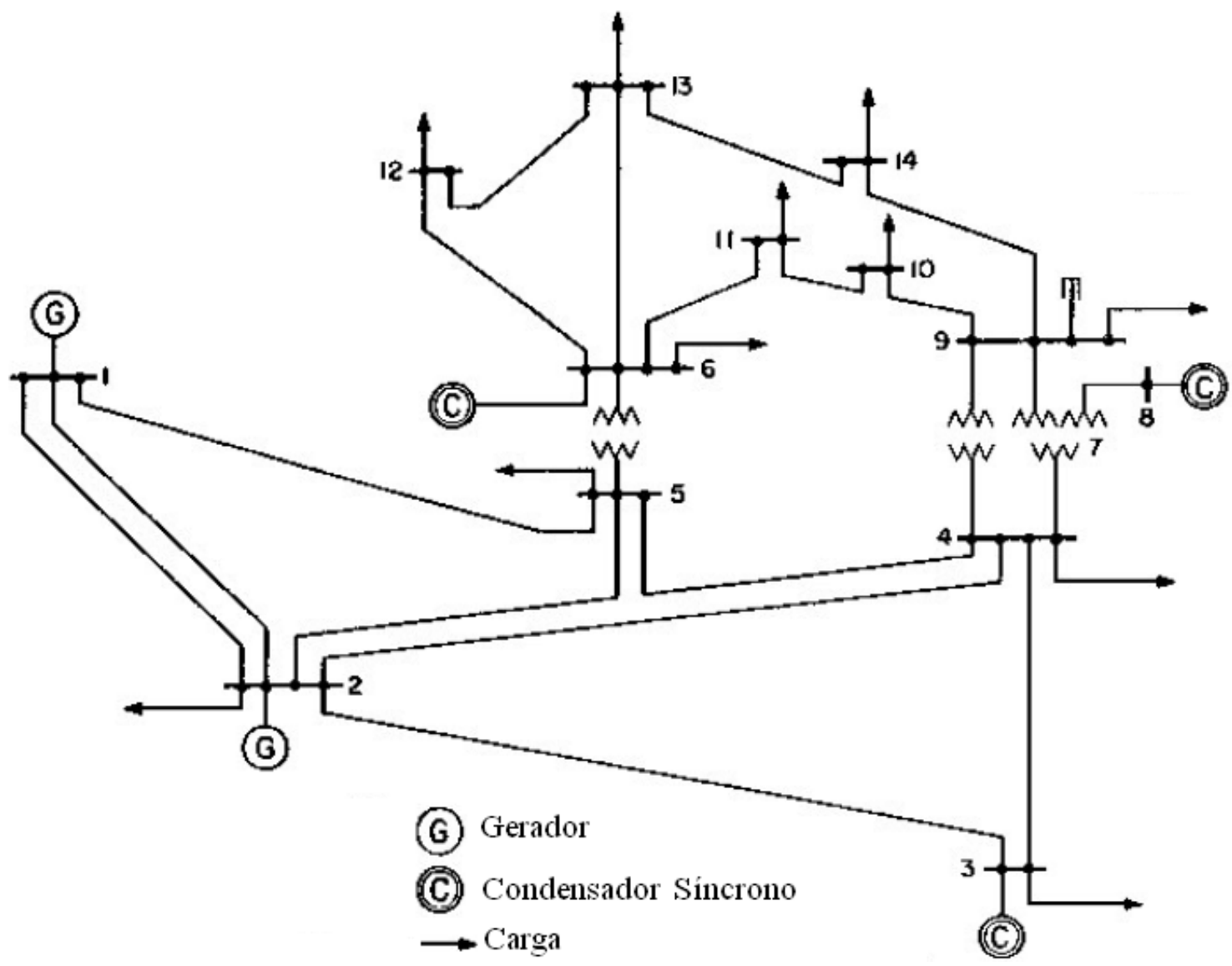

Figura 4.6: Sistema de 14 barras IEEE

A partir dos resultados de um programa de fluxo de carga (ANAREDE), construímos um plano de medição com 70 medidas, sem a presença de medidas críticas ou conjuntos críticos. Os valores dos parâmetros, bem como outras informações pertinentes à execução do fluxo de carga, para o sistema de 14 barras do IEEE, estão disponíveis no endereço eletrônico "www.ee.washington.edu/research/pstca/", e por isso não mostraremos aqui.

Os valores das 70 medidas, bem como os respectivos desvios padrão, são apresentados na Tabela 4.1. Observe que os valores apresentados naquela tabela são exatamente os determinados pelo fluxo de potência, isto é, sem acrescentar ruído aleatório algum. Os desvios padrão, lá apresentados, serão utilizados para ponderação 
das medidas, durante o processo de estimação de estado por mínimos quadrados ponderados, bem como para simulação de EGs nas mesmas, e foram determinados a partir da seguinte equação ${ }^{5}$ :

$$
3 \cdot \sigma_{i}=p r \cdot\left|\underline{z}_{i}^{f c}\right|,
$$

onde: $\sigma_{i}$ é o desvio padrão da medida $i ;\left|\underline{z}_{i}^{f c}\right|$ é o valor absoluto da medida, determinado através do fluxo de potência; e $p r$ é a precisão do medidor, que será considerada aqui igual a 5\%. As medidas tabeladas com valor NULO são medidas virtuais, e por isso seus respectivos valores de desvio padrão não foram calculados.

$\mathrm{Na}$ seqüência deste trabalho, utilizar-se-á a seguinte nomenclatura para as medidas: IA:a - medida de injeção de potência ativa na barra "a"; FA:a-b - medida de fluxo de potência ativa da barra "a" para barra "b"; IR:a - medida de injeção de potência reativa na barra "a"; FR:a-b - medida de fluxo de potência reativa da barra "a" para barra "b" e V:a - medida de magnitude de tensão da barra "a".

Tabela 4.1: Valores das medidas sem ruído e respectivos desvios padrões para o sistema de 14 barras

\begin{tabular}{c|c|c|c|c|c}
\hline \multicolumn{2}{c|}{ Medidas de Potência Ativa } & \multicolumn{3}{c}{ Medidas de Potência Reativa } \\
\hline Medida & Valor $(\mathbf{p u})$ & $\boldsymbol{\sigma}(\mathbf{p u})$ & Medida & Valor $(\mathbf{p u})$ & $\boldsymbol{\sigma}(\mathbf{p u})$ \\
\hline IA:1 & 2,32299 & 0,038733 & IR:1 & $-0,16478$ & 0,00275 \\
\hline IA:2 & 0,18312 & 0,00305 & IR:2 & 0,30877 & 0,005133 \\
\hline IA:5 & $-0,07601$ & 0,001267 & IR:5 & $-0,01599$ & 0,000267 \\
\hline IA:7 & NULO & --- & IR:7 & NULO & --- \\
\hline IA:8 & NULO & --- & IR:8 & 0,17604 & 0,002933 \\
\hline IA:9 & $-0,29542$ & 0,004917 & IR:9 & $-0,16637$ & 0,002767 \\
\hline IA:13 & $-0,1347$ & 0,00225 & IR:13 & $-0,05813$ & 0,000967 \\
\hline IA:14 & $-0,14889$ & 0,002483 & IR:14 & $-0,04961$ & 0,000833 \\
\hline FA:1-2 & 1,56837 & 0,02615 & FR:1-2 & $-0,20354$ & 0,0034 \\
\hline FA:2-3 & 0,73291 & 0,0122 & FR:2-3 & 0,0359 & 0,0006 \\
\hline FA:4-2 & $-0,54408$ & 0,009083 & FR:4-2 & 0,0301 & 0,0005 \\
\hline FA:5-2 & $-0,40571$ & 0,006767 & FR:5-2 & $-0,02111$ & 0,00035 \\
\hline FA:3-4 & $-0,23388$ & 0,003883 & FR:3-4 & 0,04487 & 0,00075 \\
\hline FA:4-5 & $-0,61131$ & 0,0102 & FR:4-5 & 0,15829 & 0,002633 \\
\hline FA:7-4 & $-0,28072$ & 0,004683 & FR:7-4 & 0,1133 & 0,0019 \\
\hline FA:9-4 & $-0,16079$ & 0,002683 & FR:9-4 & 0,01708 & 0,000283 \\
\hline FA:5-6 & 0,44023 & 0,00735 & FR:5-6 & 0,12504 & 0,002083 \\
\hline FA:6-5 & $-0,44023$ & 0,00735 & FR:6-5 & $-0,08087$ & 0,00135 \\
\hline FA:6-11 & 0,07381 & 0,001233 & FR:6-11 & 0,03549 & 0,0006 \\
\hline FA:11-6 & $-0,07326$ & 0,001217 & FR:11-6 & $-0,03432$ & 0,000567
\end{tabular}

\footnotetext{
${ }^{5}$ Essa equação foi utilizada, para as simulações com o sistema de 14 barras do IEEE, haja vista a mesma ter sido empregada em outras pesquisas desenvolvidas na área, conforme apresentado no trabalho de Castillo-Albertini (2010). Vale destacar que nesse trabalho realizou-se uma sucinta revisão de diferentes proposições para definição dos desvios padrão das medidas para efeito de estimação de estado.
} 


\begin{tabular}{|c|c|c|c|c|c|}
\hline FA:6-12 & 0,07795 & 0,0013 & FR:6-12 & 0,02508 & 0,000417 \\
\hline FA:6-13 & 0,17743 & 0,00295 & FR:6-13 & 0,07208 & 0,0012 \\
\hline FA:13-6 & $-0,17531$ & 0,002917 & FR:13-6 & $-0,0679$ & 0,001133 \\
\hline FA:7-8 & NULO & --- & FR:7-8 & $-0,17144$ & 0,002867 \\
\hline FA:8-7 & NULO & --- & FR:8-7 & 0,17604 & 0,002933 \\
\hline FA:7-9 & 0,28072 & 0,004683 & FR:7-9 & 0,05814 & 0,000967 \\
\hline FA:9-10 & 0,05206 & 0,000867 & FR:9-10 & 0,04223 & 0,0007 \\
\hline FA:10-9 & $-0,05193$ & 0,000867 & FR:10-9 & $-0,04188$ & 0,0007 \\
\hline FA:9-14 & 0,09403 & 0,001567 & FR:9-14 & 0,03595 & 0,0006 \\
\hline FA:10-11 & $-0,03801$ & 0,000633 & FR:10-11 & $-0,01597$ & 0,000267 \\
\hline FA:12-13 & 0,01601 & 0,000267 & FR:12-13 & 0,0075 & 0,000125 \\
\hline FA:14-13 & $-0,05601$ & 0,000933 & FR:14-13 & $-0,01612$ & 0,000267 \\
\hline \multicolumn{6}{|c|}{ Medidas de Tensão } \\
\hline \multicolumn{2}{|c|}{ Medida } & \multicolumn{2}{|c|}{ Valor (pu) } & \multicolumn{2}{|c|}{$\sigma(\mathrm{pu})$} \\
\hline \multicolumn{2}{|c|}{ V:1 } & \multicolumn{2}{|c|}{1,05939} & \multicolumn{2}{|c|}{0,017667} \\
\hline \multicolumn{2}{|c|}{$V: 2$} & \multicolumn{2}{|c|}{1,04436} & \multicolumn{2}{|c|}{0,017417} \\
\hline \multicolumn{2}{|c|}{$V: 3$} & \multicolumn{2}{|c|}{1,00929} & \multicolumn{2}{|c|}{0,016833} \\
\hline \multicolumn{2}{|c|}{$V: 6$} & \multicolumn{2}{|c|}{1,0692} & \multicolumn{2}{|c|}{0,017833} \\
\hline \multicolumn{2}{|c|}{$V: 8$} & \multicolumn{2}{|c|}{1,08923} & \multicolumn{2}{|c|}{0,018167} \\
\hline \multicolumn{2}{|c|}{ V:12 } & \multicolumn{2}{|c|}{1,05436} & \multicolumn{2}{|c|}{0,017583} \\
\hline
\end{tabular}

Na tabela B.1 do Anexo B, são apresentadas todas as possíveis medidas de potência e de magnitude de tensão para o sistema de 14 barras do IEEE, bem como os valores dos respectivos desvios padrões.

Para executar o estimador de estado WLS, atribuiu-se, para as medidas virtuais, a maior ponderação existente dentre todas as medidas desse sistema, ou seja, $R_{i i}^{-1}=1 /(0,000067)^{2}$.

Através da execução do estimador WLS tradicional, obtivemos as seguintes variáveis de estado estimadas (tabela 4.2):

Tabela 4.2: Variáveis de estado estimadas para o sistema de 14 barras

\begin{tabular}{c|c|c}
\hline \multirow{2}{*}{ Barra } & \multicolumn{2}{|c}{ Tensão elétrica } \\
\cline { 2 - 3 } & Magnitude (pu) & Ângulo (rad) \\
\hline $\mathbf{1}$ & 1,0594 & 0,0 \\
\hline $\mathbf{2}$ & 1,0443 & $-0,087$ \\
\hline $\mathbf{3}$ & 1,0093 & $-0,2224$ \\
\hline $\mathbf{4}$ & 1,0170 & $-0,1801$ \\
\hline $\mathbf{5}$ & 1,0188 & $-0,1532$ \\
\hline $\mathbf{6}$ & 1,0692 & $-0,2483$ \\
\hline $\mathbf{7}$ & 1,0607 & $-0,2334$ \\
\hline $\mathbf{8}$ & 1,0892 & $-0,2334$ \\
\hline $\mathbf{9}$ & 1,0551 & $-0,2609$ \\
\hline $\mathbf{1 0}$ & 1,0502 & $-0,2637$ \\
\hline $\mathbf{1 1}$ & 1,0561 & $-0,2583$ \\
\hline $\mathbf{1 2}$ & 1,0543 & $-0,2632$ \\
\hline $\mathbf{1 3}$ & 1,0495 & $-0,2646$ \\
\hline $\mathbf{1 4}$ & 1,0348 & $-0,2800$ \\
\hline
\end{tabular}


A tabela 4.3, a seguir, apresenta: o valor do índice $J(x)$; os valores das medidas estimadas; os valores dos índices $U I$; e os valores dos resíduos normalizados. Observe que o índice $U I$ não foi calculado para as medidas virtuais, uma vez que as mesmas não estão sujeitas a erros.

Tabela 4.3: Índice $J(\mathrm{x})$, valores estimados das medidas, índice $U I$ e resíduos normalizados $r^{N}$

\begin{tabular}{|c|c|c|c|c|c|c|c|}
\hline \multicolumn{8}{|c|}{ Índice $J(x)=0,00036569$} \\
\hline \multicolumn{4}{|c|}{ Medidas de Potência Ativa } & \multicolumn{4}{|c|}{ Medidas de Potência Reativa } \\
\hline Medida & $\begin{array}{c}\text { Valor } \\
\text { estimado }\end{array}$ & $\boldsymbol{U I}$ & $r^{N}$ & Medida & $\begin{array}{c}\text { Valor } \\
\text { estimado }\end{array}$ & $\boldsymbol{U} \boldsymbol{I}$ & $r^{N}$ \\
\hline IA:1 & 2,323 & 0,2827 & 0,0009 & IR:1 & $-0,1648$ & 1,4127 & $-0,0023$ \\
\hline IA:2 & 0,1831 & 6,4873 & 0,0032 & IR:2 & 0,3088 & 0,6792 & $-0,0017$ \\
\hline IA:5 & $-0,076$ & 11,5168 & $-0,016$ & IR:5 & $-0,016$ & 9,4466 & 0,151 \\
\hline IA:7 & NULO & --- & NULO & IR:7 & NULO & --- & NULO \\
\hline IA:8 & NULO & --- & NULO & IR:8 & 0,176 & 0,43 & 0,0004 \\
\hline IA:9 & $-0,2954$ & 0,7558 & $-0,0002$ & IR:9 & $-0,1664$ & 0,5676 & $-0,0036$ \\
\hline IA:13 & $-0,1347$ & 0,8338 & $-0,0002$ & IR:13 & $-0,0581$ & 0,6994 & $-0,0008$ \\
\hline IA:14 & $-0,1489$ & 0,6645 & $-0,0001$ & IR:14 & $-0,0496$ & 0,6737 & $-0,0006$ \\
\hline FA:1-2 & 1,5683 & 0,2905 & 0,0009 & FR:1-2 & $-0,2035$ & 0,579 & $-0,0005$ \\
\hline FA:2-3 & 0,7329 & 0,2477 & 0,0012 & FR:2-3 & 0,0359 & 2,2143 & $-0,0019$ \\
\hline FA:4-2 & $-0,5441$ & 0,322 & $-0,0008$ & FR:4-2 & 0,0301 & 1,186 & $-0,0011$ \\
\hline FA:5-2 & $-0,4057$ & 0,2922 & $-0,0007$ & FR:5-2 & $-0,0211$ & 1,1161 & $-0,0025$ \\
\hline FA:3-4 & $-0,2339$ & 1,3022 & $-0,004$ & FR:3-4 & 0,0449 & 2,156 & $-0,0042$ \\
\hline FA:4-5 & $-0,6113$ & 0,4903 & $-0,0011$ & FR:4-5 & 0,1583 & 0,3618 & 0,0008 \\
\hline FA:7-4 & $-0,2807$ & 0,42 & $-0,0002$ & FR:7-4 & 0,1133 & 0,4293 & 0,0024 \\
\hline FA:9-4 & $-0,1608$ & 0,4202 & $-0,0002$ & FR:9-4 & 0,0171 & 1,6329 & 0,0052 \\
\hline FA:5-6 & 0,4402 & 0,3331 & 0,0003 & FR:5-6 & 0,125 & 0,4673 & 0,003 \\
\hline FA:6-5 & $-0,4402$ & 0,3331 & $-0,0003$ & FR:6-5 & $-0,0809$ & 0,9743 & $-0,0076$ \\
\hline FA:6-11 & 0,0738 & 0,8952 & 0,0014 & FR:6-11 & 0,0355 & 0,8173 & 0,0015 \\
\hline FA:11-6 & $-0,0733$ & 0,8981 & $-0,0014$ & FR:11-6 & $-0,0343$ & 0,8882 & $-0,0017$ \\
\hline FA:6-12 & 0,0779 & 0,6026 & 0,0002 & FR:6-12 & 0,0251 & 0,8307 & 0,0002 \\
\hline FA:6-13 & 0,1774 & 0,4551 & 0,0002 & FR:6-13 & 0,0721 & 0,4307 & 0,0002 \\
\hline FA:13-6 & $-0,1753$ & 0,4501 & $-0,0002$ & FR:13-6 & $-0,0679$ & 0,455 & $-0,0004$ \\
\hline FA:7-8 & NULO & --- & NULO & FR:7-8 & $-0,1714$ & 0,417 & $-0,0004$ \\
\hline FA:8-7 & NULO & --- & NULO & FR:8-7 & 0,176 & 0,43 & 0,0003 \\
\hline FA:7-9 & 0,2807 & 0,42 & 0,0002 & FR:7-9 & 0,0581 & 1,2248 & $-0,0035$ \\
\hline FA:9-10 & 0,0521 & 0,9794 & 0,0002 & FR:9-10 & 0,0422 & 0,9307 & 0,0003 \\
\hline FA:10-9 & $-0,0519$ & 0,9736 & $-0,0002$ & FR:10-9 & $-0,0419$ & 0,9197 & $-0,0004$ \\
\hline FA:9-14 & 0,094 & 0,7581 & $-0,0003$ & FR:9-14 & 0,036 & 0,7257 & 0,0 \\
\hline FA:10-11 & $-0,038$ & 4,2529 & $-0,0028$ & FR:10-11 & $-0,016$ & 3,912 & $-0,0013$ \\
\hline FA:12-13 & 0,016 & 3,2398 & 0,0001 & FR:12-13 & 0,0075 & 3,3303 & 0,0018 \\
\hline FA:14-13 & $-0,056$ & 0,9073 & $-0,0007$ & FR:14-13 & $-0,0161$ & 1,2534 & $-0,002$ \\
\hline \multicolumn{8}{|c|}{ Medidas de Tensão } \\
\hline \multicolumn{2}{|c|}{ Medida } & \multicolumn{2}{|c|}{ Valor estimado } & \multicolumn{2}{|c|}{$U I$} & \multicolumn{2}{|c|}{$r^{N}$} \\
\hline \multicolumn{2}{|c|}{ V:1 } & \multicolumn{2}{|c|}{1,0594} & \multicolumn{2}{|c|}{0,1599} & \multicolumn{2}{|c|}{0,0016} \\
\hline \multicolumn{2}{|c|}{$\mathrm{V}: 2$} & \multicolumn{2}{|c|}{1,0443} & \multicolumn{2}{|c|}{0,1608} & \multicolumn{2}{|c|}{0,0016} \\
\hline \multicolumn{2}{|c|}{$V: 3$} & \multicolumn{2}{|c|}{1,0093} & \multicolumn{2}{|c|}{0,1669} & \multicolumn{2}{|c|}{0,0017} \\
\hline \multicolumn{2}{|c|}{ V:6 } & \multicolumn{2}{|c|}{1,0692} & \multicolumn{2}{|c|}{0,1673} & \multicolumn{2}{|c|}{0,0017} \\
\hline & & 1,0 & & 0,1 & & & \\
\hline
\end{tabular}




\begin{tabular}{c|c|c|c}
\hline $\mathbf{V}: \mathbf{1 2}$ & 1,0543 & 0,172 & 0,0017 \\
\hline
\end{tabular}

Os gráficos das figuras 4.7 e 4.8 ilustram, respectivamente, os índices UI para as medidas ativas e reativas do sistema em análise. As medidas ativas e reativas estão em gráficos distintos, devido, apenas, à limitação de espaço.

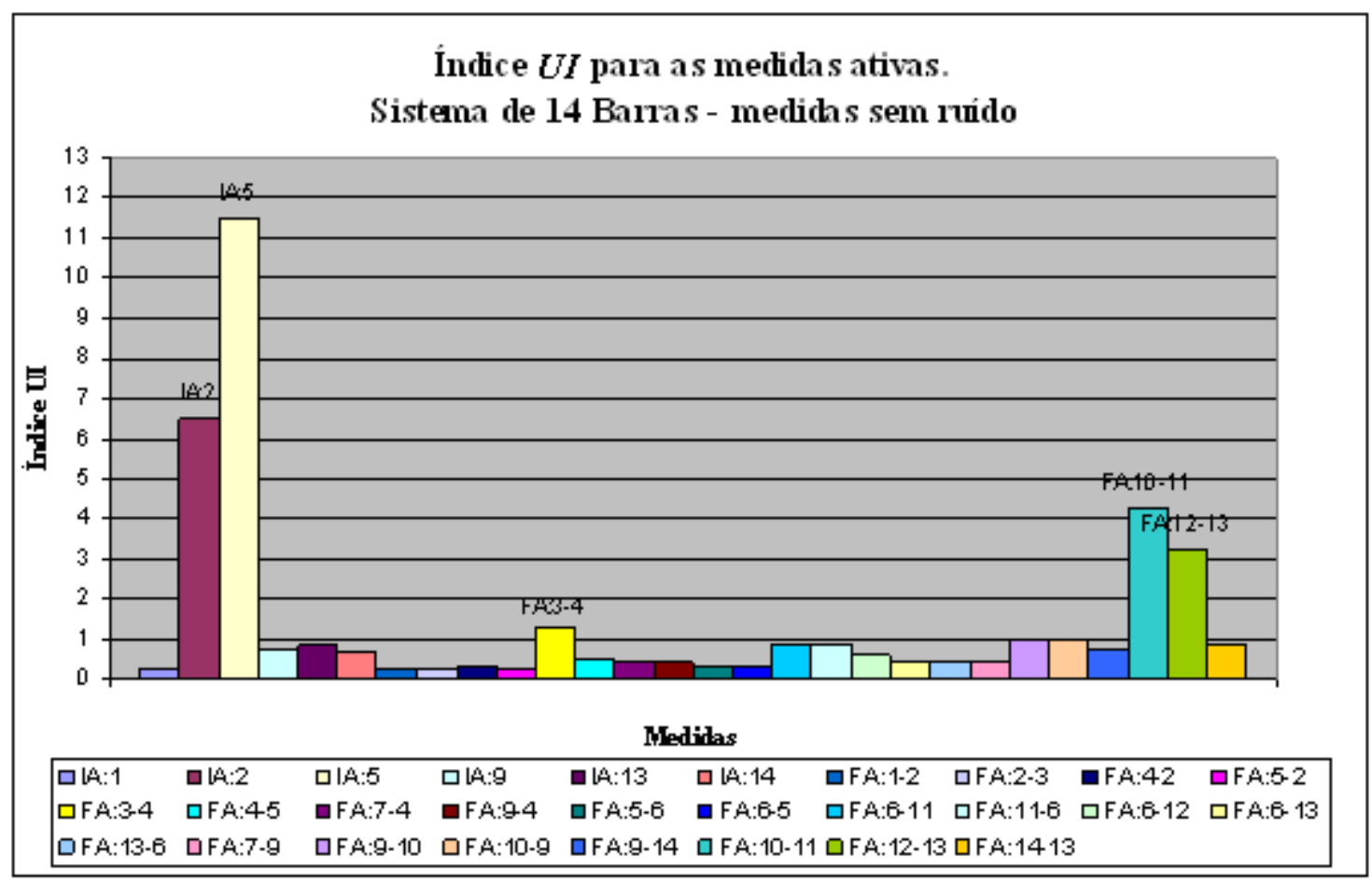

Figura 4.7: Índice UI para as medidas ativas. Sistema de 14 barras - medidas sem ruído

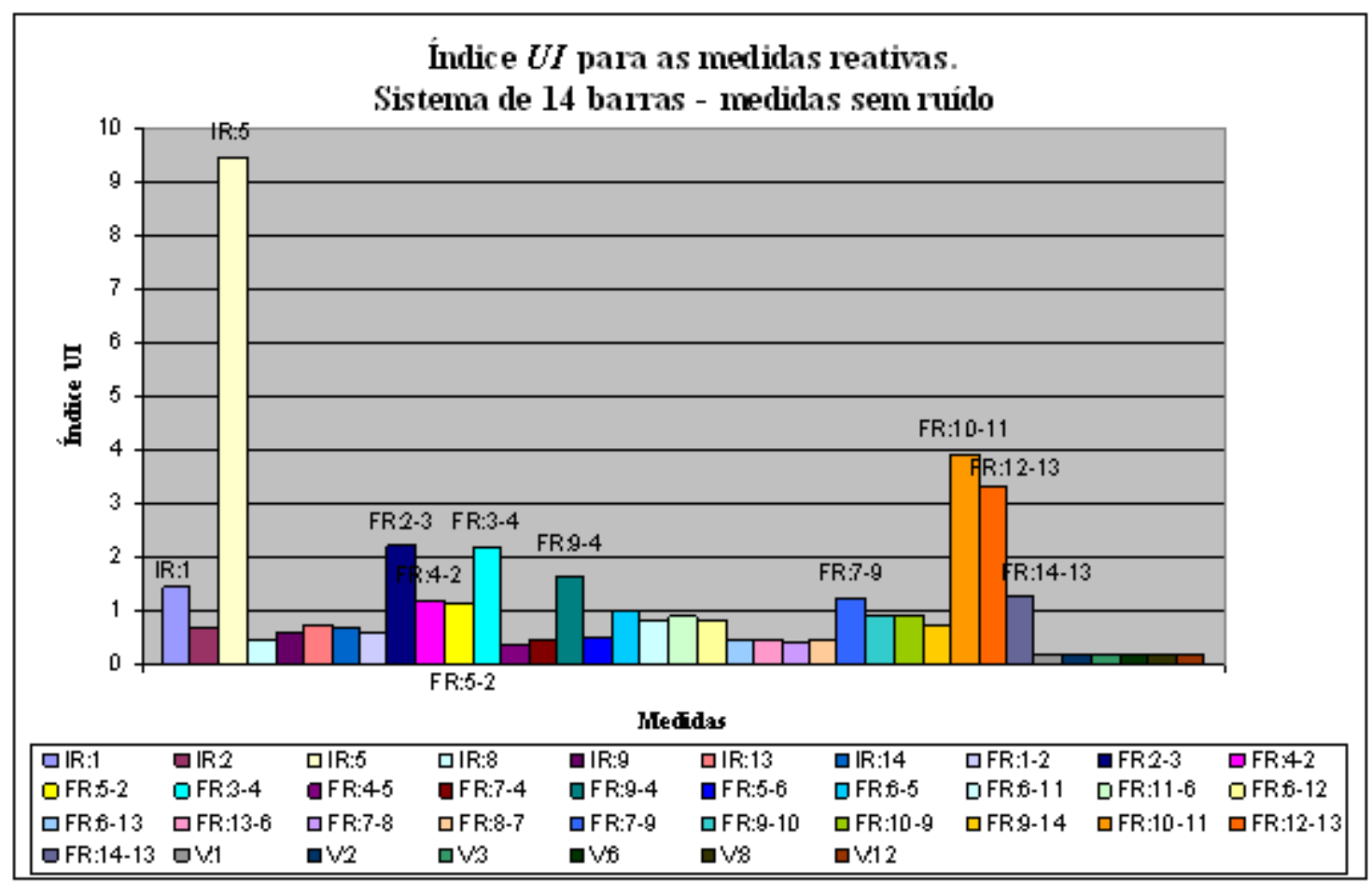

Figura 4.8: Índice $U I$ para as medidas reativas. Sistema de 14 barras - medidas sem ruído 
Através das figuras 4.7 e 4.8 , permite-se-nos verificar que as medidas em destaque são aquelas que apresentam a componente de erro não-detectável maior que a componente detectável, isto é, medidas cujos índices UI são maiores que 1. Assim, espera-se que erros em tais medidas sejam dificilmente detectados, se comparados com as demais medidas. Para demonstrar isto, faremos o que definimos como teste de identificação do nível de detecção de erro ${ }^{6}$.

A identificação do nível de detecção de erro consiste em calcular o mínimo de EG, que deve ser adicionado em cada medida, uma por uma, para que o teste do maior resíduo normalizado (ou do índice $J(x)$ ) detecte EG. Assim, se a medida $i$ apresenta nível de detecção igual a 3,5, isto significa que, para detectar-se EG na mesma, é necessário que o erro tenha magnitude de no mínimo $3,5^{*} \sigma_{i}$.

Para o sistema em análise, o limiar de detecção de EG para o índice $J(x)$ é $\lambda=\chi_{43 ;(1-0,05)}^{2} \cong 58$, considerando uma probabilidade de falso alarme $\alpha=5 \%$ e o grau de liberdade $m-n=43$. Já o limiar de detecção de EG adotado para o teste dos resíduos normalizados é $\beta=3,0$ (conforme indicado em Abur e Expósito (2004)).

A tabela 4.4, a seguir, mostra o nível de detecção para as medidas do sistema em análise. Observe que o teste do índice $J(x)$ não detectou nenhum EG, sendo que tais erros foram detectados apenas através do teste do maior resíduo normalizado.

Tabela 4.4: Identificação do nível de detecção de erro

\begin{tabular}{c|c|c|c|c|c|c|c}
\hline \multicolumn{3}{c}{ Medidas de Potência Ativa } & \multicolumn{3}{c}{ Medidas de Potência Reativa } \\
\hline Medida & $\boldsymbol{U I}$ & $\begin{array}{c}\text { Nível } \\
\text { Detecção }\end{array}$ & $\mathbf{J}(\mathbf{x})$ & Medida & $\boldsymbol{U I}$ & $\begin{array}{c}\text { Nível } \\
\text { Detecção }\end{array}$ & J(x) \\
\hline IA:1 & 0,2835 & 3,2 & 9,494 & IR:1 & 1,4182 & 5,2 & 9,081 \\
\hline IA:2 & 6,5083 & 19,8 & 9,068 & IR:2 & 0,6899 & 3,7 & 9,471 \\
\hline IA:5 & 11,5269 & 34,1 & 8,868 & IR:5 & 9,457 & 28 & 8,697 \\
\hline IA:7 & --- & --- & --- & IR:7 & --- & --- & --- \\
\hline IA:8 & --- & --- & --- & IR:8 & 0,4299 & 3,3 & 9,194 \\
\hline IA:9 & 0,7568 & 3,8 & 9,196 & IR:9 & 0,5737 & 3,5 & 9,449 \\
\hline IA:13 & 0,8338 & 4 & 9,44 & IR:13 & 0,6994 & 3,7 & 9,193 \\
\hline IA:14 & 0,6646 & 3,7 & 9,497 & IR:14 & 0,6738 & 3,7 & 9,42 \\
\hline FA:1-2 & 0,2913 & 3,2 & 9,453 & FR:1-2 & 0,5791 & 3,5 & 9,173 \\
\hline FA:2-3 & 0,248 & 3,1 & 9,072 & FR:2-3 & 2,2252 & 7,3 & 9,09 \\
\hline FA:4-2 & 0,3231 & 3,2 & 9,286 & FR:4-2 & 1,1917 & 4,7 & 9,174 \\
\hline FA:5-2 & 0,2935 & 3,2 & 9,44 & FR:5-2 & 1,1162 & 4,5 & 9,042 \\
\hline FA:3-4 & 1,3196 & 4,9 & 9,135 & FR:3-4 & 2,1772 & 7,1 & 9,063 \\
\hline FA:4-5 & 0,4912 & 3,4 & 9,34 & FR:4-5 & 0,3622 & 3,2 & 9,062 \\
\hline FA:7-4 & 0,4205 & 3,3 & 9,258 & FR:7-4 & 0,4312 & 3,3 & 9,259 \\
\hline FA:9-4 & 0,4207 & 3,3 & 9,257 & FR:9-4 & 1,6376 & 5,7 & 9,135 \\
\hline FA:5-6 & 0,3341 & 3,2 & 9,219 & FR:5-6 & 0,47 & 3,4 & 9,605 \\
\hline
\end{tabular}

\footnotetext{
${ }^{6}$ A figura C.1, do Anexo C, apresenta o algoritmo para identificação do nível de detecção.
} 


\begin{tabular}{|c|c|c|c|c|c|c|c|}
\hline FA:6-5 & 0,3341 & 3,2 & 9,219 & FR:6-5 & 0,9976 & 4,1 & 9,313 \\
\hline FA:6-11 & 0,8955 & 4,1 & 9,348 & FR:6-11 & 0,8176 & 3,9 & 9,132 \\
\hline FA:11-6 & 0,8984 & 4,1 & 9,322 & FR:11-6 & 0,8886 & 4,1 & 9,417 \\
\hline FA:6-12 & 0,6026 & 3,6 & 9,508 & FR:6-12 & 0,8307 & 4 & 9,468 \\
\hline FA:6-13 & 0,4551 & 3,3 & 9,022 & FR:6-13 & 0,4307 & 3,3 & 9,186 \\
\hline FA:13-6 & 0,4501 & 3,3 & 9,057 & FR:13-6 & 0,455 & 3,3 & 9,024 \\
\hline FA:7-8 & --- & --- & -- & FR:7-8 & 0,4171 & 3,3 & 9,282 \\
\hline FA:8-7 & --- & --- & --- & FR:8-7 & 0,4299 & 3,3 & 9,196 \\
\hline FA:7-9 & 0,4205 & 3,3 & 9,259 & FR:7-9 & 1,2312 & 3,8 & 5,885 \\
\hline FA:9-10 & 0,9794 & 4,2 & 9,005 & FR:9-10 & 0,9307 & 4,1 & 9,007 \\
\hline FA:10-9 & 0,9737 & 4,2 & 9,057 & FR:10-9 & 0,9197 & 4,1 & 9,108 \\
\hline FA:9-14 & 0,7582 & 3,8 & 9,17 & FR:9-14 & 0,7258 & 3,8 & 9,461 \\
\hline FA:10-11 & 4,2578 & 13,1 & 9,117 & FR:10-11 & 3,9174 & 12,1 & 9,095 \\
\hline FA:12-13 & 3,24 & 10,2 & 9,036 & FR:12-13 & 3,3304 & 10,5 & 9,115 \\
\hline FA:14-13 & 0,9074 & 4,1 & 9,227 & FR:14-13 & 1,2538 & 4,9 & 9,355 \\
\hline \multicolumn{8}{|c|}{ Medidas de Tensão } \\
\hline \multicolumn{2}{|c|}{ Medida } & \multicolumn{2}{|c|}{$\boldsymbol{U} \boldsymbol{I}$} & \multicolumn{2}{|c|}{$\begin{array}{c}\text { Nível } \\
\text { Detecção }\end{array}$} & \multicolumn{2}{|c|}{$\mathbf{J}(\mathbf{x})$} \\
\hline \multicolumn{2}{|c|}{$V: 1$} & \multicolumn{2}{|c|}{0,1622} & \multicolumn{2}{|c|}{3,1} & \multicolumn{2}{|c|}{9,401} \\
\hline \multicolumn{2}{|c|}{$\mathrm{V}: 2$} & \multicolumn{2}{|c|}{0,1632} & \multicolumn{2}{|c|}{3,1} & \multicolumn{2}{|c|}{9,399} \\
\hline \multicolumn{2}{|c|}{$\mathrm{V}: 3$} & \multicolumn{2}{|c|}{0,1694} & \multicolumn{2}{|c|}{3,1} & \multicolumn{2}{|c|}{9,386} \\
\hline \multicolumn{2}{|c|}{$V: 6$} & \multicolumn{2}{|c|}{0,1700} & \multicolumn{2}{|c|}{3,1} & \multicolumn{2}{|c|}{9,383} \\
\hline \multicolumn{2}{|c|}{$\mathrm{V}: 8$} & \multicolumn{2}{|c|}{0,1627} & \multicolumn{2}{|c|}{3,1} & \multicolumn{2}{|c|}{9,401} \\
\hline \multicolumn{2}{|c|}{$V: 12$} & \multicolumn{2}{|c|}{0,1747} & \multicolumn{2}{|c|}{3,1} & \multicolumn{2}{|c|}{9,372} \\
\hline
\end{tabular}

Através dos resultados da tabela 4.4, constrói-se o gráfico índice $U I$ x nível de detecção mostrado a seguir (figura 4.9):

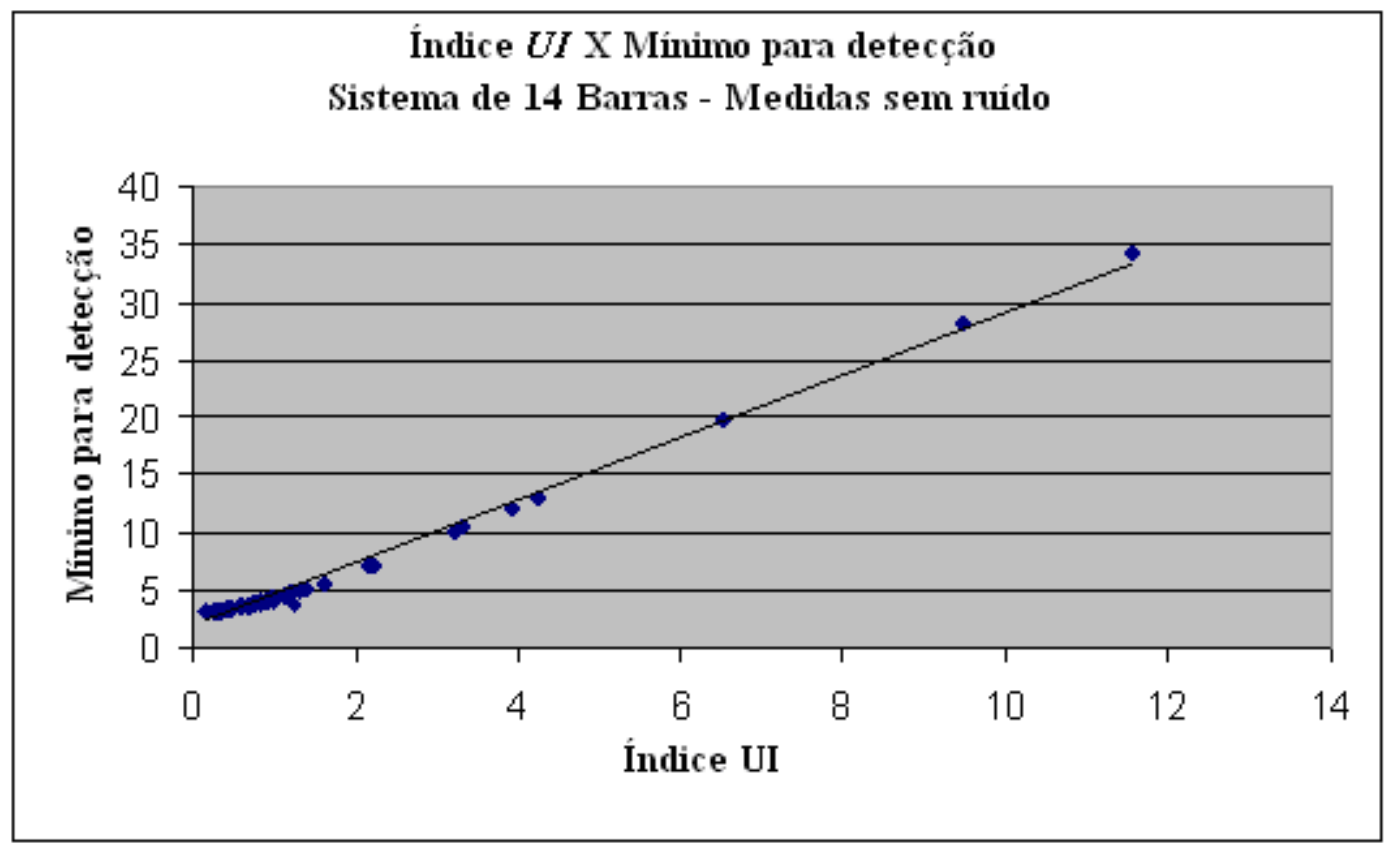

Figura 4.9: Gráfico de tendência para índice UI X Mínimo para detecção de erros em medidas.

Com base no gráfico apresentado na figura 4.9, comprova-se que quanto maior o índice $U I$ de uma medida, mais difícil será a detecção de erro grosseiro, através do teste 
dos resíduos normalizados. E ainda, através da reta de tendência, pode-se afirmar que este comportamento é praticamente linear, para o caso em que as medidas não apresentam ruídos.

\subsubsection{Comparação entre o Índice UI e os Métodos de Identificação de Medidas Ponto de Alavancamento}

Nesta subseção, mostraremos que o índice $U I$ permite uma identificação mais simples e confiável de medidas, que têm dificuldades em detectar e/ou identificar EGs, se comparado aos resultados dos métodos de identificação de medidas ponto de alavancamento, apresentados no capítulo 3 .

Para tal propósito, utilizaremos o mesmo sistema de medição da tabela 4.1 (subseção 4.3.3), e aplicaremos os métodos da matriz "chapéu" e da estatística de projeção.

A tabela 4.5, a seguir, engloba: os valores dos índices UI; os valores da diagonal principal da matriz de projeção $\left(K_{i i}\right)$; os valores da estatística de projeção $\left(P S_{i}\right)$; os graus de liberdade ( $L \mathrm{i})$; e os valores da distribuição qui-quadrada, para $L \mathrm{i}$ graus de liberdade e probabilidade de falso alarme de $0,025\left(\chi_{L i, 0,975}^{2}\right)$.

Tabela 4.5: Índices $U I$ e Valores de interesse para o teste da matriz "chapéu" e para o teste da estatística de projeção

\begin{tabular}{c|c|c|c|c|c|c|c|c|c|c|c}
\hline \multicolumn{1}{c}{ Medidas de Potência Ativa } & \multicolumn{4}{c|}{ Medidas de Potência Reativa } \\
\hline Medida & $\boldsymbol{U I}$ & $\mathbf{K i i}$ & $\boldsymbol{P S i}$ & $\boldsymbol{L i}$ & $\boldsymbol{\chi}_{L i, 0,975}^{2}$ & Medida & $\boldsymbol{U I}$ & $\boldsymbol{K i i}$ & $\boldsymbol{P S i}$ & $\boldsymbol{L i}$ & $\boldsymbol{\chi}_{L i, 0,975}^{2}$ \\
\hline IA:1 & 0,2827 & 0,074 & 3,2796 & 5 & 12,8325 & IR:1 & 1,4127 & 0,6662 & 110,1370 & 5 & 12,8325 \\
\hline IA:2 & 6,4873 & 0,9768 & 48,4505 & 9 & 19,0228 & IR:2 & 0,6792 & 0,3157 & 90,7811 & 9 & 19,0228 \\
\hline IA:5 & 11,5168 & 0,9925 & 23,9736 & 9 & 19,0228 & IR:5 & 9,4466 & 0,9889 & 282,889 & 9 & 19,0228 \\
\hline IA:7 & --- & --- & --- & --- & --- & IR:7 & --- & --- & --- & --- & --- \\
\hline IA:8 & --- & --- & --- & --- & --- & IR:8 & 0,43 & 0,156 & 32,9496 & 2 & 7,37776 \\
\hline IA:9 & 0,7558 & 0,3636 & 3,2745 & 10 & 20,4831 & IR:9 & 0,5676 & 0,2437 & 1,6638 & 10 & 20,4831 \\
\hline IA:13 & 0,8338 & 0,4101 & 22,3087 & 8 & 17,5346 & IR:13 & 0,6994 & 0,3285 & 98,2450 & 8 & 17,5346 \\
\hline IA:14 & 0,6645 & 0,3063 & 0,83850 & 6 & 14,4494 & IR:14 & 0,6737 & 0,3122 & 1,2549 & 6 & 14,4494 \\
\hline FA:1-2 & 0,2905 & 0,0778 & 3,8387 & 3 & 9,3484 & FR:1-2 & 0,579 & 0,2511 & 71,1651 & 3 & 9,3484 \\
\hline FA:2-3 & 0,2477 & 0,0578 & 2,2590 & 4 & 11,1433 & FR:2-3 & 2,2143 & 0,8306 & 121,830 & 4 & 11,1433 \\
\hline FA:4-2 & 0,322 & 0,094 & 3,5223 & 4 & 11,1433 & FR:4-2 & 1,186 & 0,5845 & 146,650 & 4 & 11,1433 \\
\hline FA:5-2 & 0,2922 & 0,0787 & 4,4876 & 4 & 11,1433 & FR:5-2 & 1,1161 & 0,5547 & 215,5348 & 4 & 11,1433 \\
\hline FA:3-4 & 1,3022 & 0,6291 & 6,4430 & 4 & 11,1433 & FR:3-4 & 2,156 & 0,823 & 96,993 & 4 & 11,1433 \\
\hline FA:4-5 & 0,4903 & 0,1938 & 1,5097 & 4 & 11,1433 & FR:4-5 & 0,3618 & 0,1158 & 1,5932 & 4 & 11,1433 \\
\hline FA:7-4 & 0,42 & 0,15 & 0,8385 & 4 & 11,1433 & FR:7-4 & 0,4293 & 0,1556 & 0,8385 & 4 & 11,1433 \\
\hline FA:9-4 & 0,4202 & 0,15 & 0,8385 & 4 & 11,1433 & FR:9-4 & 1,6329 & 0,7272 & 1,0608 & 4 & 11,1433 \\
\hline FA:5-6 & 0,3331 & 0,0999 & 0,8385 & 4 & 11,1433 & FR:5-6 & 0,4673 & 0,1792 & 30,9931 & 4 & 11,1433 \\
\hline FA:6-5 & 0,3331 & 0,0999 & 0,8385 & 4 & 11,1433 & FR:6-5 & 0,9743 & 0,487 & 46,1533 & 4 & 11,1433 \\
\hline FA:6-11 & 0,8952 & 0,4449 & 26,1800 & 4 & 11,1433 & FR:6-11 & 0,8173 & 0,4005 & 109,9181 & 4 & 11,1433 \\
\hline
\end{tabular}




\begin{tabular}{|c|c|c|c|c|c|c|c|c|c|c|c|}
\hline FA:11-6 & 0,8981 & 0,4465 & 25,9062 & 4 & 11,1433 & FR:11-6 & 0,8882 & 0,441 & 113,540 & 4 & 11,1433 \\
\hline FA:6-12 & 0,6026 & 0,2664 & 19,6052 & 4 & 11,1433 & FR:6-12 & 0,8307 & 0,4083 & 122,704 & 4 & 11,1433 \\
\hline FA:6-13 & 0,4551 & 0,1716 & 17,6359 & 4 & 11,1433 & FR:6-13 & 0,4307 & 0,1565 & 82,1473 & 4 & 11,1433 \\
\hline FA:13-6 & 0,4501 & 0,1685 & 17,2096 & 4 & 11,1433 & FR:13-6 & 0,455 & 0,1715 & 83,7972 & 4 & 11,1433 \\
\hline FA:7-8 & --- & --- & --- & --- & --- & FR:7-8 & 0,417 & 0,1482 & 31,9982 & 4 & 11,1433 \\
\hline FA:8-7 & --- & --- & --- & --- & --- & FR:8-7 & 0,43 & 0,156 & 32,9496 & 4 & 11,1433 \\
\hline FA:7-9 & 0,42 & 0,1499 & 1,2259 & 4 & 11,1433 & FR:7-9 & 1,2248 & 0,600 & 2,0014 & 4 & 11,1433 \\
\hline FA:9-10 & 0,9794 & 0,4896 & 7,2664 & 4 & 11,1433 & FR:9-10 & 0,9307 & 0,4642 & 4,1330 & 4 & 11,1433 \\
\hline FA:10-9 & 0,9736 & 0,4866 & 7,2529 & 4 & 11,1433 & FR:10-9 & 0,9197 & 0,4582 & 4,1777 & 4 & 11,1433 \\
\hline FA:9-14 & 0,7581 & 0,365 & 1,1558 & 4 & 11,1433 & FR:9-14 & 0,7257 & 0,345 & 1,6333 & 4 & 11,1433 \\
\hline FA:10-11 & 4,2529 & 0,9476 & 0,8391 & 4 & 11,1433 & FR:10-11 & 3,912 & 0,9387 & 0,9741 & 4 & 11,1433 \\
\hline FA:12-13 & 3,2398 & 0,913 & 145,9310 & 4 & 11,1433 & FR:12-13 & 3,3303 & 0,9173 & 280,889 & 4 & 11,1433 \\
\hline FA:14-13 & 0,9073 & 0,4515 & 0,9204 & 4 & 11,1433 & FR:14-13 & 1,2534 & 0,6111 & 1,0853 & 4 & 11,1433 \\
\hline \multicolumn{12}{|c|}{ Medidas de Tensão } \\
\hline \multicolumn{2}{|c|}{ Medida } & \multicolumn{2}{|c|}{$\boldsymbol{U I}$} & \multicolumn{2}{|r|}{ Kii } & \multicolumn{2}{|c|}{$P S i$} & \multicolumn{2}{|c|}{$L \mathbf{i}$} & \multicolumn{2}{|c|}{$\chi_{L i, 0,975}^{2}$} \\
\hline \multicolumn{2}{|c|}{$\mathrm{V}: 1$} & \multicolumn{2}{|c|}{0,1599} & \multicolumn{2}{|r|}{0,0249} & \multicolumn{2}{|c|}{0,838504} & \multicolumn{2}{|c|}{1} & \multicolumn{2}{|c|}{5,02389} \\
\hline \multicolumn{2}{|c|}{$\mathrm{V}: 2$} & \multicolumn{2}{|c|}{0,1608} & \multicolumn{2}{|r|}{0,0252} & \multicolumn{2}{|c|}{0,838504} & \multicolumn{2}{|c|}{1} & \multicolumn{2}{|c|}{5,02389} \\
\hline \multicolumn{2}{|c|}{$V: 3$} & \multicolumn{2}{|c|}{0,1669} & \multicolumn{2}{|r|}{0,0271} & \multicolumn{2}{|c|}{0,838504} & \multicolumn{2}{|c|}{1} & \multicolumn{2}{|c|}{5,02389} \\
\hline \multicolumn{2}{|c|}{$V: 6$} & \multicolumn{2}{|c|}{0,1673} & \multicolumn{2}{|r|}{0,0272} & \multicolumn{2}{|c|}{0,838504} & \multicolumn{2}{|c|}{1} & \multicolumn{2}{|c|}{5,02389} \\
\hline \multicolumn{2}{|c|}{$V: 8$} & \multicolumn{2}{|c|}{0,1602} & & 0,025 & 0,8385 & 04 & & 1 & & 2389 \\
\hline $\mathrm{V}: 1$ & & & & & 0,0287 & 0,8385 & 04 & & 1 & & 2389 \\
\hline
\end{tabular}

Obs. Limiar do método da matriz "chapéu": 2 n/m = 0,7714

$\mathrm{Na}$ tabela 4.5, os números em azul indicam que o valor de Kii de uma determinada medida é maior que o limiar $2 * n / m=0,7714$. Já os números em vermelho indicam que o valor de PSi de uma medida é superior ao seu limiar $\chi_{L i, 0,975}^{2}$. Ou seja, são medidas candidatas a ponto de alavancamento.

Fazendo-se uma comparação entre as medidas classificadas como ponto de alavancamento e as medidas com índice UI maior que 1 (parte não-detectável maior que parte detectável do erro), verifica-se que o método da matriz "chapéu" apresentou resultados um tanto quanto conservadores, tendo em vista que as medidas candidatas a ponto de alavancamento, por esse método, apresentaram índice $U I$ acima de 2. Assim, algumas medidas cujos resíduos não refletem a maior parte de seus erros não foram contempladas por esse método, como, por exemplo, a medida FR:9-4, cujo índice UI é 1,6376. Observe que, de acordo com a tabela 4.4, o índice de detecção de erro dessa medida é 5,7 .

Por outro lado, o método da estatística de projeção identificou muitas medidas como candidatas à medidas ponto de alavancamento. Entretanto, como pode ser observado pelos resultados da tabela 4.4, muitas dessas medidas, classificadas como ponto de alavancamento, não apresentam problemas no tocante à detecção e 
identificação de EG, como, por exemplo, a medida FR:7-8 que apresenta índice UI igual a 0,417 e índice de detecção de erro igual a 3,3 (tabela 4.4).

\subsection{Conclusões do Capítulo}

Neste capítulo foi apresentada a proposição referente ao índice de não-detecção de erro grosseiro, aqui denominado $U I$, através de uma análise geométrica do estimador de estado por mínimos quadrados ponderados.

A subseção 4.1 mostrou que a matriz de projeção $K$ projeta o vetor de medidas em $\mathfrak{R}(H)$ (imagem de $H$ ) e que a matriz $S$ projeta o vetor de medidas em $\mathfrak{R}(H)^{\perp}$ (complemento ortogonal da imagem de $H$ ).

Em decorrência dessa decomposição do espaço vetorial das medidas, foi possível, na subseção 4.2, decompor o vetor de erro das medidas em duas componentes: uma detectável e outra não-detectável. Assim, o índice $U I$ de uma medida foi definido como a relação entre a norma-2 da componente não-detectável e a norma-2 da componente detectável do erro daquela medida.

Um valor grande de $U I$, para uma determinada medida, indica que uma grande parcela do seu erro será "mascarada" no processo de estimação de estado. Desta forma, esse erro contribuirá pouco (ou quase nada) para o valor do resíduo daquela medida.

De um modo geral, o índice $U I$ nos indica que a dificuldade, ou mesmo a impossibilidade de detectar erro em uma determinada medida ocorre em razão de a componente não-detectável do erro dessa medida ser mais significante que a componente detectável.

Os resultados das simulações, apresentados na subseção 4.3.3 comprovaram que as medidas com maiores UI são aquelas cujos erros são mais difíceis de serem detectados, através do teste dos resíduos normalizados e do índice $J(\underline{x})$.

A partir das análises realizadas na seção 4.3.4, verificamos que o índice $U I$ nos possibilita uma compreensão melhor e mais completa da dificuldade de processamento de EGs, utilizando o estimador WLS, face ao conceito de medida ponto de alavancamento. Isto em razão de o índice informar o nível de mascaramento de cada uma das medidas disponíveis, independentemente de a medida mascarar muito ou pouco seus possíveis erros. Outra contribuição do índice $U I$ é apresentar uma nova forma de enxergar o problema de mascaramento de EGs, que pode ser explorada, como 
será apresentado nos capítulos 6 e 7, para desenvolvimento de metodologias mais eficientes para processamento de EGs e para projeto de sistema de medição.

Analisando a equação (4.30), apresentada na seção 4.3, verifica-se que o índice $U I$ não depende da magnitude do erro $b$, mas sim dos elementos da diagonal principal das matrizes $K$ e $S$, que, por sua vez, dependem: dos valores dos parâmetros do sistema; da redundância local e ponderação das medidas; e do valor estimado das variáveis de estado. Haja vista as estimativas dependerem dos valores das medidas, através de simulações computacionais analisar-se-á, no capítulo 5, a influência da variação dos valores das medidas no índice $U I$. 
Capítulo 4 


\section{Capítulo 5}

\section{$5 \quad$ Testes Realizados}

Neste capítulo são apresentados os testes realizados para validar os aspectos teóricos referentes ao capítulo 4. Para tal propósito, foram utilizados os sistemas testes de 14 e 30 barras do IEEE. Vários cenários de medidas, com ou sem ruído gaussiano, e, também, com medidas portadoras de EGs simples serão utilizados, para analisar a influência destes nos índices UIs das medidas.

\subsection{Simulações no Sistema de 14 Barras do IEEE}

Para os exemplos a seguir, considere o sistema de 14 barras do IEEE, ilustrado na figura 4.6, e o sistema de medição apresentado na tabela 4.1, ambos presentes no capítulo 4.

\subsubsection{Testes sem Ruído Gaussiano, mas com Erro Grosseiro Simples}

O objetivo desta subseção é analisar o desempenho dos métodos tradicionais de detecção/identificação de EGs frente à adição de EG simples, sem considerar ruído gaussiano nas medidas.

Exemplo 1: Erro grosseiro em medida com UI de valor baixo 
Considere o sistema de medição apresentado na tabela 4.1, porém agora com adição de EG de $10 \sigma$ na medida IA:1 (os valores das demais medidas são exatamente os determinados pelo fluxo de potência, isto é, sem acrescentar ruído aleatório algum).

Através da execução do estimador WLS, obtivemos os resultados tabelados a seguir (tabela 5.1). Observe que a medida portadora de EG está destacada em azul.

Tabela 5.1: Resultado numérico da adição de EG de $10 \sigma$ na medida IA:1

\begin{tabular}{|c|c|c|c|c|c|c|c|}
\hline \multicolumn{8}{|c|}{ Limiar do teste do índice $J(x): \lambda=\chi_{43}^{2}$} \\
\hline \multicolumn{8}{|c|}{ Índice $J(x)=92,6779$} \\
\hline \multicolumn{4}{|c|}{ Medidas de Potência Ativa } & \multicolumn{4}{|c|}{ Medidas de Potência Reativa } \\
\hline Medida & $\begin{array}{c}\text { Valor } \\
\text { estimado }\end{array}$ & $\boldsymbol{U I}$ & $r^{N}$ & Medida & $\begin{array}{c}\text { Valor } \\
\text { estimado }\end{array}$ & $\boldsymbol{U I}$ & $r^{N}$ \\
\hline IA:1 & 2,2946 & 0,2826 & $-9,6301$ & IR:1 & $-0,1662$ & 1,411 & 0,8723 \\
\hline IA:2 & 0,185 & 6,5105 & $-4,1418$ & IR:2 & 0,3052 & 0,6753 & 0,8404 \\
\hline IA:5 & $-0,0758$ & 11,4557 & $-2,2828$ & IR:5 & $-0,016$ & 9,446 & 0,7857 \\
\hline IA:7 & NULO & --- & NULO & IR:7 & NULO & --- & NULO \\
\hline IA:8 & NULO & --- & NULO & IR:8 & 0,1759 & 0,4297 & 0,0613 \\
\hline IA:9 & $-0,2952$ & 0,7561 & $-0,0595$ & IR:9 & $-0,1657$ & 0,5556 & $-0,2692$ \\
\hline IA:13 & $-0,1347$ & 0,8338 & $-0,0054$ & IR:13 & $-0,0581$ & 0,6994 & $-0,0255$ \\
\hline IA:14 & $-0,1489$ & 0,6645 & 0,0009 & IR:14 & $-0,0496$ & 0,6737 & 0,0131 \\
\hline FA:1-2 & 1,5488 & 0,2903 & 0,7793 & FR:1-2 & $-0,2034$ & 0,579 & $-0,0517$ \\
\hline FA:2-3 & 0,7276 & 0,2453 & 0,4486 & FR:2-3 & 0,0357 & 2,212 & 0,9893 \\
\hline FA:4-2 & $-0,5374$ & 0,3227 & $-0,7706$ & FR:4-2 & 0,0296 & 1,1875 & 1,4371 \\
\hline FA:5-2 & $-0,4014$ & 0,2931 & $-0,6676$ & FR:5-2 & $-0,021$ & 1,1141 & $-0,6512$ \\
\hline FA:3-4 & $-0,2351$ & 1,2969 & 0,5319 & FR:3-4 & 0,0444 & 2,1507 & 1,3539 \\
\hline FA:4-5 & $-0,6011$ & 0,4904 & $-1,1167$ & FR:4-5 & 0,1569 & 0,3611 & 0,5634 \\
\hline FA:7-4 & $-0,2806$ & 0,4202 & $-0,02$ & FR:7-4 & 0,1129 & 0,4243 & 0,2286 \\
\hline FA:9-4 & $-0,1607$ & 0,4203 & $-0,0223$ & FR:9-4 & 0,017 & 1,6123 & 0,7006 \\
\hline FA:5-6 & 0,4382 & 0,3336 & 0,2886 & FR:5-6 & 0,1243 & 0,4575 & 0,4078 \\
\hline FA:6-5 & $-0,4382$ & 0,3336 & $-0,2886$ & FR:6-5 & $-0,0803$ & 0,9337 & $-0,5514$ \\
\hline FA:6-11 & 0,0737 & 0,8943 & 0,1701 & FR:6-11 & 0,0354 & 0,8165 & 0,173 \\
\hline FA:11-6 & $-0,0731$ & 0,8971 & $-0,1726$ & FR:11-6 & $-0,0342$ & 0,8871 & $-0,1891$ \\
\hline FA:6-12 & 0,0779 & 0,6026 & 0,0243 & FR:6-12 & 0,0251 & 0,8307 & 0,0432 \\
\hline FA:6-13 & 0,1774 & 0,4551 & 0,0219 & FR:6-13 & 0,0721 & 0,4307 & 0,0242 \\
\hline FA:13-6 & $-0,1752$ & 0,4501 & $-0,0249$ & FR:13-6 & $-0,0679$ & 0,4549 & $-0,0398$ \\
\hline FA:7-8 & NULO & --- & NULO & FR:7-8 & $-0,1713$ & 0,4165 & $-0,0661$ \\
\hline FA:8-7 & NULO & --- & NULO & FR:8-7 & 0,1759 & 0,4297 & 0,0614 \\
\hline FA:7-9 & 0,2806 & 0,4202 & 0,0201 & FR:7-9 & 0,0584 & 1,2161 & $-0,366$ \\
\hline FA:9-10 & 0,0521 & 0,9794 & $-0,0356$ & FR:9-10 & 0,0423 & 0,9308 & $-0,0565$ \\
\hline FA:10-9 & $-0,052$ & 0,9736 & 0,0345 & FR:10-9 & $-0,0419$ & 0,9196 & 0,0528 \\
\hline FA:9-14 & 0,0941 & 0,7581 & $-0,056$ & FR:9-14 & 0,036 & 0,7256 & $-0,0974$ \\
\hline FA:10-11 & $-0,0379$ & 4,2345 & $-0,5026$ & FR:10-11 & $-0,0159$ & 3,8975 & $-0,4992$ \\
\hline FA:12-13 & 0,016 & 3,2396 & 0,04 & FR:12-13 & 0,0075 & 3,3303 & 0,0184 \\
\hline FA:14-13 & $-0,056$ & 0,907 & $-0,0889$ & FR:14-13 & $-0,0161$ & 1,2526 & $-0,1445$ \\
\hline \multicolumn{8}{|c|}{ Medidas de Tensão } \\
\hline \multicolumn{2}{|c|}{ Medida } & \multicolumn{2}{|c|}{ Valor estimado } & \multicolumn{2}{|c|}{$U I$} & \multicolumn{2}{|c|}{$r^{N}$} \\
\hline \multicolumn{2}{|c|}{ V:1 } & \multicolumn{2}{|c|}{1,0568} & \multicolumn{2}{|c|}{0,1546} & \multicolumn{2}{|c|}{0,1507} \\
\hline \multicolumn{2}{|c|}{ V:2 } & \multicolumn{2}{|c|}{1,042} & \multicolumn{2}{|c|}{0,1554} & \multicolumn{2}{|c|}{0,1387} \\
\hline
\end{tabular}




\begin{tabular}{c|c|c|c}
\hline $\mathbf{V : 3}$ & 1,007 & 0,1614 & 0,1356 \\
\hline $\mathbf{V : 6}$ & 1,067 & 0,1618 & 0,1249 \\
\hline $\mathbf{V : 8}$ & 1,0871 & 0,1549 & 0,1182 \\
\hline $\mathbf{V : 1 2}$ & 1,0521 & 0,1663 & 0,1281 \\
\hline
\end{tabular}

Como a medida portadora de EG (IA:1) apresenta índice $U I$ baixo, tanto o teste do índice $J(\mathrm{x})$, quanto o teste do maior resíduo normalizado, permitiram detectar EG, sendo que este último teste identificou corretamente a medida errônea. Além disso, verifica-se que o valor em módulo do resíduo normalizado da medida IA:1 está próximo ao valor da magnitude do erro aplicado, neste caso igual a 10.

Exemplo 2: Erro grosseiro em medida com $U I$ de valor alto

Considere o mesmo sistema de medição apresentado na tabela 4.1, porém agora com adição de EG de $10 \sigma$ na medida IA:5.

Com a execução do estimador WLS, obtivemos o resultado tabelado a seguir (tabela 5.2). A medida portadora de EG está destacada em azul.

Tabela 5.2: Resultado numérico da adição de EG de $10 \sigma$ na medida IA:5

\begin{tabular}{c|c|c|c|c|c|c|c}
\hline \multicolumn{7}{c}{ Limiar do teste do índice $J(x): \lambda=\chi_{43 ;(1-0,05)}^{2} \cong 58,0$} \\
\hline \multicolumn{7}{c}{ Índice $J(\mathrm{X})=0,7623$} \\
\hline \multicolumn{7}{c}{ Medidas de Potência Ativa } & \multicolumn{2}{c}{ Medidas de Potência Reativa } \\
\hline Medida & $\begin{array}{c}\text { Valor } \\
\text { estimado }\end{array}$ & UI & $r^{N}$ & Medida & $\begin{array}{c}\text { Valor } \\
\text { estimado }\end{array}$ & UI & $r^{N}$ \\
\hline IA:1 & 2,3153 & 0,2806 & 0,2057 & IR:1 & $-0,165$ & 1,4063 & 0,1388 \\
\hline IA:2 & 0,1831 & 6,4865 & 0,0268 & IR:2 & 0,3078 & 0,6639 & 0,2263 \\
\hline IA:5 & $-0,0634$ & 11,3898 & 0,8703 & IR:5 & $-0,016$ & 9,4473 & $-0,175$ \\
\hline IA:7 & NULO & --- & NULO & IR:7 & NULO & --- & NULO \\
\hline IA:8 & NULO & --- & NULO & IR:8 & 0,176 & 0,4293 & $-0,0024$ \\
\hline IA:9 & $-0,2954$ & 0,7553 & $-0,0134$ & IR:9 & $-0,1661$ & 0,5411 & $-0,1276$ \\
\hline IA:13 & $-0,1347$ & 0,8338 & $-0,0017$ & IR:13 & $-0,0581$ & 0,6993 & $-0,0062$ \\
\hline IA:14 & $-0,1489$ & 0,6645 & $-0,0042$ & IR:14 & $-0,0496$ & 0,6737 & $-0,0167$ \\
\hline FA:1-2 & 1,5639 & 0,2883 & 0,1772 & FR:1-2 & $-0,2032$ & 0,579 & $-0,0988$ \\
\hline FA:2-3 & 0,7322 & 0,2433 & 0,0561 & FR:2-3 & 0,0359 & 2,1974 & 0,0366 \\
\hline FA:4-2 & $-0,543$ & 0,3205 & $-0,1208$ & FR:4-2 & 0,0302 & 1,1862 & $-0,1646$ \\
\hline FA:5-2 & $-0,4033$ & 0,2912 & $-0,3783$ & FR:5-2 & $-0,0212$ & 1,114 & 0,2796 \\
\hline FA:3-4 & $-0,2342$ & 1,2756 & 0,1458 & FR:3-4 & 0,0448 & 2,1242 & 0,0932 \\
\hline FA:4-5 & $-0,6172$ & 0,4874 & 0,6429 & FR:4-5 & 0,1585 & 0,3607 & $-0,0938$ \\
\hline FA:7-4 & $-0,2807$ & 0,4198 & $-0,0095$ & FR:7-4 & 0,1133 & 0,4186 & 0,024 \\
\hline FA:9-4 & $-0,1608$ & 0,42 & $-0,0107$ & FR:9-4 & 0,0171 & 1,5937 & $-0,082$ \\
\hline FA:5-6 & 0,4413 & 0,3327 & $-0,1553$ & FR:5-6 & 0,1252 & 0,4488 & $-0,0823$ \\
\hline FA:6-5 & $-0,4413$ & 0,3327 & 0,1553 & FR:6-5 & $-0,0808$ & 0,8894 & $-0,1204$ \\
\hline FA:6-11 & 0,0738 & 0,8935 & $-0,0194$ & FR:6-11 & 0,0355 & 0,8157 & $-0,0232$ \\
\hline FA:11-6 & $-0,0733$ & 0,8962 & 0,0183 & FR:11-6 & $-0,0343$ & 0,886 & 0,0196 \\
\hline FA:6-12 & 0,0779 & 0,6026 & $-0,0022$ & FR:6-12 & 0,0251 & 0,8306 & $-0,0043$ \\
\hline FA:6-13 & 0,1774 & 0,455 & $-0,002$ & FR:6-13 & 0,0721 & 0,4307 & $-0,0046$ \\
\hline & & & & & & \\
\hline
\end{tabular}




\begin{tabular}{|c|c|c|c|c|c|c|c|}
\hline FA:13-6 & $-0,1753$ & 0,45 & 0,0007 & FR:13-6 & $-0,0679$ & 0,4547 & $-0,0019$ \\
\hline FA:7-8 & NULO & --- & NULO & FR:7-8 & $-0,1714$ & 0,4162 & $-0,0004$ \\
\hline FA:8-7 & NULO & --- & NULO & FR:8-7 & 0,176 & 0,4293 & $-0,0024$ \\
\hline FA:7-9 & 0,2807 & 0,4198 & 0,0095 & FR:7-9 & 0,0582 & 1,2038 & $-0,0684$ \\
\hline FA:9-10 & 0,0521 & 0,9793 & 0,0052 & FR:9-10 & 0,0422 & 0,9307 & 0,0284 \\
\hline FA:10-9 & $-0,0519$ & 0,9736 & $-0,0055$ & FR:10-9 & $-0,0419$ & 0,9197 & $-0,0291$ \\
\hline FA:9-14 & 0,094 & 0,758 & 0,0091 & FR:9-14 & 0,0359 & 0,7254 & 0,0188 \\
\hline FA:10-11 & $-0,038$ & 4,2184 & 0,0552 & FR:10-11 & $-0,016$ & 3,8832 & 0,0561 \\
\hline FA:12-13 & 0,016 & 3,2396 & $-0,0076$ & FR:12-13 & 0,0075 & 3,3303 & $-0,0116$ \\
\hline FA:14-13 & $-0,056$ & 0,9067 & 0,006 & FR:14-13 & $-0,0161$ & 1,2517 & 0,0043 \\
\hline \multicolumn{8}{|c|}{ Medidas de Tensão } \\
\hline \multicolumn{2}{|c|}{ Medida } & \multicolumn{2}{|c|}{ Valor estimado } & \multicolumn{2}{|c|}{$U I$} & \multicolumn{2}{|c|}{$r^{N}$} \\
\hline \multicolumn{2}{|c|}{ V:1 } & \multicolumn{2}{|c|}{1,0584} & \multicolumn{2}{|c|}{0,1478} & \multicolumn{2}{|c|}{0,0543} \\
\hline \multicolumn{2}{|c|}{$V: 2$} & \multicolumn{2}{|c|}{1,0435} & \multicolumn{2}{|c|}{0,1485} & \multicolumn{2}{|c|}{0,0528} \\
\hline \multicolumn{2}{|c|}{$V: 3$} & \multicolumn{2}{|c|}{1,0084} & \multicolumn{2}{|c|}{0,1542} & \multicolumn{2}{|c|}{0,0541} \\
\hline \multicolumn{2}{|c|}{$V: 6$} & \multicolumn{2}{|c|}{1,0683} & \multicolumn{2}{|c|}{0,1545} & \multicolumn{2}{|c|}{0,049} \\
\hline \multicolumn{2}{|c|}{$\mathrm{V}: 8$} & \multicolumn{2}{|c|}{1,0884} & \multicolumn{2}{|c|}{0,148} & \multicolumn{2}{|c|}{0,0472} \\
\hline \multicolumn{2}{|c|}{$V: 12$} & \multicolumn{2}{|c|}{1,0535} & \multicolumn{2}{|c|}{0,1588} & \multicolumn{2}{|c|}{0,0504} \\
\hline
\end{tabular}

De acordo com os resultados apresentados na tabela 5.2, os métodos tradicionais de detecção de EG não permitiram detectar a presença de EG no conjunto de medidas, já que os valores do índice $J(\mathrm{x})$ e do maior resíduo normalizado ficaram aquém dos respectivos limiares de detecção. Isto se deve ao fato de a medida portadora de EG (IA:5) apresentar índice UI elevado, ou seja, sua parcela de erro detectável é muito inferior à parcela não-detectável, acarretando valores baixos de resíduo e resíduo normalizado.

Exemplo 3: Simulações de erro grosseiro de $10 \sigma$ e $20 \sigma$

Apresentaremos a seguir, os resultados de simulações considerando EGs simples de 10 e $20 \sigma$, em cada uma das medidas do sistema de medição da tabela 4.1 (em uma medida de cada vez).

A tabela 5.3 engloba os valores do índice $J(x)$, os valores do índice $U I$ e resíduo normalizado, para a medida portadora de EG em cada caso, considerando EG simples de $10 \sigma$.

Sobre os resultados a serem apresentados, importa salientar que em vermelho distinguem-se os casos em que os métodos tradicionais de detecção/identificação de EGs falharam, e, em realce amarelo, encontram-se os casos em que apenas o teste do índice $J(\mathrm{x})$ falhou para detectar EGs. 
Tabela 5.3: Resultados numéricos da adição de erro grosseiro de $10 \sigma$ para cada medida (em uma medida de cada vez) considerando o sistema de medição da tabela 4.1

Limiar do teste do índice $J(x): \lambda=\chi_{43 ;(1-0,05)}^{2} \cong 58,0$

\begin{tabular}{|c|c|c|c|c|c|c|c|}
\hline \multicolumn{4}{|c|}{ Medidas de Potência Ativa } & \multicolumn{4}{|c|}{ Medidas de Potência Reativa } \\
\hline $\begin{array}{l}\text { Medida } \\
\text { com erro }\end{array}$ & $U I$ & $r^{N}$ & $\mathbf{J}(\mathbf{x})$ & $\begin{array}{l}\text { Medida } \\
\text { com erro }\end{array}$ & $U I$ & $r^{N}$ & $\mathbf{J}(\mathbf{x})$ \\
\hline IA:1 & 0,281 & $-9,630$ & 92,67 & IR:1 & 1,406 & $-5,794$ & 33,59 \\
\hline IA:2 & 6,491 & 1,521 & 2,31 & IR:2 & 0,665 & $-8,371$ & 69,41 \\
\hline IA:5 & 11,434 & 0,870 & 0,76 & IR:5 & 9,437 & 0,992 & 1,11 \\
\hline IA:7 & --- & --- & --- & IR:7 & --- & --- & --- \\
\hline IA: 8 & --- & --- & --- & IR:8 & 0,429 & $-9,192$ & 84,44 \\
\hline IA:9 & 0,755 & 7,975 & 63,63 & IR:9 & 0,547 & $-8,772$ & 77,13 \\
\hline IA:13 & 0,834 & 7,679 & 58,97 & IR:13 & 0,699 & $-8,196$ & 67,17 \\
\hline IA:14 & 0,665 & $-8,329$ & 69,38 & IR:14 & 0,674 & $-8,293$ & 68,78 \\
\hline FA:1-2 & 0,289 & 9,604 & 92,31 & FR:1-2 & 0,579 & $-8,655$ & 74,89 \\
\hline FA:2-3 & 0,244 & 9,733 & 94,39 & FR:2-3 & 2,197 & 4,126 & 17,03 \\
\hline FA:4-2 & 0,321 & 9,513 & 90,64 & FR:4-2 & 1,183 & 6,461 & 41,46 \\
\hline FA:5-2 & 0,292 & $-9,604$ & 92,19 & FR:5-2 & 1,114 & $-6,677$ & 44,66 \\
\hline FA:3-4 & 1,283 & $-6,119$ & 38,15 & FR:3-4 & 2,125 & 4,250 & 17,93 \\
\hline FA:4-5 & 0,489 & 8,984 & 80,72 & FR:4-5 & 0,360 & $-9,408$ & 88,49 \\
\hline FA:7-4 & 0,419 & 9,221 & 85,00 & FR:7-4 & 0,421 & $-9,186$ & 85,01 \\
\hline FA:9-4 & 0,420 & 9,220 & 84,99 & FR:9-4 & 1,603 & $-5,355$ & 28,16 \\
\hline FA:5-6 & 0,333 & $-9,488$ & 90,02 & FR:5-6 & 0,454 & $-9,157$ & 83,10 \\
\hline FA:6-5 & 0,333 & $-9,488$ & 90,03 & FR:6-5 & 0,905 & 7,331 & 55,29 \\
\hline FA:6-11 & 0,894 & $-7,456$ & 55,61 & FR:6-11 & 0,816 & $-7,748$ & 60,05 \\
\hline FA:11-6 & 0,897 & 7,442 & 55,42 & FR:11-6 & 0,886 & $-7,482$ & 56,03 \\
\hline FA:6-12 & 0,602 & 8,565 & 73,36 & FR:6-12 & 0,830 & $-7,693$ & 59,19 \\
\hline FA:6-13 & 0,455 & $-9,102$ & 82,85 & FR:6-13 & 0,431 & 9,184 & 84,35 \\
\hline FA:13-6 & 0,450 & $-9,119$ & 83,16 & FR:13-6 & 0,455 & 9,103 & 82,86 \\
\hline FA:7-8 & --- & --- & --- & FR:7-8 & 0,416 & 9,239 & 85,23 \\
\hline FA:8-7 & --- & --- & --- & FR:8-7 & 0,429 & $-9,192$ & 84,44 \\
\hline FA:7-9 & 0,419 & $-9,220$ & 84,99 & FR:7-9 & 1,208 & 6,363 & 40,74 \\
\hline FA:9-10 & 0,979 & 7,144 & 51,04 & FR:9-10 & 0,931 & 7,317 & 53,57 \\
\hline FA:10-9 & 0,974 & $-7,166$ & 51,35 & FR:10-9 & 0,919 & $-7,360$ & 54,19 \\
\hline FA:9-14 & 0,758 & $-7,970$ & 63,53 & FR:9-14 & 0,725 & $-8,095$ & 65,54 \\
\hline FA:10-11 & 4,227 & 2,303 & 5,31 & FR:10-11 & 3,890 & 2,479 & 6,21 \\
\hline FA:12-13 & 3,240 & 2,946 & 8,68 & FR:12-13 & 3,330 & $-2,875$ & 8,28 \\
\hline FA:14-13 & 0,907 & $-7,409$ & 54,90 & FR:14-13 & 1,252 & 6,242 & 38,94 \\
\hline \multicolumn{8}{|c|}{ Medidas de Tensão } \\
\hline \multicolumn{2}{|c|}{ Medida com erro } & \multicolumn{2}{|c|}{$U I$} & \multicolumn{2}{|c|}{$r^{N}$} & \multicolumn{2}{|c|}{$\mathbf{J}(\mathbf{x})$} \\
\hline \multicolumn{2}{|c|}{$\mathrm{V}: 1$} & \multicolumn{2}{|c|}{0,150} & \multicolumn{2}{|c|}{9,905} & \multicolumn{2}{|c|}{97,83} \\
\hline \multicolumn{2}{|c|}{$\mathrm{V}: 2$} & \multicolumn{2}{|c|}{0,151} & \multicolumn{2}{|c|}{$-9,900$} & \multicolumn{2}{|c|}{97,79} \\
\hline \multicolumn{2}{|c|}{$V: 3$} & \multicolumn{2}{|c|}{0,156} & \multicolumn{2}{|c|}{$-9,871$} & \multicolumn{2}{|c|}{97,63} \\
\hline \multicolumn{2}{|c|}{$V: 6$} & \multicolumn{2}{|c|}{0,157} & \multicolumn{2}{|c|}{$-9,878$} & \multicolumn{2}{|c|}{97,62} \\
\hline \multicolumn{2}{|c|}{ V:8 } & & & $-9,8$ & & & \\
\hline V: & & & & 9,8 & & & \\
\hline
\end{tabular}

Através dos resultados acima, verificamos que o teste do maior resíduo normalizado e, também, o teste do índice $J(\mathrm{x})$, falharam na detecção de EGs em 
medidas com índice UI maior ou igual a 3,240 (casos em vermelho). Vale ainda ressaltar que o teste do índice $J(\mathrm{x})$ também falhou em detectar EGs em medidas com índice $U I$ entre 0,886 e 3,240 (casos em realce amarelo).

A tabela 5.4, a seguir, apresenta os valores do índice $J(x)$, os valores do índice UI e resíduo normalizado para medida portadora de EG em cada caso, considerando EG simples de $20 \sigma$.

Tabela 5.4: Resultados numéricos da adição de EG de $20 \sigma$ para cada medida (em uma medida de cada vez) considerando o plano de medição da tabela 4.1

Limiar do teste do índice $J(x): \lambda=\chi_{43 ;(1-0,05)}^{2} \cong 58,0$

\begin{tabular}{|c|c|c|c|c|c|c|c|}
\hline \multicolumn{4}{|c|}{ Medidas de Potência Ativa } & \multicolumn{4}{|c|}{ Medidas de Potência Reativa } \\
\hline $\begin{array}{c}\text { Medida } \\
\text { com erro }\end{array}$ & $U I$ & $r^{N}$ & $\mathbf{J}(\mathbf{x})$ & $\begin{array}{c}\text { Medida } \\
\text { com erro }\end{array}$ & $U I$ & $r^{N}$ & $\mathbf{J}(\mathbf{x})$ \\
\hline IA:1 & 0,282 & $-19,251$ & 370,66 & IR:1 & 1,408 & $-11,600$ & 134,59 \\
\hline IA:2 & 6,521 & 3,041 & 9,249 & IR:2 & 0,668 & $-16,741$ & 278,39 \\
\hline IA: 5 & 11,38 & 1,768 & 3,053 & IR:5 & 9,443 & 2,195 & 4,439 \\
\hline IA: 7 & --- & --- & --- & IR:7 & --- & --- & --- \\
\hline IA:8 & --- & --- & --- & IR:8 & 0,429 & $-18,383$ & 337,84 \\
\hline IA:9 & 0,757 & 15,953 & 254,43 & IR:9 & 0,539 & $-17,569$ & 308,43 \\
\hline IA:13 & 0,834 & 15,357 & 235,83 & IR:13 & 0,699 & $-16,393$ & 268,72 \\
\hline IA:14 & 0,665 & $-16,662$ & 277,62 & IR:14 & 0,674 & $-16,590$ & 275,20 \\
\hline FA:1-2 & 0,289 & 19,210 & 369,31 & FR:1-2 & 0,579 & $-17,309$ & 299,61 \\
\hline FA:2-3 & 0,243 & 19,431 & 377,57 & FR:2-3 & 2,197 & 8,256 & 67,623 \\
\hline FA:4-2 & 0,322 & 19,037 & 362,48 & FR:4-2 & 1,188 & 12,882 & 165,44 \\
\hline FA:5-2 & 0,293 & $-19,206$ & 368,85 & FR:5-2 & 1,112 & $-13,359$ & 178,60 \\
\hline FA:3-4 & 1,289 & $-12,385$ & 153,49 & FR:3-4 & 2,128 & 8,5178 & 70,99 \\
\hline FA:4-5 & 0,489 & 17,954 & 322,59 & FR:4-5 & 0,36 & $-18,821$ & 353,99 \\
\hline FA:7-4 & 0,421 & 18,438 & 339,94 & FR:7-4 & 0,417 & $-18,506$ & 340,02 \\
\hline FA:9-4 & 0,421 & 18,437 & 339,92 & FR:9-4 & 1,587 & $-10,628$ & 112,77 \\
\hline FA:5-6 & 0,334 & $-18,976$ & 360,02 & FR:5-6 & 0,446 & $-18,250$ & 332,47 \\
\hline FA:6-5 & 0,334 & $-18,982$ & 360,18 & FR:6-5 & 0,881 & 14,576 & 220,88 \\
\hline FA:6-11 & 0,893 & $-14,923$ & 222,53 & FR:6-11 & 0,815 & $-15,503$ & 240,28 \\
\hline FA:11-6 & 0,896 & 14,897 & 221,62 & FR:11-6 & 0,886 & $-14,988$ & 224,17 \\
\hline FA:6-12 & 0,603 & 17,129 & 293,42 & FR:6-12 & 0,83 & $-15,389$ & 236,79 \\
\hline FA:6-13 & 0,455 & $-18,205$ & 331,42 & FR:6-13 & 0,431 & 18,368 & 337,39 \\
\hline FA:13-6 & 0,450 & $-18,24$ & 332,67 & FR:13-6 & 0,455 & 18,204 & 331,43 \\
\hline FA:7-8 & --- & -- & -- & FR:7-8 & 0,416 & 18,465 & 340,90 \\
\hline FA:8-7 & --- & --- & --- & FR:8-7 & 0,429 & $-18,380$ & 337,83 \\
\hline FA:7-9 & 0,421 & $-18,437$ & 339,95 & FR:7-9 & 1,203 & 12,826 & 163,08 \\
\hline FA:9-10 & 0,979 & 14,2873 & 204,13 & FR:9-10 & 0,931 & 14,6365 & 214,24 \\
\hline FA:10-9 & 0,974 & $-14,332$ & 205,41 & FR:10-9 & 0,92 & $-14,726$ & 216,80 \\
\hline FA:9-14 & 0,758 & $-15,941$ & 254,16 & FR:9-14 & 0,725 & $-16,195$ & 262,23 \\
\hline FA:10-11 & 4,214 & 4,5924 & 21,23 & FR:10-11 & 3,88 & 4,963 & 24,82 \\
\hline FA:12-13 & 3,240 & 5,889 & 34,68 & FR:12-13 & 3,331 & $-5,7511$ & 33,11 \\
\hline FA:14-13 & 0,907 & $-14,821$ & 219,66 & FR:14-13 & 1,252 & 12,4767 & 155,72 \\
\hline \multicolumn{8}{|c|}{ Medidas de Tensão } \\
\hline \multicolumn{2}{|c|}{ Medida com erro } & \multicolumn{2}{|c|}{$U I$} & \multicolumn{2}{|l|}{$r^{N}$} & \multicolumn{2}{|c|}{$\mathbf{J}(\mathbf{x})$} \\
\hline
\end{tabular}




\begin{tabular}{c|c|c|c}
\hline $\mathbf{V}: \mathbf{1}$ & 0,145 & 19,789 & 391,33 \\
\hline $\mathbf{V}: \mathbf{2}$ & 0,146 & $-19,756$ & 391,17 \\
\hline $\mathbf{V : 3}$ & 0,152 & $-19,787$ & 390,50 \\
\hline $\mathbf{V : 6}$ & 0,152 & $-19,732$ & 390,46 \\
\hline $\mathbf{V : 8}$ & 0,146 & $-19,762$ & 391,24 \\
\hline $\mathbf{V : 1 2}$ & 0,156 & 19,7403 & 390,03 \\
\hline
\end{tabular}

Com a adição de EG de $20 \sigma$ às medidas, os métodos de detecção de EGs só falharam para detectar EGs nas medidas IA:5 e IR:5, já que estas medidas (em vermelho) apresentam os maiores índices UIs, em relação às demais medidas. Além disso, o teste do maior resíduo normalizado permitiu-nos detectar e identificar EGs em medidas com índice $U I$ ente 3,240 e 6,521 (em realce amarelo), em oposição ao teste do índice $J(\mathrm{x})$, que não detectou EG nessas medidas.

Apesar de os métodos tradicionais de detecção de EGs não nos permitirem detectar EGs em algumas medidas (aquelas com índice muito alto), constatamos que, em todas as simulações, a medida com maior resíduo normalizado foi sempre aquela portadora de EG. Dessa forma, pode-se concluir, através dos resultados das tabelas 5.3 e 5.4, que os valores dos resíduos normalizados de medidas, com índice $U I$ de valor baixo, são próximos ao valor, em desvios padrão, do EG aplicado. Em contrapartida, quanto maior o índice $U I$, menor será o resíduo normalizado da medida e também o índice $J(x)$ do conjunto em análise, o que explica a dificuldade de detectar EGs, através dos métodos baseados na análise de resíduos de medidas.

\subsubsection{Testes com Ruído Gaussiano e sem Erro Grosseiro}

As simulações apresentadas anteriormente foram executadas considerando apenas medidas exatas, isto é, medidas sem ruído (valores determinados pelo fluxo de potência). Nesta subseção, se nos apresentarão simulações, considerando a adição de ruído gaussiano às medidas de potência do sistema de medição da tabela 4.1. O intuito dessa subseção foi verificar a influência de ruídos gaussianos nos índices UIs das medidas.

Assim, o vetor de medidas $\underline{z}_{i}^{m}$ é calculado da seguinte forma:

$$
\underline{z}_{i}^{m}=\underline{z}_{i}^{f c}+3 \cdot \sigma_{i} \cdot \alpha,
$$

onde: 
- $\sigma_{i}$ é o desvio padrão da medida $i$;

- $\alpha$ é uma variável aleatória, com distribuição normal padrão $N(0,1)$;

- $\underline{z}_{i}^{f c}$ é o valor obtido diretamente do programa de fluxo de carga;

- $\underline{z}_{i}^{m}$ é o valor da medida $i$.

A partir da execução do estimador WLS, para 100 amostras de medidas com ruído, obtiveram-se as médias e desvios padrões do índice $U I$, resíduo normalizado e índice $J(x)$, sendo os mesmos tabelados a seguir (tabela 5.5). Os valores de $U I$ e do resíduo normalizado das medidas virtuais não foram calculados.

Tabela 5.5: Resultados do índice UI, resíduo normalizado e índice J(x), para 100 amostras de medidas com ruído e sem EG.

\begin{tabular}{c|c|c|c|c}
\hline \multirow{2}{*}{ Medidas } & \multicolumn{2}{|c|}{ UI } & \multicolumn{2}{c}{$r^{N}$} \\
\cline { 2 - 5 } & Média & $\begin{array}{c}\text { Desvio } \\
\text { padrão }\end{array}$ & Média & $\begin{array}{c}\text { Desvio } \\
\text { padrão }\end{array}$ \\
\hline IA:1 & 0,28087 & 0,001246 & 0,10752 & 0,86471 \\
\hline IA:2 & 6,48453 & 0,012335 & 0,03821 & 0,84355 \\
\hline IA:5 & 11,4035 & 0,06951 & 0,09057 & 0,91743 \\
\hline IA:9 & 0,75553 & 0,00055 & 0,00253 & 0,90095 \\
\hline IA:13 & 0,83382 & $9,03 \mathrm{E}-05$ & 0,07235 & 0,90803 \\
\hline IA:14 & 0,66455 & 0,000123 & 0,20836 & 0,8682 \\
\hline FA:1-2 & 0,28858 & 0,001301 & $-0,0096$ & 0,87161 \\
\hline FA:2-3 & 0,2438 & 0,002374 & 0,08045 & 0,89693 \\
\hline FA:4-2 & 0,3207 & 0,001111 & 0,10476 & 0,82957 \\
\hline FA:5-2 & 0,29129 & 0,000919 & $-0,00241$ & 0,93655 \\
\hline FA:3-4 & 1,27999 & 0,013796 & 0,03529 & 0,87737 \\
\hline FA:4-5 & 0,48768 & 0,001864 & 0,01199 & 0,95791 \\
\hline FA:7-4 & 0,41993 & 0,000297 & $-0,06892$ & 0,86805 \\
\hline FA:9-4 & 0,42007 & 0,000294 & 0,03047 & 0,75901 \\
\hline FA:5-6 & 0,33288 & 0,000491 & $-0,06191$ & 0,9050 \\
\hline FA:6-5 & 0,33288 & 0,000491 & 0,05999 & 0,93314 \\
\hline FA:6-11 & 0,89363 & 0,000926 & $-0,11506$ & 0,90764 \\
\hline FA:11-6 & 0,89643 & 0,000961 & $-0,08315$ & 0,91193 \\
\hline FA:6-12 & 0,60257 & $5,41 \mathrm{E}-05$ & 0,03036 & 0,83887 \\
\hline FA:6-13 & 0,45502 & $4,74 \mathrm{E}-05$ & 0,09266 & 0,85313 \\
\hline FA:13-6 & 0,45007 & $6,99 \mathrm{E}-05$ & $-0,0051$ & 0,91081 \\
\hline FA:7-9 & 0,41991 & 0,000289 & 0,10216 & 0,91266 \\
\hline FA:9-10 & 0,97932 & $5,2 \mathrm{E}-05$ & $-0,06116$ & 0,92341 \\
\hline FA:10-9 & 0,97356 & $5,78 \mathrm{E}-05$ & $-0,06783$ & 0,9113 \\
\hline FA:9-14 & 0,75795 & 0,000118 & 0,10171 & 0,86513 \\
\hline FA:10-11 & 4,2217 & 0,017853 & 0,04678 & 0,83344 \\
\hline FA:12-13 & 3,23981 & 0,000622 & 0,04223 & 0,88788 \\
\hline FA:14-13 & 0,90682 & 0,000303 & $-0,09036$ & 0,86487 \\
\hline IR:1 & 1,40723 & 0,003655 & 0,12706 & 0,90339 \\
\hline 0,66627 & 0,007925 & 0,0579 & 0,80912 \\
\hline & & & &
\end{tabular}




\begin{tabular}{|c|c|c|c|c|}
\hline IR:5 & 9,44382 & 0,006522 & $-0,14608$ & 0,86859 \\
\hline IR:8 & 0,42936 & 0,000414 & $-0,15592$ & 0,90174 \\
\hline IR:9 & 0,54359 & 0,013889 & 0,13912 & 0,86273 \\
\hline IR:13 & 0,69929 & $9,07 \mathrm{E}-05$ & $-0,00178$ & 0,97717 \\
\hline IR:14 & 0,67371 & $7,18 \mathrm{E}-05$ & $-0,00633$ & 0,87993 \\
\hline FR:1-2 & 0,57899 & $4,32 \mathrm{E}-05$ & $-0,15438$ & 0,92306 \\
\hline FR:2-3 & 2,197 & 0,010626 & $-0,02336$ & 0,82906 \\
\hline FR:4-2 & 1,18569 & 0,003085 & $-0,00476$ & 0,91834 \\
\hline FR:5-2 & 1,11365 & 0,001441 & 0,0745 & 0,83461 \\
\hline FR:3-4 & 2,12514 & 0,018503 & $-0,01401$ & 0,83062 \\
\hline FR:4-5 & 0,36073 & 0,000705 & $-0,11782$ & 0,95477 \\
\hline FR:7-4 & 0,41954 & 0,005868 & $-0,01919$ & 0,87084 \\
\hline FR:9-4 & 1,59742 & 0,021614 & 0,07519 & 0,78768 \\
\hline FR:5-6 & 0,45063 & 0,009647 & $-0,03245$ & 0,98805 \\
\hline FR:6-5 & 0,89692 & 0,043379 & $-0,21969$ & 0,82474 \\
\hline FR:6-11 & 0,81581 & 0,000852 & 0,05683 & 0,85398 \\
\hline FR:11-6 & 0,88617 & 0,00115 & 0,05441 & 0,87592 \\
\hline FR:6-12 & 0,83059 & $9,98 \mathrm{E}-05$ & 0,00304 & 0,8334 \\
\hline FR:6-13 & 0,43064 & $4,96 \mathrm{E}-05$ & $-0,18868$ & 0,83446 \\
\hline FR:13-6 & 0,45475 & 0,000126 & $-0,21692$ & 0,87811 \\
\hline FR:7-8 & 0,41625 & 0,000507 & $-0,09437$ & 0,92768 \\
\hline FR:8-7 & 0,42936 & 0,000414 & 0,01177 & 0,94414 \\
\hline FR:7-9 & 1,20587 & 0,010935 & 0,11196 & 0,86882 \\
\hline FR:9-10 & 0,93073 & $6,67 \mathrm{E}-05$ & 0,1295 & 0,76919 \\
\hline FR:10-9 & 0,91967 & 7,39E-05 & 0,1723 & 0,7972 \\
\hline FR:9-14 & 0,72536 & 0,000253 & $-0,08641$ & 0,84069 \\
\hline FR:10-11 & 3,8859 & 0,014845 & $-0,00117$ & 0,75124 \\
\hline FR:12-13 & 3,33032 & 0,000225 & $-0,00195$ & 0,87831 \\
\hline FR:14-13 & 1,25193 & 0,000878 & $-0,03477$ & 0,77024 \\
\hline$V: 1$ & 0,14872 & 0,006644 & $-0,01003$ & 0,12681 \\
\hline $\mathrm{V}: 2$ & 0,1494 & 0,006718 & $-0,01036$ & 0,12742 \\
\hline$V: 3$ & 0,1551 & 0,006983 & $-0,01153$ & 0,13242 \\
\hline$V: 6$ & 0,15543 & 0,007029 & $-0,01062$ & 0,13288 \\
\hline $\mathrm{V}: 8$ & 0,14887 & 0,0067 & $-0,00837$ & 0,12728 \\
\hline $\mathrm{V}: 12$ & 0,15976 & 0,007225 & $-0,01131$ & 0,13636 \\
\hline \multicolumn{5}{|c|}{ Índice $J(x)$ : } \\
\hline \multicolumn{5}{|c|}{ Média $=27,5578 ;$ Desvio padrão $=6,0405$} \\
\hline
\end{tabular}

Analisando os valores das médias e respectivos desvios padrões dos resíduos normalizados e do índice $J(\mathrm{x})$, apresentados na tabela 5.5, verificar-se-á que em nenhuma das simulações foi detectado EG. Isto porque esses valores ficaram abaixo dos limiares de detecção de EGs, para esse conjunto de medidas.

Outra constatação importante é que as medidas com índice UI elevado apresentaram os maiores valores de desvio padrão de UI. Por exemplo, a medida IA:5 apresenta o maior UI médio, igual a 11,4035, e o maior desvio padrão para $U I$, igual a 0,06951 . 


\subsubsection{Identificação do Nível de Detecção de Erro Grosseiro: Medidas com Ruído}

Apresentaremos, nesta subseção, os valores médios dos resultados da identificação do nível de detecção de EG, considerando 100 amostras de ruído em medidas.

Para as simulações em questão, o limiar de detecção de EG para o índice $J(x)$ é $\lambda=\chi_{43 ;(1-0,05)}^{2} \cong 58$, considerando uma probabilidade de falso alarme $\alpha=5 \%$ e o grau de liberdade $m-n=43$. Já o limiar de detecção de EG, adotado para o teste dos resíduos normalizados, é $\beta=3,0$.

A tabela 5.6, a seguir, mostra os valores médios dos resultados do nível de detecção de EG, para as medidas do sistema em análise. Observe que o teste do índice $J(x)$ não detectou nenhum EG, sendo que tais erros foram detectados apenas através do teste do maior resíduo normalizado, ou seja, quando o valor do maior resíduo normalizado foi maior que 3 .

Tabela 5.6: Identificação do nível de detecção de EGs, para 100 amostras de medidas com ruído

\begin{tabular}{c|c|c|c|c|c|c}
\hline \multirow{2}{*}{ Medida } & \multicolumn{2}{|c|}{ UI } & \multicolumn{2}{c|}{$\begin{array}{c}\text { Nível } \\
\text { Detecc̃ão }\end{array}$} & \multicolumn{2}{c}{ J(x) } \\
\cline { 2 - 7 } & Média & $\begin{array}{c}\text { Desvio } \\
\text { Padrão }\end{array}$ & Média & $\begin{array}{c}\text { Desvio } \\
\text { Padrão }\end{array}$ & Média & $\begin{array}{c}\text { Desvio } \\
\text { Padrão }\end{array}$ \\
\hline IA:1 & 0,2809 & 0,001252 & 3,246 & 0,223571 & 35,55962 & 5,78023 \\
\hline IA:2 & 6,49047 & 0,012295 & 18,594 & 5,509694 & 34,98511 & 5,7202 \\
\hline IA:5 & 11,3961 & 0,069345 & 32,36406 & 8,407465 & 33,65461 & 6,331941 \\
\hline IA:9 & 0,75573 & 0,000603 & 3,866 & 0,575637 & 35,70108 & 5,745952 \\
\hline IA:13 & 0,83382 & $8,13 \mathrm{E}-05$ & 4,072 & 0,593241 & 35,54678 & 6,056509 \\
\hline IA:14 & 0,66458 & 0,000113 & 3,622 & 0,50042 & 35,50618 & 5,451114 \\
\hline FA:1-2 & 0,28858 & 0,001299 & 3,24 & 0,227414 & 35,78812 & 5,936004 \\
\hline FA:2-3 & 0,24339 & 0,002376 & 3,204 & 0,200967 & 35,70849 & 5,68499 \\
\hline FA:4-2 & 0,3209 & 0,001108 & 3,27 & 0,243501 & 35,65853 & 5,842378 \\
\hline FA:5-2 & 0,29152 & 0,000966 & 3,226 & 0,2268 & 35,65736 & 5,666571 \\
\hline FA:3-4 & 1,27988 & 0,015701 & 4,828 & 0,962749 & 34,94896 & 5,713611 \\
\hline FA:4-5 & 0,48777 & 0,001758 & 3,458 & 0,389037 & 35,41867 & 5,81472 \\
\hline FA:7-4 & 0,42005 & 0,000304 & 3,384 & 0,312281 & 35,77959 & 5,72729 \\
\hline FA:9-4 & 0,42019 & 0,000309 & 3,394 & 0,318399 & 35,76746 & 5,77158 \\
\hline FA:5-6 & 0,33306 & 0,000559 & 3,284 & 0,260815 & 35,78114 & 5,863845 \\
\hline FA:6-5 & 0,33306 & 0,000559 & 3,292 & 0,261186 & 35,76509 & 5,876577 \\
\hline FA:6-11 & 0,89349 & 0,00092 & 4,25 & 0,818165 & 35,23461 & 5,648707 \\
\hline FA:11-6 & 0,89626 & 0,001001 & 4,158 & 0,807688 & 35,25996 & 5,582484 \\
\hline FA:6-12 & 0,60256 & $4,98 \mathrm{E}-05$ & 3,614 & 0,453275 & 35,5078 & 5,596813 \\
\hline FA:6-13 & 0,45502 & $4,11 \mathrm{E}-05$ & 3,44 & 0,327833 & 35,76873 & 5,825239 \\
\hline FA:13-6 & 0,45007 & $6,21 \mathrm{E}-05$ & 3,41 & 0,324582 & 35,5283 & 5,721285 \\
\hline FA:7-9 & 0,42003 & 0,000312 & 3,366 & 0,319159 & 35,63396 & 5,723726 \\
\hline FA:9-10 & 0,97931 & $5,6 \mathrm{E}-05$ & 4,356 & 0,822698 & 35,23625 & 5,75315 \\
\hline FA:10-9 & 0,97358 & $6,22 \mathrm{E}-05$ & 4,274 & 0,871388 & 35,09433 & 5,667856 \\
\hline & & & & & \\
\hline
\end{tabular}




\begin{tabular}{|c|c|c|c|c|c|c|}
\hline FA:9-14 & 0,75794 & 0,000129 & 3,858 & 68596 & 35,29677 & 5,508774 \\
\hline FA:10-11 & 21913 & 0,018059 & 12,338 & 3,898893 & 34,65186 & 5,487702 \\
\hline FA:12-13 & 23981 & 00667 & 9,88 & 885841 & 34,94919 & 5,712689 \\
\hline FA:14-13 & 90681 & 0,000318 & 4,152 & 0,730363 & 35,26467 & 5,48415 \\
\hline IR:1 & 1,40695 & 0,004523 & 5,364 & 1,230383 & 34,98507 & 5,616519 \\
\hline IR:2 & 0,66584 & 0,009381 & 3,678 & 0,535522 & 35,7214 & 5,723578 \\
\hline IR:5 & 44341 & 0,008223 & 25,52002 & 7,551442 & 34,19017 & 5,660064 \\
\hline IR:8 & 42926 &, 000403 & 3,438 & 0,344298 & 35,5603 & 5,655895 \\
\hline IR:9 & 4166 & & 3,604 & 954 & 35,6 & 209 \\
\hline IR:13 & 69929 & -05 & 3,762 & 0,60616 & 35 , & \\
\hline IR:14 & 67371 & ,34E-05 & 694 & 374 & & \\
\hline FR:1-2 & 0,579 & -05 & 3,536 & 0,49 & & 903 \\
\hline FR:2-3 & 2,19678 & 0,011499 & 7,06 & 1,718703 & 34,61756 & 5,590728 \\
\hline FR:4-2 & 8585 & 834 & 4,844 & 1,1 & 35,1 & 5,813265 \\
\hline FR & 328 & 48 & 4,63 & 06 & 35,025 & 644 \\
\hline FI & 79 & 76 & 6,862 & 23 & & \\
\hline FI & & & 3,292 & & 35 , & \\
\hline FI & 57 & & 45 & & & 027 \\
\hline & 415 & & 5,672 & & & \\
\hline & & & 3,438 & & & 313 \\
\hline FF & 9069 & 59 & 4,064 & 0,6 & 35 , & 945 \\
\hline FF & 1564 & 68 & 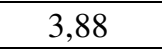 & 39 & 877 & 043 \\
\hline & 503 & & 3,968 & 38 & 36 & \\
\hline FR & 55 & 5 & 2 & 06 & 734 & 448 \\
\hline FR:6-13 & 63 & 05 & 26 & 73 & 778 & 5,975721 \\
\hline FR:13-6 & 473 & & 18 & & & 166 \\
\hline FR:7-8 & 41616 & 52 & 3,436 & & 35 , & 5,633575 \\
\hline FR:8-7 & ,42926 & 000404 & 3,44 & 0,345826 & 931 & 5,63374 \\
\hline FR:7-9 & 20443 & 237 & 4,678 & 862 & 738 & 5,757046 \\
\hline FR:9-10 & 072 & 05 & 4,076 & 0,6 & 713 & 5,793539 \\
\hline FR: & 0,91969 & & 4,138 & & 53 & 5,7 \\
\hline FR:9-14 & & & 3,77 & & & 013 \\
\hline FR:10-11 & 3,88378 & 0,015272 & 11,25 & 3,434392 & 34,82842 & 5,657283 \\
\hline FR:12-13 & 3,33038 & 0,000183 & 10,266 & 2,831005 & 34,7455 & 5,794401 \\
\hline FR:14-13 & 1,25183 & 0,000908 & 4,888 & 1,187586 & 35,16687 & 5,681397 \\
\hline $\mathrm{V} \cdot 1$ & 14758 & 0,006725 & 3,154 & 0,129817 & 36,3807 & 5,832291 \\
\hline V: & & 0,006809 & 3,154 & & 36,37857 & 5,809271 \\
\hline$V: 3$ & & 075 & 3,166 & 0,124089 & 36,42893 & 5,820515 \\
\hline$V: 6$ & 0,15427 & 0,007147 & 3,162 & 0,126155 & 36,39923 & 5,801759 \\
\hline$V: 8$ & 0,14776 & 0,006822 & 3,154 & 0,120118 & 36,36186 & 5,782948 \\
\hline$V: 12$ & 0,15855 & 0,007349 & 3,16 & 0,127128 & 36,37069 & 5,80966 \\
\hline
\end{tabular}

Através dos resultados médios apresentados na tabela 5.6, construi-se o gráfico índice $U I$ x nível de detecção mostrado a seguir (figura 5.1): 


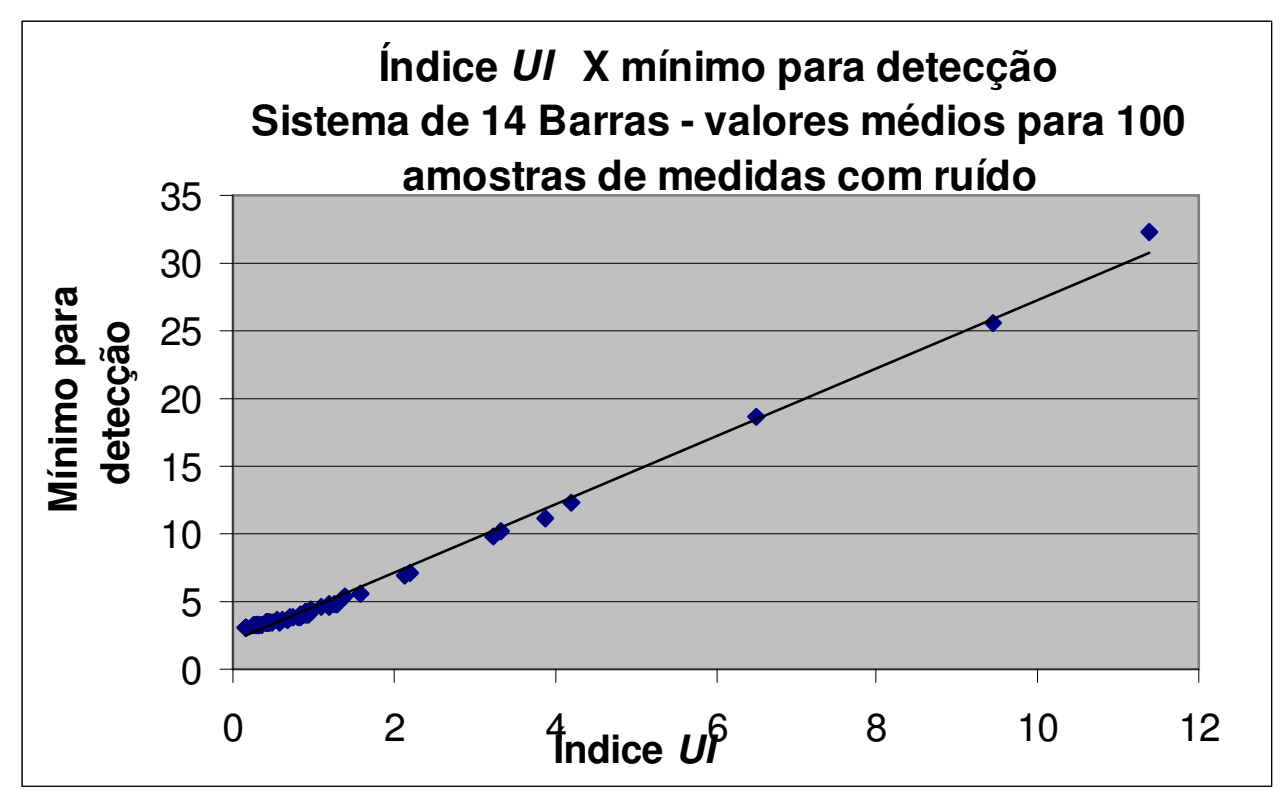

Figura 5.1: Gráfico de tendência para índice UI X Mínimo para detecção de EGs em medidas - valores médios para 100 amostras de medidas com ruído

Analisando-se o comportamento da reta de tendência acima, para os valores médios, novamente se constata que, quanto maior o índice UI de uma medida, mais difícil será a detecção de EG, através do teste dos resíduos normalizados e do índice $J(x)$. Ademais, verifica-se também o mesmo comportamento linear observado na figura 4.9 do capítulo anterior, na qual as medidas não continham ruído algum.

Com base nos valores dos desvios padrões, do nível de detecção das medidas (tabela 5.6), importa mencionar que as medidas com maiores valores foram aquelas com valores altos de índice $U I$. Por exemplo: a medida IA:2, cujo valor médio de índice $U I$ é igual a 6,49047, apresenta o valor do desvio padrão do nível de detecção igual a 5,509694. Em contrapartida, a medida IA:1, que tem índice $U I$ abaixo de 1, apresenta o valor do desvio padrão do nível de detecção igual a 0,223571. Tendo em vista que as medidas com índice $U I$ elevado apresentam os maiores valores de desvio padrão de $U I$, as mesmas apresentaram, também, os maiores valores dos desvios padrões do nível de detecção das medidas.

\subsubsection{Testes com Ruído Gaussiano e Erro Grosseiro Simples}


Apresentaremos, nesta subseção, os resultados de simulações considerando EG simples de 10 sigmas, em cada uma das medidas do sistema de medição da tabela 4.1 (um erro de cada vez), considerando 100 amostras de ruído em medidas.

A tabela 5.7, a seguir, apresenta os valores médios do índice UI, resíduo normalizado e índice $J(x)$, para a medida portadora de EG em cada caso, considerando EG simples de 10 sigmas.

Tabela 5.7: Resultados do índice $U I$, resíduo normalizado e índice J(x) para 100 amostras de medidas com ruído e com EG de 10 sigmas em cada medida

\begin{tabular}{|c|c|c|c|c|c|c|}
\hline \multirow{2}{*}{$\begin{array}{c}\text { Medida } \\
\text { com erro }\end{array}$} & \multicolumn{2}{|r|}{$\boldsymbol{U I}$} & \multicolumn{2}{|c|}{$r^{N}$} & \multicolumn{2}{|c|}{$J(\mathbf{x})$} \\
\hline & Média & $\begin{array}{l}\text { Desvio } \\
\text { Padrão }\end{array}$ & Média & $\begin{array}{l}\text { Desvio } \\
\text { Padrão }\end{array}$ & Média & $\begin{array}{l}\text { Desvio } \\
\text { Padrão }\end{array}$ \\
\hline IA:1 & 0,280784 & 0,001383651 & 9,62624 & 0,219207741 & 119,5021 & 7,420261 \\
\hline IA:2 & 6,502372 & 0,011111984 & 1,547095 & 0,837240399 & 29,93736 & 6,367706 \\
\hline IA: 5 & 11,41429 & 0,075814344 & 0,78325 & 0,90547209 & 28,14095 & 5,956948 \\
\hline IA:9 & 0,754616 & 0,000585122 & 7,91643 & 0,555270492 & 89,73033 & 10,95162 \\
\hline IA:13 & 0,833265 & $8,08728 \mathrm{E}-05$ & 7,67881 & 0,595927357 & 86,05066 & 11,21438 \\
\hline IA:14 & 0,663946 & 0,000107703 & 8,29677 & 0,475635264 & 95,82729 & 9,952838 \\
\hline FA:1-2 & 0,288435 & 0,001241323 & 9,602997 & 0,217370318 & 119,0577 & 7,492973 \\
\hline FA:2-3 & 0,243795 & 0,002798209 & 9,739348 & 0,183803907 & 121,6408 & 6,912545 \\
\hline FA:4-2 & 0,320077 & 0,001077301 & 9,4795 & 0,252544186 & 116,773 & 7,694272 \\
\hline FA:5-2 & 0,290923 & 0,000901722 & 9,56209 & 0,228264739 & 118,1595 & 7,536777 \\
\hline FA:3-4 & 1,270056 & 0,015247529 & 6,25416 & 0,613571897 & 66,18344 & 9,044084 \\
\hline FA:4-5 & 0,485936 & 0,002038742 & 8,96962 & 0,409815148 & 107,2561 & 9,316464 \\
\hline FA:7-4 & 0,419628 & 0,000311101 & 9,18638 & 0,302966523 & 111,2816 & 8,687039 \\
\hline FA:9-4 & 0,41982 & 0,000308139 & 9,17053 & 0,310717812 & 111,1746 & 8,678582 \\
\hline FA:5-6 & 0,33251 & 0,000512175 & 9,441225 & 0,258603421 & 115,9433 & 8,085702 \\
\hline FA:6-5 & 0,33246 & 0,000604027 & 9,44126 & 0,260887816 & 115,8969 & 7,985264 \\
\hline FA:6-11 & 0,894625 & 0,001053937 & 7,405714 & 0,601611915 & 81,91752 & 10,42625 \\
\hline FA:11-6 & 0,895858 & 0,000914218 & 7,4854 & 0,63285568 & 83,14871 & 10,81901 \\
\hline FA:6-12 & 0,602698 & $4,91955 \mathrm{E}-05$ & 8,622188 & 0,406073081 & 101,3642 & 9,26405 \\
\hline FA:6-13 & 0,455146 & $5,20683 \mathrm{E}-05$ & 9,149617 & 0,345660275 & 110,6614 & 9,200146 \\
\hline FA:13-6 & 0,449871 & 6,40312E-05 & 9,15166 & 0,377195604 & 110,6298 & 9,423868 \\
\hline FA:7-9 & 0,419531 & 0,000313564 & 9,191208 & 0,335729298 & 111,3118 & 8,634031 \\
\hline FA:9-10 & 0,979534 & $5,54504 \mathrm{E}-05$ & 7,062398 & 0,58958665 & 76,93182 & 9,356415 \\
\hline FA:10-9 & 0,973312 & 5,73488E-05 & 7,14679 & 0,630166068 & 78,20053 & 10,51467 \\
\hline FA:9-14 & 0,758395 & 0,000117529 & 8,051762 & 0,530817651 & 91,91522 & 10,64353 \\
\hline FA:10-11 & 4,222957 & 0,017199532 & 2,23393 & 0,81350002 & 32,50829 & 6,924346 \\
\hline FA:12-13 & 3,245468 & 0,000510868 & 2,965206 & 0,870367275 & 36,31672 & 7,737759 \\
\hline FA:14-13 & 0,906091 & 0,000332148 & 7,4526 & 0,582204687 & 82,68567 & 10,61499 \\
\hline IR:1 & 1,403991 & 0,003613585 & 5,69878 & 0,683616525 & 59,68319 & 10,04716 \\
\hline IR:2 & 0,67472 & 0,009225273 & 8,339726 & 0,438351473 & 96,45831 & 9,784634 \\
\hline IR:5 & 9,442719 & 0,006676473 & 1,17766 & 0,886788784 & 28,95762 & 6,74938 \\
\hline IR:8 & 0,429783 & 0,000456392 & 9,194189 & 0,322796664 & 111,3628 & 7,950061 \\
\hline IR:9 & 0,54284 & 0,013909084 & 8,75975 & 0,422109544 & 103,8402 & 9,664761 \\
\hline IR:13 & 0,699034 & $9,66301 \mathrm{E}-05$ & 8,17296 & 0,52806694 & 93,68598 & 9,625785 \\
\hline IR:14 & 0,673395 & 7,15979E-05 & 8,33053 & 0,477134991 & 96,41488 & 9,514209 \\
\hline FR:1-2 & 0,578885 & 3,85992E-05 & 8,67413 & 0,440457624 & 102,122 & 9,448833 \\
\hline
\end{tabular}




\begin{tabular}{|c|c|c|c|c|c|c|}
\hline FR:2-3 & 2,219078 & 0,011903599 & 4,140007 & 0,762243033 & 44,54585 & 9,470776 \\
\hline FR:4-2 & 1,191498 & 0,00302755 & 6,392469 & 0,699105605 & 68,03552 & 10,76901 \\
\hline FR:5-2 & 1,113663 & 0,001745392 & 6,67612 & 0,601581427 & 71,77666 & 9,452744 \\
\hline FR:3-4 & 2,150395 & 0,019493218 & 4,293541 & 0,783674097 & 45,92409 & 9,452085 \\
\hline FR:4-5 & 0,36112 & 0,000681798 & 9,379796 & 0,251936193 & 114,6812 & 7,363221 \\
\hline FR:7-4 & 0,419915 & 0,005863539 & 9,207545 & 0,31633025 & 111,6632 & 8,237627 \\
\hline FR:9-4 & 1,598881 & 0,021402859 & 5,293961 & 0,695937054 & 55,46936 & 9,862514 \\
\hline FR:5-6 & 0,452809 & 0,010324142 & 9,108191 & 0,328219372 & 109,5653 & 8,681145 \\
\hline FR:6-5 & 0,906129 & 0,04685379 & 7,60263 & 0,49053119 & 84,23707 & 9,052195 \\
\hline FR:6-11 & 0,816476 & 0,000920707 & 7,777996 & 0,507523211 & 87,57719 & 9,403422 \\
\hline FR:11-6 & 0,885402 & 0,001112553 & 7,46719 & 0,558477338 & 82,87729 & 10,08493 \\
\hline FR:6-12 & 0,830962 & $9,40449 \mathrm{E}-05$ & 7,682239 & 0,525432277 & 86,16161 & 10,11485 \\
\hline FR:6-13 & 0,430749 & $5,02418 \mathrm{E}-05$ & 9,143908 & 0,337320814 & 110,5565 & 8,159426 \\
\hline FR:13-6 & 0,454624 & 3493 & 75 & 0,347851197 & 110,1277 & 8,51003 \\
\hline FR:7-8 & 0,416127 & 0,000550822 & 9,27548 & 0,351203706 & 112,852 & 8,768323 \\
\hline FR:8-7 & 0,429687 & 0,000396616 & 9,220194 & 0,314118703 & 111,7851 & 8,195923 \\
\hline FR:7-9 & 1,207174 & 0,011554698 & 6,530804 & 0,654264003 & 69,78789 & 9,728976 \\
\hline FR:9-10 & 0,931143 & $5,73048 \mathrm{E}-05$ & 7,380846 & 0,59063542 & 81,77894 & 10,91723 \\
\hline FR:10-9 & 0,919224 & $5,3409 \mathrm{E}-05$ & 7,28446 & 0,59336105 & 80,30953 & 10,93884 \\
\hline FR:9-14 & 0,725908 & 0,000236848 & 8,097658 & 0,504509015 & 92,67623 & 9,517984 \\
\hline FR:10-11 & 3,886074 & 0,014093975 & 2,47711 & 0,755226493 & 33,69655 & 7,155348 \\
\hline FR:12-13 & 3,331867 & 0,000160211 & 2,889901 & 0,859107166 & 35,87475 & 8,110644 \\
\hline FR:14-13 & 1,251538 & 0,000893272 & 6,16202 & 1,414707671 & 66,94085 & 8,078498 \\
\hline V:1 & 0,14977 & 0,006619584 & 9,882532 & 0,126395694 & 125,1877 & 6,449668 \\
\hline $\mathrm{V}: 2$ & 0,150956 & 0,007763555 & 9,881736 & 0,127900278 & 125,1615 & 6,447127 \\
\hline V:3 & 0,154825 & 0,007203245 & 9,869507 & 0,133768603 & 124,9722 & 6,464539 \\
\hline V:6 & 0,155275 & 0,006607012 & 9,870066 & 0,134347083 & 124,9803 & 6,484528 \\
\hline $\mathrm{V}: 8$ & 0,150346 & 0,008001432 & 9,884296 & 0,127568223 & 125,2158 & 6,437344 \\
\hline$V: 12$ & 0,158484 & 0,006744691 & 9,86125 & 0,136142472 & 124,8371 & 6,502356 \\
\hline
\end{tabular}

Nos resultados da tabela 5.7, os casos mostrados em vermelho distinguem as medidas em que o valor médio do respectivo resíduo normalizado foi menor que 3 (limiar de detecção para o teste dos resíduos normalizados), ou seja, são os casos em que houve problemas para detecção/identificação de EGs. Tais casos correspondem às medidas com índice $U I$ elevado.

Por outro lado, os casos em realce amarelo distinguem as medidas em que o valor médio do respectivo resíduo normalizado foi maior que 3 , mas o valor médio do índice $\mathrm{J}(x)$ foi inferior a 58 (limiar de detecção para o teste do índice $\mathrm{J}(x)$ ). Como pode ser observado na tabela 5.17, esses casos correspondem às medidas cujo índice $U I$ está entre 1,598881 e 2,219078 .

\subsubsection{Análise Comparativa do Índice UI calculado adicionando ou não ruído e/ou erro grosseiro}


A tabela 5.8, abaixo, abrange os valores do índice $U I$, calculados nos cenários anteriores, ou seja, com medidas sem ruído e sem EG; sem ruído mas com EG de 10 sigmas; com ruído e sem EG; e com ruído e EG.

Tabela 5.8: Resultados do índice UI com alterações nos valores das medidas - sistema 14 barras

\begin{tabular}{|c|c|c|c|c|c|c|}
\hline \multirow{2}{*}{ Medida } & \multirow{2}{*}{$\begin{array}{c}\begin{array}{c}U I \text { sem } \\
\text { ruído e } \\
\text { sem erro }\end{array} \\
\text { valor }\end{array}$} & \multirow{2}{*}{$\begin{array}{c}\begin{array}{c}\text { UI sem } \\
\text { ruído e } \\
\text { com erro } \\
\text { de } 10 \sigma\end{array} \\
\text { valor }\end{array}$} & \multicolumn{2}{|c|}{$\begin{array}{c}\text { UI com ruído e sem } \\
\text { erro }\end{array}$} & \multicolumn{2}{|c|}{$\begin{array}{l}\text { UI com ruído e erro de } \\
\qquad 10 \sigma\end{array}$} \\
\hline & & & média & $\begin{array}{c}\text { desvio } \\
\text { padrão }\end{array}$ & média & $\begin{array}{c}\text { desvio } \\
\text { padrão }\end{array}$ \\
\hline IA:1 & 0,2827 & 0,281 & 0,28087 & 0,001246 & 0,280784 & 0,001383651 \\
\hline IA:2 & 6,4873 & 6,491 & 6,48453 & 0,012335 & 6,502372 & 0,011111984 \\
\hline IA:5 & 11,5168 & 11,434 & 11,4035 & 0,06951 & 11,41429 & 0,075814344 \\
\hline IA:9 & 0,7558 & 0,755 & 0,75553 & 0,00055 & 0,754616 & 0,000585122 \\
\hline IA:13 & 0,8338 & 0,834 & 0,83382 & $9,03 \mathrm{E}-05$ & 0,833265 & $8,08728 \mathrm{E}-05$ \\
\hline IA:14 & 0,6645 & 0,665 & 0,66455 & 0,000123 & 0,663946 & 0,000107703 \\
\hline FA:1-2 & 0,2905 & 0,289 & 0,28858 & 0,001301 & 0,288435 & 0,001241323 \\
\hline FA:2-3 & 0,2477 & 0,244 & 0,2438 & 0,002374 & 0,243795 & 0,002798209 \\
\hline FA:4-2 & 0,322 & 0,321 & 0,3207 & 0,001111 & 0,320077 & 0,001077301 \\
\hline FA:5-2 & 0,2922 & 0,292 & 0,29129 & 0,000919 & 0,290923 & 0,000901722 \\
\hline FA:3-4 & 1,3022 & 1,283 & 1,27999 & 0,013796 & 1,270056 & 0,015247529 \\
\hline FA:4-5 & 0,4903 & 0,489 & 0,48768 & 0,001864 & 0,485936 & 0,002038742 \\
\hline FA:7-4 & 0,42 & 0,419 & 0,41993 & 0,000297 & 0,419628 & 0,000311101 \\
\hline FA:9-4 & 0,4202 & 0,420 & 0,42007 & 0,000294 & 0,41982 & 0,000308139 \\
\hline FA:5-6 & 0,3331 & 0,333 & 0,33288 & 0,000491 & 0,33251 & 0,000512175 \\
\hline FA:6-5 & 0,3331 & 0,333 & 0,33288 & 0,000491 & 0,33246 & 0,000604027 \\
\hline FA:6-11 & 0,8952 & 0,894 & 0,89363 & 0,000926 & 0,894625 & 0,001053937 \\
\hline FA:11-6 & 0,8981 & 0,897 & 0,89643 & 0,000961 & 0,895858 & 0,000914218 \\
\hline FA:6-12 & 0,6026 & 0,602 & 0,60257 & $5,41 \mathrm{E}-05$ & 0,602698 & 4,91955E-05 \\
\hline FA:6-13 & 0,4551 & 0,455 & 0,45502 & $4,74 \mathrm{E}-05$ & 0,455146 & $5,20683 \mathrm{E}-05$ \\
\hline FA:13-6 & 0,4501 & 0,450 & 0,45007 & $6,99 \mathrm{E}-05$ & 0,449871 & $6,40312 \mathrm{E}-05$ \\
\hline FA:7-9 & 0,42 & 0,419 & 0,41991 & 0,000289 & 0,419531 & 0,000313564 \\
\hline FA:9-10 & 0,9794 & 0,979 & 0,97932 & $5,2 \mathrm{E}-05$ & 0,979534 & $5,54504 \mathrm{E}-05$ \\
\hline FA:10-9 & 0,9736 & 0,974 & 0,97356 & $5,78 \mathrm{E}-05$ & 0,973312 & $5,73488 \mathrm{E}-05$ \\
\hline FA:9-14 & 0,7581 & 0,758 & 0,75795 & 0,000118 & 0,758395 & 0,000117529 \\
\hline FA:10-11 & 4,2529 & 4,227 & 4,2217 & 0,017853 & 4,222957 & 0,017199532 \\
\hline FA:12-13 & 3,2398 & 3,240 & 3,23981 & 0,000622 & 3,245468 & 0,000510868 \\
\hline FA:14-13 & 0,9073 & 0,907 & 0,90682 & 0,000303 & 0,906091 & 0,000332148 \\
\hline IR:1 & 1,4127 & 1,406 & 1,40723 & 0,003655 & 1,403991 & 0,003613585 \\
\hline IR:2 & 0,6792 & 0,665 & 0,66627 & 0,007925 & 0,67472 & 0,009225273 \\
\hline IR:5 & 9,4466 & 9,437 & 9,44382 & 0,006522 & 9,442719 & 0,006676473 \\
\hline IR:8 & 0,43 & 0,429 & 0,42936 & 0,000414 & 0,429783 & 0,000456392 \\
\hline IR:9 & 0,5676 & 0,547 & 0,54359 & 0,013889 & 0,54284 & 0,013909084 \\
\hline IR:13 & 0,6994 & 0,699 & 0,69929 & $9,07 \mathrm{E}-05$ & 0,699034 & $9,66301 \mathrm{E}-05$ \\
\hline IR:14 & 0,6737 & 0,674 & 0,67371 & $7,18 \mathrm{E}-05$ & 0,673395 & 7,15979E-05 \\
\hline FR:1-2 & 0,579 & 0,579 & 0,57899 & $4,32 \mathrm{E}-05$ & 0,578885 & $3,85992 \mathrm{E}-05$ \\
\hline FR:2-3 & 2,2143 & 2,197 & 2,197 & 0,010626 & 2,219078 & 0,011903599 \\
\hline FR:4-2 & 1,186 & 1,183 & 1,18569 & 0,003085 & 1,191498 & 0,00302755 \\
\hline FR:5-2 & 1,1161 & 1,114 & 1,11365 & 0,001441 & 1,113663 & 0,001745392 \\
\hline
\end{tabular}




\begin{tabular}{c|c|c|c|c|c|c}
\hline FR:3-4 & 2,156 & 2,125 & 2,12514 & 0,018503 & 2,150395 & 0,019493218 \\
\hline FR:4-5 & 0,3618 & 0,360 & 0,36073 & 0,000705 & 0,36112 & 0,000681798 \\
\hline FR:7-4 & 0,4293 & 0,421 & 0,41954 & 0,005868 & 0,419915 & 0,005863539 \\
\hline FR:9-4 & 1,6329 & 1,603 & 1,59742 & 0,021614 & 1,598881 & 0,021402859 \\
\hline FR:5-6 & 0,4673 & 0,454 & 0,45063 & 0,009647 & 0,452809 & 0,010324142 \\
\hline FR:6-5 & 0,9743 & 0,905 & 0,89692 & 0,043379 & 0,906129 & 0,04685379 \\
\hline FR:6-11 & 0,8173 & 0,816 & 0,81581 & 0,000852 & 0,816476 & 0,000920707 \\
\hline FR:11-6 & 0,8882 & 0,886 & 0,88617 & 0,00115 & 0,885402 & 0,001112553 \\
\hline FR:6-12 & 0,8307 & 0,830 & 0,83059 & $9,98 \mathrm{E}-05$ & 0,830962 & $9,40449 \mathrm{E}-05$ \\
\hline FR:6-13 & 0,4307 & 0,431 & 0,43064 & $4,96 \mathrm{E}-05$ & 0,430749 & $5,02418 \mathrm{E}-05$ \\
\hline FR:13-6 & 0,455 & 0,455 & 0,45475 & 0,000126 & 0,454624 & 0,00013493 \\
\hline FR:7-8 & 0,417 & 0,416 & 0,41625 & 0,000507 & 0,416127 & 0,000550822 \\
\hline FR:8-7 & 0,43 & 0,429 & 0,42936 & 0,000414 & 0,429687 & 0,000396616 \\
\hline FR:7-9 & 1,2248 & 1,208 & 1,20587 & 0,010935 & 1,207174 & 0,011554698 \\
\hline FR:9-10 & 0,9307 & 0,931 & 0,93073 & $6,67 \mathrm{E}-05$ & 0,931143 & $5,73048 \mathrm{E}-05$ \\
\hline FR:10-9 & 0,9197 & 0,919 & 0,91967 & $7,39 \mathrm{E}-05$ & 0,919224 & $5,3409 \mathrm{E}-05$ \\
\hline FR:9-14 & 0,7257 & 0,725 & 0,72536 & 0,000253 & 0,725908 & 0,000236848 \\
\hline FR:10-11 & 3,912 & 3,890 & 3,8859 & 0,014845 & 3,886074 & 0,014093975 \\
\hline FR:12-13 & 3,3303 & 3,330 & 3,33032 & 0,000225 & 3,331867 & 0,000160211 \\
\hline FR:14-13 & 1,2534 & 1,252 & 1,25193 & 0,000878 & 1,251538 & 0,000893272 \\
\hline V:1 & 0,1599 & 0,150 & 0,14872 & 0,006644 & 0,14977 & 0,006619584 \\
\hline V:2 & 0,1608 & 0,151 & 0,1494 & 0,006718 & 0,150956 & 0,007763555 \\
\hline V:3 & 0,1669 & 0,156 & 0,1551 & 0,006983 & 0,154825 & 0,007203245 \\
\hline V:6 & 0,1673 & 0,157 & 0,15543 & 0,007029 & 0,155275 & 0,006607012 \\
\hline V:8 & 0,1602 & 0,150 & 0,14887 & 0,0067 & 0,150346 & 0,008001432 \\
\hline V:12 & 0,172 & 0,161 & 0,15976 & 0,007225 & 0,158484 & 0,006744691 \\
\hline & & & & & &
\end{tabular}

A partir dos resultados apresentados acima, verificamos que o índice $U I$ variou pouco, considerando as alterações nos valores das medidas em análise. Conforme será apresentado no capítulo 7 , essa informação é importante para aplicação do índice UI para projeto e fortalecimento de sistemas de medição.

A próxima seção apresentará os resultados de simulações realizadas no sistema de 30 barras do IEEE, com o intuito de ratificar os resultados e algumas conclusões, apresentadas com as simulações no sistema de 14 barras.

\subsection{Simulações no Sistema de 30 Barras IEEE}

Considere o sistema de 30 barras do IEEE, ilustrado através da figura 5.2, a seguir. 


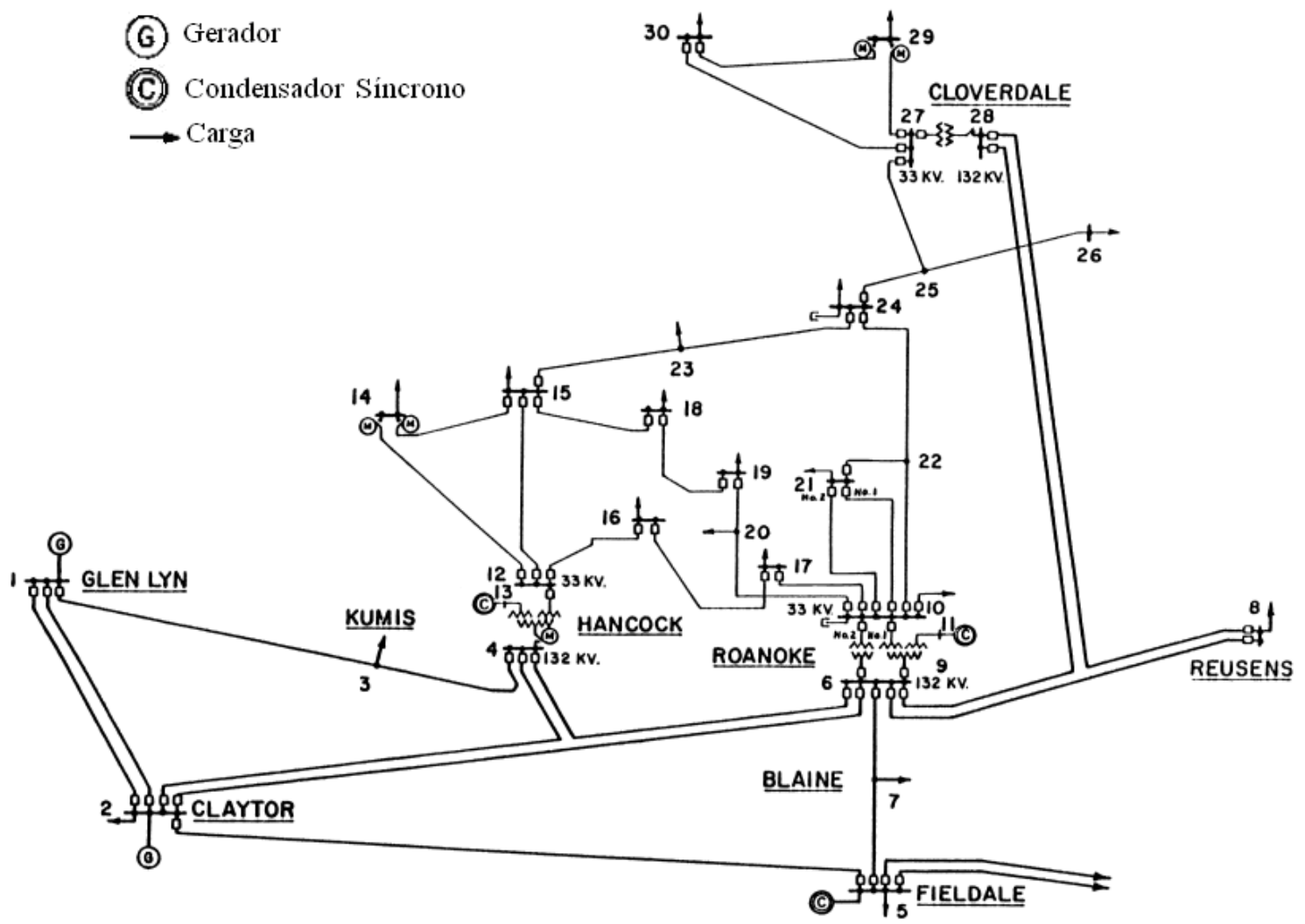

Figura 5.2: Sistema de 30 barras IEEE

A partir dos resultados de um programa de fluxo de carga (ANAREDE), construímos um sistema de medição com 122 medidas, sem a presença de medidas críticas ou conjuntos críticos. Os valores dos parâmetros, bem como outras informações pertinentes à execução do fluxo de carga, para o sistema de 30 barras do IEEE, estão disponíveis no endereço eletrônico "www.ee.washington.edu/research/pstca/", e por isso não são mostrados aqui.

Os valores das 122 medidas, bem como os respectivos desvios padrão, são apresentados na tabela 5.10. Inicialmente empregou-se, para o cálculo dos desvios padrão nas simulações com o sistema de 30 barras, a equação (4.31) utilizada nas simulações com o sistema de 14 barras. Porém, em simulações prévias verificou-se que tal procedimento ocasionava problemas de convergência do estimador, tendo em vista o mau condicionamento numérico das matrizes Ganho obtidas a partir desse procedimento.

Face ao exposto, utilizou-se, para o cálculo dos desvios padrão nas simulações com o sistema de 30 barras, a seguinte equação ${ }^{7}$ :

\footnotetext{
7 Deve-se destacar que a equação (5.1), assim como a equação (4.31), já foi empregada em outras pesquisas da área, conforme apresentado em Castillo-Albertini (2010).
} 


$$
3 \cdot \sigma_{i}=\operatorname{Pr} \cdot F E
$$

onde:

- Pr é a precisão do medidor ( $\operatorname{Pr}=0,02$ para este sistema);

- FE é o fundo de escala do medidor, sendo que os valores de $F E$ dependem da faixa de valores (em p.u.) das grandezas aferidas.

Os valores de $F E$ utilizados neste trabalho são apresentados na tabela 5.9, a seguir.

Tabela 5.9: Valores de $F E$ para o sistema de 30 barras IEEE

\begin{tabular}{c|c}
\hline Faixa de Valores & $\boldsymbol{F E}$ \\
\hline $2,00 \leq\left|z^{m}\right|$ & 2,80 \\
\hline $1,00 \leq\left|z^{m}\right|<2,00$ & 2,00 \\
\hline $0,65 \leq\left|z^{m}\right|<1,00$ & 1,00 \\
\hline $0,35 \leq\left|z^{m}\right|<0,65$ & 0,65 \\
\hline $0,20 \leq\left|z^{m}\right|<0,35$ & 0,35 \\
\hline $0,10 \leq\left|z^{m}\right|<0,20$ & 0,20 \\
\hline $0,05 \leq\left|z^{m}\right|<0,10$ & 0,10 \\
\hline $0,00 \leq\left|z^{m}\right|<0,05$ & 0,05 \\
\hline
\end{tabular}

Tabela 5.10: Valores das medidas sem ruído e respectivos desvios padrões para o sistema de 30 barras

\begin{tabular}{c|c|c|c|c|c}
\hline \multicolumn{2}{c|}{ Medidas de Potência Ativa } & \multicolumn{2}{c}{ Medidas de Potência Reativa } \\
\hline Medida & $\begin{array}{c}\text { Valor } \\
(\mathbf{p u})\end{array}$ & $\boldsymbol{\sigma}(\mathbf{p u})$ & Medida & $\begin{array}{c}\text { Valor } \\
(\mathbf{p u})\end{array}$ & $\boldsymbol{\sigma}(\mathbf{p u})$ \\
\hline IA:1 & 2,61 & 0,018667 & IR:1 & $-0,165$ & 0,001333 \\
\hline IA:2 & 0,183 & 0,001333 & IR:2 & 0,369 & 0,004333 \\
\hline IA:5 & $-0,942$ & 0,006667 & IR:5 & 0,179 & 0,001333 \\
\hline IA:6 & NULO & --- & IR:6 & NULO & --- \\
\hline IA:7 & $-0,228$ & 0,002333 & IR:7 & $-0,109$ & 0,001333 \\
\hline IA:8 & $-0,3$ & 0,002333 & IR:8 & 0,072 & 0,000667 \\
\hline IA:9 & NULO & --- & IR:9 & NULO & --- \\
\hline IA:11 & NULO & --- & IR:11 & 0,162 & 0,001333 \\
\hline IA:12 & $-0,112$ & 0,001333 & IR:12 & $-0,075$ & 0,000667 \\
\hline IA:13 & NULO & --- & IR:13 & 0,106 & 0,001333 \\
\hline IA:15 & $-0,082$ & 0,000667 & IR:15 & $-0,025$ & 0,000333 \\
\hline IA:16 & $-0,035$ & 0,000333 & IR:16 & $-0,018$ & 0,000333 \\
\hline IA:17 & $-0,09$ & 0,000667 & IR:17 & $-0,058$ & 0,000667 \\
\hline IA:19 & $-0,095$ & 0,000667 & IR:19 & $-0,034$ & 0,000333 \\
\hline IA:21 & $-0,175$ & 0,001333 & IR:21 & $-0,112$ & 0,001333 \\
\hline IA:22 & NULO & --- & IR:22 & NULO & --- \\
\hline IA:23 & $-0,032$ & 0,000333 & IR:23 & $-0,016$ & 0,000333 \\
\hline IA:24 & $-0,087$ & 0,000667 & IR:24 & $-0,067$ & 0,000667 \\
\hline & & & & & \\
\hline
\end{tabular}




\begin{tabular}{|c|c|c|c|c|c|}
\hline IA:25 & NULO & --- & IR:25 & NULO & --- \\
\hline IA:27 & NULO & --- & IR:27 & NULO & --- \\
\hline IA:28 & NULO & --- & IR:28 & NULO & --- \\
\hline IA:30 & $-0,106$ & 0,001333 & IR:30 & $-0,019$ & 0,000333 \\
\hline FA:1-2 & 1,732 & 0,013333 & FR:1-2 & $-0,211$ & 0,002333 \\
\hline FA:1-3 & 0,877 & 0,006667 & FR:1-3 & 0,046 & 0,000333 \\
\hline FA:2-4 & 0,436 & 0,004333 & FR:2-4 & 0,039 & 0,000333 \\
\hline FA:4-2 & $-0,426$ & 0,004333 & FR:4-2 & $-0,047$ & 0,000333 \\
\hline FA:2-5 & 0,824 & 0,006667 & FR:2-5 & 0,018 & 0,000333 \\
\hline FA:2-6 & 0,603 & 0,004333 & FR:2-6 & 0,004 & 0,000333 \\
\hline FA:3-4 & 0,822 & 0,006667 & FR:3-4 & $-0,036$ & 0,000333 \\
\hline FA:4-6 & 0,721 & 0,006667 & FR:4-6 & $-0,163$ & 0,001333 \\
\hline FA:4-12 & 0,442 & 0,004333 & FR:4-12 & 0,142 & 0,001333 \\
\hline FA:5-7 & $-0,148$ & 0,001333 & FR:5-7 & 0,117 & 0,001333 \\
\hline FA:6-7 & 0,381 & 0,004333 & FR:6-7 & $-0,03$ & 0,000333 \\
\hline FA:6-8 & 0,296 & 0,002333 & FR:6-8 & $-0,081$ & 0,000667 \\
\hline FA:6-9 & 0,277 & 0,002333 & FR:6-9 & $-0,082$ & 0,000667 \\
\hline FA:6-28 & 0,187 & 0,001333 & FR:6-28 & NULO & --- \\
\hline FA:8-28 & $-0,005$ & 0,000333 & FR:8-28 & $-0,004$ & 0,000333 \\
\hline FA:9-11 & NULO & --- & FR:9-11 & $-0,157$ & 0,001333 \\
\hline FA:9-10 & 0,277 & 0,002333 & FR:9-10 & 0,059 & 0,000667 \\
\hline FA:12-13 & NULO & --- & FR:12-13 & $-0,105$ & 0,001333 \\
\hline FA:12-15 & 0,179 & 0,001333 & FR:12-15 & 0,068 & 0,000667 \\
\hline FA:12-16 & 0,072 & 0,000667 & FR:12-16 & 0,034 & 0,000333 \\
\hline FA:15-18 & 0,06 & 0,000667 & FR:15-18 & 0,016 & 0,000333 \\
\hline FA:18-15 & $-0,06$ & 0,000667 & FR:18-15 & $-0,015$ & 0,000333 \\
\hline FA:15-23 & 0,05 & 0,000667 & FR:15-23 & 0,029 & 0,000333 \\
\hline FA:18-19 & 0,028 & 0,000333 & FR:18-19 & 0,006 & 0,000333 \\
\hline FA:19-20 & $-0,067$ & 0,000667 & FR:19-20 & $-0,028$ & 0,000333 \\
\hline FA:21-22 & $-0,018$ & 0,000333 & FR:21-22 & $-0,014$ & 0,000333 \\
\hline FA:22-24 & 0,057 & 0,000667 & FR:22-24 & 0,031 & 0,000333 \\
\hline FA:23-24 & 0,018 & 0,000333 & FR:23-24 & 0,012 & 0,000333 \\
\hline FA:24-25 & $-0,012$ & 0,000333 & FR:24-25 & 0,02 & 0,000333 \\
\hline FA:25-26 & 0,035 & 0,000333 & FR:25-26 & 0,024 & 0,000333 \\
\hline FA:25-27 & $-0,048$ & 0,000333 & FR:25-27 & $-0,004$ & 0,000333 \\
\hline FA:27-29 & 0,062 & 0,000667 & FR:27-29 & 0,017 & 0,000333 \\
\hline FA:27-30 & 0,071 & 0,000667 & FR:27-30 & 0,017 & 0,000333 \\
\hline FA:28-27 & 0,181 & 0,001333 & FR:28-27 & 0,05 & 0,000667 \\
\hline FA:29-30 & 0,037 & 0,000333 & FR:29-30 & 0,006 & 0,000333 \\
\hline \multicolumn{6}{|c|}{ Medidas de Tensão } \\
\hline \multicolumn{2}{|c|}{ Medida } & \multicolumn{2}{|c|}{ Valor (pu) } & \multicolumn{2}{|c|}{$\sigma(\mathbf{p u})$} \\
\hline \multicolumn{2}{|c|}{ V:1 } & \multicolumn{2}{|c|}{1,06} & \multicolumn{2}{|c|}{0,013333} \\
\hline \multicolumn{2}{|c|}{$\mathrm{V}: 2$} & \multicolumn{2}{|c|}{1,043} & \multicolumn{2}{|c|}{0,013333} \\
\hline \multicolumn{2}{|c|}{$V: 5$} & \multicolumn{2}{|c|}{1,01} & \multicolumn{2}{|c|}{0,013333} \\
\hline \multicolumn{2}{|c|}{ V:8 } & \multicolumn{2}{|c|}{1,01} & \multicolumn{2}{|c|}{0,013333} \\
\hline \multicolumn{2}{|c|}{$V: 11$} & \multicolumn{2}{|c|}{1,082} & \multicolumn{2}{|c|}{0,013333} \\
\hline \multicolumn{2}{|c|}{$V: 13$} & \multicolumn{2}{|c|}{1,071} & \multicolumn{2}{|c|}{0,013333} \\
\hline & & & & 0,0 & 333 \\
\hline $\mathrm{V}$ & & & & & 333 \\
\hline
\end{tabular}


As medidas tabeladas com valor NULO são medidas virtuais, e por isso seus respectivos valores de desvios padrão não foram calculados. Para executar o estimador de estado WLS, atribuiu-se, para as medidas virtuais, a maior ponderação existente entre as demais medidas, neste caso $R_{i i}^{-1}=1 /(0,000333)^{2}$.

Na tabela B.2 do Anexo B, são apresentadas todas as possíveis medidas de potência e magnitude de tensão, para o sistema de 30 barras do IEEE, bem como os valores dos respectivos desvios padrões.

\subsubsection{Testes sem Ruído Gaussiano e sem Erro Grosseiro}

Apresentaremos nesta subseção os resultados de simulações, considerando o sistema de medição da tabela 5.10, sem considerar ruído ou EG em medidas. Assim, o objetivo desta subseção é obter e analisar o índice $U I$ das medidas, desprezando qualquer ruído e EG.

Tabela 5.11: Valor do índice $J(\mathrm{x})$, valores estimados das medidas, índice $U I$ e resíduos normalizados

$$
r^{N} \text { - sistema de } 30 \text { barras IEEE }
$$

\begin{tabular}{c|c|c|c|c|c|c|c}
\hline \multicolumn{7}{c}{ Limiar de detecção do índice $J(\mathrm{x}): \lambda=\chi_{63 ;(1-0,05)}^{2} \cong 81$} \\
\hline \multicolumn{7}{c}{ Índice $J(\mathrm{x})=0,5165$} \\
\hline Medidas de Potência Ativa & \multicolumn{3}{c}{ Medidas de Potência Reativa } \\
\hline Valor & $\boldsymbol{U I}$ & $r^{N}$ & Medida & $\begin{array}{c}\text { Valor } \\
\text { estimado }\end{array}$ & $\boldsymbol{U I}$ & $r^{N}$ \\
\hline IA:1ida & 2,6116 & 0,393 & 0,0126 & IR:1 & $-0,1647$ & 1,0717 & $-0,0158$ \\
\hline IA:2 & 0,183 & 7,1108 & 0,0 & IR:2 & 0,3686 & 0,3596 & 0,0039 \\
\hline IA:5 & $-0,9435$ & 0,3414 & $-0,0094$ & IR:5 & 0,1787 & 0,9706 & 0,0 \\
\hline IA:6 & NULO & --- & NULO & IR:6 & NULO & --- & NULO \\
\hline IA:7 & $-0,2279$ & 1,4816 & $-0,0187$ & IR:7 & $-0,1084$ & 0,5324 & 0,0 \\
\hline IA:8 & $-0,299$ & 0,7286 & $-0,0256$ & IR:8 & 0,0718 & 1,0604 & 0 \\
\hline IA:9 & NULO & --- & NULO & IR:9 & NULO & --- & NULO \\
\hline IA:11 & NULO & --- & NULO & IR:11 & 0,1619 & 0,6237 & 0 \\
\hline IA:12 & $-0,1121$ & 3,0375 & $-0,0341$ & IR:12 & $-0,0749$ & 3,4905 & 0 \\
\hline IA:13 & NULO & --- & NULO & IR:13 & 0,1064 & 0,8817 & 0 \\
\hline IA:15 & $-0,082$ & 5,3611 & 0 & IR:15 & $-0,025$ & 6,9601 & 0 \\
\hline IA:16 & $-0,0349$ & 4,4527 & 0 & IR:16 & $-0,0182$ & 2,5653 & 0 \\
\hline IA:17 & $-0,09$ & 7,2144 & 0 & IR:17 & $-0,0583$ & 4,2606 & 0 \\
\hline IA:19 & $-0,095$ & 1,1173 & 0 & IR:19 & $-0,034$ & 1,4132 & 0 \\
\hline IA:21 & $-0,1748$ & 1,0568 & 0 & IR:21 & $-0,1117$ & 0,9257 & 0,0927 \\
\hline IA:22 & NULO & --- & NULO & IR:22 & NULO & --- & NULO \\
\hline IA:23 & $-0,032$ & 2,1209 & 0 & IR:23 & $-0,0161$ & 1,3398 & 0 \\
\hline IA:24 & $-0,0871$ & 0,993 & 0,1207 & IR:24 & $-0,067$ & 0,7781 & $-0,0495$ \\
\hline IA:25 & NULO & --- & NULO & IR:25 & NULO & --- & NULO \\
\hline
\end{tabular}




\begin{tabular}{|c|c|c|c|c|c|c|c|}
\hline IA:27 & NULO & --- & NULO & IR:27 & NULO & --- & NULO \\
\hline IA:28 & NULO & --- & NULO & IR:28 & NULO & --- & NULO \\
\hline IA:30 & $-0,106$ & 0,3866 & 0,0096 & IR:30 & $-0,019$ & 1,331 & 0 \\
\hline FA:1-2 & 1,7334 & 0,2937 & 0,0109 & FR:1-2 & $-0,2106$ & 0,402 & $-0,0084$ \\
\hline FA:1-3 & 0,8782 & 0,4348 & 0,0093 & FR:1-3 & 0,0459 & 1,4558 & 0 \\
\hline FA:2-4 & 0,4364 & 0,3421 & 0,0061 & FR:2-4 & 0,0389 & 0,7334 & 0 \\
\hline FA:4-2 & $-0,4262$ & 0,3286 & $-0,0058$ & FR:4-2 & $-0,0469$ & 1,303 & 0 \\
\hline FA:2-5 & 0,8246 & 0,2338 & 0,0092 & FR:2-5 & 0,0178 & 1,795 & 0 \\
\hline FA:2-6 & 0,6035 & 0,277 & 0,013 & FR:2-6 & 0,0044 & 0,8102 & $-0,0996$ \\
\hline FA:3-4 & 0,8209 & 2,6492 & 0,0317 & FR:3-4 & $-0,036$ & 9,2066 & 0 \\
\hline FA:4-6 & 0,7214 & 0,4274 & 0,0194 & FR:4-6 & $-0,1634$ & 0,5874 & $-0,1168$ \\
\hline FA:4-12 & 0,4424 & 0,304 & 0,0076 & FR:4-12 & 0,1431 & 0,6923 & $-0,0779$ \\
\hline FA:5-7 & $-0,1484$ & 1,3753 & 0 & FR:5-7 & 0,1165 & 0,4268 & $-0,0107$ \\
\hline FA:6-7 & 0,3818 & 0,4783 & 0,0068 & FR:6-7 & $-0,0299$ & 2,3231 & 0,1051 \\
\hline FA:6-8 & 0,2948 & 0,6012 & 0,028 & FR:6-8 & $-0,0812$ & 0,7493 & $-0,0449$ \\
\hline FA:6-9 & 0,2774 & 0,4009 & 0,0072 & FR:6-9 & $-0,0818$ & 0,8309 & $-0,0721$ \\
\hline FA:6-28 & 0,1866 & 0,41 & 0,055 & FR:6-28 & NULO & --- & NULO \\
\hline FA:8-28 & $-0,0053$ & 0,857 & 0 & FR:8-28 & $-0,0039$ & 0,3796 & $-0,0655$ \\
\hline FA:9-11 & NULO & --- & NULO & FR:9-11 & $-0,1572$ & 0,5761 & 0 \\
\hline FA:9-10 & 0,2774 & 0,4142 & 0,0068 & FR:9-10 & 0,0592 & 1,2968 & $-0,0997$ \\
\hline FA:12-13 & NULO & --- & NULO & FR:12-13 & $-0,105$ & 0,8422 & 0 \\
\hline FA:12-15 & 0,1792 & 0,5092 & 0 & FR:12-15 & 0,0682 & 0,7701 & $-0,0653$ \\
\hline FA:12-16 & 0,0723 & 0,723 & 0,0236 & FR:12-16 & 0,0336 & 1,1057 & 0 \\
\hline FA:15-18 & 0,0602 & 0,9941 & 0 & FR:15-18 & 0,0159 & 0,9969 & 0 \\
\hline FA:18-15 & $-0,0598$ & 0,9707 & 0 & FR:18-15 & $-0,0151$ & 0,9841 & 0 \\
\hline FA:15-23 & 0,0502 & 0,6156 & 0 & FR:15-23 & 0,0291 & 1,0934 & $-0,0454$ \\
\hline FA:18-19 & 0,028 & 2,8378 & 0 & FR:18-19 & 0,006 & 1,4163 & 0 \\
\hline FA:19-20 & $-0,067$ & 1,1173 & 0 & FR:19-20 & $-0,028$ & 1,4132 & 0 \\
\hline FA:21-22 & $-0,0181$ & 1,6062 & 0,074 & FR:21-22 & $-0,014$ & 1,4531 & 0 \\
\hline FA:22-24 & 0,0576 & 0,7089 & $-0,0756$ & FR:22-24 & 0,0309 & 1,2129 & $-0,1374$ \\
\hline FA:23-24 & 0,018 & 1,2868 & 0 & FR:23-24 & 0,0124 & 0,9388 & $-0,0579$ \\
\hline FA:24-25 & $-0,0121$ & 1,1629 & 0,1035 & FR:24-25 & 0,0203 & 1,018 & $-0,2731$ \\
\hline FA:25-26 & 0,0353 & 1,6757 & $-0,3923$ & FR:25-26 & 0,0239 & 1,6293 & 0,128 \\
\hline FA:25-27 & $-0,0476$ & 1,2907 & $-0,3935$ & FR:25-27 & $-0,0037$ & 0,9859 & $-0,1096$ \\
\hline FA:27-29 & 0,062 & 0,7004 & $-0,0374$ & FR:27-29 & 0,0168 & 1,0616 & 0,0847 \\
\hline FA:27-30 & 0,0709 & 0,4568 & $-0,022$ & FR:27-30 & 0,0167 & 0,4781 & 0 \\
\hline FA:28-27 & 0,1807 & 0,4263 & 0,0215 & FR:28-27 & 0,0506 & 0,4539 & $-0,2306$ \\
\hline FA:29-30 & 0,037 & 2,0916 & 0 & FR:29-30 & 0,006 & 0,6688 & 0 \\
\hline \multicolumn{8}{|c|}{ Medidas de Tensão } \\
\hline \multicolumn{2}{|c|}{ Medida } & \multicolumn{2}{|c|}{ Valor estimado } & \multicolumn{2}{|c|}{$U I$} & \multicolumn{2}{|c|}{$r^{N}$} \\
\hline \multicolumn{2}{|c|}{$V: 1$} & \multicolumn{2}{|c|}{1,0606} & \multicolumn{2}{|c|}{0,1208} & \multicolumn{2}{|c|}{0,0013} \\
\hline \multicolumn{2}{|c|}{$V: 2$} & \multicolumn{2}{|c|}{1,0436} & \multicolumn{2}{|c|}{0,12} & \multicolumn{2}{|c|}{0,0012} \\
\hline \multicolumn{2}{|c|}{$V: 5$} & \multicolumn{2}{|c|}{1,0105} & \multicolumn{2}{|c|}{0,12} & \multicolumn{2}{|c|}{0,0012} \\
\hline \multicolumn{2}{|c|}{$\mathrm{V}: 8$} & \multicolumn{2}{|c|}{1,0106} & \multicolumn{2}{|c|}{0,1212} & \multicolumn{2}{|c|}{0,0014} \\
\hline & & & & & & & \\
\hline & & & & & & & \\
\hline & & & & & & & \\
\hline & & & & & & & \\
\hline
\end{tabular}


Os gráficos das figuras 5.3 e 5.4 ilustram, respectivamente, os índices $U I$ para as medidas ativas e reativas do sistema em análise. As medidas ativas e reativas estão em gráficos distintos, devido, apenas, à limitação de espaço.

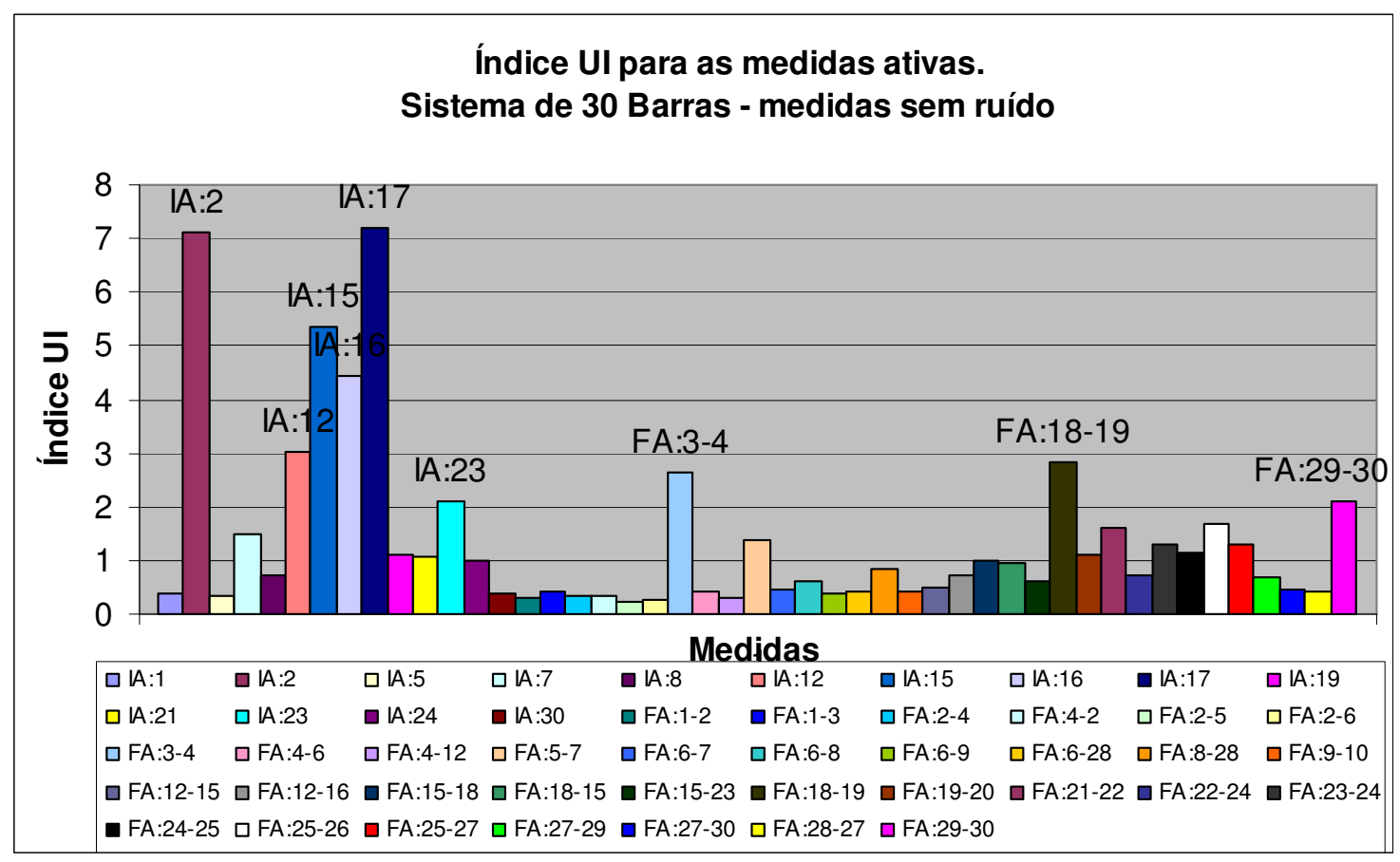

Figura 5.3: Índice $U I$ para as medidas ativas. Sistema de 30 barras - medidas sem ruído

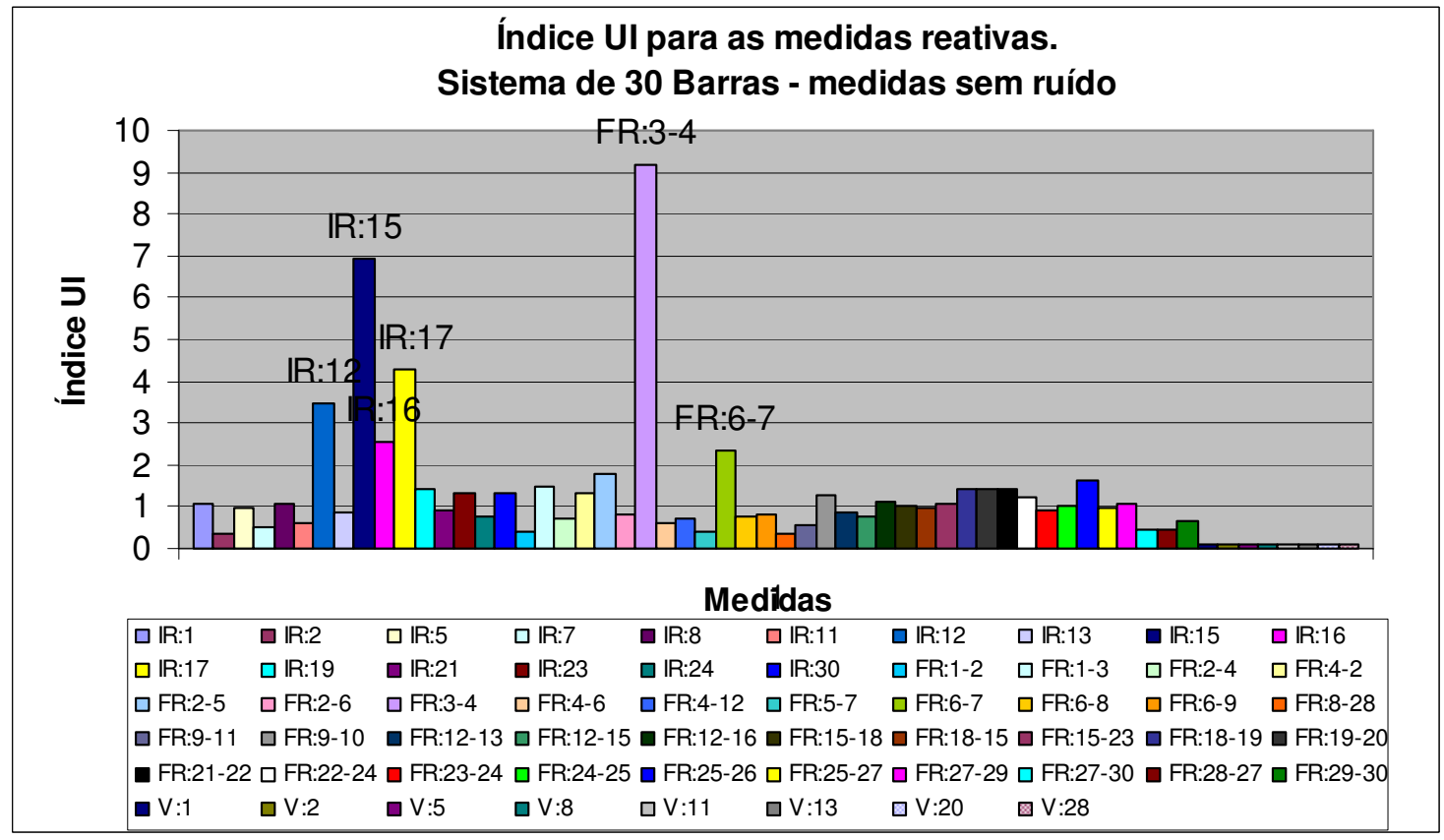

Figura 5.4: Índice UI para as medidas reativas. Sistema de 30 barras - medidas sem ruído

As medidas em evidência, mostradas nas figuras 5.3 e 5.4, são aquelas que apresentam os maiores valores numéricos de índice $U I$ (neste caso maiores que 2). Conseqüientemente, EGs nessas medidas serão mais dificilmente detectados, em relação às medidas com $U I$ de baixo valor. 
Com o intuito de ratificar a afirmação acima, na próxima subseção serão apresentados os resultados da identificação do nível de detecção de EG, para o conjunto de medidas em análise.

\subsubsection{Identificação do Nível de Detecção de Erros: medidas sem ruído}

Para a identificação do nível de detecção de EG do sistema de 30 barras em análise, o limiar de deteç̧ão de EG para o índice $J(\mathrm{x})$ é $\lambda=\chi_{63 ;(1-0,05)}^{2} \cong 81$, considerando uma probabilidade de falso alarme $\alpha=5 \%$ e o grau de liberdade $m-n=63$. Já o limiar de detecção de EG, adotado para o teste dos resíduos normalizados, é $\beta=3,0$.

A tabela 5.12, a seguir, mostra os valores dos resultados da identificação do nível de detecção, para as medidas do sistema em análise.

Tabela 5.12: Resultados numéricos da simulação do sistema de medição da tabela 5.10 - sistema de 30 barras IEEE

\begin{tabular}{c|c|c|c|c|c|c|c}
\hline \multicolumn{7}{c}{ Limiar de detecção do índice $J(x): \lambda=\chi_{63 ;(1-0,05)}^{2} \cong 81$} \\
\hline \multicolumn{7}{c|}{ Medidas de Potência Ativa } & \multicolumn{3}{c}{ Medidas de Potência Reativa } \\
\hline Medida & UI & $\begin{array}{c}\text { Nível } \\
\text { Detecção }\end{array}$ & J(x) & Medida & UI & $\begin{array}{c}\text { Nível } \\
\text { Detecção }\end{array}$ & J(x) \\
\hline IA:1 & 0,394 & 3,3 & 10,012 & IR:1 & 1,0718 & 4,4 & 9,6 \\
\hline IA:2 & 7,1385 & 21,4 & 9,515 & IR:2 & 0,3608 & 3,2 & 9,6 \\
\hline IA:5 & 0,342 & 3,2 & 9,738 & IR:5 & 0,9761 & 4,2 & 9,537 \\
\hline IA:6 & --- & --- & --- & IR:6 & --- & --- & --- \\
\hline IA:7 & 1,482 & 5,4 & 9,747 & IR:7 & 0,5328 & 3,5 & 10,056 \\
\hline IA:8 & 0,7286 & 3,7 & 9,61 & IR:8 & 1,0618 & 4,4 & 9,675 \\
\hline IA:9 & --- & --- & --- & IR:9 & --- & --- & --- \\
\hline IA:11 & --- & --- & --- & IR:11 & 0,6238 & 3,6 & 9,813 \\
\hline IA:12 & 3,0384 & 9,5 & 9,556 & IR:12 & 3,4937 & 11,1 & 9,688 \\
\hline IA:13 & --- & --- & --- & IR:13 & 0,8819 & 4 & 9,57 \\
\hline IA:15 & 5,3617 & 16,4 & 9,485 & IR:15 & 6,9668 & 20,8 & 9,456 \\
\hline IA:16 & 4,453 & 13,8 & 9,569 & IR:16 & 2,567 & 8,4 & 9,629 \\
\hline IA:17 & 7,215 & 22,1 & 9,607 & IR:17 & 4,2631 & 13,3 & 9,568 \\
\hline IA:19 & 1,1173 & 4,5 & 9,527 & IR:19 & 1,4132 & 5,2 & 9,546 \\
\hline IA:21 & 1,0568 & 4,4 & 9,74 & IR:21 & 0,9258 & 4,3 & 9,903 \\
\hline IA:22 & --- & --- & --- & IR:22 & --- & --- & --- \\
\hline IA:23 & 2,121 & 7,2 & 9,769 & IR:23 & 1,3398 & 5 & 9,569 \\
\hline IA:24 & 0,993 & 4,4 & 9,512 & IR:24 & 0,7793 & 3,8 & 9,789 \\
\hline IA:25 & ---- & --- & --- & IR:25 & --- & --- & --- \\
\hline IA:27 & ---- & --- & --- & IR:27 & --- & --- & --- \\
\hline IA:28 & ---- & --- & --- & IR:28 & --- & --- & --- \\
\hline IA:30 & 0,3866 & 3,3 & 9,933 & IR:30 & 1,3311 & 5 & 9,73 \\
\hline FA:1-2 & 0,2944 & 3,2 & 10,003 & FR:1-2 & 0,402 & 3,3 & 9,936 \\
\hline FA:1-3 & 0,4358 & 3,3 & 9,715 & FR:1-3 & 1,4558 & 5,3 & 9,639 \\
\hline
\end{tabular}




\begin{tabular}{|c|c|c|c|c|c|c|c|}
\hline FA:2-4 & 0,3429 & 3,2 & 9,711 & FR:2-4 & 0,7337 & 3,7 & 9,542 \\
\hline FA:4-2 & 0,3294 & 3,2 & 9,785 & FR:4-2 & 1,3175 & 5 & 9,773 \\
\hline FA:2-5 & 0,2342 & 3,1 & 9,679 & FR:2-5 & 1,7999 & 6,2 & 9,522 \\
\hline FA:2-6 & 0,2777 & 3,2 & 10,098 & FR:2-6 & 0,8145 & 4,1 & 9,985 \\
\hline FA:3-4 & 2,6518 & 8,5 & 9,595 & FR:3-4 & 9,2068 & 27,8 & 9,41 \\
\hline FA:4-6 & 0,4275 & 3,3 & 9,841 & FR:4-6 & 0,5883 & 3,4 & 9,79 \\
\hline FA:4-12 & 0,3042 & 3,2 & 9,936 & FR:4-12 & 0,698 & 3,8 & 9,717 \\
\hline FA:5-7 & 1,3766 & 5,1 & 9,521 & FR:5-7 & 0,427 & 3,3 & 9,667 \\
\hline FA:6-7 & 0,4785 & 3,4 & 9,965 & FR:6-7 & 2,3269 & 7,9 & 9,619 \\
\hline FA:6-8 & 0,6012 & 3,5 & 9,683 & FR:6-8 & 0,7494 & 3,7 & 9,548 \\
\hline FA:6-9 & 0,4012 & 3,3 & 9,94 & FR:6-9 & 0,831 & 3,9 & 9,952 \\
\hline FA:6-28 & 0,4102 & 3,2 & 9,61 & FR:6-28 & --- & --- & --- \\
\hline FA:8-28 & 0,857 & 4 & 9,562 & FR:8-28 & 0,382 & 3,2 & 9,839 \\
\hline FA:9-11 & --- & --- & --- & FR:9-11 & 0,5762 & 3,5 & 9,689 \\
\hline FA:9-10 & 0,4145 & 3,3 & 9,854 & FR:9-10 & 1,297 & 5,1 & 9,587 \\
\hline FA:12-13 & --- & --- & --- & FR:12-13 & 0,8424 & 4 & 9,935 \\
\hline FA:12-15 & 0,5093 & 3,4 & 9,673 & FR:12-15 & 0,7726 & 3,9 & 9,633 \\
\hline FA:12-16 & 0,7231 & 3,7 & 9,653 & FR:12-16 & 1,1074 & 4,5 & 9,758 \\
\hline FA:15-18 & 0,9941 & 4,3 & 9,808 & FR:15-18 & 0,9969 & 4,3 & 9,811 \\
\hline FA:18-15 & 0,9707 & 4,2 & 9,591 & FR:18-15 & 0,9841 & 4,3 & 9,934 \\
\hline FA: $15-23$ & 0,6156 & 3,6 & 9,928 & FR:15-23 & 1,0934 & 4,6 & 9,861 \\
\hline FA:18-19 & 2,8378 & 9,1 & 9,661 & FR:18-19 & 1,4163 & 5,2 & 9,522 \\
\hline FA:19-20 & 1,1173 & 4,5 & 9,522 & FR:19-20 & 1,4132 & 5,2 & 9,53 \\
\hline FA:21-22 & 1,6062 & 5,9 & 9,832 & FR:21-22 & 1,4531 & 5,3 & 9,653 \\
\hline FA:22-24 & 0,7089 & 3,8 & 9,659 & FR:22-24 & 1,2137 & 5 & 9,756 \\
\hline FA:23-24 & 1,287 & 4,9 & 9,721 & FR:23-24 & 0,9388 & 4,2 & 9,535 \\
\hline FA:24-25 & 1,1631 & 4,8 & 9,666 & FR:24-25 & 1,0182 & 4,7 & 9,56 \\
\hline FA:25-26 & 1,6757 & 6,7 & 9,611 & FR:25-26 & 1,6296 & 5,5 & 9,524 \\
\hline FA: $25-27$ & 1,2909 & 4,3 & 9,53 & FR:25-27 & 0,9861 & 4,1 & 9,686 \\
\hline FA:27-29 & 0,7006 & 3,8 & 9,968 & FR:27-29 & 1,0623 & 4,3 & 9,703 \\
\hline FA: $27-30$ & 0,4569 & 3,4 & 9,943 & FR:27-30 & 0,4782 & 3,3 & 9,569 \\
\hline FA: $28-27$ & 0,4265 & 3,3 & 9,862 & FR:28-27 & 0,4542 & 3,6 & 9,749 \\
\hline FA:29-30 & 2,0916 & 7 & 9,663 & FR:29-30 & 0,6689 & 3,7 & 9,872 \\
\hline \multicolumn{8}{|c|}{ Medidas de Tensão } \\
\hline \multicolumn{2}{|c|}{ Medida } & \multicolumn{2}{|c|}{$\boldsymbol{U I}$} & \multicolumn{2}{|c|}{$\begin{array}{c}\text { Nível } \\
\text { Detecção }\end{array}$} & \multicolumn{2}{|c|}{$\mathbf{J}(\mathbf{x})$} \\
\hline \multicolumn{2}{|c|}{$\mathrm{V}: 1$} & \multicolumn{2}{|c|}{0,1227} & \multicolumn{2}{|c|}{3,1} & \multicolumn{2}{|c|}{9,987} \\
\hline \multicolumn{2}{|c|}{$V: 2$} & \multicolumn{2}{|c|}{0,1219} & \multicolumn{2}{|c|}{3,1} & \multicolumn{2}{|c|}{9,991} \\
\hline \multicolumn{2}{|c|}{$V: 5$} & \multicolumn{2}{|c|}{0,1219} & \multicolumn{2}{|c|}{3,1} & \multicolumn{2}{|c|}{9,99} \\
\hline \multicolumn{2}{|c|}{$\mathrm{V}: 8$} & \multicolumn{2}{|c|}{0,1232} & \multicolumn{2}{|c|}{3,1} & \multicolumn{2}{|c|}{9,987} \\
\hline \multicolumn{2}{|c|}{$\mathrm{V}: 11$} & \multicolumn{2}{|c|}{0,12} & \multicolumn{2}{|c|}{3,1} & & \\
\hline V: & & & & 3 , & & & \\
\hline V: & & & & 3 , & & & \\
\hline V: & & & & 3 , & & & \\
\hline
\end{tabular}

Através dos resultados da tabela 5.12, constrói-se o gráfico índice $U I$ x nível de detecção mostrado a seguir (figura 5.5): 


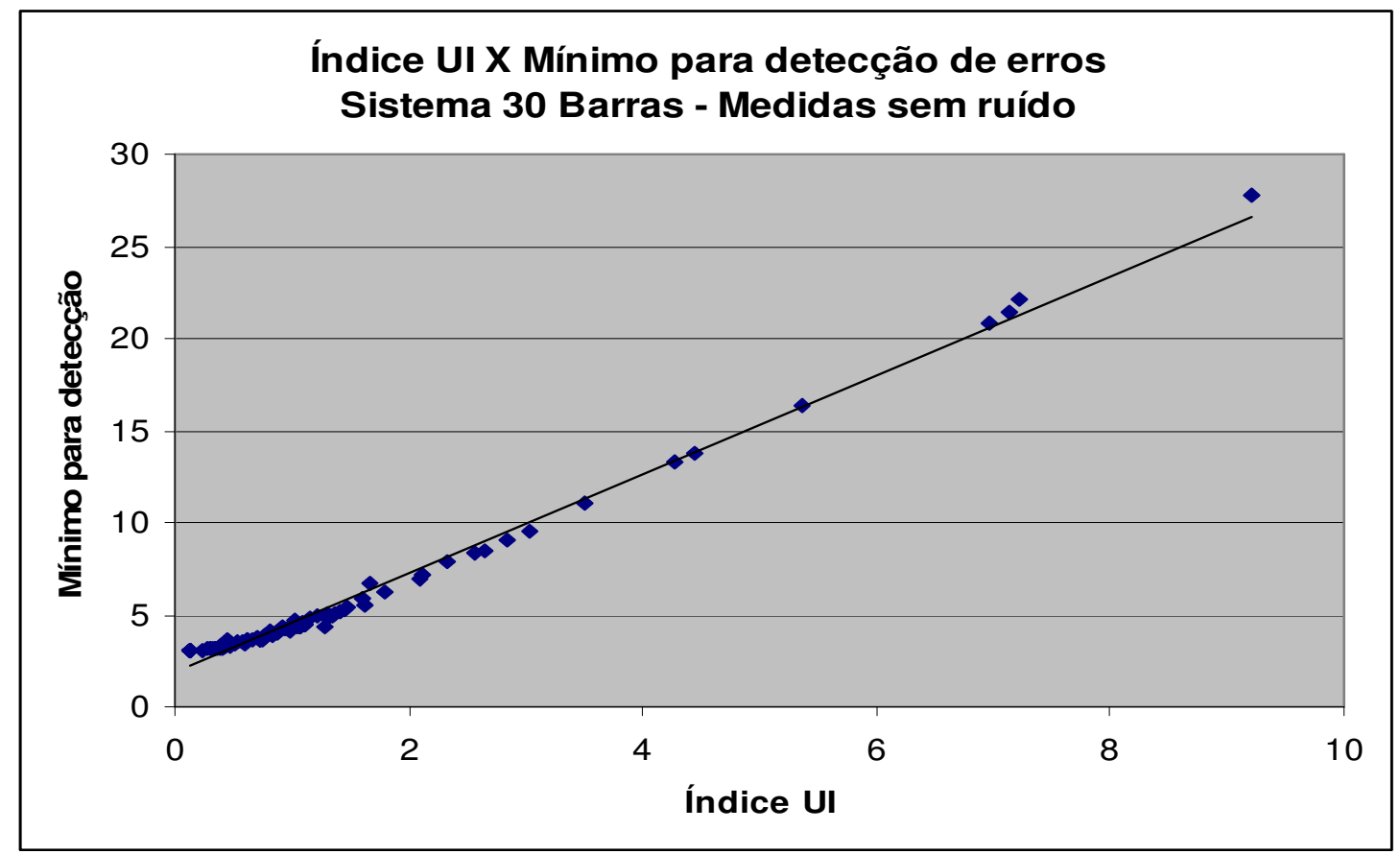

Figura 5.5: Gráfico de tendência para índice UI X Mínimo para detecção de erros em medidas. Sistema de 30 barras - medidas sem ruído

Com base no gráfico apresentado na figura 5.5, comprova-se que quanto maior o índice $U I$ de uma medida, mais difícil será a detecção de EG, através do teste dos resíduos normalizados. Além disso, assim como foi verificado para o sistema de 14 barras, a reta de tendência desse gráfico apresenta comportamento linear.

\subsubsection{Testes sem Ruído Gaussiano mas com Erro Grosseiro Simples}

O objetivo desta subseção é analisar o desempenho dos métodos tradicionais de detecção/identificação de EGs, frente à adição de EG simples, sem considerar ruído gaussiano nas medidas do sistema de 30 barras. Para tal propósito, considerar-se-ão simulações de EG simples de 10 e 20 sigmas, em cada uma das medidas do sistema de medição da tabela 5.10 (uma medida de cada vez).

Nas tabelas a seguir, os casos indicados em vermelho são aqueles em que os dois métodos tradicionais de detecção/identificação de EGs falharam e, em realce amarelo, os casos em que apenas o teste do índice $J(\mathrm{x})$ falhou para detectar EGs.

A tabela 5.13, a seguir, apresenta os valores do índice $J(x)$, os valores do índice UI e resíduo normalizado, para a medida portadora de EG em cada caso, considerando EG simples de 10 sigmas. 
Tabela 5.13: Resultados numéricos da adição de EG de $10 \sigma$ para cada medida (uma medida de cada vez) considerando o sistema de medição da tabela 5.10

Limiar de detecção do índice $J(\mathrm{x}): \lambda=\chi_{63 ;(1-0,05)}^{2} \cong 81$

\begin{tabular}{|c|c|c|c|c|c|c|c|}
\hline \multicolumn{4}{|c|}{ Medidas de Potência Ativa } & \multicolumn{4}{|c|}{ Medidas de Potência Reativa } \\
\hline $\begin{array}{l}\text { Medida } \\
\text { com erro }\end{array}$ & $U I$ & $r^{N}$ & $\mathbf{J}(\mathbf{x})$ & $\begin{array}{l}\text { Medida } \\
\text { com erro }\end{array}$ & $U I$ & $r^{N}$ & $\mathbf{J}(\mathbf{x})$ \\
\hline IA:1 & 0,4007 & $-9,285$ & 86,8057 & IR:1 & 1,0728 & $-6,8335$ & 47,1993 \\
\hline IA:2 & 7,1813 & 1,4174 & 2,5299 & IR:2 & 0,3625 & 9,4081 & 89,0364 \\
\hline IA:5 & 0,3454 & 9,451 & 89,8566 & IR:5 & 0,9922 & 7,1377 & 51,5172 \\
\hline IA:6 & --- & --- & --- & IR:6 & --- & --- & --- \\
\hline IA:7 & 1,4835 & 5,5745 & 31,5924 & IR:7 & 0,5346 & 8,8231 & 78,3841 \\
\hline IA:8 & 0,7287 & 8,0564 & 65,4293 & IR:8 & 1,0664 & $-6,8445$ & 47,3845 \\
\hline IA:9 & --- & --- & --- & IR:9 & --- & --- & --- \\
\hline IA:11 & --- & --- & --- & IR:11 & 0,624 & 8,4777 & 72,3915 \\
\hline IA:12 & 3,0403 & $-3,1639$ & 10,5183 & IR:12 & 3,5053 & 2,7708 & 8,2262 \\
\hline IA:13 & --- & --- & --- & IR:13 & 0,8827 & $-7,492$ & 56,6647 \\
\hline IA:15 & 5,3638 & 1,8516 & 3,93 & IR:15 & 6,9911 & $-1,4644$ & 2,6273 \\
\hline IA:16 & 4,4544 & 2,2088 & 5,3858 & IR:16 & 2,5737 & 3,6602 & 13,912 \\
\hline IA:17 & 7,2193 & 1,3906 & 2,4576 & IR:17 & 4,273 & $-2,2655$ & 5,5988 \\
\hline IA:19 & 1,1173 & $-6,67$ & 44,9876 & IR:19 & 1,4132 & 5,7666 & 33,8835 \\
\hline IA:21 & 1,0571 & $-6,8857$ & 47,9229 & IR:21 & 0,9263 & 7,429 & 55,7794 \\
\hline IA:22 & --- & --- & --- & IR:22 & --- & --- & --- \\
\hline IA:23 & 2,1211 & 4,2934 & 18,9526 & IR:23 & 1,3399 & $-5,9985$ & 36,5161 \\
\hline IA:24 & 0,9931 & 7,2155 & 52,5755 & IR:24 & 0,7841 & $-7,9341$ & 63,4697 \\
\hline IA:25 & --- & --- & --- & IR:25 & --- & --- & --- \\
\hline IA:27 & --- & --- & --- & IR:27 & --- & --- & --- \\
\hline IA:28 & --- & --- & --- & IR:28 & --- & --- & --- \\
\hline IA:30 & 0,3867 & 9,3365 & 87,6856 & IR:30 & 1,3318 & $-6,0395$ & 36,9962 \\
\hline FA:1-2 & 0,2987 & $-9,5805$ & 92,3178 & FR:1-2 & 0,4024 & $-9,2841$ & 86,7232 \\
\hline FA:1-3 & 0,4425 & $-9,1589$ & 84,3364 & FR:1-3 & 1,4567 & 5,6754 & 32,7377 \\
\hline FA:2-4 & 0,3482 & $-9,4419$ & 89,862 & FR:2-4 & 0,735 & 8,0858 & 65,846 \\
\hline FA:4-2 & 0,3349 & 9,4927 & 90,5964 & FR:4-2 & 1,3512 & 6,0134 & 36,6326 \\
\hline FA:2-5 & 0,237 & 9,7472 & 95,4713 & FR:2-5 & 1,8122 & 4,8683 & 23,9612 \\
\hline FA:2-6 & 0,2823 & $-9,6213$ & 93,0895 & FR:2-6 & 0,8298 & $-7,8175$ & 62,0576 \\
\hline FA:3-4 & 2,666 & $-3,5165$ & 12,8938 & FR:3-4 & 9,2087 & $-1,0204$ & 1,6365 \\
\hline FA:4-6 & 0,4278 & $-9,1748$ & 84,7111 & FR:4-6 & 0,592 & 8,5038 & 72,7976 \\
\hline FA:4-12 & 0,3041 & 9,575 & 92,2015 & FR:4-12 & 0,7217 & $-8,3378$ & 69,0518 \\
\hline FA:5-7 & 1,3796 & $-5,8829$ & 35,1136 & FR:5-7 & 0,4273 & 9,1863 & 84,8897 \\
\hline FA:6-7 & 0,4791 & $-9,0139$ & 81,7801 & FR:6-7 & 2,3346 & 4,0473 & 16,8703 \\
\hline FA:6-8 & 0,6013 & 8,598 & 74,4432 & FR:6-8 & 0,7496 & 7,9559 & 63,8349 \\
\hline FA:6-9 & 0,4012 & 9,2886 & 86,8009 & FR:6-9 & 0,8312 & 7,6155 & 58,5201 \\
\hline FA:6-28 & 0,4103 & $-9,1986$ & 85,1198 & FR:6-28 & --- & --- & --- \\
\hline FA:8-28 & 0,857 & $-7,5637$ & 57,7392 & FR:8-28 & 0,3917 & 9,2666 & 86,5048 \\
\hline FA:9-11 & --- & --- & --- & FR:9-11 & 0,5763 & 8,6684 & 75,6565 \\
\hline FA:9-10 & 0,4144 & $-9,2316$ & 85,7403 & FR:9-10 & 1,2973 & 6,0078 & 36,5622 \\
\hline FA:12-13 & --- & --- & --- & FR:12-13 & 0,8432 & 7,6387 & 58,8429 \\
\hline FA:12-15 & 0,5095 & 8,9065 & 79,848 & FR:12-15 & 0,782 & $-7,9865$ & 64,1482 \\
\hline FA:12-16 & 0,7236 & $-8,0809$ & 65,7985 & FR:12-16 & 1,1135 & 6,7236 & 45,7211 \\
\hline FA:15-18 & 0,994 & $-7,0949$ & 50,8535 & FR:15-18 & 0,9969 & 7,0854 & 50,7 \\
\hline FA:18-15 & 0,9707 & $-7,1751$ & 51,9973 & FR:18-15 & 0,9842 & $-7,1329$ & 51,3837 \\
\hline FA:15-23 & 0,6157 & $-8,5139$ & 73,0036 & FR:15-23 & 1,0934 & 6,7017 & 45,4183 \\
\hline
\end{tabular}




\begin{tabular}{|c|c|c|c|c|c|c|c|}
\hline FA:18-19 & 2,8378 & $-3,3246$ & 11,5662 & FR:18-19 & 1,4163 & $-5,7697$ & 33,7633 \\
\hline FA:19-20 & 1,1173 & 6,6686 & 44,9916 & FR:19-20 & 1,4132 & 5,7789 & 33,8751 \\
\hline FA:21-22 & 1,6062 & 5,3455 & 29,1405 & FR:21-22 & 1,4531 & 5,6341 & 32,4318 \\
\hline FA:22-24 & 0,7092 & $-8,2335$ & 68,3017 & FR:22-24 & 1,2165 & 6,2168 & 39,21 \\
\hline FA:23-24 & 1,2877 & 6,1625 & 38,4959 & FR:23-24 & 0,9388 & $-7,3487$ & 54,5179 \\
\hline FA:24-25 & 1,1631 & $-6,4178$ & 41,6944 & FR:24-25 & 1,0194 & 6,7323 & 45,7512 \\
\hline FA:25-26 & 1,6757 & $-5,5165$ & 30,8057 & FR:25-26 & 1,6307 & $-5,1012$ & 26,5324 \\
\hline FA:25-27 & 1,2909 & $-6,5206$ & 42,8648 & FR:25-27 & 0,9861 & $-7,2337$ & 52,8326 \\
\hline FA:27-29 & 0,7008 & $-8,2281$ & 68,2137 & FR:27-29 & 1,065 & $-6,7658$ & 46,3175 \\
\hline FA:27-30 & 0,457 & 9,0737 & 82,85 & FR:27-30 & 0,4785 & $-8,991$ & 81,3314 \\
\hline FA:28-27 & 0,4267 & $-9,1762$ & 84,7346 & FR:28-27 & 0,4553 & 8,8745 & 79,2088 \\
\hline FA:29-30 & 2,0916 & $-4,3093$ & 19,0916 & FR:29-30 & 0,669 & $-8,3293$ & 69,897 \\
\hline \multicolumn{8}{|c|}{ Medidas de Tensão } \\
\hline \multicolumn{2}{|c|}{ Medida com erro } & \multicolumn{2}{|c|}{$U I$} & \multicolumn{2}{|c|}{$r^{N}$} & \multicolumn{2}{|c|}{$\mathbf{J}(\mathbf{x})$} \\
\hline \multicolumn{2}{|c|}{$V: 1$} & \multicolumn{2}{|c|}{0,1306} & \multicolumn{2}{|c|}{$-9,9307$} & \multicolumn{2}{|c|}{98,9944} \\
\hline \multicolumn{2}{|c|}{$\mathrm{V}: 2$} & \multicolumn{2}{|c|}{0,1298} & \multicolumn{2}{|c|}{9,9287} & \multicolumn{2}{|c|}{99,0511} \\
\hline \multicolumn{2}{|c|}{$V: 5$} & \multicolumn{2}{|c|}{0,1298} & \multicolumn{2}{|c|}{9,9311} & \multicolumn{2}{|c|}{99,0474} \\
\hline \multicolumn{2}{|c|}{$\mathrm{V}: 8$} & \multicolumn{2}{|c|}{0,1312} & \multicolumn{2}{|c|}{$-9,9265$} & \multicolumn{2}{|c|}{98,9893} \\
\hline \multicolumn{2}{|c|}{$V: 11$} & \multicolumn{2}{|c|}{0,1277} & \multicolumn{2}{|c|}{9,9321} & \multicolumn{2}{|c|}{99,1097} \\
\hline \multicolumn{2}{|c|}{$V: 13$} & \multicolumn{2}{|c|}{0,132} & \multicolumn{2}{|c|}{$-9,9199$} & \multicolumn{2}{|c|}{98,9321} \\
\hline \multicolumn{2}{|c|}{$V: 20$} & \multicolumn{2}{|c|}{0,1364} & \multicolumn{2}{|c|}{$-9,9171$} & \multicolumn{2}{|c|}{98,8226} \\
\hline \multicolumn{2}{|c|}{$V: 28$} & 0 , & & 9,93 & & & 97 \\
\hline
\end{tabular}

Analisando os resultados da tabela 5.13, verificamos que o teste do maior resíduo normalizado e, também, o teste do índice $J(\mathrm{x})$, falharam na detecção de EGs em medidas com índice UI maior ou igual a 3,5053 (casos indicados em vermelho). Já para as medidas com índice $U I$ abaixo de 3,5053 e maior, ou igual, a 0,4553 apenas o teste do índice $J(x)$ falhou em detectar EGs (casos em realce amarelo).

A tabela 5.14, a seguir, apresenta os valores do índice $J(x)$ e os valores do índice UI e resíduo normalizado, para a medida portadora de EG em cada caso, considerando EG simples de 20 sigmas.

Tabela 5.14: Resultados numéricos da adição de EG de $20 \sigma$ para cada medida (uma medida de cada vez) considerando o sistema de medição da tabela 5.10

Limiar de detecção do índice $J(\mathrm{x}): \lambda=\chi_{63 ;(1-0,05)}^{2} \cong 81$

\begin{tabular}{c|c|c|c|c|c|c|c}
\hline \multicolumn{4}{c|}{ Medidas de Potência Ativa } & \multicolumn{3}{c}{ Medidas de Potência Reativa } \\
\hline $\begin{array}{c}\text { Medida } \\
\text { com erro }\end{array}$ & $U I$ & $r^{N}$ & $\mathbf{J}(\mathbf{x})$ & $\begin{array}{c}\text { Medida } \\
\text { com erro }\end{array}$ & $U I$ & $r^{N}$ & $\mathbf{J}(\mathbf{x})$ \\
\hline IA:1 & 0,3972 & $-18,5948$ & 346,0896 & IR:1 & 1,072 & $-13,6478$ & 186,8038 \\
\hline IA:2 & 7,1642 & 2,8066 & 8,3904 & IR:2 & 0,3613 & 18,8101 & 354,3918 \\
\hline IA:5 & 0,3437 & 18,9143 & 358,2122 & IR:5 & 0,9814 & 14,4115 & 203,6668 \\
\hline IA:6 & --- & --- & --- & IR:6 & --- & --- & --- \\
\hline IA:7 & 1,4832 & 11,1603 & 125,1459 & IR:7 & 0,5336 & 17,644 & 311,9936 \\
\hline IA:8 & 0,7287 & 16,1389 & 260,9595 & IR:8 & 1,0637 & $-13,7233$ & 188,2305 \\
\hline IA:9 & --- & --- & --- & IR:9 & --- & --- & --- \\
\hline IA:11 & --- & --- & --- & IR:11 & 0,6236 & 16,9561 & 288,0493 \\
\hline
\end{tabular}




\begin{tabular}{|c|c|c|c|c|c|c|c|}
\hline IA:12 & 3,0391 & $-6,2896$ & 40,0766 & IR:12 & 3,4985 & 5,5203 & 31,0673 \\
\hline IA:13 & --- & --- & --- & IR:13 & 0,8821 & $-15,0044$ & 225,5136 \\
\hline IA:15 & 5,3643 & 3,6747 & 14,0571 & IR:15 & 6,9765 & $\begin{array}{l}-2,8641 \\
\end{array}$ & 8,781 \\
\hline IA: 16 & 4,4537 & 4,3968 & 19,8521 & IR:16 & 2,5703 & 7,2892 & 53,6216 \\
\hline IA: 17 & 7,2173 & 2,7656 & 8,1576 & IR:17 & 4,268 & $-4,5393$ & 21,1248 \\
\hline IA:19 & 1,1173 & $-13,3393$ & 178,4277 & IR:19 & 1,4132 & 11,5563 & 133,9429 \\
\hline IA:21 & 1,057 & $-13,7587$ & 189,8304 & IR:21 & 0,926 & 14,7692 & 218,6851 \\
\hline IA:22 & --- & --- & --- & IR:22 & --- & --- & --- \\
\hline IA:23 & 2,1211 & 8,5561 & 73,7296 & IR:23 & 1,3399 & $-11,9834$ & 144,1281 \\
\hline IA:24 & 0,9931 & 14,3109 & 205,3139 & IR:24 & 0,7816 & $-15,8238$ & 250,869 \\
\hline IA:25 & --- & --- & --- & IR:25 & --- & --- & --- \\
\hline IA:27 & --- & --- & --- & IR:27 & --- & --- & --- \\
\hline IA: 28 & --- & --- & --- & IR:28 & --- & --- & --- \\
\hline IA:30 & 0,3867 & 18,663 & 348,8168 & IR:30 & 1,3314 & $-12,0523$ & 145,7801 \\
\hline FA:1-2 & 0,2964 & $-19,1713$ & 368,1138 & FR:1-2 & 0,4021 & $-18,5608$ & 345,0298 \\
\hline FA:1-3 & 0,4391 & $-18,3118$ & 335,994 & FR:1-3 & 1,4555 & 11,3197 & 128,6933 \\
\hline FA:2-4 & 0,3455 & $-18,9065$ & 358,0756 & FR:2-4 & 0,7342 & 16,1477 & 261,1147 \\
\hline FA:4-2 & 0,3321 & 18,9859 & 360,9984 & FR:4-2 & 1,332 & 12,0966 & 144,6894 \\
\hline FA:2-5 & 0,2356 & 19,4843 & 380,0055 & FR:2-5 & 1,8032 & 9,6756 & 94,1722 \\
\hline FA:2-6 & 0,2799 & $-19,2421$ & 371,2893 & FR:2-6 & 0,8209 & $-15,5722$ & 243,9302 \\
\hline FA:3-4 & 2,6613 & $\begin{array}{l}-7,1137 \\
\end{array}$ & 51,0909 & FR:3-4 & 9,2046 & $\begin{array}{l}-2,1271 \\
\end{array}$ & 5,0918 \\
\hline FA:4-6 & 0,4279 & $-18,3718$ & 338,0251 & FR:4-6 & 0,5898 & 17,1101 & 293,4955 \\
\hline FA:4-12 & 0,3041 & 19,1427 & 366,9644 & FR:4-12 & 0,7097 & $-16,3946$ & 272,3011 \\
\hline FA:5-7 & 1,3794 & $-11,7725$ & 138,9591 & FR:5-7 & 0,427 & 18,3802 & 338,3885 \\
\hline FA:6-7 & 0,479 & $-18,0341$ & 325,8095 & FR:6-7 & 2,3294 & 8,0155 & 64,4563 \\
\hline FA:6-8 & 0,6012 & 17,1679 & 295,2616 & FR:6-8 & 0,7495 & 15,9587 & 255,2081 \\
\hline FA:6-9 & 0,4011 & 18,5715 & 345,3868 & FR:6-9 & 0,8311 & 15,3064 & 234,783 \\
\hline FA:6-28 & 0,4102 & $-18,4506$ & 340,9103 & FR:6-28 & --- & --- & --- \\
\hline FA:8-28 & 0,8571 & $-15,1575$ & 230,2715 & FR:8-28 & 0,3867 & 18,5993 & 346,9686 \\
\hline FA:9-11 & --- & --- & --- & FR:9-11 & 0,5762 & 17,3335 & 300,9314 \\
\hline FA:9-10 & 0,4143 & $-18,4703$ & 341,6474 & FR:9-10 & 1,2972 & 12,1145 & 147,2706 \\
\hline FA:12-13 & --- & --- & --- & FR:12-13 & 0,8428 & 15,2824 & 234,0008 \\
\hline FA:12-15 & 0,5094 & 17,8184 & 317,9896 & FR:12-15 & 0,7768 & $-15,839$ & 252,9515 \\
\hline FA:12-16 & 0,7233 & $-16,1828$ & 262,4326 & FR:12-16 & 1,1102 & 13,4088 & 180,6887 \\
\hline FA:15-18 & 0,994 & $-14,19$ & 201,8722 & FR:15-18 & 0,9969 & 14,1642 & 201,0986 \\
\hline FA:18-15 & 0,9708 & $-14,3548$ & 206,5719 & FR:18-15 & 0,9842 & $-14,2631$ & 203,9603 \\
\hline FA:15-23 & 0,6156 & $-17,0308$ & 290,5667 & FR:15-23 & 1,0933 & 13,4478 & 181,3261 \\
\hline FA:18-19 & 2,8377 & $-6,6476$ & 44,7244 & FR:18-19 & 1,4163 & $-11,5386$ & 133,6462 \\
\hline FA:19-20 & 1,1173 & 13,3375 & 178,4209 & FR:19-20 & 1,4132 & 11,5494 & 133,9952 \\
\hline FA:21-22 & 1,6062 & 10,6362 & 113,6443 & FR:21-22 & 1,4531 & 11,3204 & 128,7211 \\
\hline FA:22-24 & 0,7091 & $-16,3922$ & 269,2324 & FR:22-24 & 1,215 & 12,5798 & 158,6848 \\
\hline FA:23-24 & 1,2875 & 12,2956 & 151,7257 & FR:23-24 & 0,9388 & $-14,6394$ & 214,8634 \\
\hline FA:24-25 & 1,1631 & $-12,941$ & 167,9601 & FR:24-25 & 1,0187 & 13,7328 & 189,0914 \\
\hline FA:25-26 & 1,6757 & $-10,6406$ & 113,6072 & FR:25-26 & 1,6301 & $-10,3327$ & 107,2549 \\
\hline FA:25-27 & 1,291 & $-12,645$ & 160,261 & FR:25-27 & 0,986 & $-14,3555$ & 206,5971 \\
\hline FA:27-29 & 0,7007 & $-16,4185$ & 270,0645 & FR:27-29 & 1,0633 & $-13,6245$ & 186,0475 \\
\hline FA:27-30 & 0,457 & 18,1693 & 330,6466 & FR:27-30 & 0,4783 & $-18,0129$ & 324,9533 \\
\hline FA:28-27 & 0,4266 & $-18,375$ & 338,1674 & FR:28-27 & 0,4547 & 17,9753 & 323,6547 \\
\hline FA:29-30 & 2,0916 & $-8,6267$ & 74,9353 & FR:29-30 & 0,6689 & $-16,6449$ & 277,5677 \\
\hline \multicolumn{8}{|c|}{ Medidas de Tensão } \\
\hline \multicolumn{2}{|c|}{ Medida com erro } & \multicolumn{2}{|c|}{$U I$} & \multicolumn{2}{|c|}{$r^{N}$} & \multicolumn{2}{|c|}{$\mathbf{J}(\mathbf{x})$} \\
\hline \multicolumn{2}{|c|}{$\mathrm{V}: 1$} & \multicolumn{2}{|c|}{0,1262} & \multicolumn{2}{|c|}{$-19,8523$} & \multicolumn{2}{|c|}{394,482} \\
\hline
\end{tabular}




\begin{tabular}{c|c|c|c}
\hline $\mathrm{V}: 2$ & 0,1254 & 19,8553 & 394,6212 \\
\hline $\mathrm{V}: 5$ & 0,1254 & 19,8619 & 394,6176 \\
\hline $\mathrm{V}: 8$ & 0,1268 & $-19,8438$ & 394,4194 \\
\hline $\mathrm{V}: 11$ & 0,1235 & 19,8576 & 394,8317 \\
\hline $\mathrm{V}: 13$ & 0,1276 & $-19,8462$ & 394,2898 \\
\hline $\mathrm{V}: 20$ & 0,1319 & $-19,8396$ & 393,8750 \\
\hline $\mathrm{V}: 28$ & 0,1271 & 19,8565 & 394,4849 \\
\hline
\end{tabular}

Com a adição de EG de $20 \sigma$ às medidas, os dois métodos de detecção de EGs falharam apenas para detectar EG nas medidas IA:2, IA:17, IR:15 e FR:3-4 (em vermelho), ou seja, um número inferior de casos com problemas de detecção de EG, se comparado aos resultados da tabela 5.13. Ademais, como pode ser visto em realce amarelo, o método do índice $J(x)$ também falhou menos em detectar EGs, se comparado aos resultados da adição de $10 \sigma$.

\subsubsection{Testes com Ruído Gaussiano e sem Erro Grosseiro}

O intuito desta subseção é verificar a influência de ruídos gaussianos nos índices UIs das medidas, para o sistema de 30 barras do IEEE. Para tal propósito, serão realizadas simulações, considerando a adição de ruído gaussiano às medidas do sistema de medição da tabela 5.10 .

Assim, o vetor de medidas $\underline{z}$ é calculado da seguinte forma:

$$
\underline{z}_{i}^{m}=\underline{z}_{i}^{f c}+3 \cdot \sigma_{i} \cdot \alpha
$$

onde:

- $\sigma_{i}$ é o desvio padrão da medida $i$;

- $\alpha$ é uma variável aleatória com distribuição normal padrão $N(0,1)$;

- $\underline{z}_{i}^{f c}$ é o valor obtido diretamente do programa de fluxo de carga;

- $\underline{z}_{i}^{m}$ é o valor da medida $i$.

A partir da execução do estimador WLS, para 100 amostras de medidas com ruído, obtiveram-se as médias e desvios padrões do índice $U I$, resíduo normalizado e índice $J(x)$, sendo os mesmos tabelados a seguir (tabela 5.15). 
Tabela 5.15: Resultados do índice $U I$, resíduo normalizado e índice J(x) para 100 amostras de medidas com ruído e sem EG

\begin{tabular}{|c|c|c|c|c|c|c|c|c|c|}
\hline \multicolumn{5}{|c|}{ Medidas de Potência Ativa } & \multicolumn{5}{|c|}{ Medidas de Potência Reativa } \\
\hline \multirow{2}{*}{ Medida } & \multicolumn{2}{|c|}{$\boldsymbol{U I}$} & \multicolumn{2}{|c|}{$r^{N}$} & \multirow{2}{*}{ Medida } & \multicolumn{2}{|c|}{$\boldsymbol{U I}$} & \multicolumn{2}{|c|}{$r^{N}$} \\
\hline & Média & $\begin{array}{l}\text { Desvio } \\
\text { padrão }\end{array}$ & ia & $\begin{array}{l}\text { Desvio } \\
\text { padrão }\end{array}$ & & Média & $\begin{array}{c}\text { Desvio } \\
\text { padrão }\end{array}$ & Média & $\begin{array}{l}\text { Desvio } \\
\text { padrão }\end{array}$ \\
\hline IA:1 & 0,395688 & 0,002227 & $-0,0288$ & 0,79733 & IR:1 & 1,072111 & 0,000552 & $-0,11791$ & 0,850648 \\
\hline IA:2 & 7,143286 & 0,020756 & 0,002405 & 0,913694 & IR:2 & 0,361094 & 0,000925 & $-0,17034$ & 0,901524 \\
\hline IA:5 & 0,342851 & 0,001162 & 0,03746 & 0,836773 & IR:5 & 0,979955 & 0,006702 & 0,047592 & 0,865344 \\
\hline IA:6 & --- & --- & --- & --- & IR:6 & --- & --- & --- & --- \\
\hline IA:7 & 1,482241 & 0,000578 & 0,040273 & 0,913312 & IR:7 & 0,533222 & 0,000669 & $-0,09495$ & 0,895398 \\
\hline IA:8 & 0,728597 & $5,02 \mathrm{E}-05$ & 0,015196 & 0,855044 & IR:8 & 1,062839 & 0,001813 & $-0,03205$ & 0,892516 \\
\hline IA:9 & --- & --- & --- & --- & IR:9 & --- & --- & --- & --- \\
\hline IA:11 & --- & --- & --- & --- & IR:11 & 0,623952 & 0,000234 & 0,031673 & 0,90651 \\
\hline IA:12 & 3,038629 & 0,000816 & $-0,06836$ & 0,923229 & IR:12 & 3,496361 & 0,004465 & 0,051485 & 0,864444 \\
\hline IA:13 & --- & --- & --- & --- & IR:13 & 0,882112 & 0,000373 & 0,019273 & 0,936795 \\
\hline IA:15 & 5,361733 & 0,001113 & 0,02456 & 0,79524 & IR:15 & 6,972302 & 0,009302 & $-0,06587$ & 0,910868 \\
\hline IA:16 & 4,45335 & 0,000547 & $-0,08939$ & 0,858037 & IR:16 & 2,568478 & 0,002497 & 0,094261 & 0,904719 \\
\hline IA:17 & 7,216082 & 0,001597 & $-0,0783$ & 0,875716 & IR:17 & 4,26524 & 0,003653 & 0,095631 & 0,899209 \\
\hline IA:19 & 1,1173 & 0 & 0,022945 & 0,847385 & IR:19 & 1,413184 & $4,43 \mathrm{E}-05$ & 0,018493 & 0,980571 \\
\hline IA:21 & 1,056897 & 0,000114 & 0,015393 & 0,833803 & IR:21 & 0,925922 & 0,000178 & 0,065757 & 0,81109 \\
\hline IA:22 & --- & --- & --- & --- & IR:22 & --- & --- & --- & --- \\
\hline IA:23 & 2,120954 & 0,00011 & 0,112838 & 0,951289 & IR:23 & 1,339839 & $6,50 \mathrm{E}-05$ & 0,08103 & 0,771166 \\
\hline IA:24 & 0,993019 & $6,15 \mathrm{E}-05$ & 0,307088 & 0,812353 & IR:24 & 0,780326 & 0,001758 & 0,124496 & 0,854601 \\
\hline IA:25 & --- & --- & --- & --- & IR:25 & --- & --- & --- & --- \\
\hline IA:27 & --- & --- & --- & --- & IR:27 & --- & --- & --- & --- \\
\hline IA:28 & --- & --- & --- & --- & IR:28 & --- & --- & --- & --- \\
\hline IA:30 & 0,386603 & $7,71 \mathrm{E}-05$ & $-0,00289$ & 0,940544 & IR:30 & 1,331303 & 0,000269 & $-0,04816$ & 0,895023 \\
\hline FA:1-2 & 0,295485 & 0,001459 & 0,0487 & 0,815392 & FR:1-2 & 0,40212 & 0,000239 & 0,014118 & 0,886737 \\
\hline FA:1-3 & 0,437481 & 0,002202 & 0,074637 & 0,855376 & FR:1-3 & 1,456269 & 0,001216 & 0,063626 & 0,818282 \\
\hline FA:2-4 & 0,344234 & 0,001757 & $-0,12292$ & 0,924154 & FR:2-4 & 734021 & 0,000512 & $-0,03939$ & 0,901396 \\
\hline FA:4-2 & 0,330783 & 0,001813 & $-0,00625$ & 0,829186 & FR:4-2 & 1,323746 & 0,014231 & $-0,04272$ & 0,951124 \\
\hline FA:2-5 & 0,234922 & 0,000952 & 0,017059 & 0,850854 & FR:2-5 & 1,802904 & 0,005443 & 0,116722 & 0,813722 \\
\hline FA:2-6 & 0,278876 & 0,001557 & 0,167754 & 0,896007 & FR:2-6 & 0,817968 & 0,005739 & $-0,06801$ & 0,8398 \\
\hline FA:3-4 & 2,654717 & 0,006008 & 0,074989 & 0,86637 & FR:3-4 & 9,20812 & 0,005522 & 0,030011 & 0,821554 \\
\hline FA:4-6 & 0,427519 & 0,00017 & 0,127372 & 0,949775 & FR:4-6 & 0,589178 & 0,001353 & $-0,1422$ & 0,767917 \\
\hline FA:4-12 & 0,304084 & $6,47 \mathrm{E}-05$ & 0,01001 & 0,969713 & FR:4-12 & 0,703328 & 0,008679 & $-0,08757$ & 0,876582 \\
\hline FA:5-7 & 1,376807 & 0,001376 & 0,060719 & 0,875867 & FR:5-7 & 0,427054 & 0,000192 & $-0,03335$ & 0,809412 \\
\hline FA:6-7 & 0,478561 & 0,000244 & $-0,06933$ & 0,886215 & FR:6-7 & 2,328473 & 0,0035 & $-0,00531$ & 0,88865 \\
\hline FA:6-8 & 0,601205 & $2,97 \mathrm{E}-05$ & 0,126761 & 0,910001 & FR:6-8 & 0,749431 & $9,40 \mathrm{E}-05$ & $-0,11867$ & 0,866396 \\
\hline FA:6-9 & 0,401066 & $9,56 \mathrm{E}-05$ & 0,00298 & 0,960465 & FR:6-9 & 0,831024 & 0,000106 & $-0,19274$ & 0,889444 \\
\hline FA:6-28 & 0,41012 & $9,85 \mathrm{E}-05$ & $-0,17703$ & 0,818722 & FR:6-28 & --- & --- & --- & --- \\
\hline FA:8-28 & 0,857002 & $3,17 \mathrm{E}-05$ & 0,084132 & 0,850657 & FR:8-28 & 0,384209 & 0,00353 & $-0,13497$ & 0,887287 \\
\hline FA:9-11 & --- & --- & --- & --- & FR:9-11 & 0,576196 & 0,000101 & 0,184555 & 0,887093 \\
\hline FA:9-10 & 0,414319 & $9,29 \mathrm{E}-05$ & 0,003921 & 0,839777 & FR:9-10 & 1,297001 & 0,000161 & $-0,01937$ & 0,845022 \\
\hline FA:12-13 & --- & --- & --- & --- & FR:12-13 & 0,842577 & 0,000308 & $-0,01826$ & 0,88475 \\
\hline FA:12-15 & 0,509334 & 7,81E-05 & 0,06326 & 0,817441 & FR:12-15 & 0,774713 & 0,003569 & $-0,13427$ & 0,954863 \\
\hline FA:12-16 & 0,723235 & 0,000175 & $-0,06637$ & 0,90448 & FR:12-16 & 1,108696 & 0,002326 & 0,080292 & 0,888373 \\
\hline FA:15-18 & 0,99408 & $7,78 \mathrm{E}-05$ & 0,128486 & 0,927588 & FR:15-18 & 0,996892 & $9,18 \mathrm{E}-05$ & 0,11462 & 0,975534 \\
\hline FA:18-15 & 0,970671 & $9,88 \mathrm{E}-05$ & 0,139634 & 0,941023 & FR:18-15 & 0,984131 & $8,37 \mathrm{E}-05$ & 0,101588 & 0,975332 \\
\hline FA:15-23 & 0,615603 & $6,43 \mathrm{E}-05$ & 0,117868 & 0,958767 & FR:15-23 & 1,093406 & $9,62 \mathrm{E}-05$ & 0,009156 & 0,84335 \\
\hline FA:18-19 & 2,837814 & $9,85 \mathrm{E}-05$ & 0,023929 & 0,847212 & FR:18-19 & 1,416296 & $7,90 \mathrm{E}-05$ & 0,017429 & 0,981663 \\
\hline
\end{tabular}




\begin{tabular}{|c|c|c|c|c|c|c|c|c|c|}
\hline FA:19-20 & 1,1173 & 0 & $-0,02321$ & 0,847062 & FR:19-20 & 1,413172 & $4,51 \mathrm{E}-05$ & $-0,01795$ & 0,981293 \\
\hline FA:21-22 & 1,606199 & $2,25 \mathrm{E}-05$ & 0,057047 & 0,819951 & FR:21-22 & 1,453095 & $2,97 \mathrm{E}-05$ & 0,001463 & 0,741743 \\
\hline FA:22-24 & 0,70896 & 0,000102 & 0,043345 & 0,864235 & FR:22-24 & 1,214274 & 0,001094 & $-0,02155$ & 0,800054 \\
\hline FA:23-24 & 1,287115 & 0,00027 & 0,110886 & 0,855343 & FR:23-24 & 0,938797 & $4,37 \mathrm{E}-05$ & $-0,0954$ & \begin{tabular}{|c|}
0,804789 \\
\end{tabular} \\
\hline FA:24-25 & 1,162962 & 0,000119 & $-0,05671$ & 0,756563 & FR:24-25 & 1,0185 & 0,000395 & $-0,38221$ & 0,771317 \\
\hline FA:25-26 & 1,675738 & $5,28 \mathrm{E}-05$ & $-0,31693$ & 0,817176 & FR:25-26 & 1,62983 & 0,000411 & 0,090672 & 0,77761 \\
\hline FA:25-27 & 1,290796 & $9,63 \mathrm{E}-05$ & $-0,39008$ & 0,902341 & FR:25-27 & 0,986059 & 0,000109 & $-0,18358$ & 0,77958 \\
\hline FA:27-29 & 0,700544 & 0,00012 & 0,062368 & 0,889736 & FR:27-29 & 1,062996 & 0,001036 & 0,150545 & 0,818322 \\
\hline FA:27-30 & 0,456898 & $6,03 \mathrm{E}-05$ & 0,042484 & 0,894862 & FR:27-30 & 0,478267 & 0,00013 & $-0,01072$ & 0,8707 \\
\hline FA:28-27 & 0,426469 & 0,000129 & $-0,09603$ & 0,858845 & FR:28-27 & 0,454433 & 0,000466 & $-0,16728$ & 0,828309 \\
\hline FA:29-30 & 2,091582 & 0,000142 & 0,010391 & 0,83245 & FR:29-30 & 0,668903 & 0,000116 & 0,011357 & 0,87952 \\
\hline \multicolumn{10}{|c|}{ Medidas de Tensão } \\
\hline \multirow{2}{*}{\multicolumn{2}{|c|}{ Medida }} & \multicolumn{4}{|c|}{$\boldsymbol{U I}$} & \multicolumn{4}{|c|}{$r^{N}$} \\
\hline & & \multicolumn{2}{|c|}{ Média } & \multicolumn{2}{|c|}{ Desvio padrão } & \multicolumn{2}{|c|}{ Média } & \multicolumn{2}{|c|}{ Desvio padrão } \\
\hline \multicolumn{2}{|c|}{$\mathrm{V}: 1$} & \multicolumn{2}{|c|}{0,124598} & \multicolumn{2}{|c|}{0,002965} & \multicolumn{2}{|c|}{$-0,00176$} & \multicolumn{2}{|c|}{0,09086} \\
\hline \multicolumn{2}{|c|}{$\mathrm{V}: 2$} & \multicolumn{2}{|c|}{0,123779} & \multicolumn{2}{|c|}{0,002947} & \multicolumn{2}{|c|}{$-0,00208$} & \multicolumn{2}{|c|}{0,09035} \\
\hline \multicolumn{2}{|c|}{$\mathrm{V}: 5$} & \multicolumn{2}{|c|}{0,12379} & \multicolumn{2}{|c|}{0,002946} & \multicolumn{2}{|c|}{$-0,00247$} & \multicolumn{2}{|c|}{0,090239} \\
\hline \multicolumn{2}{|c|}{ V:8 } & \multicolumn{2}{|c|}{0,125108} & \multicolumn{2}{|c|}{0,00298} & \multicolumn{2}{|c|}{$-0,00207$} & \multicolumn{2}{|c|}{0,091533} \\
\hline \multicolumn{2}{|c|}{$\mathrm{V}: 11$} & \multicolumn{2}{|c|}{0,121829} & \multicolumn{2}{|c|}{0,002869} & \multicolumn{2}{|c|}{$-0,00115$} & 0,0 & 895 \\
\hline $\mathrm{V}: 1$ & & 0,12 & 904 & 0,00 & 978 & 0,001 & 026 & 0,09 & 3304 \\
\hline $\mathrm{V}: 2$ & & 0,13 & 153 & 0,00 & 088 & $-0,00$ & 049 & 0,09 & 4915 \\
\hline $\mathrm{V}: 2$ & & 0,12 & 454 & 0,00 & 998 & $-0,00$ & 178 & 0,09 & 1952 \\
\hline & & & & Índice & $(\mathbf{x}):$ & & & & \\
\hline & & $\mathbf{M}$ & dia $=40$ & 694; Desv & o padrã & $=7,22781$ & & & \\
\hline
\end{tabular}

Analisando os valores das médias e respectivos desvios padrões dos resíduos normalizados e do índice $J(\mathrm{x})$, apresentados na tabela 5.15, verifica-se que em nenhuma das simulações foi detectado EG. Além disso, as medidas com índice $U I$ elevado apresentaram os maiores valores de desvio padrão de UI. Por exemplo, a medida IA:2 apresenta o maior $U I$ médio, igual a 7,143286, e o maior desvio padrão para $U I$ dentre as demais medidas, igual a 0,020756 .

\subsubsection{Identificação do Nível de Detecção de Erro Grosseiro: Medidas com Ruído}

Apresentaremos, nesta subseção, os valores médios dos resultados da identificação do nível de detecção de EG, considerando 100 amostras de ruído em medidas.

Para as simulações em questão, o limiar de detecção de EG para o índice $J(x)$ é $\lambda=\chi_{63 ;(1-0,05)}^{2} \cong 81$, considerando uma probabilidade de falso alarme $\alpha=5 \%$ e o grau de liberdade $m-n=63$. Já o limiar de detecção de EG, adotado para o teste dos resíduos normalizados, é $\beta=3,0$. 
A tabela 5.16, a seguir, mostra os valores médios dos resultados da identificação do nível de detecção, para as medidas do sistema em análise.

Tabela 5.16: Identificação do nível de detecção de erro para 100 amostras de medidas com ruído

$$
\text { Limiar de detecção do índice } J(\mathrm{x}): \lambda=\chi_{63 ;(1-0,05)}^{2} \cong 81
$$

\begin{tabular}{|c|c|c|c|c|c|c|c|c|c|c|c|c|c|}
\hline \multicolumn{7}{|c|}{ Medidas de Potência Ativa } & \multicolumn{7}{|c|}{ Medidas de Potência Reativa } \\
\hline \multirow{2}{*}{ Medida } & \multicolumn{2}{|c|}{$\boldsymbol{U I}$} & \multicolumn{2}{|c|}{$\begin{array}{c}\text { Nível } \\
\text { Detecção }\end{array}$} & \multicolumn{2}{|c|}{$J(\mathbf{x})$} & \multirow{2}{*}{ Medida } & \multicolumn{2}{|c|}{$\boldsymbol{U I}$} & \multicolumn{2}{|c|}{$\begin{array}{c}\text { Nível } \\
\text { Detecção }\end{array}$} & \multicolumn{2}{|c|}{$J(\mathbf{x})$} \\
\hline & Média & $\begin{array}{l}\text { Desvio } \\
\text { Padrão }\end{array}$ & Média & $\begin{array}{l}\text { Desvio } \\
\text { Padrão }\end{array}$ & Média & $\begin{array}{l}\text { Desvio } \\
\text { Padrão }\end{array}$ & & Iédia & \begin{tabular}{|l|} 
Desvio \\
Padrão
\end{tabular} & Iédia & $\begin{array}{l}\text { Desvio } \\
\text { Padrão }\end{array}$ & Média & $\begin{array}{l}\text { Desvio } \\
\text { Padrão }\end{array}$ \\
\hline IA:1 & 0,3950 & 0,00226 & 3,314 & 0,30650 & 48,6599 & 6,8450 & IR:1 & 1,0720 & 0,00057 & 4,498 & 1,01801 & 48,1352 & 6,78548 \\
\hline IA:2 & 7,1392 & 0,02226 & 21,162 & 6,21388 & 47,7822 & 7,0256 & IR:2 & 0,3608 & 0,00098 & 3,342 & 0,29989 & 48,6522 & 6,831747 \\
\hline IA:5 & 0,3425 & 0,00117 & 3,276 & 0,25390 & 48,6334 & 6,8890 & IR:5 & 0,9777 & 0,00696 & 4,298 & 0,74100 & 48,4621 & 6,924994 \\
\hline IA:6 & --- & --- & --- & --- & --- & --- & IR:6 & --- & --- & --- & --- & --- & --- \\
\hline IA:7 & 1,4821 & 0,0006 & 5,106 & 1,14077 & 47,9664 & 6,8167 & IR:7 & 0,5330 & 0,00066 & 3,522 & 0,39888 & 48,6196 & 6,969844 \\
\hline IA:8 & 0,7285 & 5,1E-05 & 3,776 & 0,5558 & 48,4393 & 6,9295 & IR:8 & 1,0623 & 0,00182 & 4,372 & 0,90655 & 48,1068 & 6,66442 \\
\hline IA:9 & --- & --- & --- & --- & --- & --- & IR:9 & --- & --- & --- & --- & --- & --- \\
\hline IA:11 & --- & --- & --- & --- & --- & --- & IR:11 & 0,6238 & 0,00026 & 3,716 & 0,50546 & 48,2739 & 6,640275 \\
\hline IA:12 & 3,0384 & 0,00087 & 9,638 & 2,8733 & 47,4769 & 6,7901 & IR:12 & 3,4950 & \begin{tabular}{|c|}
0,00468 \\
\end{tabular} & 10,524 & 2,83908 & 47,4526 & 6,709644 \\
\hline IA:13 & --- & --- & --- & --- & --- & --- & IR:13 & 0,8819 & 0,00037 & 3,98 & 0,71942 & 48,4027 & 7,010405 \\
\hline IA:15 & 5,3617 & 0,00116 & 15,604 & 5,11989 & 47,3981 & 6,6426 & IR:15 & 6,9695 & 0,00988 & 20,102 & 6,33372 & 47,0927 & 6,671519 \\
\hline IA:16 & 4,4531 & 0,00049 & 12,566 & 4,30738 & 47,2758 & 6,6413 & IR:16 & 2,5677 & 0,00255 & 8,404 & 2,13257 & 47,7546 & 6,840282 \\
\hline IA:17 & 7,2156 & 0,00151 & 20,22 & 6,64387 & 7,3106 & 6,6673 & IR:17 & 4,2642 & 0,00370 & 13,236 & 3,65937 & 47,5911 & 6,874755 \\
\hline IA:19 & 1,1173 & 0 & 4,574 & 1,02283 & 48,2345 & 6,9334 & IR:19 & 1,41315 & $4,1 \mathrm{E}-05$ & 5,024 & 1,16594 & 48,2659 & 6,734871 \\
\hline IA:21 & 1,0568 & 0,00011 & 4,41 & 0,79461 & 47,8818 & 6,8959 & & 0,9250 & 0,00016 & 4,31 & 0,72550 & 47,7468 & 605937 \\
\hline IA:22 & --- & --- & --- & --- & --- & --- & & -- & 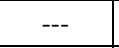 & - & --- & --- & --- \\
\hline IA:23 & 2,1209 & 0,00011 & 7,334 & 1,75235 & 47,5248 & 6,7409 & IR:23 & 1,3398 & $1 \mathrm{E}-05$ & 4,976 & 1,29249 & 47,7589 & 6,759055 \\
\hline IA:24 & 0,9930 & 5,9E-05 & 4,54 & 0,92659 & 48,0207 & 6,8745 & IR:24 & 0,7798 & 0,00181 & 3,792 & 0,66418 & 48,5086 & 6,969181 \\
\hline IA:25 & --- & --- & --- & --- & --- & --- & IR:25 & --- & --- & --- & --- & --- & --- \\
\hline IA:27 & -- & --- & --- & --- & --- & --- & IR:27 & --- & --- & --- & --- & --- & --- \\
\hline IA:28 & --- & --- & --- & --- & --- & --- & IR:28 & --- & --- & --- & --- & --- & --- \\
\hline IA:30 & 0,3866 & $7,0 \mathrm{E}-05$ & 3,368 & 0,32994 & 48,3316 & 6,5936 & IR:30 & 1,3312 & 0,00030 & 4,734 & 1,18579 & 47,8167 & 6,899495 \\
\hline FA:1-2 & 0,2950 & 0,00147 & 3,23 & 0,22984 & 48,6000 & 6,8643 & FR:1-2 & 0,4020 & 0,00025 & 3,37 & 0,33196 & 48,6629 & 6,512221 \\
\hline FA:1-3 & 0,4368 & 0,00225 & 3,366 & 0,33279 & 48,7699 & 6,9078 & FR:1-3 & 1,4561 & 0,00135 & 5,198 & 1,33862 & 47,5202 & 6,871538 \\
\hline FA:2-4 & 0,3437 & 0,00178 & 3,274 & 0,26462 & 48,5947 & 6,8647 & FR:2-4 & 0,7338 & 0,00052 & 3,8 & 0,63053 & 48,3373 & 6,557249 \\
\hline FA:4-2 & 0,3302 & 0,00184 & 3,284 & 0,26693 & 48,4355 & 6,7575 & FK:4-2 & 1,3203 & 0,01474 & 4,83 & 1,07058 & 48,1460 & 6,534829 \\
\hline FA:2-5 & 0,2346 & 0,00097 & 3,172 & 0,16822 & 48,7700 & 7,0336 & FR:2-5 & 11 & 0,00573 & 6,33 & 1,48674 & 48,0185 & 6,85872 \\
\hline FA:2-6 & 0,2784 & 0,00158 & 3,22 & 0,21367 & 48,5944 & 6,5991 & FR:2-6 & 0,8163 & \begin{tabular}{|c|}
0,00578 \\
\end{tabular} & 4,124 & 0,67016 & 48,3096 & 6,544104 \\
\hline FA:3-4 & 2,653 & 0,00681 & 8,404 & 2,35924 & 47,8467 & 6,8603 & FR:3-4 & 9,2078 & 0,00624 & 26,718 & 8,04867 & 47,1423 & 6,870454 \\
\hline FA:4-6 & 0,4275 & 0,00018 & 3,376 & 0,31046 & 48,3806 & 6,6383 & FR:4-6 & 0,5887 & 0,00131 & 3,414 & 0,36653 & 48,7013 & 6,763887 \\
\hline FA:4-12 & 0,3040 & $6,9 \mathrm{E}-05$ & 3,28 & 0,25105 & 48,5567 & 6,7350 & FR:4-12 & 0,7008 & \begin{tabular}{|l|}
0,00898 \\
\end{tabular} & 3,822 & 0,56489 & 48,5774 & 6,795018 \\
\hline FA:5-7 & 1,3766 & 0,00141 & 5,118 & 1,22502 & 48,1124 & 6,8836 & FR:5-7 & 0,4269 & 0,00021 & 3,388 & 0,33129 & 48,6367 & 6,916907 \\
\hline FA:6-7 & 0,4785 & 0,00025 & 3,36 & 0,3363 & 48,6372 & 6,6427 & FR:6-7 & 2,3275 & 0,00362 & 7,308 & 2,09596 & 47,7952 & 6,990234 \\
\hline FA:6-8 & 0,6012 & $3,0 \mathrm{E}-05$ & 3,654 & 0,40785 & 48,3263 & 6,7005 & FR:6-8 & 0,7494 & $9,1 \mathrm{E}-05$ & 3,7 & 0,54272 & 48,3493 & 6,745756 \\
\hline FA:6-9 & 0,4010 & 0,00010 & 3,398 & 0,33814 & 48,4360 & 6,6039 & FR:6-9 & 0,8310 & $8,6 \mathrm{E}-05$ & 3,914 & 0,63960 & 48,5119 & 6,938595 \\
\hline FA:6-28 & 0,4101 & 0,00011 & 3,382 & 0,30230 & 48,5207 & 6,7489 & FR:6-28 & --- & --- & --- & --- & --- & --- \\
\hline FA:8-28 & 0,8570 & $3,6 \mathrm{E}-05$ & 3,982 & 0,69404 & 48,5201 & 6,8454 & FR:8-28 & 0,3832 & 0,00358 & 3,284 & 0,26081 & 48,7700 & 6,821783 \\
\hline FA:9-11 & --- & --- & --- & --- & --- & --- & FR:9-11 & 0,5761 & 9,6E-05 & 3,604 & 0,46621 & 48,4947 & 6,77459 \\
\hline FA:9-10 & 0,4143 & 0,00010 & 3,434 & 0,32790 & 48,5661 & 6,9860 & FR:9-10 & 1,2969 & 0,00012 & 5,17 & 1,00839 & 48,1484 & 6,745351 \\
\hline FA:12-13 & --- & --- & --- & --- & --- & --- & FR:12-13 & 0,8425 & 0,00035 & 3,986 & 0,74141 & 48,5870 & 6,880398 \\
\hline FA:12-15 & 0,5093 & $8,4 \mathrm{E}-05$ & 3,474 & 0,41648 & 48,0250 & 6,5230 & FR:12-15 & 0,7736 & 0,00372 & 3,762 & 0,62325 & 47,9423 & 6,791792 \\
\hline
\end{tabular}




\begin{tabular}{|c|c|c|c|c|c|c|c|c|c|c|c|c|c|}
\hline FA:12-16 & 0,7231 & 0,00017 & 3,808 & 0,60830 & 47,8317 & 6,6467 & FR:12-16 & 1,1080 & 0,00239 & 4,408 & 1,02056 & 48,0671 & 6,921689 \\
\hline FA:15-18 & 0,9940 & $8,8 \mathrm{E}-05$ & 4,342 & 0,90488 & 48,0233 & 6,9486 & FR:15-18 & 0,9968 & \begin{tabular}{|l|}
0,00010 \\
\end{tabular} & 4,434 & \begin{tabular}{|c|}
0,79088 \\
\end{tabular} & 48,1351 & 6,816097 \\
\hline FA:18-15 & 0,9707 & 0,00011 & 4,072 & 0,80127 & 48,1717 & 6,8748 & FR:18-15 & 0,9841 & $8,5 \mathrm{E}-05$ & 4,188 & 0,77580 & 48,1703 & 6,594161 \\
\hline FA:15-23 & 0,6155 & $6,5 \mathrm{E}-05$ & 3,622 & 0,48650 & 48,3572 & 6,9216 & FR:15-23 & 1,0934 & 0,00010 & 4,632 & 0,96555 & 47,9645 & 6,748822 \\
\hline FA:18-19 & 2,8377 & 0,0001 & 8,37 & 2,46582 & 47,9025 & 7,0137 & FR:18-19 & 1,4162 & $7,8 \mathrm{E}-05$ & 5,374 & 1,10376 & 48,0237 & 6,790027 \\
\hline FA:19-20 & 1,1173 & $6,5 \mathrm{E}-05$ & 4,386 & 0,81958 & 48,3361 & 6,8041 & FR:19-20 & 1,4131 & $4,8 \mathrm{E}-05$ & 5,398 & 1,35929 & 48,1345 & 6,79987 \\
\hline FA:21-22 & 1,6062 & $2,2 \mathrm{E}-05$ & 5,708 & 1,52644 & 47,6569 & 6,9447 & FR:21-22 & 1,4530 & $4,2 \mathrm{E}-05$ & 5,09 & 1,25798 & 47,6705 & 6,686073 \\
\hline FA:22-24 & 0,7089 & 0,00011 & 3,858 & 0,59104 & 48,3925 & 6,8177 & FR:22-24 & 1,2139 & 0,00113 & 4,718 & 0,93077 & 48,0739 & 6,989724 \\
\hline FA:23-24 & 1,2870 & 0,00027 & 5,012 & 1,16958 & 47,9056 & 6,8459 & FR:23-24 & 0,9387 & $4,8 \mathrm{E}-05$ & 4,216 & 0,73782 & 48,3170 & 6,865534 \\
\hline FA:24-25 & 1,1629 & 0,00012 & 4,846 & 0,87055 & 48,1192 & 6,9453 & FR:24-25 & 1,0183 & 0,00037 & 4,7 & 0,79924 & 48,2285 & 6,875571 \\
\hline FA:25-26 & 1,6757 & $5,0 \mathrm{E}-05$ & 6,702 & 1,08711 & 47,7376 & 6,9166 & FR:25-26 & 1,6297 & \begin{tabular}{|l|}
0,00041 \\
\end{tabular} & 5,438 & \begin{tabular}{|l|}
1,18294 \\
\end{tabular} & 48,1714 & 6,86149 \\
\hline FA:25-27 & 1,2908 & 0,00010 & 4,23 & 0,92085 & 48,0123 & 6,9601 & FR:25-27 & 0,9860 & 0,00012 & 4,102 & 0,64666 & 48,4529 & 6,662873 \\
\hline FA:27-29 & 0,7005 & 0,00011 & 3,946 & 0,58593 & 48,2006 & 6,6200 & FR:27-29 & 1,0626 & 0,00110 & 4,318 & 0,8200 & 48,3621 & 6,822681 \\
\hline FA:27-30 & 0,4568 & $6,3 \mathrm{E}-05$ & 3,506 & 0,38816 & 48,5837 & 6,8151 & FR:27-30 & 0,4782 & 0,00012 & 3,366 & 0,32168 & 48,3819 & 6,80003 \\
\hline FA:28-27 & 0,4264 & 0,00014 & 3,458 & 0,34500 & 48,4513 & 6,6336 & FR:28-27 & 0,4542 & 0,00050 & 3,592 & 0,28698 & 48,5289 & 6,827373 \\
\hline FA:29-30 & 2,0915 & 0,00015 & 7,22 & 1,78693 & 47,8465 & 6,7482 & FR:29-30 & 0,6688 & 0,00011 & 3,818 & 0,53036 & 48,2020 & 6,921818 \\
\hline \multicolumn{14}{|c|}{ Medidas de Tensão } \\
\hline \multirow{2}{*}{\multicolumn{2}{|c|}{ Medida }} & \multicolumn{4}{|c|}{$\boldsymbol{U I}$} & \multicolumn{4}{|c|}{$\begin{array}{c}\text { Nível } \\
\text { Detecção }\end{array}$} & \multicolumn{4}{|c|}{$J(\mathbf{x})$} \\
\hline & & \multicolumn{2}{|c|}{ Média } & \multicolumn{2}{|c|}{ Desvio Padrão } & \multicolumn{2}{|c|}{ Média } & \multicolumn{2}{|c|}{ Desvio Padrão } & \multicolumn{2}{|c|}{ Média } & \multicolumn{2}{|c|}{ Desvio Padrão } \\
\hline \multicolumn{2}{|c|}{ V:1 } & \multicolumn{2}{|c|}{0,123685} & \multicolumn{2}{|c|}{0,003064} & \multicolumn{2}{|c|}{3,114} & \multicolumn{2}{|c|}{0,11461} & \multicolumn{2}{|c|}{49,300} & \multicolumn{2}{|c|}{6,818319} \\
\hline \multicolumn{2}{|c|}{$\mathrm{V}: 2$} & \multicolumn{2}{|c|}{0,122875} & \multicolumn{2}{|c|}{0,00305} & \multicolumn{2}{|c|}{3,114} & \multicolumn{2}{|c|}{0,11461} & \multicolumn{2}{|c|}{49,30104} & \multicolumn{2}{|c|}{6,819286} \\
\hline \multicolumn{2}{|c|}{$\mathrm{V}: 5$} & \multicolumn{2}{|c|}{0,122887} & \multicolumn{2}{|c|}{0,003049} & \multicolumn{2}{|c|}{3,108} & 0,11 & 8646 & 49,2 & 7661 & 6,79 & 3745 \\
\hline $\mathrm{V}: 8$ & & 0,12 & 93 & 0,00 & 3086 & & 112 & 0,11 & 8305 & 49,2 & 9168 & 6,81 & 1879 \\
\hline $\mathrm{V}: 1$ & & 0,12 & 951 & 0,002 & 2966 & &, 11 & 0,11 & 032 & 49,2 & 7578 & 6,83 & 033 \\
\hline $\mathrm{V}: 1$ & & 0,12 & 997 & 0,00 & 3068 & & 112 & 0,11 & 1265 & 49,2 & 2979 & 6,79 & 1661 \\
\hline $\mathrm{V}: 2$ & & 0,12 & 213 & 0,00 & 3188 & & 112 & 0,11 & 8305 & 49,2 & 2827 & 6,84 & 7888 \\
\hline $\mathrm{V}: 28$ & & 0,12 & 537 & 0,003 & 3102 & & 112 & 0,11 & 8305 & 49,2 & 9302 & 6,81 & 0839 \\
\hline
\end{tabular}

De acordo com a tabela 5.16, as medidas que apresentaram os maiores desvios padrões para o nível de detecção são aquelas com valores altos de índice UI. Por exemplo: a medida IA:2, cujo valor médio de índice $U I$ é igual a 7,1392, apresenta o valor do desvio padrão do nível de detecção igual a 6,21388. Já a medida IA:1, que tem índice $U I$ abaixo de 1, apresenta o valor do desvio padrão do nível de detecção igual a 0,30650 .

Através dos resultados da tabela 5.16, constrói-se o gráfico índice UI x nível de detecção mostrado a seguir (figura 5.6): 


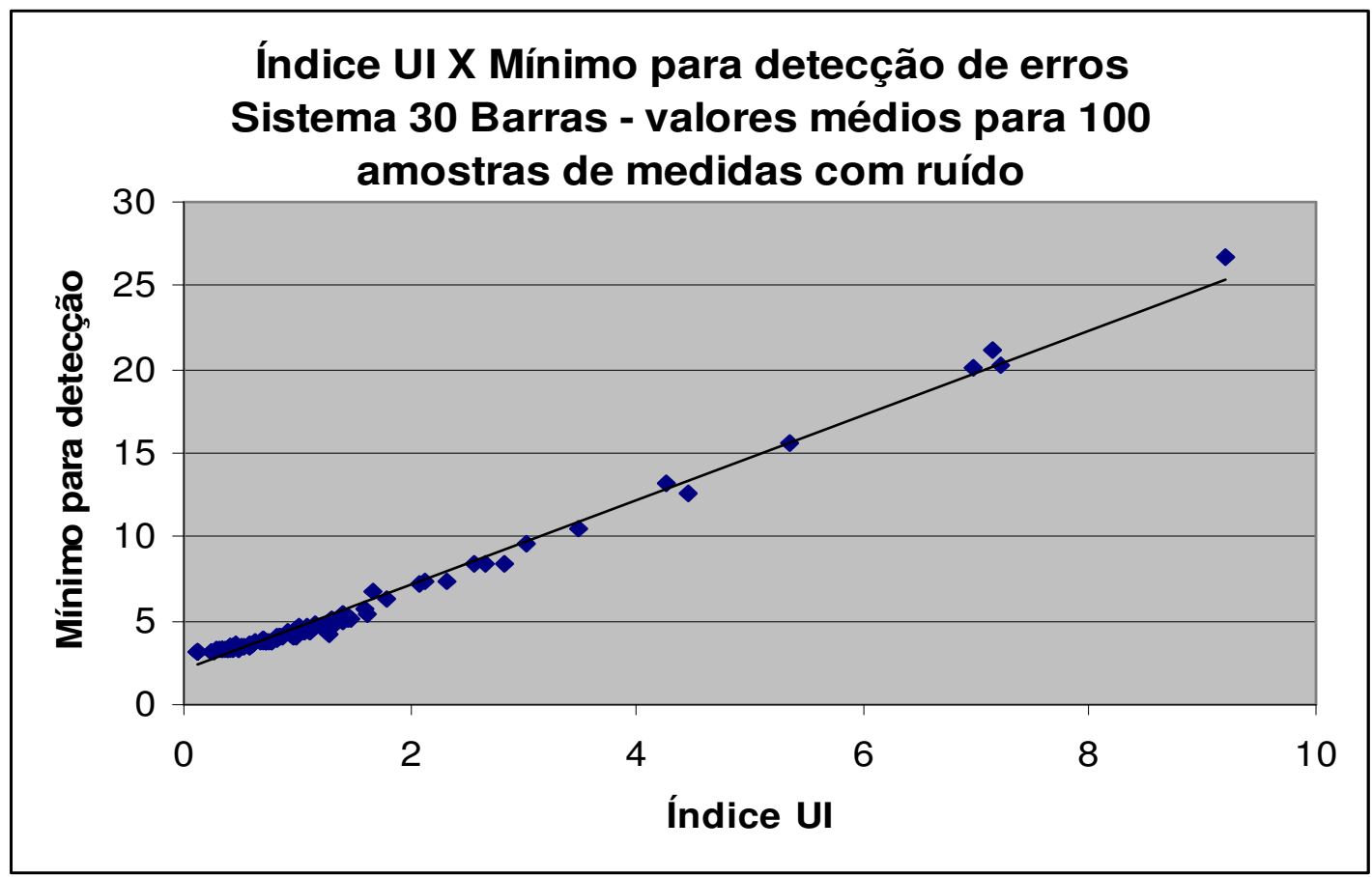

Figura 5.6: Gráfico de tendência para índice UI X Mínimo para detecção de EGs em medidas valores médios para 100 amostras de medidas com ruído

Tanto os resultados da tabela 5.16, quanto o gráfico da figura 5.6, ratificam que as medidas com maiores índices UIs terão mais dificuldade para detectar EGs.

\subsubsection{Testes com Ruído Gaussiano e Erro Grosseiro Simples}

Apresentaremos nesta subseção os resultados de simulações, considerando EG simples de 10 sigmas, em cada uma das medidas do sistema de medição da tabela 5.11 (uma medida de cada vez), considerando 100 amostras de ruído em medidas.

A tabela 5.17, a seguir, apresenta os valores médios do índice $U I$, resíduo normalizado e índice $J(x)$, para a medida portadora de EG em cada caso, considerando EG simples de 10 sigmas. 
Tabela 5.17: Resultados do índice UI, resíduo normalizado e índice J(x) para 100 amostras de medidas com ruído e com EG de 10 sigmas em cada medida

Limiar de detecção do índice $J(\mathrm{x}): \lambda=\chi_{63 ;(1-0,05)}^{2} \cong 81$

\begin{tabular}{|c|c|c|c|c|c|c|c|c|c|c|c|c|c|}
\hline \multicolumn{7}{|c|}{ Medidas de Potência Ativa } & \multicolumn{7}{|c|}{ Medidas de Potência Reativa } \\
\hline \multirow{2}{*}{$\begin{array}{c}\text { Medida } \\
\text { com } \\
\text { erro }\end{array}$} & \multicolumn{2}{|c|}{$\boldsymbol{U I}$} & \multicolumn{2}{|c|}{$r^{N}$} & \multicolumn{2}{|c|}{$J(\mathbf{x})$} & \multirow{2}{*}{$\begin{array}{c}\text { Medida } \\
\text { com } \\
\text { erro }\end{array}$} & \multicolumn{2}{|c|}{$\boldsymbol{U I}$} & \multicolumn{2}{|c|}{$r^{N}$} & \multicolumn{2}{|c|}{$J(\mathbf{x})$} \\
\hline & Média & $\begin{array}{c}\text { Desvio } \\
\text { Padrão }\end{array}$ & Média & $\begin{array}{c}\text { Desvio } \\
\text { Padrão }\end{array}$ & Média & $\begin{array}{c}\text { Desvio } \\
\text { Padrão }\end{array}$ & & Média & $\begin{array}{c}\text { Desvio } \\
\text { Padrão }\end{array}$ & Média & $\begin{array}{l}\text { Desvio } \\
\text { Padrão }\end{array}$ & Média & $\begin{array}{l}\text { Desvio } \\
\text { Padrão }\end{array}$ \\
\hline IA:1 & 0,39458 & 0,00259 & 9,3417 & 0,31039 & 127,183 & 9,34555 & IR:1 & 1,073 & 0,00056 & $-6,913$ & 0,5987 & 87,8512 & 10,825 \\
\hline IA: 2 & 7,1456 & 0,02196 & 1,4011 & 0,89438 & 42,3759 & 8,04296 & IR:2 & 0,3618 & 0,00105 & 9,40425 & 0,27874 & 128,112 & 8,9514 \\
\hline IA:5 & 0,34257 & 0,00129 & $-9,4646$ & 0,28644 & 129,404 & 8,79309 & IR:5 & 0,9843 & 0,00778 & 7,1546 & 0,60447 & 91,2557 & 11,407 \\
\hline IA:6 & --- & --- & --- & --- & --- & --- & IR:6 & --- & --- & --- & --- & --- & --- \\
\hline IA:7 & 1,4812 & 0,00053 & $-5,6218$ & 0,7373 & 71,7557 & 10,8095 & IR:7 & 0,533 & 0,0007 & $-8,8078$ & 0,37944 & 117,358 & 9,2557 \\
\hline IA:8 & 0,72847 & $5,0 \mathrm{E}-05$ & $-8,1223$ & 0,46683 & 105,909 & 9,86041 & IR:8 & 1,0625 & 0,00184 & 6,86418 & 0,64985 & 87,1912 & 10,852 \\
\hline IA:9 & --- & --- & --- & --- & --- & --- & IR:9 & --- & --- & --- & --- & --- & --- \\
\hline IA:11 & --- & --- & --- & --- & --- & --- & IR:11 & 0,6248 & 0,00027 & 8,48962 & 0,44531 & 111,902 & 10,045 \\
\hline IA:12 & 3,0381 & 0,00083 & $-3,1783$ & 0,88599 & 50,4754 & 9,77967 & IR:12 & 3,4941 & 0,00506 & $-2,7227$ & 0,82308 & 47,7921 & 8,534 \\
\hline IA:13 & --- & --- & --- & --- & --- & --- & IR:13 & 0,8834 & 0,00039 & 7,59498 & 0,57491 & 97,5872 & 10,505 \\
\hline IA:15 & 5,35957 & 0,00114 & $-1,8109$ & 0,77376 & 43,6923 & 7,54396 & IR:15 & 6,9697 & 0,0104 & $-1,4813$ & 0,90667 & 42,6143 & 7,7142 \\
\hline IA:16 & 4,45242 & 0,00056 & $-2,2943$ & 0,84633 & 45,6808 & 7,94055 & IR:16 & 2,5664 & 0,00257 & $-3,5715$ & 0,79432 & 53,0073 & 9,3203 \\
\hline IA:17 & 7,21367 & 0,00187 & $-1,4617$ & 0,86782 & 42,5624 & 7,49277 & IR:17 & 4,2616 & 0,00372 & $-2,1868$ & 0,90107 & 45,2272 & 8,1446 \\
\hline IA:19 & 1,11728 & $4,0 \mathrm{E}-05$ & $-6,6046$ & 0,64469 & 83,7703 & 10,0896 & IR:19 & 1,413 & $4,0 \mathrm{E}-05$ & $-5,7388$ & 0,77688 & 73,0204 & 11,178 \\
\hline IA:21 & 1,05691 & 0,00012 & $-6,8682$ & 0,57048 & 87,2467 & 11,019 & IR:21 & 0,9259 & 0,00018 & $-7,2833$ & 0,56788 & 93,1641 & 11,323 \\
\hline IA:22 & --- & --- & --- & --- & --- & --- & IR:22 & --- & --- & --- & --- & --- & --- \\
\hline IA:23 & 2,12029 & $9,5 \mathrm{E}-05$ & $-4,1826$ & 0,89718 & 57,8281 & 10,64 & IR:23 & 1,3394 & $6,3 \mathrm{E}-05$ & $-6,0394$ & 0,66455 & 76,7676 & 10,819 \\
\hline IA:24 & 0,99273 & $5,5 \mathrm{E}-05$ & $-6,8677$ & 0,57198 & 87,1859 & 11,3094 & IR:24 & 0,78 & 0,00186 & $-7,8727$ & 0,50325 & 101,915 & 9,5317 \\
\hline IA:25 & --- & --- & --- & --- & --- & --- & IR:25 & --- & --- & --- & --- & --- & --- \\
\hline IA:27 & --- & --- & --- & --- & --- & --- & IR:27 & -- & --- & --- & --- & --- & -- \\
\hline IA:28 & --- & --- & --- & --- & --- & --- & IR:28 & --- & --- & --- & --- & --- & --- \\
\hline IA:30 & 0,38639 & 7,4E-05 & $-9,3178$ & 0,32761 & 126,498 & 9,03113 & IR:30 & 1,3298 & 0,00026 & $-6,0859$ & 0,76771 & 77,2746 & 11,429 \\
\hline FA:1-2 & 0,29499 & 0,00157 & 9,6323 & 0,22423 & 132,617 & 8,45604 & FR:1-2 & 0,4025 & 0,00022 & $-9,2929$ & 0,32585 & 126,131 & 9,6532 \\
\hline FA:1-3 & 0,43592 & 0,00225 & 9,2157 & 0,33762 & 124,766 & 9,1967 & FR:1-3 & 1,4603 & 0,00123 & 5,67407 & 0,71349 & 72,473 & 11,062 \\
\hline FA:2-4 & 0,34352 & 0,00167 & 9,4916 & 0,25441 & 129,74 & 8,47865 & FR:2-4 & 0,7343 & 0,0005 & 7,99497 & 0,53508 & 103,829 & 11,433 \\
\hline FA:4-2 & 0,33008 & 0,00197 & $-9,5419$ & 0,2576 & 130,875 & 8,60376 & FR:4-2 & 1,306 & 0,01282 & $-5,9774$ & 0,70522 & 75,869 & 11,78 \\
\hline FA:2-5 & 0,23478 & 0,00103 & 9,7623 & 0,1 & 13 & 7, & FI & 1,8072 & 592 & 4,95413 & 0,70465 & 64,8152 & 9,7658 \\
\hline FA:2-6 & 0,27861 & 0,00166 & 9,6941 & 0,22131 & 133,635 & 7,98474 & FR:2-6 & 0,8163 & 0,00577 & 7,6008 & 0,45807 & 97,7459 & 10,141 \\
\hline FA:3-4 & 2,69308 & 0,00628 & 3,6003 & 0,85635 & 53,3838 & 10,1077 & FR:3-4 & 9,2033 & 0,00553 & $-1,0623$ & 0,80635 & 41,5458 & 7,2598 \\
\hline FA:4-6 & 0,42758 & 0,00017 & 9,2139 & 0,34166 & 124,546 & 8,81908 & FR:4-6 & 0,5887 & 0,00143 & $-8,749$ & 0,37925 & 116,526 & 9,6904 \\
\hline FA:4-12 & 0,30402 & $6,0 \mathrm{E}-05$ & 9,5799 & 0,25349 & 131,352 & 8,50015 & FR:4-12 & 0,6998 & 0,00992 & 8,0799 & 0,52088 & 105,344 & 10,609 \\
\hline FA:5-7 & 1,37508 & 0,00125 & $-5,7927$ & 0,6424 & 73,6453 & 9,73355 & FR:5-7 & 0,4272 & 0,00019 & 9,14918 & 0,33427 & 123,614 & 9,2664 \\
\hline FA:6-7 & 0,47869 & 0,00025 & 9,0319 & 0,3799 & 121,382 & 9,4053 & FR:6-7 & 2,3281 & 0,00348 & $-3,9433$ & 0,80787 & 55,8564 & 8,9773 \\
\hline FA:6-8 & 0,60122 & $4,1 \mathrm{E}-05$ & 8,6484 & 0,3636 & 114,536 & 9,91165 & FR:6-8 & 0,7493 & $9,1 \mathrm{E}-05$ & $-8,0908$ & 0,5695 & 105,464 & 11,169 \\
\hline FA:6-9 & 0,40093 & 0,00010 & 9,2587 & 0,32811 & 125,362 & 9,4702 & FR:6-9 & 0,8306 & 0,0001 & $-7,8456$ & 0,5815 & 101,515 & 11,674 \\
\hline FA:6-28 & 0,41003 & 0,00010 & 9,2646 & 0,26208 & 125,653 & 8,40133 & FR:6-28 & --- & --- & --- & --- & --- & --- \\
\hline FA:8-28 & 0,85691 & $3,0 \mathrm{E}-05$ & $-7,5801$ & 0,52728 & 97,456 & 10,8264 & FR:8-28 & 0,3835 & 0,0037 & $-9,399$ & 0,28807 & 128,069 & 8,7562 \\
\hline FA:9-11 & --- & --- & --- & --- & --- & --- & FR:9-11 & 0,576 & $9,5 \mathrm{E}-05$ & $-8,6175$ & 0,42171 & 114,069 & 9,7376 \\
\hline FA:9-10 & 0,41419 & $9,9 \mathrm{E}-05$ & 9,2162 & 0,32917 & 124,795 & 9,56311 & FR:9-10 & 1,2966 & 0,00014 & 6,02906 & 0,71198 & 76,5977 & 11,231 \\
\hline FA:12-13 & --- & --- & --- & --- & --- & --- & FR:12-13 & 0,8416 & 0,00031 & $-7,7458$ & 0,54034 & 99,9564 & 10,867 \\
\hline FA:12-15 & 0,50931 & $8,4 \mathrm{E}-05$ & 8,9213 & 0,3604 & 119,499 & 8,63403 & FR:12-15 & 0,7736 & 0,00361 & 7,85533 & 0,55431 & 101,51 & 10,665 \\
\hline FA:12-16 & 0,72339 & 0,00021 & 8,043 & 0,50214 & 104,57 & 10,4834 & FR:12-16 & 1,1083 & 0,00259 & 6,80566 & 0,57617 & 86,3059 & 11,31 \\
\hline FA:15-18 & 0,99463 & $6,5 \mathrm{E}-05$ & 7,0917 & 0,6684 & 90,3129 & 12,5206 & FR:15-18 & 0,9976 & $8,3 \mathrm{E}-05$ & 7,2097 & 0,59802 & 91,8237 & 10,897 \\
\hline FA:18-15 & 0,96994 & $8,6 \mathrm{E}-05$ & $-7,0458$ & 0,58202 & 89,5293 & 10,5544 & FR:18-15 & 0,9834 & 7,3E-05 & $-7,1532$ & 0,63624 & 91,0641 & 11,534 \\
\hline
\end{tabular}




\begin{tabular}{|c|c|c|c|c|c|c|c|c|c|c|c|c|c|}
\hline FA:15-23 & 0,61581 & $6,4 \mathrm{E}-05$ & 8,4994 & 0,49179 & 112,003 & 10,8161 & FR:15-23 & 1,0941 & $8,0 \mathrm{E}-05$ & 6,84065 & 0,63645 & 86,937 & 10,545 \\
\hline FA:18-19 & 2,83879 & $4,3 \mathrm{E}-05$ & 3,3231 & 0,77166 & 51,3671 & 9,39101 & FR:18-19 & 1,4171 & $5,9 \mathrm{E}-05$ & 5,76553 & 0,76089 & 73,3033 & 11,535 \\
\hline FA:19-20 & 1,1173 & 0 & $-6,6507$ & 0,67322 & 84,4186 & 11,8171 & FR:19-20 & 1,4134 & $4,8 \mathrm{E}-05$ & $-5,7751$ & 0,77117 & 73,4328 & 11,559 \\
\hline FA:21-22 & 1,60611 & $2,8 \mathrm{E}-05$ & $-5,1938$ & 0,67329 & 67,1949 & 9,30637 & FR:21-22 & 1,453 & $2,1 \mathrm{E}-05$ & $-5,6896$ & 0,61848 & 72,6736 & 10,531 \\
\hline FA:22-24 & 0,70914 & 0,00012 & 8,1619 & 0,49129 & 106,558 & 11,015 & FR:22-24 & 1,2146 & 0,00124 & 6,3065 & 0,64799 & 79,9861 & 10,122 \\
\hline FA:23-24 & 1,2874 & 0,00028 & 6,1222 & 0,72058 & 77,7054 & 11,3306 & FR:23-24 & 0,939 & $3,6 \mathrm{E}-05$ & 7,20665 & 0,54234 & 92,0232 & 10,697 \\
\hline FA:24-25 & 1,1623 & $9,4 \mathrm{E}-05$ & $-6,5168$ & 0,54065 & 82,6359 & 10,6334 & FR:24-25 & 1,0192 & 0,00043 & 6,67604 & 0,49215 & 84,5215 & 10,317 \\
\hline FA:25-26 & 1,6756 & $3,3 \mathrm{E}-05$ & 4,7446 & 0,60552 & 62,559 & 9,40545 & FR:25-26 & 1,6295 & 0,00046 & 5,35715 & 0,62967 & 68,9311 & 9,0749 \\
\hline FA:25-27 & 1,29051 & $9,7 \mathrm{E}-05$ & $-6,4964$ & 0,62943 & 82,0799 & 10,7047 & FR:25-27 & 0,9857 & 0,00011 & $-7,2895$ & 0,47772 & 93,1769 & 10,378 \\
\hline FA:27-29 & 0,70049 & 0,00011 & 8,1528 & 0,47146 & 106,348 & 9,97367 & FR:27-29 & 1,0628 & 0,00125 & 6,93596 & 0,51282 & 88,1226 & 11,126 \\
\hline FA:27-30 & 0,45686 & $5,8 \mathrm{E}-05$ & 9,0683 & 0,34923 & 122,008 & 9,08044 & FR:27-30 & 0,4784 & 0,00014 & 9,04774 & 0,32878 & 121,666 & 9,6882 \\
\hline FA:28-27 & 0,42631 & 0,00012 & 9,1756 & 0,32828 & 124,007 & 8,82774 & FR:28-27 & 0,4543 & 0,00053 & 8,87433 & 0,28232 & 118,577 & 9,4204 \\
\hline FA:29-30 & 2,09289 & $8,9 \mathrm{E}-05$ & 4,3336 & 0,75788 & 59,1074 & 10,0837 & FR:29-30 & 0,6694 & 0,00011 & 8,34823 & 0,48695 & 109,607 & 11,101 \\
\hline \multicolumn{14}{|c|}{ Medidas de Tensão } \\
\hline \multirow{2}{*}{\multicolumn{2}{|c|}{$\begin{array}{l}\text { Medida com } \\
\text { erro }\end{array}$}} & \multicolumn{4}{|c|}{$\boldsymbol{U I}$} & \multicolumn{4}{|c|}{$r^{N}$} & \multicolumn{4}{|c|}{$J(\mathbf{x})$} \\
\hline & & \multicolumn{2}{|c|}{ Média } & \multicolumn{2}{|c|}{ Desvio Padrão } & \multicolumn{2}{|c|}{ Média } & \multicolumn{2}{|c|}{ Desvio Padrão } & \multicolumn{2}{|c|}{ Média } & \multicolumn{2}{|c|}{ Desvio Padrão } \\
\hline \multicolumn{2}{|c|}{ V:1 } & \multicolumn{2}{|c|}{0,12361} & \multicolumn{2}{|c|}{0,002682} & \multicolumn{2}{|c|}{9,9232} & \multicolumn{2}{|c|}{0,09093} & \multicolumn{2}{|c|}{138,915} & \multicolumn{2}{|c|}{7,31773} \\
\hline \multicolumn{2}{|c|}{$\mathrm{V}: 2$} & \multicolumn{2}{|c|}{0,12311} & \multicolumn{2}{|c|}{0,002861} & \multicolumn{2}{|c|}{9,9243} & \multicolumn{2}{|c|}{0,09074} & \multicolumn{2}{|c|}{138,928} & \multicolumn{2}{|c|}{7,3165} \\
\hline \multicolumn{2}{|c|}{$\mathrm{V}: 5$} & \multicolumn{2}{|c|}{0,12282} & \multicolumn{2}{|c|}{0,002604} & \multicolumn{2}{|c|}{9,9236} & \multicolumn{2}{|c|}{0,09057} & 13 & & 7,32 & \\
\hline $\mathbf{V}$ & & 0,12 & & 0,00 & 173 & 9, & 22 & & 925 & 138 & & 7,3 & \\
\hline V: & & 0,1 & & 0,00 & 053 & 9,9 & 264 & 0,0 & 024 & 138 & & 7,30 & \\
\hline V: & & 0,12 & & 0,00 & 138 & 9,9 & 246 & 0,0 & 397 & 138 & & 7,30 & \\
\hline V: & & 0,12 & & 0,00 & 497 & 9,9 & 178 & 0,0 & 9589 & 138 & & 7,3 & \\
\hline V: & & 0,12 & & 0,00 & 191 & 9,9 & 216 & 0,0 & 257 & 138 & 395 & 7,32 & 196 \\
\hline
\end{tabular}

Analisando os resultados da tabela 5.17, verificamos que o teste do maior resíduo normalizado e, também, o teste do índice $J(\mathrm{x})$ falharam na detecção de EGs em medidas com índice $U I$ elevado (casos em vermelho).

Por outro lado, os casos em amarelo distinguem as medidas em que o valor médio do respectivo resíduo normalizado foi maior que 3, mas o valor médio do índice $\mathrm{J}(x)$ foi inferior a 81 (limiar de detecção para o teste do índice $\mathrm{J}(x)$ ). Como pode ser observado na tabela 5.17, esses casos correspondem às medidas cujo índice $U I$ está entre 1,2146 e 3,0381 .

\subsubsection{Análise Comparativa do Índice UI calculado adicionando ou não ruído e/ou erro grosseiro}

Com o intuito de analisar a variação do índice $U I$, com relação às alterações nos valores das medidas, a tabela 5.18 apresenta os valores do índice $U I$, calculados nos casos anteriores, sendo os mesmos divididos da seguinte forma:

- Caso 1: medidas sem ruído e sem EG; 
- Caso 2: medidas sem ruído, mas com adição de EG simples de $10 \sigma$ em cada medida;

- Caso 3: medidas com ruído, mas sem adição de EG;

- Caso 4: medidas com ruído e com adição de EG simples de $10 \sigma$, em cada medida.

Tabela 5.18: Resultados do índice UI, com alterações nos valores das medidas - sistema de 30 barras

\begin{tabular}{|c|c|c|c|c|c|c|c|c|c|c|c|c|c|}
\hline \multicolumn{7}{|c|}{ Medidas de Potência Ativa } & \multicolumn{7}{|c|}{ Medidas de Potência Reativa } \\
\hline \multirow{2}{*}{ Medida } & \multirow{2}{*}{$\begin{array}{c}\text { Caso 1: } \\
\text { UI } \\
\text { valor }\end{array}$} & \multirow{2}{*}{$\begin{array}{c}\text { Caso 2: } \\
\text { UI } \\
\text { valor }\end{array}$} & \multicolumn{2}{|c|}{$\begin{array}{c}\text { Caso 3: } \\
\text { UI } \\
\end{array}$} & \multicolumn{2}{|c|}{$\begin{array}{c}\text { Caso 4: } \\
U I \\
\end{array}$} & \multirow{2}{*}{ Medida } & \multirow{2}{*}{$\begin{array}{c}\begin{array}{c}\text { Caso 1: } \\
\text { UI }\end{array} \\
\text { valor }\end{array}$} & \multirow{2}{*}{$\begin{array}{c}\text { Caso 2: } \\
\text { UI }\end{array}$} & \multicolumn{2}{|c|}{$\begin{array}{c}\text { Caso 3: } \\
\text { UI } \\
\end{array}$} & \multicolumn{2}{|c|}{$\begin{array}{c}\text { Caso 4: } \\
\text { UI }\end{array}$} \\
\hline & & & Média & Desvio & Média & Desvio & & & & Média & Desvio & Média & Desvio \\
\hline IA:1 & 0,394 & 0,401 & 0,39569 & 0,00223 & 0,39458 & 0,00259 & IR:1 & 1,0718 & 1,0728 & 1,07211 & 0,00055 & 1,073 & 0,00056 \\
\hline IA:2 & 7,1385 & 7,181 & 7,14329 & 0,02076 & 7,1456 & 0,02196 & IR:2 & 0,3608 & 0,3625 & 0,36109 & 0,00093 & 0,3618 & 0,00105 \\
\hline IA:5 & 0,342 & 0,345 & 0,34285 & 0,00116 & 0,34257 & 0,00129 & IR:5 & 0,9761 & 0,9922 & 0,97996 & 0,0067 & 0,9843 & 0,00778 \\
\hline IA:6 & --- & --- & --- & --- & --- & --- & IR:6 & --- & --- & --- & --- & --- & --- \\
\hline IA:7 & 1,482 & 1,484 & 1,48224 & 0,00058 & 1,4812 & 0,00053 & IR:7 & 0,5328 & 0,5346 & 0,53322 & 0,00067 & 0,533 & 0,0007 \\
\hline IA:8 & 0,7286 & 0,729 & 0,7286 & $5,02 \mathrm{E}-05$ & 0,72847 & $5,00 \mathrm{E}-05$ & IR:8 & 1,0618 & 1,0664 & 1,06284 & 0,00181 & 1,0625 & 0,00184 \\
\hline IA:9 & --- & --- & --- & --- & --- & --- & IR:9 & --- & --- & --- & --- & --- & --- \\
\hline IA:11 & --- & --- & --- & --- & --- & --- & IR:11 & 0,6238 & 0,624 & 0,62395 & 0,00023 & 0,6248 & 0,00027 \\
\hline IA:12 & 3,0384 & 3,04 & 3,03863 & 0,00082 & 3,0381 & 0,00083 & IR:12 & 3,4937 & 3,5053 & 3,49636 & 0,00447 & 3,4941 & 0,00506 \\
\hline IA:13 & --- & --- & --- & --- & --- & --- & IR:13 & 0,8819 & 0,8827 & 0,88211 & 0,00037 & 0,8834 & 0,00039 \\
\hline IA:15 & 5,3617 & 5,364 & 5,36173 & 0,00111 & 5,35957 & 0,00114 & IR:15 & 6,9668 & 6,9911 & 6,9723 & 0,0093 & 6,9697 & 0,0104 \\
\hline IA:16 & 4,453 & 4,454 & 4,45335 & 0,00055 & 4,45242 & 0,00056 & IR:16 & 2,567 & 2,5737 & 2,56848 & 0,0025 & 2,5664 & 0,00257 \\
\hline IA:17 & 7,215 & 7,219 & 7,21608 & 0,0016 & 7,21367 & 0,00187 & IR:17 & 4,2631 & 4,273 & 4,26524 & 0,00365 & 4,2616 & 0,00372 \\
\hline IA:19 & 1,1173 & 1,117 & 1,1173 & 0 & 1,11728 & $4,00 \mathrm{E}-05$ & IR:19 & 1,4132 & 1,4132 & 1,41318 & $4,43 \mathrm{E}-05$ & 1,413 & $4,00 \mathrm{E}-05$ \\
\hline IA:21 & 1,0568 & 1,057 & 1,0569 & 0,00011 & 1,05691 & 0,00012 & IR:21 & 0,9258 & 0,9263 & 0,92592 & 0,00018 & 0,9259 & 0,00018 \\
\hline IA:22 & --- & --- & --- & --- & --- & --- & IR:22 & --- & --- & --- & --- & --- & --- \\
\hline IA: 23 & 2,121 & 2,121 & 2,12095 & 0,00011 & 2,12029 & $9,50 \mathrm{E}-05$ & IR:23 & 1,3398 & 1,3399 & 1,33984 & $6,50 \mathrm{E}-05$ & 1,3394 & $6,30 \mathrm{E}-05$ \\
\hline IA:24 & 0,993 & 0,993 & 0,99302 & $6,15 \mathrm{E}-05$ & 0,99273 & $5,50 \mathrm{E}-05$ & IR:24 & 0,7793 & 0,7841 & 0,78033 & 0,00176 & 0,78 & 0,00186 \\
\hline IA:25 & --- & --- & --- & --- & --- & --- & IR:25 & --- & --- & --- & -- & --- & --- \\
\hline IA:27 & --- & --- & --- & --- & --- & --- & IR:27 & --- & --- & --- & --- & --- & --- \\
\hline IA:28 & --- & --- & --- & --- & --- & --- & IR:28 & --- & --- & --- & --- & --- & --- \\
\hline IA:30 & 0,3866 & 0,387 & 0,3866 & 7,71E-05 & 0,38639 & $7,40 \mathrm{E}-05$ & IR:30 & 1,3311 & 1,3318 & 1,3313 & 0,00027 & 1,3298 & 0,00026 \\
\hline FA:1-2 & 0,2944 & 0,299 & 0,29549 & 0,00146 & 0,29499 & 0,00157 & FR:1-2 & 0,402 & 0,4024 & 0,40212 & 0,00024 & 0,4025 & 0,00022 \\
\hline FA:1-3 & 0,4358 & 0,443 & 0,43748 & 0,0022 & 0,43592 & 0,00225 & FR:1-3 & 1,4558 & 1,4567 & 1,45627 & 0,00122 & 1,4603 & 0,00123 \\
\hline FA:2-4 & 0,3429 & 0,348 & 0,34423 & 0,00176 & 0,34352 & 0,00167 & FR:2-4 & 0,7337 & 0,735 & 0,73402 & 0,00051 & 0,7343 & 0,0005 \\
\hline FA:4-2 & 0,3294 & 0,335 & 0,33078 & 0,00181 & 0,33008 & 0,00197 & FR:4-2 & 1,3175 & 1,3512 & 1,32375 & 0,01423 & 1,306 & 0,01282 \\
\hline FA:2-5 & 0,2342 & 0,237 & 0,23492 & 0,00095 & 0,23478 & 0,00103 & FR:2-5 & 1,7999 & 1,8122 & 1,8029 & 0,0 & 1,8072 & 0,00592 \\
\hline FA:2-6 & 0,2777 & 0,282 & 0,27888 & 0,00156 & 0,27861 & 0,00166 & FR:2-6 & 0,8145 & 0,8298 & 0,81797 & 0,00574 & 0,8163 & 0,00577 \\
\hline FA:3-4 & 2,6518 & 2,666 & 2,65472 & 0,00601 & 2,69308 & 0,00628 & FR:3-4 & 9,2068 & 9,2087 & 9,20812 & 0,00552 & 9,2033 & 0,00553 \\
\hline FA:4-6 & 0,4275 & 0,428 & 0,42752 & 0,00017 & 0,42758 & 0,00017 & FR:4-6 & 0,5883 & 0,592 & 0,58918 & 0,00135 & 0,5887 & 0,00143 \\
\hline FA:4-12 & 0,3042 & 0,304 & 0,30408 & $6,47 \mathrm{E}-05$ & 0,30402 & $6,00 \mathrm{E}-05$ & FR:4-12 & 0,698 & 0,7217 & 0,70333 & 0,00868 & 0,6998 & 0,00992 \\
\hline FA:5-7 & 1,3766 & 1,38 & 1,37681 & 0,00138 & 1,37508 & 0,00125 & FR:5-7 & 0,427 & 0,4273 & 0,42705 & 0,00019 & 0,4272 & 0,00019 \\
\hline FA:6-7 & 0,4785 & 0,479 & 0,47856 & 0,00024 & 0,47869 & 0,00025 & FR:6-7 & 2,3269 & 2,3346 & 2,32847 & 0,0035 & 2,3281 & 0,00348 \\
\hline FA:6-8 & 0,6012 & 0,601 & 0,60121 & 2,97E-05 & 0,60122 & 4,10E-05 & FR:6-8 & 0,7494 & 0,7496 & 0,74943 & $9,40 \mathrm{E}-05$ & 0,7493 & $9,10 \mathrm{E}-05$ \\
\hline FA:6-9 & 0,4012 & 0,401 & 0,40107 & $9,56 \mathrm{E}-05$ & 0,40093 & 0,0001 & FR:6-9 & 0,831 & 0,8312 & 0,83102 & 0,00011 & 0,8306 & 0,0001 \\
\hline FA:6-28 & 0,4102 & 0,41 & 0,41012 & $9,85 \mathrm{E}-05$ & 0,41003 & 0,0001 & FR:6-28 & -- & --- & --- & -- & --- & --- \\
\hline
\end{tabular}




\begin{tabular}{|c|c|c|c|c|c|c|c|c|c|c|c|c|c|}
\hline FA:8-28 & 0,857 & 0,857 & 0,857 & $3,17 \mathrm{E}-05$ & 0,85691 & $3,00 \mathrm{E}-05$ & FR:8-28 & 0,382 & 0,3917 & 0,38421 & 0,00353 & 0,3835 & 0,0037 \\
\hline FA:9-11 & --- & --- & --- & --- & --- & --- & FR:9-11 & 0,5762 & 0,5763 & 0,5762 & 0,0001 & 0,576 & $9,50 \mathrm{E}-05$ \\
\hline FA:9-10 & 0,4145 & 0,414 & 0,41432 & $9,29 \mathrm{E}-05$ & 0,41419 & $9,90 \mathrm{E}-05$ & FR:9-10 & 1,297 & 1,2973 & 1,297 & 0,00016 & 1,2966 & 0,00014 \\
\hline FA:12-13 & --- & --- & --- & --- & --- & --- & FR:12-13 & 0,8424 & 0,8432 & 0,84258 & 0,00031 & 0,8416 & 0,00031 \\
\hline FA:12-15 & 0,5093 & 0,51 & 0,50933 & $7,81 \mathrm{E}-05$ & 0,50931 & $8,40 \mathrm{E}-05$ & FR:12-15 & 0,7726 & 0,782 & 0,77471 & 0,00357 & 0,7736 & 0,00361 \\
\hline FA:12-16 & 0,7231 & 0,724 & 0,72324 & 0,00018 & 0,72339 & 0,00021 & FR:12-16 & 1,1074 & 1,1135 & 1,1087 & 0,00233 & 1,1083 & 0,00259 \\
\hline FA:15-18 & 0,9941 & 0,994 & 0,99408 & $7,78 \mathrm{E}-05$ & 0,99463 & $6,50 \mathrm{E}-05$ & FR:15-18 & 0,9969 & 0,9969 & 0,99689 & $9,18 \mathrm{E}-05$ & 0,9976 & $8,30 \mathrm{E}-05$ \\
\hline FA:18-15 & 0,9707 & 0,971 & 0,97067 & $9,88 \mathrm{E}-05$ & 0,96994 & $8,60 \mathrm{E}-05$ & FR:18-15 & 0,9841 & 0,9842 & 0,98413 & $8,37 \mathrm{E}-05$ & 0,9834 & 7,30E-05 \\
\hline FA:15-23 & 0,6156 & 0,616 & 0,6156 & $6,43 \mathrm{E}-05$ & 0,61581 & $6,40 \mathrm{E}-05$ & FR:15-23 & 1,0934 & 1,0934 & 1,09341 & $9,62 \mathrm{E}-05$ & 1,0941 & $8,00 \mathrm{E}-05$ \\
\hline FA:18-19 & 2,8378 & 2,838 & 2,83781 & $9,85 \mathrm{E}-05$ & 2,83879 & $4,30 \mathrm{E}-05$ & FR:18-19 & 1,4163 & 1,4163 & 1,4163 & $7,90 \mathrm{E}-05$ & 1,4171 & $5,90 \mathrm{E}-05$ \\
\hline FA:19-20 & 1,1173 & 1,117 & 1,1173 & 0 & 1,1173 & 0 & FR:19-20 & 1,4132 & 1,4132 & 1,41317 & $4,51 \mathrm{E}-05$ & 1,4134 & $4,80 \mathrm{E}-05$ \\
\hline FA:21-22 & 1,6062 & 1,606 & 1,6062 & $2,25 \mathrm{E}-05$ & 1,60611 & $2,80 \mathrm{E}-05$ & FR:21-22 & 1,4531 & 1,4531 & 1,4531 & $2,97 \mathrm{E}-05$ & 1,453 & $2,10 \mathrm{E}-05$ \\
\hline FA:22-24 & 0,7089 & 0,709 & 0,70896 & 0,0001 & 0,70914 & 0,00012 & FR:22-24 & 1,2137 & 1,2165 & 1,21427 & 0,00109 & 1,2146 & 0,00124 \\
\hline FA:23-24 & 1,287 & 1,288 & 1,28712 & 0,00027 & 1,2874 & 0,00028 & FR:23-24 & 0,9388 & 0,9388 & 0,9388 & $4,37 \mathrm{E}-05$ & 0,939 & $3,60 \mathrm{E}-05$ \\
\hline FA:24-25 & 1,1631 & 1,163 & 1,16296 & 0,00012 & 1,1623 & $9,40 \mathrm{E}-05$ & FR:24-25 & 1,0182 & 1,0194 & 1,0185 & 0,0004 & 1,0192 & 0,00043 \\
\hline FA:25-26 & 1,6757 & 1,676 & 1,67574 & $5,28 \mathrm{E}-05$ & 1,6756 & $3,30 \mathrm{E}-05$ & FR:25-26 & 1,6296 & 1,6307 & 1,62983 & 0,00041 & 1,6295 & 0,00046 \\
\hline FA:25-27 & 1,2909 & 1,291 & 1,2908 & $9,63 \mathrm{E}-05$ & 1,29051 & $9,70 \mathrm{E}-05$ & FR:25-27 & 0,9861 & 0,9861 & 0,98606 & 0,00011 & 0,9857 & 0,00011 \\
\hline FA:27-29 & 0,7006 & 0,701 & 0,70054 & 0,00012 & 0,70049 & 0,00011 & FR:27-29 & 1,0623 & 1,065 & 1,063 & 0,00104 & 1,0628 & 0,00125 \\
\hline FA:27-30 & 0,4569 & 0,457 & 0,4569 & $6,03 \mathrm{E}-05$ & 0,45686 & $5,80 \mathrm{E}-05$ & FR:27-30 & 0,4782 & 0,4785 & 0,47827 & 0,00013 & 0,4784 & 0,00014 \\
\hline FA:28-27 & 0,4265 & 0,427 & 0,42647 & 0,00013 & 0,42631 & 0,00012 & FR:28-27 & 0,4542 & 0,4553 & 0,45443 & 0,00047 & 0,4543 & 0,00053 \\
\hline FA:29-30 & 2,0916 & 2,092 & 2,09158 & 0,00014 & 2,09289 & $8,90 \mathrm{E}-05$ & FR:29-30 & 0,6689 & 0,669 & 0,6689 & 0,00012 & 0,6694 & 0,00011 \\
\hline \multicolumn{14}{|c|}{ Medidas de Tensão } \\
\hline \multirow{2}{*}{\multicolumn{2}{|c|}{ Medida }} & \multicolumn{2}{|c|}{$\begin{array}{c}\text { Caso 1: } \\
\text { UI }\end{array}$} & \multicolumn{2}{|c|}{$\begin{array}{c}\text { Caso 2: } \\
\text { UI } \\
\end{array}$} & \multicolumn{4}{|c|}{$\begin{array}{c}\text { Caso 3: } \\
\text { UI }\end{array}$} & \multicolumn{4}{|c|}{$\begin{array}{c}\text { Caso 4: } \\
\text { UI }\end{array}$} \\
\hline & & \multicolumn{2}{|c|}{ valor } & \multicolumn{2}{|c|}{ valor } & \multicolumn{2}{|c|}{ Média } & \multicolumn{2}{|c|}{ Desvio } & \multicolumn{2}{|c|}{ Média } & \multicolumn{2}{|c|}{ Desvio } \\
\hline \multicolumn{2}{|c|}{ V:1 } & \multicolumn{2}{|c|}{0,1227} & \multicolumn{2}{|c|}{0,131} & \multicolumn{2}{|c|}{0,1246} & \multicolumn{2}{|c|}{0,00297} & \multicolumn{2}{|c|}{0,12361} & \multicolumn{2}{|c|}{0,00268} \\
\hline \multicolumn{2}{|c|}{$\mathrm{V}: 2$} & \multicolumn{2}{|c|}{0,1219} & \multicolumn{2}{|c|}{0,13} & \multicolumn{2}{|c|}{0,12378} & \multicolumn{2}{|c|}{0,00295} & \multicolumn{2}{|c|}{0,12311} & \multicolumn{2}{|c|}{0,00286} \\
\hline V: & & & 219 & 0 , & & 0,12 & 2379 & 0,00 & & 0,12 & 282 & & 26 \\
\hline V: & & & 232 & 0,1 & & 0,12 & 2511 & 0,00 & & 0,12 & 365 & & 317 \\
\hline $\mathrm{V}:$ & & & 12 & 0,1 & & 0,12 & 2183 & 0,00 & & 0,1 & & & 305 \\
\hline V: & & & 24 & 0,1 & & 0,1 & 259 & 0,00 & & 0,12 & 509 & & 314 \\
\hline $\mathrm{V}:$ & & & 282 & 0,1 & & 0,13 & 3015 & 0,00 & & 0,12 & 901 & & 335 \\
\hline $\mathrm{V}:$ & & & 235 & 0,1 & & 0,12 & 2545 & 0,0 & & 0,12 & 394 & & 319 \\
\hline
\end{tabular}

Assim como mostrado nas simulações no sistema de 14 barras, os resultados da tabela 5.18 mostram que o índice UI variou pouco, considerando as alterações nos valores das medidas em análise. Isso se deve ao fato de o índice $U I$ de uma medida não depender apenas dos valores das medidas, mas de outros fatores que influenciam as matrizes $K$ e $S$. Dentre estes fatores destacam-se os parâmetros da rede elétrica e a ponderação das medidas. 


\subsection{Conclusões do Capítulo}

Neste capítulo foram apresentados os resultados de algumas simulações, visando a validar os aspectos teóricos do capítulo 4. Para tal propósito, vários cenários de medidas, com ou sem ruído gaussiano e, também, com medidas portadoras de EGs simples, foram utilizados nos sistemas testes de 14 e 30 barras do IEEE.

De um modo geral, através dos resultados, verificou-se que os valores dos resíduos normalizados de medidas portadoras de EGs, com índice $U I$ de valor baixo, são próximos ao valor da magnitude do erro aplicado. Em contrapartida, quanto maior o índice $U I$, menor será o resíduo normalizado da medida e também o índice $J(x)$ do conjunto em análise.

No capítulo 4 concluiu-se que o índice $U I$ depende dos elementos da diagonal principal das matrizes $K$ e $S$, que, por sua vez, dependem dos valores dos parâmetros do sistema, bem como da redundância local, ponderação e valores das medidas. Analisando os valores do índice $U I$, calculados neste capítulo adicionando ou não ruído e/ou EG, constatamos que os mesmos variaram pouco com tais alterações nos valores das medidas. Dessa forma, pode-se concluir que os fatores que mais influenciam, no valor do índice $U I$ de medidas redundantes, são:

- Os valores dos parâmetros do sistema;

- A Ponderação e redundância local das medidas. 


\section{Capítulo 6}

\section{Aplicação do Índice de Não-Detecção de Erros para Detecção e Identificação de Erros Grosseiros}

Neste capítulo é apresentada uma aplicação possível do índice $U I$, para prover uma melhora substancial no processo de detecção e identificação de erros grosseiros simples. Para tal propósito, as seções deste capítulo estão divididas da seguinte forma:

- A seção 6.1 englobará todo o embasamento teórico necessário para mostrar que é possível utilizar o índice $U I$, para a detecção e identificação de EGs, além de exibir os resultados de várias simulações, nos sistemas testes de 14 e 30 barras do IEEE;

- A seção 6.2 apresentará as conclusões relevantes deste capítulo.

\subsection{Utilização do Índice UI para Detecção e Identificação de Erros Grosseiros}

Nesta seção mostrar-se-á que, através do índice UI, é possível obter uma estimativa do EG de uma medida. Para isto, basta calcular o índice UI e o resíduo para todas as medidas disponíveis.

Conforme apresentado anteriormente, no capítulo 4, o resíduo de uma medida corresponde apenas à componente detectável do erro da mesma $\left(\left|\underline{r}_{i}\right| \cong\left\|\underline{w}_{D i}\right\|_{R^{-1}}\right)$ e o índice UI de uma medida corresponde à relação entre a componente não-detectável e detectável do seu erro (equação (4.26)). Assim, o índice UI pode ser utilizado para estimar a componente não-detectável do erro de uma medida, através do resíduo da 
mesma (componente detectável), que é obtido através do estimador de estado WLS. Desta forma, através do índice UI torna-se possível estimar o erro da medida, isto é, torna-se possível compor o erro total da medida a partir da parte detectável (resíduo) e da parte não detectável (obtida através do resíduo e do índice $U I$ ) do erro. Denominaremos essa composição do erro como "erro composto".

Lembrando que as duas componentes do vetor de erros são ortogonais entre si (equação (4.18)) e da definição do índice $U I$ (equação (4.26), obtemos o erro composto para cada medida $i\left(e c_{\mathrm{i}}\right)$, da seguinte forma:

$$
\begin{aligned}
& \underline{e c}_{i}^{2}=\left\|\underline{w}_{U i}\right\|_{R^{-1}}^{2}+\left\|\underline{w}_{D i}\right\|_{R^{-1}}^{2} \\
& \underline{e c}_{i}=\sqrt{\left\|\underline{w}_{U i}\right\|_{R^{-1}}^{2}+\left\|\underline{w}_{D i}\right\|_{R^{-1}}^{2}} \\
& \underline{e c}_{i}=\sqrt{\left(U I_{i} \cdot\left\|\underline{w}_{D i}\right\|_{R^{-1}}\right)^{2}+\left\|\underline{w}_{D i}\right\|_{R^{-1}}^{2}} \\
& \underline{e c}_{i}=\sqrt{\left(U I_{i} \cdot \underline{r}_{i}\right)^{2}+\underline{r}_{i}^{2}} \\
& \underline{e c}_{i}=\sqrt{\left(U I_{i}^{2}+1\right) \cdot \underline{r}_{i}^{2}} \\
& \underline{e c}_{i}=\underline{r}_{i} \cdot \sqrt{\left(U I_{i}^{2}+1\right)}
\end{aligned}
$$

Agora, considerando que a $i$-ésima medida seja portadora de erro grosseiro e que as demais estejam isentas de qualquer erro ou ruído, isto é,

$$
\underline{z}^{\text {erro }}=\underline{z}^{\text {erro }} \underline{w}^{\text {en }}
$$

com $\underline{w}^{\text {erro }}=b \cdot \sigma_{i} \cdot[0, \ldots, 0,1,0, \ldots, 0]^{t}$, podemos calcular o valor esperado do erro composto $E\left\{\underline{e c}_{i}\right\}$, através do seguinte desenvolvimento:

$$
\begin{aligned}
& E\left\{e c_{i}\right\}=E\left\{\underline{r}_{i} \cdot \sqrt{\left(U I_{i}^{2}+1\right)}\right\}=\sqrt{\left(U I_{i}^{2}+1\right)} \cdot E\left\{\underline{r}_{i}\right\} \\
& E\left\{e c_{i}\right\}=\sqrt{\left(U I_{i}^{2}+1\right)} \cdot E\left\{\underline{w}_{i}^{\text {erro }} \cdot S_{i i}\right\} \\
& E\left\{e c_{i}\right\}=\sqrt{\left(U I_{i}^{2}+1\right)} \cdot b \cdot \sigma_{i} \cdot S_{i i}
\end{aligned},
$$

como a expressão $\sqrt{\left(U I_{i}^{2}+1\right)}$ pode ser simplificada por

$$
\begin{aligned}
& \sqrt{\left(U I_{i}^{2}+1\right)}=\sqrt{\frac{K_{i i}}{\left(1-K_{i i}\right)}+1}=\sqrt{\frac{K_{i i}+1-K_{i i}}{\left(1-K_{i i}\right)}} \\
& \sqrt{\left(U I_{i}^{2}+1\right)}=\sqrt{\frac{1}{\left(1-K_{i i}\right)}}=\sqrt{\frac{R_{i i}}{\Omega_{i i}}}=\frac{\sigma_{i}}{\sqrt{\Omega_{i i}}}
\end{aligned},
$$

finalmente temos 


$$
\begin{aligned}
& E\left\{e c_{i}\right\}=\frac{\sigma_{i}}{\sqrt{\Omega_{i i}}} \cdot b \cdot \sigma_{i} \cdot S_{i i}=\frac{b \cdot R_{i i} \cdot S_{i i}}{\sqrt{\Omega_{i i}}} \\
& E\left\{e c_{i}\right\}=\frac{b \cdot \Omega_{i i}}{\sqrt{\Omega_{i i}}}=b \cdot \sqrt{\Omega_{i i}}
\end{aligned}
$$

Com base na expressão (6.5), torna-se possível obter o erro composto em desvio padrão para cada medida $i\left(e_{\sigma \mathrm{i}}\right)$ da seguinte forma:

$$
\begin{aligned}
& \underline{e}_{\sigma i}=\frac{\underline{e c_{i}}}{\sqrt{\Omega_{i i}}}=\frac{\underline{r}_{i} \cdot \sqrt{\left(U I_{i}^{2}+1\right)}}{\sqrt{\Omega_{i i}}} . \\
& \underline{e}_{\sigma i}=\underline{r}_{i}^{N} \cdot \sqrt{\left(U I_{i}^{2}+1\right)}
\end{aligned} .
$$

sendo $r_{i}^{N}$ o resíduo normalizado da medida $i$ (definido no capítulo 4).

Com base nos resultados apresentados até aqui, fica fácil verificar que o valor esperado do erro composto, em desvio padrão, $E\left\{\underline{e}_{\sigma i}\right\}$ para a medida $i$, portadora de erro grosseiro, torna-se:

$$
\begin{aligned}
& E\left\{e_{\sigma i}\right\}=E\left\{\underline{r}_{i}^{N} \cdot \sqrt{\left(U I_{i}^{2}+1\right)}\right\}=E\left\{\frac{\underline{r}_{i}}{\sqrt{\Omega_{i i}}} \cdot \sqrt{\left(U I_{i}^{2}+1\right)}\right\} \\
& E\left\{\underline{e}_{\sigma i}\right\}=E\left\{\frac{e c_{i}}{\sqrt{\Omega_{i i}}}\right\}=\frac{1}{\sqrt{\Omega_{i i}}} \cdot E\left\{\underline{e c}_{i}\right\} \\
& E\left\{\underline{e}_{\sigma i}\right\}=\frac{1}{\sqrt{\Omega_{i i}}} \cdot b \cdot \sqrt{\Omega_{i i}}=b
\end{aligned}
$$

Conseqüentemente, através do erro composto em desvio $\left(e_{\sigma i}\right)$, torna-se possível estimar o erro da medida errônea $\left(\underline{\hat{w}}_{i}\right)$, por meio de:

$$
\underline{\hat{w}}_{i}=\underline{e}_{\sigma i} \cdot \underline{\sigma}_{i},
$$

e, por conseguinte, torna-se possível corrigir o valor da medida:

$$
\underline{z}_{i}^{\text {corrigido }}=\underline{z}_{i}^{\text {erro }}-\underline{\hat{w}}_{i}=\underline{z}_{i}^{\text {erro }}-\underline{e}_{\sigma i} \cdot \underline{\sigma}_{i} .
$$

Face ao exposto, acreditamos que, através do índice $U I$, seja possível aumentar a eficiência do processo de detecção e identificação de EGs através da análise dos resíduos normalizados.

Conforme mostrado na seção 4.3 do capítulo 4, medidas com elevado índice $U I$ podem apresentar resíduos normalizados pequenos, mesmo quando as mesmas são portadoras de EGs. Em situações como essa, provavelmente o teste dos resíduos normalizados iria falhar, mesmo quando apenas uma das medidas seja portadora de EG. 
$\mathrm{Na}$ literatura sobre estimação de estado já foi demonstrado que, quando apenas uma das medidas é portadora de EG (erro grosseiro simples), e as demais são medidas perfeitas (sem ruído), a medida portadora de EG vai apresentar o maior resíduo normalizado em módulo (MONTICELLI e GARCIA, 1983; ABUR e EXPÓSITO, 2004).

Quando o EG ocorre em uma medida com elevado índice $U I$, o valor do seu resíduo normalizado será o maior de todos, mas provavelmente menor que o limiar estabelecido para detecção de EG, pois a componente não-detectável do erro dessa medida é bem maior que a componente detectável, mascarando assim o EG. Nesses casos, o limiar para detecção de EG deve ser comparado com o valor do erro composto em desvio padrão, tendo em vista que o mesmo contempla as duas componentes do erro. Se o erro composto em desvio for maior que o limiar, o mesmo pode ser utilizado ainda para corrigir o valor da medida, conforme apresentado na equação (6.9). Assim, ao invés de eliminar a medida portadora de EG, seria possível corrigir o seu valor, para, em seguida, re-estimar as variáveis de estado.

Em contrapartida, se o índice $U I$ da medida portadora de EG for pequeno, maior será a componente detectável do erro. Nesse caso, provavelmente o teste do resíduo normalizado funcionará de forma adequada, tanto para detectar quanto para identificar a medida com EG, e a contribuição do índice UI seria para correção do valor da medida.

Tendo em vista as possibilidades de utilização do índice $U I$, supracitadas, vamos propor, na próxima subseção, um algoritmo para prover uma melhora substancial ao processo de detecção e identificação de medidas, portadoras de EGs simples.

\subsubsection{Algoritmo para Detecção e Identificação de Erros Grosseiros Simples}

O algoritmo para detecção e identificação de EGs, a ser apresentado, pode ser visto como uma continuação do algoritmo mostrado na subseção 4.3.2, em que o índice UI é determinado. Assim, utilizando-se o estimador WLS convencional, faça:

Passo 1: Determine o valor estimado das variáveis de estado $(\underline{\hat{x}})$, através da solução iterativa da equação (2.17) (equação normal);

Passo 2: Calcule as matrizes $K$ e $(I-K)$ considerando $\underline{\hat{x}}$, ou calcule apenas os elementos da diagonal principal dessas matrizes;

Passo 3: Calcule o resíduo normalizado $r_{i}^{N}$ para cada medida $i$ e o índice $U I$, isto é: 


$$
\begin{gathered}
r_{i}^{N}=\frac{r_{i}}{\sqrt{\Omega_{i i}}}, \text { para } i=1, \ldots, m ; \\
U I_{i}=\frac{\sqrt{\left(K_{i i}\right)}}{\sqrt{\left(1-K_{i i}\right)}}, \text { para } i=1, \ldots, m .
\end{gathered}
$$

Passo 4: Através dos resíduos normalizados e dos índices UIs, calcule o erro composto em desvio padrão $e_{\sigma i}$ para cada medida $i$, utilizando a equação (6.6):

$$
\underline{e}_{\sigma i}=\underline{r}_{i}^{N} \cdot \sqrt{\left(U I_{i}^{2}+1\right)}, \text { para } i=1, \ldots, m .
$$

Passo 5: Para a medida com maior resíduo normalizado em módulo, verifique se o módulo de seu erro composto em desvio padrão é maior que certo limiar de detecção pré-estabelecido $\rho$ (pode ser o mesmo limiar do teste dos resíduos normalizados), ou seja:

- Se $\left|e_{\sigma i}\right|>\rho$, esta medida $i$ apresenta EG; vá ao passo 6 .

- Se $\left|e_{\sigma i}\right| \leq \rho$, aceita-se a hipótese de que não exista EG; fim.

Passo 6: Corrija o valor da medida identificada no passo 5, através da equação (6.9):

$$
\underline{z}_{i}^{\text {corrigido }}=\underline{z}_{i}^{\text {erro }}-\underline{\hat{w}}_{i}=\underline{z}_{i}^{\text {erro }}-\underline{e}_{\sigma i} \cdot \underline{\sigma}_{i} ;
$$

volte ao passo 1.

Nota 6.1: Para o cálculo da equação (6.9), o sinal (positivo ou negativo) do $\underline{e}_{\sigma i}$ deve ser levado em conta, e não apenas sua magnitude. Assim:

- Se $\underline{e}_{\sigma i}$ for negativo, $\underline{z}_{i}^{\text {corrigido }}=\underline{z}_{i}+\left|\underline{e}_{\sigma i}\right| \cdot \underline{\sigma}_{i} ;$

- Se $\underline{e}_{\sigma i}$ for positivo, $\underline{z}_{i}^{\text {corrigido }}=\underline{z}_{i}-\left|\underline{e}_{\sigma i}\right| \cdot \underline{\sigma}_{i}$.

\subsubsection{Aplicação do Algoritmo para Detecção e Identificação de Erros Grosseiros sem acrescentar Ruído Gaussiano às Medidas: Exemplos}

\section{$\underline{\text { Sistema de } 14 \text { barras }}$}

Para os exemplos a seguir, considere o sistema de 14 barras do IEEE, e o limiar de detecção de erro grosseiro $\rho=3,0$ (o mesmo limiar do teste dos resíduos normalizados). 
Exemplo 1: Medidas sem ruído e adição de EG simples, em medida com UI de valor baixo

Considere o sistema de medição apresentado na tabela 4.1, porém agora com adição de EG de $10 \sigma$ na medida IA:1 (os valores das demais medidas são exatamente os determinados pelo fluxo de potência, isto é, sem acrescentar ruído aleatório algum).

Através da execução do algoritmo apresentado na subseção anterior, obtivemos os resultados tabelados a seguir (tabela 6.1):

Tabela 6.1: Resultados numéricos da adição de EG de $10 \sigma$ na medida IA:1

\begin{tabular}{|c|c|c|c|c|c|c|c|c|c|}
\hline \multicolumn{5}{|c|}{ Medidas de Potência Ativa } & \multicolumn{5}{|c|}{ Medidas de Potência Reativa } \\
\hline Medida & $\begin{array}{c}\text { Valor } \\
\text { estimado }\end{array}$ & $U I$ & $r^{N}$ & $e_{\sigma}$ & Medida & $\begin{array}{c}\text { Valor } \\
\text { estimado }\end{array}$ & $U I$ & $r^{N}$ & $e_{\sigma}$ \\
\hline IA: 1 & 2,2946 & 0,2826 & $-9,6301$ & $-10,0073$ & IR:1 & $-0,1662$ & 1,411 & 0,8723 & 1,5086 \\
\hline IA:2 & 0,185 & 6,5105 & $-4,1418$ & $-27,2818$ & IR:2 & 0,3052 & 0,6753 & 0,8404 & 1,014 \\
\hline IA:5 & $-0,0758$ & 11,4557 & $-2,2828$ & $-26,25$ & IR:5 & $-0,016$ & 9,446 & 0,7857 & 7,4631 \\
\hline IA: 7 & NULO & --- & NULO & --- & IR:7 & NULO & --- & NULO & --- \\
\hline IA:8 & NULO & --- & NULO & --- & IR:8 & 0,1759 & 0,4297 & 0,0613 & 0,0668 \\
\hline IA:9 & $-0,2952$ & 0,7561 & $-0,0595$ & $-0,0746$ & IR:9 & $-0,1657$ & 0,5556 & $-0,2692$ & $-0,308$ \\
\hline IA:13 & $-0,1347$ & 0,8338 & $-0,0054$ & $-0,0071$ & IR:13 & $-0,0581$ & 0,6994 & $-0,0255$ & $-0,0312$ \\
\hline IA:14 & $-0,1489$ & 0,6645 & 0,0009 & 0,0011 & IR:14 & $-0,0496$ & 0,6737 & 0,0131 & 0,0158 \\
\hline FA:1-2 & 1,5488 & 0,2903 & 0,7793 & 0,8114 & FR:1-2 & $-0,2034$ & 0,579 & $-0,0517$ & $-0,0598$ \\
\hline FA:2-3 & 0,7276 & 0,2453 & 0,4486 & 0,4619 & FR:2-3 & 0,0357 & 2,212 & 0,9893 & 2,4017 \\
\hline FA:4-2 & $-0,5374$ & 0,3227 & $-0,7706$ & $-0,8097$ & FR:4-2 & 0,0296 & 1,1875 & 1,4371 & 2,2311 \\
\hline FA:5-2 & $-0,4014$ & 0,2931 & $-0,6676$ & $-0,6956$ & FR:5-2 & $-0,021$ & 1,1141 & $-0,6512$ & $-0,9749$ \\
\hline FA:3-4 & $-0,2351$ & 1,2969 & 0,5319 & 0,8711 & FR:3-4 & 0,0444 & 2,1507 & 1,3539 & 3,2112 \\
\hline FA:4-5 & $-0,6011$ & 0,4904 & $-1,1167$ & $-1,2438$ & FR:4-5 & 0,1569 & 0,3611 & 0,5634 & 0,599 \\
\hline FA:7-4 & $-0,2806$ & 0,4202 & $-0,02$ & $-0,0217$ & FR:7-4 & 0,1129 & 0,4243 & 0,2286 & 0,2483 \\
\hline FA:9-4 & $-0,1607$ & 0,4203 & $-0,0223$ & $-0,0241$ & FR:9-4 & 0,017 & 1,6123 & 0,7006 & 1,3292 \\
\hline FA:5-6 & 0,4382 & 0,3336 & 0,2886 & 0,3042 & FR:5-6 & 0,1243 & 0,4575 & 0,4078 & 0,4484 \\
\hline FA:6-5 & $-0,4382$ & 0,3336 & $-0,2886$ & $-0,3042$ & FR:6-5 & $-0,0803$ & 0,9337 & $-0,5514$ & $-0,7543$ \\
\hline FA:6-11 & 0,0737 & 0,8943 & 0,1701 & 0,2282 & FR:6-11 & 0,0354 & 0,8165 & 0,173 & 0,2233 \\
\hline FA:11-6 & $-0,0731$ & 0,8971 & $-0,1726$ & $-0,2319$ & FR:11-6 & $-0,0342$ & 0,8871 & $-0,1891$ & $-0,2528$ \\
\hline FA:6-12 & 0,0779 & 0,6026 & 0,0243 & 0,0283 & FR:6-12 & 0,0251 & 0,8307 & 0,0432 & 0,0562 \\
\hline FA:6-13 & 0,1774 & 0,4551 & 0,0219 & 0,0241 & FR:6-13 & 0,0721 & 0,4307 & 0,0242 & 0,0263 \\
\hline FA:13-6 & $-0,1752$ & 0,4501 & $-0,0249$ & $-0,0273$ & FR:13-6 & $-0,0679$ & 0,4549 & $-0,0398$ & $-0,0437$ \\
\hline FA:7-8 & NULO & --- & NULO & --- & FR:7-8 & $-0,1713$ & 0,4165 & $-0,0661$ & $-0,0716$ \\
\hline FA:8-7 & NULO & --- & NULO & --- & FR:8-7 & 0,1759 & 0,4297 & 0,0614 & 0,0669 \\
\hline FA:7-9 & 0,2806 & 0,4202 & 0,0201 & 0,0218 & FR:7-9 & 0,0584 & 1,2161 & $-0,366$ & $-0,5762$ \\
\hline FA:9-10 & 0,0521 & 0,9794 & $-0,0356$ & $-0,0499$ & FR:9-10 & 0,0423 & 0,9308 & $-0,0565$ & $-0,0772$ \\
\hline FA:10-9 & $-0,052$ & 0,9736 & 0,0345 & 0,0481 & FR:10-9 & $-0,0419$ & 0,9196 & 0,0528 & 0,0717 \\
\hline FA:9-14 & 0,0941 & 0,7581 & $-0,056$ & $-0,0703$ & FR:9-14 & 0,036 & 0,7256 & $-0,0974$ & $-0,1203$ \\
\hline FA:10-11 & $-0,0379$ & 4,2345 & $-0,5026$ & $-2,1869$ & FR:10-11 & $-0,0159$ & 3,8975 & $-0,4992$ & $-2,0087$ \\
\hline FA:12-13 & 0,016 & 3,2396 & 0,04 & 0,1357 & FR:12-13 & 0,0075 & 3,3303 & 0,0184 & 0,0641 \\
\hline FA:14-13 & $-0,056$ & 0,907 & $-0,0889$ & $-0,12$ & FR:14-13 & $-0,0161$ & 1,2526 & $-0,1445$ & $-0,2316$ \\
\hline
\end{tabular}

\begin{tabular}{c|c|c|c|c}
\hline \multicolumn{5}{|c|}{ Medidas de Tensão } \\
\hline Medida & Valor estimado & $\boldsymbol{U I}$ & $r^{N}$ & $\boldsymbol{e}_{\sigma}$ \\
\hline $\mathbf{V : 1}$ & 1,0568 & 0,1546 & 0,1507 & 0,1525 \\
\hline $\mathbf{V : 2}$ & 1,042 & 0,1554 & 0,1387 & 0,1403 \\
\hline $\mathbf{V : 3}$ & 1,007 & 0,1614 & 0,1356 & 0,1373 \\
\hline
\end{tabular}




\begin{tabular}{c|c|c|c|c}
\hline $\mathbf{V : 6}$ & 1,067 & 0,1618 & 0,1249 & 0,1265 \\
\hline $\mathbf{V : 8}$ & 1,0871 & 0,1549 & 0,1182 & 0,1196 \\
\hline $\mathbf{V : 1 2}$ & 1,0521 & 0,1663 & 0,1281 & 0,1299 \\
\hline
\end{tabular}

A partir dos resultados apresentados na tabela 6.1, verifica-se que o maior resíduo normalizado em módulo é o da medida IA:1 (em azul), cujo valor é 9,6301. Como o erro composto em desvio padrão dessa medida é maior que o limiar de detecção, isto é:

$$
\left|e_{\sigma I A: 1}\right|=10,0073>\rho=3,0,
$$

considera-se então a medida IA:1 como portadora de erro grosseiro.

Através da equação (6.9), calcula-se o valor corrigido da medida IA:1:

$$
\begin{aligned}
& z_{\text {IA:1 }}^{\text {corrigido }}=z_{I A: 1}-e_{\sigma \text { IA:1 }} \cdot \sigma_{I A: 1} \\
& z_{\text {IA:1 }}^{\text {corrigido }}=1,93566-(-10,0073) \cdot 0,03873 . \\
& z_{\text {IA:1 }}^{\text {corrigido }}=2,3232
\end{aligned}
$$

Após a correção realizada no valor da medida IA:1, aplicamos novamente o algoritmo de detecção de EGs. Como resultado dessa nova simulação, verificamos que o erro composto em desvio, para a medida com maior resíduo normalizado (medida IR:5, $\left.\left|r_{I R: 5}^{N}\right|=0,0473\right)$ vale: $\left|e_{\sigma I R: 5}\right|=0,4493$. Como este valor é menor que o limiar $\rho=3,0$, conclui-se que o sistema de medição não apresenta mais EG.

Vale ressaltar que o valor sem EG e sem ruído da medida IA:1 é 2,32299 p.u., e o valor estimado dessa medida, após a identificação e correção do erro, foi de 2,3230, ou seja, um valor muito próximo ao considerado "correto".

Exemplo 2: Medidas sem ruído e adição de EG simples, em medida com UI de valor alto

Considere o mesmo sistema de medição apresentado na tabela 4.1, porém agora com adição de EG de $10 \sigma$ na medida IA:5.

Com a execução do algoritmo apresentado na subseção anterior, obtivemos os resultados tabelados a seguir (tabela 6.2): 
Tabela 6.2: Resultados numéricos da adição de EG de 10 б na medida IA:5

\begin{tabular}{|c|c|c|c|c|c|c|c|c|c|}
\hline \multicolumn{5}{|c|}{ Medidas de Potência Ativa } & \multicolumn{5}{|c|}{ Medidas de Potência Reativa } \\
\hline Medida & $\begin{array}{c}\text { Valor } \\
\text { estimado }\end{array}$ & $\boldsymbol{U I}$ & $r^{N}$ & $e_{\sigma}$ & Medida & $\begin{array}{c}\text { Valor } \\
\text { estimado }\end{array}$ & $\boldsymbol{U I}$ & $r^{N}$ & $e_{\sigma}$ \\
\hline IA:1 & 2,3153 & 0,2806 & 0,2057 & 0,2137 & IR:1 & $-0,165$ & 1,4063 & 0,1388 & 0,2396 \\
\hline IA:2 & 0,1831 & 6,4865 & 0,0268 & 0,1759 & IR:2 & 0,3078 & 0,6639 & 0,2263 & 0,2716 \\
\hline IA:5 & $-0,0634$ & 11,3898 & 0,8703 & 9,9509 & IR:5 & $-0,016$ & 9,4473 & $-0,175$ & $-1,6623$ \\
\hline IA:7 & NULO & --- & NULO & --- & IR:7 & NULO & --- & NULO & --- \\
\hline IA:8 & NULO & --- & NULO & --- & IR:8 & 0,176 & 0,4293 & $-0,0024$ & $-0,0026$ \\
\hline IA:9 & $-0,2954$ & 0,7553 & $-0,0134$ & $-0,0168$ & IR:9 & $-0,1661$ & 0,5411 & $-0,1276$ & $-0,1451$ \\
\hline IA:13 & $-0,1347$ & 0,8338 & $-0,0017$ & $-0,0022$ & IR:13 & $-0,0581$ & 0,6993 & $-0,0062$ & $-0,0076$ \\
\hline IA:14 & $-0,1489$ & 0,6645 & $-0,0042$ & $-0,0051$ & IR:14 & $-0,0496$ & 0,6737 & $-0,0167$ & $-0,0201$ \\
\hline FA:1-2 & 1,5639 & 0,2883 & 0,1772 & 0,1844 & FR:1-2 & $-0,2032$ & 0,579 & $-0,0988$ & $-0,1141$ \\
\hline FA:2-3 & 0,7322 & 0,2433 & 0,0561 & 0,0578 & FR:2-3 & 0,0359 & 2,1974 & 0,0366 & 0,0883 \\
\hline FA:4-2 & $-0,543$ & 0,3205 & $-0,1208$ & $-0,1269$ & FR:4-2 & 0,0302 & 1,1862 & $-0,1646$ & $-0,2553$ \\
\hline FA:5-2 & $-0,4033$ & 0,2912 & $-0,3783$ & $-0,394$ & FR:5-2 & $-0,0212$ & 1,114 & 0,2796 & 0,4185 \\
\hline FA:3-4 & $-0,2342$ & 1,2756 & 0,1458 & 0,2363 & FR:3-4 & 0,0448 & 2,1242 & 0,0932 & 0,2188 \\
\hline FA:4-5 & $-0,6172$ & 0,4874 & 0,6429 & 0,7152 & FR:4-5 & 0,1585 & 0,3607 & $-0,0938$ & $-0,0997$ \\
\hline FA:7-4 & $-0,2807$ & 0,4198 & $-0,0095$ & $-0,0103$ & FR:7-4 & 0,1133 & 0,4186 & 0,024 & 0,0261 \\
\hline FA:9-4 & $-0,1608$ & 0,42 & $-0,0107$ & $-0,0116$ & FR:9-4 & 0,0171 & 1,5937 & $-0,082$ & $-0,1543$ \\
\hline FA:5-6 & 0,4413 & 0,3327 & $-0,1553$ & $-0,1637$ & FR:5-6 & 0,1252 & 0,4488 & $-0,0823$ & $-0,0902$ \\
\hline FA:6-5 & $-0,4413$ & 0,3327 & 0,1553 & 0,1637 & FR:6-5 & $-0,0808$ & 0,8894 & $-0,1204$ & $-0,1611$ \\
\hline FA:6-11 & 0,0738 & 0,8935 & $-0,0194$ & $-0,026$ & FR:6-11 & 0,0355 & 0,8157 & $-0,0232$ & $-0,0299$ \\
\hline FA:11-6 & $-0,0733$ & 0,8962 & 0,0183 & 0,0246 & FR:11-6 & $-0,0343$ & 0,886 & 0,0196 & 0,0262 \\
\hline FA:6-12 & 0,0779 & 0,6026 & $-0,0022$ & $-0,0025$ & FR:6-12 & 0,0251 & 0,8306 & $-0,0043$ & $-0,0056$ \\
\hline FA:6-13 & 0,1774 & 0,455 & $-0,002$ & $-0,0022$ & FR:6-13 & 0,0721 & 0,4307 & $-0,0046$ & $-0,005$ \\
\hline FA:13-6 & $-0,1753$ & 0,45 & 0,0007 & 0,0008 & FR:13-6 & $-0,0679$ & 0,4547 & $-0,0019$ & $-0,0021$ \\
\hline FA:7-8 & NULO & --- & NULO & --- & FR:7-8 & $-0,1714$ & 0,4162 & $-0,0004$ & $-0,0004$ \\
\hline FA:8-7 & NULO & --- & NULO & --- & FR:8-7 & 0,176 & 0,4293 & $-0,0024$ & $-0,0026$ \\
\hline FA:7-9 & 0,2807 & 0,4198 & 0,0095 & 0,0103 & FR:7-9 & 0,0582 & 1,2038 & $-0,0684$ & $-0,107$ \\
\hline FA:9-10 & 0,0521 & 0,9793 & 0,0052 & 0,0073 & FR:9-10 & 0,0422 & 0,9307 & 0,0284 & 0,0388 \\
\hline FA:10-9 & $-0,0519$ & 0,9736 & $-0,0055$ & $-0,0077$ & FR:10-9 & $-0,0419$ & 0,9197 & $-0,0291$ & $-0,0395$ \\
\hline FA:9-14 & 0,094 & 0,758 & 0,0091 & 0,0114 & FR:9-14 & 0,0359 & 0,7254 & 0,0188 & 0,0233 \\
\hline FA:10-11 & $-0,038$ & 4,2184 & 0,0552 & 0,2392 & FR:10-11 & $-0,016$ & 3,8832 & 0,0561 & 0,225 \\
\hline FA:12-13 & 0,016 & 3,2396 & $-0,0076$ & $-0,0259$ & FR:12-13 & 0,0075 & 3,3303 & $-0,0116$ & $-0,0402$ \\
\hline FA:14-13 & $-0,056$ & 0,9067 & 0,006 & 0,0081 & FR:14-13 & $-0,0161$ & 1,2517 & 0,0043 & 0,0069 \\
\hline
\end{tabular}

\begin{tabular}{c|c|c|c|c}
\hline \multicolumn{5}{|c|}{ Medidas de Tensão } \\
\hline Medida & Valor estimado & $\boldsymbol{U I}$ & $r^{N}$ & $\boldsymbol{e}_{\sigma}$ \\
\hline $\mathbf{V : 1}$ & 1,0584 & 0,1478 & 0,0543 & 0,0549 \\
\hline $\mathbf{V : 2}$ & 1,0435 & 0,1485 & 0,0528 & 0,0534 \\
\hline $\mathbf{V : 3}$ & 1,0084 & 0,1542 & 0,0541 & 0,0548 \\
\hline $\mathbf{V : 6}$ & 1,0683 & 0,1545 & 0,049 & 0,0495 \\
\hline $\mathbf{V : 8}$ & 1,0884 & 0,148 & 0,0472 & 0,0477 \\
\hline $\mathbf{V : 1 2}$ & 1,0535 & 0,1588 & 0,0504 & 0,051 \\
\hline
\end{tabular}

A partir dos resultados da tabela 6.2, verificamos que o erro composto em desvio, para a medida com maior resíduo normalizado (medida IA:5, $\left|r_{I A: 5}^{N}\right|=0,8703$ ), vale $\left|e_{\sigma_{I A: 5}}\right|=9,9506$, sendo maior que o limiar $\rho=3,0$. 
Através da equação (6.9), calculamos o valor corrigido da medida IA:5:

$$
\begin{aligned}
& z_{\text {IA:5 }}^{\text {corrigido }}=z_{I A: 5}-e_{\sigma \text { IA:5 }} \cdot \sigma_{I A: 5} \\
& z_{\text {IA:5 }}^{\text {corigido }}=-0,0633036-(9,9506) \cdot 0,001267 . \\
& z_{\text {IA:5 }}^{\text {corrigido }}=-0,075911
\end{aligned}
$$

Nota 6.2: $\mathrm{O}$ valor sem erro e sem ruído da medida IA:5 é -0,07601 p.u..

Após a correção realizada no valor da medida IA:5, aplicamos novamente o algoritmo de detecção de EGs. Como resultado dessa nova simulação, verificamos que o erro composto em desvio padrão para a medida com maior resíduo normalizado (medida IR:5, $\left|r_{I R: 5}^{N}\right|=0,0317$ ) vale: $\left|e_{\sigma \text { IR:5 }}\right|=0,3015$. Portanto, o conjunto de medição não apresenta mais EG.

Exemplo 3: Simulações de EG simples de 10 sigmas e 20 sigmas em cada uma das medidas

Apresentaremos, neste exemplo, os resultados de simulações considerando EG simples de 10 e 20 sigmas, em cada uma das medidas do sistema de medição da tabela 4.1 (uma de cada vez e as demais sem erro e sem ruído). Os resultados após a correção do EG não serão mostrados, já que em todos os casos a correção foi satisfatória, o suficiente para que nenhum EG fosse detectado depois das correções.

A tabela 6.3, a seguir, apresenta os valores do índice $U I$, do resíduo normalizado e do erro composto em desvio padrão, para a medida portadora de EG em cada caso, considerando erro grosseiro simples de 10 sigmas.

Tabela 6.3: Resultados numéricos da adição de EG de $10 \sigma$ para cada medida (uma de cada vez) considerando o sistema de medição 1

Limiar do teste do índice $J(x): \lambda=\chi_{43 ;(1-0,05)}^{2} \cong 58,0$

\begin{tabular}{c|c|c|c|c|c|c|c|c|c}
\hline \multicolumn{4}{|c}{ Medidas de Potência Ativa } & \multicolumn{5}{c}{ Medidas de Potência Reativa } \\
\hline $\begin{array}{c}\text { Medida } \\
\text { com erro }\end{array}$ & $U I$ & $r^{N}$ & $e_{\sigma}$ & $\mathbf{J}(\mathbf{x})$ & $\begin{array}{c}\text { Medida } \\
\text { com erro }\end{array}$ & $U I$ & $r^{N}$ & $e_{\sigma}$ & J(x) \\
\hline IA:1 & 0,281 & $-9,630$ & $-10,007$ & 92,67 & IR:1 & 1,406 & $-5,794$ & $-9,984$ & 33,59 \\
\hline IA:2 & 6,491 & 1,521 & 9,996 & 2,31 & IR:2 & 0,665 & $-8,371$ & $-10,082$ & 69,41 \\
\hline IA:5 & 11,434 & 0,870 & 9,951 & 0,76 & IR:5 & 9,437 & 0,992 & 9,434 & 1,11 \\
\hline IA:7 & --- & --- & -- & --- & IR:7 & --- & --- & --- & -- \\
\hline IA:8 & --- & --- & --- & --- & IR:8 & 0,429 & $-9,192$ & $-10,006$ & 84,44 \\
\hline IA:9 & 0,755 & 7,975 & 9,996 & 63,63 & IR:9 & 0,547 & $-8,772$ & $-9,979$ & 77,13 \\
\hline IA:13 & 0,834 & 7,679 & 10,001 & 58,97 & IR:13 & 0,699 & $-8,196$ & $-10,001$ & 67,17 \\
\hline IA:14 & 0,665 & $-8,329$ & $-9,999$ & 69,38 & IR:14 & 0,674 & $-8,293$ & $-9,999$ & 68,78 \\
\hline
\end{tabular}




\begin{tabular}{|c|c|c|c|c|c|c|c|c|c|}
\hline FA:1-2 & 0,289 & 9,604 & 9,990 & 92,31 & FR:1-2 & 0,579 & $-8,655$ & -10.000 & 74,89 \\
\hline FA:2-3 & 0,244 & 9,733 & 10,037 & 94,39 & FR:2-3 & 2,197 & 4,126 & 10,032 & 17,03 \\
\hline FA:4-2 & 0,321 & 9,513 & 9,984 & 90,64 & FR:4-2 & 1,183 & 6,461 & 10,084 & 41,46 \\
\hline FA:5-2 & 0,292 & $-9,604$ & $-10,003$ & 92,19 & FR:5-2 & 1,114 & $-6,677$ & $-9,988$ & 44,66 \\
\hline FA:3-4 & 1,283 & $-6,119$ & $-9,782$ & 38,15 & FR:3-4 & 2,125 & 4,250 & 10,134 & 17,93 \\
\hline FA:4-5 & 0,489 & 8,984 & 10,003 & 80,72 & FR:4-5 & 0,360 & $-9,408$ & $-10,002$ & 88,49 \\
\hline FA:7-4 & 0,419 & 9,221 & 10,003 & 85,00 & FR:7-4 & 0,421 & $-9,186$ & $-9,926$ & 85,01 \\
\hline FA:9-4 & 0,420 & 9,220 & 10,002 & 84,99 & FR:9-4 & 1,603 & $-5,355$ & $-10,178$ & 28,16 \\
\hline FA:5-6 & 0,333 & $-9,488$ & $-10,002$ & 90,02 & FR:5-6 & 0,454 & $-9,157$ & $-10,089$ & 83,10 \\
\hline FA:6-5 & 0,333 & $-9,488$ & $-9,998$ & 90,03 & FR:6-5 & 0,905 & 7,331 & 9,734 & 55,29 \\
\hline FA:6-11 & 0,894 & $-7,456$ & $-9,993$ & 55,61 & FR:6-11 & 0,816 & $-7,748$ & $-9,997$ & 60,05 \\
\hline FA:11-6 & 0,897 & 7,442 & 9,994 & 55,42 & FR:11-6 & 0,886 & $-7,482$ & $-9,992$ & 56,03 \\
\hline FA:6-12 & 0,602 & 8,565 & 9,999 & 73,36 & FR:6-12 & 0,830 & $-7,693$ & $-9,998$ & 59,19 \\
\hline FA:6-13 & 0,455 & $-9,102$ & $-9,999$ & 82,85 & FR:6-13 & 0,431 & 9,184 & 9,999 & 84,35 \\
\hline FA:13-6 & 0,450 & $-9,119$ & $-9,999$ & 83,16 & FR:13-6 & 0,455 & 9,103 & 10.000 & 82,86 \\
\hline FA:7-8 & --- & --- & --- & --- & FR:7-8 & 0,416 & 9,239 & 10,014 & 85,23 \\
\hline FA:8-7 & --- & --- & --- & --- & FR:8-7 & 0,429 & $-9,192$ & $-10,006$ & 84,44 \\
\hline FA:7-9 & 0,419 & $-9,220$ & $-10,001$ & 84,99 & FR:7-9 & 1,208 & 6,363 & 9,915 & 40,74 \\
\hline FA:9-10 & 0,979 & 7,144 & 9,999 & 51,04 & FR:9-10 & 0,931 & 7,317 & 9,998 & 53,57 \\
\hline FA:10-9 & 0,974 & $-7,166$ & $-10,000$ & 51,35 & FR:10-9 & 0,919 & $-7,360$ & $-9,997$ & 54,19 \\
\hline FA:9-14 & 0,758 & $-7,970$ & $-9,999$ & 63,53 & FR:9-14 & 0,725 & $-8,095$ & $-9,997$ & 65,54 \\
\hline FA:10-11 & 4,227 & 2,303 & 9,994 & 5,31 & FR:10-11 & 3,890 & 2,479 & 9,915 & 6,21 \\
\hline FA:12-13 & 3,240 & 2,946 & 10,006 & 8,68 & FR:12-13 & 3,330 & $-2,875$ & $-9,994$ & 8,28 \\
\hline FA:14-13 & 0,907 & $-7,409$ & $-9,998$ & 54,90 & FR:14-13 & 1,252 & 6,242 & 10,005 & 38,94 \\
\hline \multicolumn{10}{|c|}{ Medidas de Tensão } \\
\hline \multicolumn{2}{|c|}{ Medida com erro } & \multicolumn{2}{|c|}{$U I$} & \multicolumn{2}{|c|}{$r^{N}$} & \multicolumn{2}{|c|}{$e_{\sigma}$} & \multicolumn{2}{|c|}{$\mathbf{J}(\mathbf{x})$} \\
\hline \multicolumn{2}{|c|}{ V:1 } & \multicolumn{2}{|c|}{0,150} & \multicolumn{2}{|c|}{9,905} & \multicolumn{2}{|c|}{10,029} & \multicolumn{2}{|c|}{97,83} \\
\hline \multicolumn{2}{|c|}{$V: 2$} & \multicolumn{2}{|c|}{0,151} & \multicolumn{2}{|c|}{$-9,900$} & \multicolumn{2}{|c|}{$-10,022$} & \multicolumn{2}{|c|}{97,79} \\
\hline \multicolumn{2}{|c|}{$V: 3$} & \multicolumn{2}{|c|}{0,156} & \multicolumn{2}{|c|}{$-9,871$} & \multicolumn{2}{|c|}{$-9,981$} & \multicolumn{2}{|c|}{97,63} \\
\hline \multicolumn{2}{|c|}{ V:6 } & \multicolumn{2}{|c|}{0,157} & \multirow{2}{*}{\multicolumn{2}{|c|}{$\begin{array}{l}-9,878 \\
-9,886\end{array}$}} & \multicolumn{2}{|c|}{$-9,997$} & \multicolumn{2}{|c|}{97,62} \\
\hline \multicolumn{2}{|c|}{$\mathrm{V}: 8$} & \multicolumn{2}{|c|}{0,150} & & & & & & \\
\hline $\mathrm{V}:$ & & & 61 & & 877 & & 04 & & \\
\hline
\end{tabular}

A tabela 6.4, a seguir, apresenta os valores do índice $J(x)$ e os valores do índice UI, resíduo normalizado e erro composto em desvio padrão, para a medida portadora de EG, em cada caso, considerando EG simples de 20 sigmas.

Tabela 6.4: Resultados numéricos da adição de EG de $20 \sigma$ para cada medida (uma de cada vez) considerando o sistema de medição 1

Limiar do teste do índice $J(x): \lambda=\chi_{43 ;(1-0,05)}^{2} \cong 58,0$

\begin{tabular}{c|c|c|c|c|c|c|c|c|c}
\hline \multicolumn{9}{c}{ Medidas de Potência Ativa } & \multicolumn{5}{c}{ Medidas de Potência Reativa } \\
\hline $\begin{array}{c}\text { Medida } \\
\text { com erro }\end{array}$ & $U I$ & $r^{N}$ & $\boldsymbol{e}_{\sigma}$ & $\mathbf{J}(\mathbf{x})$ & $\begin{array}{c}\text { Medida } \\
\text { com erro }\end{array}$ & $U I$ & $r^{N}$ & $\boldsymbol{e}_{\sigma}$ & $\mathbf{J}(\mathbf{x})$ \\
\hline IA:1 & 0,282 & $-19,251$ & $-19,999$ & 370,66 & IR:1 & 1,408 & $-11,600$ & $-19,969$ & 134,59 \\
\hline IA:2 & 6,521 & 3,041 & 20,047 & 9,249 & IR:2 & 0,668 & $-16,741$ & $-20,088$ & 278,39 \\
\hline IA:5 & 11,38 & 1,768 & 20,432 & 3,053 & IR:5 & 9,443 & 2,195 & 20,853 & 4,439 \\
\hline IA:7 & --- & --- & --- & -- & IR:7 & -- & --- & --- & --- \\
\hline IA:8 & --- & --- & --- & --- & IR:8 & 0,429 & $-18,383$ & $-20,003$ & 337,84 \\
\hline IA:9 & 0,757 & 15,953 & 20,014 & 254,43 & IR:9 & 0,539 & $-17,569$ & $-20,022$ & 308,43 \\
\hline IA:13 & 0,834 & 15,357 & 20,005 & 235,83 & IR:13 & 0,699 & $-16,393$ & $-19,999$ & 268,72 \\
\hline
\end{tabular}




\begin{tabular}{|c|c|c|c|c|c|c|c|c|c|}
\hline IA:14 & 0,665 & $-16,662$ & $-19,995$ & 277,62 & IR:14 & 0,674 & $-16,590$ & $-19,998$ & 275,20 \\
\hline FA:1-2 & 0,289 & 19,210 & 19,983 & 369,31 & FR:1-2 & 0,579 & $-17,309$ & $-20,000$ & 299,61 \\
\hline FA:2-3 & 0,243 & 19,431 & 19,999 & 377,57 & FR:2-3 & 2,197 & 8,256 & 20,304 & 67,623 \\
\hline FA:4-2 & 0,322 & 19,037 & 20,003 & 362,48 & FR:4-2 & 1,188 & 12,882 & 20,114 & 165,44 \\
\hline FA:5-2 & 0,293 & $-19,206$ & $-19,998$ & 368,85 & FR:5-2 & 1,112 & $-13,359$ & $-19,984$ & 178,60 \\
\hline FA:3-4 & 1,289 & $-12,385$ & $-19,866$ & 153,49 & FR:3-4 & 2,128 & 8,5178 & 20,638 & 70,99 \\
\hline FA:4-5 & 0,489 & 17,954 & 20,001 & 322,59 & FR:4-5 & 0,36 & $-18,821$ & $-20,011$ & 353,99 \\
\hline FA:7-4 & 0,421 & 18,438 & 20,004 & 339,94 & FR:7-4 & 0,417 & $-18,506$ & $-20,146$ & 340,02 \\
\hline FA:9-4 & 0,421 & 18,437 & 20,004 & 339,92 & FR:9-4 & 1,587 & $-10,628$ & $-20,012$ & 112,77 \\
\hline FA:5-6 & 0,334 & $-18,976$ & $-20,006$ & 360,02 & FR:5-6 & 0,446 & $-18,250$ & $-20,028$ & 332,47 \\
\hline FA:6-5 & 0,334 & $-18,982$ & $-20,005$ & 360,18 & FR:6-5 & 0,881 & 14,576 & 19,247 & 220,88 \\
\hline FA:6-11 & 0,893 & $-14,923$ & $-20,008$ & 222,53 & FR:6-11 & 0,815 & $-15,503$ & $-20,002$ & 240,28 \\
\hline FA:11-6 & 0,896 & 14,897 & 20,034 & 221,62 & FR:11-6 & 0,886 & $-14,988$ & $-20,037$ & 224,17 \\
\hline FA:6-12 & 0,603 & 17,129 & 20,001 & 293,42 & FR:6-12 & 0,83 & $-15,389$ & $-19,997$ & 236,79 \\
\hline FA:6-13 & 0,455 & $-18,205$ & $-19,999$ & 331,42 & FR:6-13 & 0,431 & 18,368 & 20,001 & 337,39 \\
\hline FA:13-6 & 0,450 & $-18,24$ & $-19,999$ & 332,67 & FR:13-6 & 0,455 & 18,204 & 20,000 & 331,43 \\
\hline FA:7-8 & --- & --- & --- & --- & FR:7-8 & 0,416 & 18,465 & 20,005 & 340,90 \\
\hline FA:8-7 & --- & --- & --- & --- & FR:8-7 & 0,429 & $-18,380$ & $-19,997$ & 337,83 \\
\hline FA:7-9 & 0,421 & $-18,437$ & $-20,001$ & 339,95 & FR:7-9 & 1,203 & 12,826 & 20,177 & 163,08 \\
\hline FA:9-10 & 0,979 & 14,2873 & 20,001 & 204,13 & FR:9-10 & 0,931 & 14,6365 & 20,004 & 214,24 \\
\hline FA:10-9 & 0,974 & $-14,332$ & $-19,996$ & 205,41 & FR:10-9 & 0,92 & $-14,726$ & $-19,998$ & 216,80 \\
\hline FA:9-14 & 0,758 & $-15,941$ & $-19,994$ & 254,16 & FR:9-14 & 0,725 & $-16,195$ & $-19,998$ & 262,23 \\
\hline FA:10-11 & 4,214 & 4,5924 & 19,859 & 21,23 & FR:10-11 & 3,88 & 4,963 & 19,862 & 24,82 \\
\hline FA:12-13 & 3,240 & 5,889 & 20,030 & 34,68 & FR:12-13 & 3,331 & $-5,7511$ & $-19,981$ & 33,11 \\
\hline FA:14-13 & 0,907 & $-14,821$ & $-19,992$ & 219,66 & FR:14-13 & 1,252 & 12,4767 & 19,997 & 155,72 \\
\hline \multicolumn{10}{|c|}{ Medidas de Tensão } \\
\hline \multicolumn{2}{|c|}{ Medida com erro } & \multicolumn{2}{|c|}{$U I$} & \multicolumn{2}{|c|}{$r^{N}$} & \multicolumn{2}{|c|}{$e_{\sigma}$} & \multicolumn{2}{|c|}{$\mathbf{J}(\mathbf{x})$} \\
\hline \multicolumn{2}{|c|}{$\mathrm{V}: 1$} & \multicolumn{2}{|c|}{0,145} & \multicolumn{2}{|c|}{19,789} & \multicolumn{2}{|c|}{20,012} & \multicolumn{2}{|c|}{391,33} \\
\hline \multicolumn{2}{|c|}{$V: 2$} & \multicolumn{2}{|c|}{0,146} & \multicolumn{2}{|c|}{$-19,756$} & \multicolumn{2}{|c|}{$-19,953$} & \multicolumn{2}{|c|}{391,17} \\
\hline \multicolumn{2}{|c|}{$\mathrm{V}: 3$} & \multicolumn{2}{|c|}{0,152} & \multicolumn{2}{|c|}{$-19,787$} & \multicolumn{2}{|c|}{$-20,052$} & \multicolumn{2}{|c|}{390,50} \\
\hline \multicolumn{2}{|c|}{$\mathrm{V}: 6$} & \multicolumn{2}{|c|}{0,152} & \multicolumn{2}{|c|}{$-19,732$} & \multicolumn{2}{|c|}{$-19,945$} & \multicolumn{2}{|c|}{390,46} \\
\hline \multicolumn{2}{|c|}{$V: 8$} & \multicolumn{2}{|c|}{0,146} & & 762 & & 965 & & \\
\hline $\mathrm{V}$ & & 0 , & & & 7403 & & 980 & & 03 \\
\hline
\end{tabular}

Conforme pode ser visto através das tabelas 6.3 e 6.4 , os valores de $e_{\sigma}$ obtidos estão muito próximos ao valor da magnitude do EG, aplicado em cada caso. Assim, detectamos e identificamos todos os EGs simples de forma correta para os casos em análise, mesmo para medidas com índice $U I$ de alto valor. Entretanto, vale ressaltar que os testes acima foram realizados sem adicionar ruídos gaussianos às medidas. Os testes considerando medidas com ruídos gaussianos serão apresentados na subseção 6.1.4.

\subsubsection{Comparação entre o erro composto em desvio padrão e o teste- $\hat{b}$}

Na subseção 3.3.3, do capítulo 3, apresentamos o teste- $\hat{b}$ (MONTICELLI e GARCIA, 1983) para detecção e identificação de EGs. Através de uma análise 
comparativa entre a equação de $\hat{b}_{i}$ (equação 3.38) e a equação do erro composto em desvio $e_{\sigma i}$ (equação 6.6), verifica-se serem as mesmas iguais, ou seja:

$$
\begin{aligned}
& \underline{e}_{\sigma i}=\underline{r}_{i}^{N} \cdot \sqrt{\left(U I_{i}^{2}+1\right)}=\underline{r}_{i}^{N} \cdot \sqrt{\frac{K_{i i}}{\left(1-K_{i i}\right)}+1} \\
& \underline{e}_{\sigma i}=\underline{r}_{i}^{N} \cdot \sqrt{\frac{K_{i i}+1-K_{i i}}{\left(1-K_{i i}\right)}}=\underline{r}_{i}^{N} \cdot \sqrt{\frac{1}{\left(1-K_{i i}\right)}} . \\
& \underline{e}_{\sigma i}=\underline{r}_{i}^{N} \cdot \sqrt{\frac{R_{i i}}{\Omega_{i i}}}=\frac{\underline{r}_{i}^{N} \cdot \sigma_{i}}{\sqrt{\Omega_{i i}}}=\underline{\hat{b}}_{i}
\end{aligned}
$$

A partir dessa constatação, verifica-se também a igualdade entre o algoritmo da subseção 6.1.2 e o apresentado em Monticelli e Garcia (1983). Dessa forma, aplicando o teste- $\hat{b}$ nos exemplos apresentados na seção anterior, os resultados obtidos seriam os mesmos.

Vale ressaltar, entretanto, que a obtenção do erro composto em desvio padrão é apenas uma das aplicações que vislumbramos para o índice UI. No próximo capítulo e na conclusão destacaremos outras. Além disso, conforme apresentamos nas seções anteriores, existe toda uma sustentação teórica, através de uma análise geométrica da formulação do estimador de estado WLS, demonstrando que o índice UI possibilita a classificação das medidas de acordo com suas características de não refletirem grande parcela de seus erros, nos resíduos do estimador WLS. Esse é um dos diferenciais entre o índice $U I$ e o teste- $\hat{b}$, proposto em Monticelli e Garcia (1983).

Entretanto, considerando que o caminho percorrido para obtenção do $\hat{b}$ e do $e_{\sigma i}$ são distintos, a igualdade das equações dos mesmos serve apenas para validá-los, ou melhor, para validar o $e_{\sigma i}$, uma vez que o teste- $\hat{b}$ já vem sendo utilizado há alguns anos, para melhoria da análise de EGs. Lembremo-nos ainda de que, em Monticelli e Garcia (1983), a idéia era determinar um valor para medida suspeita de EG que iria zerar o resíduo da mesma no processo de estimação de estado.

Agregando a análise do $e_{\sigma i}$, com a informação oriunda do índice $U I$, verificamos a possibilidade da obtenção de uma metodologia para processamento mais eficiente de EGs que o teste- $\hat{b}$. Observe que, em algumas situações práticas, o teste- $\hat{b}$ e a análise direta do $e_{\sigma i}$ podem falhar. Neste ponto, devemos chamar a atenção para o fato de que os testes apresentados na seção anterior não correspondem à realidade prática, isto é, no mundo real as medidas não são perfeitas, mas possuem ruídos. 
Como será mostrado a seguir, quando adicionarmos ruído às medidas, o teste- $\hat{b}$ e a análise direta do $e_{\sigma i}$ podem falhar pois, em algumas situações a medida portadora de EG não apresenta o maior resíduo normalizado. Outra situação que representa uma limitação para aplicação dessas técnicas é a baixa redundância local de medidas, que leva ao aparecimento de conjuntos críticos de medidas. Em situações como essa, agregando a análise do $e_{\sigma i}$ com a informação dada pelo índice $U I$, torna-se ainda possível, em algumas situações, o processamento de EGs.

A seguir, apresentamos algumas situações em que o teste- $\hat{b}$ e a análise direta do $e_{\sigma i}$ falham, e, ao mesmo tempo, mostraremos como utilizar o índice UI para contornar esses problemas.

Exemplo 1: EG aplicado em medida pertencente a um conjunto crítico

Para facilitar os nossos estudos, neste exemplo será considerado o modelo linear (ou CC) do estimador de estado WLS e o sistema de duas barras e duas linhas apresentado na figura 6.1.

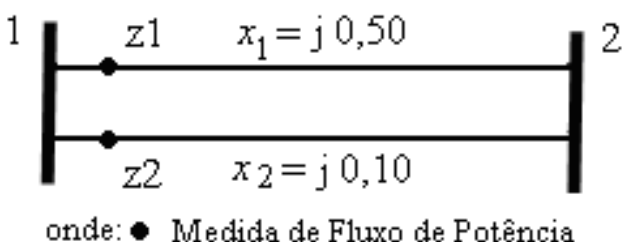

Figura 6.1: Sistema de duas barras e duas linhas

Adotando-se o ângulo de fase da tensão da barra 2 como referência $\left(\theta_{2}=0 \mathrm{rad}\right)$; a matriz Jacobiana do sistema torna-se:

$$
H=z_{z_{2}}\left[\begin{array}{c}
\theta_{1} \\
10
\end{array}\right] .
$$

Considerando que os desvios padrão das medidas sejam iguais a 1, isto é, $\sigma 1=$ $\sigma 2=1$, temos: $R^{-1}=I$.

Considerando, ainda, que o valor "verdadeiro" do ângulo da tensão da barra 1 seja $\theta_{1}^{v}=0,175 \mathrm{rad}$, então o vetor de medidas “verdadeiras", isto é, sem ruído, que na prática é desconhecido, será $\underline{z}^{v}=H \cdot \underline{\theta}_{1}^{v}=\left[\begin{array}{lll}0,35 & 1,75\end{array}\right]^{t}$. A partir desses valores 
"verdadeiros", vamos analisar como se dá o processamento de EG, quando o mesmo ocorre em cada uma das medidas disponíveis neste exemplo.

a) Erro em z1

Adicionando-se um EG de magnitude $9 \sigma 1$ à medida $\mathrm{zl}$ e estimando-se as variáveis de estado através do estimador linear WLS, obtemos:

$$
\begin{aligned}
& \hat{\theta}_{1}^{\text {erro } 1}=\left(H^{t} \cdot R^{-1} \cdot H\right)^{-1} \cdot H^{t} \cdot R^{-1} \cdot \underline{z}^{\text {errol }} . \\
& \hat{\theta}_{1}^{\text {errol }}=0,3481 \mathrm{rad}
\end{aligned}
$$

Através dos cálculos do índice $U I$, do resíduo normalizado, do erro composto em desvio padrão e $\hat{b}$, obtemos os resultados tabelados a seguir (tabela 6.5):

Tabela 6.5: Resultados numéricos da adição de EG de 9 бi à medida z1

\begin{tabular}{c|c|c|c|c}
\hline \multicolumn{5}{c}{ Limiar do teste do índice $J(x): \lambda=\chi_{1 ;(1-0,05)}^{2} \cong 5,02$} \\
\hline \multicolumn{5}{c}{ Índice $J(\mathrm{x})=77,8846$} \\
\hline Medida & $\boldsymbol{U I}$ & $r^{N}$ & $e_{\sigma i}$ & $\hat{b}$ \\
\hline $\mathbf{z 1}$ & 0,20 & 8,8252 & 9,00 & 9,00 \\
\hline $\mathbf{z 2}$ & 5,00 & $-8,8252$ & $-45,00$ & $-45,00$ \\
\hline
\end{tabular}

Como se nos permite ver na tabela 6.5 , os resíduos normalizados das medidas z1 e z2 são iguais em módulo e superiores ao limiar de detecção $(\rho=3,0)$. Assim, embora tenha sido detectada a presença de EG, não é possível, através da análise do resíduo normalizado, do teste- $\hat{b}$ e da análise direta do $e_{\sigma i}$, identificar a medida portadora de EG (observe que a medida com maior valor de $\hat{b}$, ou $e_{\sigma i}$, é a medida z2, que não possui EG). Deve-se destacar que esse problema já era esperado, tendo em vista que as medidas z1 e z2 constituem um conjunto crítico de medidas.

Analisemos agora os índices $U I$ das medidas z1 e z2. A medida z1 apresenta índice $U I$ baixo $(0,20)$, ou seja, a parte detectável do erro dessa medida é muito maior que a parte não-detectável. Já a medida z2 apresenta $U I$ com valor alto (igual a 5). Dessa forma, podemos dizer que, através da análise dos resíduos normalizados, erros na medida z1 serão mais facilmente detectáveis que erros na medida z2. Assim, tendo em vista que ambas as medidas têm resíduo normalizado maior que o limiar para detecção, poderíamos afirmar que z1 é provavelmente a medida portadora de EG, e não a medida z2, pois, se o erro fosse na medida z2, o resíduo normalizado da mesma provavelmente não seria maior que o limiar para detecção de EG. 
Após identificarmos o erro em z1, através da interpretação geométrica do índice $U I$, iremos retirar o efeito desse erro através da equação (6.9):

$$
\begin{aligned}
& z 1^{\text {novo }}=z 1^{\text {erro }}-e_{\sigma z 1} \cdot \sigma 1=9,35-(9,00) \cdot 1 . \\
& z 1^{\text {novo }}=0,35 \text { p.u. }
\end{aligned}
$$

Estimando-se novamente as variáveis de estado e calculando as variáveis de interesse, obtemos:

$$
\hat{\theta}_{1}^{\text {novo }}=0,175 \mathrm{rad} ; J(\underline{\hat{x}})=0,0 ; \underline{r}=\left[\begin{array}{l}
0,0 \\
0,0
\end{array}\right] \text { e } \underline{r}^{N}=\left[\begin{array}{l}
0,0 \\
0,0
\end{array}\right],
$$

e, portanto, o efeito do erro foi retirado com êxito da medida z1.

\section{b) Erro em z2}

Adicionando-se um EG de magnitude $9 \sigma 2$ à medida z2 e estimando-se novamente as variáveis de estado, através do estimador linear WLS, obtemos $\hat{\theta}_{1}^{\text {erro } 2}=1,0404 \mathrm{rad}$.

Através dos cálculos do índice $U I$, do resíduo normalizado, do erro composto em desvio padrão e $\hat{b}$, obtemos os resultados tabelados a seguir (tabela 6.6):

Tabela 6.6: Resultados numéricos da adição de EG de 9 бi à medida z2

\begin{tabular}{c|c|c|c|c}
\hline \multicolumn{5}{c}{ Limiar do teste do índice $J(x): \lambda=\chi_{1 ;(1-0,05)}^{2} \cong 5,02$} \\
\hline \multicolumn{5}{c}{$J(\mathrm{x})=3,1154$} \\
\hline Medida & $\boldsymbol{U I}$ & $r^{N}$ & $e_{\sigma i}$ & $\hat{b}$ \\
\hline $\mathbf{z 1}$ & 0,20 & $-1,7650$ & $-1,80$ & $-1,80$ \\
\hline $\mathbf{z 2}$ & 5,00 & 1,7650 & 9,00 & 9,00 \\
\hline
\end{tabular}

Assim como no item (a) deste exemplo, as medidas z1 e z2 apresentam resíduos normalizados iguais em módulo, o que dificulta a aplicação do teste- $\hat{b}$ e da análise direta do $e_{\sigma i}$. Nesta situação, como o EG foi aplicado em z2, e esta medida apresenta valor alto de $U I$, seu resíduo normalizado apresenta valor bem inferior à magnitude do erro aplicado. Tendo em vista que essa medida apresenta erro composto em desvio padrão maior que o limiar para detecção de erro grosseiro $(\rho=3,0)$, temos então um indicativo de que o EG está em z2, e não em z1.

Através da equação (6.9), retira-se o efeito do erro na medida z2: 


$$
\begin{aligned}
& z 2^{\text {novo }}=z 2^{\text {erro }}-e_{\sigma z 2} \cdot \sigma 2=10,75-(9,00) \cdot 1 \\
& z 2^{\text {novo }}=1,75 \text { p.u. }
\end{aligned}
$$

Estimando-se novamente os estados e calculando as variáveis de interesse, obtemos:

$$
\hat{\theta}_{1}^{\text {novo }}=0,175 \mathrm{rad} ; J(\underline{\hat{x}})=0,0 ; \underline{r}=\left[\begin{array}{c}
0,0 \\
0,0
\end{array}\right] \text { e } \underline{r}^{N}=\left[\begin{array}{l}
0,0 \\
0,0
\end{array}\right],
$$

e portanto, o efeito do erro foi retirado com êxito da medida z2.

Nota 6.3: A interpretação geométrica da aplicação de EG em z1 e em z2 pode ser visualizada através da figuras 4.4 e 4.5 (seção 4.3.1, capítulo 4), respectivamente.

Exemplo 2: Simulação no sistema de 14 barras do IEEE

Considere agora o sistema de 14 barras IEEE e o sistema de medição apresentado na tabela 4.1, porém agora sem as medidas IA:2, IR:2, FA:2-3, FR:2-3 e FR:3-4, e adicionando as medidas IA:4 (valor medido: -0,4780 pu; desvio padrão: 0,007967) e IR:4 (valor medido: 0,0390 pu; desvio padrão: 0,00065).

a) Erro grosseiro em IA:4:

Neste exemplo, será adicionado EG de $10 \sigma$ à medida IA:4, e os valores considerados para todas as demais medidas não apresentam ruído algum. Através do estimador de estado não-linear WLS, estimam-se as variáveis de estado e calculam-se os resíduos normalizados para todas as medidas. A tabela 6.7 apresenta o índice $U I$, o resíduo normalizado, o erro composto em desvio padrão e o $\hat{b}$, das cinco medidas que apresentaram os maiores resíduos normalizados em módulo.

Tabela 6.7: Resultados numéricos da adição de EG de 10 б à medida IA:4

\begin{tabular}{c|c|c|c|c}
\hline \multicolumn{5}{c}{ Medidas com maiores resíduos normalizados } \\
\hline Medida & $\boldsymbol{U I}$ & $r^{N}$ & $e_{\sigma}$ & $\hat{b}$ \\
\hline IA:4 & 1,4065 & $-5,8169$ & $-10,0390$ & $-10,0390$ \\
\hline FA:3-4 & 3,2860 & $-5,8162$ & $-19,9775$ & $-19,9775$ \\
\hline FA:4-5 & 0,3805 & 2,3298 & 2,4928 & 2,4928 \\
\hline FA:5-2 & 0,5166 & 2,1396 & 2,4083 & 2,4083 \\
\hline FA:4-2 & 0,4186 & 2,1264 & 2,3051 & 2,3051 \\
\hline
\end{tabular}


Observe, na tabela 6.7, que os resíduos normalizados das medidas IA:4 e FA:3-4 são quase iguais (diferença no quarto número após a vírgula). Isto se deve ao fato de as mesmas formarem um conjunto crítico de medidas. Mesmo assim, o EG foi identificado na medida IA:4, já que o seu resíduo normalizado foi ligeiramente maior. Entretanto, os resíduos normalizados dessas medidas poderiam ser idênticos (até uma certa precisão), bem como será mostrado no caso (b) deste exemplo. Em tais situações, importa analisar os índices UIs das duas medidas suspeitas e verificar quais das medidas apresentam mais facilidade (ou dificuldade) em detectar EG.

b) Erro grosseiro em FA:3-4:

Neste exemplo adiciona-se EG de $10 \sigma$ à medida FA:3-4, e os valores considerados para todas as demais medidas não apresentam ruído algum. A tabela 6.8 apresenta o índice $U I$, o resíduo normalizado, o erro composto em desvio padrão e o $\hat{b}$, das cinco medidas que apresentaram os maiores resíduos normalizados em módulo.

Tabela 6.8: Resultados numéricos da adição de EG de 10 б na medida FA:3-4

\begin{tabular}{c|c|c|c|c}
\hline \multicolumn{5}{c}{ Medidas com maiores resíduos normalizados } \\
\hline Medida & $\boldsymbol{U I}$ & $r^{N}$ & $e_{\sigma}$ & $\hat{b}$ \\
\hline IA:4 & 1,4031 & $-2,9213$ & $-5,0335$ & $-5,0335$ \\
\hline FA:3-4 & 3,2596 & $-2,9213$ & $-9,9603$ & $-9,9603$ \\
\hline FA:4-5 & 0,3783 & 1,1751 & 1,2564 & 1,2564 \\
\hline FA:5-2 & 0,5160 & 1,0778 & 1,2128 & 1,2128 \\
\hline FA:4-2 & 0,4178 & 1,0718 & 1,1616 & 1,1616 \\
\hline
\end{tabular}

Conforme pode ser visto na tabela 6.8, os resíduos normalizados de IA:4 e FA:34 são iguais (até o quarto número após a vírgula), inviabilizando a aplicação do teste- $\hat{b}$ e da análise direta do $e_{\sigma}$. Dessa forma, deve-se analisar o índice $U I$ das duas medidas para tentar identificar a medida portadora de EG.

As medidas FA:3-4 e IA:4 apresentam o maior resíduo normalizado em módulo, porém, o valor desse resíduo normalizado em módulo é relativamente baixo (abaixo do limiar de detecção para o teste do maior resíduo normalizado, $\rho=3,0$ ). Assim, o EG provavelmente está na medida com maior $U I$ entre as duas, que neste caso é a medida FA:3-4. Observe que, dentre as medidas FA:3-4 e IA:4, o erro composto em desvio padrão é maior em FA:3-4 e o valor está muito próximo ao valor da magnitude do erro aplicado. 
Se as duas medidas apresentassem índice $U I$ muito próximos ou iguais, ficaria praticamente impossível identificar a medida portadora de EG, entretanto a detecção do erro seria ainda possível.

\subsubsection{Testes com Ruído Gaussiano Adicionado às Medidas}

Nesta subseção serão apresentados resultados oriundos de simulações com 100 amostras de medidas com ruído gaussiano adicionado às medidas, e com EG simples.

Inicialmente, consideramos o limiar de detecção de EG para o teste do $e_{\sigma}$ como $\rho=3,0$. Entretanto, através de simulações prévias, verificamos que esse valor do limiar de detecção não é apropriado, quando se consideram ruídos gaussianos em medidas, sendo necessário um valor maior. Assim, escolhemos $\rho=4,0$, como sugerido em Monticelli e Garcia (1983) para o teste- $\hat{b}$.

A seguir, apresentaremos os resultados de simulações de EG simples, utilizando o sistema de 14 barras do IEEE e, em seguida, os resultados utilizando o sistema de 30 barras do IEEE.

\section{a) Testes com ruído gaussiano e erro grosseiro simples: Sistema de 14 Barras IEEE}

A tabela 6.9, a seguir, apresenta os valores médios do índice $U I$, resíduo normalizado e erro composto em desvio padrão de simulações considerando EG simples de 10 sigmas em cada uma das medidas (uma de cada vez) do sistema de medição da tabela 4.1, considerando 100 amostras de medidas com ruído.

Tabela 6.9: Valores médios e desvios padrões do índice $U I$, resíduo normalizado e erro composto em desvio padrão para 100 amostras de medidas com ruído e com EG simples de 10 sigmas em cada medida

\begin{tabular}{|c|c|c|c|c|c|c|}
\hline \multirow{2}{*}{$\begin{array}{l}\text { Medida } \\
\text { com erro }\end{array}$} & \multicolumn{2}{|r|}{$\boldsymbol{U I}$} & \multicolumn{2}{|c|}{$r^{N}$} & \multicolumn{2}{|c|}{$e_{\sigma}$} \\
\hline & Média & $\begin{array}{l}\text { Desvio } \\
\text { Padrão }\end{array}$ & Média & $\begin{array}{l}\text { Desvio } \\
\text { Padrão }\end{array}$ & Média & $\begin{array}{l}\text { Desvio } \\
\text { Padrão }\end{array}$ \\
\hline IA:1 & 0,280784 & 0,001383651 & 9,62624 & 0,219207741 & 9,998531 & 0,22821875 \\
\hline IA:2 & 6,502372 & 0,011111984 & 1,547095 & 0,837240399 & 10,31891 & 5,23477384 \\
\hline IA: 5 & 11,41429 & 0,075814344 & 0,78325 & 0,90547209 & 11,24952 & 7,79556421 \\
\hline IA:9 & 0,754616 & 0,000585122 & 7,91643 & 0,555270492 & 9,917573 & 0,69660118 \\
\hline IA:13 & 0,833265 & $8,08728 \mathrm{E}-05$ & 7,67881 & 0,595927357 & 9,995258 & 0,7760325 \\
\hline IA:14 & 0,663946 & 0,000107703 & 8,29677 & 0,475635264 & 9,958957 & 0,5712619 \\
\hline FA:1-2 & 0,288435 & 0,001241323 & 9,602997 & 0,217370318 & 9,994491 & 0,22678235 \\
\hline FA:2-3 & 0,243795 & 0,002798209 & 9,739348 & 0,183803907 & 10,02467 & 0,1902469 \\
\hline
\end{tabular}




\begin{tabular}{|c|c|c|c|c|c|c|}
\hline FA:4-2 & 0,320077 & 0,001077301 & 9,4795 & 0,252544186 & 9,95326 & 0,26564287 \\
\hline FA:5-2 & 0,290923 & 0,000901722 & 9,56209 & 0,228264739 & 9,958523 & 0,23789421 \\
\hline FA:3-4 & 1,270056 & 0,015247529 & 6,25416 & 0,613571897 & 10,11061 & 1,00130952 \\
\hline FA:4-5 & 0,485936 & 0,002038742 & 8,96962 & 0,409815148 & 9,972589 & 0,45592476 \\
\hline FA:7-4 & 0,419628 & 0,000311101 & 9,18638 & 0,302966523 & 9,962419 & 0,32882267 \\
\hline FA:9-4 & 0,41982 & 0,000308139 & 9,17053 & 0,310717812 & 9,945911 & 0,33715944 \\
\hline FA:5-6 & 0,33251 & 0,000512175 & 9,441225 & 0,258603421 & 9,949473 & 0,27278005 \\
\hline FA:6-5 & 0,33246 & 0,000604027 & 9,44126 & 0,260887816 & 9,949372 & 0,27529723 \\
\hline FA:6-11 & 0,894625 & 0,001053937 & 7,405714 & 0,601611915 & 9,936759 & 0,80694502 \\
\hline FA:11-6 & 0,895858 & 0,000914218 & 7,4854 & 0,63285568 & 10,04985 & 0,84946146 \\
\hline FA:6-12 & 0,602698 & 4,91955E-05 & 8,622188 & 0,406073081 & 10,06707 & 0,47405425 \\
\hline FA:6-13 & 0,455146 & $5,20683 \mathrm{E}-05$ & 9,149617 & 0,345660275 & 10,05276 & 0,37971901 \\
\hline FA:13-6 & 0,449871 & $6,40312 \mathrm{E}-05$ & 9,15166 & 0,377195604 & 10,03511 & 0,41376322 \\
\hline FA:7-9 & 0,419531 & 0,000313564 & 9,191208 & 0,335729298 & 9,967298 & 0,36427652 \\
\hline FA:9-10 & 0,979534 & $5,54504 \mathrm{E}-05$ & 7,062398 & 0,58958665 & 9,88606 & 0,82523376 \\
\hline FA:10-9 & 0,973312 & $5,73488 \mathrm{E}-05$ & 7,14679 & 0,630166068 & 9,973146 & 0,87950701 \\
\hline FA:9-14 & 0,758395 & 0,000117529 & 8,051762 & 0,530817651 & 10,10537 & 0,66588751 \\
\hline FA:10-11 & 4,222957 & 0,017199532 & 2,23393 & 0,81350002 & 9,695666 & 3,53141062 \\
\hline FA:12-13 & 3,245468 & 0,000510868 & 2,965206 & 0,870367275 & 10,06989 & 2,95564428 \\
\hline FA:14-13 & 0,906091 & 0,000332148 & 7,4526 & 0,582204687 & 10,05691 & 0,78601872 \\
\hline IR:1 & 1,403991 & 0,003613585 & 5,69878 & 0,683616525 & 9,823003 & 1,17786171 \\
\hline IR:2 & 0,67472 & 0,009225273 & 8,339726 & 0,438351473 & 10,06108 & 0,53760644 \\
\hline IR:5 & 9,442719 & 0,006676473 & 1,17766 & 0,886788784 & 11,99632 & 7,17538551 \\
\hline IR:8 & 0,429783 & 0,000456392 & 9,194189 & 0,322796664 & 10,00736 & 0,35116132 \\
\hline IR:9 & 0 , & 084 & 75 & 09544 & 667 & 1542 \\
\hline IR:13 & 0, & 55 & 96 & 4 & 55 & 5945 \\
\hline IR:14 & 0,673395 & 7,15979E-05 & 8,33053 & 0,477134991 & 10,04327 & 0,57541849 \\
\hline FR:1-2 & 0,578885 & $3,85992 \mathrm{E}-05$ & 8,67413 & 0,440457624 & 10,02267 & 0,50897181 \\
\hline FR:2-3 & 2,219078 & 0,011903599 & 4,140007 & 0,762243033 & 10,07666 & 1,85511116 \\
\hline FR:4-2 & 1,191498 & 0,00302755 & 6,392469 & 0,699105605 & 9,943444 & 1,08555584 \\
\hline FR:5-2 & 1,113663 & 0,001745392 & 6,67612 & 0,601581427 & 9,992572 & 0,90164634 \\
\hline FR:3-4 & 2,150395 & 0,019493218 & 4,293541 & 0,783674097 & 10,18323 & 1,86410194 \\
\hline FR:4-5 & 0,36112 & 0,000681798 & 9,379796 & 0,251936193 & 9,972651 & 0,2680697 \\
\hline FR:7-4 & 0,419915 & 0,005863539 & 9,207545 & 0,31633025 & 9,986608 & 0,34644699 \\
\hline FR:9-4 & 1,598881 & 0,021402859 & 5,293961 & 0,695937054 & 9,985647 & 1,33182227 \\
\hline FR:5-6 & 0,452809 & 0,010324142 & 9,108191 & 0,328219372 & 9,9989 & 0,3654147 \\
\hline FR:6-5 & 0,906129 & 0,04685379 & 7,60263 & 0,49053119 & 10,26739 & 0,76761445 \\
\hline FR:6-11 & 0,816476 & 0,000920707 & 7,777996 & 0,507523211 & 10,04125 & 0,65533236 \\
\hline FR:11-6 & 0,885402 & 0,001112553 & 7,46719 & 0,558477338 & 9,97351 & 0,74630012 \\
\hline FR:6-12 & 0,830962 & 9,40449E-05 & 7,682239 & 0,525432277 & 9,988399 & 0,68299006 \\
\hline FR:6-13 & 0,430749 & $5,02418 \mathrm{E}-05$ & 9,143908 & 0,337320814 & 9,956142 & 0,36721616 \\
\hline FR:13-6 & 0,454624 & 0,00013493 & 9,12475 & 0,347851197 & 10,02346 & 0,38224088 \\
\hline FR:7-8 & 0,416127 & 0,000550822 & 9,27548 & 0,351203706 & 10,04652 & 0,38066834 \\
\hline FR:8-7 & 0,429687 & 0,000396616 & 9,220194 & 0,314118703 & 10,03533 & 0,34174505 \\
\hline FR:7-9 & 1,207174 & 0,011554698 & 6,530804 & 0,654264003 & 10,23879 & 1,03922799 \\
\hline FR:9-10 & 0,931143 & $5,73048 \mathrm{E}-05$ & 7,380846 & 0,59063542 & 10,08512 & 0,80683562 \\
\hline FR:10-9 & 0,919224 & $5,3409 \mathrm{E}-05$ & 7,28446 & 0,59336105 & 9,894478 & 0,80615788 \\
\hline FR:9-14 & 0,725908 & 0,000236848 & 8,097658 & 0,504509015 & 10,00622 & 0,62310245 \\
\hline FR:10-11 & 3,886074 & 0,014093975 & 2,47711 & 0,755226493 & 9,94136 & 3,03567353 \\
\hline FR:12-13 & 3,331867 & 0,000160211 & 2,889901 & 0,859107166 & 10,05304 & 2,98834878 \\
\hline FR:14-13 & 1,251538 & 0,000893272 & 6,16202 & 1,414707671 & 10,08946 & 0,89629517 \\
\hline $\mathrm{V}: 1$ & 0,14977 & 0,006619584 & 9,882532 & 0,126395694 & 9,992975 & 0,12899829 \\
\hline
\end{tabular}




\begin{tabular}{c|c|c|c|c|c|c}
\hline $\mathbf{V : 2}$ & 0,150956 & 0,007763555 & 9,881736 & 0,127900278 & 9,993996 & 0,131383 \\
\hline $\mathbf{V : 3}$ & 0,154825 & 0,007203245 & 9,869507 & 0,133768603 & 9,98737 & 0,13809533 \\
\hline $\mathbf{V : 6}$ & 0,155275 & 0,006607012 & 9,870066 & 0,134347083 & 9,988577 & 0,13879413 \\
\hline $\mathbf{V : 8}$ & 0,150346 & 0,008001432 & 9,884296 & 0,127568223 & 9,995706 & 0,13120412 \\
\hline $\mathbf{V : 1 2}$ & 0,158484 & 0,006744691 & 9,86125 & 0,136142472 & 9,98455 & 0,13904955 \\
\hline
\end{tabular}

Através dos resultados apresentados na tabela 6.9, verificamos que as medidas com índice $U I$ alto (em vermelho) são aquelas que proporcionam os maiores desvios padrões para $e_{\sigma}$. Isto quer dizer que, quanto maior for o índice $U I$ de uma determinada medida, tanto menos precisa será a correção da medida, através do seu respectivo $e_{\sigma}$ (ou teste- $\hat{b}$ ), quando se consideram medidas com ruído.

Observe ainda, que os resultados tabelados acima são aqueles calculados para as medidas portadoras de erro grosseiro, independentemente de serem identificadas ou não como as medidas com maiores resíduos normalizados. Os resultados após as correções das medidas, consideradas suspeitas de serem portadoras de erros grosseiros, não serão apresentados, já que em algumas simulações ocorreram falhas para detectar ou identificar a correta medida portadora de EG.

As medidas com índice $U I$ menor que 1 tiveram uma baixíssima incidência de casos com problemas de identificação do EG e nenhuma incidência de caso com problema de detecção, através do resíduo normalizado convencional. Por outro lado, as medidas com índice $U I$ acima de 1 tiveram o maior número de casos com falhas na detecção e, também, na identificação do EG.

A tabela 6.10 apresenta um relatório dos casos em que houve falha para detectar ou identificar EG, considerando 100 amostras de medidas com ruído e EG simples de 10 sigmas, em cada uma das amostras.

Tabela 6.10: Dados do número de casos em que houve falha na detecção ou identificação de erros para 100 amostras de medidas com ruído e adição de erro grosseiro de 10 sigmas em cada medida

\begin{tabular}{c|c|c|c|c}
\hline \multirow{2}{*}{$\begin{array}{c}\text { Medida } \\
\text { com erro }\end{array}$} & \multirow{2}{*}{ UI médio } & \multicolumn{3}{|c}{ Número de casos com falhas } \\
\cline { 3 - 5 } & & $\begin{array}{c}\text { Falhas p/ } \\
\text { Detecção (\%) }\end{array}$ & $\begin{array}{c}\text { Falhas p/ } \\
\text { Identificação (\%) }\end{array}$ & $\begin{array}{c}\text { Falhas } \\
\text { totais (\%) }\end{array}$ \\
\hline IA:2 & 6,502372 & 64 & 14 & 78 \\
\hline IA:5 & 11,41429 & 84 & 10 & 94 \\
\hline FA:3-4 & 1,270056 & 0 & 6 & 6 \\
\hline FA:9-10 & 0,979534 & 0 & 10 & 10 \\
\hline FA:10-9 & 0,973312 & 0 & 11 & 11 \\
\hline FA:10-11 & 4,222957 & 44 & 11 & 55 \\
\hline FA:12-13 & 3,245468 & 20 & 7 & 27 \\
\hline IR:1 & 1,403991 & 0 & 1 & 1 \\
\hline
\end{tabular}




\begin{tabular}{|c|c|c|c|c|}
\hline IR:5 & 9,442719 & 78 & 12 & 90 \\
\hline FR:2-3 & 2,219078 & 0 & 39 & 39 \\
\hline FR:4-2 & 1,191498 & 0 & 1 & 1 \\
\hline FR:5-2 & 1,113663 & 0 & 2 & 2 \\
\hline FR:3-4 & 2,150395 & 0 & 30 & 30 \\
\hline FR:6-12 & 0,830962 & 0 & 7 & 7 \\
\hline FR:9-10 & 0,931143 & 0 & 1 & 1 \\
\hline FR:10-11 & 3,886074 & 34 & 11 & 45 \\
\hline FR:12-13 & 3,331867 & 29 & 13 & 42 \\
\hline
\end{tabular}

A partir dos resultados apresentados acima (tabela 6.10), podemos qualificar as medidas desse sistema levando-se em conta o índice $U I$ e o número de casos falhos para detecção ou identificação de EG, quando existe erro simples de magnitude 10 sigmas. Assim, poderíamos dividir as medidas desse sistema da seguinte forma:

Tabela 6.11: Divisão das medidas considerando o índice $U I$ e a possibilidade de falhas para detecção e identificação de erro grosseiro simples de 10 sigmas - sistema de 14 barras do IEEE

\begin{tabular}{|c|c|c|c|}
\hline \multirow{2}{*}{$\begin{array}{c}\text { Faixa de valores do } \\
\text { Índice } U I \text { p/medidas }\end{array}$} & $\begin{array}{c}|c| \\
\text { Sem problema p/detecção } \\
\text { ou identificação de erro }\end{array}$ & $\begin{array}{c}\text { Problema } \\
\text { p/identificação de erro }\end{array}$ & $\begin{array}{c}\text { Problema p/detecção } \\
\text { de erro }\end{array}$ \\
\hline$U I<0,830962$ & $\mathrm{X}$ & $\mathrm{X}$ & \\
\hline $0,830962<U I<3,24546$ & & $\mathrm{X}$ & $\mathrm{X}$ \\
\hline$U I>3,24546$ & & & \\
\hline
\end{tabular}

Na tabela 6.11, a divisão das medidas em três faixas de valores de UI pode ser utilizada para qualificar as medidas de maneira global. Desse modo, medidas com UI menor que 0,830962 (em verde) podem ser qualificadas como medidas muito boas para detecção e identificação de EG simples. As medidas que apresentam $U I$ entre 0,830962 e 3,24546 (em amarelo) podem ser qualificadas como medidas com possibilidade de problemas de identificação de EG simples. Já as medidas com UI maior que 3,24546 (em vermelho) podem ser qualificadas como medidas ruins, tanto para detecção, quanto para identificação de EG simples.

\section{b) Testes com ruído gaussiano e erro grosseiro simples: Sistema de 30 Barras IEEE}

A tabela 6.12, a seguir, apresenta os valores médios do índice UI, resíduo normalizado e erro composto em desvio padrão de simulações, considerando EG 
simples de 10 sigmas, em cada uma das medidas (uma de cada vez) do sistema de medição da tabela 5.10, considerando ainda 100 amostras de medidas com ruído.

Tabela 6.12: Valores médios e desvios padrões do índice $U I$, resíduo normalizado e erro composto em desvio padrão para 100 amostras de medidas com ruído e com EG de 10 sigmas em cada medida -

sistema de 30 barras do IEEE

\begin{tabular}{|c|c|c|c|c|c|c|c|c|c|c|c|c|c|}
\hline \multicolumn{7}{|c|}{ Medidas de Potência Ativa } & \multicolumn{7}{|c|}{ Medidas de Potência Reativa } \\
\hline \multirow{2}{*}{$\begin{array}{c}\text { Medida } \\
\text { com } \\
\text { erro }\end{array}$} & \multicolumn{2}{|c|}{$U I$} & \multicolumn{2}{|c|}{$r^{N}$} & \multicolumn{2}{|c|}{$e_{\sigma}$} & \multirow{2}{*}{$\begin{array}{c}\text { Medida } \\
\text { com } \\
\text { erro }\end{array}$} & \multicolumn{2}{|c|}{$\boldsymbol{U I}$} & \multicolumn{2}{|c|}{$r^{N}$} & \multicolumn{2}{|c|}{$e_{\sigma}$} \\
\hline & Média & $\begin{array}{l}\text { Desvio } \\
\text { Padrão }\end{array}$ & Média & $\begin{array}{l}\text { Desvio } \\
\text { Padrão }\end{array}$ & Média & $\begin{array}{l}\text { Desvio } \\
\text { Padrão }\end{array}$ & & Média & $\begin{array}{l}\text { Desvio } \\
\text { Padrão }\end{array}$ & Média & $\begin{array}{l}\text { Desvio } \\
\text { Padrão }\end{array}$ & Média & $\begin{array}{l}\text { Desvio } \\
\text { Padrão }\end{array}$ \\
\hline IA:1 & 0,39458 & 0,00259 & 9,3417 & 0,31039 & 10,04268 & 0,33452 & IR:1 & 1,073 & 0,00056 & $-6,913$ & 0,5987 & $-10,1398$ & 0,87746 \\
\hline IA:2 & 7,1456 & 0,02196 & 1,4011 & 0,89438 & 10,1093 & 6,45567 & IR:2 & 0,3618 & 0,00105 & 9,40425 & 0,27874 & 10,00083 & 0,29537 \\
\hline IA:5 & 0,34257 & 0,00129 & $-9,4646$ & 0,28644 & $-10,0045$ & 0,30295 & IR:5 & 0,9843 & 0,00778 & 7,1546 & 0,60447 & 10,03906 & 0,84846 \\
\hline IA:6 & --- & --- & --- & --- & --- & --- & IR:6 & --- & --- & --- & --- & --- & --- \\
\hline IA:7 & 1,4812 & 0,00053 & $-5,6218$ & 0,7373 & $-10,0471$ & 1,31809 & IR:7 & 0,533 & 0,0007 & $-8,8078$ & 0,37944 & $-9,98071$ & 0,43003 \\
\hline IA:8 & 0,72847 & $5,0 \mathrm{E}-05$ & $-8,1223$ & 0,46683 & $-10,0489$ & 0,57760 & IR:8 & 1,0625 & 0,00184 & 6,86418 & 0,64985 & 10,01537 & 0,94814 \\
\hline IA:9 & --- & --- & --- & --- & $\begin{array}{l}-- \\
-\end{array}$ & --- & IR:9 & --- & --- & --- & --- & --- & --- \\
\hline IA:11 & --- & --- & --- & --- & --- & --- & IR:11 & 0,6248 & 0,00027 & 8,48962 & 0,44531 & 10,01063 & 0,52437 \\
\hline IA:12 & 3,0381 & 0,00083 & $-3,1783$ & 0,88599 & $-10,1655$ & 83327 & IR:12 & 3,4941 & 0,00506 & $-2,7227$ & 0,82308 & $-9,89615$ & 2,99412 \\
\hline IA:13 & --- & --- & --- & --- & --- & --- & IR:13 & 0,8834 & 0,00039 & 7,59498 & 0,57491 & 10,13385 & 0,76655 \\
\hline IA:15 & 5,35957 & 0,00114 & 1,8109 & 0,77376 & $-9,87311$ & 21867 & IR:15 & 6,9697 & 0,0104 & $-1,4813$ & 0,90667 & $-10,4306$ & 6,38635 \\
\hline IA:16 & 4,45242 & 0,00056 & $-2,2943$ & 0,84633 & $-10,4695$ & 3,86213 & IR:16 & 2,5664 & 0,00257 & $-3,5715$ & 0,79432 & $-9,83709$ & 2,18733 \\
\hline IA: 17 & 7,21367 & 0,00 & 7 & 0,86 & -10 & 8 & 47 & 16 & 72 & 68 & 0,9 & 72 & 3,94454 \\
\hline IA:19 & 1,117 & $4,0 \mathrm{E}-05$ & $-6,6$ & 0,64469 & $-9,90$ & 0,9 & 19 & 1,413 & -05 & $\begin{array}{l}-5,7388 \\
\end{array}$ & 0,77688 & $-9,93422$ & 1,34486 \\
\hline IA:21 & 1,05691 & 0,00012 & $-6,8682$ & 0,57048 & $-9,99335$ & 0,83002 & IR:21 & 0,9259 & 0,00018 & $-7,2833$ & 0,56788 & $-9,92566$ & 0,77398 \\
\hline IA:22 & --- & --- & --- & --- & --- & --- & IR:22 & --- & --- & --- & --- & --- & --- \\
\hline IA:23 & 2,12029 & $9,5 \mathrm{E}-05$ & $-4,1826$ & 0,89718 & $-9,80519$ & 2,10338 & IR:23 & 1,3394 & $6,3 \mathrm{E}-05$ & $-6,0394$ & 0,66455 & $-10,0951$ & 1,11093 \\
\hline IA:24 & 0,99273 & 5,5E-05 & $-6,8677$ & 0,57198 & $-9,6771$ & 0,80609 & IR:24 & 0,78 & 0,00186 & $-7,8727$ & 0,50325 & $-9,98421$ & 0,63930 \\
\hline IA:25 & --- & --- & --- & --- & --- & --- & IR:25 & --- & --- & --- & $\begin{array}{l}-- \\
\end{array}$ & --- & --- \\
\hline IA:27 & --- & --- & --- & --- & --- & --- & IR:27 & --- & --- & --- & --- & --- & --- \\
\hline IA:28 & --- & --- & --- & --- & --- & --- & IR:28 & --- & --- & --- & --- & --- & --- \\
\hline IA:30 & 0,38639 & 7,4E-05 & $-9,3178$ & 0,32761 & $-9,98915$ & 0,35137 & IR:30 & 1,3298 & 0,00026 & $-6,0859$ & 0,76771 & $-10,1261$ & 1,27775 \\
\hline FA:1-2 & 0,29499 & 0,00157 & 9,6323 & 0,22423 & 10,0426 & 23358 & $: 1-2$ & 0,4025 & 0,00022 & $-9,2929$ & 0,32585 & $-10,0173$ & 0,35096 \\
\hline FA:1-3 & 0,43592 & 0,00225 & 9,2157 & 0,33762 & 10,05335 & 37006 & 10 & 1,4603 & 0,00123 & 5,67407 & 0,71349 & 10,04247 & 1,26192 \\
\hline FA:2-4 & 0,34352 & 0 & 9,4916 & 0,25 & 03598 & 25 & FR:2-4 & 43 & 05 & 7,99497 & 508 & 9,918756 & 0,66403 \\
\hline FA:4-2 & 0,33008 & 0,00197 & $-9,5419$ & 0,2576 & $-10,0483$ & 0,27217 & FR:4-2 & 1,306 & 0,01282 & $-5,9774$ & 0,70522 & $-9,83306$ & 1,17122 \\
\hline FA:2-5 & 0,23478 & 0,00103 & 9,7623 & 0,19942 & 10,02772 & 0,20447 & FR:2-5 & 1,8072 & 0,00592 & 4,95413 & 0,70465 & 10,23167 & 1,45159 \\
\hline FA:2-6 & 0,27861 & 0,00166 & 9,6941 & 0,22131 & 10,06335 & 0,22938 & FR:2-6 & 0,8163 & 0,00577 & 7,6008 & 0,45807 & 9,811751 & 0,59188 \\
\hline FA:3-4 & 2,69308 & 0,00628 & 3,6003 & 0,85635 & 10,34205 & 2,45819 & FR:3-4 & 9,2033 & 0,00553 & $-1,0623$ & 0,80635 & $-9,83378$ & 7,46423 \\
\hline FA:4-6 & 0,42758 & 0,00017 & 9,2139 & 0,34166 & 10,02083 & 0,37147 & FR:4-6 & 0,5887 & 0,00143 & $-8,749$ & 0,37925 & $-10,1524$ & 0,44120 \\
\hline FA:4-12 & 0,30402 & $6,0 \mathrm{E}-05$ & 9,5799 & 0,25349 & 10,01279 & 0,26500 & FR:4-12 & 0,6998 & 0,00992 & 8,0799 & 0,52088 & 9,862607 & 0,64433 \\
\hline FA:5-7 & 1,37508 & 0,00125 & $-5,7927$ & 0,6424 & $-9,84911$ & 1,09370 & FR:5-7 & 0,4272 & 0,00019 & 9,14918 & 0,33427 & 9,949053 & 0,36327 \\
\hline FA:6-7 & 0,47869 & 0,00025 & 9,0319 & 0,3799 & 10,01337 & 0,42099 & FR:6-7 & 2,3281 & 0,00348 & $-3,9433$ & 0,80787 & $-9,99145$ & 2,04788 \\
\hline FA:6-8 & 0,60122 & 4,1E-05 & 8,6484 & 0,3636 & 10,09115 & 0,42424 & FR:6-8 & 0,7493 & $9,1 \mathrm{E}-05$ & $-8,0908$ & 0,5695 & $-10,1101$ & 0,71170 \\
\hline FA:6-9 & 0,40093 & 0,00010 & 9,2587 & 0,32811 & 9,975132 & 0,35362 & FR:6-9 & 0,8306 & 0,0001 & $-7,8456$ & 0,5815 & $-10,199$ & 0,75621 \\
\hline FA:6-28 & 0,41003 & 0,00010 & 9,2646 & 0,26208 & 10,01317 & 0,28330 & FR:6-28 & --- & --- & --- & --- & --- & --- \\
\hline FA:8-28 & 0,85691 & $3,0 \mathrm{E}-05$ & $-7,5801$ & 0,52728 & $-9,98248$ & 0,69441 & FR:8-28 & 0,3835 & 0,0037 & $-9,399$ & 0,28807 & $-10,0664$ & 0,30702 \\
\hline FA:9-11 & --- & --- & --- & --- & --- & --- & FR:9-11 & 0,576 & $9,5 \mathrm{E}-05$ & $-8,6175$ & 0,42171 & $-9,94468$ & 0,48674 \\
\hline FA:9-10 & 0,41419 & 9,9E-05 & 9,2162 & 0,32917 & 9,975459 & 0,35635 & FR:9-10 & 1,2966 & 0,00014 & 6,02906 & 0,71198 & 9,872028 & 1,16595 \\
\hline FA:12-13 & --- & --- & --- & --- & --- & -- & FR:12-13 & 0,8416 & 0,00031 & $-7,7458$ & 0,54034 & $-10,124$ & 0,70668 \\
\hline
\end{tabular}




\begin{tabular}{|c|c|c|c|c|c|c|c|c|c|c|c|c|c|}
\hline FA:12-15 & 0,50931 & $8,4 \mathrm{E}-05$ & 8,9213 & 0,3604 & 10,01171 & 0,40446 & FR:12-15 & 0,7736 & 0,00361 & 7,85533 & 0,55431 & 9,931433 & 0,70141 \\
\hline FA:12-16 & 0,72339 & 0,00021 & 8,043 & 0,50214 & 9,926829 & 0,61959 & FR:12-16 & 1,1083 & 0,00259 & 6,80566 & 0,57617 & 10,1594 & 0,86068 \\
\hline FA:15-18 & 0,99463 & $6,5 \mathrm{E}-05$ & 7,0917 & 0,6684 & 10,00231 & 0,94245 & FR:15-18 & 0,9976 & $8,3 \mathrm{E}-05$ & 7,2097 & 0,59802 & 10,18356 & 0,84439 \\
\hline FA:18-15 & 0,96994 & $8,6 \mathrm{E}-05$ & $-7,0458$ & 0,58202 & $-9,81565$ & 0,81112 & FR:18-15 & 0,9834 & $7,3 \mathrm{E}-05$ & $-7,1532$ & 0,63624 & $-10,0328$ & 0,89266 \\
\hline FA:15-23 & 0,61581 & $6,4 \mathrm{E}-05$ & 8,4994 & 0,49179 & 9,981716 & 0,57739 & FR:15-23 & 1,0941 & $8,0 \mathrm{E}-05$ & 6,84065 & 0,63645 & 10,13941 & 0,94309 \\
\hline FA:18-19 & 2,83879 & $4,3 \mathrm{E}-05$ & 3,3231 & 0,77166 & 10,00182 & 2,32244 & FR:18-19 & 1,4171 & $5,9 \mathrm{E}-05$ & 5,76553 & 0,76089 & 9,999593 & 1,31944 \\
\hline FA:19-20 & 1,1173 & 0 & $-6,6507$ & 0,67322 & $-9,97243$ & 1,00943 & FR:19-20 & 1,4134 & $4,8 \mathrm{E}-05$ & $-5,7751$ & 0,77117 & $-9,99877$ & 1,33510 \\
\hline FA:21-22 & 1,60611 & $2,8 \mathrm{E}-05$ & $-5,1938$ & 0,67329 & $-9,82657$ & 1,27386 & FR:21-22 & 1,453 & $2,1 \mathrm{E}-05$ & $-5,6896$ & 0,61848 & $-10,0358$ & 1,09092 \\
\hline FA:22-24 & 0,70914 & 0,00012 & 8,1619 & 0,49129 & 10,00582 & 0,60215 & FR:22-24 & 1,2146 & 0,00124 & 6,3065 & 0,64799 & 9,922053 & 1,01906 \\
\hline FA:23-24 & 1,2874 & 0,00028 & 6,1222 & 0,72058 & 9,980102 & 1,17454 & FR:23-24 & 0,939 & $3,6 \mathrm{E}-05$ & 7,20665 & 0,54234 & 9,885727 & 0,74385 \\
\hline FA:24-25 & 1,1623 & $9,4 \mathrm{E}-05$ & $-6,5168$ & 0,54065 & $-9,99212$ & 0,82916 & FR:24-25 & 1,0192 & 0,00043 & 6,67604 & 0,49215 & 9,532476 & 0,7020 \\
\hline FA:25-26 & 1,6756 & $3,3 \mathrm{E}-05$ & 4,7446 & 0,60552 & 9,258209 & 1,18165 & FR:25-26 & 1,6295 & 0,00046 & 5,35715 & 0,62967 & 10,24225 & 1,20407 \\
\hline FA:25-27 & 1,29051 & $9,7 \mathrm{E}-05$ & $-6,4964$ & 0,62943 & $-10,606$ & 1,02778 & FR:25-27 & 0,9857 & 0,00011 & $-7,2895$ & 0,47772 & $-10,2352$ & 0,67090 \\
\hline FA:27-29 & 0,70049 & 0,00011 & 8,1528 & 0,47146 & 9,954101 & 0,57560 & FR:27-29 & 1,0628 & 0,00125 & 6,93596 & 0,51282 & 10,12148 & 0,74885 \\
\hline FA:27-30 & 0,45686 & $5,8 \mathrm{E}-05$ & 9,0683 & 0,34923 & 9,96992 & 0,38393 & FR:27-30 & 0,4784 & 0,00014 & 9,04774 & 0,32878 & 10,02973 & 0,36440 \\
\hline FA:28-27 & 0,42631 & 0,00012 & 9,1756 & 0,32828 & 9,974597 & 0,35701 & FR:28-27 & 0,4543 & 0,00053 & 8,87433 & 0,28232 & 9,747306 & 0,31017 \\
\hline FA:29-30 & 2,09289 & $8,9 \mathrm{E}-05$ & 4,3336 & 0,75788 & 10,05191 & 1,75774 & FR:29-30 & 0,6694 & 0,00011 & 8,34823 & 0,48695 & 10,04612 & 0,58560 \\
\hline
\end{tabular}

\begin{tabular}{|c|c|c|c|c|c|c|}
\hline \multicolumn{7}{|c|}{ Medidas de Tensão } \\
\hline \multirow{2}{*}{$\begin{array}{l}\text { Medida com } \\
\text { erro }\end{array}$} & \multicolumn{2}{|c|}{$\boldsymbol{U I}$} & \multicolumn{2}{|c|}{$r^{N}$} & \multicolumn{2}{|c|}{$e_{\sigma}$} \\
\hline & Média & Desvio Padrão & Média & Desvio Padrão & Média & Desvio Padrão \\
\hline V:1 & 0,12361 & 0,002682 & 9,9232 & 0,09093 & 9,998797 & 0,09187 \\
\hline $\mathrm{V}: 2$ & 0,12311 & 0,002861 & 9,9243 & 0,09074 & 9,999243 & 0,09191 \\
\hline V:5 & 0,12282 & 0,002604 & 9,9236 & 0,09057 & 9,998244 & 0,09155 \\
\hline $\mathrm{V}: 8$ & 0,12365 & 0,003173 & 9,922 & 0,0925 & 9,997553 & 0,09402 \\
\hline V:11 & 0,1203 & 0,003053 & 9,9264 & 0,09024 & 9,997959 & 0,09149 \\
\hline V:13 & 0,12509 & 0,003138 & 9,9246 & 0,09397 & 10,00205 & 0,09565 \\
\hline $\mathrm{V}: 20$ & 0,12901 & 0,003497 & 9,9178 & 0,09589 & 10,00003 & 0,09753 \\
\hline $\mathrm{V}: 28$ & 0,12394 & 0,003191 & 9,9216 & 0,09257 & 9,997536 & 0,09407 \\
\hline
\end{tabular}

Obs.: As medidas em que não foram calculados $U I, r^{\mathrm{N}}$ e $e_{\sigma}$ são aquelas cujos valores são nulos.

Através dos resultados acima, confirmamos aquilo que constatamos na simulação anterior, ou seja, as medidas com índice $U I$ alto (em vermelho) são aquelas que proporcionam os maiores desvios padrões para $e_{\sigma}$. Logo, quanto maior for o índice UI de uma determinada medida, menos precisa será a correção da mesma, através do seu respectivo $e_{\sigma}$ (ou teste- $\hat{b}$ ), quando se consideram medidas com ruído.

A tabela 6.13 apresenta um relatório dos casos em que houve falha para detectar ou identificar EG, considerando 100 amostras de medidas com ruído e EG simples de 10 sigmas em cada uma das amostras. 
Tabela 6.13: Dados do número de casos em que houve falha na detecção ou identificação de erros para 100 amostras de medidas com ruído e adição de EG de 10 sigmas em cada medida

\begin{tabular}{|c|c|c|c|c|}
\hline \multirow{2}{*}{$\begin{array}{l}\text { Medida } \\
\text { com erro }\end{array}$} & \multirow{2}{*}{ UI médio } & \multicolumn{3}{|c|}{ Número de casos com falhas } \\
\hline & & $\begin{array}{c}\text { Falhas p/ } \\
\text { Detecção (\%) }\end{array}$ & $\begin{array}{c}\text { Falhas p/ } \\
\text { Identificação (\%) }\end{array}$ & $\begin{array}{c}\text { Falhas } \\
\text { totais }(\%)\end{array}$ \\
\hline IA:2 & 7,1392 & 66 & 13 & 79 \\
\hline IA: 7 & 1,4821 & 0 & 2 & 2 \\
\hline IA:12 & 3,0384 & 17 & 14 & 31 \\
\hline IA:15 & 5,3617 & 55 & 20 & 75 \\
\hline IA:16 & 4,4531 & 43 & 35 & 78 \\
\hline IA:17 & 7,2156 & 73 & 20 & 93 \\
\hline IA:19 & 1,1173 & 0 & 72 & 72 \\
\hline IA:21 & 1,0568 & 0 & 17 & 17 \\
\hline IA:23 & 2,1209 & 2 & 12 & 14 \\
\hline FA:3-4 & 2,653 & 5 & 11 & 16 \\
\hline FA:5-7 & 1,3766 & 0 & 1 & 1 \\
\hline FA:12-15 & 0,5093 & 0 & 1 & 1 \\
\hline FA:12-16 & 0,7231 & 0 & 22 & 22 \\
\hline FA:15-18 & 0,994 & 0 & 19 & 19 \\
\hline FA:18-15 & 0,9707 & 0 & 15 & 15 \\
\hline FA:15-23 & 0,6155 & 0 & 1 & 1 \\
\hline FA:18-19 & 2,8377 & 15 & 41 & 56 \\
\hline FA:19-20 & 1,1173 & 0 & 78 & 78 \\
\hline FA:21-22 & 1,6062 & 0 & 27 & 27 \\
\hline FA:25-26 & 1,6757 & 0 & 1 & 1 \\
\hline FA:29-30 & 2,0915 & 0 & 5 & 5 \\
\hline IR:8 & 1,0623 & 0 & 1 & 1 \\
\hline IR:12 & 3,495 & 25 & 38 & 63 \\
\hline IR:15 & 6,9695 & 69 & 24 & 93 \\
\hline IR:16 & 2,5677 & 7 & 59 & 66 \\
\hline IR:17 & 4,2642 & 44 & 43 & 87 \\
\hline IR:19 & 1,41315 & 0 & 45 & 45 \\
\hline IR:21 & 0,9258 & 0 & 7 & 7 \\
\hline IR:23 & 1,3398 & 0 & 9 & 9 \\
\hline FR:1-3 & 1,4561 & 0 & 18 & 18 \\
\hline FR:2-5 & 1,8011 & 1 & 0 & 1 \\
\hline FR:3-4 & 9,2078 & 78 & 16 & 94 \\
\hline FR:6-7 & 2,3275 & 1 & 13 & 14 \\
\hline FR:12-15 & 0,7736 & 0 & 2 & 2 \\
\hline FR:12-16 & 1,108 & 0 & 31 & 31 \\
\hline FR:15-18 & 0,9968 & 0 & 22 & 22 \\
\hline FR:18-15 & 0,9841 & 0 & 20 & 20 \\
\hline FR:15-23 & 1,0934 & 0 & 4 & 4 \\
\hline FR:18-19 & 1,4162 & 0 & 73 & 73 \\
\hline FR:19-20 & 1,4131 & 0 & 63 & 63 \\
\hline FR:21-22 & 1,453 & 0 & 12 & 12 \\
\hline
\end{tabular}




\begin{tabular}{|c|c|c|c|c|}
\hline FR:25-26 & 1,6297 & 0 & 1 & 1 \\
\hline
\end{tabular}

Com base nos resultados apresentados na Tabela 6.13 e levando-se em conta o índice $U I$ e o número de casos falhos para detecção ou identificação de erro, podemos dividir as medidas do sistema em análise, de acordo com a tabela 6.14, a seguir:

Tabela 6.14: Divisão das medidas considerando o índice $U I$ e a possibilidade de falhas para detecção e identificação de EG simples de 10 sigmas - sistema de 30 barras IEEE

\begin{tabular}{|c|c|c|c|}
\hline \multirow{2}{*}{$\begin{array}{c}\text { Faixa de valores do } \\
\text { Índice } U I \text { p/medidas }\end{array}$} & $\begin{array}{c}|c| \\
\text { Sem problema p/detecção } \\
\text { ou identificação de erro }\end{array}$ & $\begin{array}{c}\text { Problema } \\
\text { p/identificação de erro }\end{array}$ & $\begin{array}{c}\text { Problema p/detecção } \\
\text { de erro }\end{array}$ \\
\hline$U I<0,9258^{*}$ & $\mathrm{X}$ & $\mathrm{X}$ & \\
\hline $0,9258<U I<2,5677$ & & $\mathrm{X}$ & $\mathrm{X}$ \\
\hline$U I>2,5677$ & & & \\
\hline
\end{tabular}

*Obs.: Para a faixa de valores do índice $U I$, os valores de corte foram escolhidos considerando uma incidência mínima de $5 \%$ de falha. Por exemplo, para a primeira faixa de valores (em verde), o valor de corte foi 0,9258, já que a medida IR:21 correspondeu àquela que apresentou menor índice $U I$, com incidência de falha para identificação maior que 5\%, mais precisamente igual a 7\%. Já para o valor de corte superior referente à faixa amarela, a medida IR:16 foi a que apresentou menor índice $U I$, com incidência de falha para detecção maior que $5 \%$, neste caso igual a $7 \%$.

A diferença entre as faixas de valores, apresentadas nas tabelas 6.11 e 6.14 , pode ser explicada pelo fato de os sistemas elétricos em análise (14 e 30 barras IEEE) serem distintos e apresentarem conjuntos de medidas também distintos. Assim, a divisão das medidas através do índice $U I$, levando-se em conta a dificuldade em detectar/identificar possíveis EG, será diferente para cada sistema elétrico e conjunto de medidas associado. Entretanto, fica evidente com os resultados apresentados até o momento que valores ideais para o índice $U I$ são abaixo de 1 , isto é, a parte detectável do erro maior que a parte não-detectável. 


\subsection{Conclusões do Capítulo}

Neste capítulo apresentamos em detalhes uma das possíveis aplicações do índice $U I$, para prover uma melhora substancial no processo de estimação de estado em sistemas elétricos de potência.

Através da seção 6.1, mostrou-se que é possível, utilizando o índice UI, obter uma estimativa da magnitude do erro de medida para sistemas de medição com uma medida com EG e as demais com ruído gaussiano, mesmo para medidas com UI elevado. Denominamos essa estimativa como erro composto em desvio padrão $\left(e_{\sigma}\right)$, e ela depende tanto do resíduo normalizado quanto do índice $U I$ da medida.

Em decorrência dessa estimativa do erro, ao invés de eliminar-se a medida portadora de EG, torna-se possível corrigir o valor da medida errônea para, em seguida, re-estimarmos as variáveis de estado, assim como proposto em Monticelli e Garcia (1983).

Através dos testes apresentados na seção 6.1.2, verificamos a eficácia do algoritmo proposto, aplicado em sistemas de medição sem adicionar ruído gaussiano às medidas. Por outro lado, com a adição de ruído gaussiano (seção 6.1.4), constatamos que o algoritmo pode falhar, tanto na detecção, quanto na identificação de EGs, para medidas cujo índice $U I$ é alto. Destaca-se que, da mesma forma que o algoritmo proposto, o teste- $\hat{b}$, proposto em Monticelli e Garcia (1983), também falha para as situações supracitadas. Porém, como ilustrado na seção 6.1.3, haja vista o índice $U I$ permitir a classificação das medidas, de acordo com as suas características de não refletirem seus erros nos resíduos do estimador WLS, vislumbra-se que o mesmo pode ser utilizado para possibilitar o processamento de EGs em situações em que a análise, pura e simplesmente, do erro composto em desvio padrão e do teste- $\hat{b}$ falha.

No próximo capítulo, mostraremos como o índice $U I$ pode ser utilizado para o projeto ou fortalecimento de sistemas de medição, para efeito de estimação de estado. 


\section{Capítulo 7}

\section{Utilização do Índice de Não-Detecção de Erros para Projeto ou}

\section{Fortalecimento de Sistemas de Medição}

Neste capítulo mostraremos que o índice UI pode ser utilizado para locação de medidores, tanto na fase de projeto de novos sistemas de medição, quanto para o fortalecimento de sistemas de medição já existentes. As seções deste capítulo estão divididas da seguinte forma:

- A seção 7.1 apresentará exemplos elucidativos, mostrando a influência da redundância local de medidas sobre os índices UIs.

- Na seção 7.2, apresentaremos a primeira proposta de algoritmo para projeto ou fortalecimento de sistemas de medição, baseado no índice $U I$ das medidas.

- A seção 7.3 apresentará uma segunda proposta de algoritmo para projeto de sistemas de medição, baseado não apenas no índice $U I$, mas também no custo do sistema de medição.

- As conclusões principais do capítulo estão na seção 7.4.

Vale destacar que considerar-se-ão apenas medidores de potência ativa e reativa e de magnitude de tensão.

\subsection{Influência da Redundância de Medidas sobre o Índice de Não-Detecção de Erros}

Nesta seção mostraremos que é possível utilizar o índice UI para o fortalecimento e projeto de sistemas de medição, em sistemas elétricos de potência. 
Conforme descrito anteriormente, quanto maior o índice $U I$ de uma determinada medida, mais difícil será a detecção de EG na mesma. Tal situação é indesejável à monitoração dos sistemas elétricos de potência. Assim sendo, fica evidente a necessidade de diminuir o índice $U I$ das medidas para prover um aumento na capacidade de detecção e identificação de EGs, num sistema de medição já existente, ou ainda, projetar novos sistemas de medição, tendo como objetivo a minimização do índice $U I$ das medidas.

Como o índice UI está diretamente relacionado com o nível de redundância local das medidas, um modo de diminuir-se o índice $U I$ pode ser através da inserção de medidas, próximas àquela com índice alto. Dada esta constatação, apresentaremos exemplos elucidativos mostrando a influência da redundância local de medidas sobre os índices UIs.

Como veremos a seguir, os dois primeiros exemplos mostram situações em que novas medidas são adicionadas ao sistema de medição existente, com o intuito de diminuir o índice UI de medidas com índice alto. Já o terceiro exemplo, mostra a influência da retirada de medidas com índice alto, em um sistema de medição bem redundante.

\section{Exemplos}

Para os exemplos que se seguem, considerar-se-á o sistema de 14 barras do IEEE associado ao sistema de medição da tabela 4.1 (capítulo 4) e os valores dos índices UIs das respectivas medidas, apresentados na tabela 4.3.

Exemplo 1: Diminuição do índice UI, para as medidas FA:10-11 e FR:10-11

O objetivo deste exemplo é mostrar que a adição de medidas redundantes pode diminuir significativamente o índice $U I$ de uma medida com índice alto. Além disso, o novo valor do índice UI pode ficar abaixo de 1 . Vale ressaltar, que valores desejáveis de UI são abaixo de 1, como vimos pelos resultados de simulações de erros simples no capítulo 5 e no capítulo 6.

Através dos resultados da tabela 4.3, observa-se que as medidas FA:10-11 e FR:10-11 apresentam índice $U I$ de valor alto, respectivamente, 4,2529 e 3,9120.

Verifica-se, também, que várias medidas de potência ainda podem ser instaladas, de modo a aumentar a redundância local, tais como: FA:11-10; FR:11-10; IA:10; IR:10; IA:11 e IR:11. 
A tabela 7.1, a seguir, mostra a modificação gradual nos valores dos índices UIs, das medidas FA:10-11 e FR:10-11, frente à adição de várias medidas próximas a elas, e também mostra o índice $U I$ das novas medidas. Os valores das medidas a serem adicionadas podem ser vistos na tabela B.1 do Anexo B.

Tabela 7.1: Variação do índice $U I$ das medidas FA:10-11 e FR:10-11 e, também, das novas medidas adicionadas

\begin{tabular}{l|c|c|c|c|c|c|c|c}
\hline \multirow{2}{*}{ Caso } & \multicolumn{7}{|c}{ Medidas próximas a FA:10-11 e FR:10-11 } \\
\hline & FA:10-11 & FR:10-11 & FA:11-10 & FR:11-10 & IA:10 & IR:10 & IA:11 & IR:11 \\
\cline { 2 - 10 } & UI & UI & UI & UI & UI & UI & UI & UI \\
\hline Situação inicial & $4,2529 *$ & 3,9120 & - & - & - & - & - & - \\
\hline Adição de FA:11-10 & $0,9681 * *$ & 3,8974 & 0,9786 & - & - & - & - & - \\
\hline Adição de FR:11-10 & 0,9679 & 0,9642 & 0,9784 & 0,9737 & - & - & - & - \\
\hline Adição de IA:10 & 0,9016 & 0,9640 & 0,9108 & 0,9735 & 0,5034 & - & - & - \\
\hline Adição de IR:10 & 0,9016 & 0,9314 & 0,9107 & 0,9403 & 0,5034 & 0,5337 & - & - \\
\hline Adição de IA:11 & 0,7590 & 0,9319 & 0,7655 & 0,9401 & 0,4889 & 0,5337 & 1,6099 & - \\
\hline Adição de IR:11 & 0,7587 & 0,7938 & 0,7653 & 0,7994 & 0,4888 & 0,5315 & 1,6093 & 1,4364 \\
\hline * Os valores em vermelho indicam índices UIs maiores que 1. & & & & \\
** Os valores em verde indicam índices UIs menores que 1. &
\end{tabular}

Como pode ser visualizado através dos resultados da tabela 7.1, os índices UIs das medidas FA:10-11 e FR:10-11 diminuíram, à medida que novas medidas redundantes foram adicionadas ao conjunto de medidas. Confira o gráfico da figura 7.1, a seguir, que apresenta a variação dos UIs daquelas medidas, com a adição gradual das medidas FA:11-10, FR:11-10, IA:10, IR:10, IA:11 e IR:11.

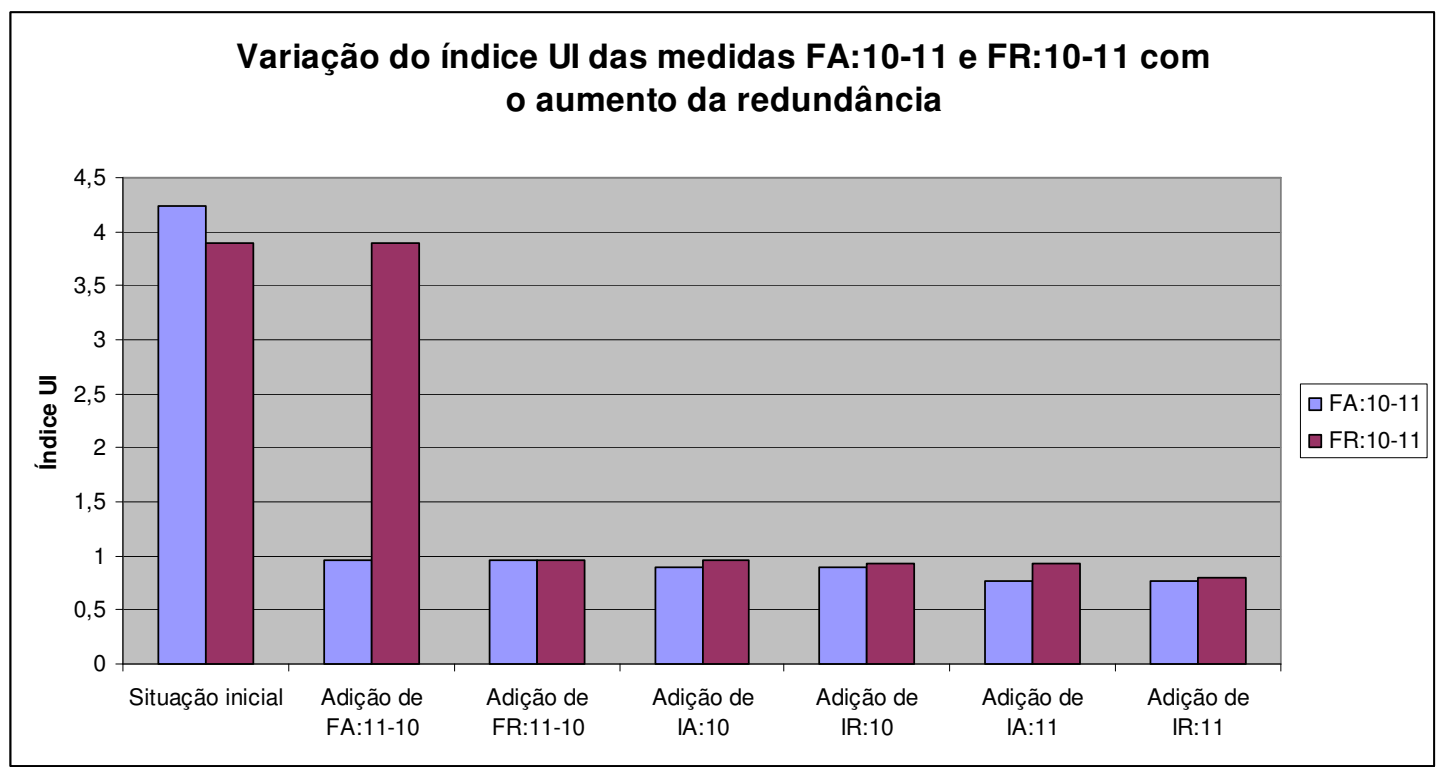

Figura 7.1: Variação do índice UI das medidas FA:10-11 e FR:10-11com o aumento da redundância local 
Importa observar que a simples adição da medida FA:11-10 fez o índice $U I$ da medida FA:10-11 passar de 4,2529 para 0,9681, representando uma modificação bem significativa. Por outro lado, a adição da medida FA:11-10 diminuiu pouquíssimo o índice $U I$ da medida FR:10-11 (igual a 3,8974).

Com a adição de FR:11-10, o índice UI de FR:10-11 ficou abaixo de 1, mais precisamente igual a 0,9642. Neste caso, poderíamos parar de acrescentar mais medidas redundantes, já que as medidas inseridas também apresentaram índice $U I$ abaixo de 1 , como observado na tabela 7.1. Continuando com a adição de novas medidas, verificouse que a inserção das medidas IA:11 e IR:11 fez diminuir ainda mais os UIs de FA:1011 e FR:11-10, entretanto, as mesmas (IA:11 e IR:11) apresentaram índices acima de 1 (em vermelho), o que é indesejável. Portanto, a adição das medidas IA:11 e IR:11 não é recomendada. Aliás, neste caso, recomenda-se a instalação das medidas FA:11-10, FR:11-10, IA:10 e IR:10.

A despeito do exemplo acima, o aumento da redundância local de uma medida não implica numa diminuição tão significativa do índice $U I$ para algumas medidas. Isto porque o índice UI não depende somente da redundância local das medidas, mas também de outros fatores como, por exemplo, a ponderação da medida e os parâmetros do sistema, conforme apresentado na conclusão do capítulo 5.

Com o intuito de exemplificar a afirmação acima, segue a seguir mais um exemplo.

Exemplo 2: Diminuição do índice $U I$ para as medidas IA:5 e IR:5

A partir da verificação dos índices UIs presentes na tabela 4.3, observa-se que as medidas IA:5 e IR:5 apresentam índice UI muito alto, cujos respectivos valores são 11,5168 e 9,4466 .

Nas proximidades das medidas em análise, ainda podem ser instaladas as seguintes medidas: FA:1-5 e FR:1-5; FA:5-1 e FR:5-1; FA:2-5 e FR:2-5; FA:4-5 e FR:4-5; IA:6 e IR:6; IA:4 e IR:4; V:5.

Com a adição gradativa das medidas candidatas a serem instaladas e, por último, de todas as medidas de potência e de tensão do sistema de 14 barras, obtivemos as variações do índice $U I$ das medidas em análise, as quais podem ser vistas através da tabela 7.2 e da figura 7.2. 
Tabela 7.2: Variação do índice UI das medidas IA:5 e IR:5 com a adição de novas medidas

\begin{tabular}{|c|c|c|}
\hline & Medidas & Análise \\
\hline \multirow{2}{*}{ Caso } & IA:5 & IR:5 \\
\hline & $\boldsymbol{U I}$ & $\boldsymbol{U I}$ \\
\hline Situação inicial & 11,5168 & 9,4466 \\
\hline Adição de FA:1-5 e FR:1-5 & 9,9052 & 9,4233 \\
\hline Adição de FA:5-1 e FR:5-1 & 9,5277 & 8,6255 \\
\hline Adição de FA:2-5 e FR:2-5 & 9,2380 & 8,1157 \\
\hline Adição de FA:4-5 e FR:4-5 & 7,7399 & 6,9225 \\
\hline Adição de IA:6 e IR:6 & 7,2176 & 6,5541 \\
\hline Adição IA:4 e IR:4 & 6,3561 & 5,0805 \\
\hline Adição de V:5 & 6,2153 & 5,0696 \\
\hline Adição de todas medidas* & 5,6955 & 3,6061 \\
\hline
\end{tabular}

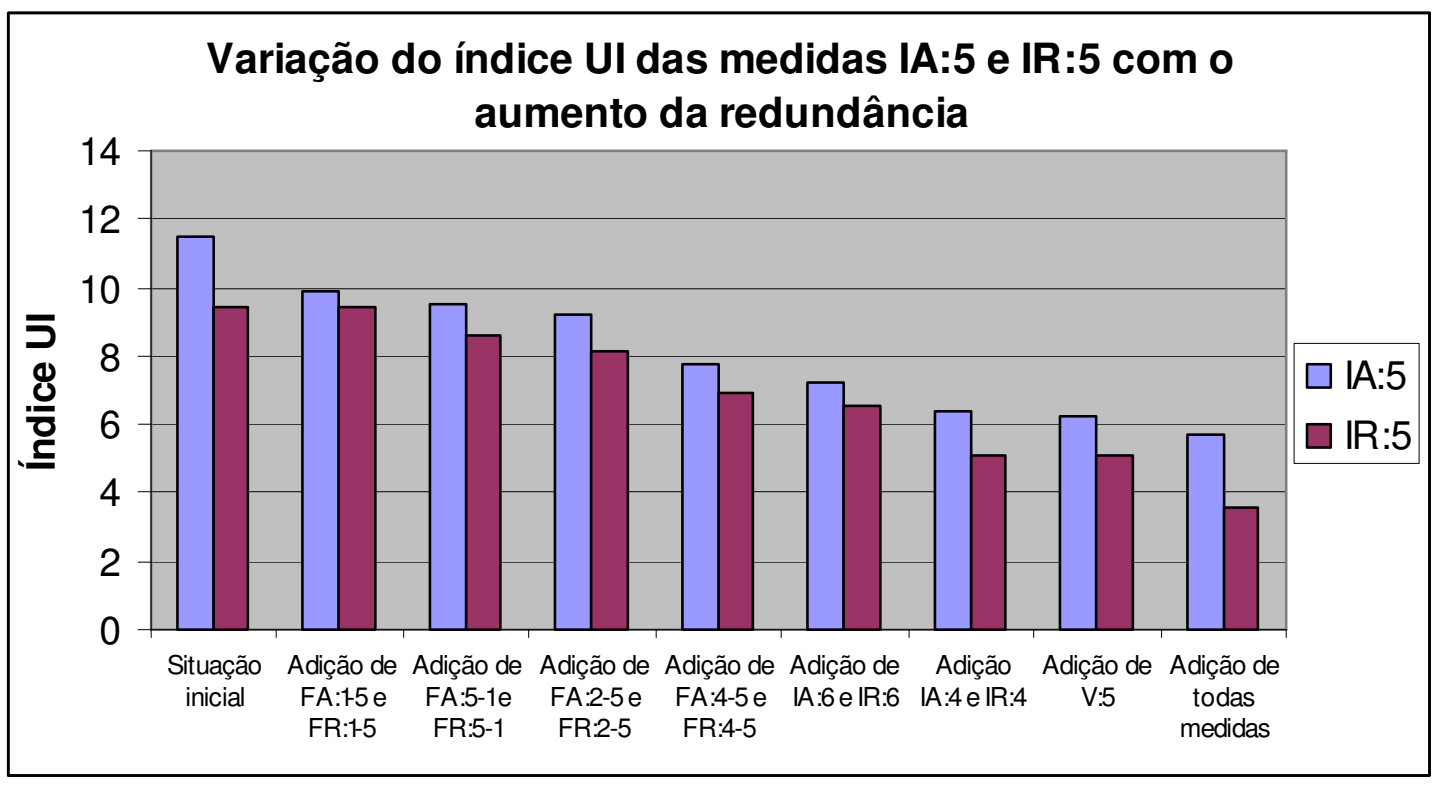

Figura 7.2: Variação do índice $U I$ das medidas IA:5 e IR:5, com o aumento da redundância

Neste exemplo, ficou evidente que a adição de todas as medidas de potência e de tensão não foram suficientes para reduzir o índice $U I$ das medidas IA:5 e IR:5 a valores inferiores a 1. Além disso, outras medidas também apresentaram $U I$ acima de 1, com a instalação de todas as medidas, dentre as quais podemos citar: IA:2, IA:11, IR:4 e FR:49 (veja as figuras 7.3 e 7.4). Como dito anteriormente, isto se deve ao fato de que o índice $U I$ de uma medida não depende apenas da redundância local da mesma, mas também de outros fatores, como sua ponderação e os parâmetros do sistema. 
Os gráficos das figuras 7.3 e 7.4, a seguir, apresentam os valores de $U I$, considerando a instalação de todas as medidas de potência e de tensão, para o sistema de 14 barras do IEEE.

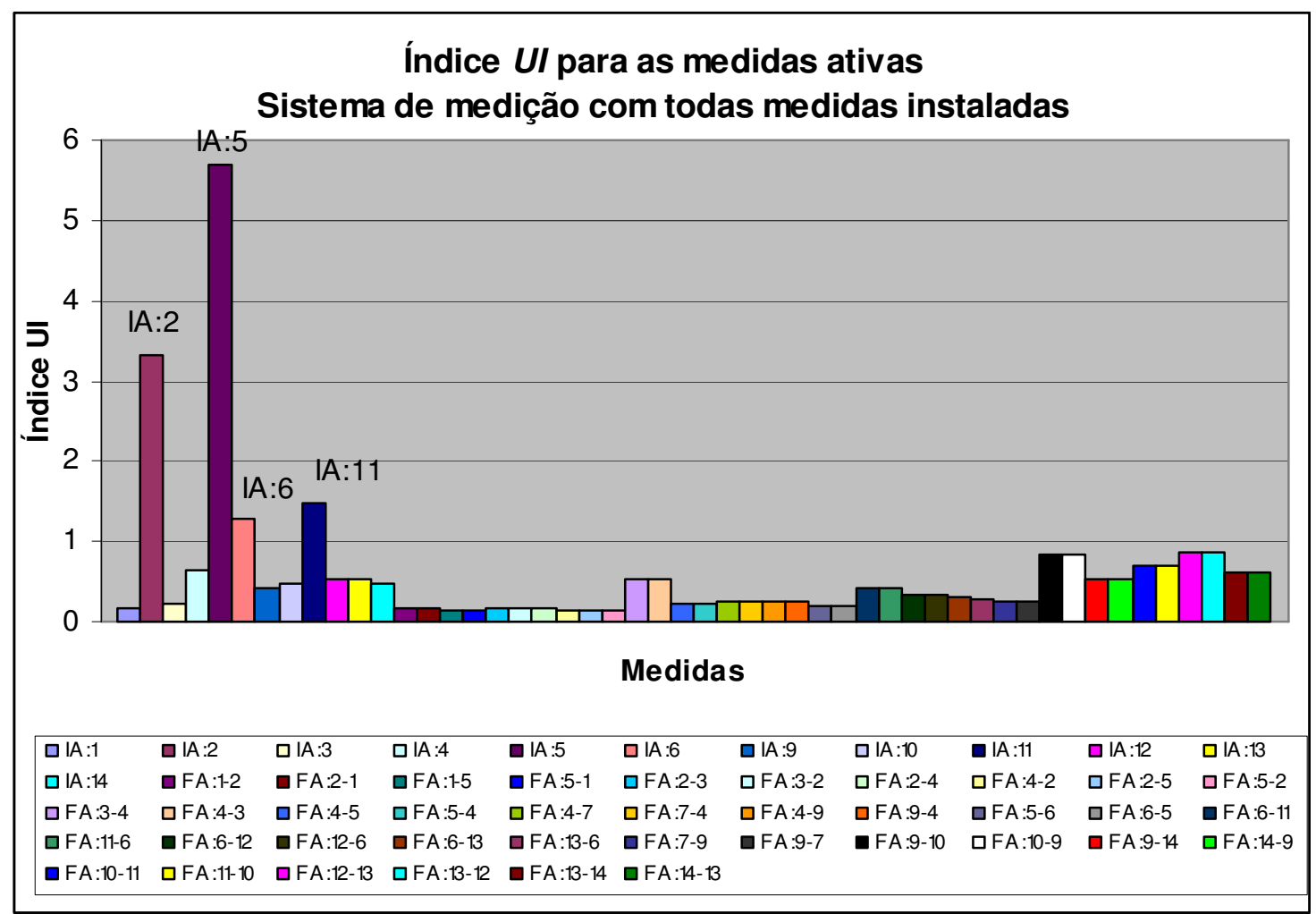

Figura 7.3: Índice $U I$ das medidas ativas após a instalação de todas as medidas de potência e tensão

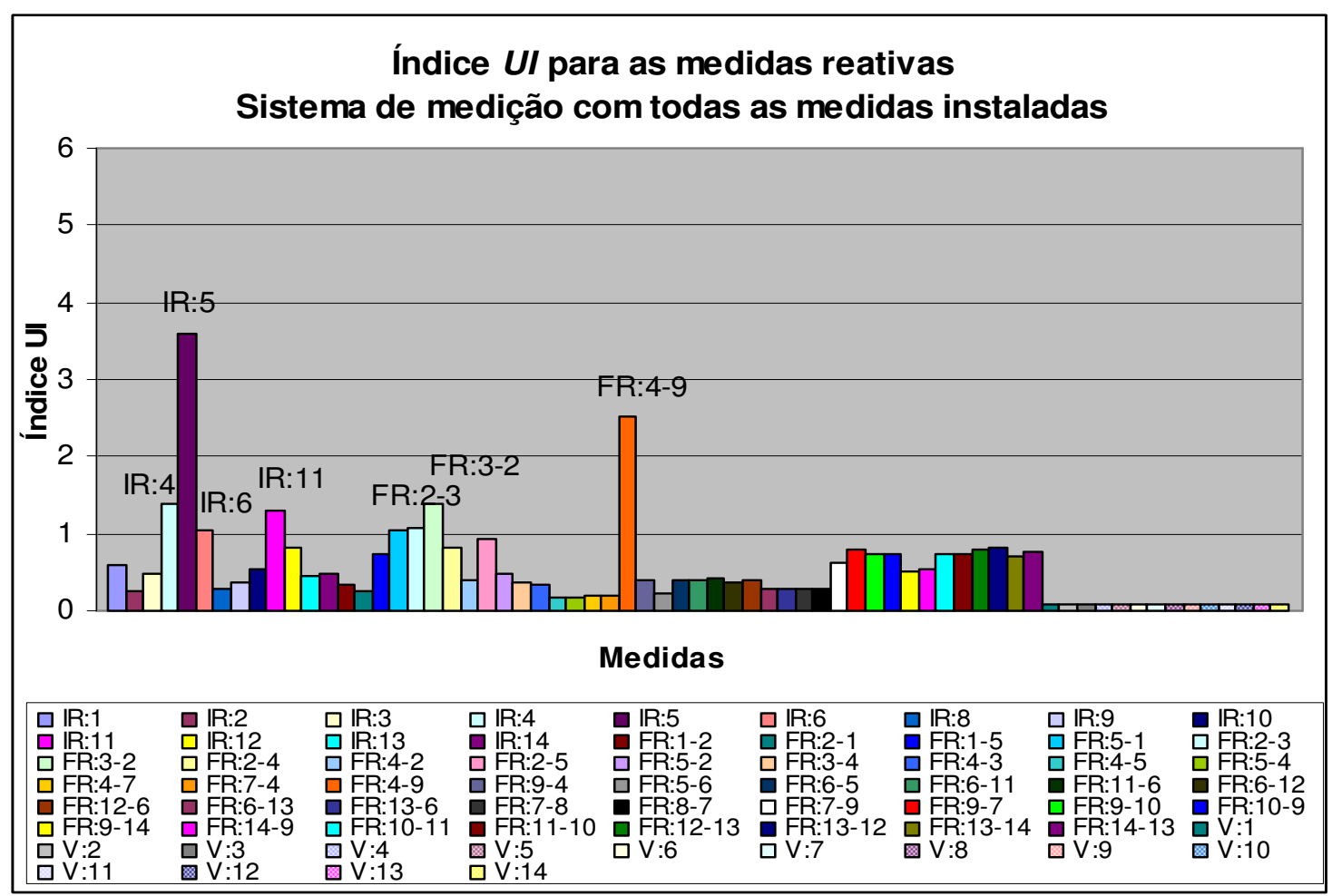

Figura 7.4: Índice $U I$ das medidas reativas após a instalação de todas as medidas de potência e tensão 
Embora os valores de UI para algumas medidas não sejam menores do que 1, a adição de todas as medidas melhorou muito os valores dos índices UIs, se comparados aos respectivos valores presentes nos gráficos das figuras 4.7 e 4.8. Isto mostra, mais uma vez, que o aumento da redundância melhora o índice $U I$ das medidas.

O próximo exemplo mostrará uma situação em que são retiradas as medidas com maiores índices $U I$, desde que isso não dê origem a medidas críticas e conjuntos críticos de medidas.

Exemplo 3: Retirada de medidas com índice $U I$ alto

Neste exemplo, simularemos a retirada das medidas com elevados índices UIs, após a instalação de todas as medidas possíveis no sistema de 14 barras do IEEE, tomando como base os resultados verificados através das figuras 7.3 e 7.4 (exemplo 2).

A partir dos valores dos índices UIs presentes nas figuras 7.3 e 7.4 , fica fácil observar que os maiores UIs são das medidas: IA:2, IA:5, IA:6; IA:11, IR:4, IR:5, IR:11, FR:3-2 e FR:4-9. Logo, estas medidas foram escolhidas para serem retiradas do sistema de medição.

Depois da atualização do sistema de medição do sistema de 14 barras do IEEE, calculamos os índices UIs para todas as medidas, os quais são apresentados nas figuras 7.5 e 7.6 a seguir.

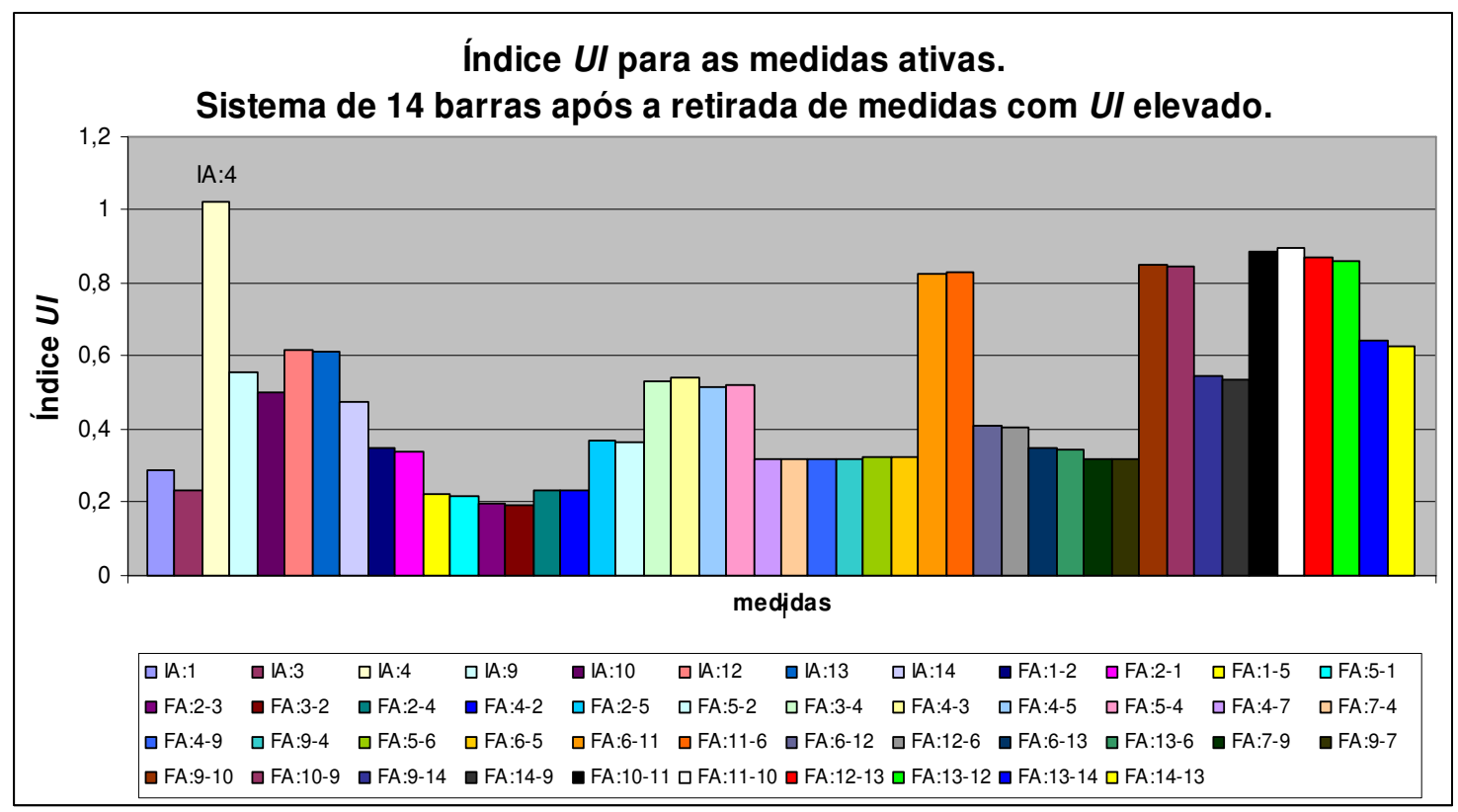

Figura 7.5: Índice $U I$ das medidas ativas após a retirada de medidas com $U I$ elevado 


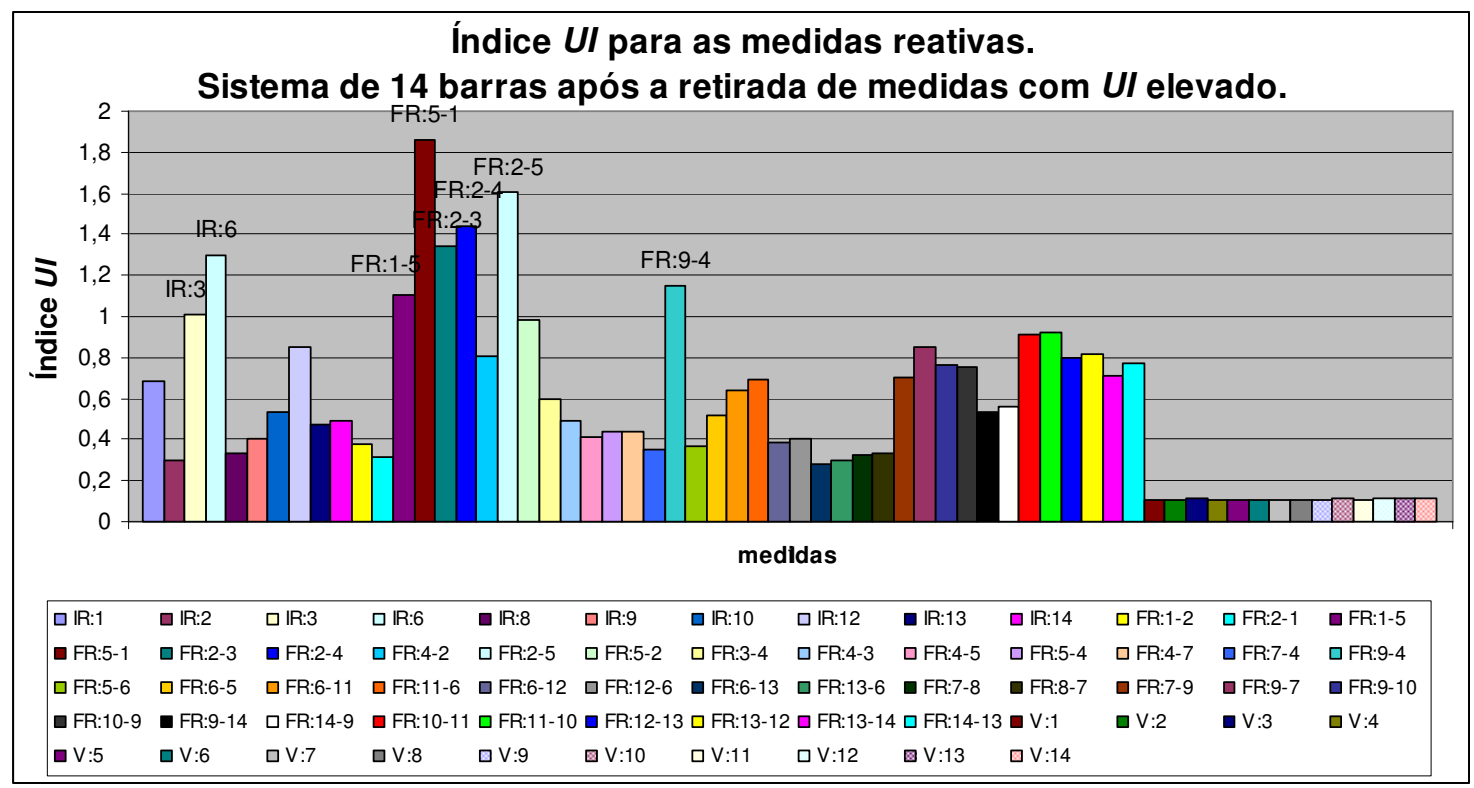

Figura 7.6: Índice $U I$ das medidas reativas após a retirada de medidas com $U I$ elevado

Os resultados apresentados no gráfico da figura 7.5 mostram que os valores dos índices UIs de quase todas as medidas ativas ficaram abaixo de 1 , com exceção da medida IA:4, com índice no valor de 1,0241. Por outro lado, algumas medidas reativas ainda tiveram o índice $U I$ na faixa de valores entre 1 e 2 , dentre as quais podemos citar FR:5-1 e FR:2-5, que apresentaram UI acima de 1,5.

Note, através dos resultados das figuras 7.5 e 7.6, que os índices UIs de algumas medidas ficaram maiores se comparados aos respectivos valores apresentados nas figuras 7.3 e 7.4. Por exemplo, a medida FR:5-1 apresentava índice UI de 1,0536 e, agora, apresenta índice $U I$ de 1,8561. Além disso, através da simulação da retirada de FR:5-1 (que não será apresentada), o índice $U I$ de FR:1-5 piorou, ficou acima de 2. Esta situação mostra que a escolha da medida a ser retirada de um sistema de medição deve ser tomada de forma cautelosa, uma vez que a retirada de uma medida aumentará o índice das demais redundantes a ela, podendo ser um aumento de baixa magnitude ou não (o que implicaria em prejuízo para detecção e identificação de EGs).

Nas próximas seções, serão propostos dois algoritmos para o projeto e/ou fortalecimento de sistemas de medição, baseado na redundância e no índice $U I$ das medidas.

Os algoritmos propostos precisam dos valores dos índices $U I$ das medidas, que por sua vez dependem do valor estimado das variáveis de estado $(\underline{\hat{x}})$, conforme algoritmo apresentado na seção 4.3.2, do capítulo 4. Dessa forma, para aplicar os 
algoritmos propostos, faz-se necessário a execução de um estimador de estado WLS. Conseqüentemente, torna-se necessária a realização de um estudo inicial de fluxo de carga, para o sistema que será analisado. Os resultados desse estudo serão utilizados para obtenção dos valores das medidas que serão consideradas para estimação de estado e cálculo dos índices UI. Vale lembrar que, de acordo com os testes apresentados no capítulo 4, os índices $U I$ variam pouco com a variação dos valores das medidas.

\subsection{Algoritmo para Projeto ou Fortalecimento de Sistemas de Medição: Algoritmo 1}

Mostraremos, nesta seção, o primeiro algoritmo proposto para projeto de sistemas de medição, baseado no índice $U I$ das medidas, que também pode ser utilizada para o fortalecimento de sistemas de medição já existentes.

A idéia que norteia o algoritmo é considerar, inicialmente, que todas as medidas do sistema elétrico em análise estão disponíveis. Em seguida, retirar-se-ão as medidas que apresentarem $U I$ acima de certo limiar pré-estabelecido $(L)$, levando-se em conta: ausência de medidas críticas e de conjuntos críticos de medidas.

\section{Algoritmo para Projeto de Sistemas de Medição: Algoritmo 1}

Passo 0: Faça a leitura dos parâmetros da rede elétrica e dos valores obtidos com o fluxo de potência. Estabeleça o limiar inicial de corte $L$ (preferencialmente entre 1 e 3 ) para o UI das medidas, o valor do incremento $\Delta$ (preferencialmente 0,5 ) e inicie o contador de teste $(\mathrm{T})$ em $\mathrm{T}=1$;

Passo 1: Considere como instaladas todas as medidas possíveis de potência e de magnitude de tensão da rede elétrica em questão;

Passo 2: Calcule o índice $U I$ das medidas (algoritmo da seção 4.3.2). Os valores das medidas utilizados para calcular os índices $U I$ são obtidos a partir do estudo de fluxo de carga previamente realizado;

Passo 3: Identifique as medidas que apresentarem $U I$ maior que o limiar $L^{\prime}$ ( $L$ mais uma tolerância), sendo:

$$
L^{\prime}=1,1 \cdot L \text {. }
$$

Caso todas medidas apresentem $U I<L^{\prime}$, fim do processo; caso contrário, prossiga ao próximo passo; 
Passo 4: Descarte as medidas com $U I>L$ ' e verifique a observabilidade e redundância do novo plano de medição. Caso o sistema não seja mais observável, ou alguma medida seja crítica ou faça parte de algum conjunto crítico (isto é, nível de redundância NR menor que 2), aumente o limiar $L(L=L+\Delta)$; incremente $\mathrm{T}(\mathrm{T}=\mathrm{T}+1)$ e volte ao passo 1; caso contrário, vá ao passo 2.

A seguir, apresentamos o fluxograma do algoritmo 1:

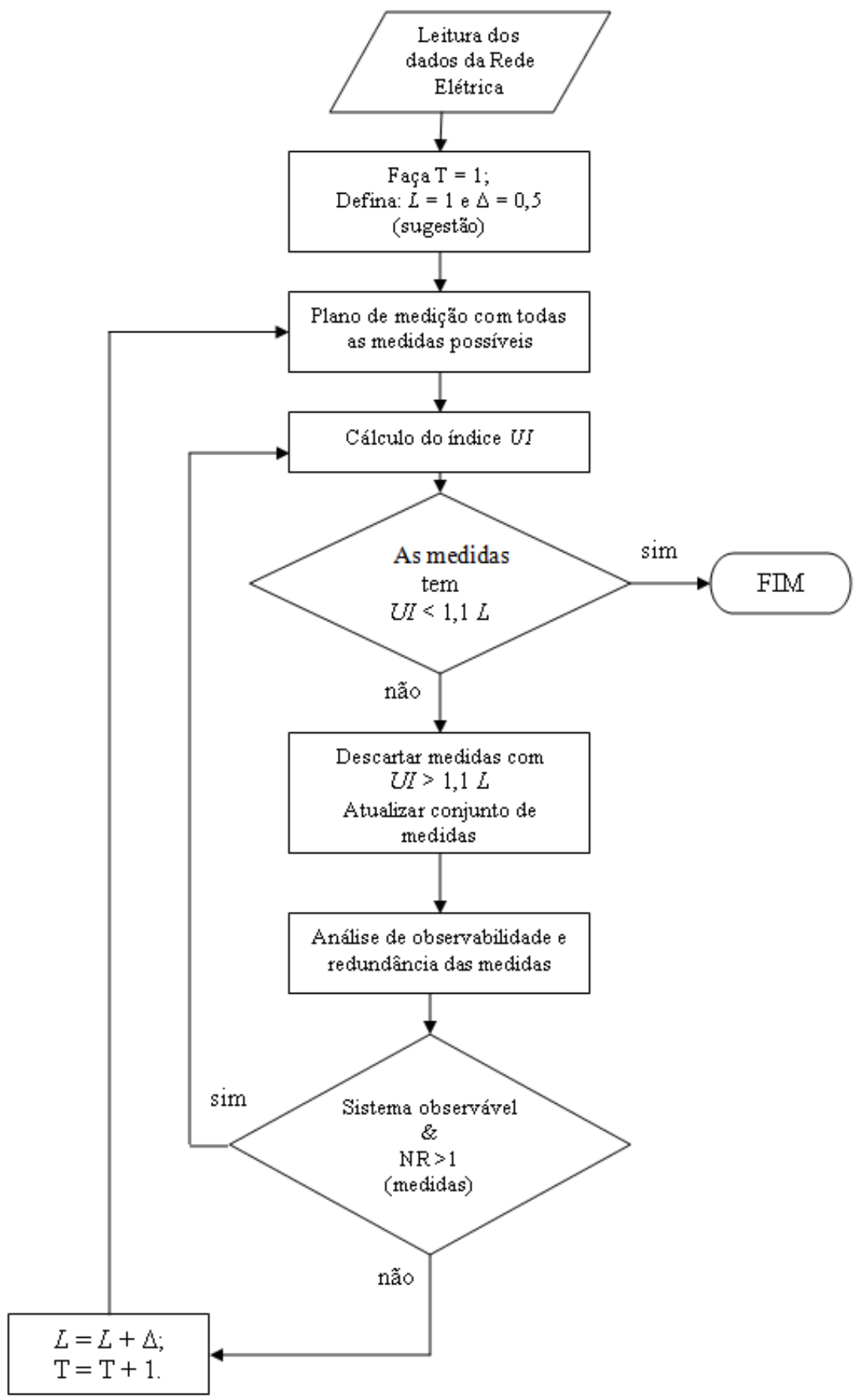

Figura 7.7: Fluxograma do algoritmo 1 para o projeto de sistemas de medição 


\section{Observações:}

i) Para o descarte de medidas de potência, consideramos a retirada do par (ativo e reativo), mesmo que uma das medidas do par esteja com índice $U I$ menor que $L^{\prime}$;

ii) Utilizamos o valor do limiar $L$ ', com $10 \%$ a mais do valor de $L$, porque essa diferença não é tão significativa em termos de detecção de erros grosseiros; além disso, poderíamos utilizar apenas o valor de $L$ como limiar de corte, ou seja, em termos de nosso algoritmo teríamos $L^{\prime}=L$;

iii) $\mathrm{O}$ incremento " $\Delta$ " também pode ser ajustado conforme o interesse do projetista. Neste trabalho utilizamos $\Delta=0,5$, mas poderíamos utilizar um valor inferior a este, como, por exemplo, $\Delta=0,1$;

iv) Para a análise de observabilidade e redundância de medidas, utilizamos a metodologia baseada na análise da matriz ${H_{\Delta}}^{\mathrm{t}}$, apresentada em London Jr. et al. (2007).

\subsubsection{Aplicação do Algoritmo 1 ao Sistema de 14 Barras do IEEE}

\section{Resultado}

Passo 0: Inicialmente fazemos a leitura dos dados do sistema de 14 barras do IEEE e do resultado da simulação de um fluxo de carga. Em seguida, definimos: $L=1,0 ; \Delta=0,5 \mathrm{e}$ iniciamos o contador de teste $\mathrm{T}=1$.

$$
\underline{\mathrm{T}=1 \mathrm{e} L=1,0}
$$

Passo 1: Consideramos o sistema de medição com todos os medidores possíveis instalados;

Passo 2: Calculamos o índice $U I$ de todas as medidas.

Passo 3: Medidas com UI maior que $1,1 L\left(L^{\prime}=1,10\right)$ (tabela 7.3):

Tabela 7.3: Medidas com índice UI maior que 1,1 L considerando todos os medidores instalados

\begin{tabular}{c|c}
\hline Medida & $\boldsymbol{U I}$ \\
\hline IA:2 & 3,3177 \\
\hline IA:5 & 5,6955 \\
\hline IA:6 & 1,2848 \\
\hline IA:11 & 1,4805 \\
\hline IR:4 & 1,3903 \\
\hline IR:5 & 3,6061 \\
\hline IR:11 & 1,3056 \\
\hline FR:3-2 & 1,3956 \\
\hline
\end{tabular}




\begin{tabular}{l|l} 
FR:4-9 & 2,5273 \\
\hline
\end{tabular}

Passo 4: Com base nos resultados da tabela acima, descartamos os pares de medidas de potência (ativa e reativa) I:2, I:4, I:5, I:6, I:11, F:3-2 e F:4-9. Após a atualização do conjunto de medidas, não houve a formação de conjuntos críticos e não se tornou crítica nenhuma medida, logo continuamos o processo.

Passo 2': Com o conjunto de medidas atualizado, calculamos o índice UI das medidas.

Passo 3': Medidas com UI maior que 1,1 L (tabela 7.4):

Tabela 7.4: Medidas com índice $U I$ maior que $1,1 \mathrm{~L}$

\begin{tabular}{c|c}
\hline Medida & $\boldsymbol{U I}$ \\
\hline FR:5-1 & 2,1155 \\
\hline FR:2-3 & 1,3777 \\
\hline FR:2-4 & 1,5148 \\
\hline FR:2-5 & 1,6476 \\
\hline FR:9-4 & 1,2099 \\
\hline
\end{tabular}

Passo 4': Descartamos os pares de medidas de potência (ativa e reativa) F:5-1, F:2-3, F:2-4, F:2-5 e F:9-4 . Após a atualização do conjunto de medidas, não houve a formação de conjuntos críticos e nenhuma medida se tornou crítica, logo continuamos o processo.

Passo 2"': Com o conjunto de medidas atualizado, calculamos o índice UI das mesmas.

Passo 3'': Medidas com UI maior que L' (tabela 7.5):

Tabela 7.5: Medidas com índice $U I$ maior que $1,1 \mathrm{~L}$

\begin{tabular}{c|c}
\hline Medida & $\boldsymbol{U I}$ \\
\hline IR:3 & 1,1233 \\
\hline FR:1-5 & 1,8573 \\
\hline FR:4-2 & 1,1163 \\
\hline FR:5-2 & 1,7736 \\
\hline
\end{tabular}

Passo 4': Descartamos os pares de medidas de potência (ativa e reativa) I:3, F:1-5, F:42 e F:5-2. Após a atualização do conjunto de medidas, verificamos que as medidas IA:1 e IR:1 são críticas.

Com isso, concluímos que não é possível obter, através do algoritmo 1, um sistema de medição em que todas as medidas tenham $U I<1,10$ e que não apresente conjuntos críticos. 
Com base nesse resultado, aumentamos o limiar $L$ para 1,50; incrementamos $\mathrm{T}$ para 2 e voltamos ao passo 1.

$$
\underline{\mathrm{T}=2 \mathrm{e} L=1,50}
$$

Passo 1: Consideramos o sistema de medição com todas as medidas possíveis instaladas;

Passo 2: Calculamos o índice $U I$ de todas as medidas (na realidade não calculamos, pois já tínhamos este resultado armazenado para $\mathrm{T}=1$ ).

Passo 3: Medidas com UI maior que 1,1 $L\left(L^{\prime}=1,65\right)$ (tabela 7.6):

Tabela 7.6: Medidas com índice UI maior que 1,65, considerando todas as medidas instaladas

\begin{tabular}{c|c}
\hline Medida & $\boldsymbol{U I}$ \\
\hline IA:2 & 3,3177 \\
\hline IA:5 & 5,6955 \\
\hline IR:5 & 3,6061 \\
\hline FR:4-9 & 2,5273 \\
\hline
\end{tabular}

Passo 4: Com base nos resultados da tabela acima, descartamos os pares de medidas de potência (ativa e reativa) I:2, I:5 e F:4-9. Após a atualização do conjunto de medidas, não houve a formação de conjuntos críticos e nenhuma medida se tornou crítica, logo continuamos o processo.

Passo 2': Com o conjunto de medidas atualizado calculamos o índice UI das mesmas;

Passo 3': Medidas com UI maior que L' (tabela 7.7):

Tabela 7.7: Medidas com índice $U I$ maior que 1,65

\begin{tabular}{c|c}
\hline Medida & $\boldsymbol{U I}$ \\
\hline IR:4 & 2,4630 \\
\hline FR:5-1 & 2,0353 \\
\hline
\end{tabular}

Passo 4': Descartamos os pares de medidas de potência: I:4 e F:5-1. Com a atualização do conjunto de medidas, não houve a formação de conjuntos críticos e nenhuma medida se tornou crítica, logo continuamos o processo.

Passo 2": Com o conjunto de medidas atualizado, calculamos o índice UI das medidas; Passo 3"': Nenhuma medida apresentou UI > L'. Fim do Processo.

As medidas que restaram compõem o sistema de medição sem medidas críticas e conjuntos críticos, e ainda, as medidas apresentam $U I$ menor que o limiar 1,65. Veja a tabela 7.8 , e as figuras 7.8 e 7.9 : 
Tabela 7.8: Medidas do plano de medição resultante e respectivos valores de UI (sistema de 14 barras)

\begin{tabular}{|c|c|c|c|c|c|}
\hline \multicolumn{2}{|c|}{$\begin{array}{c}\text { Medidas de Potência } \\
\text { Ativa }\end{array}$} & \multicolumn{2}{|c|}{$\begin{array}{l}\text { Medidas de Potência } \\
\text { Reativa }\end{array}$} & \multicolumn{2}{|c|}{$\begin{array}{c}\text { Medidas } \\
\text { deTensão }\end{array}$} \\
\hline Medida & Indice UI & Medida & Indice UI & Medida & Índice UI \\
\hline IA: 1 & 0,3894 & IR:1 & 0,8651 & $V: 1$ & 0,1064 \\
\hline IA:3 & 0,2378 & IR:3 & 0,5122 & $V: 2$ & 0,1089 \\
\hline IA:6 & 1,5583 & IR:6 & 1,2367 & $V: 3$ & 0,1127 \\
\hline IA:7 * & --- & IR:7 & --- & $V: 4$ & 0,11 \\
\hline IA:8 & --- & IR:8 & 0,3364 & $V: 5$ & 0,109 \\
\hline IA:9 & 0,5069 & IR:9 & 0,4074 & $V: 6$ & 0,1097 \\
\hline IA:10 & 0,4872 & IR:10 & 0,53 & V:7 & 0,1093 \\
\hline IA:11 & 1,5042 & IR:11 & 1,3229 & $\mathrm{~V}: 8$ & 0,1057 \\
\hline IA:12 & 0,5576 & IR:12 & 0,8479 & V:9 & 0,1112 \\
\hline IA:13 & 0,5587 & IR:13 & 0,4729 & V:10 & 0,1124 \\
\hline IA:14 & 0,4768 & IR:14 & 0,4833 & V:11 & 0,1115 \\
\hline FA:1-2 & 0,4763 & FR:1-2 & 0,5056 & V:12 & 0,1125 \\
\hline FA:2-1 & 0,4605 & FR:2-1 & 0,7392 & V:13 & 0,1134 \\
\hline FA:1-5 & 0,2707 & FR:1-5 & 1,133 & V:14 & 0,1162 \\
\hline FA:2-3 & 0,1975 & FR:2-3 & 1,312 & & \\
\hline FA:3-2 & 0,1956 & FR:3-2 & 1,5644 & & \\
\hline FA:2-4 & 0,2556 & FR:2-4 & 1,5297 & & \\
\hline FA:4-2 & 0,2519 & FR:4-2 & 0,7437 & & \\
\hline FA:2-5 & 0,3757 & FR:2-5 & 1,6408 & & \\
\hline FA:5-2 & 0,3702 & FR:5-2 & 0,9936 & & \\
\hline FA:3-4 & 0,5687 & FR:3-4 & 0,4773 & & \\
\hline FA:4-3 & 0,5792 & FR:4-3 & 0,4243 & & \\
\hline FA:4-5 & 0,6826 & FR:4-5 & 0,4066 & & \\
\hline FA:5-4 & 0,6906 & FR:5-4 & 0,4363 & & \\
\hline FA:4-7 & 0,2905 & FR:4-7 & 0,4496 & & \\
\hline FA:7-4 & 0,2905 & FR:7-4 & 0,3538 & & \\
\hline FA:9-4 & 0,2905 & FR:9-4 & 1,1295 & & \\
\hline FA:5-6 & 0,2307 & FR:5-6 & 0,3295 & & \\
\hline FA:6-5 & 0,2307 & FR:6-5 & 0,5267 & & \\
\hline FA:6-11 & 0,4326 & FR:6-11 & 0,4119 & & \\
\hline FA:11-6 & 0,4335 & FR:11-6 & 0,4369 & & \\
\hline FA:6-12 & 0,3616 & FR:6-12 & 0,3885 & & \\
\hline FA:12-6 & 0,36 & FR:12-6 & 0,4028 & & \\
\hline FA:6-13 & 0,3075 & FR:6-13 & 0,2795 & & \\
\hline FA:13-6 & 0,3044 & FR:13-6 & 0,2949 & & \\
\hline FA:7-8 & --- & FR:7-8 & 0,327 & & \\
\hline FA:8-7 & --- & FR:8-7 & 0,3364 & & \\
\hline FA:7-9 & 0,2905 & FR:7-9 & 0,704 & & \\
\hline FA:9-7 & 0,2905 & FR:9-7 & 0,8551 & & \\
\hline FA:9-10 & 0,8406 & FR:9-10 & 0,7485 & & \\
\hline FA:10-9 & 0,8363 & FR:10-9 & 0,7408 & & \\
\hline FA:9-14 & 0,5347 & FR:9-14 & 0,516 & & \\
\hline FA:14-9 & 0,5268 & FR:14-9 & 0,5391 & & \\
\hline FA:10-11 & 0,7016 & FR:10-11 & 0,7383 & & \\
\hline FA:11-10 & 0,7073 & FR:11-10 & 0,743 & & \\
\hline FA:12-13 & 0,8692 & FR:12-13 & 0,8019 & & \\
\hline FA:13-12 & 0,8598 & FR:13-12 & 0,8178 & & \\
\hline FA:13-14 & 0,6309 & FR:13-14 & 0,7026 & & \\
\hline
\end{tabular}




\begin{tabular}{l|c|c|c|c|c}
\hline FA:14-13 & 0,6158 & FR:14-13 & 0,7605 & & \\
\hline * Obs.: Os valores de $U I$ das medidas nulas não foram calculados. \\
\hline
\end{tabular}

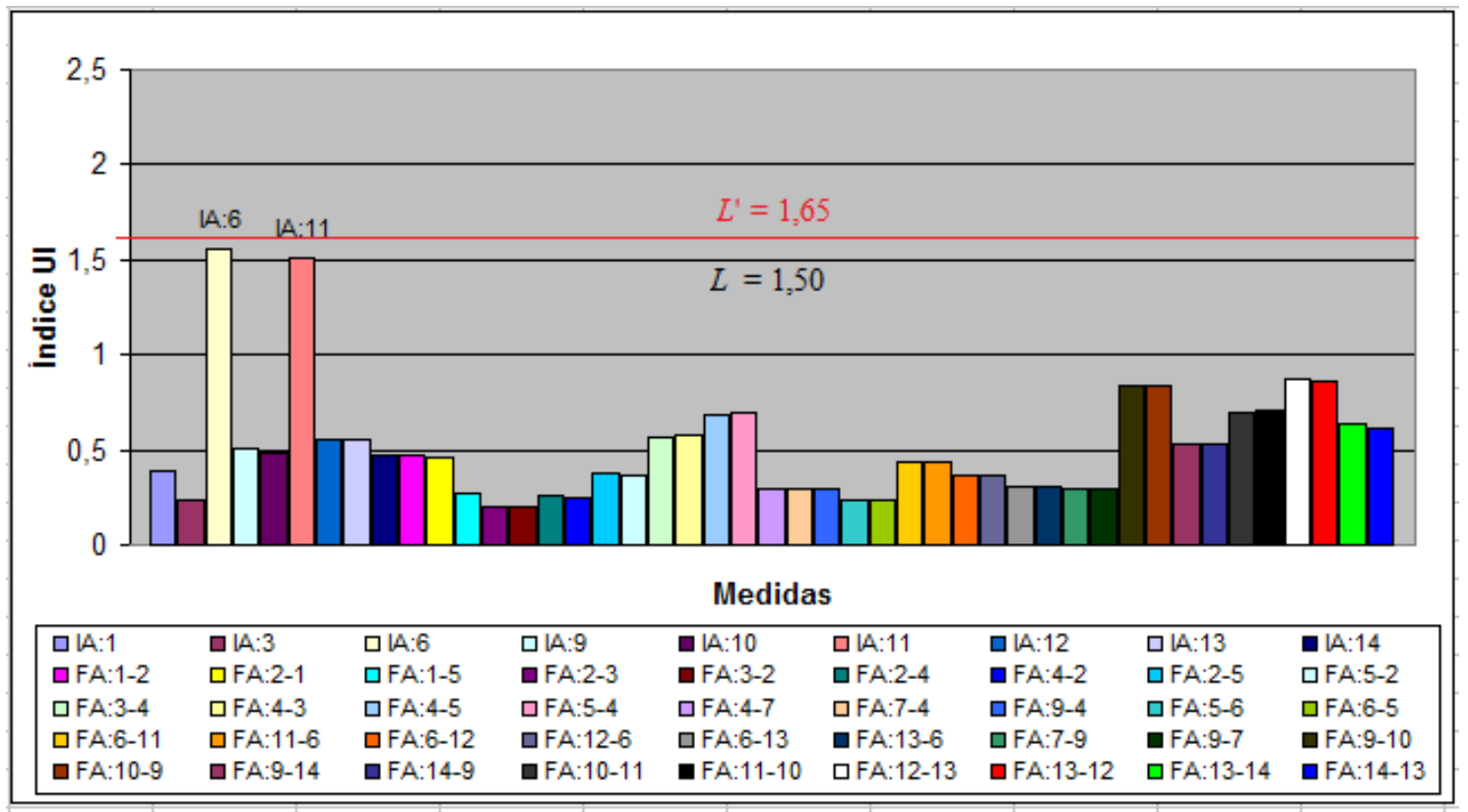

Figura 7.8: Índice $U I$ das medidas ativas do sistema de medição obtido com o Algoritmo 1 - Sistema de 14 barras IEEE

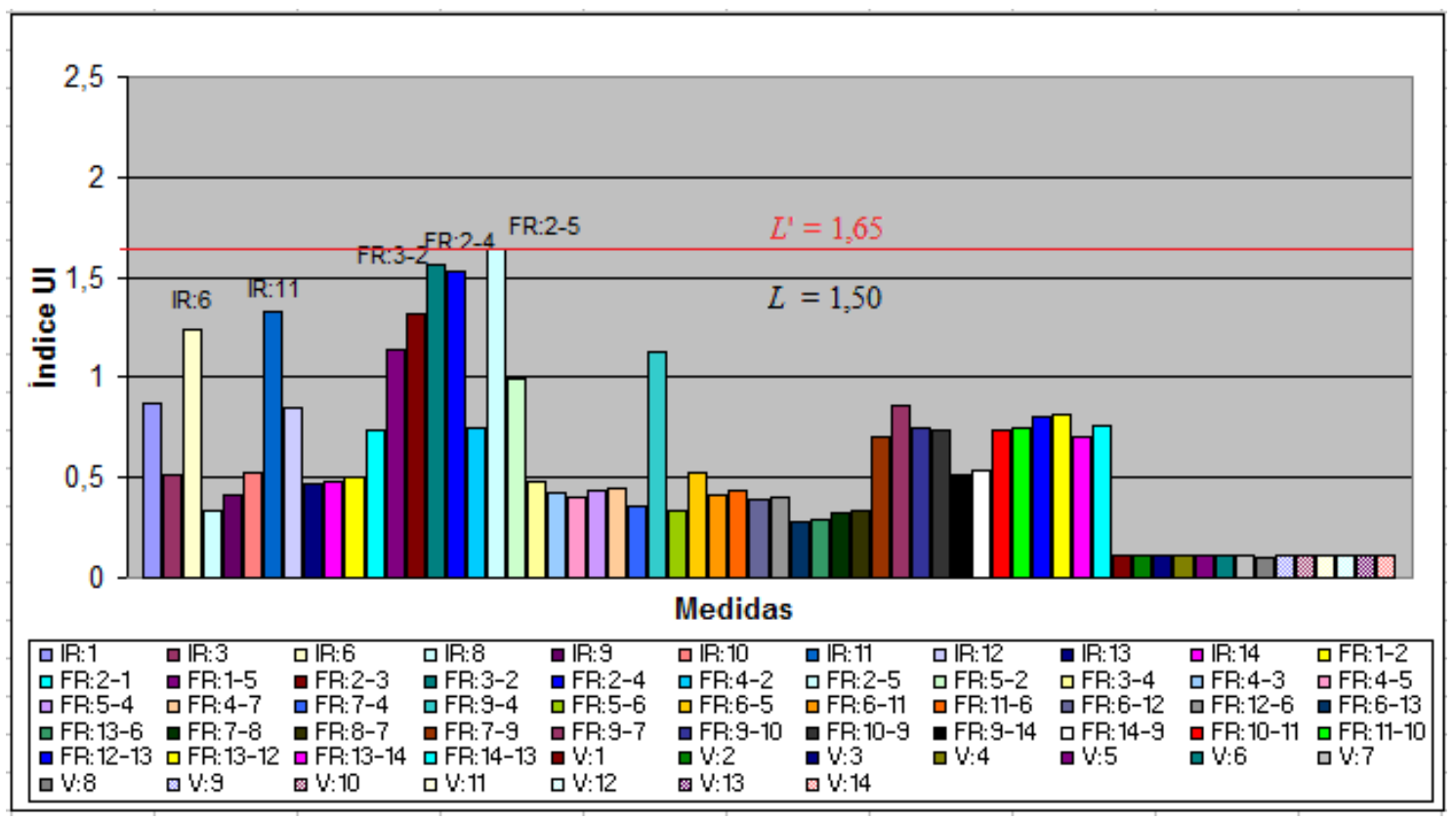

Figura 7.9: Índice UI das medidas reativas do sistema de medição obtido com o Algoritmo 1 - Sistema de 14 barras IEEE

Analisando o sistema de medição obtido acima, percebemos que menos de $10 \%$ do total de medidas apresentam índice $U I$ acima de 1 , que representa um bom resultado. 
Apesar de essa minoria de medidas apresentarem UI acima de 1, a capacidade de detecção de erro grosseiro em tais medidas não chega a ser ruim, já que o limiar $L$ ' fícou restrito a 1,65 .

\subsubsection{Aplicação do Algoritmo 1 sobre o Sistema de 30 Barras do IEEE}

\section{Resultado}

Passo 0: Inicialmente fazemos a leitura dos dados do sistema de 30 barras do IEEE e do resultado da simulação de um fluxo de carga. Em seguida, definimos: $L=1,0 ; \Delta=0,5 \mathrm{e}$ iniciamos o contador de teste $\mathrm{T}=1$.

$$
\underline{\mathrm{T}=1 \mathrm{e} L=1,0}
$$

Passo 1: Consideramos o sistema de medição com todas as medidas possíveis instaladas;

Passo 2: Calculamos o índice $U I$ de todas as medidas.

Passo 3: Medidas com UI maior que $L^{\prime}\left(L^{\prime}=1,10\right)$ (tabela 7.9):

Tabela 7.9: Medidas com índice $U I$ maior que 1,1 considerando todas as medidas instaladas

\begin{tabular}{c|c}
\hline Medida & $\boldsymbol{U I}$ \\
\hline IA:2 & 4,5631 \\
\hline IA:3 & 9,4658 \\
\hline IA:4 & 5,7414 \\
\hline IA:10 & 1,9456 \\
\hline IA:15 & 1,1823 \\
\hline IA:16 & 1,349 \\
\hline IA:18 & 1,2607 \\
\hline IA:20 & 1,6858 \\
\hline IA:23 & 1,3443 \\
\hline IA:29 & 1,2042 \\
\hline IR:3 & 1,179 \\
\hline IR:4 & 2,5255 \\
\hline IR:10 & 2,4069 \\
\hline IR:12 & 1,4372 \\
\hline IR:15 & 1,5858 \\
\hline FR:2-5 & 1,3372 \\
\hline
\end{tabular}

Passo 4: Descartamos os pares de medidas de potência (ativa e reativa) I:2, I:3, I:4, I:10, I:12, I:15, I:16, I:18, I:20, I:23, I:29 e F:2-5. Após a atualização do conjunto de medidas, não houve a formação de conjuntos críticos e nenhuma medida se tornou crítica, logo continuamos o processo.

Passo 2': Com o conjunto de medidas atualizado, calculamos o índice $U I$ das medidas;

Passo 3': Medidas com UI maior que L' (tabela 7.10): 


\begin{tabular}{c|c}
\hline Medida & $\boldsymbol{U I}$ \\
\hline FR:1-3 & 1,3633 \\
\hline FR:3-1 & 1,5579 \\
\hline FR:3-4 & 2,0238 \\
\hline
\end{tabular}

Passo 4': Descartamos os pares de medidas de potência F:1-3, F:3-1 e F:3-4. Após a atualização do conjunto de medidas, não houve a formação de conjuntos críticos e nenhuma medida se tornou crítica, logo continuamos o processo.

Passo 2'": Com o conjunto de medidas atualizado, calculamos o índice UI das medidas;

Passo 3': Medidas com UI maior que L' (tabela 7.11):

Tabela 711: Medidas com índice UI maior que 1,1

\begin{tabular}{c|c}
\hline Medida & $\boldsymbol{U I}$ \\
\hline FA:4-3 & 7,3880 \\
\hline IR:1 & 1,8774 \\
\hline FR:4-3 & 17,6226 \\
\hline
\end{tabular}

Passo 4': Descartamos os pares de medidas de potência I:1 e F:4-3. Após a atualização do conjunto de medidas, verificamos que o sistema não é observável com a retirada de I:1 (IA:1 e IR:1) e F:4-3 (FA:4-3 e FR:4-3).

Com base neste resultado, aumentamos o limiar $L$ para 1,50; incrementamos T para 2 e voltamos ao passo 1 .

$$
\underline{\mathrm{T}=2 \mathrm{e} L=1,5}
$$

Passo 1: Consideramos todas as medidas possíveis instaladas;

Passo 2: Calculamos o índice $U I$ de todas as medidas.

Passo 3: Medidas com UI maior que $L^{\prime}\left(L^{\prime}=1,65\right)$ (tabela 7.12):

Tabela 7.12: Medidas com índice UI maior que 1,65 considerando todas as medidas instaladas

\begin{tabular}{c|c}
\hline Medida & $\boldsymbol{U I}$ \\
\hline IA:2 & 4,5631 \\
\hline IA:3 & 9,4658 \\
\hline IA:4 & 5,7414 \\
\hline IA:10 & 1,9456 \\
\hline IA:20 & 1,6858 \\
\hline IR:4 & 2,5255 \\
\hline IR:10 & 2,4069 \\
\hline
\end{tabular}

Passo 4: Descartamos os pares de medidas de potência I:2, I:3, I:4, I:10 e I:20. Após a atualização do conjunto de medidas, não houve a formação de conjuntos críticos e nenhuma medida se tornou crítica, logo continuamos o processo. 
Passo 2': Com o conjunto de medidas atualizado calculamos o índice $U I$ das mesmas;

Passo 3': Medidas com UI maior que L' (tabela 7.13):

Tabela 7.13: Medidas com índice UI maior que 1,65

\begin{tabular}{c|c}
\hline Medida & $\boldsymbol{U I}$ \\
\hline FR:3-4 & 2,0206 \\
\hline
\end{tabular}

Passo 4': Descartamos o par de medidas de potência F:3-4. Após a atualização do conjunto de medidas, não houve a formação de conjuntos críticos e nenhuma medida tornou-se crítica, logo continuamos o processo.

Passo 2"': Com o conjunto de medidas atualizado, calculamos o índice UI das mesmas;

Passo 3": Medidas com UI maior que L' (tabela 7.14):

Tabela 7.14: Medidas com índice UI maior que 1,65

\begin{tabular}{c|c}
\hline Medida & $\boldsymbol{U I}$ \\
\hline FA:4-3 & 1,8909 \\
\hline FR:4-3 & 3,2762 \\
\hline
\end{tabular}

Passo 4": Descartamos o par de medidas de potência F:4-3. Após a atualização do conjunto de medidas, não houve a formação de conjuntos críticos e nenhuma medida se tornou crítica, logo continuamos o processo.

Passo 2",: Com o conjunto de medidas atualizado, calculamos o índice UI das mesmas;

Passo 3"': Medidas com UI maior que $L$ ' (tabela 7.15):

Tabela 7.15: Medidas com índice UI maior que 1,65

\begin{tabular}{c|c}
\hline Medida & $\boldsymbol{U I}$ \\
\hline FR:1-3 & 3,5277 \\
\hline FR:3-1 & 3,9499 \\
\hline
\end{tabular}

Passo 4": Descartamos os pares de medidas de potência F:1-3 e F:3-1. Após a atualização do conjunto de medidas, verificamos que as medidas IA:1 e IR:1 são críticas.

Assim, aumentamos o limiar $L$ para 2,0; incrementamos T para 3 e voltamos ao passo 1 .

$$
\underline{\mathrm{T}=3 \text { e } L=2,0}
$$

Passo 1: Consideramos todas as medidas possíveis instaladas;

Passo 2: Calculamos o índice $U I$ de todas as medidas.

Passo 3: Medidas com UI maior que $1,1 L^{\prime}\left(L^{\prime}=2,20\right)$ (tabela 7.16): 
Tabela 7.16: Medidas com índice UI maior que 2,20 considerando todas as medidas instaladas

\begin{tabular}{c|c}
\hline Medida & $\boldsymbol{U I}$ \\
\hline IA:2 & 4,5631 \\
\hline IA:3 & 9,4658 \\
\hline IA:4 & 5,7414 \\
\hline IR:4 & 2,5255 \\
\hline IR:10 & 2,4069 \\
\hline
\end{tabular}

Passo 4: Descartamos os pares de medidas de potência I:2, I:3, I:4 e I:10. Após a atualização do conjunto de medidas, não houve a formação de conjuntos críticos e nenhuma medida se tornou crítica, logo continuamos o processo.

Passo 2': Com o conjunto de medidas atualizado, calculamos o índice UI das mesmas;

Passo 3': Nenhuma medida apresentou $U I>L$ '. Fim do Processo.

As medidas restantes compõem o plano de medição sem medidas críticas e conjuntos críticos, sendo que as medidas apresentam $U I$ menor que o limiar $L$ '. As medidas e os respectivos valores de $U I$ estão na tabela B.3 do Anexo B.

As figuras 7.10 e 7.11, a seguir, mostram o índice $U I$ das medidas ativas e reativas, respectivamente.

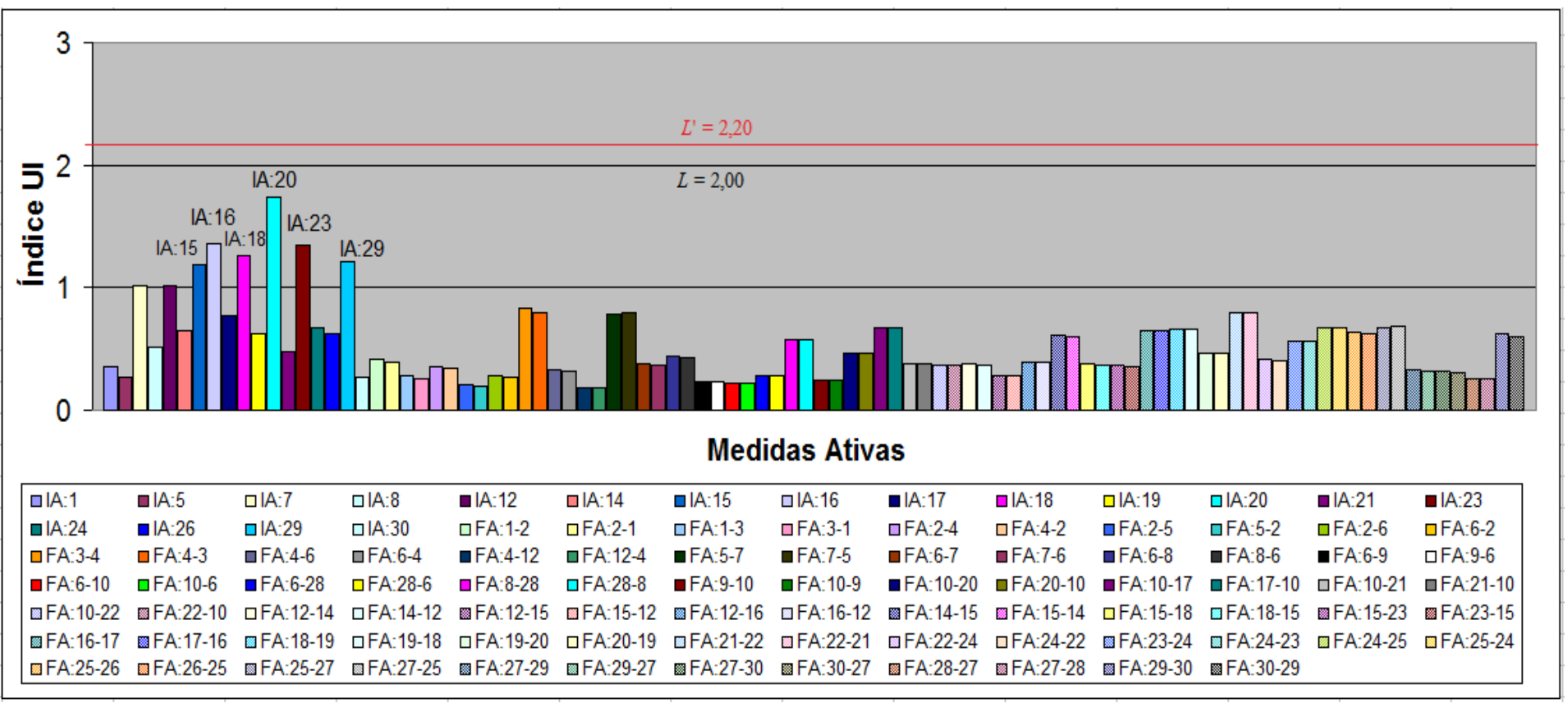

Figura 7.10: Índice $U I$ das medidas ativas do sistema de medição obtido com o Algoritmo 1 - Sistema de 30 barras IEEE 


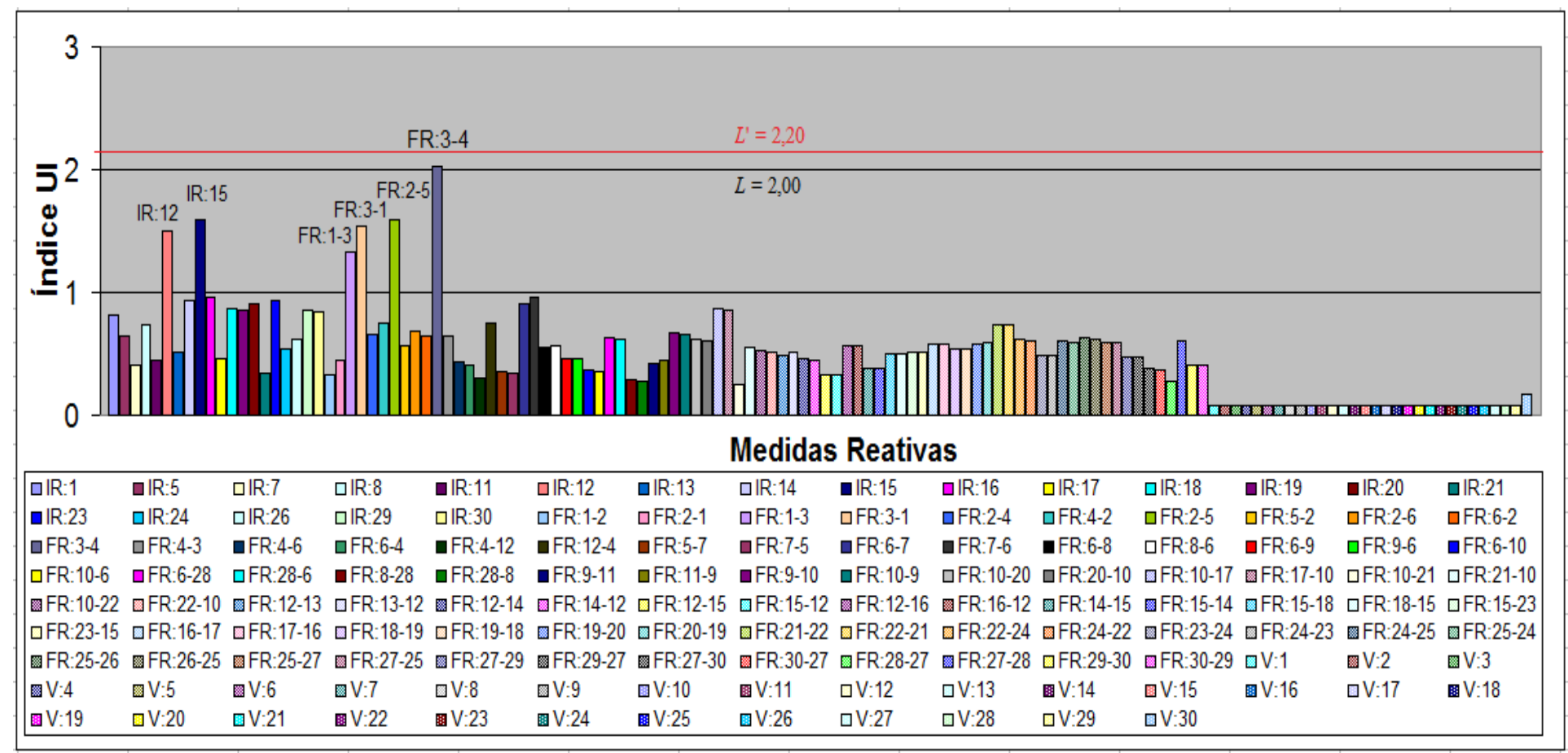

Figura 7.11: Índice $U I$ das medidas reativas do sistema de medição obtido com o Algoritmo 1 - Sistema de 30 barras IEEE

Através dos gráficos das figuras 7.10 e 7.11 , vemos que o sistema de medição obtido apresenta apenas 14 medidas com índice UI maior que 1, o que representa menos de $7 \%$ do total das medidas. Além disso, poucas medidas tem UI próximo ao limiar $L$ ' $(2,20)$.

Importa observar que, durante a aplicação do algoritmo ao sistema de 30 barras, tentamos utilizar o limiar $L$ como 1 e, em seguida, 1,5. Entretanto, para tais valores, não nos foi possível garantir que as medidas atendessem a nossos critérios de projeto, isto é, medidas com nível de redundância maior que 1 e $U I$ menor que $1,1 L$.

A próxima seção apresentará uma outra proposta de algoritmo, baseada no índice $U I$ das medidas.

\subsection{Algoritmo para Projeto ou Fortalecimento de Sistemas de Medição: Algoritmo 2}

Como se nos permite observar, através dos resultados da aplicação do Algoritmo 1, muitos medidores devem ser adicionadas à rede elétrica em análise, com o intuito de garantir: medidas com índice $U I$ abaixo do limiar estabelecido; ausência de conjuntos críticos e medidas críticas. Assim, o custo para implantação do sistema de medição obtido com o Algoritmo 1 pode ser elevado. 
Para obtenção de sistemas de medição confiáveis (observáveis e isentos de medidas críticas e conjuntos críticos de medidas) de baixo custo, já foram desenvolvidas diversas metodologias que minimizam o número de medidores a serem instalados (SOUZA et al., 2005; COSER et al., 2006; VIGLIASSI et al., 2009).

Propomos então outro algoritmo, que possibilita a obtenção de confiáveis sistemas de medição minimizando o número de medidores a serem instalados. A diferença desse algoritmo, para as metodologias supracitadas, é que o mesmo não considera como candidatas as medidas que possuírem UI elevados. Dessa forma, o algoritmo proposto possibilita a obtenção de sistemas de medição confiáveis, de baixo custo, formados por medidas com índices $U I$ menores que um valor pré-determinado.

O algoritmo proposto contempla três etapas:

Etapa 1: consiste na pré-seleção das medidas candidatas as serem instaladas no sistema elétrico em questão. Isto é, das medidas que, se instaladas, não apresentarão índice $U I$ maior que um valor pré-determinado. Para realizar essa tarefa, basta aplicar o Algoritmo 1 proposto anteriormente. As medidas candidatas a serem instaladas são aquelas pertencentes ao sistema de medição resultante da aplicação do Algoritmo 1.

Etapa 2: A partir das medidas selecionadas como candidatas na etapa 1, obtenha um sistema de medição confiável. Para isso, utilizaremos a metodologia desenvolvida em Vigliassi et al. (2009), apresentada de forma sucinta no Anexo D. Ademais, podemos obter um sistema de medição confiável através de um processo iterativo, compreendendo a adição de medidas e a verificação da redundância das mesmas, até a ausência de medidas críticas e de conjuntos críticos de medidas.

Etapa 3: Inicialmente verifica-se o $U I$ de todas as medidas do sistema de medição resultante da Etapa 2. Em seguida, serão adicionadas medidas redundantes àquelas com $U I$ acima do limiar estabelecido $L 2$, sendo $L 2 \geq L$.

A seguir, apresentaremos o segundo algoritmo proposto para projeto de sistemas de medição, que também pode ser utilizado para fortalecimento de novos sistemas. 
Etapa 1: Aplique o Algoritmo 1 à rede elétrica a ser analisada. O sistema de medição resultante indicará as medidas candidatas a serem instaladas.

Etapa 2: Obtenha um sistema de medição confiável, considerando apenas as medidas classificadas como candidatas na Etapa 1.

Etapa 3: Esta etapa compreende alguns passos:

Passo 0: Escolha um limiar $L 2$, igual ou maior ao valor de $L(L 2 \geq L$ ) utilizado na Etapa 1, e considere o sistema de medição obtido com a Etapa 2.

Passo 1: Calcule o índice $U I$ do sistema de medição em análise e identifique as medidas com índice $U I$ maior que $1,1 \mathrm{~L} 2$. Se todas as medidas apresentarem $U I<1,1$ L2, pare; caso contrário, siga ao passo 2.

Passo 2: Dentre as medidas identificadas no passo anterior $(U I>1,1 \quad L 2)$, escolha a medida com menor $U I$, e verifique onde novos medidores podem ser instalados próximos à mesma, considerando as medidas selecionadas na Etapa 1, de modo a aumentar a sua redundância local.

Observação: A partir de simulações computacionais verificamos que, escolhendo a medida com menor $U I$ (dentre aquelas com $U I>1,1 L 2$ ), seleciona-se um número menor de medidores para obtenção de um sistema de medição formado apenas por medidas com UI menor que um valor pré-estabelecido.

Passo 3: Adicione uma ou mais das medidas candidatas selecionadas no passo 2 ao sistema de medição em questão e volte ao passo 1 .

Com o intuito de elucidar melhor o Algoritmo 2 para projeto de sistemas de medição, a figura 7.12 mostra o fluxograma deste algoritmo. 


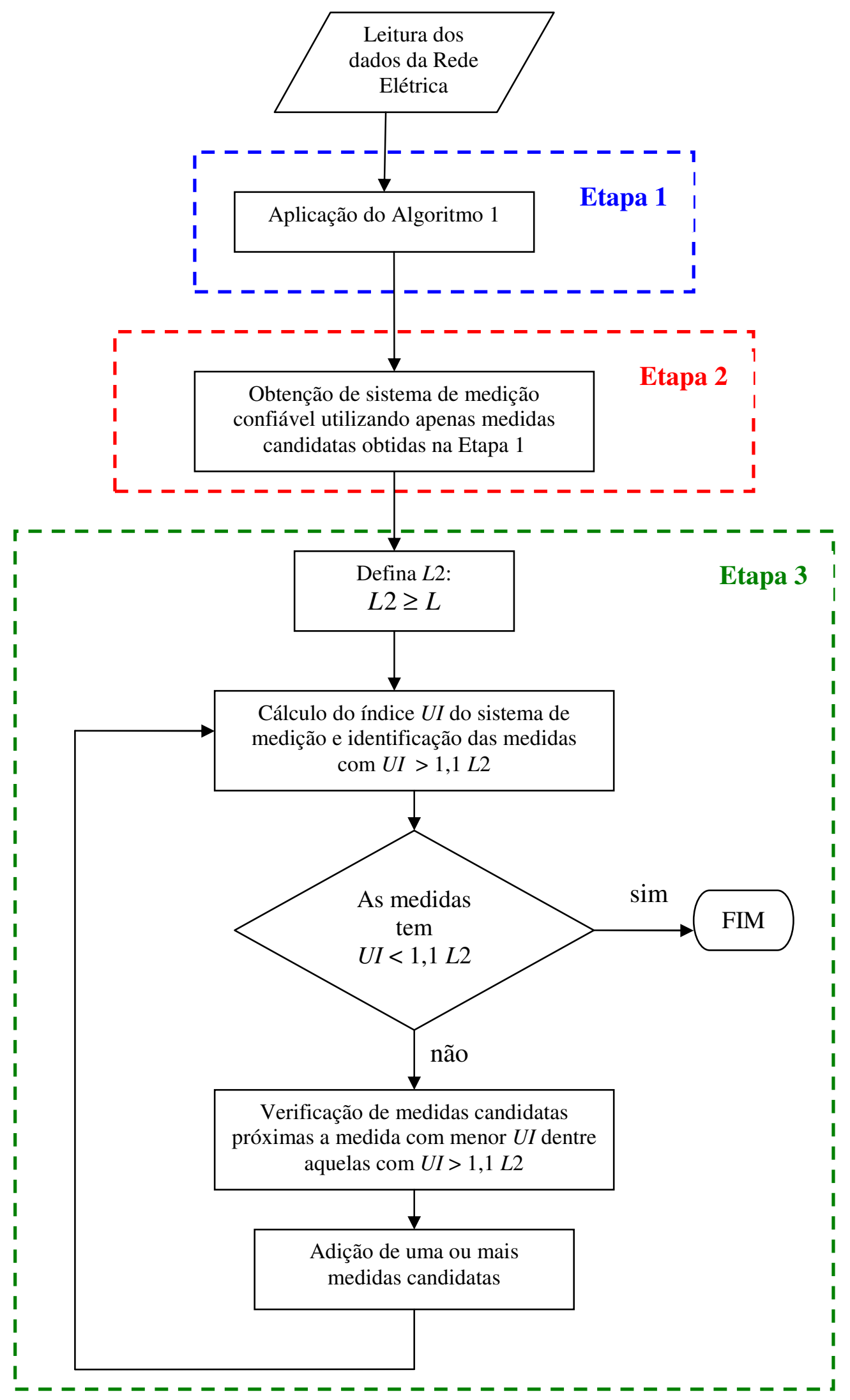

Figura 7.12: Fluxograma do algoritmo 2 para o projeto de sistemas de medição 


\subsubsection{Aplicação do Algoritmo 2 sobre o Sistema de 14 Barras do IEEE}

\section{$\underline{\text { Resultado }}$}

Etapa 1: Com a aplicação do Algoritmo 1, obtivemos os resultados já apresentados na subseção 7.2.1. Assim, as medidas candidatas são aquelas mostradas na tabela 7.8. Nessa situação o valor do limiar $L$ utilizado é 1,50.

Etapa 2: Considerando apenas as medidas candidatas selecionadas na Etapa 1, e utilizando o algoritmo apresentado em Vigliassi et al. (2009), construímos um sistema de medição contendo medidas com nível de redundância maior que 1. Veja a tabela 7.17 a seguir:

Tabela 7.17: Sistema de medição confiável para sistema de 14 barras do IEEE

\begin{tabular}{c|c}
\hline $\begin{array}{c}\text { Medidas de } \\
\text { Potência Ativa }\end{array}$ & $\begin{array}{c}\text { Medidas de } \\
\text { Potência Reativa }\end{array}$ \\
\hline IA:1 & IR:1 \\
\hline IA:3 & IR:3 \\
\hline IA:7 & IR:7 \\
\hline IA:8 8 IR:8 \\
\hline IA:9 & IR:9 \\
\hline IA:10 & IR:10 \\
\hline IA:13 & IR:13 \\
\hline FA:1-5 & FR:1-5 \\
\hline FA:5-2 & FR:5-2 \\
\hline FA:4-3 & FR:4-3 \\
\hline FA:5-4 & FR:5-4 \\
\hline FA:4-7 & FR:4-7 \\
\hline FA:5-6 & FR:5-6 \\
\hline FA:6-13 & FR:6-13 \\
\hline FA:13-6 & FR:13-6 \\
\hline FA:7-8 & FR:7-8 \\
\hline FA:8-7 & FR:8-7 \\
\hline FA:7-9 & FR:7-9 \\
\hline FA:9-7 & FR:9-7 \\
\hline FA:10-9 & FR:10-9 \\
\hline FA:10-11 & FR:10-11 \\
\hline FA:11-10 & FR:11-10 \\
\hline FA:12-13 & FR:12-13 \\
\hline FA:13-12 & FR:13-12 \\
\hline FA:13-14 & FR:13-14 \\
\hline Medidas de Tensão \\
\hline & \\
\hline & \\
\hline & \\
\hline & \\
\hline & \\
\hline &
\end{tabular}


Etapa 3: Primeiramente escolhemos o limiar $L 2$, de modo a satisfazer à condição $L 2 \geq L$. Para tanto, escolhemos $L 2=2,0$.

Observação: Através de simulações prévias, considerando L2 como 1,50, obtivemos um sistema de medição muito parecido ao obtido com o Algoritmo 1, razão pela qual não apresentaremos o sistema de medição resultante. Neste caso, escolhemos $L 2$ como 2,0, com o intuito de obtermos um sistema de medição bem diferente daquele obtido através do algoritmo 1 , cujo limiar $L$ era 1,50.

Passo 1 (I): Calculamos UI para as medidas do sistema de medição da etapa 2, chamado de caso base. Índices $U I$ acima de 2,20 ( $\left.L 2^{\prime}=1,1 L 2\right)$ :

Tabela 7.18: Medidas com UI maior que 2,20 (caso base)

\begin{tabular}{c|c}
\hline Medida & Indice UI \\
\hline FA:5-4 & 4,5376 \\
\hline FA:13-14 & 2,9634 \\
\hline IR:1 & 2,8684 \\
\hline IR:9 & 2,8074 \\
\hline FR:1-5 & 2,4899 \\
\hline FR:5-2 & 4,7894 \\
\hline FR:5-4 & 4,6981 \\
\hline FR:5-6 & 4,5926 \\
\hline FR:13-14 & 4,3902 \\
\hline
\end{tabular}

Passo 2 (I): Medida com menor UI: FR:1-5

Passo 3 (I): (Adição 1) Para mostrar que as medidas de magnitude de tensão não alteram de forma significativa o UI das medidas de potência, adicionaremos, neste exemplo, as medidas: V:2, V:4, V:5 e V:6.

Passo 1 (II): Após a adição 1, temos:

Tabela 7.19: Medidas com UI maior que 2,20 (adição 1)

\begin{tabular}{c|c}
\hline Medida & Indice UI \\
\hline FA:5-4 & 4,3039 \\
\hline FA:13-14 & 2,943 \\
\hline IR:1 & 2,5669 \\
\hline FR:1-5 & 2,2429 \\
\hline FR:5-2 & 4,7295 \\
\hline FR:5-4 & 4,3021 \\
\hline FR:5-6 & 3,5034 \\
\hline FR:13-14 & 4,2983 \\
\hline
\end{tabular}

Passo 2 (II): Medida com menor UI: FR:1-5

Passo 3 (II): (Adição 2) Adição de F:1-2 
Observação: F:a-b indica o par de medida de fluxo de potência ativa (FA:a-b) e de potência reativa (FR:a-b) da barra "a" para "b"; e I:a indica o par de medida de injeção de potência ativa (IA:a) e de potência reativa (IR:a) na barra "a".

Passo 1 (III): Após a adição 2, temos:

Tabela 7.20: Medidas com UI maior que 2,20 (adição 2)

\begin{tabular}{c|c}
\hline Medida & Indice UI \\
\hline FA:5-4 & 4,4241 \\
\hline FA:13-14 & 2,935 \\
\hline FR:1-5 & 2,2314 \\
\hline FR:5-2 & 4,618 \\
\hline FR:5-4 & 4,2141 \\
\hline FR:5-6 & 3,3242 \\
\hline FR:13-14 & 4,2809 \\
\hline
\end{tabular}

Passo 2 (III): Medida com menor UI: FR:1-5

Passo 3 (III): (Adição 3) Adição de F:2-1

Passo 1 (IV): Após a adição 3, temos:

Tabela 7.21: Medidas com UI maior que 2,20 (adição 3)

\begin{tabular}{c|c}
\hline Medida & Indice UI \\
\hline FA:5-4 & 4,3069 \\
\hline FA:13-14 & 2,9327 \\
\hline FR:5-2 & 4,5692 \\
\hline FR:5-4 & 4,1791 \\
\hline FR:5-6 & 3,2189 \\
\hline FR:13-14 & 4,267 \\
\hline
\end{tabular}

Passo 2 (IV): Medida com menor UI: FA:13-14

Passo 3 (IV): (Adição 4) Adição de F:14-13

Passo 1 (V): Após a adição 4, temos:

Tabela 7.22: Medidas com UI maior que 2,20 (adição 4)

\begin{tabular}{l|r}
\hline Medida & Indice UI \\
\hline FA:5-4 & 4,4125 \\
\hline FR:5-2 & 4,602 \\
\hline FR:5-4 & 4,0966 \\
\hline FR:5-6 & 3,088 \\
\hline
\end{tabular}

Passo 2 (V): Medida com menor UI: FR:5-6

Passo 3 (V): (Adição 5) Adição de F:6-5

Passo 1 (VI): Após a adição 5, temos:

Tabela 7.23: Medidas com UI maior que 2,20 (adição 5)

\begin{tabular}{l|r}
\hline Medida & \multicolumn{1}{|c}{ Indice UI } \\
\hline FA:5-4 & 4,2221 \\
\hline
\end{tabular}




\begin{tabular}{l|l}
\hline FR:5-2 & 4,5871 \\
\hline FR:5-4 & 4,0761 \\
\hline
\end{tabular}

Passo 2 (VI): Medida com menor UI: FR:5-4

Passo 3 (VI): (Adição 6) Adição de F:4-5

Passo 1 (VII): Após a adição 6, temos:

Tabela 7.24: Medidas com UI maior que 2,20 (adição 6)

\begin{tabular}{c|c}
\hline Medida & Indice UI \\
\hline FR:5-2 & 4,5345 \\
\hline
\end{tabular}

Passo 2 (VII): Medida com menor UI: FR:5-2

Passo 3 (VII): (Adição 7) Adição de F:2-5

Passo1 (VIII): Após a adição 7, temos:

Tabela 7.25: Medidas com UI maior que 2,20 (adição 7)

\begin{tabular}{c|c}
\hline Medida & Indice UI \\
\hline FR:2-5 & 2,9119 \\
\hline
\end{tabular}

Passo 2 (VIII): Medida com menor UI: FR:2-5

Passo 3 (VIII): (Adição 8) Adição de F:2-4

Passo 1 (IX): Após a adição 8, nenhuma medida apresentou $U I>2,20$. Fim do processo.

A tabela 7.26, a seguir, mostra a variação do índice $U I$ das medidas (apenas daquelas cujo valor inicial estava acima de 2,20), com a adição de novas medidas redundantes.

Tabela 7.26: Variação do índice $U I$ de medidas (cujo valor inicial estava acima de 2,20) com a adição de novas medidas

\begin{tabular}{c|c|c|c|c|c|c|c|c|c}
\hline & \multicolumn{7}{|c}{ Índice UI para cada situação } \\
\hline Medida & Inicial & Adição 1 & Adição 2 & Adição 3 & Adição 4 & Adição 5 & Adição 6 & Adição 7 & Adição 8 \\
\hline IR:9 & $2,8074 *$ & 1,9993 & 1,8964 & 1,822 & 1,7534 & 1,5938 & 1,8593 & 1,4913 & 1,4409 \\
\hline IR:1 & 2,8684 & 2,5669 & 1,1657 & 0,9829 & 0,9835 & 0,9831 & 0,983 & 0,8767 & 0,8727 \\
\hline FR:1-5 & 2,4899 & 2,2429 & 2,2314 & 2,1323 & 2,1585 & 2,1099 & 2,1266 & 1,1709 & 1,1586 \\
\hline FA:13-14 & 2,9634 & 2,943 & 2,935 & 2,9327 & 0,9576 & 0,957 & 0,9572 & 0,9567 & 0,9565 \\
\hline FR:13-14 & 4,3902 & 4,2983 & 4,2809 & 4,267 & 0,9302 & 0,9296 & 0,9299 & 0,9295 & 0,9294 \\
\hline FR:5-6 & 4,5926 & 3,5034 & 3,3242 & 3,2189 & 3,088 & 0,7959 & 0,7956 & 0,7956 & 0,7954 \\
\hline FA:5-4 & 4,5376 & 4,3039 & 4,4241 & 4,3069 & 4,4125 & 4,2221 & 0,9718 & 0,9708 & 0,8949 \\
\hline FR:5-4 & 4,6981 & 4,3021 & 4,2141 & 4,1791 & 4,0966 & 4,0761 & 1,0845 & 1,0813 & 0,5102 \\
\hline FR:5-2 & 4,7894 & 4,7295 & 4,618 & 4,5692 & 4,602 & 4,5871 & 4,5345 & 1,8766 & 1,7931 \\
\hline FR:2-5** & - & - & - & - & - & - & - & 2,9119 & 1,8022 \\
\hline
\end{tabular}

* Os valores em vermelho e em verde indicam, respectivamente, índice $U I$ maior que 2,20 e menor que 2,20.

** Medida que foi adicionada durante a etapa 3 e que apresentou índice $U I$ maior que 2,20. 
As figuras 7.13 e 7.14 ilustram as medidas e os UIs do sistema de medição obtido com a aplicação do Algoritmo 2.

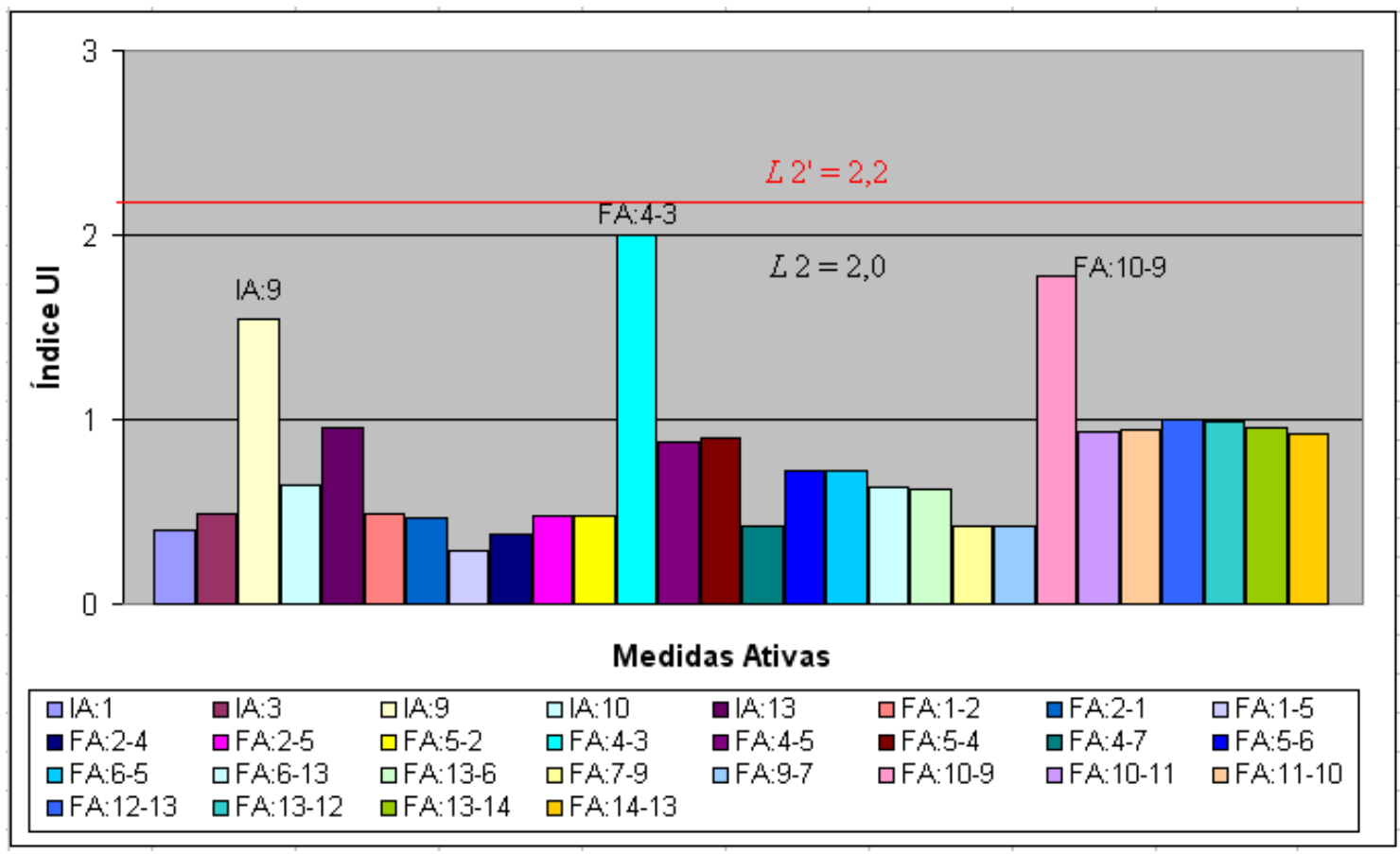

Figura 7.13: Índice $U I$ das medidas ativas do sistema de medição obtido com o

Algoritmo 2 - Sistema de 14 barras IEEE

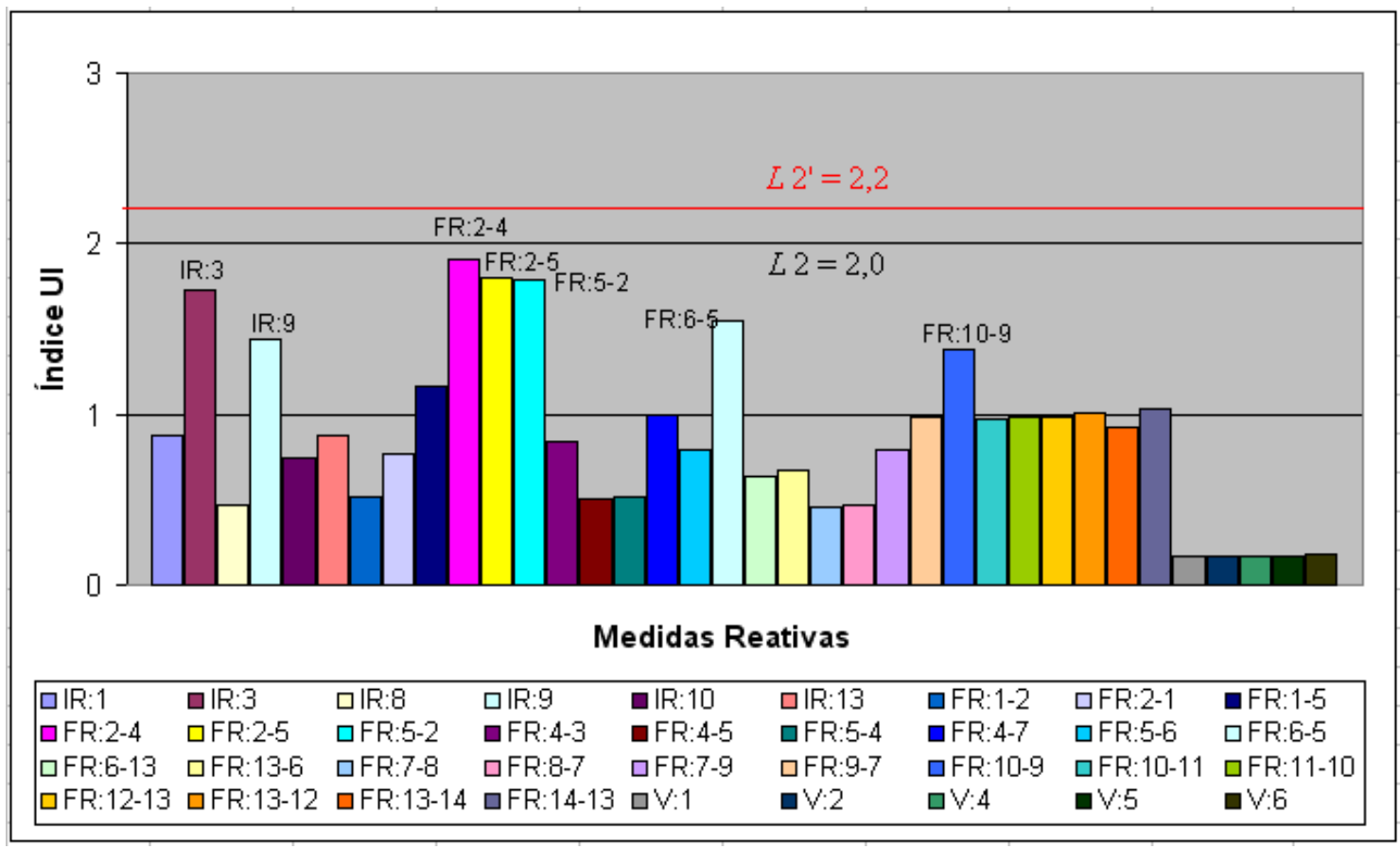

Figura 7.14: Índice $U I$ das medidas reativas do sistema de medição obtido com o Algoritmo 2 - Sistema de 14 barras IEEE 
Fazendo uma comparação entre os resultados obtidos com o Algoritmo 1 e os resultados obtidos com o Algoritmo 2, verifica-se que o número de medidores selecionados para instalação pelo Algoritmo 2 foi inferior ao obtido pelo Algoritmo 1.

Importa novamente ressaltar, que na simulação acima, escolhemos $L 2$ como 2,0 com o intuito de obtermos um sistema de medição bem diferente daquele obtido através do Algoritmo 1, cujo limiar $L$ era 1,50. Dadas estas considerações, sugerimos a utilização inicial do limiar $L 2$ igual a $L$; caso o sistema de medição resultante não satisfaça aos interesses do projetista ou da empresa, aumente esse limiar L2. Note que, quanto maior for $L 2$, pior será a capacidade de detecção e identificação de erros grosseiros.

\subsubsection{Aplicação do Algoritmo 2 ao Sistema de 30 Barras do IEEE}

\section{Resultado}

Etapa 1: Através da aplicação do Algoritmo 1, as medidas selecionadas como candidatas são aquelas mostradas na tabela B.3 do anexo B. Nessa situação, o valor do limiar $L$ utilizado foi 2,0.

Etapa 2: Considerando apenas as medidas candidatas selecionadas na Etapa 1, e utilizando o algoritmo apresentado em Vigliassi et al. (2009), construímos um sistema de medição confiável. As medidas obtidas nesta etapa estão na tabela 7.27:

Tabela 7.27: Medidas do sistema de medição obtido após a Etapa 2 e respectivos valores de $U I$ (sistema de 30 barras)

\begin{tabular}{c|c|c|c|c|c}
\hline $\begin{array}{c}\text { Medidas de Potência } \\
\text { Ativa }\end{array}$ & \multicolumn{2}{c|}{$\begin{array}{c}\text { Medidas de Potência } \\
\text { Reativa }\end{array}$} & \multicolumn{2}{c}{$\begin{array}{c}\text { Medidas de } \\
\text { Tensão }\end{array}$} \\
\hline Medida & Índice UI & Medida & Índice UI & Medida & Índice II \\
\hline IA:1 & 0,7327 & IR:1 & 1,7648 & V:1 & 0,1389 \\
\hline IA:5 & 0,383 & IR:5 & 1,1026 & V:5 & 0,1393 \\
\hline IA:6* & --- & IR:6 & --- & V:7 & 0,1405 \\
\hline IA:7 & 1,5106 & IR:7 & 0,5522 & V:13 & 0,14 \\
\hline IA:8 & 0,7294 & IR:8 & 1,0617 & V:21 & 0,1442 \\
\hline IA:9 & --- & IR:9 & -- & V:26 & 0,149 \\
\hline IA:11 & --- & IR:11 & 0,529 & & \\
\hline IA:13 & --- & IR:13 & 0,7162 & & \\
\hline IA:14 & 0,9539 & IR:14 & 1,4018 & & \\
\hline IA:17 & 1,1095 & IR:17 & 0,6938 & & \\
\hline IA:19 & 1,113 & IR:19 & 1,4057 & & \\
\hline
\end{tabular}




\begin{tabular}{|c|c|c|c|c|c|}
\hline IA:21 & 0,6677 & IR:21 & 0,5243 & & \\
\hline IA:22 & --- & IR:22 & --- & & \\
\hline IA:24 & 0,9359 & IR:24 & 0,8081 & & \\
\hline IA:25 & --- & IR:25 & --- & & \\
\hline IA:26 & 0,8434 & IR:26 & 0,8386 & & \\
\hline IA:27 & --- & IR:27 & --- & & \\
\hline IA:28 & --- & IR:28 & --- & & \\
\hline FA:1-2 & 1,3917 & FR:1-2 & 0,5886 & & \\
\hline FA:1-3 & 0,4026 & FR:1-3 & 3,9131 & & \\
\hline FA:3-1 & 0,3848 & FR:3-1 & 4,2845 & & \\
\hline FA:2-4 & 0,7534 & FR:2-4 & 1,356 & & \\
\hline FA:2-5 & 0,3131 & FR:2-5 & 2,0933 & & \\
\hline FA:2-6 & 0,5428 & FR:2-6 & 0,9135 & & \\
\hline FA:4-6 & 0,57 & FR:4-6 & 0,664 & & \\
\hline FA:4-12 & 0,3522 & FR:4-12 & 0,7697 & & \\
\hline FA:5-7 & 1,5294 & FR:5-7 & 0,4346 & & \\
\hline FA:6-7 & 0,4987 & FR:6-7 & 2,4143 & & \\
\hline FA:6-8 & 0,602 & FR:6-8 & 0,7501 & & \\
\hline FA:6-9 & 0,3791 & FR:6-9 & 0,6651 & & \\
\hline FA:6-10 & 0,3723 & FR:6-10 & 0,6008 & & \\
\hline FA:6-28 & 0,4076 & FR:6-28 & --- & & \\
\hline FA:8-28 & 0,8519 & FR:8-28 & 0,3976 & & \\
\hline FA:9-11 & --- & FR:9-11 & 0,4914 & & \\
\hline FA:11-9 & --- & FR:11-9 & 0,529 & & \\
\hline FA:9-10 & 0,3913 & FR:9-10 & 1,0186 & & \\
\hline FA:10-20 & 1,5439 & FR:10-20 & 1,8004 & & \\
\hline FA:10-17 & 1,0477 & FR:10-17 & 1,8958 & & \\
\hline FA:10-21 & 0,5156 & FR:10-21 & 0,3763 & & \\
\hline FA:10-22 & 0,4871 & FR:10-22 & 0,8704 & & \\
\hline FA:12-13 & --- & FR:12-13 & 0,689 & & \\
\hline FA:13-12 & --- & FR:13-12 & 0,7162 & & \\
\hline FA:12-14 & 0,634 & FR:12-14 & 0,6773 & & \\
\hline FA:12-15 & 0,7585 & FR:12-15 & 1,004 & & \\
\hline FA:12-16 & 1,5237 & FR:12-16 & 1,9278 & & \\
\hline FA:14-15 & 1,1885 & FR:14-15 & 0,6622 & & \\
\hline FA:15-18 & 1,3967 & FR:15-18 & 1,6445 & & \\
\hline FA:15-23 & 1,5003 & FR:15-23 & 2,0701 & & \\
\hline FA:16-17 & 2,0813 & FR:16-17 & 1,4856 & & \\
\hline FA:18-19 & 2,3932 & FR:18-19 & 1,2212 & & \\
\hline FA:19-20 & 1,0736 & FR:19-20 & 1,2801 & & \\
\hline FA:21-22 & 1,4848 & FR:21-22 & 1,2635 & & \\
\hline FA:22-24 & 0,5805 & FR:22-24 & 0,9507 & & \\
\hline FA:23-24 & 1,5323 & FR:23-24 & 1,0793 & & \\
\hline FA:24-25 & 1,0821 & FR:24-25 & 0,9445 & & \\
\hline FA:25-26 & 0,8697 & FR:25-26 & 0,864 & & \\
\hline FA:25-27 & 1,1874 & FR:25-27 & 0,9241 & & \\
\hline FA:27-29 & 0,6942 & FR:27-29 & 1,0756 & & \\
\hline FA:27-30 & 0,4834 & FR:27-30 & 0,7729 & & \\
\hline FA:28-27 & 0,4209 & FR:28-27 & 0,4426 & & \\
\hline FA:29-30 & 3,1439 & FR:29-30 & 1,5716 & & \\
\hline
\end{tabular}


Etapa 3: Primeiramente escolhemos o limiar $L 2$, de modo a satisfazer à condição $L 2 \geq L$. Desse modo, escolhemos $L 2=2,0$.

Passo 1 (I): Calculamos UI para as medidas do sistema de medição da etapa 2, sendo que os valores podem ser visualizados através da tabela 7.27.

Com base nesses resultados, os índices $U I$ acima de 2,20 são tabelados a seguir:

Tabela 7.28: Medidas com UI maior que 2,20 (caso base)

\begin{tabular}{l|r}
\hline Medida & Indice UI \\
\hline FA:18-19 & 2,3932 \\
\hline FA:29-30 & 3,1439 \\
\hline FR:1-3 & 3,9131 \\
\hline FR:3-1 & 4,2845 \\
\hline FR:6-7 & 2,4143 \\
\hline
\end{tabular}

Passo 2 (I): Medida com menor UI: FA:18-19

Passo 3 (I): (Adição 1) Adição de F:19-18

Passo 1 (II): Após a adição 1, temos:

Tabela 7.29: Medidas com UI maior que 2,20 (adição 1)

\begin{tabular}{l|r}
\hline Medida & Indice UI \\
\hline FA:29-30 & 3,1439 \\
\hline FR:1-3 & 3,9193 \\
\hline FR:3-1 & 4,2924 \\
\hline FR:6-7 & 2,4154 \\
\hline
\end{tabular}

Passo 2 (II): Medida com menor UI: FR:6-7

Passo 3 (II): (Adição 2) Adição de F:7-6

Passo 1 (III): Após a adição 2, temos:

Tabela 7.30: Medidas com UI maior que 2,20 (adição 2)

\begin{tabular}{l|r}
\hline Medida & \multicolumn{1}{|l}{ Indice UI } \\
\hline FA:29-30 & 3,1439 \\
\hline FR:1-3 & 3,8788 \\
\hline FR:3-1 & 4,2352 \\
\hline
\end{tabular}

Passo 2 (III): Medida com menor UI: FA:29-30

Passo 3 (III): (Adição 3) Adição de F:30-29

Passo 1 (IV): Após a adição 3, temos:

Tabela 7.31: Medidas com UI maior que 2,20 (adição 3)

\begin{tabular}{l|r}
\hline Medida & \multicolumn{1}{|c}{ Indice UI } \\
\hline FR:1-3 & 3,8909 \\
\hline FR:3-1 & 4,2522 \\
\hline
\end{tabular}

Passo 2 (IV): Medida com menor UI: FR:1-3 
Passo 3 (IV): (Adição 4) Adição de F:2-1

Passo 1 (V): Após a adição 4, obtemos:

Tabela 7.32: Medidas com UI maior que 2,20 (adição 4)

\begin{tabular}{l|r}
\hline Medida & Indice UI \\
\hline FR:1-3 & 3,6175 \\
\hline FR:3-1 & 4,1939 \\
\hline
\end{tabular}

Passo 2 (V): Medida com menor UI: FR:1-3

Passo 3 (V): (Adição 5) Adição de F:2-1

Passo 1 (VI): Após a adição 5, obtemos:

Tabela 7.33: Medidas com UI maior que 2,20 (adição 5)

\begin{tabular}{c|c}
\hline Medida & Indice UI \\
\hline FA:3-4 & 2,4156 \\
\hline FR:3-4 & 7,2919 \\
\hline
\end{tabular}

Passo 2 (VI): Medida com menor UI: FA:3-4

Passo 3 (VI): (Adição 6) Adição de F:4-3

Passo 1 (VII): Após a adição 6, nenhuma medida apresentou $U I>2,20$. Fim do processo.

As medidas restantes compõem o sistema de medição obtido com a aplicação do Algoritmo 2, sendo que todas as medidas satisfazem aos seguintes aspectos de projeto: i) apresentam Nível de Redundância maior que $1(\mathrm{NR}>1)$; ii) apresentam índice $U I$ menor que o limiar estabelecido $L 2$ ' $(U I<2,2)$.

A seguir, apresentamos a variação do índice $U I$ das medidas (apenas daquelas cujo valor inicial estava acima de 2,20), com a adição de novas medidas redundantes.

Tabela 7.34: Variação do índice UI de medidas (cujo valor inicial estava acima de 2,20) com a adição de novas medidas

\begin{tabular}{c|c|c|c|c|c|c|c}
\hline \multirow{2}{*}{ Medida } & \multicolumn{7}{c}{ Índice UI para cada situação } \\
\cline { 2 - 8 } & Inicial & Adição 1 & Adição 2 & Adição 3 & Adição 4 & Adição 5 & Adição 6 \\
\hline FA:18-19 & $2,3932 *$ & 0,9253 & 0,9253 & 0,9253 & 0,9253 & 0,9253 & 0,9253 \\
\hline FR:6-7 & 2,4143 & 2,4154 & 0,9547 & 0,9551 & 0,9534 & 0,9441 & 0,9434 \\
\hline FA:29-30 & 3,1439 & 3,1439 & 3,1439 & 0,9682 & 0,9682 & 0,9681 & 0,9682 \\
\hline FR:1-3 & 3,9131 & 3,9193 & 3,8788 & 3,8909 & 3,6175 & 1,4159 & 1,3752 \\
\hline FR:3-1 & 4,2845 & 4,2924 & 4,2352 & 4,2522 & 4,1939 & 2,1944 & 2,1755 \\
\hline FA:3-4** & - & - & - & - & - & 2,4156 & 0,8403 \\
\hline FR:3-4** & - & - & - & - & - & 7,2919 & 2,0320 \\
\hline * Os valores em vermelho e em verde indicam, respectivamente, índice UI maior que 2,20 e menor que 2,20. \\
** Medida que foi adicionada durante a etapa 3 e que apresentou índice UI maior que 2,20. \\
\hline
\end{tabular}


As figuras 7.15 e 7.16 mostram, respectivamente, o índice $U I$ das medidas ativas e reativas do sistema de medição obtido.

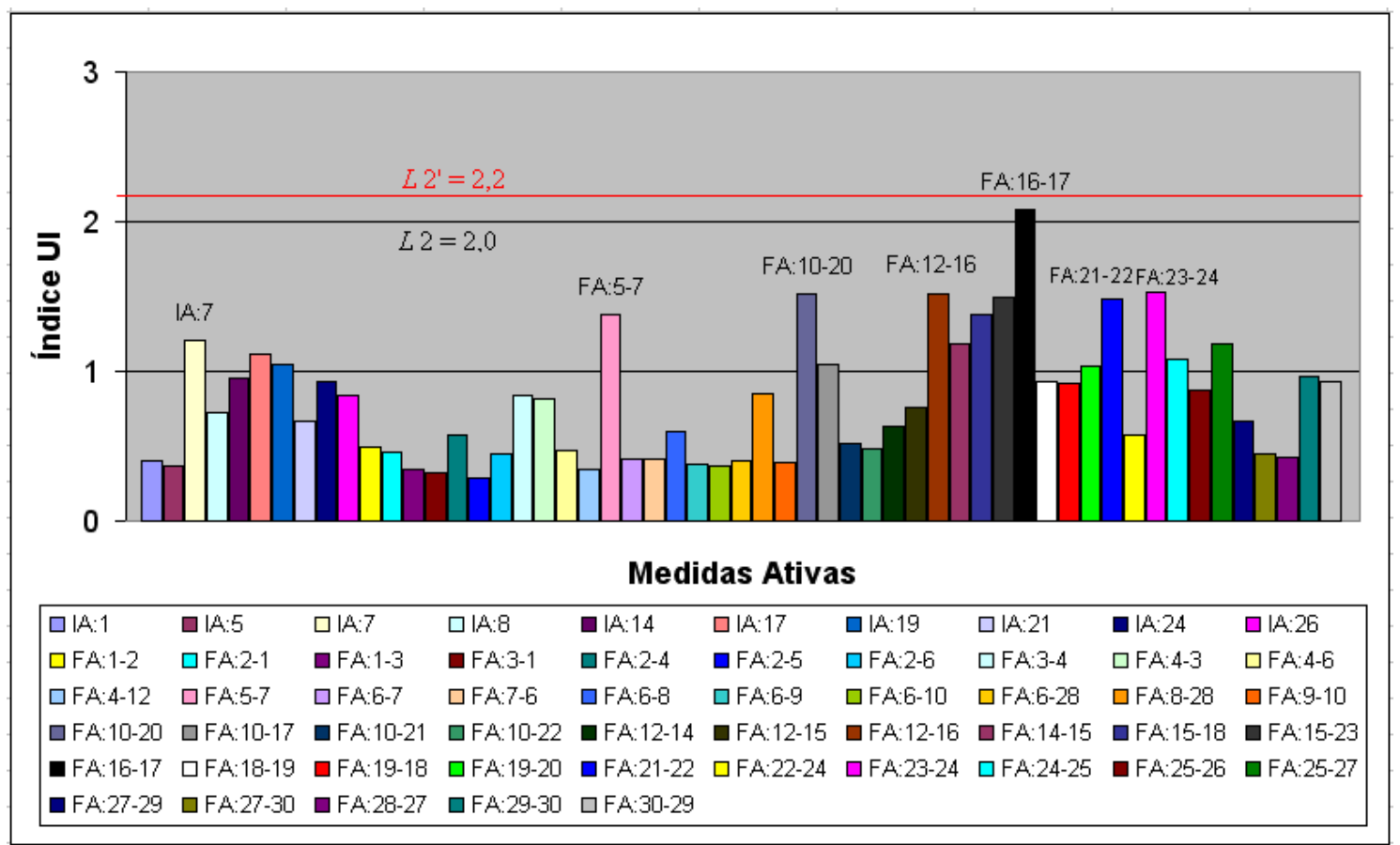

Figura 7.15: Índice $U I$ das medidas ativas do sistema de medição obtido com o

Algoritmo 2 - Sistema de 30 barras IEEE

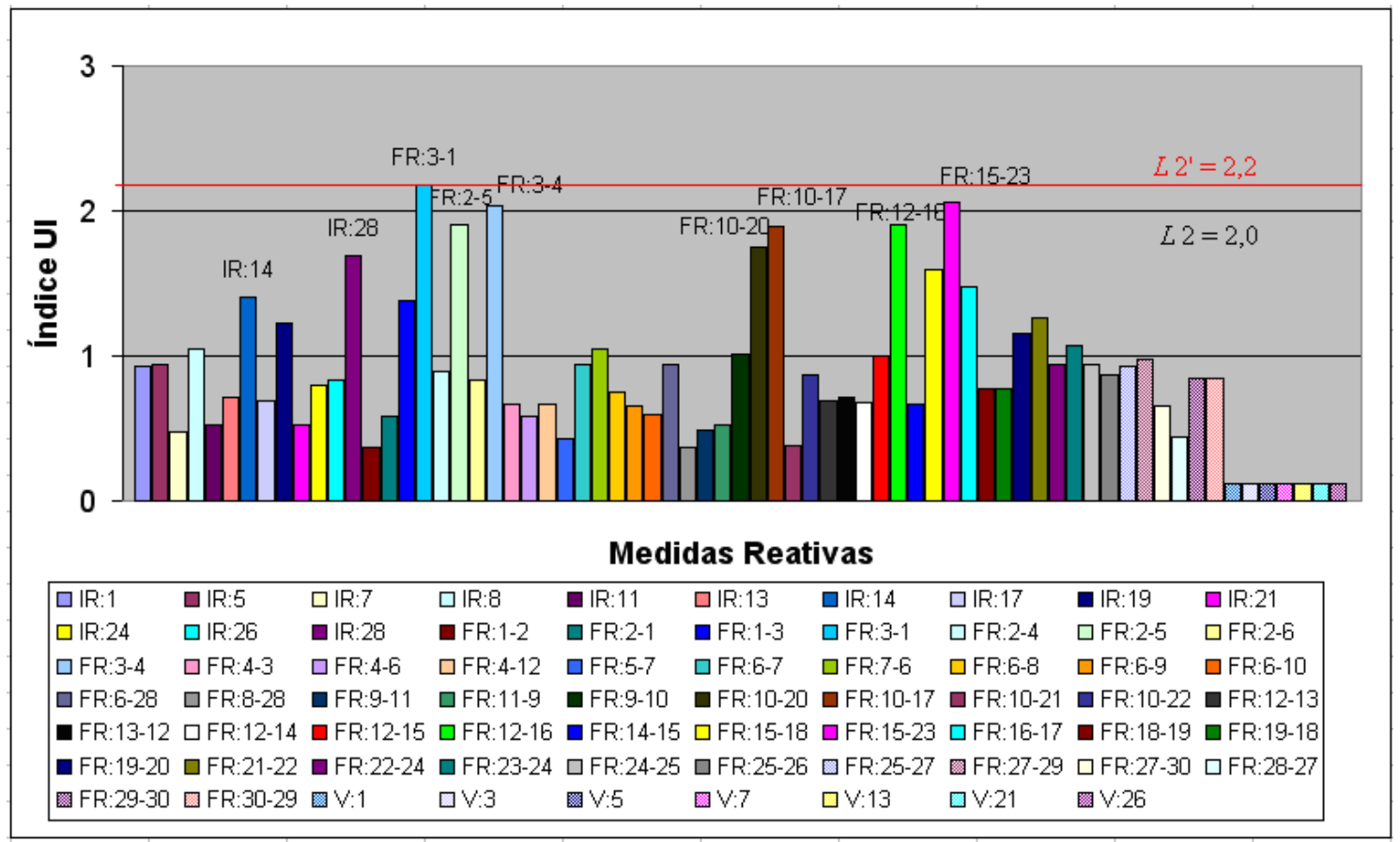

Figura 7.16: Índice UI das medidas reativas do sistema de medição obtido com o

Algoritmo 2 - Sistema de 30 barras IEEE 
Comparando o sistema de medição obtido acima com o sistema obtido através do Algoritmo 1, depara-se-nos uma redução expressiva no número de medidas a serem alocadas através do Algoritmo 2, mesmo o limiar $L 2$ sendo igual ao limiar $L(L=2,0)$. Para se ter uma idéia dessa redução, importa salientar que o sistema de medição obtido pelo Algoritmo 1 apresenta 246 medidas; já o sistema de medição obtido pelo Algoritmo 2 apresenta 141 medidas.

\subsection{Conclusões do Capítulo}

De um modo geral, mostramos neste capítulo a influência da adição e retirada de medidas ao índice $U I$, e apresentamos ainda dois algoritmos para projeto de sistemas de medição.

Na seção 7.1, alguns exemplos elucidativos foram expostos, para mostrar que o índice $U I$ de determinada medida decresce com o aumento da redundância local. Além disto, mostramos que a adição de todas as medidas redundantes a uma determinada medida, não garante que seu índice $U I$ fique menor que "1". Isto porque, o índice $U I$ não depende somente da redundância local das medidas, mas também de outros fatores como, por exemplo, a ponderação da medida e os parâmetros do sistema de transmissão.

Na seção 7.2, foi apresentado o primeiro algoritmo proposto para projeto de sistemas de medição, baseado no índice $U I$ das medidas, que também pode ser utilizado para o fortalecimento de sistemas de medição já existentes. A idéia que norteia esse algoritmo, Algoritmo 1, é considerar, inicialmente, que estão disponíveis todas as medidas do sistema elétrico em análise. Em seguida, retirar-se-ão as medidas que apresentarem $U I$ acima de certo limiar pré-estabelecido $(L)$, sob a condição da ausência de medidas críticas e de conjuntos críticos de medidas.

Através das simulações executadas com os sistemas de 14 e 30 barras do IEEE, verificamos, passo-a-passo, como o Algoritmo 1 funciona. Além disso, vimos que o ajuste no limiar $L$ é importante para garantir o fim do processamento iterativo do método, quando os critérios de projeto não são alcançados com um determinado valor $L$.

Uma questão importante a ser destacada é que, por meio do Algoritmo 1, muitas medidas podem ser selecionadas para serem instaladas na rede elétrica em análise. Deste modo, o custo para implantação do sistema de medição, obtido com o Algoritmo 1 , pode ser elevado. 
Caso haja restrição orçamentária para a implantação de novos sistemas de medição, ou fortalecimento de sistemas de medição já existentes, apresentamos, na seção 7.3, outro algoritmo (Algoritmo 2), cujo propósito é diminuir o número de medidas a serem instaladas, em relação aos sistemas de medição obtidos pelo Algoritmo 1.

Fazendo uma análise comparativa dos resultados obtidos pelos algoritmos propostos, verifica-se que os sistemas de medição confiáveis obtidos pelo Algoritmo 2 possuem realmente um número menor de medidores. Isso ficou ainda mais evidente comparando os sistemas de medição confiáveis, obtidos por ambos os algoritmos, para o sistema de 30 barras do IEEE.

Com base nos testes apresentados neste capítulo, sugerimos, para aplicação do Algoritmo 2, a utilização inicial do limiar $L 2$ sendo igual a $L$; e caso o sistema de medição resultante não satisfaça aos interesses do projetista ou da empresa, aumente esse limiar $L 2$. Note que, quanto maior for $L 2$, pior será a capacidade de detecção e identificação de EGs.

Embora não tenha sido apresentado nenhum exemplo de aplicação dos algoritmos propostos para fortalecimento de sistemas de medição já existentes, os mesmos podem ser aplicados para esse fim. Bastaria considerar os medidores já instalados, como medidas já existentes para aplicação dos algoritmos. Importa destacar que o processo de remoção (adição) de medidas candidatas para instalação, pelo Algoritmo 1 (Algoritmo 2), pode ser melhorado através da aplicação de alguma técnica de busca otimizada. Como, por exemplo, metas-heurísticas. Porém, isso ficará para um trabalho futuro. 


\section{Capítulo 8}

\section{Conclusões}

\subsection{Conclusões}

A contribuição principal deste trabalho foi a proposição de um índice para qualificar as medidas, de um sistema elétrico de potência, de acordo com as suas características de não refletirem grande parcela de seus erros nos resíduos do processo de estimação de estado, por mínimos quadrados ponderados. Consequientemente, tornase possível mensurar a dificuldade em detectar erros grosseiros nas mesmas, através do teste do resíduo normalizado ou do índice $\mathrm{J}(\mathrm{x})$ associado ao estimador de estado WLS. O índice proposto foi denominado Índice de Não-Detecção de Erros, ou simplesmente índice $U I$, desenvolvendo-se a partir de uma análise geométrica do estimador de estado WLS.

Através da decomposição do espaço vetorial das medidas demonstrou-se, no capítulo 4, que o índice UI de uma medida indica, de certa forma, a proximidade da mesma para imagem da matriz Jacobiana. Quanto maior o índice UI de uma medida, mais próxima a mesma estará da imagem da matriz Jacobiana.

Analisando o estimador de estado WLS através de projeções ortogonais, demonstrou-se, também no capítulo 4, que grande parcela dos erros em medidas que apresentam índices $U I$ elevados, isto é, medidas que estão próximas da imagem da matriz Jacobiana, não aparece nos seus resíduos. Isto é, tais erros são, de certa forma, "mascarados". 
Importa lembrar que a medida crítica é o caso limite de medidas cujos erros são "mascarados", isto é, ela pertence à imagem da matriz Jacobiana, possui índice UI infinito e seu resíduo é igual a zero, independente de a mesma ter ou não EG.

O conjunto de medidas com elevado $U I$ contém as medidas críticas e, em geral, as medidas ponto de alavancamento. Entretanto, a partir das análises apresentadas no capítulo 4, verifica-se que o índice $U I$ possibilita uma compreensão mais ampla e simples do problema de detecção e identificação de EGs, utilizando o estimador WLS, do que o conceito de medida ponto de alavancamento.

As análises comparativas dos valores dos índices $U I$, calculados adicionando ou não ruído e/ou EGs nas medidas, demonstraram que os valores dos índices $U I$ variam pouco com alterações nos valores das medidas. Isto porque os índices $U I$ dependem principalmente de outros fatores, tais como:

- os valores dos parâmetros do sistema elétrico;

- a ponderação e redundância local das medidas.

No capítulo 6, apresentamos uma aplicação do índice $U I$, que possibilita uma melhora no processo de estimação de estado, no tocante à detecção e identificação de EGs simples. Primeiramente, mostramos que é possível, através do índice $U I$, obter uma estimativa da magnitude do erro de medida, para sistemas de medição contendo EG simples, mesmo para medidas com UI elevado. Logo, ao invés de eliminarmos a medida portadora de EG, foi possível corrigir o valor da mesma para, em seguida, re-estimar as variáveis de estado, assim como proposto em Monticelli e Garcia (1983). Com isso, propusemos um algoritmo para melhorar a detecção e identificação de EGs simples, baseado na análise do resíduo normalizado e do erro composto em desvio padrão.

Através dos testes apresentados no capítulo 6, verificamos a eficácia do algoritmo proposto, aplicado em sistemas de medição sem adicionar ruído gaussiano às medidas. Por outro lado, com a adição de ruído gaussiano, constatamos que o algoritmo pode falhar, tanto na detecção, quanto na identificação de EGs, para medidas cujo índice $U I$ é alto. Destaca-se que, da mesma forma que o algoritmo proposto, o teste- $\hat{b}$, proposto em Monticelli e Garcia (1983), também falha para as situações supracitadas.

Posteriormente, no capítulo 7, mostramos a influência da adição e retirada de medidas ao índice $U I$, e ainda, foram propostos dois algoritmos para projeto ou fortalecimento de sistemas de medição. O intuito desses algoritmos é a obtenção de sistemas de medição confiáveis (observáveis e sem medidas críticas e conjuntos críticos) formados apenas por medidas com $U I$ menor que um valor pré-estabelecido. 
A idéia que norteia o primeiro algoritmo proposto, Algoritmo 1, é considerar, inicialmente, que estão disponíveis todas as medidas do sistema elétrico em análise. Em seguida, retirar-se-ão as medidas que apresentarem UI acima de certo limiar préestabelecido, sob a condição da ausência de medidas críticas e de conjuntos críticos de medidas.

Os testes realizados, ilustrados no capítulo 7, demonstram a eficiência do Algoritmo 1, pois o mesmo possibilitou a obtenção de sistemas de medição confiáveis, formados por medidas com índices $U I$ menores que um valor pré-estabelecido. Porém, o custo para implantação desses sistemas de medição pode ser elevado, tendo em vista o elevado número de medidores selecionados para instalação pelo Algoritmo 1.

Para possibilitar a obtenção de sistemas de medição confiáveis, levando em consideração não apenas o índice $U I$ das medidas, mas também o número de medidores selecionados para instalação, foi proposto então o Algoritmo 2.

Considerando como medidas candidatas para instalação, apenas aquelas com $U I$ abaixo de um valor pré-estabelecido, o Algoritmo 2 possibilita a obtenção de confiáveis sistemas de medição, formados por medidas com baixos índices $U I$, minimizando o número de medidas selecionadas para instalação.

Os resultados apresentados no capítulo 7 demonstram que os sistemas de medição confiáveis, obtidos pelo Algoritmo 2, possuem realmente um número menor de medidores em relação àqueles obtidos pelo Algoritmo 1.

\subsection{Perspectivas Futuras}

Como vimos no capítulo 7, o processo de remoção (adição) de medidas candidatas para instalação, pelo Algoritmo 1 (Algoritmo 2), pode ser melhorado através da aplicação de alguma técnica de busca otimizada. Como, por exemplo, metasheurísticas.

Assim, umas de nossas perspectivas futuras é o desenvolvimento de uma técnica, provavelmente baseada em algoritmos evolutivos multi-objetivos, para guiar a busca por medidas candidatas realizada pelos algoritmos propostos no capítulo 7 para projeto e fortalecimento de sistemas de medição. 
Além das aplicações do índice UI, apresentadas nos capítulos 6 e 7, vislumbramos outras aplicações. A seguir, faremos uma breve descrição dessas outras aplicações.

\section{i) Utilização do Índice UI, para Detecção e Identificação de Erros Grosseiros}

\section{Simples e Múltiplos}

Através de estudos e testes preliminares, verificamos que o índice $U I$ pode ser utilizado para detectar e identificar EGs simples e múltiplos de forma mais eficiente, porém não exatamente da forma apresentada no capítulo 6 .

Embora o trabalho esteja em fase de investigação, averiguamos que os EGs múltiplos podem ser detectados/identificados, quando associados às medidas com índice UI baixo. A partir disto, pretendemos também estender e direcionar as nossas pesquisas à análise de EGs múltiplos.

\section{ii) Utilização do Índice UI para Aprimorar o teste HTI}

Conforme visto no capítulo 3, a eficiência do teste HTI depende do conjunto reduzido escolhido, ou conjunto de medidas suspeitas de estarem com EGs. A escolha desse conjunto geralmente está associada ao teste dos resíduos normalizados e, portanto, medidas com índice UI alto e portadoras de EGs (que apresentam baixos valores de resíduos normalizados) poderiam estar fora dessa escolha.

A partir dessa constatação, verificamos ser possível utilizar o índice UI para aprimorar a escolha do conjunto suspeito para o teste HTI e, também, utilizar o índice UI para avaliar as estimativas finais obtidas. 


\section{Referências Bibliográficas}

AAM, S.; HOLTEN, L.; GJERDE, O. (1983). Design of the measurement system for state estimation in the Norwegian high-voltage transmission network. IEEE Transactions on Power Apparatus and Systems, Vol. PAS-102, No. 12, p. 3769-3777, Dezembro.

ABUR, A. \& EXPÓSITO, A.G. (2004). Power system state estimation: theory and implementation. Marcel \& Dekker Publishers, Nova York, EUA.

ABUR, A.; MAGNAGO, H. (1999). Optimal meter placement for maintaining observability during single branch outages. IEEE Transaction on Power Systems, Vol.14, No. 4, p.1273-1278, Novembro.

ABUR, A. and EXPOSITO, A.G. (1996). Detecting Multiple Solutions in State Estimation in the Presence of Current Magnitude Measurements. 1996 IEEE/PES Winter Meeting, Baltimore, Paper No: 300-4 PWRS. Janeiro.

ALLEMONG, J.J.; RADU, L.; SASSON, A.M. (1982). A Fast and Reliable State Estimator Algorithm for AEP's New Control Center, IEEE Transactions on Power Apparatus and Systems, Vol. PAS-101, pp. 933-944, Abril.

ALSAÇ, O.; VEMPATI, N.; STOTT, B.; MONTICELLI, A. (1998). Generalized State Estimation. IEEE Transaction on Power Systems, Vol.13, No. 3, p.1069-1075, 
Agosto. (DOI: 10.1109/59.709101).

AYRES, M.; HALEY, P.H. (1986). Bad data groups in power system state estimation. IEEE Transaction on Power Systems, Vol.1, No. 3, p.1-9, Agosto.

ASADA, E.N. (2004). Análise de Observabilidade e Processamento de Erros Grosseiros Conformativos Utilizando a Metaheurística Busca Tabu na Estimação de Estado Generalizada. Tese de Doutorado, Faculdade de Engenharia Elétrica e de Computação (FEEC), Universidade Estadual de Campinas (UNICAMP), Brasil.

ASCMONEIT, F., DENZEL, D., GRAG, R.; SCHELlSTEDE, G, (1976). Development of an Optimal State Estimator And Implementation in a Real Time Computer System, CIGRE Meeting, Paris.

AZEVEDO, G. P.; OLIVEIRA FILHO, A. L. (2001). Control Centers with Open Archictectures. IEEE Computer Applications in Power Transaction on Power, p.2732, Outubro.

BALDICK, R.; CLEMENTS, K.A.; PINJO-DZIGAL, Z.; DAVIS, P.W. (1997). Implementing Nonquadratic Objective Functions for State Estimation and Bad Data Rejection. IEEE Transactions on Power Systems, Vol. 12, No. 1, p. 376-382, Fevereiro. (DOI: 10.1109/59.575722).

BALU, N.; BERTRAM, T.; BOSE, A.; BRANDWAJN, V.; CAUlEY, G.; CURTICE, D.; FOUAD, A.; FINK, L.; LAUBY, M.G.; WOLLENBERG, B.F.; WRUBEL, J.N. (1992). On-Line Power System Security Analysis. Proceendings of the IEEE. Vol. 80, No. 2, p.262-282, Fevereiro. (DOI: 10.1109/5.123296).

BARAN, E.M.; ZHU, J.; ZHU, H.; GARREN, K.E. (1995). A meter placement method for state esrtimation. IEEE Transactions on Power Systems, Vol.10, No. 3, p.1704-1710, Agosto.

BRETAS, N.G. (1989). An iterative dynamic state estimation and bad data 
processing. International Journal of Electrical Power \& Energy System, Vol. 11, p. 70-74, Janeiro.

BRETAS, N.G. (1996). Network Observability: A theory based on triangular factorization and path graph concepts - part I. International conference on Automatic

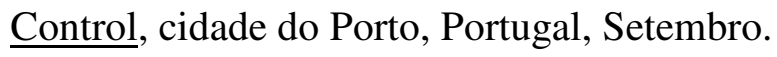

BRETAS, N.G.; LONDON J., J.B.A. (1998). Network observability: The critical measurement identification using the symbolic Jacobian matrix. International Conference on Power System Technology Proceedings, Beijing, China, Vol. 2, p. 1222-1226, Agosto.

BRETAS, N.G.; LONDON Jr., J.B.A.; ALBERTO, L.F.C.; BRETAS, A.S. (2005). A Topological Approach to the Identification of Critical Measurements in PowerSystem State Estimation. IEEE Transactions on Circuits and Systems. Vol. 52, No. 1, p. 139 - 147, Janeiro.

BRETAS, N.B.; LONDON JR.; J.B.A.; ALBERTO, L.F.C.; BENEDITO, R.A.S. (2009). Geometrical Approach on Masked Gross Errors for Power Systems State Estimation. In Proceedings of 2009 IEEE Power and Energy Society General Meeting, Calgary, Canada, p. 1-7. 26-30 July. (DOI: 10.1109/PES.2009.5275941).

CASTILlO, E.; CONEJO, A.J.; PRUNEDA, R.E. e SOlARES, C. (2006). Observability Analysis in State Estimation: A Unified Numerical Approach. IEEE Transactions on Power Systems, Vol. 21, No. 2, p.877-887, Maio.

CASTILLO-ALBERTINI, M. R. M. (2010). Metodologia para Depuração off-line de Parâmetros Série e Shunt de Linhas de Transmissão Através de Diversas Amostras de Medidas. Tese de Doutorado, Escola de Engenharia de São Carlos (EESC), Universidade de São Paulo (USP), Brasil. (disponível em: http://www.teses.usp.br/teses/disponiveis/18/18154/tde-20102010-105622/pt-br.php).

CEASE, T. W.; FELDHAUS, B. (1999). Real-time monitoring of the TVA power 
system. IEEE Comput. Appl. Power, Vol. 7, No. 3, p. 47-51, Julho.

CHEN, R.L. (1990). A fast integer algorithm for observability analysis using network topology. IEEE Transactions on Power Systems, Vol.5, No. 3, p. 1001-1009, Agosto.

CHENIAE, M.G.; MILI, L.; ROUSSEEUW, P.J. (1996). Identification of multiple interacting bad data via power system decomposition. IEEE Transactions on Power Systems, Vol.11, No. 3, p. 1555-1563, Agosto.

CLEMENTS, K. A.; DAVIS, P. W. (1986). Multiple Bad Data Detectability and Identifiability: A Geometric Approach. IEEE Transactions on Power Delivery, Vol. 1, No. 3, p.355-360, Julho (DOI: 10.1109/TPWRD.1986.4308015).

CLEMENTS, K. A. ; KRUMPHOLZ, G. R. ; DAVIS, P. W. (1981). Power system state estimation residual analysis: an algorithm using network topology. IEEE Transactions on Power Apparatus and Systems, Vol.PAS 100, No. 4, p.1779- 1787, Abril.

CLEMENTS, K.A.; KRUMPHOLZ, G.R.; DAVIS, P.W. (1982). State estimation measurement system reliability evaluation - an efficient algorithm based on topology observability theory. IEEE Transactions on Power Apparatus and Systems, Vol. PAS-101, No. 4, p.997- 1004, Abril.

CLEMENTS, K.A.; KRUMPHOLZ, G.R.; DAVIS, P.W. (1983). Power system state estimation with measurement deficiency: an observability/measurement placement algorithm". IEEE Transactions on Power Apparatus and Systems, Vol PAS-102, No. 7, p.2012- 2020, Julho.

CONTAXIS, G.C.; KORRES, G.N. (1988). A reduced model for power system observability analysis and restoration. IEEE Transactions on Power Systems, Vol. 3, No. 4, p. 1411-1417, Novembro.

COSER, J.; SIMÕES-COSTA, A.; ROLIM, J.G. (2005). Metering Scheme 
Optimization with Emphasis on Ensuring Bad-Data Processing Capability. IEEE Transactions on Power Systems, Vol. 21, No. 4, p. 1903-1911, Novembro. (DOI: 10.1109/TPWRS.2006.881137).

COUTTO FILHO, M.B.D.; LEITE da SILVA, A.M.; FALCÃO, D.M. (1990). Bibliography on power system state estimation (1968-1989). IEEE Transactions on Power Systems, Vol. 5, No. 3, p. 950-961, Agosto.

COUTTO FILHO, M.B.D.; SOUZA, J.C.S. (2009). Forecasting-Aided State Estimation - Part I: Panorama. IEEE Transactions on Power Systems, Vol. 24, No. 4, p. 1667-1677, Setembro. (DOI: 10.1109/TPWRS.2009.2030295).

COUTTO FILHO, M.B.D.; SOUZA, J.C.S.; OLIVEIRA, F.M.F.; SCHILLING, M.Th. (2001). Identifying critical measurements and sets for power system state estimation. Proceedings do IEEE Porto PowerTech, paper EDT2-147, p. 1-6, Porto, Portugal, Setembro.

COUTTO FILHO, M.B.D.; DUNCAN GLOVER, J.; LEITE da SILVA, A.M. (1993). State estimators with forecasting capability. $11^{\text {th }}$ PSCC Proc., Vol. II, p. 689695, França, Agosto.

COUTTO FILHO, M.B.D.; LEITE da SILVA, A.M.; CANTERA, J.M.C.; SILVA, R.A. (1989). Information debugging for real-time power systems monitoring. IEE Proc.C, Vol. 136, p. 145-152, Maio.

CRAINIC, E.D.; HORISBERGER, H.P.; DO, X.D.; MUKHEDKAR, D. (1990). Power network observability: The assessement of the measurement system strength. IEEE Transactions on Power Systems, Vol. 5, No. 4, p. 1267- 1285, Novembro.

ÇELIK, M.K.; ABUR, A. (1992). A Robust WLAV State Estimator Using Transformations. IEEE Transactions on Power Systems, Vol. 7, No. 1, p. 106-113, Fevereiro. 
DEBS, A.S. \& LARSON, R.E. (1970). A dynamic estimator for tracking the state of a power system. IEEE Transactions on Power Apparatus and Systems, Vol. PAS-89, No. 7, p. 1670-1678, setembro/outubro.

DY LIACCO, T. E. (1974). Real-Time Computer Control of Power Systems. Proceedings of the IEEE, Vol. 62, No. 7, p.884-891, Julho.

DY LIACCO, T. E. (2002). Control Centers Are Here To Stay. IEEE Computer Applications in Power, Vol. 15, No. 4, p.18-23, Outubro. (DOI: 10.1109/MCAP.2002.1046107).

EBRAHIMIAN, R.; BALDICK, R. (2001). State Estimator Condition Number Analysis. IEEE Transactions on Power Systems, Vol. 16, No. 2, p.273-279, Maio. (DOI: 10.1109/59.918298).

EHRENSPERGER, J.G. (2004). Sistemas de medição fasorial sincronizada: análise do estado da arte e aplicações no monitoramento de sistemas de energia elétrica. Dissertação de Mestrado. Departamento de Engenharia Elétrica, Universidade Federal de Santa Catarina (UFSC), Florianópolis, Brasil.

EXPOSITO, A. G. \& ABUR A. (1998). Generalized Observability Analysis and Measurement Classification. IEEE Transactions on Power Systems, Vol. 13, No. 3, p. 1090-1095, Agosto.

FALCÃO, D.M.; COOKE, P.A.; BRAMELLER, A. (1982). Power system tracking state estimation and bad data processing. IEEE Transactions on Power Apparatus and Systems, Vol. PAS-101,No. 2, p. 325-333, fevereiro.

FALCÃO, D.M.; e ARIAS, M. (1994). State Estimation and Observability Analysis Based on Echelon Forms of the Linearized Measurement Models. IEEE Transactions On Power Systems, Vol. 9(2), pp. 979-987, Maio. (DOI: 10.1109/59.317647). 
GARCIA, A.; MONTICELlI, A.; ABREU, P. (1979). Fast decoupled state estimation and bad data processing. IEEE Transactions on Power Apparatus and

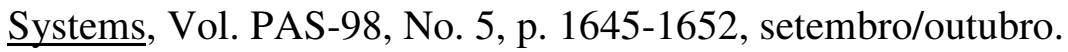

GJELSVIK, A.; AAM, S.; HOLTEN, L. (1985). Hachtel's Augmented Matrix Method - a Rapid Method Improving Numerical Stability in Power System Static State Estimation. IEEE Transactions on Power Apparatus and Systems, Vol. PAS104, No. 11, p. 2987-2993, Novembro. (DOI: 10.1109/TPAS.1985.31893).

GOLUB, G.H.; VAN LOAN, C.F. (1996). Matrix Computations. The Johns Hopkins University Press. Third edition. Baltimore, Maryland, USA.

GOU, B. e ABUR A. (2000). A direct numerical method for observability analysis. IEEE Trans. Power Systems, VOL. 15, No. 2, p.625-630, Maio.

GOU, B. e ABUR A. (2001). An Improved Measurement Placement Algorithm for Network Observability. IEEE Trans. Power Systems, VOL. 16, No. 4, p.819-824, novembro.

GOU, B. (2006). Jacobian Matrix-Based Observability Analysis for State Estimation. IEEE TRANSACTIONS ON POWER SYSTEMS, VOL. 21, No. 1, p.348-356, fevereiro.

GOUVÊA, J.P.S. \& SIMÕES-COSTA, A.J.A.(1998). Um método ortogonal com restrições de igualdade e desigualdade para a estimação de estados em sistemas de potência. Proceedings of XII Brazilian Automatic Control Conference, Vol. 6, pp. 2137-2142, Uberlândia, Brasil, setembro.

HABIBALLAH, I.O. and IRVING, M.R. (2001). Observability analysis for state estimation using linear programming. IEE Proc.-Gener. Transm. Distr. Vol 148 No 2 p.142-145, março.

HANDSCHIN, E.; BONGERS, C. (1972). Theoretical and practical considerations in 
the design of state estimators for eletric power systems. In Computerized Operation of Power Systems, S. C. Savulescu, Editor, Elsevier, p. 104 - 136.

HANDSCHIN, E.; SCHWEPPE, F.C.; KOHLAS, J.; FIECHTER, A. (1975). Bad data analysis for power systems state estimation. IEEE Transactions on Power Apparatus and Systems, Vol. 94, No. 2, p. 329-337, Março/Abril. <disponível: http://ieeexplore.ieee.org/stamp/stamp.jsp?tp=\&arnumber=1601460>.

HOLTEN, L.; GJELSVIK, A.; AAM, S.; WU, F.F.; LIU, W.H.E. (1988). Comparison of Different Methods for State Estimation. IEEE Transactions on Power Apparatus and Systems, Vol. 3, No. 4, p. 1798-1806, Novembro. (DOI: $\underline{10.1109 / 59.192998)}$.

HORISBERGER, H.P.; RICHARD, J.C.; ROSSIER, C. (1976). A fast decoupled static state estimator for electric power systems. IEEE Transactions on Power Apparatus and Systems, Vol. 95, No. 1, p. 208-215, Janeiro/Fevereiro.

HUBER, P.J. (1964). Robust Estimation of a Location Parameter. Annals Math. Statist. Vol. 35, p. 73-101.

KORRES, G.N.; CONTAXIS, G.C. (1991a). A reduced model for bad data processing in state estimation. IEEE Transaction on Power Systems, Vol. 6, No. 2, p.550-557, Maio.

KORRES, G.N.; CONTAXIS, G.C. (1991b). Identification and updating of minimaly dependent sets of measurements in state estimation. IEEE Transaction on Power Systems, Vol. 6, No. 3, p.999-1005, Agosto.

KORRES, G.N.; CONTAXIS, G.C. (1994). A tool for the evaluation and selection of state estimator measurement schemes. IEEE Transaction on Power Systems, Vol. 9, No. 2, p.1110-1116, Maio.

KORRES G. N.; KATSIKAS P. J.; CLEMENTS K. A. e DAVIS P. W. (2003). 
Numerical observability analysis based on network graph theory. IEEE Transaction on Power Systems, Vol. 18, No. 3, p. 1035-1045, Agosto.

KRUMPHOLZ, G.R.; CLEMENTS, K.A.; DAVIS, P.W. (1980). Power system observability: a practical algorithm using network topology. IEEE Transactions on Power Apparatus and Systems, Vol. PAS-99, No. 4, p. 1534- 1542, Julho - Agosto.

LEITE da SILVA, A.M.; COUTTO FILHO, M.B.D.; CANTERA, J.M.C. (1987). An efficient dynamic state estimation algorithm including bad data processing. IEEE Transactions on Power Systems, Vol.2, No. 4, p.1050-1058, Novembro.

LEITE da SILVA, A.M.; COUTTO FILHO, M.B.D.; QUEIROZ, J.F. (1983). State forecasting in electric power systems. IEE Proc.C, Vol. 130, p. 237-244, setembro.

LONDON Jr., J.B.A. (2000). Identificação do nível de redundância das medidas de um sistema de potência, para efeito da Estimação de seus estados. Tese Doutorado. Universidade de São Paulo, Escola de Engenharia de São Carlos, Departamento de Engenharia Elétrica.

LONDON Jr., J.B.A.; ALBERTO, L.F.C. e BRETAS, N.G. (2001). Identificação do nível de redundância das medidas para efeito de estimação de estado em sistemas de potência". Revista Controle \& Automação, Vol. 12, No. 2, pp.141-147, maio/ Junho/ Julho/ Agosto.

LONDON Jr., J.B.A.; ALBERTO, L.F.C. \& BRETAS, N.G. (2007). Analysis of Measurement Set Qualitative Characteristics for State Estimation Purposes. IET Generation, Transmission \& Distribution (formerly IEE Proceedings. Generation, Transmission \& Distribution), Vol.1, p. 39-45, Janeiro.

LONDON Jr., J.B.A.; BRETAS, A.S. \& BRETAS, N.G. (2004). Algorithms to solve qualitative problems in power system state estimation. International Journal of Electrical Power \& Energy Systems, Vol. 26/8, p.583-592. 
LONDON Jr., J.B.A.; BRITO, G.L.R.; BRETAS, N.G. (2002). Método para locação de medidores e UTRs para efeito de estimação de estado. Anais do XIV Congresso Brasileiro de Automática, pp. 629-635, Natal, RN.

LONDON Jr., J.B.A.; BRITO, G.L.R.; BRETAS, N.G. (2003). Method for meter and RTU placement for state estimation purposes. In Proc. IEEE Bologna Power Tech. Conf., Bologna, Italy, Junho.

LONDON Jr., J.B.A.; PIERETI, S.A.R.; BENEDITO, R.A.S.; BRETAS, N.G. (2009). Redundancy and Observability Analysis of Conventional and PMU Measurements. IEEE Transactions on Power Systems, Vol. 24, No. 3, p. 1629-1630, Agosto. (DOI: 10.1109/TPWRS.2009.2021195).

MASIELLO, R.D.; SCHWEPPE, F.C. (1971). A tracking static state estimator. IEEE Transactions on Power Apparatus and Systems, Vol. PAS-90, No. 3, p. 1025-1033, maio/junho.

MERRIL, H.M.; SCHWEPPE, F.C. (1971). Bad Data Suppression in Power System Static State Estimation. IEEE Transactions on Power Apparatus and Systems. Vol. 90, No. 6, p. 2718-2725, Novembro. (DOI: 10.1109/TPAS.1971.292925).

MEZA E. B. M.; COUTTO FILlO M. B. do; STACCHINI de SOUZA J.; SCHILLING TH. M. (2006). Estimação de Parâmetros de redes elétricas. $\underline{X}$ Simpósio de Especialistas em Planejamento da Operação e Expansão Elétrica, Maio.

MILI, L.; PHANIRAJ, V.; ROUSSEEUW, P.J. (1991). Least Median of Squares Estimation in Power Systems. IEEE Transactions on Power Systems. Vol. 6, No. 2, p. 511-523, Maio. (DOI: 10.1109/59.76693).

MILI, L.; CHENIAE, M.; VICHARE, N.; ROUSSEEUW, P. (1996). Robust state estimation based on projection statistics. IEEE Transactions on Power Systems, Vol. 11, No. 2, p. 1118-112. 
MILI, L.; VAN CUTSEM, Th.; RIBBENS-PAVELLA, M. (1984). Hypothesis Testing Identification: A New Method for Bad Data Analysis in Power System State Estimation. IEEE Transactions on Power Apparatus and Systems, Vol. 103, No. 11, p. 3239-3252, Novembro. (DOI: 10.1109/TPAS.1984.318561).

MILI, L.; VAN CUTSEM, Th.; RIBBENS-PAVELLA, M. (1985). Bad data identification methods in power system state estimation- A comparative study. IEEE Transactions on Power Apparatus and Systems, Vol. PAS-104, No. 11, p. 3037-3049, Novembro.

MONTICELLI, A. J. (1983). Fluxo de Carga em Redes de Energia Elétrica. Edgard Blucher, São Paulo, Brasil.

MONTICELLI, A. (1993a). The Impact of Modeling Short Circuit Branches in State Estimation. IEEE Transactions on Power Systems, Vol. 8, No. 1, p. 364-370. (DOI: $\underline{10.1109 / 59.221219)}$.

MONTICELLI, A. (1993b). Modeling Circuit Breakers in Weighted Least Squares State Estimation. IEEE Transactions on Power Systems, Vol. 8, No. 3, p. 1143-1149. (DOI: $10.1109 / 59.260883$ ).

MONTICELLI, A. (1999). State Estimation in Electric Power Systems: A Generalized Approach. Kluwer Academic Publishers, Massachusetts, USA.

MONTICELLI, A. (2000). Electric Power System State Estimation. Proceedings of the IEEE, Vol. 88, No. 2, Fevereiro. (DOI: 10.1109/5.824004).

MONTICELLI, A.; GARCIA, A. (1983). Reliable bad data processing for real-time state estimation. IEEE Transactions on Power Apparatus and Systems, Vol. 102, No. 5, p. 1126-1139, Maio.

MONTICELlI, A.; GARCIA, A. (1990). Fast Decoupled State Estimators. IEEE Transactions on Power Systems, Vol. 5, No. 2, p. 556-564, Maio. (DOI: 
$\underline{10.1109 / 59.54566)}$.

MONTICELLI, A.; GARCIA, A. (1991). Modeling Zero Impedance Branches in Power System State Estimation. IEEE Transactions on Power Systems, Vol. 6, No. 4, p. 1561-1570, Novembro. (DOI: 10.1109/59.117003).

MONTICELlI, A.; GARCIA, A.; SLUTSKER, I.W. (1992). Handling discardable measurements in power system state estimation. IEEE Transaction on Power Systems, Vol. 7, No. 3, p.1333-1340, Agosto.

MONTICELLI, A.; MURARI, C.A.F.; WU, F.F. (1985). A Hybrid State Estimator: Solving Normal Equations By Orthogonal Transformations. IEEE Transactions on Power Apparatus and Systems, Vol. PAS-104, No. 12, p.3460-3468, Fevereiro. (DOI: $\underline{10.1109 / T P A S .1985 .318896)}$.

MONTICELLI, A.; WU, F.F. (1985a). Network observability: Identification of observable islands and measurement placement. IEEE Transaction on Power Apparatus and Systems, Vol. PAS-104, No. 5, p. 1035-1041, Maio.

MONTICELLI, A.; WU, F.F. (1985b). Network observability: Theory. IEEE Transaction on Power Apparatus and Systems, Vol. PAS-104, No. 5, p. 1042-1048, Maio.

MONTICELLI, A.; WU, F.F. (1986). Observability analysis for orthogonal transformation based state estimation. IEEE Transactions on Power Systems, Vol.1, No. 1, p. 201-208, Fevereiro.

MONTICELLI, A.; WU, F.F.; YEN, M. (1986). Multiple Bad Data Identification for State Estimation by Combinatorial Optimization. IEEE Transactions on Power Delivery, Vol.1, No. 3, p. 361-369, Julho. (DOI: 10.1109/TPWRD.1986.4308016).

MOREIRA, E. M. (2006). Estimação de estado em sistemas elétricos de potência: programa para análise e atualização das características qualitativas de conjuntos de 
medidas. Dissertação Mestrado. Universidade de São Paulo, Escola de Engenharia de São Carlos, Departamento de Engenharia Elétrica.

MORI, H.; TSUZUKI, S. (1991). A fast method for topological observability analysis using a minimum spanning tree technique. IEEE Transactions on Power Systems, Vol. 6, No. 2, p. 491 - 500, Maio.

MOUNIR, Y.; RABIN, J.; EL-BITAR, I.; WAKED (2001). A PC Based state estimator interfaced with a remote terminal unit placement algorithm. IEEE Transactions on Power Systems. Vol. 16, No. 2, p.210-215.

NUCERA, R.R.; GILLES, M.L. (1991). Observability analisys: A new topological algorithm. IEEE Transactions on Power Systems, Vol. 6, No. 2, p. 466 - 475, Maio.

PARK, Y.M.; MOON, Y.H.; CHOO, J.B.; KWON, T.W. (1988). Design a reliable measurement system for state estimation. IEEE Transactions on Power Systems, Vol.3, No. 3, p.830-836, Agosto.

PHADKE, A.G. (1993). Synchronized Phasor Measurements in Power Systems. IEEE Computer Applications in Power, Vol. 6, No. 2, p. 10-15, Abril. (DOI: $\underline{10.1109 / 67.207465)}$.

PHADKE, A.G. (2002). Synchronized phasor measurements - a historical overview. In Proceedings of IEEE Power Eng. Soc. Asia Pacific Transmission Distribution Conf. Exhib., Oct. 6-10, Vol. 1, p. 476-479, Outubro.

PHADKE, A.G.; THORP, J.S.; KARIMI, K.J. (1986). State estimation with phasor measurements. IEEE Transactions on Power Systems, Vol. 1, No. 1, p. 233-240, Fevereiro.

PIRES, R. C.; SIMÕES-COSTA, A. ; MILI, L. (1999). Iteratively reweighted leastsquares state estimation through givens rotations. IEEE Transactions on Power

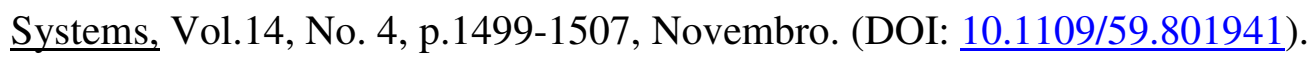


QUARTERONI, A.; SACCO, R.; SALERI, F. (2000). Numerical Mathematics. Springer Verlag New York, NY, USA.

QUINTANA, V.H.; SIMÕES-COSTA, A.; MANDEL, A. (1982a). Power system topological observability using a direct graph-theoretic approach. IEEE Transactions on Power Apparatus and Systems, Vol. PAS-101, No. 3, p. 617- 626, Março.

ROUSSEAUW, P.; VAN CUTSEM, Th.; DY LIACCO, T.E. (1990). Whither dynamic state estimation. Electrical Power \& Energy Systems, Vol. 12, p.237-244, Abril.

ROUSSEEUW, P.J.; LEROY, A.M. (1987). Robust Regression and Outilier Detection. Ed. John Wiley, New York, USA.

ROY, L.; MOHAMED, T.A. (1997). Fast super decoupled state estimation for power systems. IEEE Transactions on Power Systems, Vol.12, No. 4, p.1597-1603, Novembro.

SCHWEPPE, F. C.; WILDES, J. and ROM, D. (1968). Power system static state estimation, Power Syst. Eng. Group, M.I.T. Rep. 10, Novembro.

SCHWEPPE, F.C (1970). Power system static-state estimation, part III: Implementation. IEEE Transactions on Power Apparatus and Systems, Vol. PAS-89, No. 1, p. 130-135, Janeiro.

SCHWEPPE, F.C.; DOUGLAS, B.R. (1970). Power system static-state estimation, part II: Approximate model. IEEE Transactions on Power Apparatus and Systems, Vol. PAS-89, No. 1, p. 125-130, Janeiro.

SCHWEPPE, F.C.; WILDES, J. (1970). Power system static-state estimation, part I: Exact model. IEEE Transactions on Power Apparatus and Systems, Vol. PAS-89, No. 1, p. 120-125, Janeiro. 
SCHWEPPE, F.C.; HANDSCHIN, E.J. (1974). Static State Estimation in Eletric Power Systems. IEEE Transactions on Power Apparatus and Systems, Vol. 62, No. 7, p. 972-982, Julho.(http://ieeexplore.ieee.org/stamp/stamp.jsp?tp=\&arnumber=1451479).

SHIVAKUMAR, N.R.; JAIN, A. (2008). A Review of Power System Dynamic State Estimation Techniques. In Proc. of Power System Technology and IEEE Power India Conference. p. 1-6, New Delhi, India. (DOI: 10.1109/ICPST.2008.4745312).

SIMÕES-COSTA, A.; PIAZZA, T.S.; MANDEL, A. (1990). Qualitative methods to solve qualitative problems in power system state estimation. IEEE Transactions on Power Systems, Vol. 5, No. 3, p. 941-949, Agosto.

SIMÕES-COSTA, A.; QUINTANA, V.H. (1981-a). A Robust Numerical Technique for Power System State Estimation. IEEE Transactions on Power Apparatus and Systems, Vol. PAS-100, No. 2, p. 691-698, Fevereiro. (DOI: $\underline{\text { 10.1109/TPAS.1981.316920). }}$.

SIMÕES-COSTA, A.; QUINTANA, V.H. (1981-b). An Orthogonal Row Processing Algorithm for Power System Sequential State Estimation.. IEEE Transactions on Power Apparatus and Systems, Vol. PAS-100, No. 8, p. 3791-3800, Agosto. (DOI: 10.1109/TPAS.1981.317022).

SIMÕES-COSTA, A.; SALGADO, R. (2002). Análise Estática de Segurança de Sistemas Elétricos de Potência. Notas de aula UFSC, Setembro.

SLUTSKER, I.W. (1989). Bad data identification in power system state estimation based on measurement compensation and linear residual calculation. IEEE Transactions on Power Systems, Vol. 4, No. 1, p. 53-60, Fevereiro.

SLUTSKER, I.W.; SCUDDER, J.M. (1987). Network observability analysis through measurement Jacobian matrix reduction. IEEE Transactions on Power Systems, Vol. 2, No. 2, p. 331- 337, Maio. 
SOUZA, J.C.S.; COUTTO FILHO, M.B.D.; SCHILLING, M.Th. (2005). Optimal Metering Systems for Monitoring Power Networks Under Multiple Topological Scenarios. IEEE Transactions on Power Systems, Vol. 20, No. 4, p.1700-1708, Novembro. (DOI: 10.1109/TPWRS.2005.857941)

SOUZA, J.C.M.; LEITE da SILVA, A.M.; ALVES da SILVA, A.P. (1996). Data debugging for real-time power system monitoring based on pattern analysis. IEEE Transaction on Power Systems, Vol.11, No. 3, p.1592-1599, Agosto.

THORP, J.S.; PHADKE, A.G.; KARIMI, K.J. (1985). Real time voltage-phasor measurements for static state estimation. IEEE Transactions on. Power Apparatus and Systems, Vol. PAS-104, No. 11, p. 3098-3104, Novembro.

TINNEY, W.F.; BRANDWAJN, V.; CHAN, S.M. (1985). Sparse vector methods. IEEE Transactions on Power Apparatus and Systems, Vol. PAS-104, No. 2, p. 295301, Fevereiro.

VIGLIASSI, M.P.; LONDON JR., J.B.A.; DELBEM, A.; BRETAS, N.G. (2009). Metering system planning for state estimation via evolutionary algorithm and $H_{\Delta}$ matrix. In Proceedings of 2009 IEEE Bucharest Power Tech Conference, p. 1-6, Bucharest, Romania, June 28th - July 2nd. (DOI: 10.1109/PTC.2009.5281902).

WU, F. F.; MOSLEHI, K.; BOSE, A. (2005). Power System Control Centers: Past, Present, and Future. Proceedings of the IEEE, Vol. 93, No. 11, p.1890-1908, Novembro.

WU, F. F. (1988). Real-time network security monitoring, assessment and optimization. Electrical Power and Energy Systems, Vol. 10, No 2, p.83-100, Abril.

ZARCO, P.; EXPÓSITO, A. G. (2000). Power System Parameter Estimation: A Survey. IEEE Transactions on Power Systems. Vol.15, No. 1, p.216-222, Fevereiro.

ZHOU, M.; CENTENO, V.A.; PHADKE, A.G.; HU, Y.; NOVOSEL, D.; VOLSKIS, 
H.A.R. (2008). In Proceedings of Third International Conference on Electric Utilily Deregulation and Restructuring and Power Technologies - DRPT 2008, p. 28622867, Abril. (DOI: 10.1109/DRPT.2008.4523897).

ZHOU, M.; CENTENO, V.A.; THORP, J.S.; PHADKE, A.G. (2006). An Alternative for Including Phasor Measurements in State Estimators. IEEE Transactions on Power Systems, Vol. 21, No. 4, p. 1930-1937, Novembro.

ZIVANOVIC, R.; CAIRNS, C. (1996). Implementation of PMU technology in state estimation: an overview. In Proceeding of IEEE 4th AFRICON, Vol. 2, p. 10061011, Setembro.

ZOLLENKOPF, K. (1971). Bi-factorization-basic computation algorithm and programming techniques. In: Large sparse sets of linear equations. Editado por Reid, J.K., N. York, Academic Press, p. 75-97. 


\section{Anexo A}

\section{A Demonstrações Matemáticas não Apresentadas no Capítulo 4}

Será apresentado neste Anexo todo o desenvolvimento matemático necessário para a obtenção das equações 4.28 e 4.29 .

Como ponto de partida, temos a seguinte expressão para o índice $U I$ de uma medida $i$ (equação 4.27 do capítulo 4):

$$
U I_{i}=\frac{\left\|\underline{w}_{U}^{i}\right\|_{R^{-1}}}{\left\|\underline{w}_{D}^{i}\right\|_{R^{-1}}}=\frac{b \cdot \sqrt{\left(K \cdot \underline{\delta}^{i}\right)^{t} \cdot R^{-1} \cdot\left(K \cdot \underline{\delta}^{i}\right)}}{b \cdot \sqrt{\left((I-K) \cdot \underline{\delta}^{i}\right)^{t} \cdot R^{-1} \cdot\left((I-K) \cdot \underline{\delta}^{i}\right)}} .
$$

Primeiramente, provaremos que o numerador da equação acima se reduz a equação 4.28, aqui designada por (A.2):

$$
\left\|\underline{w}_{U}^{i}\right\|_{R^{-1}}=b \cdot \sqrt{\left(K \cdot \underline{\delta}^{i}\right)^{t} \cdot R^{-1} \cdot\left(K \cdot \underline{\delta}^{i}\right)}=b \cdot \sigma_{i}^{-1} \cdot \sqrt{K_{i i}} ;
$$

em seguida, mostraremos que o denominador de (A.1) é igual a equação 4.29, aqui chamada de (A.3):

$$
\left\|\underline{w}_{D}^{i}\right\|_{R^{-1}}=b \cdot \sqrt{\left((I-K) \cdot \underline{\delta}^{i}\right)^{t} \cdot R^{-1} \cdot\left((I-K) \cdot \underline{\delta}^{i}\right)}=b \cdot \sigma_{i}^{-1} \cdot \sqrt{\left(1-K_{i i}\right)} .
$$

\section{Prova da Equação (A.2)}

Através de uma simples operação matricial, podemos escrever o numerador da equação $A .1$ como:

$$
\left\|\underline{w}_{U}^{i}\right\|_{R^{-1}}=b \cdot \sqrt{\left(K \cdot \underline{\delta}^{i}\right)^{t} \cdot R^{-1} \cdot\left(K \cdot \underline{\delta}^{i}\right)}=b \cdot \sqrt{\underline{\delta}^{i t} \cdot\left(K^{t} \cdot R^{-1} \cdot K\right) \cdot \underline{\delta}^{i}} .
$$

Como $\underline{\delta}^{i}=[0, \ldots, 0,1,0, \ldots, 0]^{t}$, a seguinte expressão é válida:

$$
\underline{\delta}^{i t} \cdot B \cdot \underline{\delta}^{i}=B_{i i},
$$

sendo $B$ uma matriz quadrada cuja ordem é igual ao número de linhas de $\underline{\delta}^{i}$. 
A partir da equação $A .5$, verificamos que a equação $A .4$ pode ser reescrita como:

$$
\left\|\underline{w}_{U}^{i}\right\|_{R^{-1}}=b \cdot \sqrt{\underline{\delta}^{i t} \cdot\left(K^{t} \cdot R^{-1} \cdot K\right) \cdot \underline{\delta}^{i}}=b \cdot \sqrt{\left(K^{t} \cdot R^{-1} \cdot K\right)_{i i}} ;
$$

por sua vez, a expressão matricial $K^{t} \cdot R^{-1} \cdot K$ ainda pode ser simplificada utilizando a propriedade P.4 (apresentada no capítulo 3), isto é,

$$
K^{t} \cdot R^{-1} \cdot K=R^{-1} \cdot K .
$$

Por fim, substituindo a equação $A .7$ em $A .6$, chegamos à forma simplificada da equação $A .2$ (ou equação 4.28):

$$
\begin{aligned}
& \left\|\underline{w}_{U}^{i}\right\|_{R^{-1}}=b \cdot \sqrt{\left(K^{t} \cdot R^{-1} \cdot K\right)_{i i}}=b \cdot \sqrt{\left(R^{-1} \cdot K\right)_{i i}} \\
& \left\|\underline{w}_{U}^{i}\right\|_{R^{-1}}=b \cdot \sqrt{\frac{1}{\sigma_{i}^{2}} \cdot K_{i i}}=b \cdot \sigma_{i}^{-1} \cdot \sqrt{K_{i i}}
\end{aligned}
$$

\section{Prova da Equação $(\boldsymbol{A . 3})$}

A prova da equação $A .3$ é similar à apresentada acima, veja a seguir.

Através de operações matriciais sobre o denominador da equação $A .1 \mathrm{e}$ utilizando a propriedade P.7 (apresentada no capítulo 3), temos o seguinte desenvolvimento:

$$
\begin{aligned}
& \left\|\underline{w}_{D}^{i}\right\|_{R^{-1}}=b \cdot \sqrt{\left((I-K) \cdot \underline{\delta}^{i}\right)^{t} \cdot R^{-1} \cdot\left((I-K) \cdot \underline{\delta}^{i}\right)}=b \cdot \sqrt{\underline{\delta}^{i t} \cdot\left(S^{t} \cdot R^{-1} \cdot S\right) \cdot \underline{\delta}^{i}} \\
& \left\|\underline{w}_{D}^{i}\right\|_{R^{-1}}=b \cdot \sqrt{\left(S^{t} \cdot R^{-1} \cdot S\right)_{i i}}=b \cdot \sqrt{\left(R^{-1} \cdot S\right)_{i i}} \\
& \left\|\underline{w}_{D}^{i}\right\|_{R^{-1}}=b \cdot \sqrt{\frac{1}{\sigma_{i}^{2}} \cdot S_{i i}}=b \cdot \sigma_{i}^{-1} \cdot \sqrt{S_{i i}}=b \cdot \sigma_{i}^{-1} \cdot \sqrt{\left(1-K_{i i}\right)}
\end{aligned} .
$$




\section{Anexo B}

\section{B Tabelas com Dados e Resultados dos Sistemas Testes}

Tabela B.1: Valores de todas as medidas e respectivos desvios padrões para o sistema de 14 barras

\begin{tabular}{|c|c|c|c|c|c|}
\hline \multicolumn{3}{|c|}{ Medidas de Potência Ativa } & \multicolumn{3}{|c|}{ Medidas de Potência Reativa } \\
\hline Medida & $\begin{array}{c}\text { Valor } \\
\text { (pu) }\end{array}$ & $\sigma(\mathbf{p u})$ & Medida & $\begin{array}{c}\text { Valor } \\
\text { (pu) }\end{array}$ & $\sigma(\mathbf{p u})$ \\
\hline IA:1 & 2,32221 & 0,038733 & IR:1 & $-0,16444$ & 0,00275 \\
\hline IA:2 & 0,18301 & 0,00305 & IR:2 & 0,30848 & 0,005133 \\
\hline IA:3 & $-0,94283$ & 0,0157 & IR:3 & 0,06091 & 0,001017 \\
\hline IA:4 & $-0,47508$ & 0,007967 & IR:4 & 0,03939 & 0,00065 \\
\hline IA:5 & $-0,07606$ & 0,001267 & IR:5 & $-0,01597$ & 0,000267 \\
\hline IA:6 & $-0,11179$ & 0,001867 & IR:6 & 0,05181 & 0,000867 \\
\hline IA:7 & 0,0 & --- & IR:7 & 0,0 & --- \\
\hline IA:8 & 0,0 & --- & IR:8 & 0,17631 & 0,002933 \\
\hline IA:9 & $-0,29613$ & 0,004917 & IR:9 & $-0,16628$ & 0,002767 \\
\hline IA:10 & $-0,08993$ & 0,0015 & IR:10 & $-0,0578$ & 0,000967 \\
\hline IA:11 & $-0,03504$ & 0,000583 & IR:11 & $-0,01807$ & 0,0003 \\
\hline IA:12 & $-0,06107$ & 0,001017 & IR:12 & $-0,01616$ & 0,000267 \\
\hline IA:13 & $-0,13477$ & 0,00225 & IR:13 & $-0,05835$ & 0,000967 \\
\hline IA:14 & $-0,1486$ & 0,002483 & IR:14 & $-0,04968$ & 0,000833 \\
\hline FA:1-2 & 1,56779 & 0,02615 & FR:1-2 & $-0,2033$ & 0,0034 \\
\hline FA:2-1 & $-1,52482$ & 0,025433 & FR:2-1 & 0,27604 & 0,004617 \\
\hline FA:1-5 & 0,75443 & 0,012583 & FR:1-5 & 0,03885 & 0,00065 \\
\hline FA:5-1 & $-0,72681$ & 0,012117 & FR:5-1 & 0,02198 & 0,000367 \\
\hline FA:2-3 & 0,73249 & 0,0122 & FR:2-3 & 0,03582 & 0,0006 \\
\hline FA:3-2 & $-0,70922$ & 0,011817 & FR:3-2 & 0,01601 & 0,000267 \\
\hline FA:2-4 & 0,56064 & 0,00935 & FR:2-4 & $-0,01531$ & 0,000258 \\
\hline FA:4-2 & $-0,5439$ & 0,009083 & FR:4-2 & 0,02999 & 0,0005 \\
\hline FA:2-5 & 0,4147 & 0,006917 & FR:2-5 & 0,01192 & 0,0002 \\
\hline FA:5-2 & $-0,40567$ & 0,006767 & FR:5-2 & $-0,02118$ & 0,00035 \\
\hline FA:3-4 & $-0,23361$ & 0,003883 & FR:3-4 & 0,0449 & 0,00075 \\
\hline FA:4-3 & 0,23738 & 0,00395 & FR:4-3 & $-0,04844$ & 0,0008 \\
\hline FA:4-5 & $-0,61066$ & 0,0102 & FR:4-5 & 0,15821 & 0,002633 \\
\hline FA:5-4 & 0,61579 & 0,010283 & FR:5-4 & $-0,14201$ & 0,002367 \\
\hline FA:4-7 & 0,2811 & 0,004683 & FR:4-7 & $-0,09635$ & 0,001617 \\
\hline FA:7-4 & $-0,2811$ & 0,004683 & FR:7-4 & 0,11343 & 0,0019 \\
\hline FA:4-9 & 0,161 & 0,002683 & FR:4-9 & $-0,00402$ & 0,000067 \\
\hline FA:9-4 & $-0,161$ & 0,002683 & FR:9-4 & 0,01712 & 0,000283 \\
\hline FA:5-6 & 0,44064 & 0,00735 & FR:5-6 & 0,12524 & 0,002083 \\
\hline FA:6-5 & $-0,44064$ & 0,00735 & FR:6-5 & $-0,08099$ & 0,00135 \\
\hline FA:6-11 & 0,0737 & 0,001233 & FR:6-11 & 0,0354 & 0,0006 \\
\hline FA:11-6 & $-0,07315$ & 0,001217 & FR:11-6 & $-0,03424$ & 0,000567 \\
\hline
\end{tabular}




\begin{tabular}{|c|c|c|c|c|c|}
\hline FA:6-12 & 0,07784 & 0,0013 & FR:6-12 & 0,02515 & 0,000417 \\
\hline FA:12-6 & $-0,07712$ & 0,001283 & FR:12-6 & $-0,02365$ & 0,0004 \\
\hline FA:6-13 & 0,1773 & 0,00295 & FR:6-13 & 0,07224 & 0,0012 \\
\hline FA:13-6 & $-0,17518$ & 0,002917 & FR:13-6 & $-0,06806$ & 0,001133 \\
\hline FA:7-8 & 0,0 & --- & FR:7-8 & $-0,17169$ & 0,002867 \\
\hline FA:8-7 & 0,0 & --- & FR:8-7 & 0,17631 & 0,002933 \\
\hline FA:7-9 & 0,2811 & 0,004683 & FR:7-9 & 0,05827 & 0,000967 \\
\hline FA:9-7 & $-0,2811$ & 0,004683 & FR:9-7 & $-0,05021$ & 0,000833 \\
\hline FA:9-10 & 0,05208 & 0,000867 & FR:9-10 & 0,04227 & 0,0007 \\
\hline FA:10-9 & $-0,05195$ & 0,000867 & FR:10-9 & $-0,04193$ & 0,0007 \\
\hline FA:9-14 & 0,09389 & 0,001567 & FR:9-14 & 0,03608 & 0,0006 \\
\hline FA:14-9 & $-0,09274$ & 0,00155 & FR:14-9 & $-0,03362$ & 0,000567 \\
\hline FA:10-11 & $-0,03798$ & 0,000633 & FR:10-11 & $-0,01587$ & 0,000267 \\
\hline FA:11-10 & 0,0381 & 0,000633 & FR:11-10 & 0,01617 & 0,000267 \\
\hline FA:12-13 & 0,01605 & 0,000267 & FR:12-13 & 0,0075 & 0,000125 \\
\hline FA:13-12 & $-0,01599$ & 0,000267 & FR:13-12 & $-0,00744$ & 0,000123 \\
\hline FA:13-14 & 0,0564 & 0,000933 & FR:13-14 & 0,01715 & 0,000283 \\
\hline FA:14-13 & $-0,05586$ & 0,000933 & FR:14-13 & $-0,01605$ & 0,000267 \\
\hline \multicolumn{6}{|c|}{ Medidas de Tensão } \\
\hline \multicolumn{2}{|c|}{ Medida } & \multicolumn{2}{|c|}{ Valor (pu) } & \multicolumn{2}{|c|}{$\sigma(\mathbf{p u})$} \\
\hline \multicolumn{2}{|c|}{ V:1 } & \multicolumn{2}{|c|}{1,05941} & \multicolumn{2}{|c|}{0,017667} \\
\hline \multicolumn{2}{|c|}{$V: 2$} & \multicolumn{2}{|c|}{1,04438} & \multicolumn{2}{|c|}{0,017417} \\
\hline \multicolumn{2}{|c|}{$V: 3$} & \multicolumn{2}{|c|}{1,00933} & \multicolumn{2}{|c|}{0,016833} \\
\hline \multicolumn{2}{|c|}{$V: 4$} & \multicolumn{2}{|c|}{1,01704} & \multicolumn{2}{|c|}{0,016967} \\
\hline \multicolumn{2}{|c|}{$V: 5$} & \multicolumn{2}{|c|}{1,01887} & \multicolumn{2}{|c|}{0,017} \\
\hline \multicolumn{2}{|c|}{$V: 6$} & \multicolumn{2}{|c|}{1,06918} & \multicolumn{2}{|c|}{0,017833} \\
\hline \multicolumn{2}{|c|}{ V:7 } & \multicolumn{2}{|c|}{1,0608} & \multicolumn{2}{|c|}{0,0177} \\
\hline \multicolumn{2}{|c|}{ V:8 } & \multicolumn{2}{|c|}{1,08931} & \multicolumn{2}{|c|}{0,018167} \\
\hline \multicolumn{2}{|c|}{ V:9 } & & & & \\
\hline & & & & & \\
\hline & & & & & \\
\hline & & & & & \\
\hline & & & & & \\
\hline & & & & & \\
\hline
\end{tabular}


Tabela B2: Valores de todas as medidas e respectivos desvios padrões para o sistema de 30 barras

\begin{tabular}{|c|c|c|c|c|c|}
\hline \multicolumn{3}{|c|}{ Medidas de Potência Ativa } & \multicolumn{3}{|c|}{ Medidas de Potência Reativa } \\
\hline Medida & Valor (pu) & $\sigma(\mathbf{p u})$ & Medida & Valor (pu) & $\sigma(\mathbf{p u})$ \\
\hline IA:1 & 2,61 & 0,018667 & IR:1 & $-0,165$ & 0,001333 \\
\hline IA:2 & 0,183 & 0,001333 & IR:2 & 0,369 & 0,004333 \\
\hline IA:3 & $-0,024$ & 0,000333 & IR:3 & $-0,012$ & 0,000333 \\
\hline IA:4 & $-0,076$ & 0,000667 & IR:4 & $-0,016$ & 0,000333 \\
\hline IA:5 & $-0,942$ & 0,006667 & IR:5 & 0,179 & 0,001333 \\
\hline IA:6 & 0,0 & --- & IR:6 & 0,0 & --- \\
\hline IA:7 & $-0,228$ & 0,002333 & IR:7 & $-0,109$ & 0,001333 \\
\hline IA:8 & $-0,3$ & 0,002333 & IR:8 & 0,072 & 0,000667 \\
\hline IA:9 & 0,0 & --- & IR:9 & 0,0 & --- \\
\hline IA:10 & $-0,058$ & 0,000667 & IR:10 & $-0,02$ & 0,000333 \\
\hline IA:11 & 0,0 & 0,000333 & IR:11 & 0,162 & 0,001333 \\
\hline IA:12 & $-0,112$ & 0,001333 & IR:12 & $-0,075$ & 0,000667 \\
\hline IA:13 & 0,0 & --- & IR:13 & 0,106 & 0,001333 \\
\hline IA:14 & $-0,062$ & 0,000667 & IR:14 & $-0,016$ & 0,000333 \\
\hline IA:15 & $-0,082$ & 0,000667 & IR:15 & $-0,025$ & 0,000333 \\
\hline IA:16 & $-0,035$ & 0,000333 & IR:16 & $-0,018$ & 0,000333 \\
\hline IA:17 & $-0,09$ & 0,000667 & IR:17 & $-0,058$ & 0,000667 \\
\hline IA:18 & $-0,032$ & 0,000333 & IR:18 & $-0,009$ & 0,000333 \\
\hline IA:19 & $-0,095$ & 0,000667 & IR:19 & $-0,034$ & 0,000333 \\
\hline IA:20 & $-0,022$ & 0,000333 & IR:20 & $-0,007$ & 0,000333 \\
\hline IA:21 & $-0,175$ & 0,001333 & IR:21 & $-0,112$ & 0,001333 \\
\hline IA:22 & 0,0 & --- & IR:22 & 0,0 & --- \\
\hline IA:23 & $-0,032$ & 0,000333 & IR:23 & $-0,016$ & 0,000333 \\
\hline IA:24 & $-0,087$ & 0,000667 & IR:24 & $-0,067$ & 0,000667 \\
\hline IA: 25 & 0,0 & --- & IR:25 & 0,0 & --- \\
\hline IA:26 & $-0,035$ & 0,000333 & IR:26 & $-0,023$ & 0,000333 \\
\hline IA:27 & 0,0 & --- & IR:27 & 0,0 & --- \\
\hline IA:28 & 0,0 & --- & IR:28 & 0,0 & --- \\
\hline IA:29 & $-0,024$ & 0,000333 & IR:29 & $-0,009$ & 0,000333 \\
\hline IA:30 & $-0,106$ & 0,001333 & IR:30 & $-0,019$ & 0,000333 \\
\hline FA:1-2 & 1,732 & 0,013333 & FR:1-2 & $-0,211$ & 0,002333 \\
\hline FA:2-1 & $-1,68$ & 0,013333 & FR:2-1 & 0,308 & 0,002333 \\
\hline FA:1-3 & 0,877 & 0,006667 & FR:1-3 & 0,046 & 0,000333 \\
\hline FA:3-1 & $-0,846$ & 0,006667 & FR:3-1 & 0,024 & 0,000333 \\
\hline FA:2-4 & 0,436 & 0,004333 & FR:2-4 & 0,039 & 0,000333 \\
\hline FA:4-2 & $-0,426$ & 0,004333 & FR:4-2 & $-0,047$ & 0,000333 \\
\hline FA:2-5 & 0,824 & 0,006667 & FR:2-5 & 0,018 & 0,000333 \\
\hline FA:5-2 & $-0,794$ & 0,006667 & FR:5-2 & 0,062 & 0,000667 \\
\hline FA:2-6 & 0,603 & 0,004333 & FR:2-6 & 0,004 & 0,000333 \\
\hline FA:6-2 & $-0,584$ & 0,004333 & FR:6-2 & 0,015 & 0,000333 \\
\hline FA:3-4 & 0,822 & 0,006667 & FR:3-4 & $-0,036$ & 0,000333 \\
\hline FA:4-3 & $-0,813$ & 0,006667 & FR:4-3 & 0,052 & 0,000667 \\
\hline FA:4-6 & 0,721 & 0,006667 & FR:4-6 & $-0,163$ & 0,001333 \\
\hline FA:6-4 & $-0,715$ & 0,006667 & FR:6-4 & 0,176 & 0,001333 \\
\hline FA:4-12 & 0,442 & 0,004333 & FR:4-12 & 0,142 & 0,001333 \\
\hline FA:12-4 & $-0,442$ & 0,004333 & FR:12-4 & $-0,096$ & 0,000667 \\
\hline FA:5-7 & $-0,148$ & 0,001333 & FR:5-7 & 0,117 & 0,001333 \\
\hline FA:7-5 & 0,149 & 0,001333 & FR:7-5 & $-0,133$ & 0,001333 \\
\hline FA:6-7 & 0,381 & 0,004333 & FR:6-7 & $-0,03$ & 0,000333 \\
\hline
\end{tabular}




\begin{tabular}{|c|c|c|c|c|c|}
\hline FA:7-6 & $-0,377$ & 0,004333 & FR:7-6 & 0,024 & 0,000333 \\
\hline FA:6-8 & 0,296 & 0,002333 & FR:6-8 & $-0,081$ & 0,000667 \\
\hline FA:8-6 & $-0,294$ & 0,002333 & FR:8-6 & 0,076 & 0,000667 \\
\hline FA:6-9 & 0,277 & 0,002333 & FR:6-9 & $-0,082$ & 0,000667 \\
\hline FA:9-6 & $-0,277$ & 0,002333 & FR:9-6 & 0,098 & 0,000667 \\
\hline FA:6-10 & 0,158 & 0,001333 & FR:6-10 & 0,002 & 0,000333 \\
\hline FA:10-6 & $-0,158$ & 0,001333 & FR:10-6 & 0,011 & 0,000333 \\
\hline FA:6-28 & 0,187 & 0,001333 & FR:6-28 & 0,0 & 0,000333 \\
\hline FA:28-6 & $-0,186$ & 0,001333 & FR:28-6 & $-0,011$ & 0,000333 \\
\hline FA:8-28 & $-0,005$ & 0,000333 & FR:8-28 & $-0,004$ & 0,000333 \\
\hline FA:28-8 & 0,005 & 0,000333 & FR:28-8 & $-0,04$ & 0,000333 \\
\hline FA:9-11 & 0,0 & --- & FR:9-11 & $-0,157$ & 0,001333 \\
\hline FA:11-9 & 0,0 & --- & FR:11-9 & 0,162 & 0,001333 \\
\hline FA:9-10 & 0,277 & 0,002333 & FR:9-10 & 0,059 & 0,000667 \\
\hline FA:10-9 & $-0,277$ & 0,002333 & FR:10-9 & $-0,051$ & 0,000667 \\
\hline FA:10-20 & 0,09 & 0,000667 & FR:10-20 & 0,037 & 0,000333 \\
\hline FA:20-10 & $-0,089$ & 0,000667 & FR:20-10 & $-0,035$ & 0,000333 \\
\hline FA:10-17 & 0,053 & 0,000667 & FR:10-17 & 0,044 & 0,000333 \\
\hline FA:17-10 & $-0,053$ & 0,000667 & FR:17-10 & $-0,044$ & 0,000333 \\
\hline FA:10-21 & 0,158 & 0,001333 & FR:10-21 & 0,100 & 0,001333 \\
\hline FA:21-10 & $-0,157$ & 0,001333 & FR:21-10 & $-0,098$ & 0,000667 \\
\hline FA:10-22 & 0,076 & 0,000667 & FR:10-22 & 0,046 & 0,000333 \\
\hline FA:22-10 & $-0,076$ & 0,000667 & FR:22-10 & $-0,045$ & 0,000333 \\
\hline FA:12-13 & 0,0 & --- & FR:12-13 & $-0,105$ & 0,001333 \\
\hline FA:13-12 & 0,0 & --- & FR:13-12 & 0,106 & 0,001333 \\
\hline FA:12-14 & 0,079 & 0,000667 & FR:12-14 & 0,024 & 0,000333 \\
\hline FA:14-12 & $-0,078$ & 0,000667 & FR:14-12 & $-0,022$ & 0,000333 \\
\hline FA:12-15 & 0,179 & 0,001333 & FR:12-15 & 0,068 & 0,000667 \\
\hline FA:15-12 & $-0,177$ & 0,001333 & FR:15-12 & $-0,064$ & 0,000667 \\
\hline FA:12-16 & 0,072 & 0,000667 & FR:12-16 & 0,034 & 0,000333 \\
\hline FA:16-12 & $-0,072$ & 0,000667 & FR:16-12 & $-0,032$ & 0,000333 \\
\hline FA:14-15 & 0,016 & 0,000333 & FR:14-15 & 0,006 & 0,000333 \\
\hline FA:15-14 & $-0,016$ & 0,000333 & FR:15-14 & $-0,006$ & 0,000333 \\
\hline FA:15-18 & 0,06 & 0,000667 & FR:15-18 & 0,016 & 0,000333 \\
\hline FA:18-15 & $-0,06$ & 0,000667 & FR:18-15 & $-0,015$ & 0,000333 \\
\hline FA:15-23 & 0,05 & 0,000667 & FR:15-23 & 0,029 & 0,000333 \\
\hline FA:23-15 & $-0,05$ & 0,000667 & FR:23-15 & $-0,028$ & 0,000333 \\
\hline FA:16-17 & 0,037 & 0,000333 & FR:16-17 & 0,014 & 0,000333 \\
\hline FA:17-16 & $-0,037$ & 0,000333 & FR:17-16 & $-0,014$ & 0,000333 \\
\hline FA:18-19 & 0,028 & 0,000333 & FR:18-19 & 0,006 & 0,000333 \\
\hline FA:19-18 & $-0,028$ & 0,000333 & FR:19-18 & $-0,006$ & 0,000333 \\
\hline FA:19-20 & $-0,067$ & 0,000667 & FR:19-20 & $-0,028$ & 0,000333 \\
\hline FA:20-19 & 0,067 & 0,000667 & FR:20-19 & 0,028 & 0,000333 \\
\hline FA:21-22 & $-0,018$ & 0,000333 & FR:21-22 & $-0,014$ & 0,000333 \\
\hline FA:22-21 & 0,018 & 0,000333 & FR:22-21 & 0,014 & 0,000333 \\
\hline FA:22-24 & 0,057 & 0,000667 & FR:22-24 & 0,031 & 0,000333 \\
\hline FA:24-22 & $-0,057$ & 0,000667 & FR:24-22 & $-0,03$ & 0,000333 \\
\hline FA:23-24 & 0,018 & 0,000333 & FR:23-24 & 0,012 & 0,000333 \\
\hline FA:24-23 & $-0,018$ & 0,000333 & FR:24-23 & $-0,012$ & 0,000333 \\
\hline FA:24-25 & $-0,012$ & 0,000333 & FR:24-25 & 0,02 & 0,000333 \\
\hline FA:25-24 & 0,012 & 0,000333 & FR:25-24 & $-0,02$ & 0,000333 \\
\hline FA:25-26 & 0,035 & 0,000333 & FR:25-26 & 0,024 & 0,000333 \\
\hline
\end{tabular}




\begin{tabular}{l|c|c|c|c|c}
\hline FA:26-25 & $-0,035$ & 0,000333 & FR:26-25 & $-0,023$ & 0,000333 \\
\hline FA:25-27 & $-0,048$ & 0,000333 & FR:25-27 & $-0,004$ & 0,000333 \\
\hline FA:27-25 & 0,048 & 0,000333 & FR:27-25 & 0,004 & 0,000333 \\
\hline FA:27-29 & 0,062 & 0,000667 & FR:27-29 & 0,017 & 0,000333 \\
\hline FA:29-27 & $-0,061$ & 0,000667 & FR:29-27 & $-0,015$ & 0,000333 \\
\hline FA:27-30 & 0,071 & 0,000667 & FR:27-30 & 0,017 & 0,000333 \\
\hline FA:30-27 & $-0,069$ & 0,000667 & FR:30-27 & $-0,014$ & 0,000333 \\
\hline FA:28-27 & 0,181 & 0,001333 & FR:28-27 & 0,05 & 0,000667 \\
\hline FA:27-28 & $-0,181$ & 0,001333 & FR:27-28 & $-0,037$ & 0,000333 \\
\hline FA:29-30 & 0,037 & 0,000333 & FR:29-30 & 0,006 & 0,000333 \\
\hline FA:30-29 & $-0,037$ & 0,000333 & FR:30-29 & $-0,005$ & 0,000333 \\
\hline
\end{tabular}

Medidas de Tensão

\begin{tabular}{|c|c|c|}
\hline Medida & Valor (pu) & $\sigma(\mathrm{pu})$ \\
\hline V:1 & 1,06 & 0,013333 \\
\hline$V: 2$ & 1,043 & 0,013333 \\
\hline$V: 3$ & 1,021 & 0,013333 \\
\hline$V: 4$ & 1,012 & 0,013333 \\
\hline$V: 5$ & 1,01 & 0,013333 \\
\hline V:6 & 1,01 & 0,013333 \\
\hline V:7 & 1,002 & 0,013333 \\
\hline$V: 8$ & 1,01 & 0,013333 \\
\hline V:9 & 1,051 & 0,013333 \\
\hline V:10 & 1,045 & 0,013333 \\
\hline V:11 & 1,082 & 0,013333 \\
\hline V:12 & 1,057 & 0,013333 \\
\hline V:13 & 1,071 & 0,013333 \\
\hline V:14 & 1,042 & 0,013333 \\
\hline V:15 & 1,038 & 0,013333 \\
\hline V:16 & 1,044 & 0,013333 \\
\hline V:17 & 1,04 & 0,013333 \\
\hline V:18 & 1,028 & 0,013333 \\
\hline V:19 & 1,026 & 0,013333 \\
\hline V:20 & 1,03 & 0,013333 \\
\hline V:21 & 1,033 & 0,013333 \\
\hline V:22 & 1,033 & 0,013333 \\
\hline$V: 23$ & 1,027 & 0,013333 \\
\hline$V: 24$ & 1,022 & 0,013333 \\
\hline V:25 & 1,017 & 0,013333 \\
\hline$V: 26$ & 1 & 0,013333 \\
\hline V:27 & 1,023 & 0,013333 \\
\hline$V: 28$ & 1,007 & 0,013333 \\
\hline V:29 & 1,003 & 0,013333 \\
\hline V:30 & 0,992 & 0,006667 \\
\hline
\end{tabular}


Tabela B.3: Medidas do sistema de medição obtido pelo Algoritmo 1 e respectivos valores de $U I$ (sistema de 30 barras)

\begin{tabular}{|c|c|c|c|c|c|}
\hline \multicolumn{2}{|c|}{$\begin{array}{c}\text { Medidas de Potência } \\
\text { Ativa }\end{array}$} & \multicolumn{2}{|c|}{$\begin{array}{c}\text { Medidas de Potência } \\
\text { Reativa }\end{array}$} & \multicolumn{2}{|c|}{$\begin{array}{l}\text { Medidas de } \\
\text { Tensão }\end{array}$} \\
\hline Medida & Índice $U I$ & Medida & Índice $U I$ & Medida & Índice $U I$ \\
\hline IA:1 & 0,3543 & IR:1 & 0,8215 & V:1 & 0,075 \\
\hline IA:5 & 0,2665 & IR:5 & 0,6443 & $V: 2$ & 0,0754 \\
\hline IA:6* & --- & IR:6 & --- & $V: 3$ & 0,0742 \\
\hline IA:7 & 1,0123 & IR:7 & 0,4137 & $V: 4$ & 0,0745 \\
\hline IA:8 & 0,5158 & IR:8 & 0,7382 & $V: 5$ & 0,0751 \\
\hline IA:9 & --- & IR:9 & --- & $V: 6$ & 0,0755 \\
\hline IA:11 & --- & IR:11 & 0,4481 & V:7 & 0,0758 \\
\hline IA:12 & 1,0113 & IR:12 & 1,4999 & V:8 & 0,0755 \\
\hline IA:13 & --- & IR:13 & 0,5075 & V:9 & 0,0771 \\
\hline IA:14 & 0,6437 & IR:14 & 0,9407 & V:10 & 0,0781 \\
\hline IA:15 & 1,192 & IR:15 & 1,5903 & V:11 & 0,0755 \\
\hline IA:16 & 1,3637 & IR:16 & 0,9607 & V:12 & 0,0782 \\
\hline IA:17 & 0,7769 & IR:17 & 0,4571 & V:13 & 0,0765 \\
\hline IA:18 & 1,2614 & IR:18 & 0,873 & V:14 & 0,0791 \\
\hline IA:19 & 0,6199 & IR:19 & 0,8567 & V:15 & 0,0793 \\
\hline IA:20 & 1,7401 & IR:20 & 0,9082 & V:16 & 0,0786 \\
\hline IA:21 & 0,4762 & IR:21 & 0,3402 & V:17 & 0,0786 \\
\hline IA:22 & --- & IR:22 & --- & V:18 & 0,0797 \\
\hline IA:23 & 1,3464 & IR:23 & 0,9369 & V:19 & 0,0798 \\
\hline IA:24 & 0,679 & IR:24 & 0,544 & V:20 & 0,0795 \\
\hline IA:25 & --- & IR:25 & --- & V:21 & 0,0791 \\
\hline IA:26 & 0,6228 & IR:26 & 0,6207 & V:22 & 0,079 \\
\hline IA:27 & --- & IR:27 & --- & $V: 23$ & 0,0799 \\
\hline IA:28 & --- & IR:28 & --- & $V: 24$ & 0,0801 \\
\hline IA:29 & 1,214 & IR:29 & 0,8546 & $V: 25$ & 0,0801 \\
\hline IA:30 & 0,2643 & IR:30 & 0,847 & V:26 & 0,0818 \\
\hline FA:1-2 & 0,4151 & FR:1-2 & 0,323 & V:27 & 0,0796 \\
\hline FA:2-1 & 0,3885 & FR:2-1 & 0,4534 & $V: 28$ & 0,0758 \\
\hline FA:1-3 & 0,2769 & FR:1-3 & 1,3346 & V:29 & 0,0812 \\
\hline FA:3-1 & 0,2617 & FR:3-1 & 1,5459 & V:30 & 0,166 \\
\hline FA:2-4 & 0,3584 & FR:2-4 & 0,6562 & & \\
\hline FA:4-2 & 0,3428 & FR:4-2 & 0,7441 & & \\
\hline FA:2-5 & 0,2072 & FR:2-5 & 1,5871 & & \\
\hline FA:5-2 & 0,1965 & FR:5-2 & 0,5607 & & \\
\hline FA:2-6 & 0,287 & FR:2-6 & 0,6847 & & \\
\hline FA:6-2 & 0,2726 & FR:6-2 & 0,6496 & & \\
\hline FA:3-4 & 0,8275 & FR:3-4 & 2,0206 & & \\
\hline FA:4-3 & 0,7995 & FR:4-3 & 0,6491 & & \\
\hline FA:4-6 & 0,3254 & FR:4-6 & 0,4338 & & \\
\hline FA:6-4 & 0,3192 & FR:6-4 & 0,406 & & \\
\hline FA:4-12 & 0,1847 & FR:4-12 & 0,3066 & & \\
\hline FA:12-4 & 0,1847 & FR:12-4 & 0,7456 & & \\
\hline FA:5-7 & 0,7785 & FR:5-7 & 0,3511 & & \\
\hline FA:7-5 & 0,7969 & FR:7-5 & 0,3364 & & \\
\hline FA:6-7 & 0,3813 & FR:6-7 & 0,9079 & & \\
\hline FA:7-6 & 0,3729 & FR:7-6 & 0,9653 & & \\
\hline FA:6-8 & 0,4376 & FR:6-8 & 0,5584 & & \\
\hline FA:8-6 & 0,434 & FR:8-6 & 0,5638 & & \\
\hline
\end{tabular}




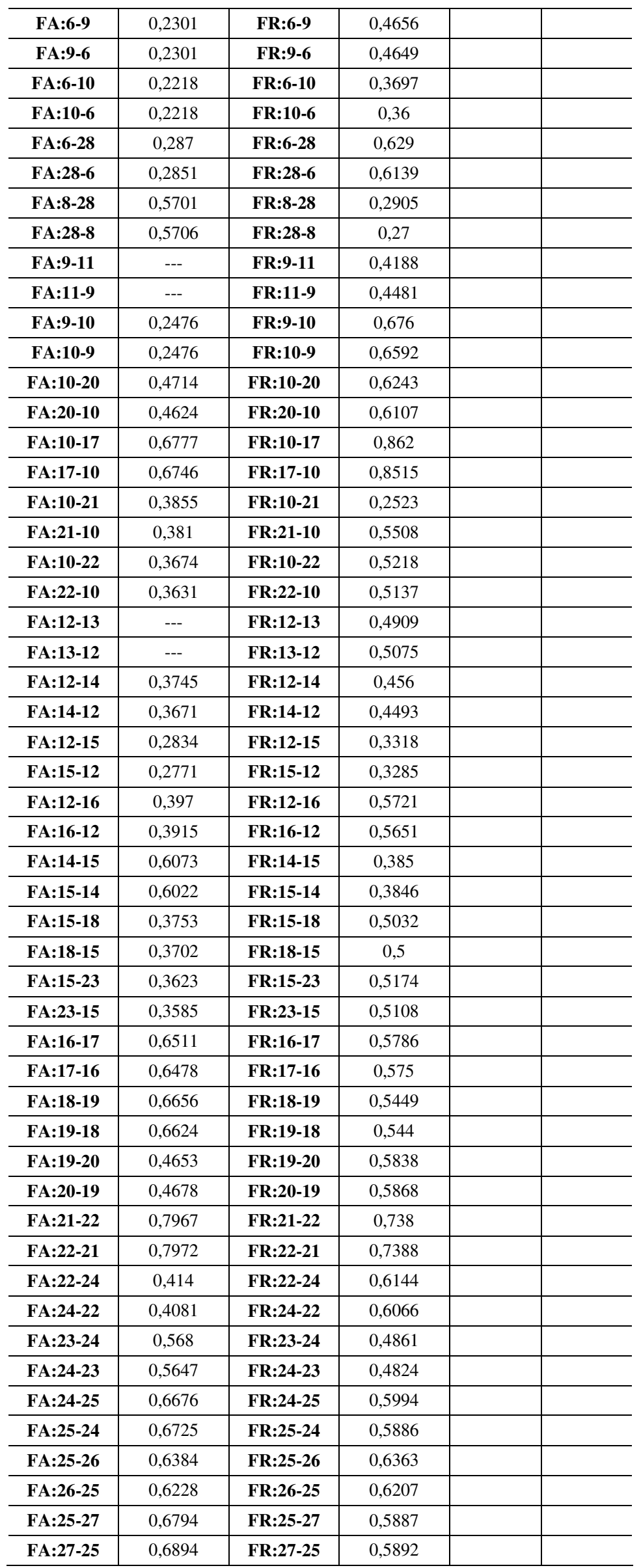




\begin{tabular}{l|c|c|c|l|l}
\hline FA:27-29 & 0,3271 & FR:27-29 & 0,4801 & & \\
\hline FA:29-27 & 0,3179 & FR:29-27 & 0,4734 & & \\
\hline FA:27-30 & 0,3216 & FR:27-30 & 0,376 & & \\
\hline FA:30-27 & 0,3065 & FR:30-27 & 0,3689 & & \\
\hline FA:28-27 & 0,2583 & FR:28-27 & 0,2782 & & \\
\hline FA:27-28 & 0,2583 & FR:27-28 & 0,6019 & & \\
\hline FA:29-30 & 0,6204 & FR:29-30 & 0,4124 & & \\
\hline FA:30-29 & 0,6054 & FR:30-29 & 0,4096 & & \\
\hline * Obs.: Os valores de $U I$ das medidas nulas não foram calculados.
\end{tabular}

* Obs.: Os valores de $U I$ das medidas nulas não foram calculados. 
Tabela B.4: Medidas do sistema de medição obtido pelo Algoritmo 2 e respectivos valores de $U I$ (sistema de 14 barras)

\begin{tabular}{|c|c|c|c|c|c|}
\hline \multicolumn{2}{|c|}{$\begin{array}{c}\text { Medidas de Potência } \\
\text { Ativa }\end{array}$} & \multicolumn{2}{|c|}{$\begin{array}{c}\text { Medidas de Potência } \\
\text { Reativa }\end{array}$} & \multicolumn{2}{|c|}{$\begin{array}{c}\text { Medidas } \\
\text { deTensão }\end{array}$} \\
\hline Medida & Índice $U I$ & Medida & Índice $U I$ & Medida & Índice $U I$ \\
\hline IA:1 & 0,395 & IR:1 & 0,8727 & V:1 & 0,1666 \\
\hline IA:3 & 0,4835 & IR:3 & 1,7276 & $V: 2$ & 0,1699 \\
\hline IA:7* & --- & IR:7 & --- & V:4 & 0,1722 \\
\hline IA:8 & --- & IR:8 & 0,4712 & V:5 & 0,1718 \\
\hline IA:9 & 1,5469 & IR:9 & 1,4409 & $V: 6$ & 0,1788 \\
\hline IA:10 & 0,6476 & IR:10 & 0,7425 & & \\
\hline IA:13 & 0,958 & IR:13 & 0,8739 & & \\
\hline FA:1-2 & 0,4848 & FR:1-2 & 0,513 & & \\
\hline FA:2-1 & 0,4692 & FR:2-1 & 0,7701 & & \\
\hline FA:1-5 & 0,2915 & FR:1-5 & 1,1586 & & \\
\hline FA:2-4 & 0,375 & FR:2-4 & 1,9125 & & \\
\hline FA:2-5 & 0,4801 & FR:2-5 & 1,8022 & & \\
\hline FA:5-2 & 0,4738 & FR:5-2 & 1,7931 & & \\
\hline FA:4-3 & 1,9962 & FR:4-3 & 0,841 & & \\
\hline FA:4-5 & 0,8824 & FR:4-5 & 0,5079 & & \\
\hline FA:5-4 & 0,8949 & FR:5-4 & 0,5102 & & \\
\hline FA:4-7 & 0,4204 & FR:4-7 & 0,9967 & & \\
\hline FA:5-6 & 0,7261 & FR:5-6 & 0,7954 & & \\
\hline FA:6-5 & 0,7261 & FR:6-5 & 1,5527 & & \\
\hline FA:6-13 & 0,628 & FR:6-13 & 0,6306 & & \\
\hline FA:13-6 & 0,6199 & FR:13-6 & 0,6679 & & \\
\hline FA:7-8 & --- & FR:7-8 & 0,4565 & & \\
\hline FA:8-7 & --- & FR:8-7 & 0,4712 & & \\
\hline FA:7-9 & 0,4203 & FR:7-9 & 0,79 & & \\
\hline FA:9-7 & 0,4203 & FR:9-7 & 0,979 & & \\
\hline FA:10-9 & 1,7789 & FR:10-9 & 1,3778 & & \\
\hline FA:10-11 & 0,9342 & FR:10-11 & 0,9708 & & \\
\hline FA:11-10 & 0,9442 & FR:11-10 & 0,9815 & & \\
\hline FA:12-13 & 1,0027 & FR:12-13 & 0,9848 & & \\
\hline FA:13-12 & 0,99 & FR:13-12 & 1,0061 & & \\
\hline FA:13-14 & 0,9565 & FR:13-14 & 0,9294 & & \\
\hline FA:14-13 & 0,9255 & FR:14-13 & 1,0282 & & \\
\hline
\end{tabular}


Tabela B.5: Medidas do sistema de medição obtido pelo Algoritmo 2 e respectivos valores de $U I$ (sistema de 30 barras)

\begin{tabular}{|c|c|c|c|c|c|}
\hline \multicolumn{2}{|c|}{$\begin{array}{c}\text { Medidas de Potência } \\
\text { Ativa }\end{array}$} & \multicolumn{2}{|c|}{$\begin{array}{c}\text { Medidas de Potência } \\
\text { Reativa }\end{array}$} & \multicolumn{2}{|c|}{$\begin{array}{c}\text { Medidas de } \\
\text { Tensão } \\
\end{array}$} \\
\hline Medida & Índice $U I$ & Medida & Índice $U I$ & Medida & $\begin{array}{c}\text { Índice } \\
U I\end{array}$ \\
\hline IA:1 & 0,4029 & IR:1 & 0,9311 & V:1 & 0,116 \\
\hline IA:5 & 0,3645 & IR:5 & 0,9454 & $V: 3$ & 0,1149 \\
\hline IA:6 * & --- & IR:6 & --- & V:5 & 0,116 \\
\hline IA:7 & 1,2107 & IR:7 & 0,4716 & V:7 & 0,1171 \\
\hline IA:8 & 0,7281 & IR:8 & 1,0499 & V:13 & 0,1165 \\
\hline IA:9 & --- & IR:9 & --- & $\mathrm{V}: 21$ & 0,1199 \\
\hline IA:11 & --- & IR:11 & 0,5284 & V:26 & 0,124 \\
\hline IA:13 & --- & IR:13 & 0,7162 & & \\
\hline IA:14 & 0,9537 & IR:14 & 1,4018 & & \\
\hline IA:17 & 1,1095 & IR:17 & 0,6936 & & \\
\hline IA:19 & 1,051 & IR:19 & 1,2244 & & \\
\hline IA:21 & 0,6675 & IR:21 & 0,5241 & & \\
\hline IA:22 & --- & IR:22 & --- & & \\
\hline IA:24 & 0,9358 & IR:24 & 0,793 & & \\
\hline IA:25 & --- & IR:25 & --- & & \\
\hline IA:26 & 0,8434 & IR:26 & 0,8381 & & \\
\hline IA:27 & --- & IR:27 & --- & & \\
\hline IA:28 & --- & IR:28 & --- & & \\
\hline FA:1-2 & 0,4926 & FR:1-2 & 0,3635 & & \\
\hline FA:2-1 & 0,4607 & FR:2-1 & 0,5782 & & \\
\hline FA:1-3 & 0,3404 & FR:1-3 & 1,3752 & & \\
\hline FA:3-1 & 0,3251 & FR:3-1 & 2,1755 & & \\
\hline FA:2-4 & 0,5792 & FR:2-4 & 0,8971 & & \\
\hline FA:2-5 & 0,2849 & FR:2-5 & 1,9054 & & \\
\hline FA:2-6 & 0,4467 & FR:2-6 & 0,8355 & & \\
\hline FA:3-4 & 0,8403 & FR:3-4 & 2,032 & & \\
\hline FA:4-3 & 0,8117 & FR:4-3 & 0,6699 & & \\
\hline FA:4-6 & 0,4743 & FR:4-6 & 0,5777 & & \\
\hline FA:4-12 & 0,3451 & FR:4-12 & 0,6696 & & \\
\hline FA:5-7 & 1,3783 & FR:5-7 & 0,429 & & \\
\hline FA:6-7 & 0,4178 & FR:6-7 & 0,9434 & & \\
\hline FA:7-6 & 0,4086 & FR:7-6 & 1,0417 & & \\
\hline FA:6-8 & 0,6009 & FR:6-8 & 0,7457 & & \\
\hline FA:6-9 & 0,3782 & FR:6-9 & 0,6578 & & \\
\hline FA:6-10 & 0,3715 & FR:6-10 & 0,5968 & & \\
\hline FA:6-28 & 0,4065 & FR:6-28 & 0,9426 & & \\
\hline FA:8-28 & 0,8517 & FR:8-28 & 0,3708 & & \\
\hline FA:9-11 & --- & FR:9-11 & 0,4911 & & \\
\hline FA:11-9 & --- & FR:11-9 & 0,5284 & & \\
\hline FA:9-10 & 0,3905 & FR:9-10 & 1,0168 & & \\
\hline FA:10-20 & 1,5213 & FR:10-20 & 1,751 & & \\
\hline FA:10-17 & 1,0472 & FR:10-17 & 1,8909 & & \\
\hline FA:10-21 & 0,5154 & FR:10-21 & 0,3761 & & \\
\hline FA:10-22 & 0,4869 & FR:10-22 & 0,8691 & & \\
\hline FA:12-13 & --- & FR:12-13 & 0,689 & & \\
\hline FA:13-12 & --- & FR:13-12 & 0,7162 & & \\
\hline
\end{tabular}




\begin{tabular}{l|c|c|c|l|l}
\hline FA:12-14 & 0,6334 & FR:12-14 & 0,6764 & & \\
\hline FA:12-15 & 0,757 & FR:12-15 & 0,9986 & & \\
\hline FA:12-16 & 1,5145 & FR:12-16 & 1,9083 & & \\
\hline FA:14-15 & 1,1871 & FR:14-15 & 0,6612 & & \\
\hline FA:15-18 & 1,3749 & FR:15-18 & 1,5972 & & \\
\hline FA:15-23 & 1,4948 & FR:15-23 & 2,0574 & & \\
\hline FA:16-17 & 2,0773 & FR:16-17 & 1,4774 & & \\
\hline FA:18-19 & 0,9253 & FR:18-19 & 0,7744 & & \\
\hline FA:19-18 & 0,9196 & FR:19-18 & 0,7726 & & \\
\hline FA:19-20 & 1,0307 & FR:19-20 & 1,1583 & & \\
\hline FA:21-22 & 1,4845 & FR:21-22 & 1,2628 & & \\
\hline FA:22-24 & 0,58 & FR:22-24 & 0,9458 & & \\
\hline FA:23-24 & 1,5292 & FR:23-24 & 1,0715 & & \\
\hline FA:24-25 & 1,0819 & FR:24-25 & 0,9423 & & \\
\hline FA:25-26 & 0,8696 & FR:25-26 & 0,8636 & & \\
\hline FA:25-27 & 1,187 & FR:25-27 & 0,9232 & & \\
\hline FA:27-29 & 0,665 & FR:27-29 & 0,9749 & & \\
\hline FA:27-30 & 0,444 & FR:27-30 & 0,654 & & \\
\hline FA:28-27 & 0,42 & FR:28-27 & 0,4386 & & \\
\hline FA:29-30 & 0,9682 & FR:29-30 & 0,8468 & & \\
\hline FA:30-29 & 0,9358 & FR:30-29 & 0,8398 & & \\
\hline
\end{tabular}

* Obs.: Os valores de $U I$ das medidas nulas não foram calculados. 


\section{Anexo C}

\section{Fluxograma do Algoritmo para Identificação do Nível de Detecção}

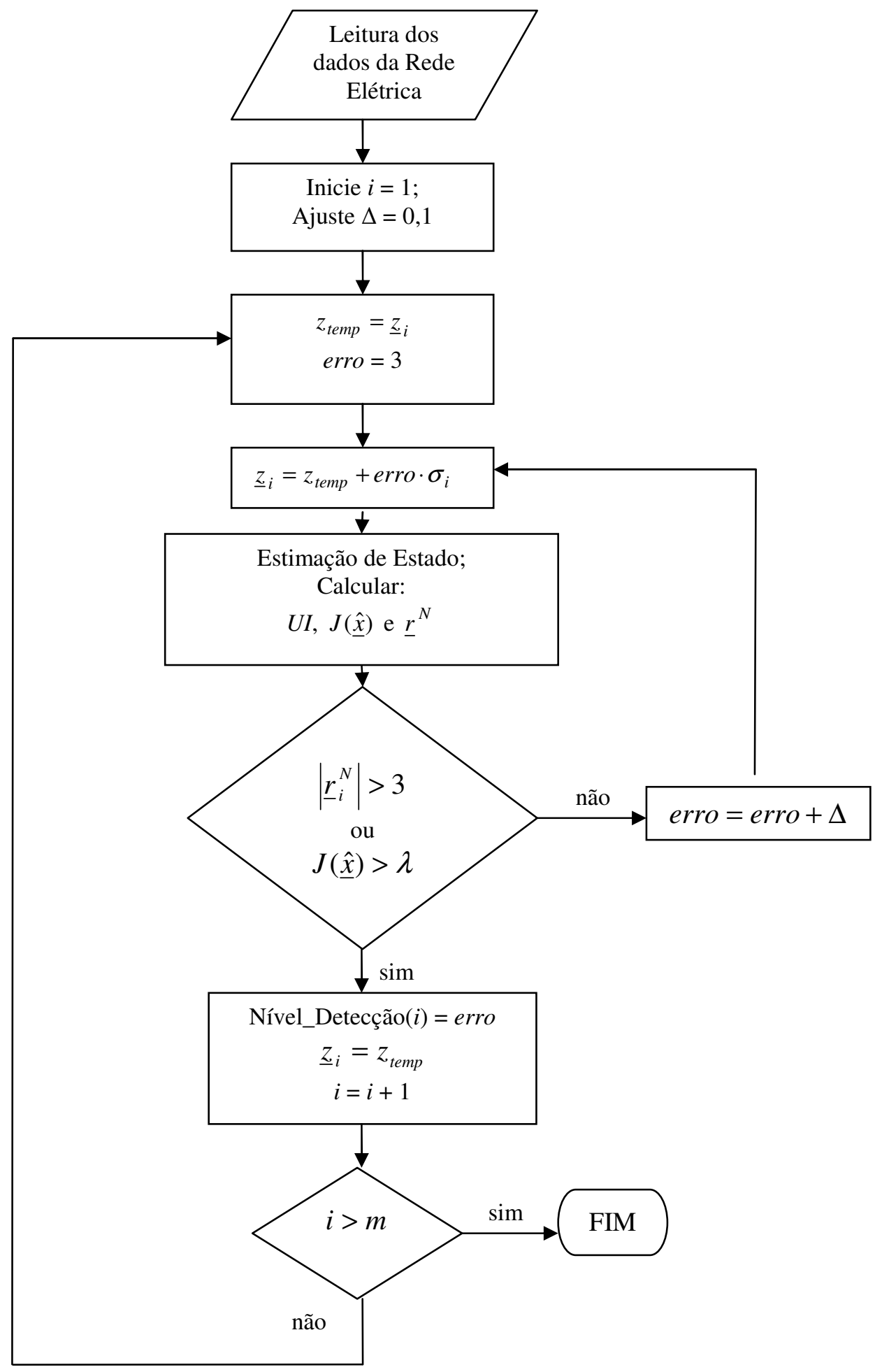

Figura C.1: Fluxograma do algoritmo para Identificação do Nível de Detecção 
Sendo:

$z_{\text {temp }}:$ a variável que acumula o valor da $i$-ésima medida sem erro $\left(z_{\text {temp }}=\underline{z}_{i}\right)$;

$\underline{z}_{i}$ : o $i$-ésimo elemento do vetor de medidas $\underline{z}$, que pode estar sem erro $\left(\underline{z}_{i}=\underline{z}_{i}^{l f}\right)$ ou com erro $\left(\underline{z}_{i}=z_{\text {temp }}+\right.$ erro $\left.\cdot \sigma_{i}\right)$.

Observação: Importa salientar que o valor de $\underline{z}_{i}^{l f}$ é aquele obtido através de estudo de fluxo de potência. 


\section{Anexo D}

\section{Projeto de Sistemas de Medição Confiáveis Através de Algoritmos Evolutivos e da Matriz $\boldsymbol{H}_{\Delta}^{\mathrm{t}}$}

Neste Anexo apresenta-se a metodologia para projeto e fortalecimento de sistemas de medição proposta no trabalho de Vigliassi et al. (2009), que se baseia em Algoritmos Evolutivos (AEs) e na análise da estrutura da matriz $H_{\Delta}{ }^{\mathrm{t}}$.

Esta metodologia utiliza a análise da estrutura da matriz $H_{\Delta}{ }^{\mathrm{t}}$ para obtenção de sistemas de medição confiáveis; e faz uso de um algoritmo evolutivo para encontrar o sistema de medição confiável ${ }^{8}$ de menor custo de investimento. Essa abordagem utiliza uma função de fitness que mede o custo da instalação de medidores a partir de um determinado sistema de medição confiável.

Uma vantagem relevante dessa metodologia é a sua estratégia para a obtenção de sistemas de medição confiáveis (SMCs). Uma codificação indireta do cromossomo representando uma ordem preferencial para instalação de medidores combinada com as propriedades da matriz $H_{\Delta}{ }^{\mathrm{t}}$ garante ao algoritmo evolutivo a geração somente de soluções factíveis, isto é, SMCs.

Nas próximas seções deste Anexo apresentar-se-á, de forma sucinta, a metodologia proposta em Vigliassi et al. (2009).

\section{D.1 Embasamento Teórico}

Nesta seção, será apresentado o método para projeto de SMCs, proposto por London Jr. et al. (2002), bem como conceitos importantes de técnicas de Computação

\footnotetext{
${ }^{8}$ No capítulo 7 desta tese, os sistemas de medição confiáveis foram definidos como sistemas observáveis e isentos de medidas críticas e conjuntos críticos de medidas. Já em Vigliassi et al. (2009) considerou-se também a ausência de Unidades Terminais Remotas (UTRs) críticas.
} 
Evolutiva. Vale destacar que o método proposto em Vigliassi et al. (2009) baseia-se na análise da matriz $H_{\Delta}{ }^{\mathrm{t}}$, conforme desenvolvido em London Jr. et al. (2002).

Assim como nos demais trabalhos, relacionados a projeto e fortalecimento de sistemas de medição, considera-se o processo de estimação de estado linear. Isto em razão de já ter sido demonstrado que tal processo pode ser adotado para análise de observabilidade e de redundância de medidas (MONTICELLI e WU, 1985).

\section{D.1.1 Método para fortalecimento e projeto de sistemas de medição via matriz $H_{\Delta}{ }^{t}$}

Considerando o modelo linear $P-\theta$ e um sistema observável, a matriz $H_{\Delta}{ }^{\mathrm{t}}$ apresenta a seguinte estrutura (LONDON Jr. et al., 2002):

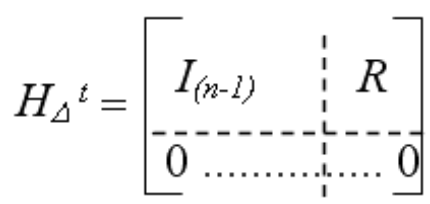

sendo:

$I$ - submatriz identidade, de dimensão $(n-1) \times(n-1)$;

$R$ - submatriz de dimensão $(n-1) \times[m-(n-1)]$;

$n$ - número de barras do sistema;

$m$ - número de medidas.

Observação 1: A última linha de $H_{\Delta}{ }^{\mathrm{t}}$ corresponde à barra escolhida como referência angular.

A matriz $H_{\Delta}{ }^{\mathrm{t}}$ é obtida através de um processo de fatoração triangular da matriz Jacobiana transposta $\left(H^{\mathrm{t}}\right)$. Esse processo de fatoração é realizado através da combinação das linhas da matriz $H^{\mathrm{t}}$, que correspondem às variáveis de estado do sistema. Logo, a matriz $H_{\Delta}{ }^{\mathrm{t}}$ relaciona as medidas com variáveis de estado equivalentes, que são combinações das variáveis de estado do sistema.

As medidas correspondentes às colunas da submatriz $I$ são chamadas de medidas Básicas, em razão de serem suficientes para tornar o sistema observável. As demais medidas são chamadas de medidas Suplementares. 
Os elementos não nulos, que aparecem em uma linha da matriz $H_{\Delta}{ }^{\mathrm{t}}$, indicam as medidas que fornecem informação do estado equivalente correspondente àquela linha. Logo, as " $p$ " medidas, correspondentes às colunas dos $p$ elementos não nulos, que aparecem em uma linha de $H_{\Delta}{ }^{\mathrm{t}}$, formam os chamados conjuntos $p$-críticos de medidas.

Em London Jr. et al. (2004), definiu-se um conjunto $p$-crítico de medidas como conjunto de " $p$ " medidas, associadas a um sistema elétrico observável, no qual a perda de todas essas " $p$ " medidas torna o sistema não observável. Além disso, a remoção de qualquer conjunto de $k$ medidas, pertencentes a um conjunto $p$-crítico, com $k<p$, não causa a perda da observabilidade do sistema. Através dessa definição, pode-se verificar que:

- Para $p=1$, o conjunto $p$-crítico é a medida crítica;

- Para $p=2$, par crítico de medidas;

- Para $p=3$, trio crítico de medidas; e assim por diante.

A análise direta da estrutura da matriz $H_{\Delta}{ }^{\mathrm{t}}$ possibilita a identificação dos conjuntos $p$-críticos que possuem apenas uma medida Básica. Para verificar como se realiza a identificação dos conjuntos $p$-críticos que possuem mais de uma medida Básica, vide London Jr. et al. (2007).

Através dos conjuntos $p$-críticos de medidas, identifica-se o nível de redundância (NR) de cada uma das medidas disponíveis. Neste contexto, uma medida tem NR igual a $(p-1)$, se o conjunto $p$-crítico, com menor número de medidas, a que ela pertencer, possuir $p$ medidas. Assim, por exemplo, a medida crítica tem NR igual a "0" e uma medida que aparece em pelo menos um par crítico tem NR igual a “1”, e assim por diante.

Em relação ao número de medidores e Unidades Terminais Remotas (UTRs) de um sistema de medição já existente, o método proposto por London Jr. et al. (2002) possibilita o seu fortalecimento de duas maneiras:

(i) Através da seleção e instalação de medidas candidatas: consiste na instalação de medidores novos, em usinas e subestações que já possuem UTRs e alguns medidores;

(ii) Através da seleção e instalação de UTRs candidatas: consiste na instalação de UTRs e de medidores, em usinas e subestações previamente desprovidas desses equipamentos.

Este método trabalha em três fases distintas, sendo: 
Fase 1: Análise e restauração da observabilidade: consiste em verificar se o sistema de potência é observável, considerando o sistema de medição já existente. Caso não o seja, o método permite determinar onde devem ser instalados medidores e/ou UTRs, para tornar o sistema de potência observável como um todo. Esse procedimento é realizado via fatoração triangular da matriz $H^{\mathrm{t}}$, que é formada a partir da análise de uma lista contendo todas as medidas instaladas e candidatas a serem instaladas em um sistema;

Fase 2: Análise e restauração da redundância de medidas: o objetivo desta fase é a obtenção de um sistema de medição formado apenas por medidas com NR maior que 1 , pois, considerando a definição de NR apresentada anteriormente, para tal sistema de medição permitisse-nos afirmar que não existem medidas críticas nem mesmo conjuntos críticos de medidas;

Fase 3: Identificando e aumentando a redundância das UTRs críticas: inicialmente verifica-se a existência de UTRs críticas, no sistema de medição resultante da execução das fases 1 e 2 . Caso não exista UTR crítica, esse sistema de medição é confiável e a análise se encerra. Caso contrário, o método permitirá determinar onde devem ser instalados medidores e/ou UTRs para tornar, as UTRs críticas, redundantes.

Observação 2: No capítulo 7 desta tese (sobre projeto de sistemas de medição) não levamos em conta a Fase 3 apresentada acima, ou seja, consideramos sistemas de medição confiáveis como sistemas de medição sem medidas críticas e conjuntos críticos de medidas, sem nos preocuparmos com UTRs críticas.

\section{D.1.2 Computação Evolutiva (CE)}

$\mathrm{Na}$ natureza existem diversas soluções funcionais para um grande número de problemas. Estas soluções estão presentes nas mais diversas espécies de seres vivos e são facilmente observadas, como, por exemplo, as abelhas que se organizam para construir uma colméia, facilitando a sobrevivência do enxame.

Técnicas tradicionais de computação não são eficientes para resolver um grande número de problemas, como por exemplo, problemas de otimização. $\mathrm{O}$ homem pode ser considerado um dos mais poderosos processos de otimização natural. Baseado em sua evolução foram desenvolvidos algoritmos auto-adaptativos capazes de resolver um 
grande número de problemas práticos. Algoritmos inspirados na $\mathrm{CE}$ apresentam uma sequiência de procedimentos gerais que podem ser adaptados a cada problema. Os dois aspectos destacados a seguir são fundamentais para o desempenho de uma abordagem evolutiva:

Codificação dos indivíduos em representação genotípica, em que cada indivíduo representa um candidato à solução de um dado problema (cromossomo);

Uma função de aptidão que indica o valor de adequação de cada indivíduo, mostrando o quão próximo está este valor da solução procurada, dentro do contexto de cada problema (fitness).

Gerando-se várias soluções possíveis temos um conjunto de cromossomos, chamado de população. A otimização ocorre nessas populações em etapas que chamamos de gerações. Uma geração é um conjunto de cromossomos que serão avaliados segundo um critério de seleção. No processo de avaliação, cada indivíduo recebe um score (aptidão), que será utilizado na seleção dos indivíduos melhores adaptados. Existem muitos métodos para a seleção, dentre os quais os mais utilizados são o método da Roleta e o Elitismo. No método da Roleta, os indivíduos com maior aptidão têm maior probabilidade de serem escolhidos, enquanto no Elitismo, os indivíduos com maior aptidão serão sempre escolhidos. Após a seleção dos mais adaptados é produzida uma nova geração, excluindo os indivíduos menos adaptados e criando novos indivíduos (filhos). Na criação são usados basicamente dois operadores:

1. Crossover: recombinação de características dos pais. Na figura D.1 temos um exemplo simples de crossover. Um pai dando origem a um filho através de uma divisão e inversão de posições.

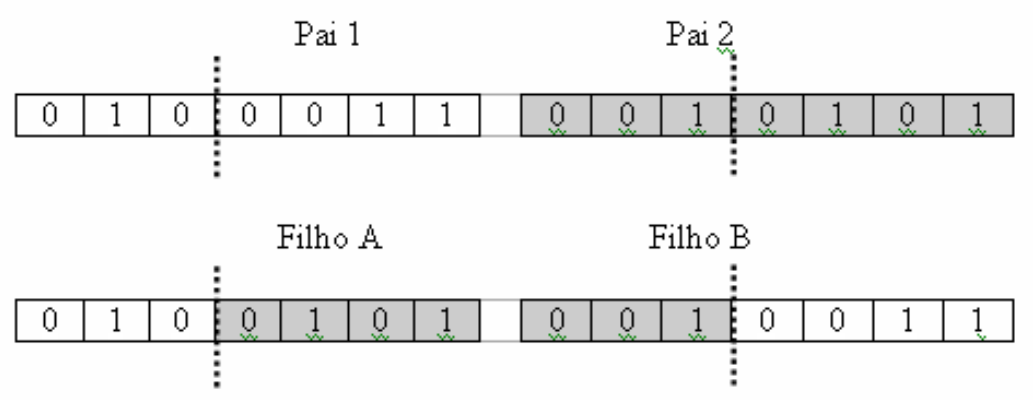

Figura D.1: Exemplo de crossover.

Existem várias maneiras diferentes para gerar filhos a partir de crossover (crossover de um ponto, dois pontos, multiponto e uniforme). 
2. Mutação: introduz diversidade genética na população. Ela altera aleatoriamente um ou mais genes de um cromossomo. Essa diversidade faz com que o $\mathrm{AE}$ tenha um espaço de busca maior, normalmente não explorado somente através de crossover. Se não a utilizarmos ficamos sujeitos a uma solução que pode não ser a ideal, é o que chamamos de ótimo local, ou seja, uma população que evoluiu em um pequeno campo de busca, não atingindo o ótimo global. A figura D.2 mostra uma evolução que atinge o Ótimo Global.

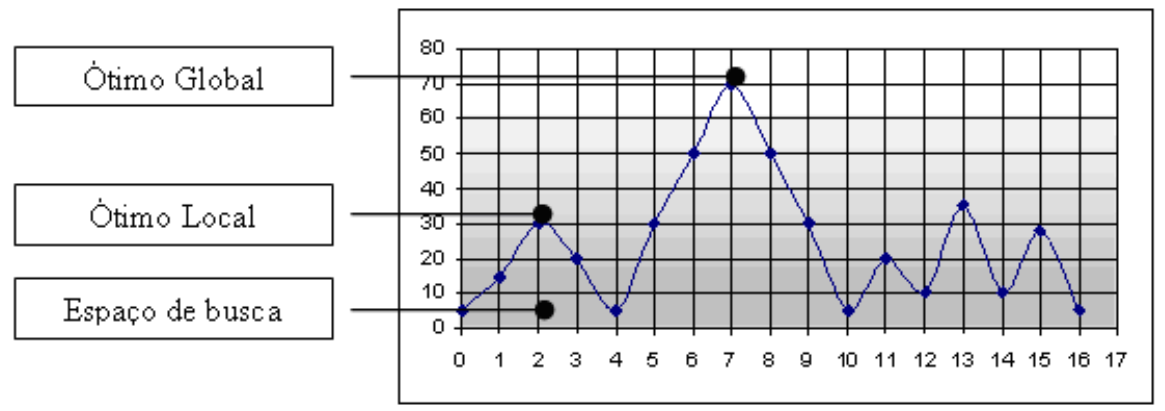

Figura D.2: Ótimo local, global e espaço de busca.

Note que a mutação deve ser suficiente para alternar entre os espaços de busca locais.

\section{D.2 Metodologia Proposta em Vigliassi et al. (2009)}

A metodologia proposta em Vigliassi et al. (2009) pode ser utilizada tanto para o projeto de um sistema de medição novo e confiável, quanto para análise e fortalecimento de um sistema de medição já existente. Entretanto, será mostrado como a metodologia possibilita a realização da primeira tarefa.

A metodologia em questão baseia-se no método desenvolvido por London Jr. et al. (2002). Conforme apresentado na seção anterior, tal método possibilita a obtenção de um sistema de medição confiável, a partir da análise de uma lista contendo todos os medidores candidatos a serem instalados em um sistema, com a indicação da correspondente UTR candidata. Analisando essa lista, o método seleciona os medidores e UTRs que devem ser instalados, para obtenção de um sistema de medição confiável. Isso é realizado através da obtenção e análise da matriz $H_{\Delta}{ }^{\mathrm{t}}$. Dependendo da seqüência com que os medidores candidatos estão dispostos na lista, o método fornece confiáveis sistemas de medição selecionando, para instalação, números distintos de medidores e 
UTRs. Em razão de o custo de um sistema de medição depender do número de medidores e UTRs instalados, a idéia dessa metodologia é a seguinte:

Passo 1: Através de AE geram-se diversas listas (indivíduos) contendo todos os medidores candidatos, sendo que, em cada uma das listas, esses medidores aparecem em uma ordem distinta;

Passo 2: Cada uma das listas (indivíduos), geradas no passo anterior, é analisada pelo método proposto por London Jr. et al. (2002), que fornece, como resultado, o número de medidores e UTRs que devem ser instalados para obtenção de um sistema de medição confiável. De acordo com esse número de medidores e UTRs calcula-se o custo do sistema de medição obtido (função de aptidão), que será o parâmetro a ser analisado para definir a solução "ótima” do problema, isto é, para definir qual das listas (indivíduo) possibilitou a obtenção do sistema de medição confiável mais barato.

Face ao exposto verifica-se que na metodologia proposta em Vigliassi et al. (2009), o AE vai possibilitar a obtenção de um sistema de medição de baixo custo, "otimizando" a sequiência com que as medidas candidatas serão analisadas pelo método proposto por London Jr. et al. (2002).

\section{D.2.1 Representação do Problema}

A representação do $\mathrm{AE}$ utilizada na metodologia em questão é:

- Cromossomo: cada indivíduo é um array, cujas posições correspondem a cada um dos medidores possíveis de serem instalados no sistema. A diferença entre os indivíduos é a seqüência com que os medidores estão ordenados no array. O tamanho de cada um dos indivíduos é igual ao número total de medidores possíveis de serem instalados no sistema $\left(m_{\text {total }}\right)$.

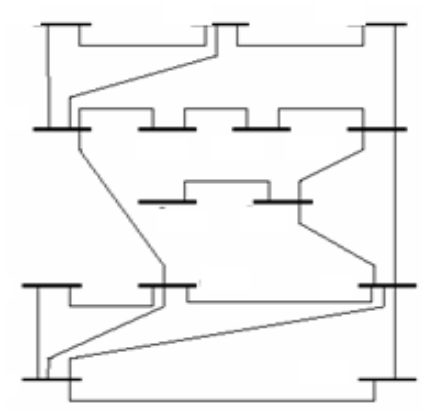

Cromossomo:| Medidor 1| |Medidor 14| (...) |Medidor $m_{\text {total }} \mid$

Figura D.3: Sistema de 14 barras IEEE e seu cromossomo. 
Tomando como exemplo o sistema IEEE de 14 barras, ilustrado na figura D.3, o cromossomo seria representado por um array de 52 posições, onde 38 seriam medidores de fluxo (dois em cada linha) e 14 de injeção (um em cada barra).

- População: a população é de tamanho fixo e não se altera durante a execução do AE.

- $\quad$ Função de fitness $(f)$ : o correto funcionamento do AE depende principalmente da determinação dessa função. Neste trabalho ela é definida da seguinte forma:

$$
f=c m * \text { numMed }+ \text { cutr } * \text { numUtr }
$$

onde:

$\mathrm{cm}=$ custo do medidor;

numMed $=$ número de medidores instalados;

cutr $=$ custo da UTR;

numUtr = número de UTRs instaladas;

Diferente do que é feito nos métodos propostos para projeto e atualização de sistemas de medição, baseados na aplicação de sistemas inteligentes, apresentados em (SOUZA et al., 2005; COSER et al., 2006, VIGLIASSI et al., 2007), na metodologia proposta em Vigliassi et al. (2009) não são atribuídas penalidades para não observância dos requisitos de confiabilidade de um sistema de medição (observabilidade e ausência de MC, CCM e UTR crítica). Isto em razão de todos os indivíduos possibilitarem o atendimento de todos aqueles quesitos.

Os custos de medidores e UTRs utilizados os mesmos considerados em (COSER et al., 2006) isto é, 4,50 e 100,00 unidades monetárias (UM) para medidores e UTRs, respectivamente.

- Seleção: os indivíduos são selecionados através do Elitismo. Dessa maneira, com certeza, os melhores indivíduos de cada geração sobrevivem.

- Operadores genéticos: para a reprodução dos indivíduos foi utilizado o crossover com um ponto (metade do cromossomo). Esse será o ponto de separação utilizado pelo crossover para gerar os novos indivíduos, segundo a seqüência abaixo:

\begin{tabular}{l|l|l|} 
Pai A & 1 & 2 \\
Pai B & 3 & 4 \\
Filho A & 1 & 4 \\
Filho B & 3 & 2 \\
\hline
\end{tabular}


Os melhores resultados foram encontrados realizando o crossover entre o melhor indivíduo da população e todos os demais. Para obter mais detalhes, bem como exemplos de aplicação da metodologia apresentada neste anexo, veja a referência Vigliassi et al. (2009). 


\section{Anexo E}

\section{E Publicações do Autor Durante o Período de Doutoramento}

\section{E. 1 Publicações oriundas desta Tese}

BRETAS, N.B.; LONDON JR.; J.B.A.; ALBERTO, L.F.C.; BENEDITO, R.A.S. (2009). Geometrical Approach on Masked Gross Errors for Power Systems State Estimation. In Proceedings of 2009 IEEE Power and Energy Society General Meeting, 26-30 July, p. 1-7. Calgary, AB, Canada. (DOI: 10.1109/PES.2009.5275941).

BRETAS, N.B.; LONDON JR.; J.B.A.; ALBERTO, L.F.C.; BENEDITO, R.A.S. (2009). Geometrical Approaches for Gross Errors Analysis in Power Systems State Estimation. In Proceedings of 2009 IEEE Bucharest Power Tech Conference, June 28th - July 2nd, p. 1-7. Bucharest, Romania. (DOI: 10.1109/PTC.2009.5281845).

LONDON Jr., J.B.A.; BENEDITO, R.A.S.; PIERETI, S.A.R.; DELBEM A.C.B.; ALBERTO, L.F.C.; BRETAS, N.G. (2008). Estimação de Estado em Sistemas Elétricos de Potência: Medidas que Escondem Erro e Configurador de Redes. Em: X EDAO Encontro para Debates de Assuntos de Operação, São Paulo.

\section{E. 2 Publicações sobre Estimação de Estado em Sistemas Elétricos de Potência}

BENEDITO, R.A.S; LONDON Jr., J.B.A.; BRETAS, N.G. (2009). Análise de Observabilidade em Sistemas Elétricos de Potência: Identificação de Ilhas Observáveis através de Caminhos de Fatoração Associados à Matriz Jacobiana. Anais do XI $\underline{\text { SEPOPE - Simpósio de Especialistas em Planejamento da Operação e Expansão }}$ Elétrica. Belém, Março. 
BENEDITO, R.A.S; LONDON Jr., J.B.A.; BRETAS, N.G. (2009). A Unified Algorithm for Observability and Redundancy Analysis. Proceedings of the 2009 IEEE Bucharest Power Tech Conference, p.1-7, Romênia.

BENEDITO, R.A.S.; MOREIRA, E.M.; LONDON Jr., J.B.A.; BRETAS, N.G. (2008). Observability Analysis Based on Path Graph Concepts and Triangular Factorization of the Jacobian Matrix. Proceedings of the IEEE PES Transmission and Distribution Conference and Exposition: Latin America, p. 456-462, Bogotá.

LONDON Jr., J.B.A.; PIERETI, S.A.R.; BENEDITO, R.A.S.; BRETAS, N.G. (2009). Redundancy and Observability Analysis of Conventional and PMU Measurements. IEEE Transactions on Power Systems, Vol. 24, No. 3, p. 1629-1630, Agosto. (DOI: 10.1109/TPWRS.2009.2021195).

TECCHIO, P.V.; BENEDITO, R.A.S.; ALBERTO, L.F.C. (2010). The Behavior of WLS State Estimator Near the Maximum Loadability Point of Power Systems. Proceedings of the IEEE PES General Meeting 2010, Minneapolis-USA, 25 a 29 de Julho.

BORGES, G.; BENEDITO, R.A.S.; LONDON Jr., J.B.A.; BRETAS, N.G. (2010). Estimação de Estado em Sistemas Elétricos de Potência: Metodologia para Análise de Observabilidade Considerando Medidas Convencionais e Fasoriais. Anais do XVIII CBA - Congresso Brasileiro de Automática, p. 4560-4566, Bonito-MS, 12 a 16 de Setembro.

THEODORO, E.; BENEDITO, R.A.S; ALBERTO, L.F.C. (2010). Método Rápido para Identificação de Ilhamento em Sistemas Elétricos de Potência. Anais do XVIII CBA Congresso Brasileiro de Automática, p.2405-2412, Bonito-MS, 12 a 16 de Setembro. 
